\title{
A Review of the Graphite Irradiation Creep Data from the "OC-Series" of Experiments
}

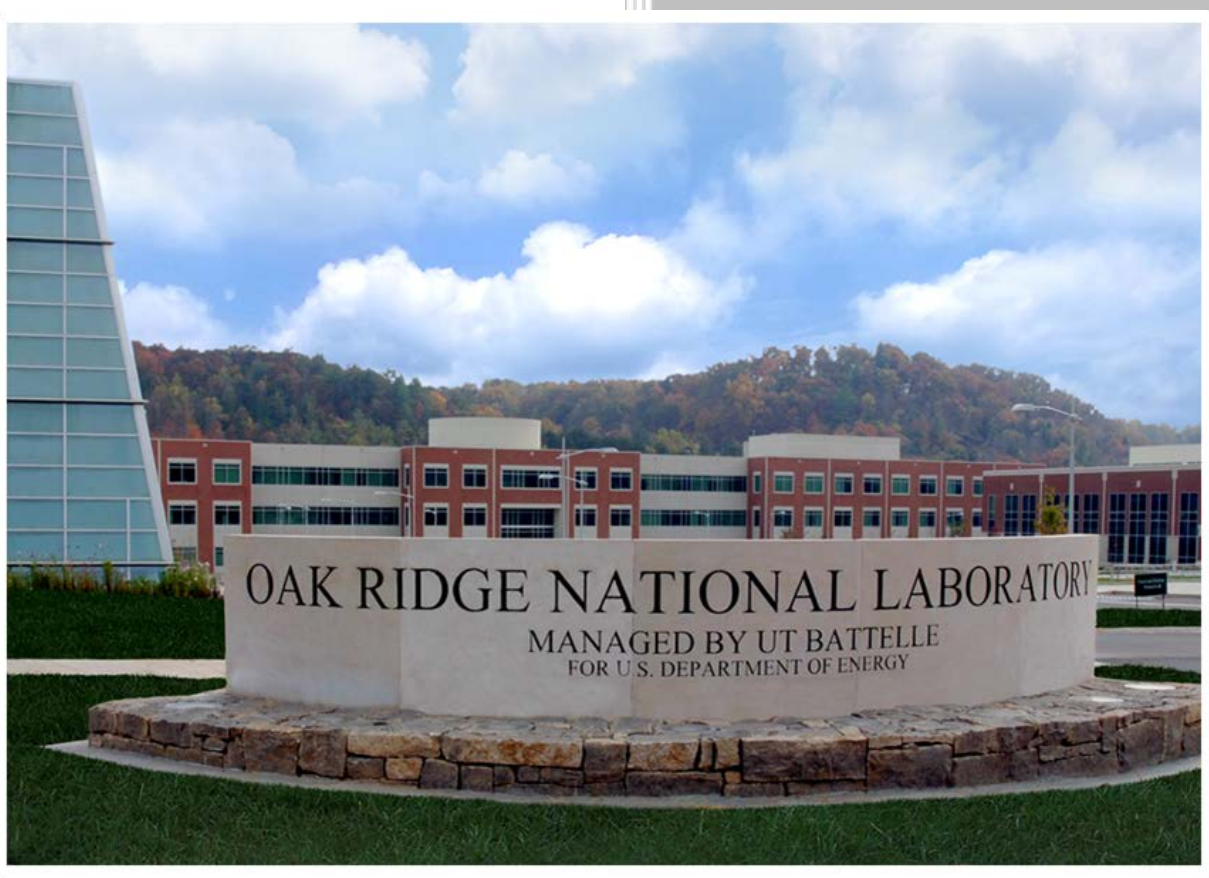

Approved for public release. Distribution is unlimited.
Mark A. Davies

Marad Ltd.

and

Timothy D. Burchell

Oak Ridge National Laboratory

December 2012 


\title{
DOCUMENT AVAILABILITY
}

Reports produced after January 1, 1996, are generally available free via US Department of Energy (DOE) SciTech Connect.

\section{Website http://www.osti.gov/scitech/}

Reports produced before January 1, 1996, may be purchased by members of the public from the following source:

\author{
National Technical Information Service \\ 5285 Port Royal Road \\ Springfield, VA 22161 \\ Telephone 703-605-6000 (1-800-553-6847) \\ TDD 703-487-4639 \\ Fax 703-605-6900 \\ E-mail info@ntis.gov \\ Website http://www.ntis.gov/help/ordermethods.aspx
}

Reports are available to DOE employees, DOE contractors, Energy Technology Data Exchange representatives, and International Nuclear Information System representatives from the following source:

Office of Scientific and Technical Information

PO Box 62

Oak Ridge, TN 37831

Telephone 865-576-8401

Fax 865-576-5728

E-mail reports@osti.gov

Website http://www.osti.gov/contact.html

This report was prepared as an account of work sponsored by an agency of the United States Government. Neither the United States Government nor any agency thereof, nor any of their employees, makes any warranty, express or implied, or assumes any legal liability or responsibility for the accuracy, completeness, or usefulness of any information, apparatus, product, or process disclosed, or represents that its use would not infringe privately owned rights. Reference herein to any specific commercial product, process, or service by trade name, trademark, manufacturer, or otherwise, does not necessarily constitute or imply its endorsement, recommendation, or favoring by the United States Government or any agency thereof. The views and opinions of authors expressed herein do not necessarily state or reflect those of the United States Government or any agency thereof. 


\title{
A REVIEW OF THE GRAPHITE IRRADIATION CREEP DATA FROM THE “OC-SERIES” OF EXPERIMENTS
}

\author{
Mark A. Davies \\ MARAD Co. Ltd. \\ and \\ Timothy D. Burchell \\ Oak Ridge National Laboratory
}

December, 2012

\author{
Prepared by \\ OAK RIDGE NATIONAL LABORATORY \\ Oak Ridge, TN 3782-31-6283 \\ managed by \\ UT-BATTELLE \\ for the \\ USDEPARTMENT OF ENERGY \\ under contract DE-AC05-00OR22725
}




\section{Contents}

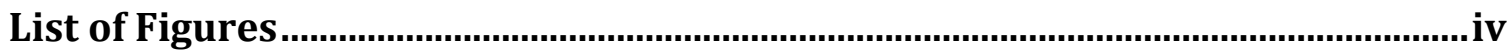

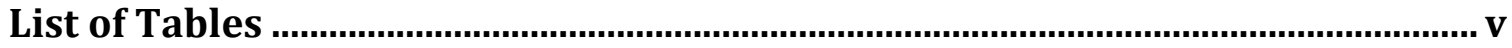

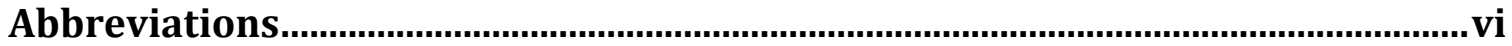

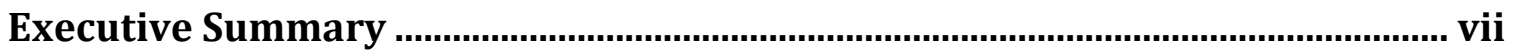

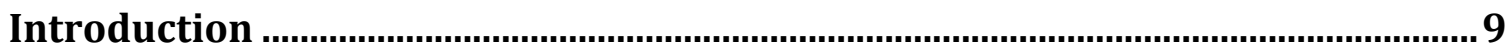

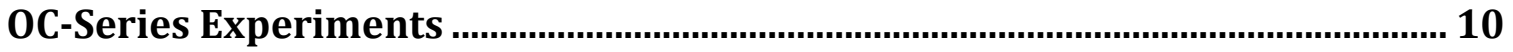

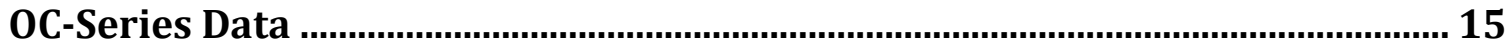

Analysis of Available Data ................................................................................. 24

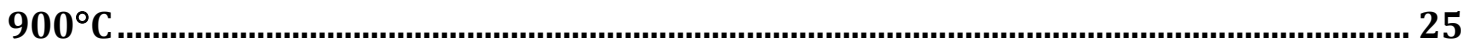

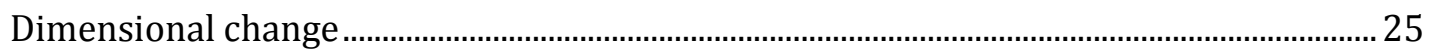

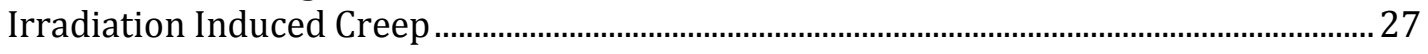

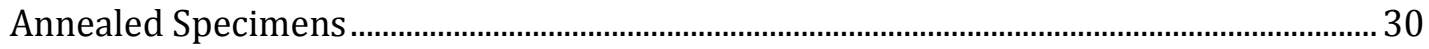

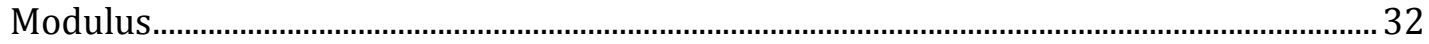

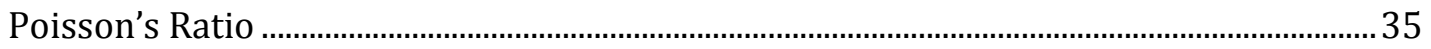

Coefficient of Thermal Expansion ............................................................................................36

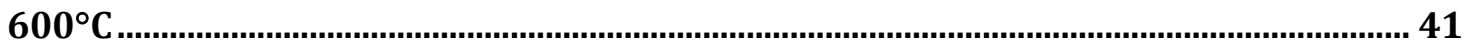

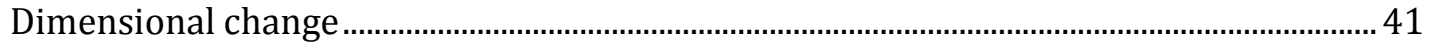

Irradiation Induced Creep ................................................................................................................. 43

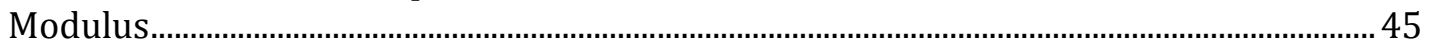

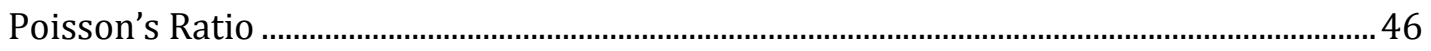

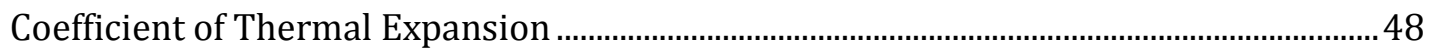

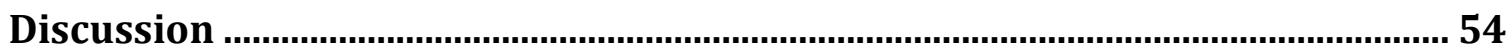

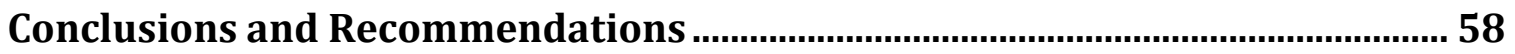

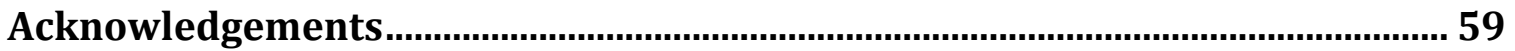

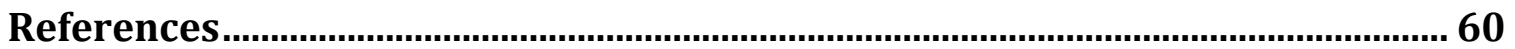

Appendix A $900^{\circ} \mathrm{C}$ Specimen Characterisation Archive Data.................................61

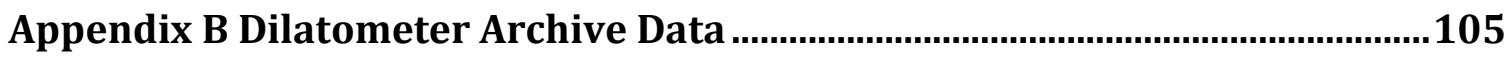

OC3 Dilatometer Data ............................................................................................105

OC2 Dilatometer Data ............................................................................................130

OC4 Dilatometer Data ...............................................................................................156

Appendix C Creep Corrections ........................................................................172

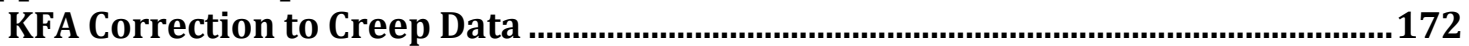

UK correction to creep data ..........................................................................................174

Discussion ...............................................................................................................175

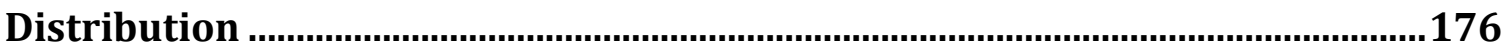


ORNL/TM-2009/176

\section{List of Figures}

Figure 1: Stressed Column Cross Section

Figure 2: Unstressed Column Cross Section

Figure 3: $900^{\circ} \mathrm{C}$ Direct Dimensional Change Data

Figure 4: $900^{\circ} \mathrm{C}$ Lateral Dimensional Change Data

Figure 5: $900^{\circ} \mathrm{C}$ Volume Change Data

Figure 6: $900^{\circ} \mathrm{C}$ Direct Creep Data

Figure 7: $900^{\circ} \mathrm{C}$ Lateral Creep Data

Figure 8: $900^{\circ} \mathrm{C}$ Normalized Direct Creep Data

Figure 9: Direct Recovery of Annealed Specimens from OC-3

Figure 10: Lateral Recovery of Annealed Specimens from OC-3

Figure 11: Normalized Direct Recovery of Annealed Specimens from OC-3

Figure 12: Young's and Shear Modulii at $900^{\circ} \mathrm{C}$

Figure 13: Young's and Shear Modulii at $900^{\circ} \mathrm{C}$ with specimen history

Figure 14: Fractional change of Modulii at $900^{\circ} \mathrm{C}$ with specimen history

Figure 15: Effect of Creep strain on Poisson's ratio at $900^{\circ} \mathrm{C}$ (Kennedy)

Figure 16: Effect of Creep strain on Poisson's ratio at $900^{\circ} \mathrm{C}$

Figure 17: Effect of Creep Strain on the Mean Coefficient of thermal Expansion $(\alpha=\mathrm{A}+\mathrm{BT})$ (Kennedy)

Figure 18: Pre-Irradiated Dilatometer Data from OC-1 and OC-3

Figure 19: Post-Irradiated Dilatometer Data from OC-1

Figure 20: Effect of External Load on CTE (800)

Figure 21: $600^{\circ} \mathrm{C}$ Direct dimensional change data

Figure 22: $600^{\circ} \mathrm{C}$ Lateral Dimensional Change Data

Figure 23: $600^{\circ} \mathrm{C}$ Volume Change Data

Figure 24: $600^{\circ} \mathrm{C}$ Direct Creep Data

Figure 25: $600^{\circ} \mathrm{C}$ Lateral Creep Data

Figure 26: $600^{\circ} \mathrm{C}$ Normalized Direct Creep Data

Figure 27: Young's Modulus at $600^{\circ} \mathrm{C}$

Figure 28: Shear Modulus at $600^{\circ} \mathrm{C}$

Figure 29: Effect of Creep strain on Poisson's ratio at $600^{\circ} \mathrm{C}$ (Kennedy)

Figure 30: Effect of external load on Poisson's ratio at $600^{\circ} \mathrm{C}$

Figure 31: OC2 \& OC4 - Effect of Creep Strain on the Mean Coefficient of thermal Expansion $(\alpha=\mathrm{A}+\mathrm{BT})$ (Kennedy)

Figure 32: Post-Irradiated Dilatometer Data from OC-2

Figure 33: Post-Irradiated Dilatometer Data from OC-4

Figure 34: Effect of External Load on CTE (500) 


\section{List of Tables}

Table 1: OC-Series Experimental Schedule

Table 2: Graphite Materials for the OC-1 Capsule

Table 3: $900^{\circ} \mathrm{C}$ Experiment - Specimen Irradiation Plan

Table 4: $900^{\circ} \mathrm{C}$ Experiments - Mobasheran Data

Table 5: $600^{\circ} \mathrm{C}$ Experiments - Mobasheran Data

Table 6: Important Documents recovered from ORNL OC-Series Archive

Table 7: $900^{\circ} \mathrm{C}$ Experiments - ORNL Archive Data

Table 8: $600^{\circ} \mathrm{C}$ Experiments - ORNL Archive Data 


\section{Abbreviations}

esu elastic strain unit

CTE Coefficient of Thermal Expansion

FRG Federal Republic of Germany

OC ORR-Creep

ORNL Oak Ridge National Laboratory

ORR Oak Ridge Research Reactor

UK United Kingdom

US United States 


\section{Executive Summary}

The original OC-Series experiments planned to irradiate 3 sets of 28 stressed specimens along with their reference specimens to incremental fluences of $1,2,4$ and $8\left(10^{25} \mathrm{n} / \mathrm{m}^{2}\right.$ $\mathrm{E}>0.18 \mathrm{MeV}$ ) at $600^{\circ} \mathrm{C}, 900^{\circ} \mathrm{C}$ and $1250^{\circ} \mathrm{C}$. Due to funding constraints the OC-Series was cut short and 2 sets of 28 stressed specimens were irradiated at $900^{\circ} \mathrm{C}$ and $600^{\circ} \mathrm{C}$ respectively.

Some of the data from these experiments were reported in extended abstracts at a Carbon Conference (Kennedy et al, 1977: Kennedy and Eatherly, 1979). Moreover, the OC series creep data was reviewewd and succesfully modelled (with modification to creep strain for dimensional changes) by Kelly and Burchell (1994), and Burchell (2008). The data presented some challenges to the accepted approach to irradiation induced creep in graphite adopted in the UK, specifically lateral creep strain behaviour and the effect of irradiation induced creep strain on material properties, e.g. Coefficient of Thermal Expansion (CTE) and Poisson’s Ratio.

A recent review of irradiation induced creep (Davies \& Bradford, 2004) included an anlaysis of the available OC-series data (Mobasheran, 1985) and led to a request to ORNL for an examination of the original OC-Series dataset. An initial search of the ORNL archive revealed additional data from the OC-Series experiment including previously unknown irradiation annealing experiments.

The OC-Series archive at ORNL was interrogated and a number of important datafiles were recovered. This project re-analyzed some of the available data from the OC-Series archive and some important findings were made.

1. Previously unknown irradiation annealing data from the OC-Series archive indicates that there is substantial recovery of dimensional change well in excess of values normally attributed to primary creep. Some over recovery in dimensional change was observed and it is recommended that this is investigated further.

2. The $900^{\circ} \mathrm{C}$ Modulus data revealed some unexpected stressed specimen history behavior when compared to reference history behavior. It was not possible to examine the $600^{\circ} \mathrm{C}$ in the same way as there were only two irradiation capsules spanning the fluences where the changes at $900^{\circ} \mathrm{C}$ become apparent. It is recommended that efforts are made to find or evaluate the unirradiated $600^{\circ} \mathrm{C}$ Modulus data.

3. When the CTE were re-evaluated from dilatometer expansion data a systematic offset was found in the CTE data. This was subsequently attributed to a use of a quartz reference standard for expansion correction.

4. Whilst subject to significant scatter, the CTE data appear to have saturated at $600^{\circ} \mathrm{C}$ but not necessarily at $900^{\circ} \mathrm{C}$. It is recommended that the CTE data are investigated further.

5. The CTE data appear to be well represented by the UK theory. 
6. Previously quoted (Kennedy et al, 1977: Kennedy and Eatherly, 1979: Brocklehurst and Kelly, 1989) Poisson's ratio and CTE dependence on creep have been demonstrated to be ill founded and as a result should not be considered further.

7. During the archive search substantial data were found on other experiments such as the ORR series and Petten (US/FRG exchange - H-451 \& ATR-2E). It is recommended that these are examined in more detail. 
ORNL/TM-2009/176

\section{Introduction}

The OC-Series graphite irradiation creep experiments were conducted in the early 1970s in the Oak Ridge Research Reactor (ORR) at ORNL. The OC Series consisted of 5 experiments, Capsules 1, 3 and 5 were irradiated at $900^{\circ} \mathrm{C}$ and Capsules 2 and 4 were irradiated at $600^{\circ} \mathrm{C}$. Each capsule contained four columns of specimens, two loaded in compression and two un-loaded. The loaded columns had specimens of different diameter to generate two stress levels, 13.8 MPa and 20.7 MPa.

Some of the data from these experiments were presented in extended abstracts at a Carbon Conference (Kennedy et al, 1977: Kennedy and Eatherly, 1979). The data presented some challenges to the accepted approach to irradiation induced creep in graphite adopted in the UK, specifically lateral creep strain behaviour and the effect of irradiation induced creep strain on material properties, e.g. CTE and Poisson's Ratio.

A recent review of irradiation induced creep (Davies \& Bradford, 2004) included an anlaysis of the available OC-series data (Mobasheran, 1990) and led to a request to ORNL for an examination of the original OC-Series dataset. An initial search of the ORNL archive revealed additional data from the OC-Series experiment including previously unknown irradiation annealing experiments.

This report presents a re-analysis of the available data from the OC-Series archive. 
ORNL/TM-2009/176

\section{OC-Series Experiments}

A series of 12 irradiation experiments were planned to evaluate irradiation induced creep characteristics of a number of graphites when exposed to elevated temperatures and high fast fluences (Senn et al, 1977).

Three graphites considered of interest to past and present reactor designers were chosen. A small number of specimens were machined from $\mathrm{H}-327$, which was used as the material for the core structures in Fort St Vrain. A small number of AXF-8QBG1 specimens were included to extend the existing creep database to high fluence. The majority of the test specimens are $\mathrm{H}-451$, a near isotropic medium grain graphite and one of the principal contenders for the proposed 1000 MW(e) HTGR application at that time and core fuel and moderator block replacement graphite in the Fort St Vrain HTGR.

The original series planned to irradiate 3 sets of 28 stressed specimens, $15.24 \mathrm{~mm}$ diameter by $25.4 \mathrm{~mm}$ long to incremental fluences of $1,2,4$ and $810^{25} \mathrm{n} / \mathrm{m}^{2}(\mathrm{E}>0.18$ $\mathrm{MeV}$ ) at $900^{\circ} \mathrm{C}, 600^{\circ} \mathrm{C}$ and $1250^{\circ} \mathrm{C}$. The original schedule of experiments is shown in Table 1.

\begin{tabular}{|l|c|c|c|c|}
\hline & \multicolumn{4}{|c|}{ Fluence $10^{25} \mathrm{n} / \mathrm{m}^{2}(\mathrm{E}>0.18 \mathrm{MeV})$} \\
\hline Temperature & 1.2 & 2.4 & 4.8 & 9.6 \\
\hline & & & & OC-8 \\
\hline $900^{\circ} \mathrm{C}$ & OC-1 & OC-4 & OC-6 & OC-10 \\
\hline $600^{\circ} \mathrm{C}$ & OC-2 & OC-5 & OC-7 & OC-12 \\
\hline $1250^{\circ} \mathrm{C}$ & OC-3 & OC-9 & OC-11 & O
\end{tabular}

Table 1: OC-Series Experimental Schedule

A compressive load of $13.79 \mathrm{MPa}$ was applied to 20 specimens in each capsule via a metal bellows, which was expanded by gas pressure against the specimen columns. 8 of the specimens in each column had reduced diameters to increase the compressive load to 20.68 MPa. Two unloaded columns were included in each capsule to act as reference specimens for the loaded specimens. Figure 1 shows the stressed column cross section of the in-core portion of the OC-Series capsule. Figure 2 shows the unstressed column cross section. 
ORNL/TM-2009/176

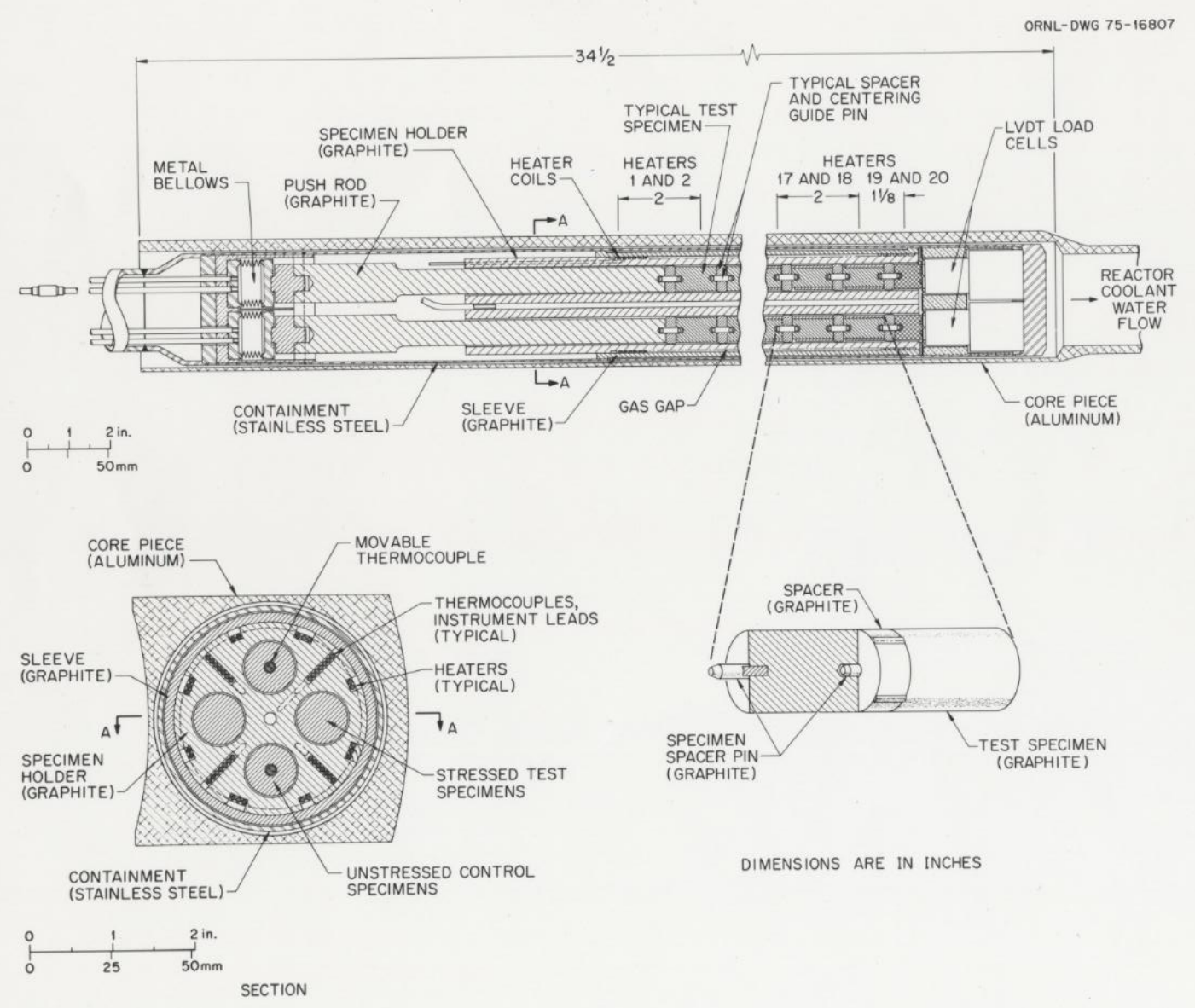

Figure 1: Stressed Column Cross Section 


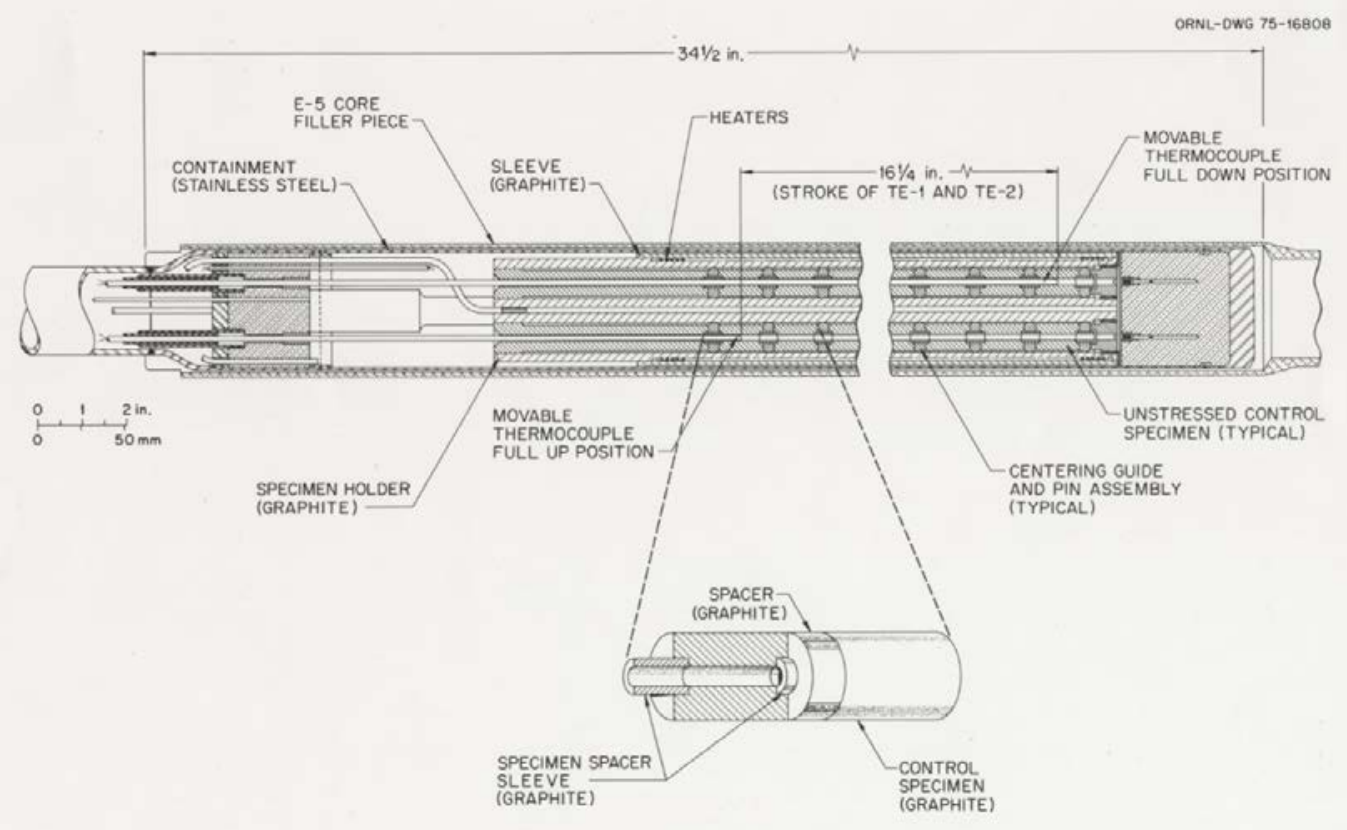

Figure 2: Unstressed Column Cross Section

The OC-Series Capsules included movable centerline thermocouples located in the reference columns, load cells to monitor the applied load and a computerized temperature control system to maintain accurate temperatures over the $0.508 \mathrm{~m}$ column length.

Table 2 shows the graphite materials for the OC-1 capsule indicating the location material, orientation and load. It is interesting to note that specimens of $\mathrm{H}-451$ (parallel) were chosen with low, intermediate and high Modulus for positions 6, 7 and 8. No reference could be found as to why this was done. 
ORNL/TM-2009/176

\begin{tabular}{|c|c|c|c|c|c|c|c|}
\hline \multirow[b]{3}{*}{ Position } & \multicolumn{7}{|c|}{ TO BE OPERATED AT $900^{\circ} \mathrm{C}$} \\
\hline & & \multicolumn{3}{|c|}{ Column "E" } & \multicolumn{3}{|c|}{ Column "W" } \\
\hline & Grade & Orientation & Stress & Modulus & Orientation & Stress & Modulus \\
\hline 1 & AXF-8QBGI & Isometric & & & Isometric & & \\
\hline 2 & $\mathrm{H}-327$ & ॥ & & & II & & \\
\hline 3 & $\mathrm{H}-451$ & $\|$ & & & $\|$ & & \\
\hline 4 & $\mathrm{H}-451$ & ॥ & High & & ॥ & High & \\
\hline 5 & $\mathrm{H}-451$ & ॥ & & & $\|$ & & \\
\hline 6 & $\mathrm{H}-\overline{451}$ & II & & $\overline{\text { Low }}$ & ॥ & & $\overline{\text { Low }}$ \\
\hline 7 & $\mathrm{H}-451$ & II & & High & II & & $\mathrm{High}$ \\
\hline 8 & $\mathrm{H}-451$ & ॥ & & $\begin{array}{l}\text { Inter- } \\
\text { mediate }\end{array}$ & II & & $\begin{array}{l}\text { Inter- } \\
\text { mediate }\end{array}$ \\
\hline 9 & $\mathrm{H}-451$ & Radial & & & Radial & & \\
\hline 10 & $\mathrm{H}-\overline{451}$ & II & High & & II & $\overline{\mathrm{High}}$ & \\
\hline 11 & $\mathrm{H}-451$ & ॥ & & & II & & \\
\hline 12 & $\mathrm{H}-451$ & II & High & & II & High & \\
\hline 13 & $\mathrm{H}-327$ & 1 & & & 1 & & \\
\hline 14 & AXF-8QBGI & Isometric & & & Isometric & & \\
\hline
\end{tabular}

Table 2: Graphite Materials for the OC-1 Capsule

Table 3 shows the planned series of experiments for the $900^{\circ} \mathrm{C}$ irradiation. The Table clearly indicates that some specimens were planned to be irradiation annealed at various stages throughout the program to obtain data on the recovery of primary creep. 
ORNL/TM-2009/176

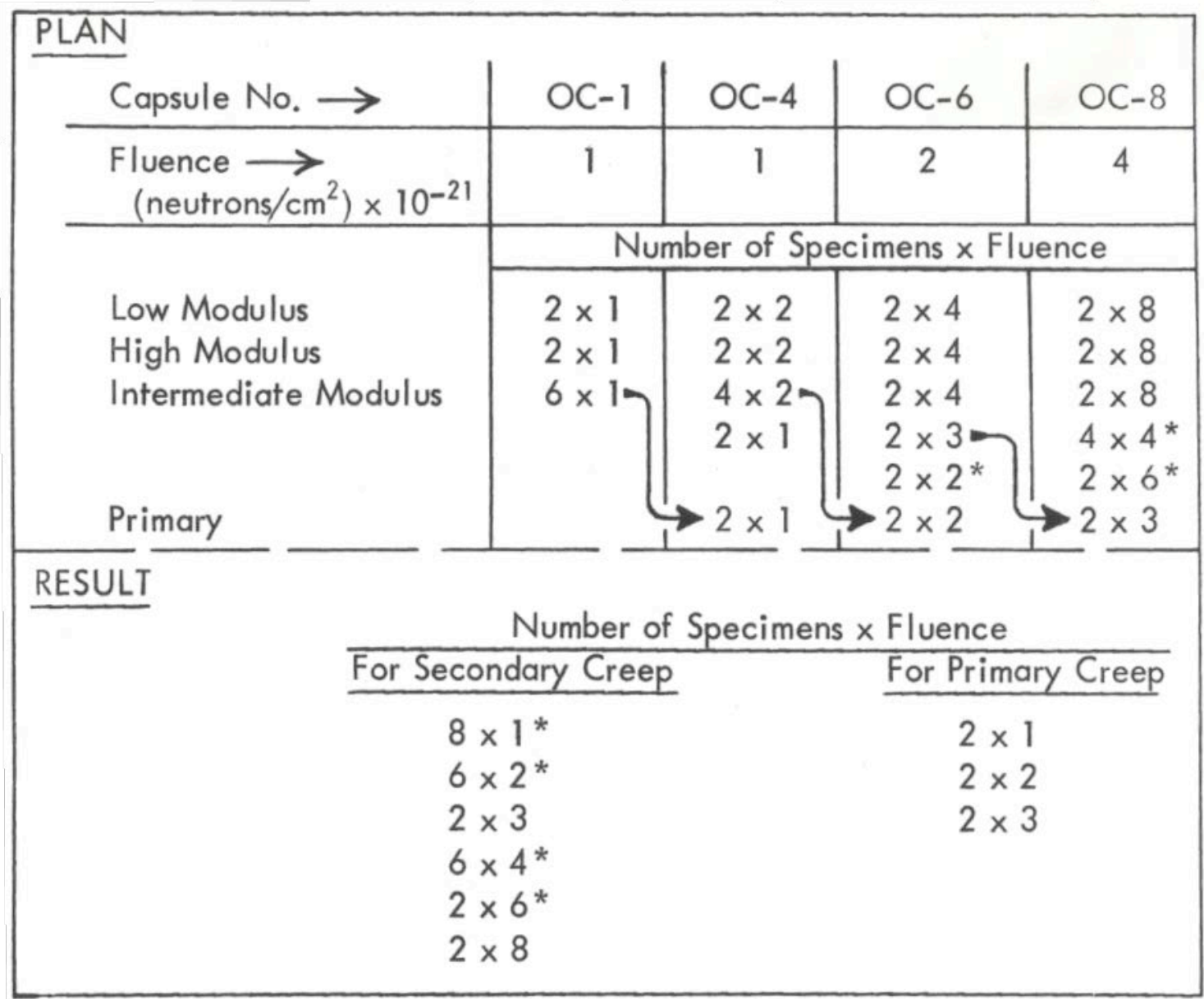

*Could use to introduce second graphite type.

Table 3: $900^{\circ} \mathrm{C}$ experiment - Specimen Irradiation Plan

The original schedule was never achieved and although a $1250^{\circ} \mathrm{C}$ capsule was designed no creep irradiations at this temperature were ever carried out. The reduced schedule consisted of 3 capsules, OC- 1 , OC-3 and OC-5 irradiated at $900^{\circ} \mathrm{C}$ to a maximum cumulative fluence of $0.5310^{25} \mathrm{n} / \mathrm{m}^{2}$ (E > $50 \mathrm{keV}$ ) and 2 capsules, OC-2 and OC-4 irradiated at $600^{\circ} \mathrm{C}$ to a maximum cumulative fluence of $0.51310^{25} \mathrm{n} / \mathrm{m}^{2}(\mathrm{E}>50 \mathrm{keV})$. Capsule OC-3 contained some irradiation anneal specimens previously irradiated in OC1. 


\section{OC-Series Data}

Historically it appears that the best available source for the OC-Series experimental data was contained in a University of Tennessee Ph.D. Thesis (Mobasheran, 1990). The data available from the Ph.D. is included in this report in Tables 4 and 5 for completeness. Interestingly the data contained within the Ph.D. did not include any annealed speciemen data. 


\begin{tabular}{|c|c|c|c|c|c|c|c|c|c|c|c|c|c|c|c|}
\hline Observation & Graphite & Specimen & $\begin{array}{c}\text { Length } \\
\text { (\%) }\end{array}$ & $\begin{array}{c}\text { Diameter } \\
\text { (\%) }\end{array}$ & $\begin{array}{c}\text { Volume } \\
\text { (\%) }\end{array}$ & $\begin{array}{l}\text { Density } \\
\left(\mathrm{g} / \mathrm{cm}^{3}\right)\end{array}$ & $\begin{array}{c}\text { Fluence } \\
(>50 \mathrm{keV})\end{array}$ & $\begin{array}{l}\text { Unirradiated } \\
\text { Sonic } \\
\text { Young's } \\
\text { Modulus } \\
\text { (GPa) }\end{array}$ & $\begin{array}{l}\text { Irradiated } \\
\text { Sonic } \\
\text { Young's } \\
\text { Modulus } \\
\text { (GPa) }\end{array}$ & $\begin{array}{l}\text { Irradiated } \\
\text { Sonic } \\
\text { Shear } \\
\text { Modulus } \\
\text { (GPa) }\end{array}$ & $\begin{array}{l}\text { Resistivity } \\
\text { Side } \\
(\mu \mathrm{Ohm} \cdot \mathrm{m})\end{array}$ & $\begin{array}{c}\text { Resistivity } \\
\text { Ends } \\
\text { ( } \mu \text { Ohm.m) }\end{array}$ & $\begin{array}{c}\text { CTE } \\
(500) \\
\left({ }^{\circ} \mathrm{C}^{-1} 10^{-6}\right)\end{array}$ & $\begin{array}{c}\text { Axial } \\
\text { Creep } \\
(\%)\end{array}$ & $\begin{array}{c}\text { Axial } \\
\text { Creep } \\
(\% / \text { lesu) }\end{array}$ \\
\hline 1 & $40-A-C$ & 1101.1090 & -0.0930 & -0.0180 & -0.1740 & 1.7020 & 0.0640 & 8.2740 & 10.9000 & 4.4800 & 14.2600 & & 3.8900 & 0.0000 & 0.0000 \\
\hline 2 & $40-A-C$ & 1103.0970 & -0.0730 & 0.0240 & -0.0400 & 1.7090 & 0.0800 & 8.4119 & 11.0800 & 4.5800 & 15.8400 & & 3.8500 & 0.0000 & 0.0000 \\
\hline 3 & $40-A-C$ & 1104.1120 & -0.1100 & 0.0140 & -0.0880 & 1.7230 & 0.085 & 8.61675 & 11.8300 & 4.8700 & 16.0400 & & 3.8200 & 0.0000 & 0.0000 \\
\hline 4 & $40-A-C$ & 1105.0990 & -0.0900 & -0.0370 & -0.1910 & 1.7040 & 0.095 & 8.34295 & 11.5400 & 4.7800 & 16.3400 & & 3.7400 & 0.0000 & 0.0000 \\
\hline 5 & $40-A-C$ & 1108.0860 & \begin{tabular}{|l|}
-0.1200 \\
\end{tabular} & 0.0120 & 0.1100 & 1.7060 & 0.113 & 8.89455 & 11.4900 & 4.7300 & 16.4600 & & 4.2800 & 0.0000 & 0.0000 \\
\hline 6 & $40-A-C$ & 1110.1140 & -0.1300 & 0.0350 & -0.0760 & 1.7190 & 0.115 & 8.49085 & 11.9000 & 4.9200 & 15.8300 & & 4.0400 & 0.0000 & 0.0000 \\
\hline 7 & $40-A-C$ & 1111.1010 & -0.1270 & 0.0400 & 0.0050 & 1.7030 & 0.113 & 8.34295 & 11.2600 & 4.6500 & 16.4600 & & 4.1600 & 0.0000 & 0.0000 \\
\hline 8 & $40-A-C$ & 1112.1160 & -0.1270 & 0.0310 & -0.0850 & 1.7170 & 0.109 & 8.68770 & 11.7200 & 4.8000 & 15.2700 & & 4.1600 & 0.0000 & 0.0000 \\
\hline 9 & $40-A-C$ & 1201.1110 & -0.0870 & -0.0310 & -0.1970 & 1.7020 & 0.064 & 8.34295 & 11.0600 & 4.5700 & 13.4800 & & 3.8100 & 0.0000 & 0.0000 \\
\hline 10 & $40-A-C$ & 1203.0980 & \begin{tabular}{|l|}
-0.0970 \\
\end{tabular} & 0.0170 & -0.0890 & 1.7040 & 0.080 & 8.41190 & 11.2300 & 4.6900 & 15.4500 & & 4.0300 & 0.0000 & 0.0000 \\
\hline 11 & $40-A-C$ & 1204.1130 & -0.0870 & 0.0250 & -0.0650 & 1.7210 & 0.085 & 8.54980 & 11.8600 & 4.8900 & 13.3800 & & 4.2100 & 0.0000 & 0.0000 \\
\hline 12 & $40-A-C$ & 1205.1000 & -0.1150 & 0.0220 & 0.0400 & 1.7030 & 0.095 & 8.34295 & 11.3900 & 4.7400 & 14.8700 & & 3.8700 & 0.0000 & 0.0000 \\
\hline 13 & $40-A-C$ & 1208.0870 & -0.1100 & 0.0180 & -0.0420 & 1.7060 & 0.113 & 8.48085 & 11.6200 & 4.8200 & 16.6000 & & 3.7900 & 0.0000 & 0.0000 \\
\hline 14 & $40-A-C$ & 1210.1150 & -0.1050 & 0.0240 & -0.1450 & 1.7220 & 0.115 & 8.54980 & 11.9100 & 4.9300 & 18.0200 & & 3.9200 & 0.0000 & 0.0000 \\
\hline 15 & $40-A-C$ & 1211.1020 & -0.1180 & 0.0370 & -0.0610 & 1.7040 & 0.113 & 8.34295 & 11.4900 & 4.7400 & 15.5400 & & 3.6900 & 0.0000 & 0.0000 \\
\hline 16 & $40-A-C$ & 1212.1170 & -0.0970 & 0.0430 & -0.0330 & 1.7050 & 0.109 & 8.41190 & 11.3400 & 4.7000 & 14.2400 & & 3.9600 & 0.0000 & 0.0000 \\
\hline 17 & $40-A-C$ & 3101.1240 & -0.1800 & -0.1190 & -0.4800 & 1.7190 & 0.140 & 8.96350 & 13.1100 & 5.4200 & 18.2000 & 21.7000 & 3.9000 & 0.0000 & 0.0000 \\
\hline 18 & $40-A-C$ & 3103.0970 & -0.3950 & -0.2220 & -1.1080 & 1.7270 & 0.260 & 8.27400 & 13.9500 & 5.8100 & 20.9000 & 26.3000 & 3.9900 & 0.0000 & 0.0000 \\
\hline 19 & $40-A-C$ & 3112.1260 & -0.2200 & -0.1320 & -0.5490 & 1.7200 & 0.240 & 8.96350 & 13.4700 & 5.5700 & 18.5000 & 17.7000 & 4.0000 & 0.0000 & 0.0000 \\
\hline 20 & $40-A-C$ & 3201.1110 & -0.3120 & -0.2100 & -1.0300 & 1.7200 & 0.210 & 8.34295 & 13.3000 & 5.5000 & 19.6000 & 20.0000 & 3.6000 & 0.0000 & 0.0000 \\
\hline 21 & $40-A-C$ & 3203.2060 & -0.1900 & -0.1190 & -0.4960 & 1.7060 & 0.170 & 8.96350 & 12.9900 & 5.4400 & 18.2000 & 18.4000 & 4.2100 & 0.0000 & 0.0000 \\
\hline 22 & $40-A-C$ & 3204.1250 & -0.1950 & -0.1210 & -0.4970 & 1.7210 & 0.190 & 8.96350 & 13.3300 & 5.5500 & 18.4000 & 18.4000 & 4.2000 & 0.0000 & 0.0000 \\
\hline 23 & $40-A-C$ & 3205.2070 & -0.2380 & -0.1330 & -0.5770 & 1.7080 & 0.210 & 8.96350 & 13.2100 & 5.5300 & 18.6000 & 19.2000 & 4.3500 & 0.0000 & 0.0000 \\
\hline 24 & $40-A-C$ & 3208.0870 & -0.5350 & -0.2930 & -1.3470 & 1.7300 & 0.350 & 8.48085 & 14.3500 & 6.0400 & 20.9000 & 21.3000 & 4.0000 & 0.0000 & 0.0000 \\
\hline 25 & $40-A-C$ & 3210.1150 & -0.5220 & -0.2830 & -1.4270 & 1.7410 & 0.370 & 8.54980 & 14.4900 & 5.9900 & 19.9000 & 20.4000 & 3.9900 & 0.0000 & 0.0000 \\
\hline 26 & $40-A-C$ & 3211.1020 & -0.4650 & -0.2530 & -1.2950 & 1.7250 & 0.370 & 8.34295 & 14.2200 & 5.9300 & 20.5000 & 21.2000 & 4.1400 & 0.0000 & 0.0000 \\
\hline 27 & $40-A-C$ & 3212.1170 & -0.4000 & -0.2210 & -1.1340 & 1.7210 & 0.350 & 8.41190 & 13.9800 & 5.8300 & 20.6000 & 20.8000 & 3.8900 & 0.0000 & 0.0000 \\
\hline 28 & $40-A-C$ & 5101.1240 & $\mid-0.3130$ & -0.1900 & -0.7860 & 1.7240 & 0.230 & 8.96350 & 15.8500 & 7.0300 & 21.1000 & 28.2000 & 3.9900 & 0.0000 & 0.0000 \\
\hline 29 & $40-A-C$ & 5103.0970 & -0.7100 & -0.4190 & -1.7130 & 1.7380 & 0.370 & 8.41190 & 16.7300 & 6.6100 & 23.0000 & 30.4000 & 4.3300 & 0.0000 & 0.0000 \\
\hline
\end{tabular}




\begin{tabular}{|c|c|c|c|c|c|c|c|c|c|c|c|c|c|c|c|}
\hline Observation & Graphite & Specimen & $\begin{array}{c}\text { Length } \\
\text { (\%) }\end{array}$ & $\begin{array}{l}\text { Diameter } \\
\text { (\%) }\end{array}$ & $\begin{array}{c}\text { Volume } \\
\text { (\%) }\end{array}$ & $\begin{array}{l}\text { Density } \\
\left(\mathrm{g} / \mathrm{cm}^{3}\right)\end{array}$ & $\begin{array}{c}\text { Fluence } \\
\text { (>50 keV) }\end{array}$ & $\begin{array}{l}\text { Unirradiated } \\
\text { Sonic } \\
\text { Young's } \\
\text { Modulus } \\
\text { (GPa) }\end{array}$ & $\begin{array}{l}\text { Irradiated } \\
\text { Sonic } \\
\text { Young's } \\
\text { Modulus } \\
\text { (GPa) }\end{array}$ & $\begin{array}{l}\text { Irradiated } \\
\text { Sonic } \\
\text { Shear } \\
\text { Modulus } \\
\text { (GPa) }\end{array}$ & $\begin{array}{l}\text { Resistivity } \\
\text { Side } \\
(\mu \mathrm{Ohm} . \mathrm{m})\end{array}$ & $\begin{array}{l}\text { Resistivity } \\
\text { Ends } \\
(\mu \mathrm{Ohm} \cdot \mathrm{m})\end{array}$ & $\begin{array}{c}\text { CTE } \\
(500) \\
\left({ }^{\circ} \mathrm{C}^{-1} 10^{-6}\right)\end{array}$ & $\begin{array}{c}\text { Axial } \\
\text { Creep } \\
(\%)\end{array}$ & $\begin{array}{c}\text { Axial } \\
\text { Creep } \\
\text { (\%/esu) }\end{array}$ \\
\hline 30 & $40-A-C$ & 5104.1120 & -0.2470 & -0.1070 & -0.3270 & 1.7270 & 0.200 & 8.61875 & 16.9600 & 6.5700 & 21.3000 & 26.8000 & 3.9300 & 0.0000 & 0.0000 \\
\hline 31 & $40-A-C$ & \begin{tabular}{|l|}
5105.0990 \\
\end{tabular} & \begin{tabular}{|c|}
-0.2600 \\
\end{tabular} & -0.1860 & -0.9350 & 1.7170 & 0.230 & 8.34295 & 15.2100 & 6.2300 & 22.8000 & 29.1000 & 4.2900 & 0.0000 & 0.0000 \\
\hline 32 & $40-A-C$ & 5106.0660 & -0.3220 & -0.1650 & -0.7390 & 1.7170 & 0.270 & 8.69455 & 16.9700 & 6.7000 & 22.2000 & 29.1000 & 4.6400 & 0.0000 & 0.0000 \\
\hline 33 & $40-A-C$ & \begin{tabular}{|l|l}
5110.1140 \\
\end{tabular} & -0.3200 & -0.1510 & -0.6950 & 1.7290 & 0.270 & 8.48085 & 16.0800 & 6.7400 & 21.9000 & 21.0000 & 4.9300 & 0.0000 & 0.0000 \\
\hline 34 & $40-A-C$ & \begin{tabular}{|c|}
5111.1010 \\
\end{tabular} & -0.2900 & -0.1290 & -0.5500 & 1.7120 & 0.270 & 8.34295 & 16.1000 & 6.4400 & 22.3000 & 22.7000 & 4.8400 & 0.0000 & 0.0000 \\
\hline 35 & $40-A-C$ & 5112.1260 & 0.0000 & 0.0000 & 0.0000 & 1.7310 & 0.490 & 8.96350 & 16.3900 & 6.3100 & 22.1000 & 21.4000 & 4.1100 & 0.0000 & 0.0000 \\
\hline 36 & $40-A-C$ & 5201.1110 & -0.5220 & -0.4610 & -1.6530 & 1.7230 & 0.300 & 8.34295 & 14.8200 & 6.0500 & 22.5000 & 22.6000 & 3.9900 & 0.0000 & 0.0000 \\
\hline 37 & $40-A-C$ & 5203.2060 & \begin{tabular}{|l|}
-0.4700 \\
\end{tabular} & -0.2500 & -1.1170 & 1.7160 & 0.280 & 8.96350 & 14.2200 & 5.8400 & 21.3000 & 22.4000 & 4.0400 & 0.0000 & 0.0000 \\
\hline 38 & $40-A-C$ & \begin{tabular}{|l|}
5204.1250 \\
\end{tabular} & \begin{tabular}{|c|}
-0.4830 \\
\end{tabular} & -0.2830 & -1.1650 & 1.7320 & 0.310 & 8.96350 & 14.6400 & 5.9900 & 21.3000 & 21.3000 & 3.9800 & 0.0000 & 0.0000 \\
\hline 39 & $40-A-C$ & 5205.2070 & -0.5780 & -0.3290 & -1.3660 & 1.7220 & 0.341 & 8.96350 & 14.5400 & 5.9900 & 21.8000 & 22.2000 & 4.0400 & 0.0000 & 0.0000 \\
\hline 40 & $40-A-C$ & 5206.0870 & \begin{tabular}{|l|}
-1.0690 \\
\end{tabular} & -0.5960 & -2.3830 & 1.7470 & 0.520 & 8.48085 & 15.7500 & 6.4400 & 22.8000 & 23.5000 & 3.8300 & 0.0000 & 0.0000 \\
\hline 41 & $40-A-C$ & 5210.1150 & \begin{tabular}{|l|}
-0.9950 \\
\end{tabular} & -0.5680 & -2.3630 & 1.7610 & 0.530 & 8.54980 & 16.1600 & 6.6300 & 22.3000 & 19.3000 & 3.9600 & 0.0000 & 0.0000 \\
\hline 42 & $40-A-C$ & 5211.1020 & \begin{tabular}{|l|}
-0.8950 \\
\end{tabular} & -0.5030 & -2.1280 & 1.7390 & 0.520 & 8.34295 & 15.7700 & 6.5100 & 22.9000 & 23.2000 & 4.1900 & 0.0000 & 0.0000 \\
\hline 43 & $40-A-C$ & \begin{tabular}{|l|}
5212.1170 \\
\end{tabular} & \begin{tabular}{|l|}
-0.7670 \\
\end{tabular} & -0.4430 & -1.8570 & 1.7360 & 0.500 & 8.41190 & 15.1500 & 6.2000 & 23.0000 & 23.4000 & 4.5500 & 0.0000 & 0.0000 \\
\hline 44 & $40-A-2$ & 1301.0460 & -0.6530 & 0.1500 & -0.3600 & 1.7140 & 0.064 & 8.20505 & 10.3900 & 4.6700 & 15.8000 & 15.9000 & 4.5900 & -0.5630 & -0.0408 \\
\hline 45 & $40-A-2$ & \begin{tabular}{|c|}
1303.0340 \\
\end{tabular} & \begin{tabular}{|l|}
-0.7070 \\
\end{tabular} & 0.1970 & -0.3310 & 1.7130 & 0.080 & 8.27400 & 11.2500 & 4.7300 & 17.3000 & 17.5000 & 4.5400 & -0.6220 & -0.0451 \\
\hline 46 & $40-A-2$ & 1305.0360 & \begin{tabular}{|c|}
-0.7400 \\
\end{tabular} & 0.2060 & -0.3460 & 1.7120 & 0.095 & 8.20505 & 11.3200 & 4.7900 & 17.6000 & 18.2000 & \begin{tabular}{l|l|}
4.5300 \\
\end{tabular} & -0.6150 & -0.0446 \\
\hline 47 & $40-A-2$ & \begin{tabular}{|l}
1308.0230 \\
\end{tabular} & -0.8020 & 0.2000 & -0.4180 & 1.7230 & 0.113 & 8.61675 & 11.8900 & 4.9600 & 18.2000 & 18.2000 & \begin{tabular}{|l|l|}
4.5600 \\
\end{tabular} & -0.6870 & -0.0498 \\
\hline 48 & $40-A-2$ & 1311.0360 & \begin{tabular}{|l|}
-0.7800 \\
\end{tabular} & 0.1860 & -0.4340 & 1.7140 & 0.113 & 8.27400 & 11.3700 & 0.2243 & 17.5000 & 18.0000 & \begin{tabular}{|l|l}
4.5400 \\
\end{tabular} & -0.6600 & -0.0479 \\
\hline 49 & $40-A-2$ & 3301.0460 & -1.4500 & 0.2030 & -1.0970 & 1.7270 & 0.210 & 8.20505 & 13.5000 & 5.6600 & $\sim 5.20$ & 20.8000 & 4.3900 & -1.1400 & -0.0827 \\
\hline 50 & $40-A-2$ & 3303.0340 & \begin{tabular}{|l|}
-1.7000 \\
\end{tabular} & 0.2460 & -1.2600 & 1.7230 & 0.170 & 8.27400 & 13.3000 & 5.5100 & 20.5000 & 20.4000 & \begin{tabular}{|l|l}
4.6500 \\
\end{tabular} & -1.3000 & -0.0943 \\
\hline 51 & $40-A-2$ & 3305.1720 & \begin{tabular}{|l|}
-1.3940 \\
\end{tabular} & 0.2170 & -1.0070 & 1.7300 & 0.310 & 8.82560 & 13.1800 & 5.6600 & 19.2000 & 21.4000 & \begin{tabular}{|l|}
4.5100 \\
\end{tabular} & -1.1540 & -0.0837 \\
\hline 52 & $40-A-2$ & 3308.0230 & -2.0020 & 0.2470 & -1.5660 & 1.7420 & 0.360 & 8.61875 & 13.9100 & 5.8100 & 20.0000 & 20.3000 & 4.5000 & -1.4620 & -0.1060 \\
\hline 53 & $40-A-2$ & 3311.0380 & -1.9000 & 0.2040 & -1.5620 & 1.7340 & 0.370 & 8.27400 & 13.0500 & 5.3400 & 21.1000 & 20.1000 & \begin{tabular}{l|l|}
4.9700 \\
\end{tabular} & -1.4300 & -0.1037 \\
\hline 54 & $40-A-2$ & \begin{tabular}{|c|}
3403.1710 \\
\end{tabular} & -1.3610 & 0.1940 & -1.0360 & 1.7190 & 0.170 & 8.62560 & 11.4200 & 4.5100 & 19.1000 & 23.4000 & \begin{tabular}{|l|l|}
4.9200 \\
\end{tabular} & -1.1710 & -0.0849 \\
\hline 55 & $40-A-2$ & 3405.1730 & -1.4960 & 0.1790 & -1.1570 & 1.7240 & 0.210 & 8.82560 & 11.7000 & 4.6700 & 19.8000 & 20.0000 & \begin{tabular}{l|}
4.3500 \\
\end{tabular} & -1.2560 & -0.0911 \\
\hline 56 & $40-A-2$ & 5301.0460 & \begin{tabular}{|l|}
-2.0700 \\
\end{tabular} & 0.1010 & -1.9790 & 1.7420 & 0.230 & 8.2005 & 14.8900 & 6.2200 & 22.7000 & 22.4000 & \begin{tabular}{|l|l}
4.7400 \\
\end{tabular} & -1.5500 & -0.1124 \\
\hline 57 & $40-A-2$ & 5303.0340 & \begin{tabular}{|l|}
-2.4570 \\
\end{tabular} & 0.1330 & -2.3300 & 1.7470 & 0.3700 & 8.2740 & 15.3300 & 6.4400 & 23.6000 & 23.0000 & 4.9500 & -1.7470 & -0.1267 \\
\hline 58 & $40-A-2$ & 5305.1720 & -2.3350 & 0.1280 & -2.1910 & 1.7380 & 0.3400 & 8.8256 & 14.4600 & 6.0500 & 23.2000 & 22.7000 & 5.0000 & -1.7450 & -0.1265 \\
\hline 59 & $40-A-2$ & 5308.0230 & -2.9970 & 0.0430 & -3.0860 & 1.7690 & 0.5200 & 8.6188 & 16.1300 & 6.7600 & 23.9000 & 23.1000 & 5.1000 & -1.9270 & -0.1397 \\
\hline
\end{tabular}




\begin{tabular}{|c|c|c|c|c|c|c|c|c|c|c|c|c|c|c|c|}
\hline Observation & Graphite & Specimen & $\begin{array}{c}\text { Length } \\
\text { (\%) }\end{array}$ & $\begin{array}{c}\text { Diameter } \\
\text { (\%) }\end{array}$ & $\begin{array}{c}\text { Volume } \\
\text { (\%) }\end{array}$ & $\begin{array}{l}\text { Density } \\
\left(\mathrm{g} / \mathrm{cm}^{3}\right)\end{array}$ & $\begin{array}{c}\text { Fluence } \\
\text { (>50 keV) }\end{array}$ & $\begin{array}{l}\text { Unirradiated } \\
\text { Sonic } \\
\text { Young's } \\
\text { Modulus } \\
\text { (GPa) }\end{array}$ & $\begin{array}{l}\text { Irradiated } \\
\text { Sonic } \\
\text { Young's } \\
\text { Modulus } \\
\text { (GPa) }\end{array}$ & $\begin{array}{l}\text { Irradiated } \\
\text { Sonic } \\
\text { Shear } \\
\text { Modulus } \\
\text { (GPa) }\end{array}$ & $\begin{array}{l}\text { Resistivity } \\
\text { Side } \\
(\mu \mathrm{Ohm} . \mathrm{m})\end{array}$ & $\begin{array}{l}\text { Resistivity } \\
\text { Ends } \\
(\mu \mathrm{Ohm} \cdot \mathrm{m})\end{array}$ & $\begin{array}{c}\text { CTE } \\
(500) \\
\left({ }^{\circ} \mathrm{C}^{-1} 10^{-6}\right)\end{array}$ & $\begin{array}{c}\text { Axial } \\
\text { Creep } \\
(\%)\end{array}$ & $\begin{array}{c}\text { Axial } \\
\text { Creep } \\
\text { (\%/esu) }\end{array}$ \\
\hline 60 & $40-A-2$ & 5311.0380 & -2.7910 & 0.0720 & -2.8130 & 1.7550 & 0.5200 & 8.2740 & 15.4800 & 6.4400 & 23.5000 & 23.2000 & 5.2900 & -1.8910 & -0.1371 \\
\hline 61 & $40-A-2$ & 5403.1710 & -2.1900 & 0.1070 & -2.0820 & 1.7360 & 0.2800 & 8.8256 & 14.1300 & 5.8800 & 22.3000 & 22.2000 & 4.8900 & -1.7200 & -0.1247 \\
\hline 62 & $40-A-2$ & 5405.1730 & -2.6030 & 0.0580 & -2.5810 & 1.7480 & 0.3400 & 8.8256 & 14.6200 & 6.1100 & 23.0000 & 22.7000 & 4.8300 & -2.0130 & -0.1460 \\
\hline 63 & $40-A-3$ & 1304.0490 & -0.9960 & 0.2170 & -0.5990 & 1.7350 & 0.0850 & 8.4809 & 11.8200 & 5.0700 & 17.5000 & 18.5000 & 4.5700 & -0.8960 & -0.0433 \\
\hline 64 & $40-A-3$ & 1310.0510 & \begin{tabular}{|l|}
-1.0870 \\
\end{tabular} & 0.2240 & -0.6780 & 1.7340 & 0.1150 & 8.4809 & 11.8400 & 5.0000 & 18.1000 & 19.1000 & 4.7100 & -0.9400 & -0.0454 \\
\hline 65 & $40-A-3$ & 1312.0530 & \begin{tabular}{|l|}
-1.0200 \\
\end{tabular} & 0.2140 & -0.6210 & 1.7310 & 0.1090 & 8.4809 & 11.5200 & 4.9200 & 17.1000 & 18.2000 & 4.3500 & -0.9050 & -0.0438 \\
\hline 66 & $40-A-3$ & 3304.0610 & -1.7920 & 0.4280 & -1.0130 & 1.7360 & 0.1900 & 8.8256 & 12.9800 & 5.5300 & 19.8000 & 22.0000 & 5.1000 & -1.5970 & -0.0772 \\
\hline 67 & $40-A-3$ & 3310.0510 & \begin{tabular}{|l|}
-2.6990 \\
\end{tabular} & 0.4690 & -1.8220 & 1.7540 & 0.3700 & 8.4809 & 13.8800 & 5.9400 & 21.9000 & 19.5000 & 5.4500 & -2.2900 & -0.1107 \\
\hline 68 & $40-A-3$ & 3312.0530 & \begin{tabular}{|l|}
-2.3950 \\
\end{tabular} & 0.4430 & -1.5520 & 1.7480 & 0.3500 & 8.4809 & 13.1000 & 5.4100 & 21.5000 & 19.7000 & 5.3200 & $\mid-1.9950$ & -0.0965 \\
\hline 69 & $40-A-3$ & 3404.0620 & -1.9420 & 0.4490 & -1.1410 & 1.7380 & 0.1900 & 8.8946 & 11.8100 & 4.7300 & 20.5000 & 24.7000 & 5.1400 & -1.7470 & -0.0645 \\
\hline 70 & $40-A-3$ & 5304.0610 & -2.9220 & 0.3520 & -2.3900 & 1.7590 & 0.3100 & 8.8256 & 14.6000 & 6.2500 & 23.9000 & 23.0000 & 5.5900 & -2.4420 & -0.1161 \\
\hline 71 & $40-A-3$ & 5310.0510 & -3.8840 & 0.3040 & -3.5290 & 1.7830 & 0.5300 & 8.4809 & 16.2700 & 6.9200 & 25.5000 & 24.0000 & 5.8000 & -2.8840 & -0.1394 \\
\hline 72 & $40-A-3$ & 5312.0530 & \begin{tabular}{|l|}
-3.4420 \\
\end{tabular} & 0.3210 & -3.0070 & 1.7550 & 0.5000 & 8.4809 & 15.8000 & 6.7000 & 25.2000 & 23.8000 & 5.6600 & -2.7720 & -0.1340 \\
\hline 73 & $40-A-3$ & 5404.0620 & \begin{tabular}{|l|}
-3.2560 \\
\end{tabular} & 0.3210 & -2.8200 & 1.7660 & 0.3100 & 8.8946 & 14.9000 & 6.3800 & 25.2000 & 23.0000 & 5.3600 & -2.7760 & -0.1342 \\
\hline 74 & $40-R-C$ & 1109.0910 & \begin{tabular}{|l|}
-0.0770 \\
\end{tabular} & 0.0170 & -0.0570 & 1.6990 & 0.1160 & 7.6535 & 10.3200 & 4.4700 & 16.2900 & & 4.6800 & 0.0000 & 0.0000 \\
\hline 75 & 40-R-C & 1209.0920 & -0.0650 & 0.0220 & -0.0390 & 1.7040 & 0.1160 & 7.5845 & 10.5200 & 4.5400 & 19.7600 & & 4.5100 & 0.0000 & 0.0000 \\
\hline 76 & $40-R-C$ & 3109.0950 & -0.1700 & -0.2400 & -0.7280 & 1.7120 & 0.2600 & 7.6535 & 11.7300 & 5.0100 & 20.7000 & 23.7000 & 4.7000 & 0.0000 & 0.0000 \\
\hline 77 & $40-R-C$ & 3209.0920 & \begin{tabular}{|l|}
-0.3350 \\
\end{tabular} & -0.3570 & -1.4760 & 1.7230 & .3700 & 7.5845 & 12.5600 & 5.3700 & 21.5000 & 19.9000 & 4.6500 & $0 . .000$ & 0.0000 \\
\hline 78 & 40-R-C & 5109.0950 & \begin{tabular}{|l|}
-0.4100 \\
\end{tabular} & -0.5670 & -1.7120 & 1.7280 & 0.4200 & 7.6535 & 13.3300 & 5.6000 & 24.1000 & 29.1000 & 4.4100 & $0 . .000$ & $0 . .00000$ \\
\hline 79 & 40-R-C & 5209.0920 & \begin{tabular}{|l|}
-0.6780 \\
\end{tabular} & -0.7090 & -2.3410 & 1.7430 & 0.5300 & 7.5845 & 14.3200 & 6.1300 & 23.9000 & 21.9000 & 4.4500 & 0.0000 & 0.0000 \\
\hline 80 & $40-R-2$ & 1309.0280 & \begin{tabular}{|l|}
-0.8500 \\
\end{tabular} & 0.1830 & -0.5020 & 1.7130 & 0.1160 & 7.5845 & 10.5700 & 4.6300 & 16.5000 & 17.5000 & 5.1900 & -0.7600 & -0.0566 \\
\hline 81 & $40-R-2$ & 3309.0280 & \begin{tabular}{|l|}
-2.0870 \\
\end{tabular} & 0.1510 & -1.8470 & 1.7370 & 0.3700 & 7.5845 & 12.2500 & 5.1700 & 21.8000 & 20.9000 & 5.5600 & \begin{tabular}{|l|} 
\\
\end{tabular} & -0.1267 \\
\hline 82 & $40-R-2$ & 5309.0280 & -2.9770 & -0.0170 & -3.1870 & 1.7590 & 0.5300 & 7.5845 & 14.6000 & 6.2800 & 24.5000 & 22.2000 & 6.1600 & -2.2970 & -0.1666 \\
\hline 83 & $41-A-C$ & 1106.0760 & -0.0800 & 0.0210 & -0.0520 & 1.7130 & 0.1010 & 8.3430 & 11.7700 & 4.8400 & 16.3500 & & 3.7800 & 0.0000 & 0.0000 \\
\hline 84 & $41-A-C$ & 1206.0770 & \begin{tabular}{|l|}
-0.0970 \\
\end{tabular} & 0.0010 & -0.1140 & 1.7120 & 0.1010 & 8.4119 & 11.7500 & 4.9000 & 15.3600 & & 3.7900 & 0.0000 & 0.0000 \\
\hline 85 & $41-A-C$ & 3106.0800 & \begin{tabular}{|l|}
-0.3670 \\
\end{tabular} & -0.1110 & -0.8040 & 1.7060 & 0.2250 & 8.8946 & 13.1500 & 5.3700 & 19.9000 & 24.7000 & 3.9500 & 0.000 & 0.0000 \\
\hline 86 & $41-A-C$ & 3206.0760 & \begin{tabular}{|l|}
-0.4400 \\
\end{tabular} & -0.3300 & -1.1400 & 1.7310 & 0.2100 & 8.3430 & 14.2100 & 5.6600 & 20.3000 & 21.1000 & 4.2000 & 0.0000 & 0.0000 \\
\hline 87 & $41-A-C$ & 5106.0800 & \begin{tabular}{|l|}
-0.7110 \\
\end{tabular} & -0.3750 & -1.6280 & 1.7200 & 0.3600 & 8.8940 & 16.2900 & 6.3700 & 23.6000 & 30.6000 & 4.1600 & 0.0000 & 0.0000 \\
\hline 88 & $41-A-C$ & 5206.0760 & -0.8000 & -0.5930 & -2.1990 & 1.7500 & 0.3600 & 8.3430 & 15.7000 & 6.3800 & 22.2000 & 22.8000 & 3.6000 & 0.0000 & 0.0000 \\
\hline 89 & $41-A-2$ & 1306.0130 & -0.7850 & 0.2000 & -0.3990 & 1.7120 & 0.1010 & 8.6188 & 11.7000 & 4.9800 & 17.3000 & 17.6000 & 4.5200 & -0.700 & -0.0508 \\
\hline
\end{tabular}




\begin{tabular}{|c|c|c|c|c|c|c|c|c|c|c|c|c|c|c|c|}
\hline Observation & Graphite & Specimen & $\begin{array}{c}\text { Length } \\
\text { (\%) }\end{array}$ & $\begin{array}{c}\text { Diameter } \\
\text { (\%) }\end{array}$ & $\begin{array}{c}\text { Volume } \\
\text { (\%) }\end{array}$ & $\begin{array}{l}\text { Density } \\
\left(\mathrm{g} / \mathrm{cm}^{3}\right)\end{array}$ & $\begin{array}{c}\text { Fluence } \\
\text { (>50 keV) }\end{array}$ & $\begin{array}{l}\text { Unirradiated } \\
\text { Sonic } \\
\text { Young's } \\
\text { Modulus } \\
\text { (GPa) }\end{array}$ & $\begin{array}{l}\text { Irradiated } \\
\text { Sonic } \\
\text { Young's } \\
\text { Modulus } \\
\text { (GPa) }\end{array}$ & $\begin{array}{l}\text { Irradiated } \\
\text { Sonic } \\
\text { Shear } \\
\text { Modulus } \\
\text { (GPa) }\end{array}$ & $\begin{array}{l}\text { Resistivity } \\
\text { Side } \\
\text { ( } \mu \text { Ohm.m) }\end{array}$ & $\begin{array}{l}\text { Resistivity } \\
\text { Ends } \\
(\mu \mathrm{Ohm} \cdot \mathrm{m})\end{array}$ & $\begin{array}{c}\text { CTE } \\
(500) \\
\left({ }^{\circ} \mathrm{C}^{-1} 10^{-6}\right)\end{array}$ & $\begin{array}{c}\text { Axial } \\
\text { Creep } \\
(\%)\end{array}$ & $\begin{array}{c}\text { Axial } \\
\text { Creep } \\
\text { (\%/esu) }\end{array}$ \\
\hline 90 & 41-A-2 & 3306.0130 & -1.9430 & 0.2360 & -1.5210 & 1.7390 & 0.3300 & 8.6188 & 14.1500 & 5.9800 & 20.6000 & 20.4000 & 4.5900 & -1.5030 & -0.1070 \\
\hline 91 & $41-A-2$ & 5306.0130 & -2.8430 & 0.0280 & -2.8750 & 1.7620 & 0.4600 & 8.6188 & 15.8600 & 6.6300 & 23.9000 & 22.8000 & 4.9300 & -2.0880 & -0.1514 \\
\hline 92 & 41-A2-C & 1107.0810 & \begin{tabular}{|l|}
-0.0950 \\
\end{tabular} & 0.0390 & -0.0340 & 1.7280 & 0.1110 & 8.8946 & 12.2500 & 5.0800 & 16.0500 & & 3.6600 & 0.0000 & 0.0000 \\
\hline 93 & 41-A2-C & 1207.0820 & \begin{tabular}{|l|}
-0.0900 \\
\end{tabular} & 0.0190 & -0.0690 & 1.7260 & 0.1110 & 8.6877 & 12.3000 & 5.1100 & 15.7700 & & 3.7300 & 0.0000 & 0.0000 \\
\hline 94 & 41-A2-C & 3107.0850 & \begin{tabular}{|l|}
-0.2515 \\
\end{tabular} & -0.1680 & -3.7030 & 1.7300 & 0.2400 & 8.3430 & 14.2100 & 5.9200 & 16.6000 & 23.7000 & 3.9000 & 0.0000 & 0.0000 \\
\hline 95 & 41-A2-C & 3207.0820 & -0.4410 & -0.2820 & -1.3460 & 1.7440 & 0.3500 & 8.6877 & 14.9600 & 6.2000 & 19.7000 & 20.3000 & 4.1500 & 0.0000 & 0.0000 \\
\hline 96 & 41-A2-C & 5107.0850 & -0.5980 & -0.3790 & -1.4930 & 1.7440 & 0.3900 & 8.3430 & 17.2400 & 6.8000 & 22.3000 & 29.1000 & 3.8800 & 0.0000 & 0.0000 \\
\hline 97 & 41-A2-C & 5207.0820 & -0.8950 & -0.5540 & -2.2240 & 1.7630 & 0.5000 & 8.6877 & 16.7700 & 6.9100 & 21.9000 & 22.2000 & 3.8100 & 0.0000 & 0.0000 \\
\hline 98 & $41-A 2-2$ & 1307.0180 & -0.6870 & 0.1960 & -0.3080 & 1.7390 & 0.1110 & 8.9635 & 12.6200 & 5.2700 & 19.7000 & 17.4000 & 4.5900 & -0.5970 & -0.0433 \\
\hline 99 & $41-\mathrm{A} 2-2$ & 3307.0180 & \begin{tabular}{|l|}
-1.7600 \\
\end{tabular} & 0.2170 & 0.0000 & 1.7560 & 0.3500 & 8.9635 & 14.7700 & 6.1700 & 19.7000 & 20.9000 & 4.7900 & -1.3100 & -0.0950 \\
\hline 100 & $41-\mathrm{A} 2-2$ & 5307.0180 & -2.6720 & 0.0360 & -2.7280 & 1.7800 & 0.5000 & 8.9635 & 17.1900 & 7.2600 & 22.5000 & 22.1000 & 4.8000 & \begin{tabular}{|l|} 
\\
\end{tabular} & -0.1265 \\
\hline 101 & $3-A-C$ & 1102.0680 & -0.1820 & 0.0010 & -0.2160 & 1.7680 & 0.0720 & 12.1352 & 10.7200 & 4.2800 & 10.6300 & & 1.5700 & 0.0000 & 0.0000 \\
\hline 102 & $3-A-C$ & 1202.0660 & -0.1450 & 0.0400 & -0.0900 & 1.7730 & 0.0720 & 12.2731 & 11.8300 & 4.9900 & 8.4200 & & 1.9000 & 0.0000 & 0.0000 \\
\hline 103 & $3-A-C$ & 3102.0690 & \begin{tabular}{|l|}
-0.2720 \\
\end{tabular} & -0.1390 & -0.6290 & 1.7730 & 0.1600 & 12.8937 & 17.6600 & 7.3600 & 16.9000 & 26.6000 & 1.7500 & 0.0000 & 0.0000 \\
\hline 104 & $3-A-C$ & 3202.0660 & -0.4820 & -0.1790 & -1.0490 & 1.7880 & 0.2300 & 12.1352 & 17.9900 & 7.2200 & 16.5000 & 23.2000 & 2.1500 & 0.0000 & 0.0000 \\
\hline 105 & $3-A-C$ & 5102.0690 & -0.2820 & -0.2260 & -0.7550 & 1.7750 & 0.2600 & 12.8937 & 17.5100 & 6.6800 & 20.9000 & 34.9000 & 1.7900 & 0.0000 & 0.0000 \\
\hline 106 & $3-A-C$ & 5202.0660 & \begin{tabular}{|l|}
-0.7970 \\
\end{tabular} & -0.2780 & -1.4950 & 1.7980 & 0.3300 & 12.1352 & 14.5700 & 5.3200 & 20.7000 & 27.9000 & 1.6000 & 0.0000 & 0.0000 \\
\hline 107 & $3-A-2$ & 1302.0050 & -0.6000 & 0.1110 & -0.3920 & 1.7760 & 0.0720 & 11.9284 & 11.0100 & 4.5700 & 15.4000 & 18.8000 & 1.8800 & -0.4500 & -0.0326 \\
\hline 108 & 3-A-2 & 3302.0050 & \begin{tabular}{|l|}
-1.3570 \\
\end{tabular} & 0.1210 & -1.1290 & 1.7900 & 0.2300 & 11.9284 & 15.7600 & 6.3900 & 15.7000 & 16.4000 & 2.5000 & -0.8770 & -0.0636 \\
\hline 109 & $3-A-2$ & 5302.0050 & -1.9590 & 0.0820 & -1.8560 & 1.8020 & 0.3300 & 11.9284 & 18.8900 & 7.3500 & 21.3000 & 25.6000 & 2.6500 & -1.159 & -0.0841 \\
\hline 110 & $3-A-2$ & 1113.0070 & -0.0650 & 0.0610 & -0.0530 & 1.7600 & 0.0970 & 5.8608 & 6.8200 & 3.4300 & 21.1300 & & 3.3600 & 0.0000 & 0.0000 \\
\hline 111 & $3-A-2$ & 1213.0710 & \begin{tabular}{|l|}
-0.0430 \\
\end{tabular} & 0.0540 & -0.0490 & 1.7700 & 0.0970 & 5.8608 & 8.2180 & 3.4600 & 18.6300 & & 3.4100 & 0.0000 & 0.0000 \\
\hline 112 & $3-A-2$ & 3113.0740 & \begin{tabular}{|l|}
-0.1350 \\
\end{tabular} & -0.1370 & -0.4770 & 1.7720 & 0.2100 & 5.8608 & 10.5200 & 7.4600 & 18.9000 & 15.6000 & & 0.0000 & 0.0000 \\
\hline 113 & $3-R-C$ & 3213.0710 & -0.2230 & -0.2290 & -0.9550 & 1.7850 & 0.2900 & 5.8608 & 8.5200 & & 22.8000 & 17.4000 & 3.5600 & 0.0000 & 0.0000 \\
\hline 114 & $3-R-C$ & 5113.0740 & -0.2450 & -0.3040 & -0.9720 & 1.7810 & 0.3300 & 8.3430 & 10.3800 & 4.2800 & 22.9000 & 22.7000 & 3.7100 & 0.0000 & 0.0000 \\
\hline 115 & $3-R-C$ & 5213.0710 & -0.3750 & -0.5300 & -1.5810 & 1.7990 & 0.4100 & 5.8608 & 11.0200 & 4.5500 & 22.5000 & 19.7000 & 3.5500 & 0.0000 & 0.0000 \\
\hline 116 & $3-R-2$ & 1313.0070 & -1.1100 & 0.1600 & -0.8130 & 1.7750 & 0.0970 & 7.1708 & 6.4570 & 3.8100 & 16.4000 & 15.9000 & 4.2400 & -1.0550 & -0.0765 \\
\hline 117 & 3-R-2 & 3313.0070 & -2.0170 & 0.1540 & -1.7430 & 1.7920 & 0.2900 & 7.1708 & 9.7900 & 6.7100 & 23.3000 & 21.9000 & 5.0700 & \begin{tabular}{|l|}
-1.7970 \\
\end{tabular} & -0.1303 \\
\hline
\end{tabular}

Table 4: $900^{\circ} \mathrm{C}$ OC Experiments - Mobasheran Data (Mobasheran, 1990) 
ORNL/TM-2009/176

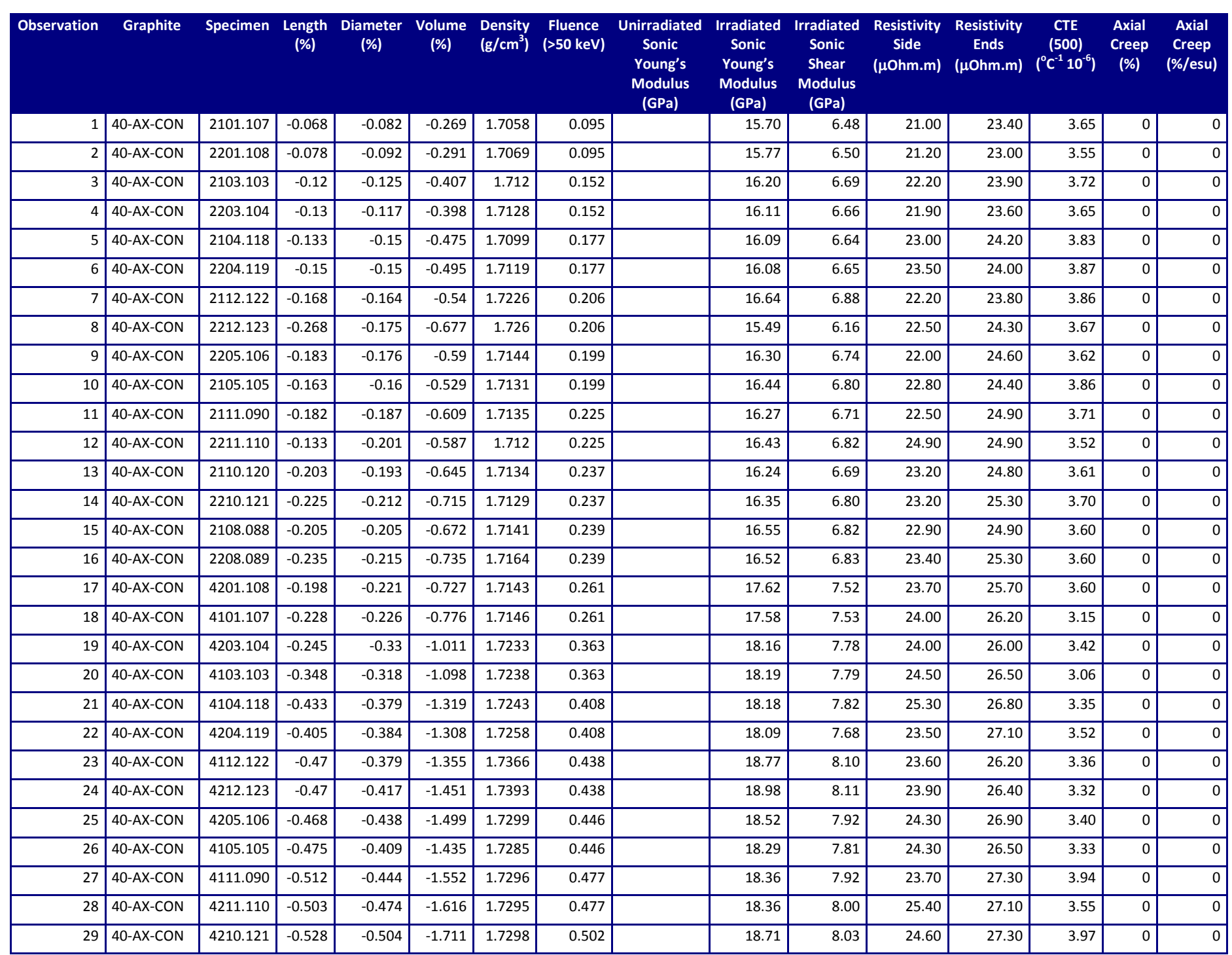




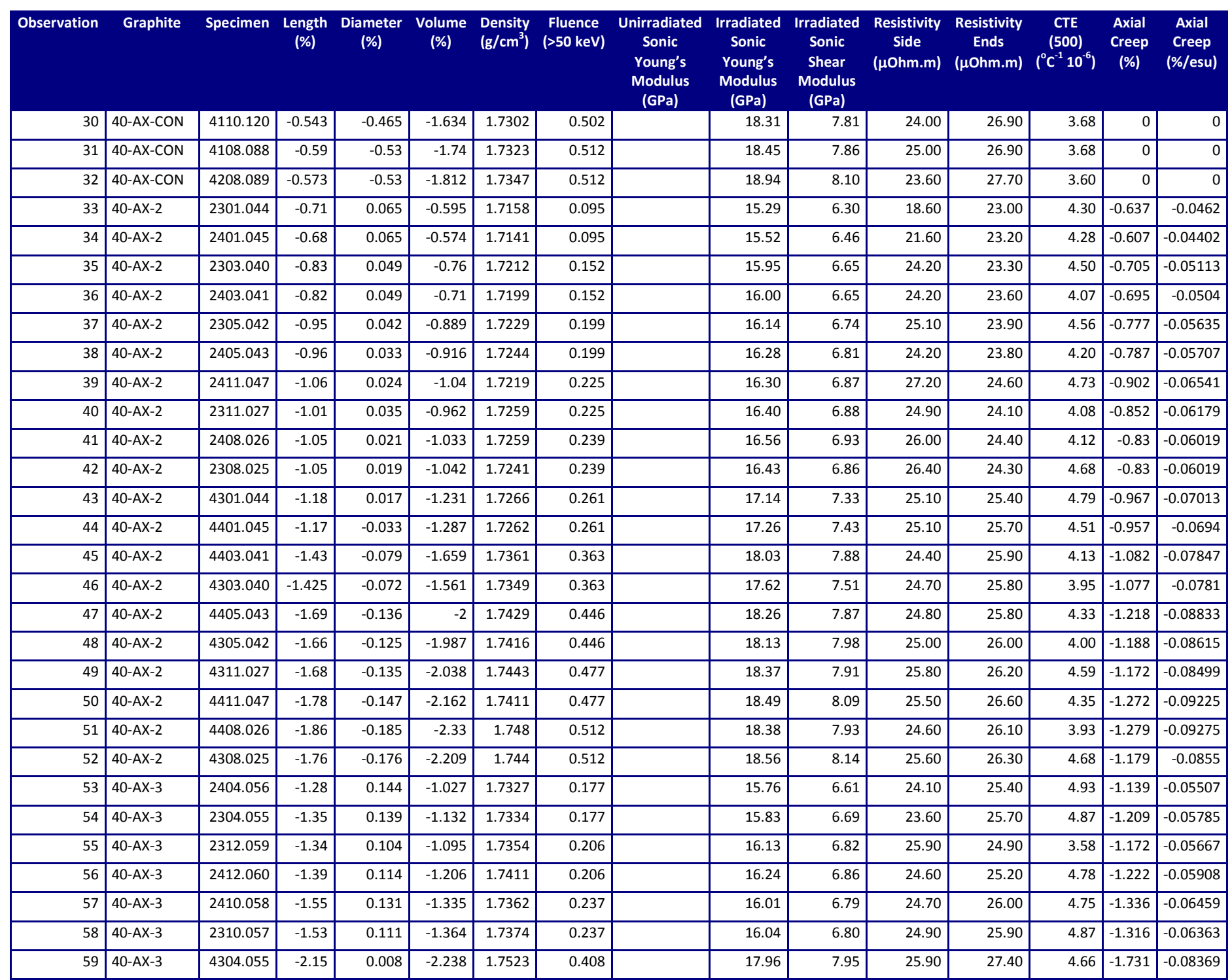




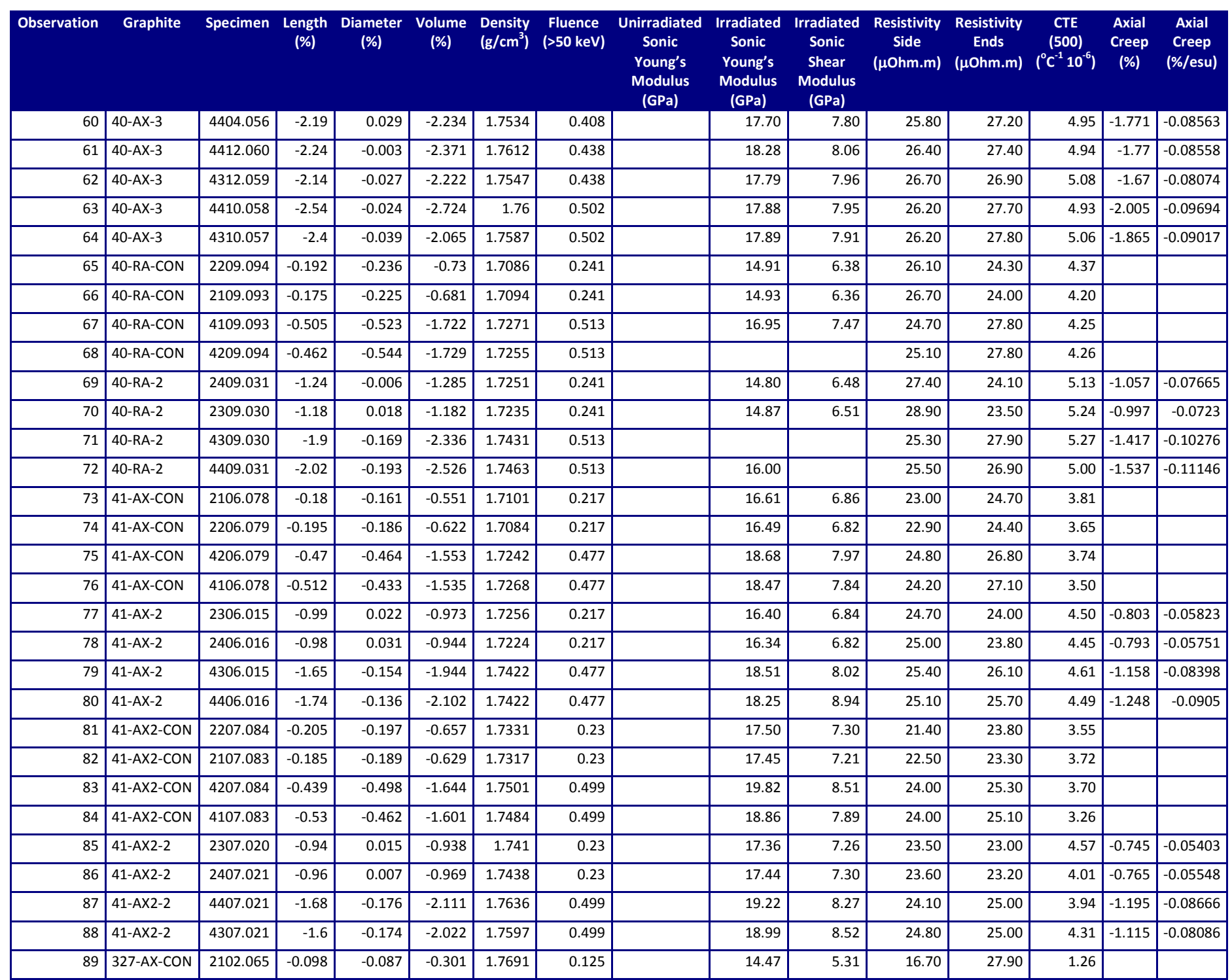




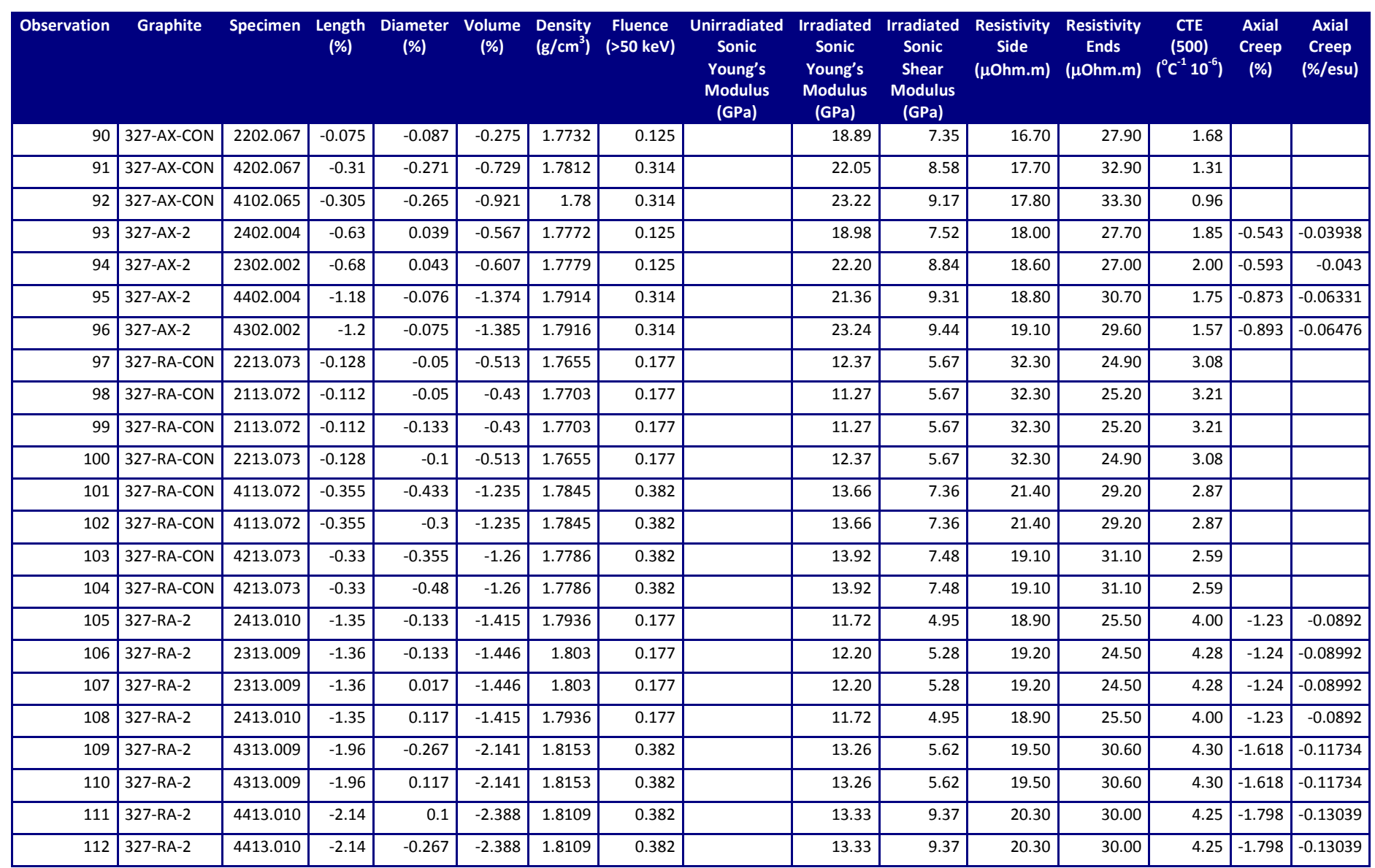

Table 5: $600^{\circ} \mathrm{C}$ OC Experiments - Mobasheran Data (Mobasheran, 1990) 
The OC-Series archive at ORNL was interrogated and a number of important documents were recovered. Three types of documents were recovered and these are summarised in Table 6.

\begin{tabular}{|lccccc|}
\hline & Pre & OC1 & $900^{\circ} \mathrm{C}$ & & OC3 \\
CPO & No & Yes & Yes & Yes \\
Datasheet & Yes & Yes & Part & No \\
Extension & No & Yes & Yes & No \\
& & & $600^{\circ} \mathrm{C}$ & & \\
& Pre & OC2 & OC4 & \\
CPO & No & Yes & Yes & \\
Datasheet & No & No & No & \\
Extension & No & Yes & Yes & \\
\hline
\end{tabular}

Table 6: Important Documents recovered from ORNL OC-Series Archive

Firstly, computer database printouts (CPO) for both the $900^{\circ} \mathrm{C}$ and $600^{\circ} \mathrm{C}$ experiments were discovered. The data from the $900^{\circ} \mathrm{C}$ series of experiments is presented in Table 7 . The data from $600^{\circ} \mathrm{C}$ series of experiments is presented in Table 8.

It should be noted that no hardcopy computer printout was uncovered for the precharacterization of either series of experiments. However hand written datasheets for the $900^{\circ} \mathrm{C}$ series of experiments were discovered which contained some pre-characterization data. Due to the format this data is presented as a series of tabular sheets in Appendix A.

The computer printouts were compared electronically with the data tables obtained from the Ph.D. Thesis (Mobasheran, 1990). Following some corrections to the Ph.D. tables the datasets were self-consistent. The computer printouts provided some additional data such as annealed specimen data and Poisson's Ratio. Tables of hand written thermal expansion data were discovered for OC3, OC2 and OC4. These are presented in a series of Tables in Appendix B.

In addition, several data storage disks (3.5”) have been found in the archive. It is anticipated that these disks could reveal additional data and any data manipulations that were carried out. However to date these disks have proved unreadable. It is recommended that efforts are continued to read these disks and uncover missing data.

\section{Analysis of Available Data}

All data are plotted as a function of neutron fluence $\left(10^{26} \mathrm{n} / \mathrm{m}^{2}[\mathrm{E}>50 \mathrm{keV}]\right)$. 
ORNL/TM-2009/176

\section{$900^{\circ} \mathrm{C}$}

\section{Dimensional change}

The dimensional change data, length and diameter have been calculated using engineering strain. The original dimensional change data were re-calculated using large strain formulation and as expected no significant difference was observed at these modest strains.

The direct and lateral dimensional change data at $900^{\circ} \mathrm{C}$ are presented in Figure 3 and 4 . A little surprisingly there is some overlap in the different stressed datasets. This can in part, be explained by the overstressing of specimens in Column 4 during OC-1. The specimens could have been subjected to a considerable over stress for some period during the irradiation and this is reflected in the enhanced dimensional change over Column 3 specimens shown in Figures 3 and 4.

In Figure 4 the lateral data show little or no separation at low fluence which may highlight some concerns surrounding the lateral data - i.e. is the fluence correct? This should be investigated further. It is also interesting to note that the higher stress in Column 4 could indicate earlier reduction in the rate of change of the lateral creep data although there is obviously very limited data. This requires further investigation.

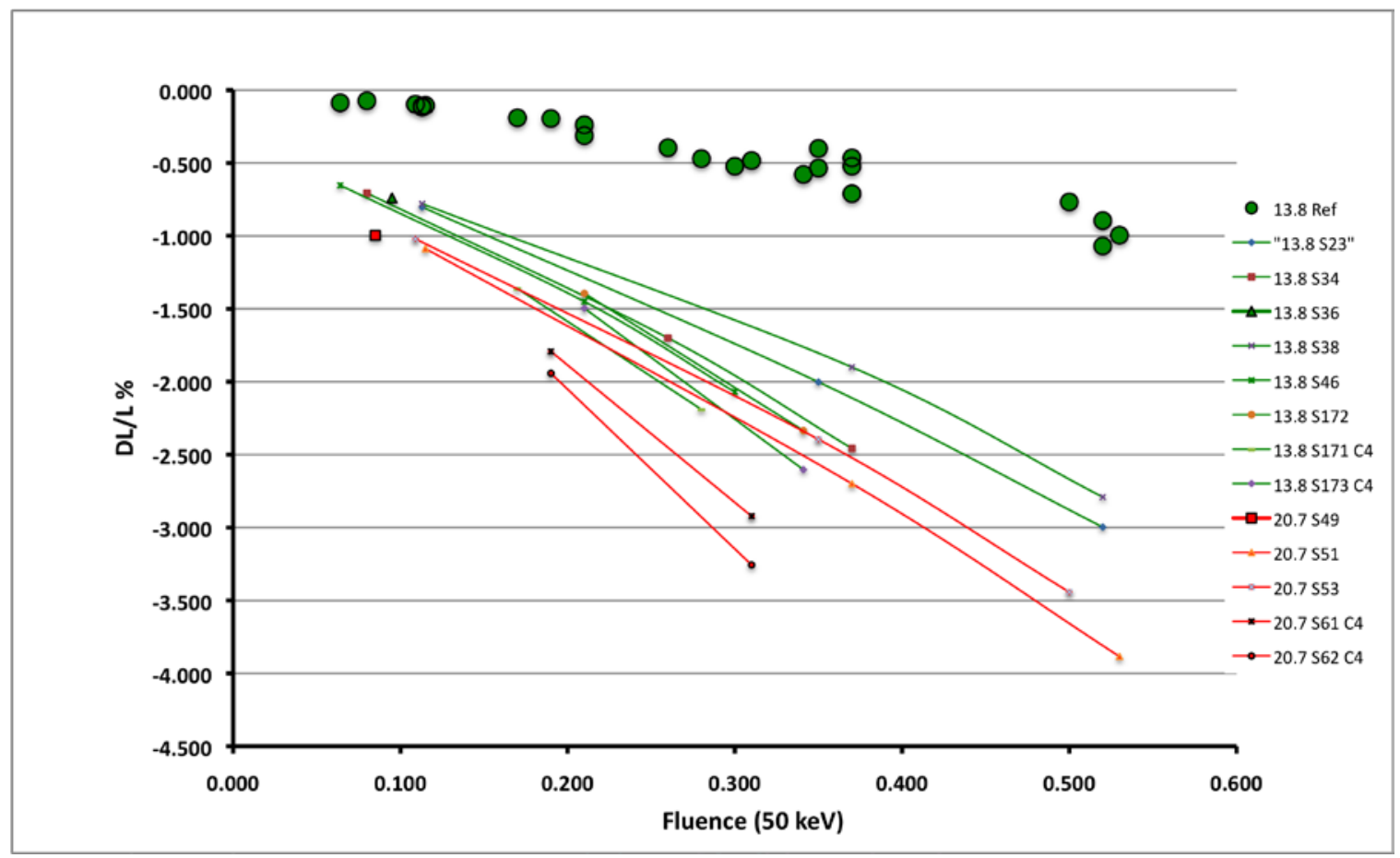

Figure 3: $900^{\circ} \mathrm{C}$ Direct Dimensional Change Data 
ORNL/TM-2009/176

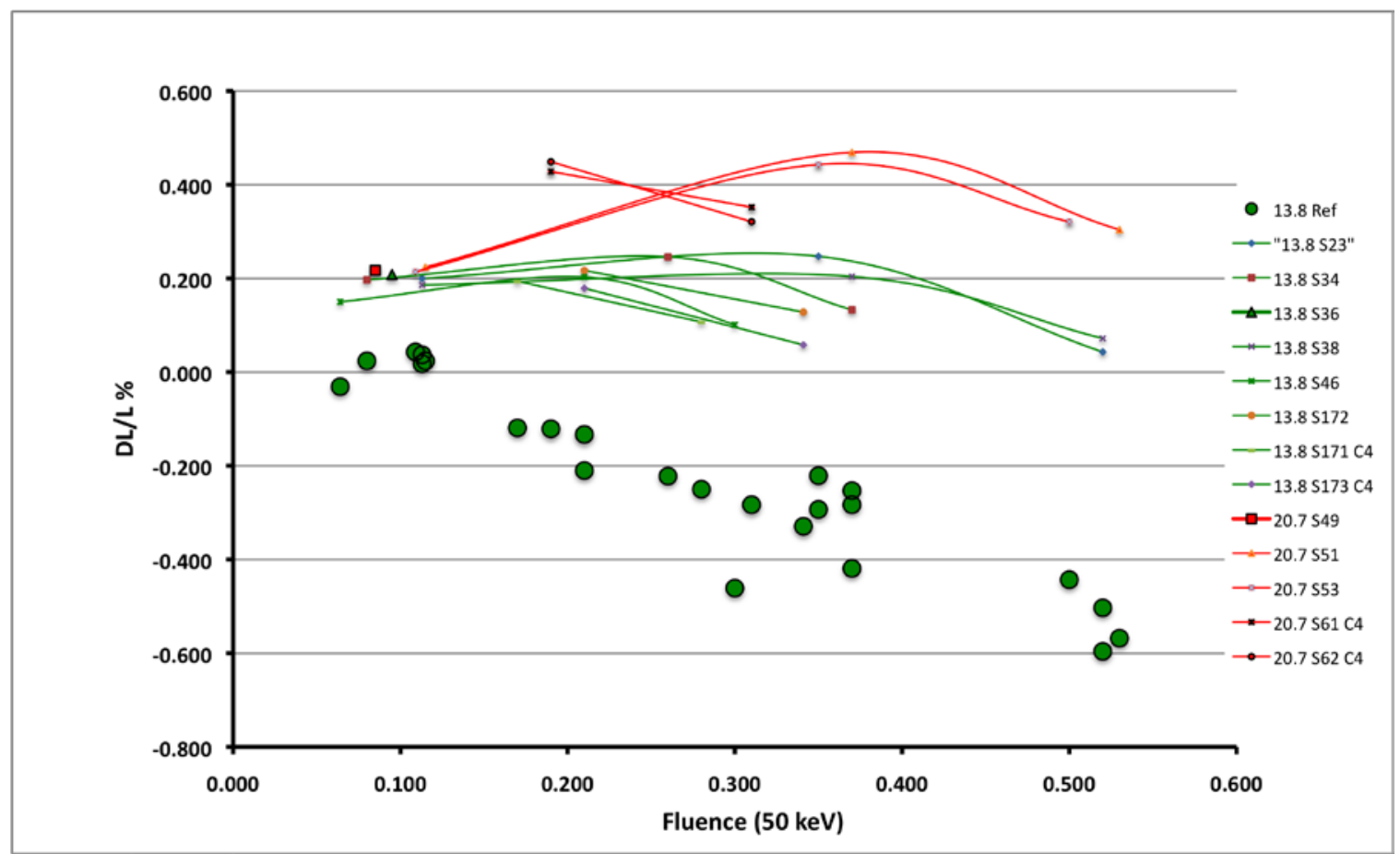

Figure 4: $900^{\circ} \mathrm{C}$ Lateral Dimensional Change Data

The volume data are shown in Figure 5 based on the diameter and length changes quoted above. However it should be noted that the original volume data quoted by Mobasheran (1990) included a correction due to the internal hole drilled in the reference specimens to allow thermal couple insertion and for the spacer holes drilled in the stressed specimens. This slight modification to volume change was required to determine the true density as it was used in the derivation of the Modulii via the density. The data are very scattered and it is difficult to draw any conclusions from the data. 


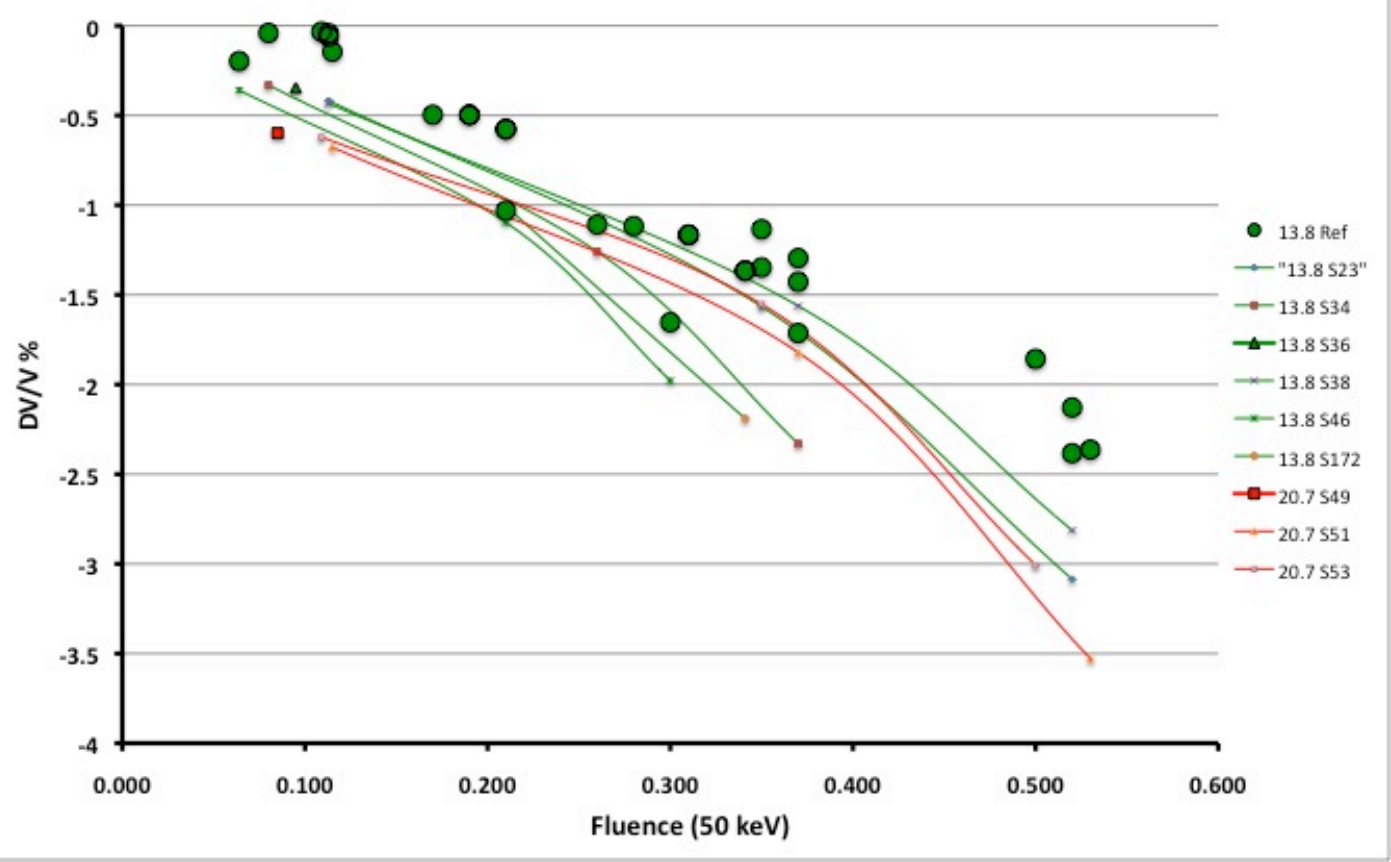

Figure 5: $\mathbf{9 0 0}^{\circ} \mathrm{C}$ Volume Change Data

\section{Irradiation Induced Creep}

The quoted creep data has been derived by the difference between the stressed and reference specimens at the same position (axial height) within the column. No corrections appear to have been made to the measured data; however, it is not known which reference specimens were used in the original analysis. It was also observed that some difference in fluence at various positions throughout the capsules were observed between the reference and stressed specimens. This requires resolution, i.e. is there any significant difference at a given axial position?

In this re-analysis Stressed Column 3 has been paired with Reference Column 1 and Stressed Column 4 has been paired with Reference Column 2. The creep strain has also been calculated assuming the German KFA correction for the changes in CTE and Modulus (See Appendix C). Apart from the very low fluences a modest correction ( 2\%) was applied to the measured data as a result of the KFA correction. Any lateral creep data has been presented without correction as there is insufficient data to make the corrections. The direct and lateral data are presented in Figures 6 and 7 and show the high creep strains predicted for the Column 4 data from OC-1. As a result of the uncertainty associated with these data they have not been considered further in this analysis but perhaps could be revisited in the future. 
ORNL/TM-2009/176

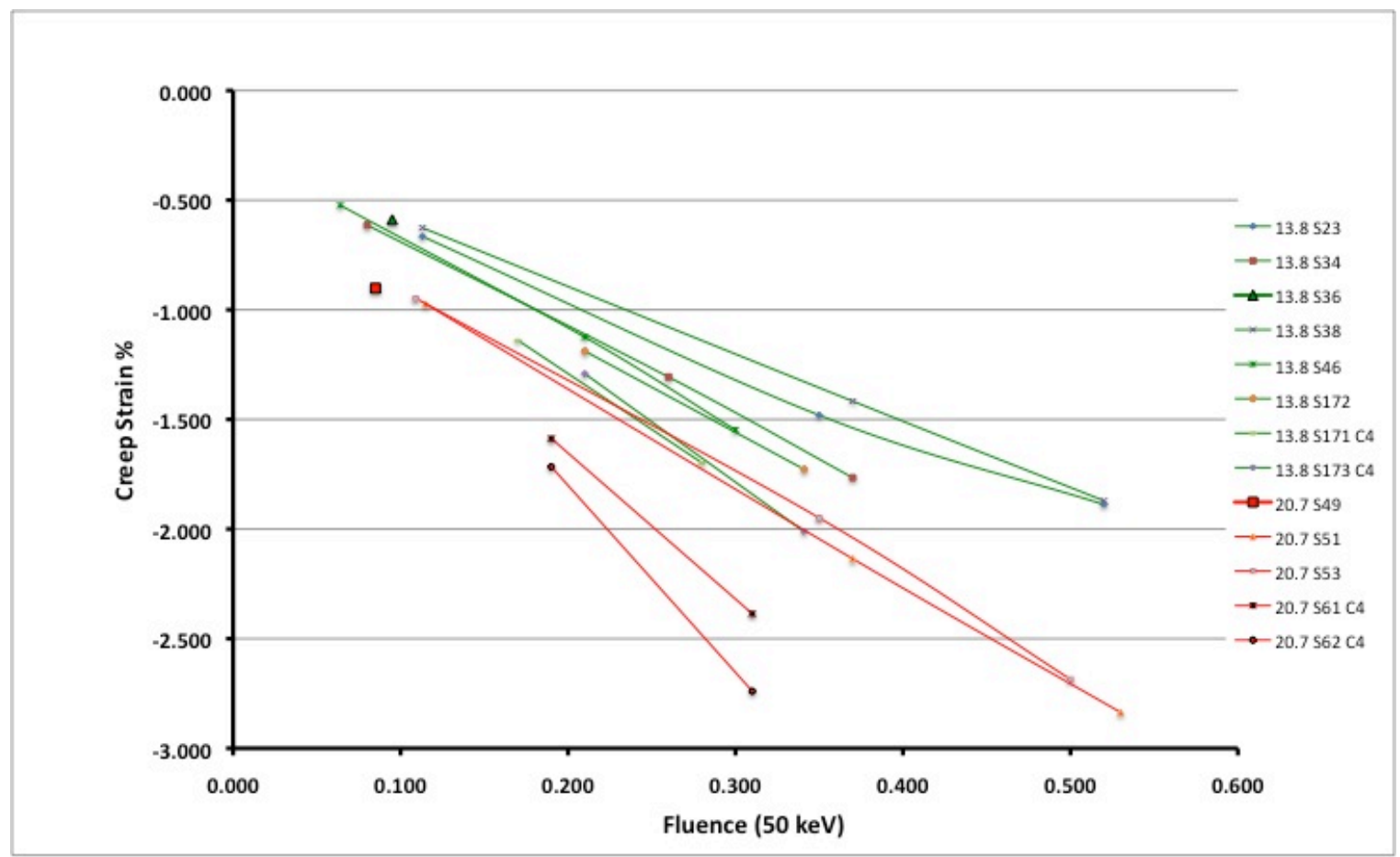

Figure 6: $900^{\circ} \mathrm{C}$ Direct Creep Data

In Figure 7 the lateral creep data show little or no separation at low fluence which may indicate little or no lateral creep for these specimens. It could also indicate that there could be some concerns surrounding the data i.e. is the fluence correct? Also shown in Figure 7 is the C4 data indicating that lateral creep has saturated earlier or at a lower level which would not be expected if the specimens were significantly overloaded. This requires further investigation. 
ORNL/TM-2009/176

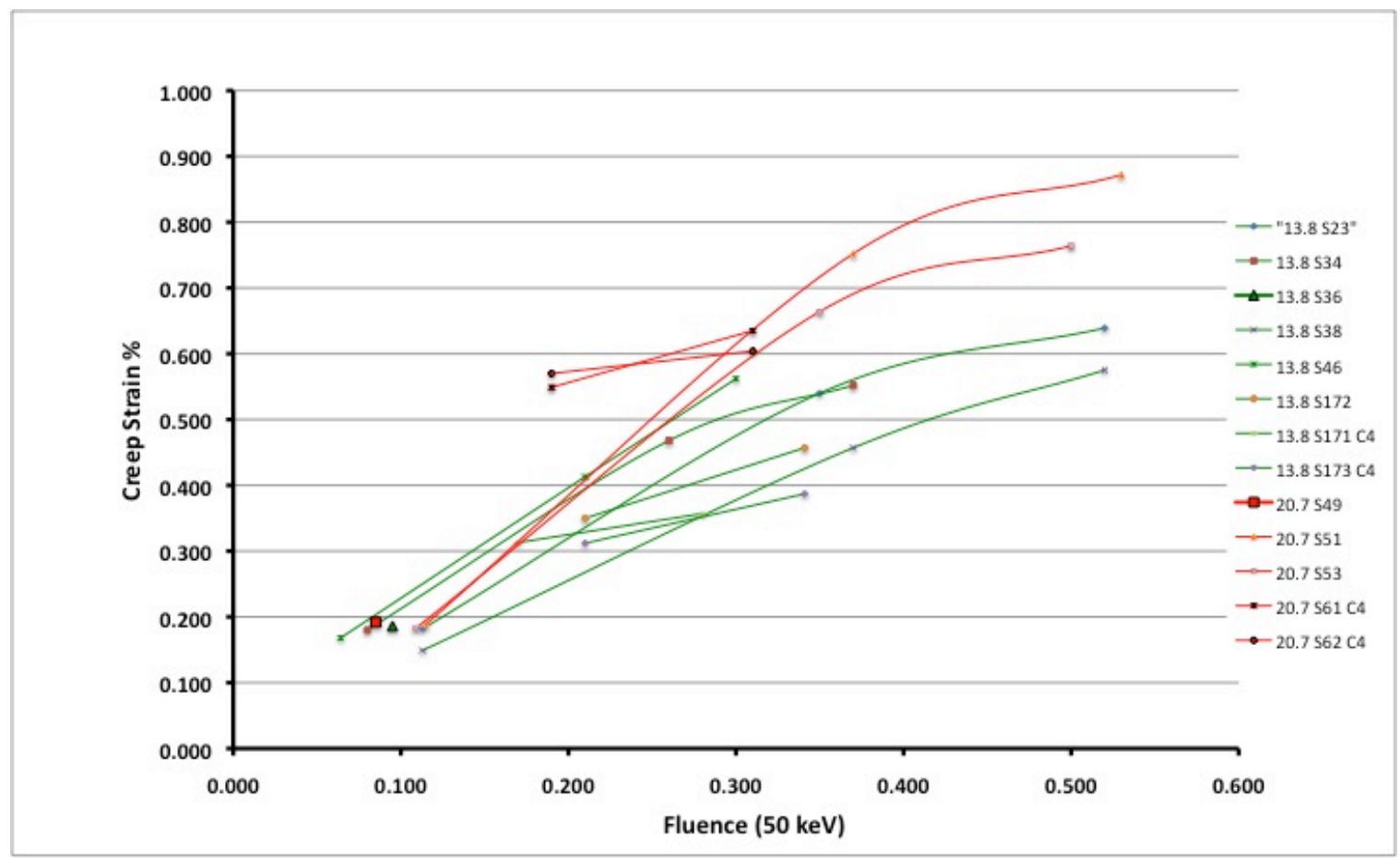

Figure 7: $900^{\circ} \mathrm{C}$ Lateral Creep Data

The direct creep data have been normalized by initial elastic strain and are expressed in \%/esu (elastic strain units) as shown in Figure 8. The normalization has used the initial dynamic Modulus. The data shows that the higher stress data lie slightly below the lower stress values. It also shows considerable variation in the 13.8 MPa dataset. This may be due to the temperature variation exhibited in OC-1 at some axial positions in the Capsule. Also the UK assumes the initial Static Modulus is used which would affect the esu calculated and the correction applied. This may also reduce the scatter in the results. These issues may be resolved with further investigation. 


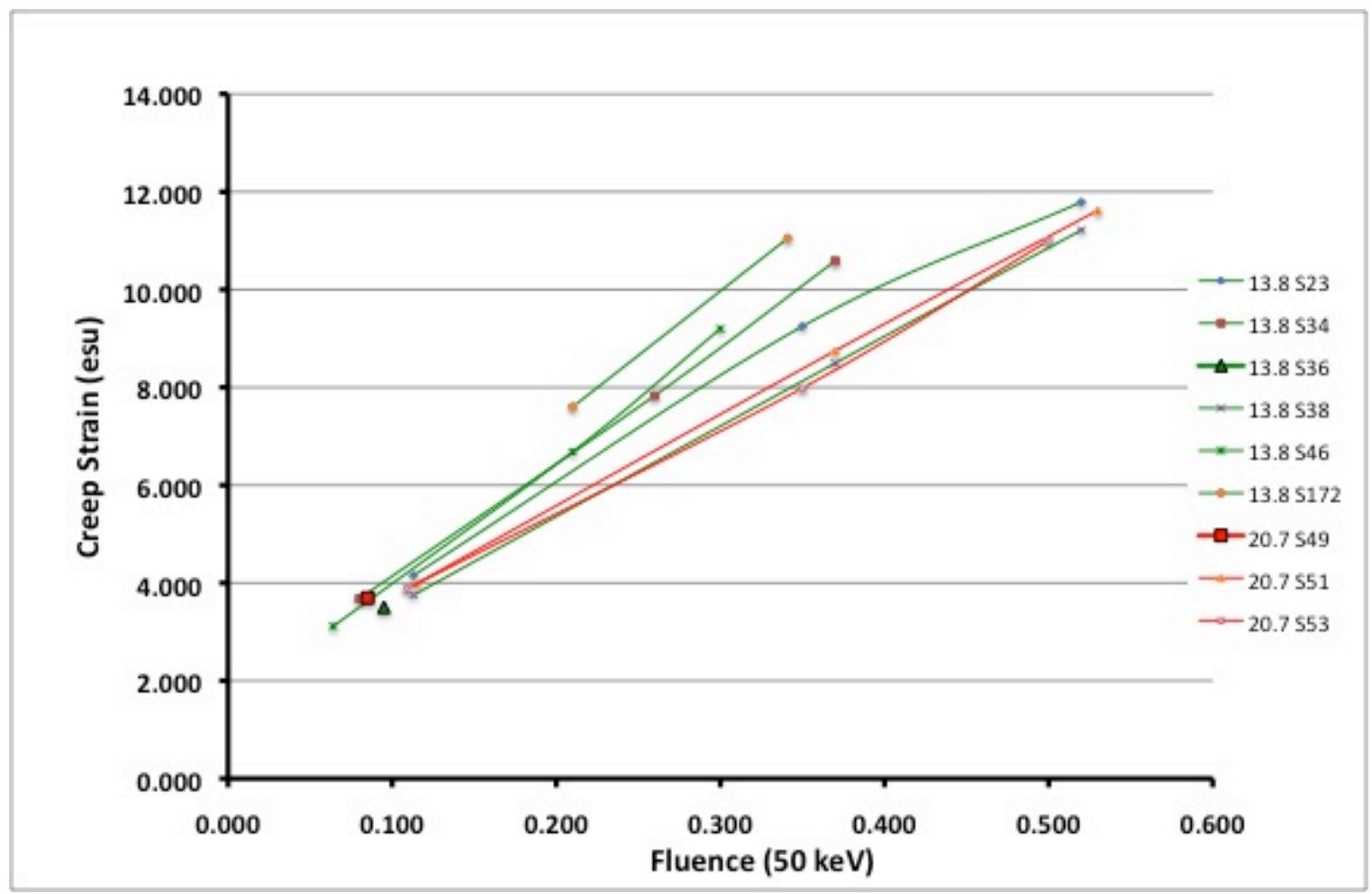

Figure 8: $\mathbf{9 0 0}^{\circ} \mathrm{C}$ Normalized Direct Creep Data

\section{Annealed Specimens}

Five specimens that were irradiated under load in OC-1 were subsequently irradiated without load in OC-3, i.e. the crept specimens were annealed to recover primary creep. The length change results of the anneal experiment are shown in Figure 9 and Figure 10 shows the equivalent results for diameter change.

The figures clearly illustrate the total recovery of creep strain, in fact some of the annealed data exhibit considerable recovery beyond the reference data set. This "over recovery" has been observed in thermal annealing experiments of unstressed PGA graphite and has also been observed in UK unstressed experiments where specimens have been re-irradiated at different irradiation temperatures and overshoot in the dimensional change has been observed. This requires further consideration.

Unfortunately, three of the five annealed specimens were loaded in column 4 of capsule OC-1 and were therefore subject to significant overload. As discussed earlier the data obtained from the column 4 specimens is somewhat uncertain and has been excluded from further consideration at this time. Therefore only two of the annealed specimens, one 13.8 MPa and one 20.7 MPa, were loaded in Column 3 of Capsule OC-1 and annealed in OC-3. Figure 11 compares the normalized creep strain from these specimens with the main $\mathrm{H}-451$ dataset. The results are remarkable and show that approximately 4 esu are recovered substantially in excess of the 1 esu traditionally attributed to primary creep. (NB if UK static Modulus is used this drops to $~ 3.5$ esu). It should be noted that these annealing results indicate there is apparently no secondary or "irrecoverable" creep established at these low fluences. 
Further work is required to investigate the overstressed specimens that were subsequently annealed. Efforts should be made to investigate the Disks for material property measurements of annealed samples.

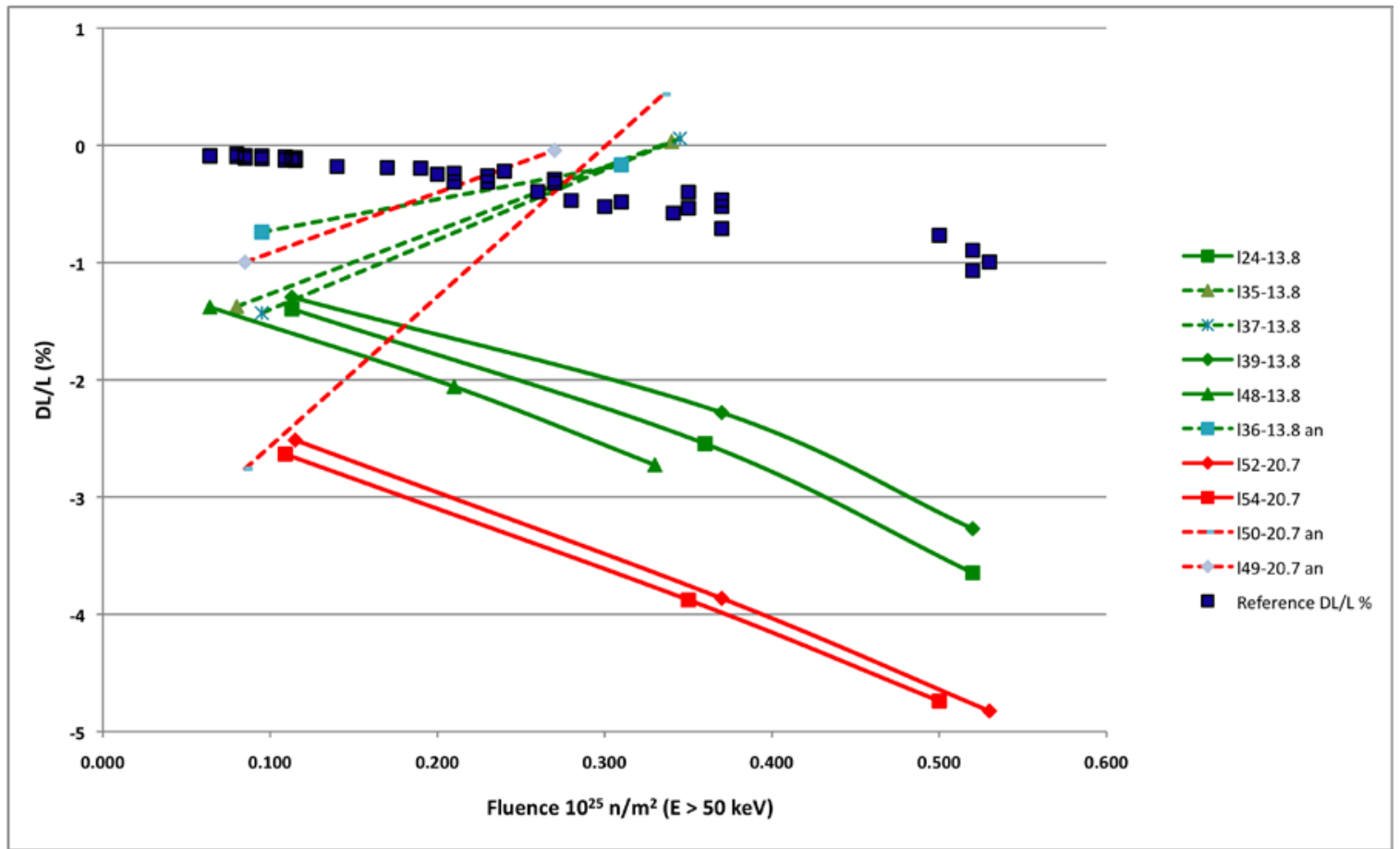

Figure 9: Direct Recovery of Annealed Specimens from OC-3

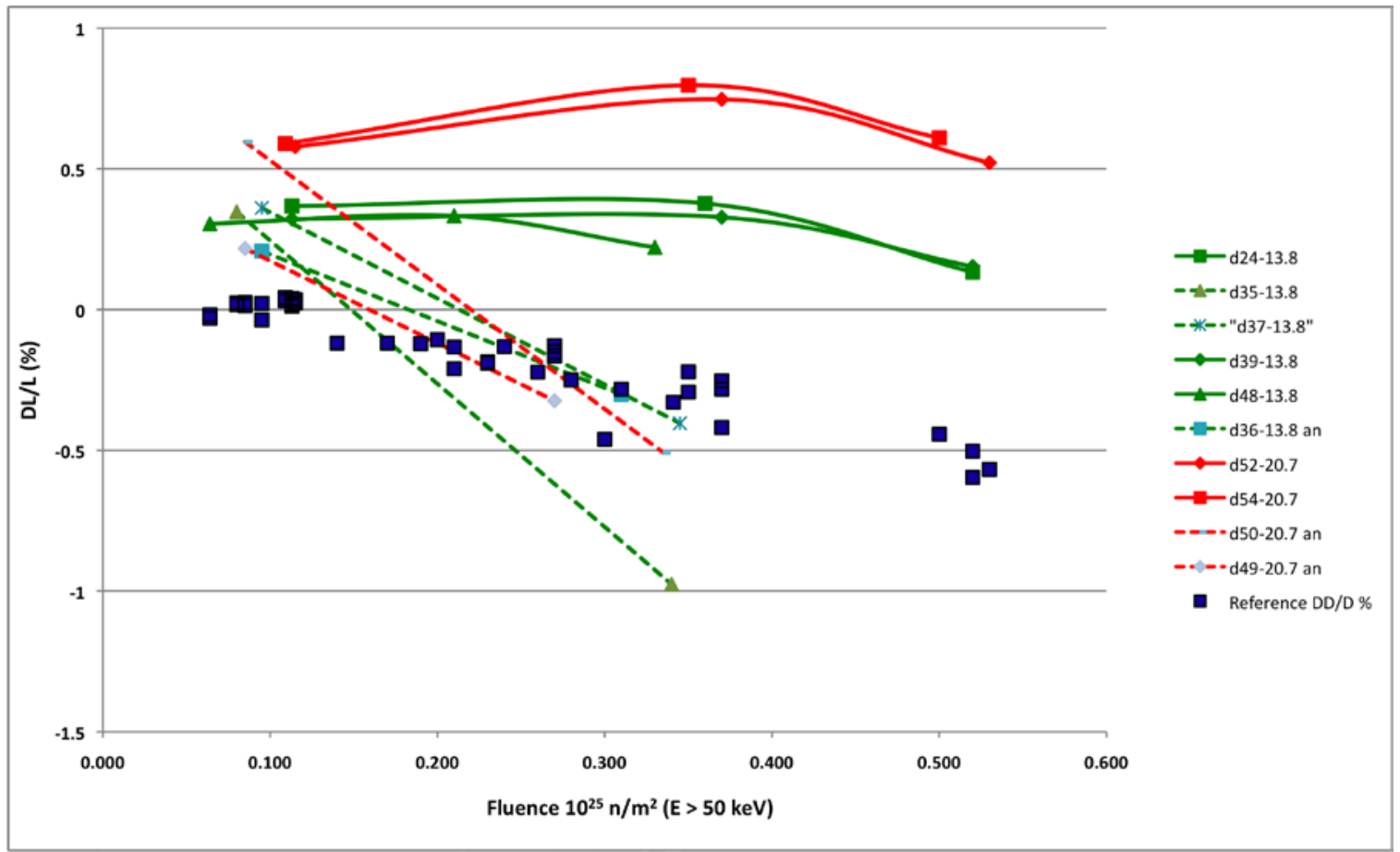

Figure 10: Lateral Recovery of Annealed Specimens from OC-3 


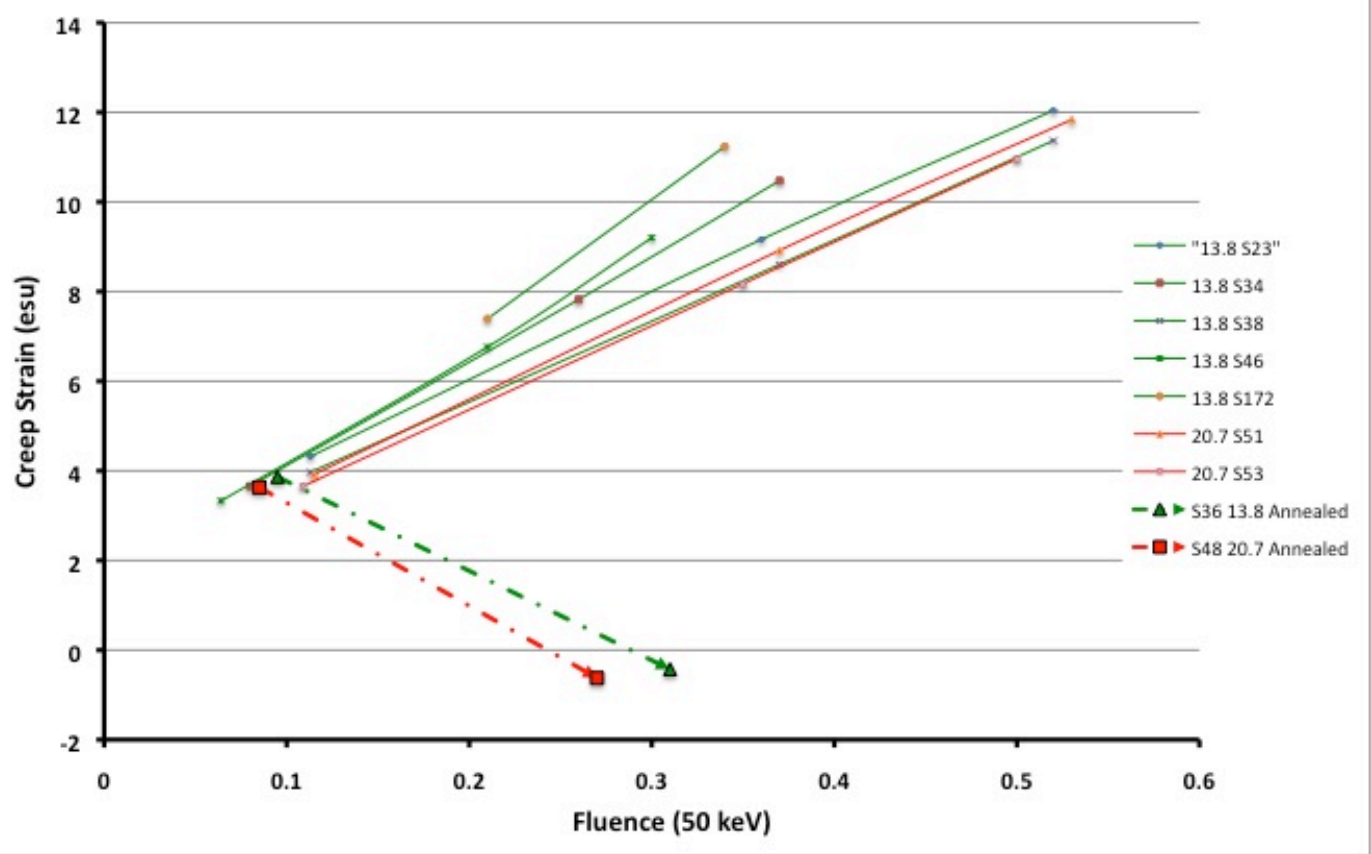

Figure 11: Normalized Direct Recovery of Annealed Specimens from OC-3

\section{Modulus}

The Irradiated Modulus data at $900^{\circ} \mathrm{C}$ are presented in Figure 12. The data are separated into Reference and Stressed specimens and appear to indicate no dependence of either Modulus on creep strain at these fluences.

From Figure 12 it would appear that the data are saturating towards the higher fluence. Figure 13 represents the data with the irradiation history of each sample shown and indicates that both Modulii are increasing possibly at a different rate to the reference samples. This is reinforced in Figure 14 where the data are evaluated as fractional change in Modulii and indicate a different behaviour. This requires further investigation. 
ORNL/TM-2009/176

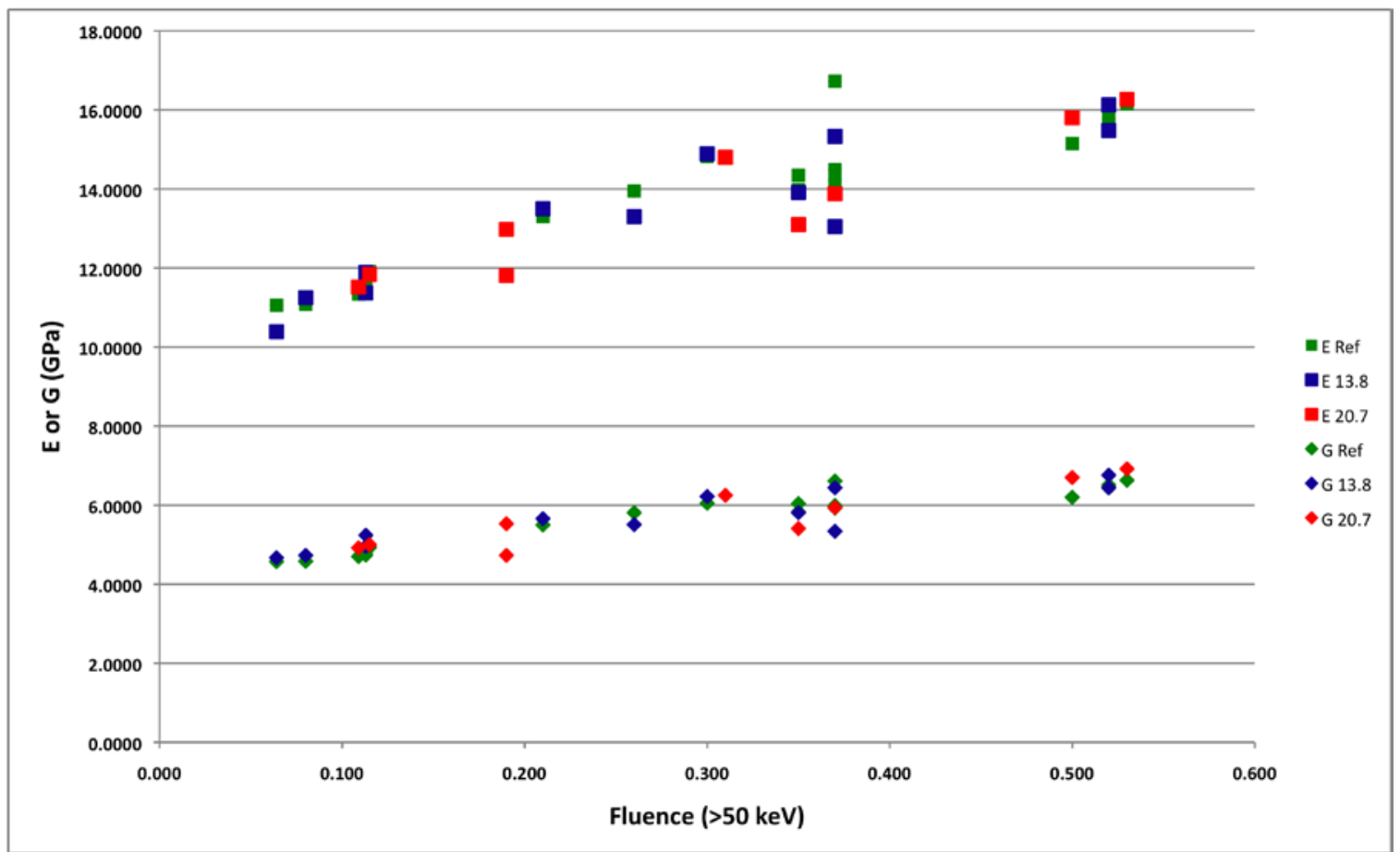

Figure 12: Young's and Shear Modulii at $900^{\circ} \mathrm{C}$

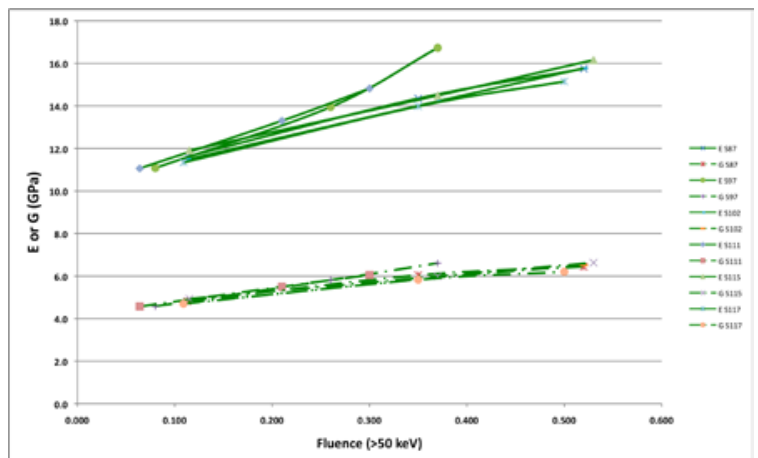

a) Reference

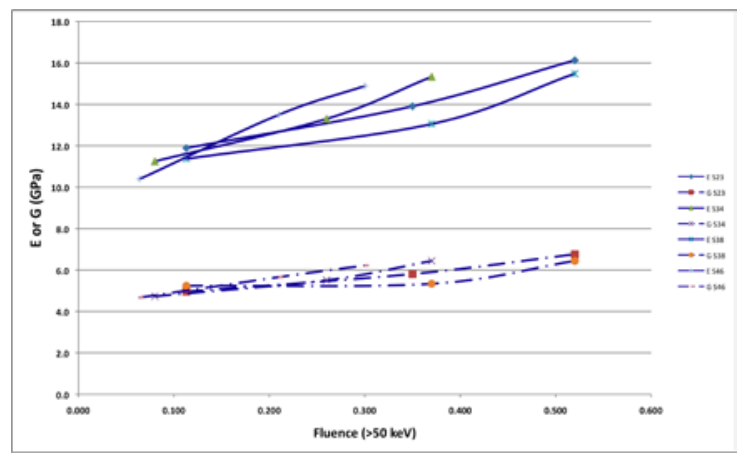

b) $13.8 \mathrm{MPa}$ 
ORNL/TM-2009/176

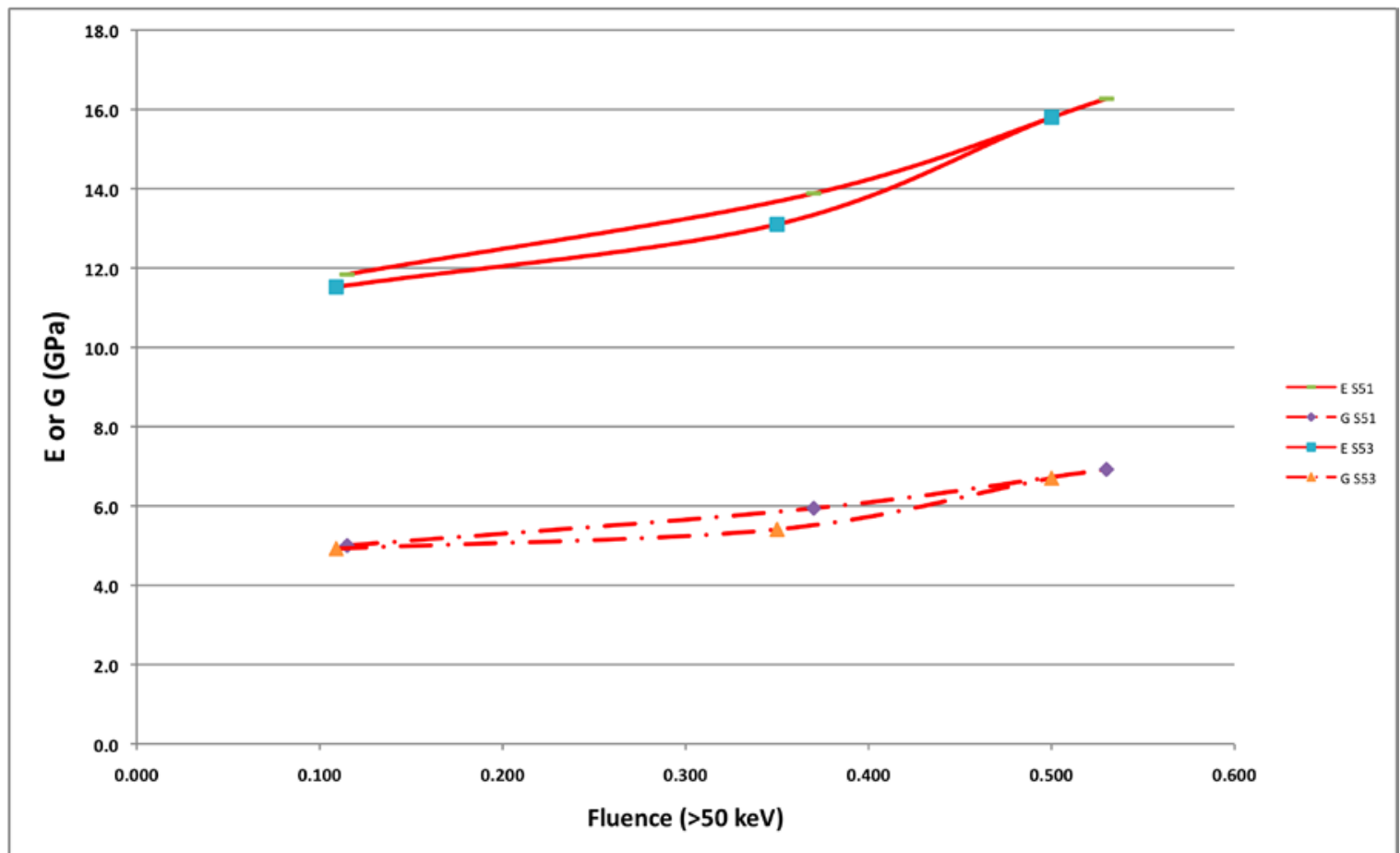

c) $20.7 \mathrm{MPa}$

Figure 13: Young's and Shear Modulii at $900^{\circ} \mathrm{C}$ with specimen history

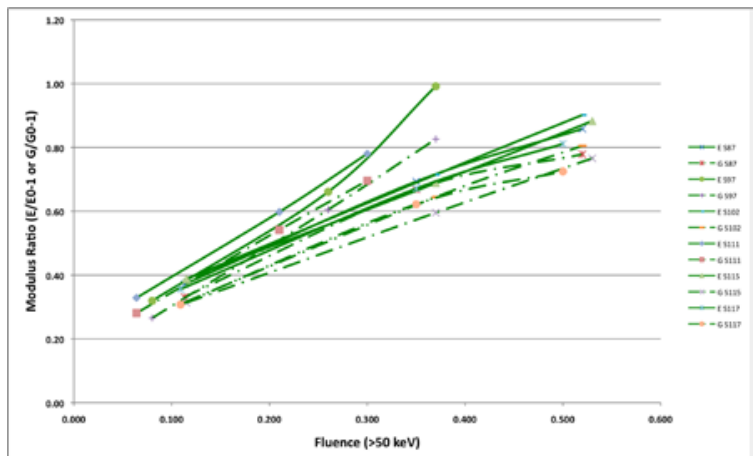

a) Reference

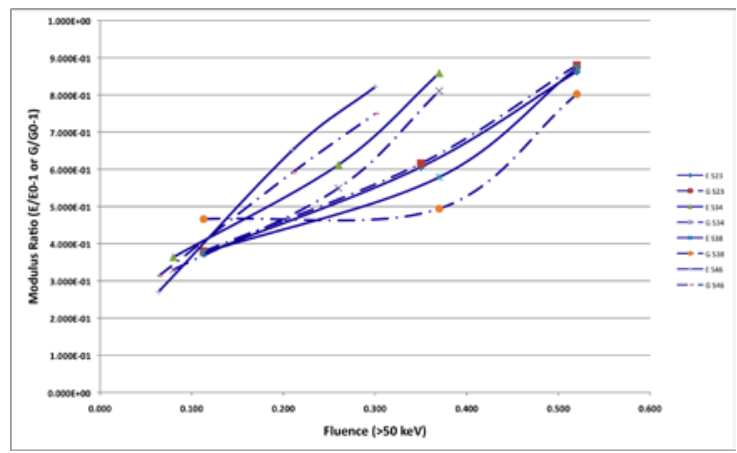

b) $13.8 \mathrm{MPa}$ 


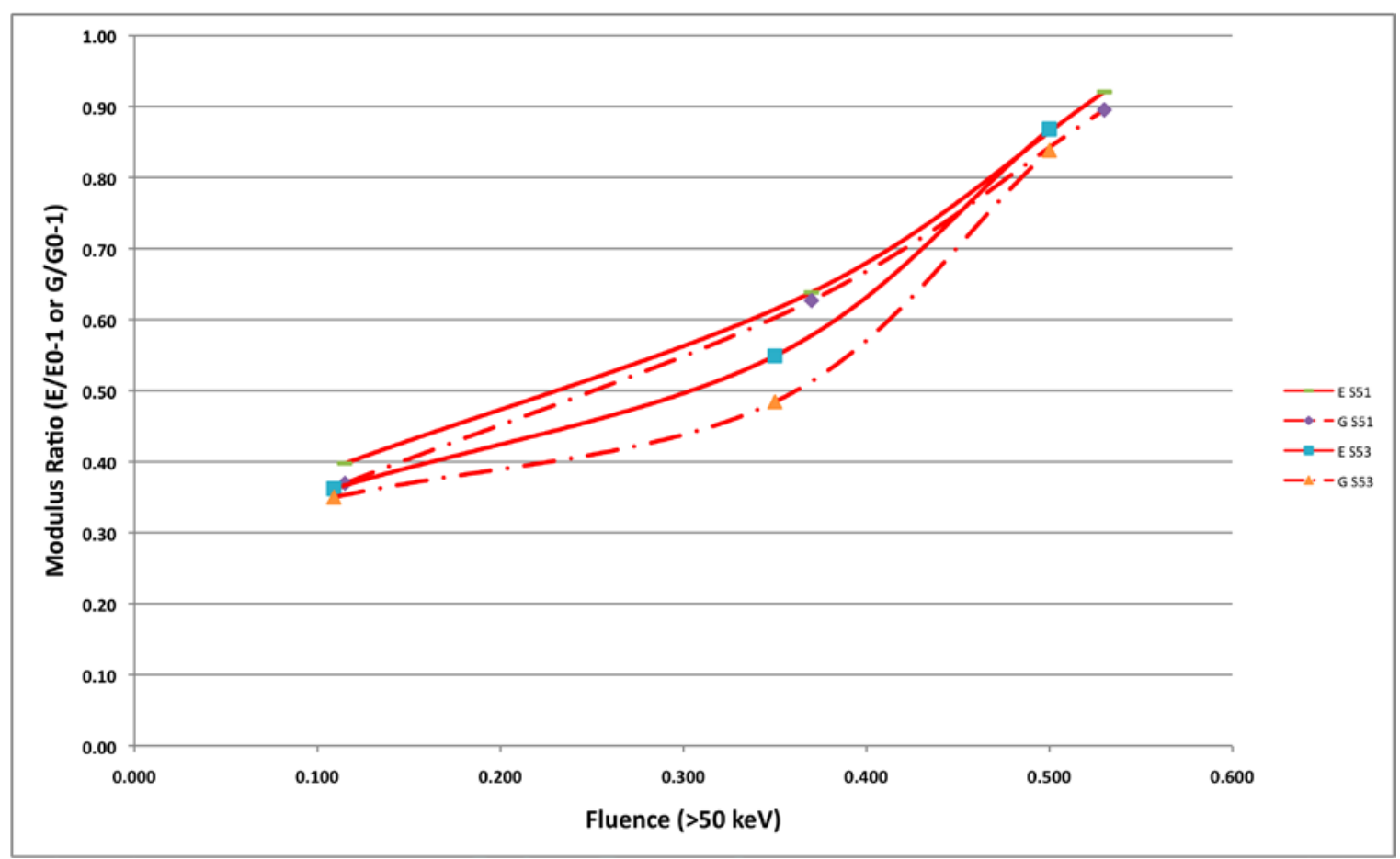

c) $20.7 \mathrm{MPa}$

Figure 14: Fractional change of Modulii at $900^{\circ} \mathrm{C}$ with specimen history

\section{Poisson's Ratio}

There is limited Poisson's Ratio data reported in the computer print out which appears to be at odds with the data published in the ORNL annual report and the data presented by Kennedy et al (1977) where it was claimed that Poisson's ratio of H-451 was substantially reduced as a result of creep strain for H-451. The H-451 Kennedy et al (1977) data $\left(900^{\circ} \mathrm{C}\right)$ are reported in Figure 15 . The US data presented in the UKAEA review (Brocklehurst and Kelly, 1989) are shown in Figure 15 and 29.

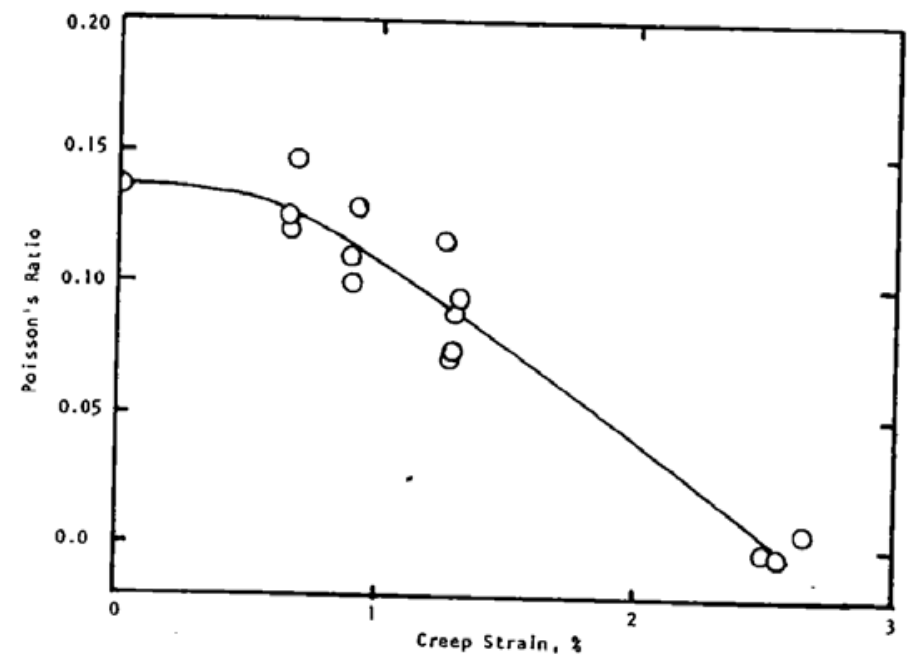

Figure 15: Effect of Creep strain on Poisson's ratio at $900^{\circ} \mathrm{C}$ (Kennedy et al, 1977) 
It has been found that this graph has been mislabeled and is $\mathrm{H}-327$ not $\mathrm{H}-451$. The $\mathbf{H}-327$ data presented in this Figure should be confirmed as this data is not contained within the computer printouts. Additional H-451 data may also be sourced from the computer disks.

The H-451 unirradiated Poisson's ratio has been determined from quoted sonic velocities in the datasheets (Table 9) and this is presented in Figure 16 along with the quoted $\mathrm{H}-451$ irradiated data (OC1 only). Apart from Specimen 23 the data all exhibit an increase of Poisson's ratio with irradiation and compressive creep strain in contrast to the data presented in Figure 15. This begs the question does tensile creep strain reduce Poisson's ratio.

The increase in Poisson's ratio of the stressed data (Specimen 23 aside) may be slightly less than the reference data. Now that the unirradiated Poisson's ratio have been determined for the specimens it may be possible to determine irradiated Poisson's ratio without knowing the sonic velocities. This should be investigated further to provide additional information on Poisson's ratio at higher irradiation fluences and creep strains.

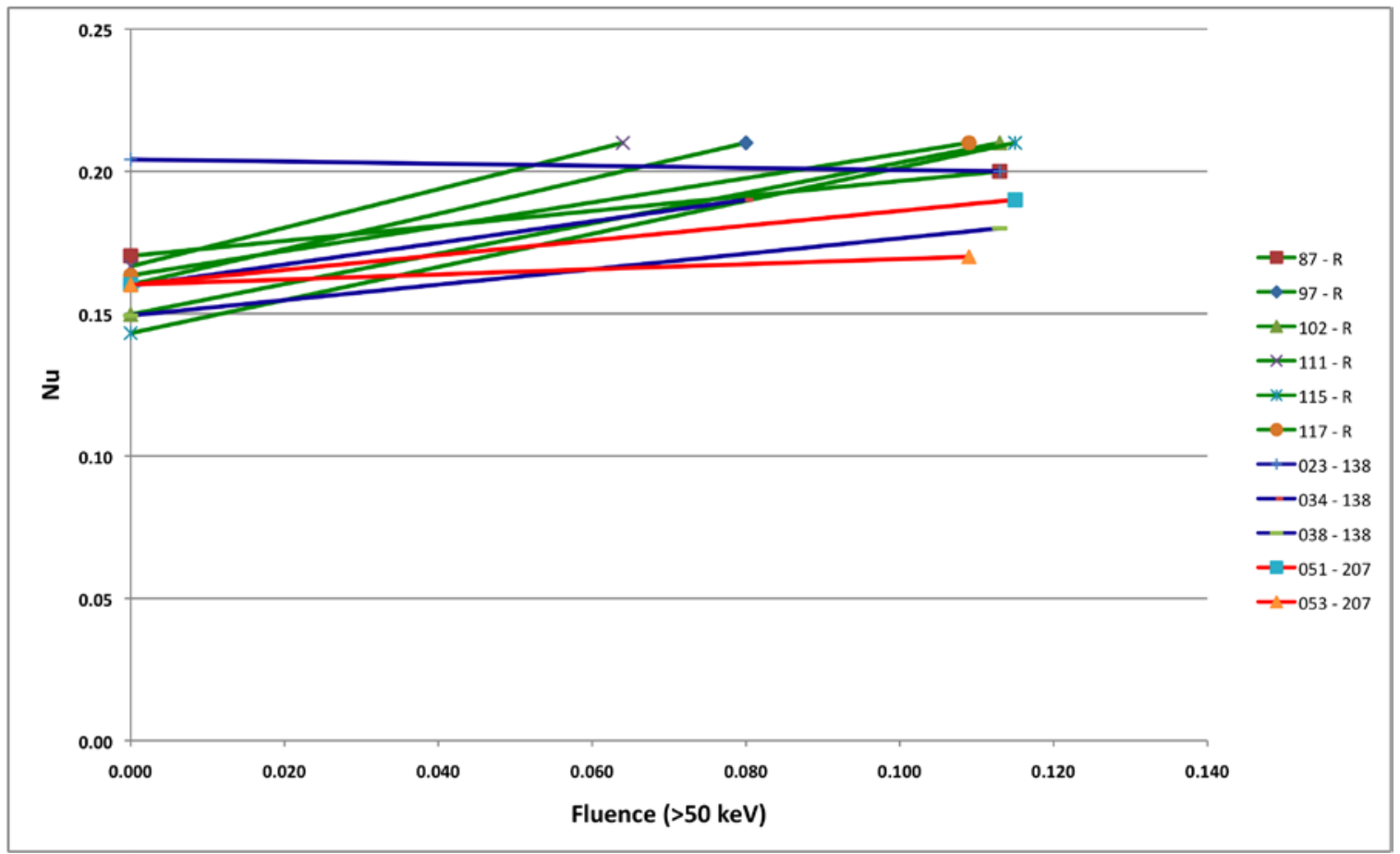

Figure 16: Effect of Creep strain on Poisson's ratio at $900^{\circ} \mathrm{C}$

\section{Coefficient of Thermal Expansion}

Figure 17 shows the representation of the CTE dilatometer data from the OC-1, 3 and 5 series of experiments presented at the 1979 Carbon Conference (Kennedy and Eatherly, 1979). The dilatometer data has been evaluated by eye as a linear function of temperature with $\mathrm{A}$ the intercept (i.e. the mean $\mathrm{CTE}$ at $0^{\circ} \mathrm{C}$ ) and $\mathrm{B}$ the slope. In Figure 17, A is 
presented as $\mathrm{A} / \mathrm{A}_{0}$ which is believed to be ratio of the stressed CTE to the unstressed CTE and $\mathrm{B}$ is presented as the slope determined from the stressed specimen. This approach is considered questionable as the evaluation is subjective and the CTE data are non-linear.

Figure 17 shows that $\mathrm{A} / \mathrm{A}_{0}$ and $\mathrm{B}$ are relatively flat for all grades up to $2 \%$ creep strain and the previous interpretation of these results is significantly influenced by the creep strain values above $2 \%$. These high creep strain values are believed to be specimens that were subject to a significant over load in column 4 during experiment OC- 1 and as such the results may be misleading. This requires further investigation outside the scope of this report.

Additionally, the unstressed value of B, i.e. at zero creep strain, estimated from the dilatometer data is approximately $2 \mathrm{E}-9 \mathrm{~K}^{-2}$ which is higher than the trend line drawn in Figure 17. Therefore using the data as presented in Figure 17 and ignoring both the drawn trend line and the high strain values would tend to suggest that the temperature dependence of CTE is independent of creep strain for an irradiation temperature of $900^{\circ} \mathrm{C}$.

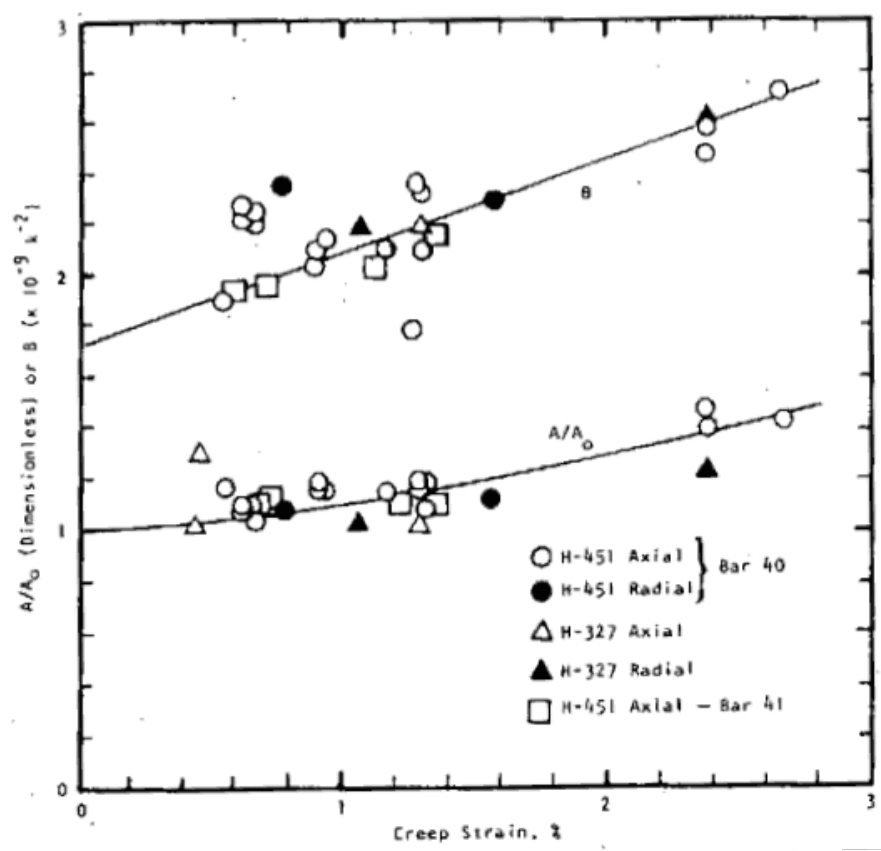

Figure 17: Effect of Creep Strain on the Mean Coefficient of thermal Expansion $(\alpha=A+B T)$ (Kennedy and Eatherly, 1977)

The dilatometer data from OC-1 and OC-3 have been re-assessed from the available extension data and are presented in Appendix A and Appendix B. The determination of the mean $\mathrm{CTE}$ at $800^{\circ} \mathrm{C}$ from the extension data were similar in trend to previously quoted values but systematically offset by $0.5 \mathrm{E}-6 /{ }^{\circ} \mathrm{C}$. The systematic offset is be due to a the dilatometer measurement technique using quartz as the reference material. The CTE of Quartz is relatively temperature insensitive and this would lead to a fixed offset in the 
determination of the graphite CTE. For the purposes of this reassessment a fixed CTE offset of $0.5 \mathrm{E}-6 /{ }^{\circ} \mathrm{C}$ has been included.

Figure 18 shows the pre-irradiated dilatometer data for $\mathrm{H}-451$ specimens in the OC-1, OC-3 and OC-5 experiments. No post experiment data dilatometer data exists for experiment OC-5. The data indicate a significant variability in the mean CTE, which is consistent across the temperature range.

The UK developed a theory to derive the mean CTE at any temperature from the mean CTE over the $20^{\circ} \mathrm{C}$ to $120^{\circ} \mathrm{C}$ temperature range and the temperature dependence of the crystal CTEs, $\alpha_{c}$ and $\alpha_{a}$ up to the temperature of interest (Tsang/Marsden, CTE Science). The UK methodology assumes that any change in structure associated with CTE will manifest itself at the lower temperature range and the temperature dependence remains unaffected. Figure 18 shows that the body of pre-irradiated data can be well represented assuming the UK theoretical prediction of the temperature dependence of mean CTE (black dotted line).

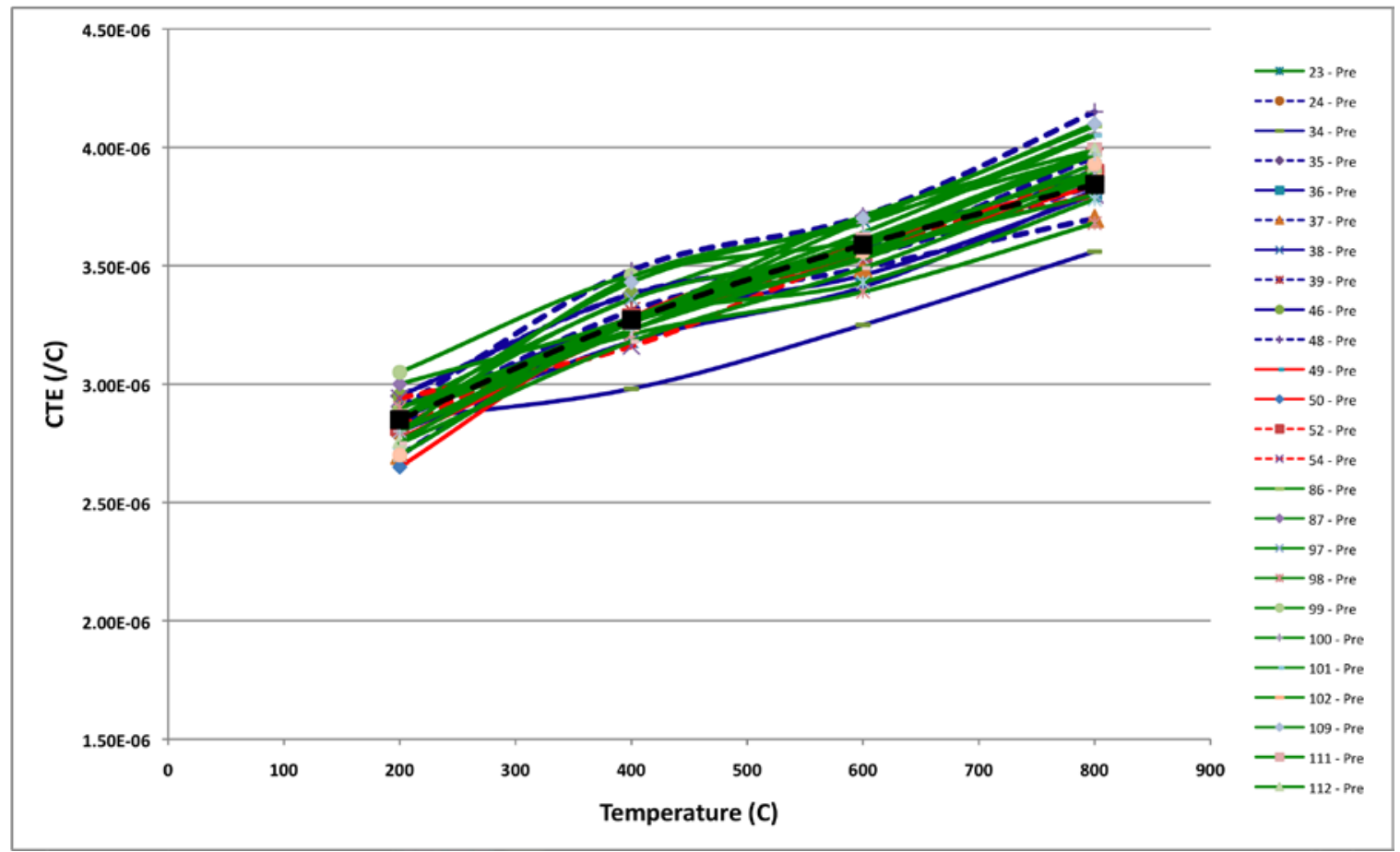

Figure 18: Pre-Irradiated Dilatometer Data from OC-1 and OC-3

Figure 19 shows the post-irradiated dilatometer data for $\mathrm{H}-451$ specimens in the OC-1 experiment. The data are split into reference and stressed specimens. The reference data indicate a similar variability in the mean CTE to the pre-irradiated data and are well represented by the UK theory. There are fewer specimens available in the stressed figures and the predictions based on the UK theory appear to under predict the temperature dependence at 13.8 MPa but represent the data reasonably well at 20.7 MPa. This requires further consideration. 
Two additional datasets are shown in Figure 19 which were found in the extension datasheets but are not referenced in the computer printouts or the Ph.D. They have been presented as 20.7 MPa data but the history of the specimens is not known. The data exhibit a similar trend to the other stressed data but have a higher CTE. It may be that additional specimens have been irradiated but not reported. This requires further investigation.

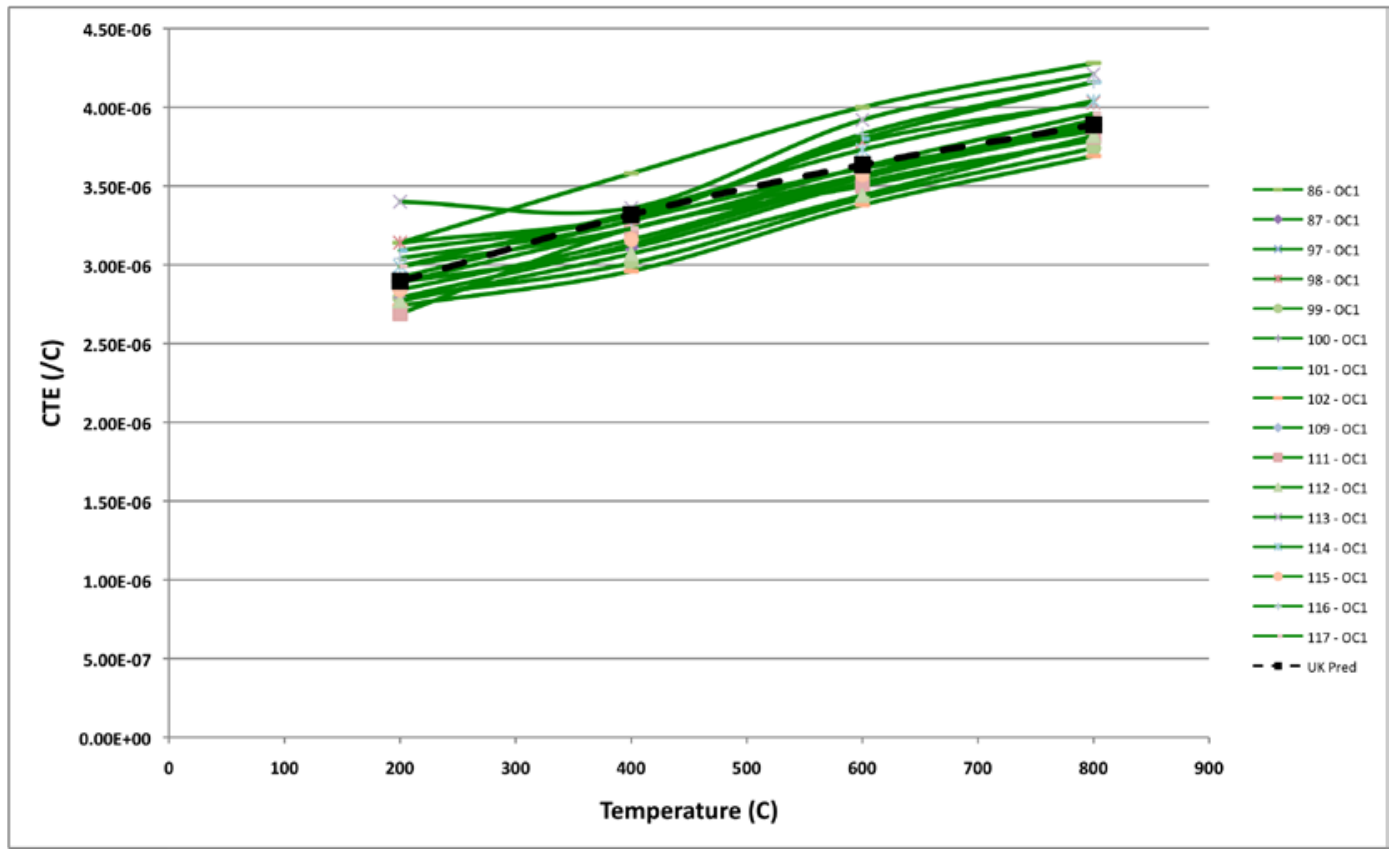

a) Reference

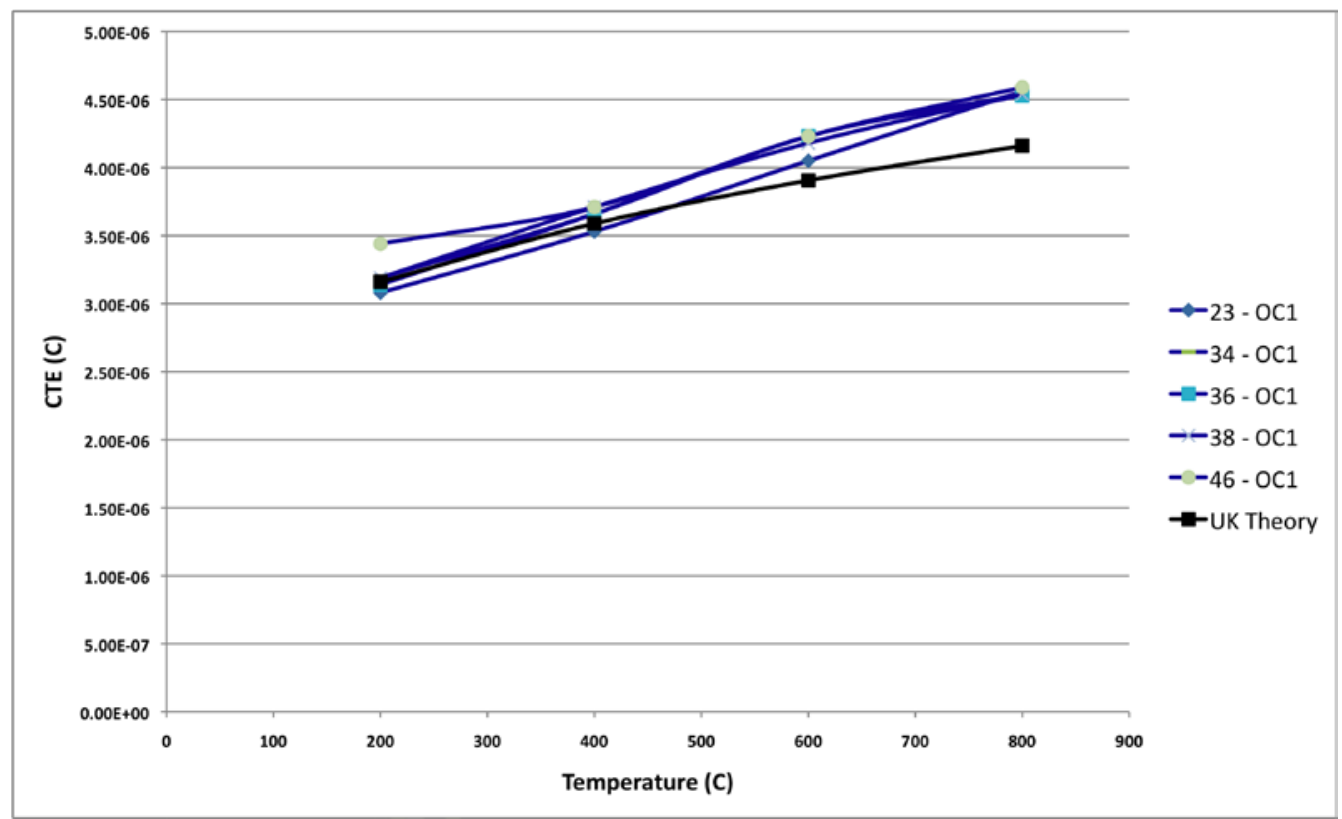

b) $13.8 \mathrm{MPa}$ 


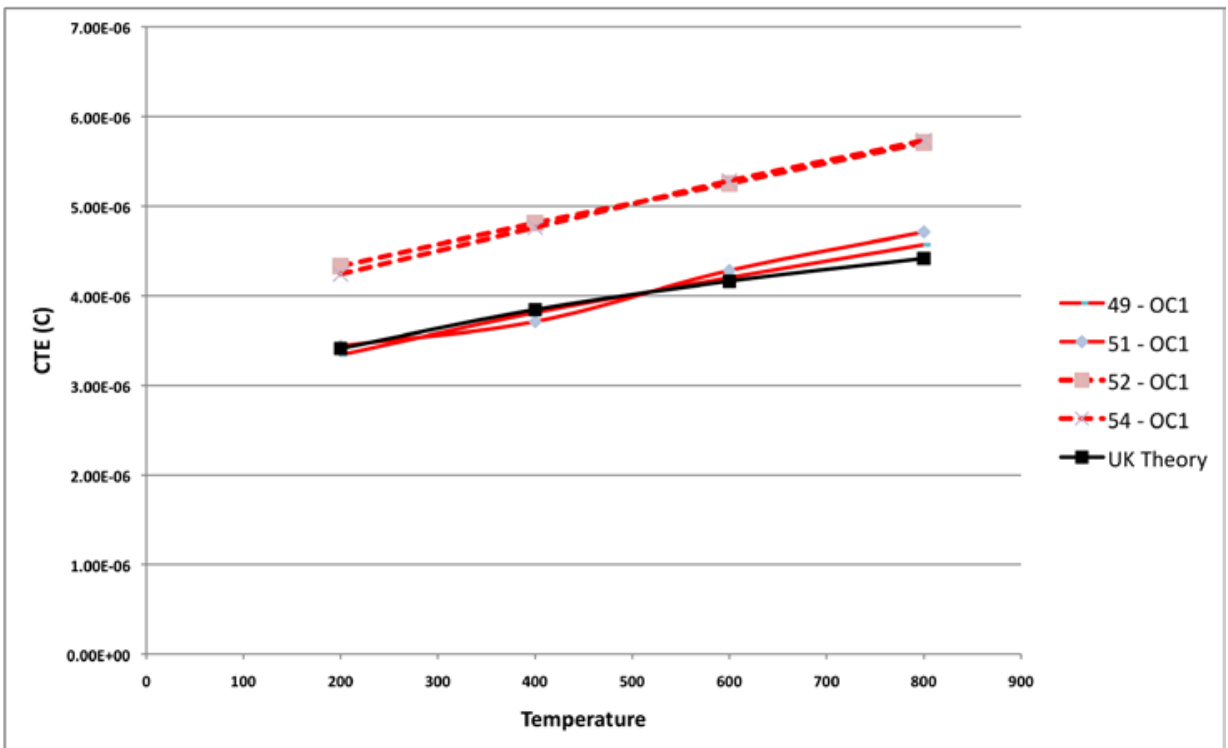

c) $20.7 \mathrm{MPa}$

Figure 19: Post-Irradiated Dilatometer Data from OC-1

Figure 20 shows the effect of the external load on the mean CTE measured at $800^{\circ} \mathrm{C}$.

There is considerable scatter in the unstressed CTE and some overlap of stressed data at low fluence. The 13.8 MPa data exhibit some scatter which may be out of trend with the 20.7 MPa data. This should be investigated further.

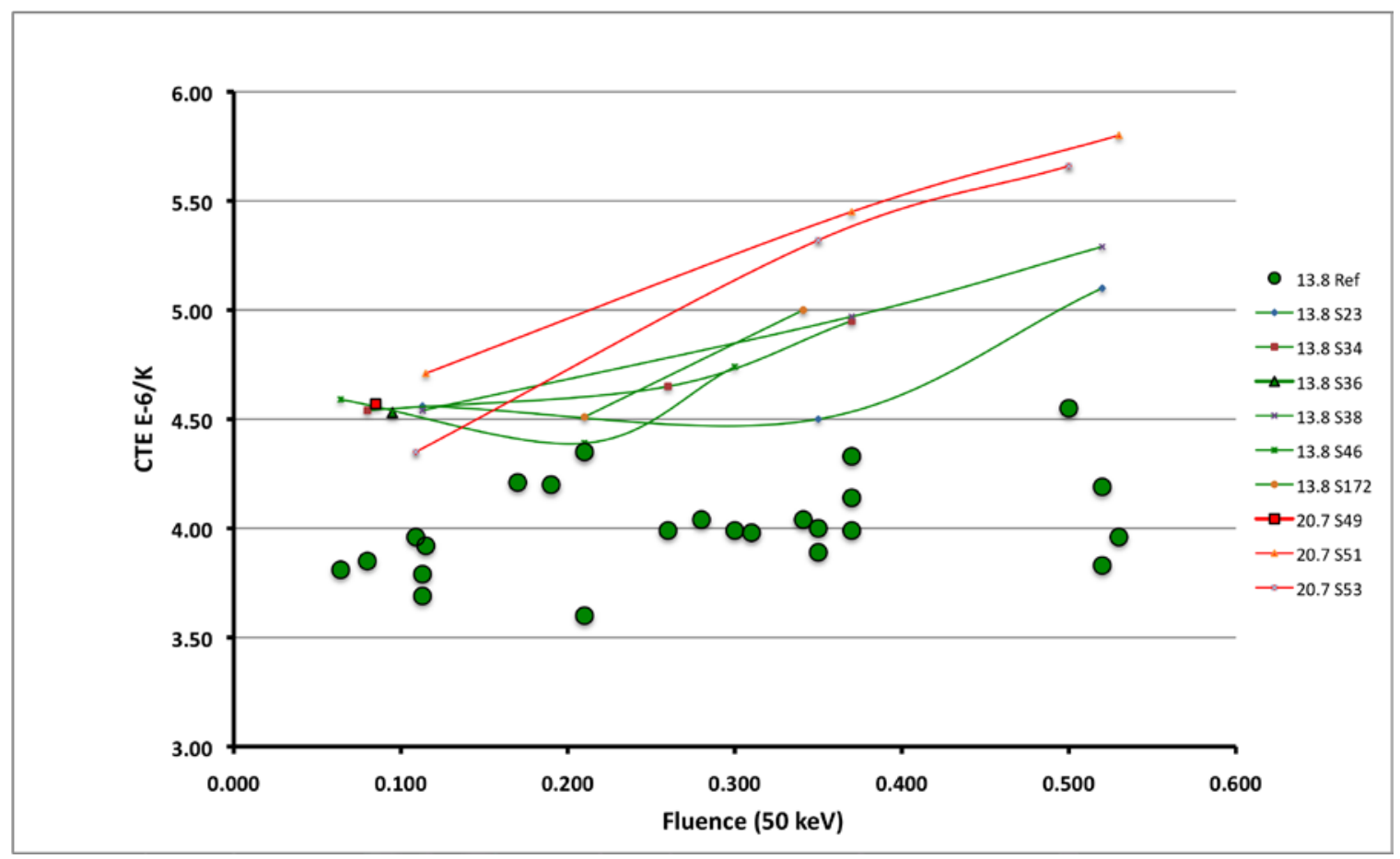

Figure 20: Effect of External Load on CTE (800) 
ORNL/TM-2009/176

$600^{\circ} \mathrm{C}$

\section{Dimensional change}

The dimensional change data, length and diameter have been re-calculated using engineering strain. The dimensional change data were re-calculated using large strain formulation and as expected no significant difference was observed at these modest strains.

The direct and lateral dimensional change data at $600^{\circ} \mathrm{C}$ are presented in Figure 21 and 22. The data exhibits little scatter and clear separation between the $13.8 \mathrm{MPa}$ and 20.7 MPa specimens.

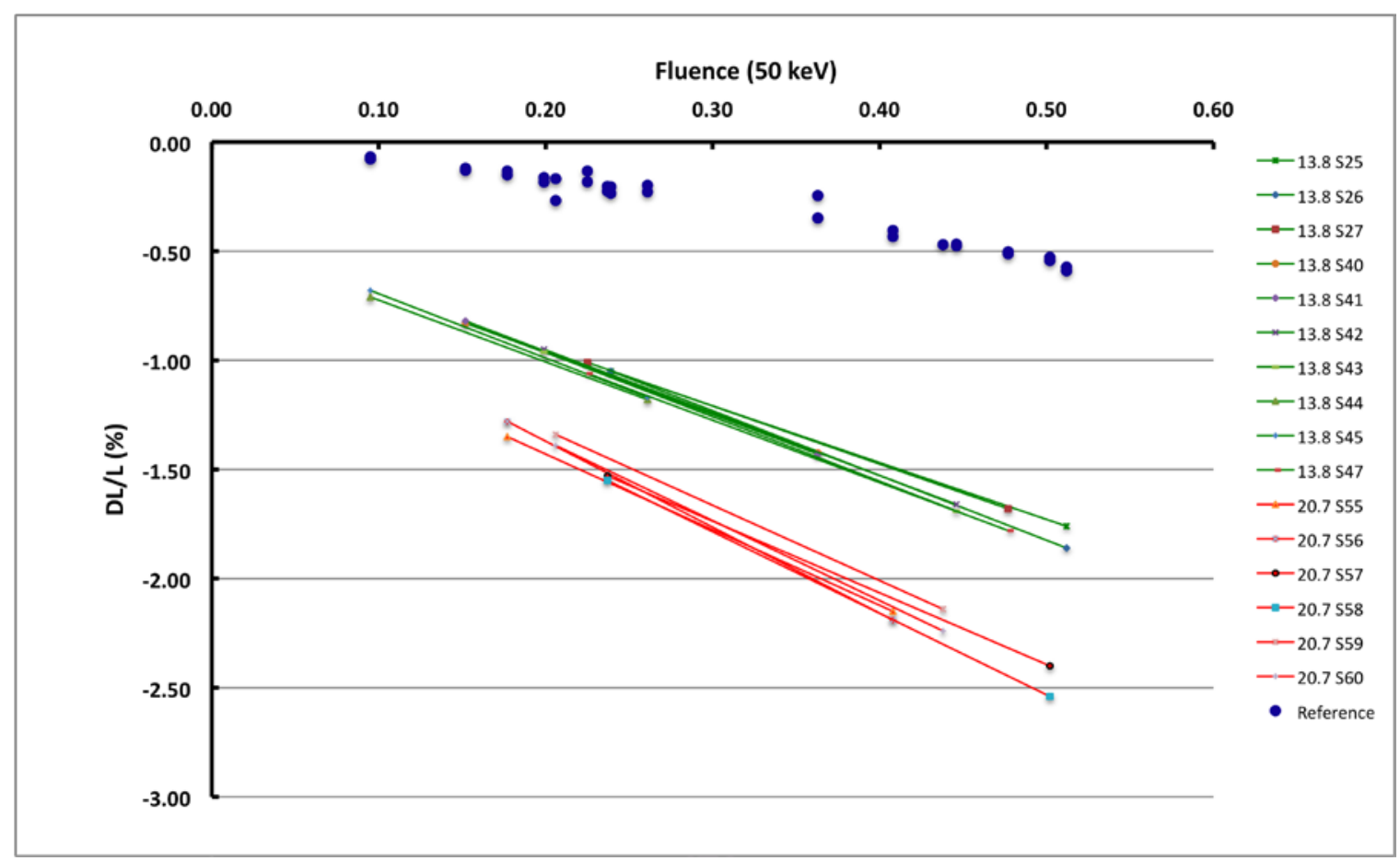

Figure 21: $600^{\circ} \mathrm{C}$ Direct dimensional change data 
ORNL/TM-2009/176

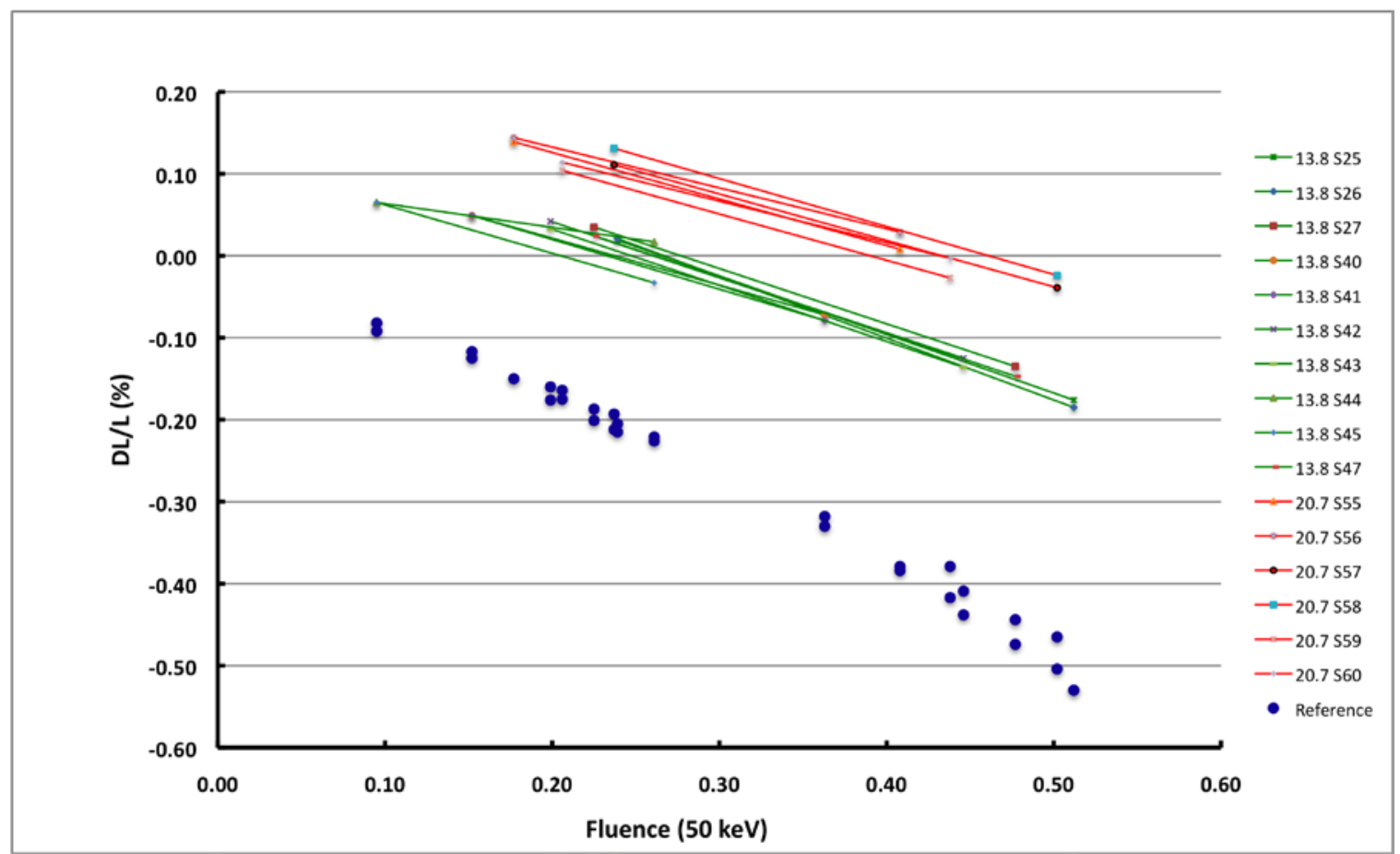

Figure 22: $600^{\circ} \mathrm{C}$ Lateral Dimensional Change Data

The volume data used to determine the change in density of the specimen was modified for the internal hole drilled in the reference specimens to allow thermal couple insertion and for the spacer holes drilled in the stressed specimens. The modified volume change was used in the derivation of the Modulii via the density. The modified Volume is shown in Figure 23. In contrast to the $900^{\circ} \mathrm{C}$ data the data are well ordered and appear to indicate significant volume change at low fluence (or low creep strain) followed by a period of relatively low volume change. 


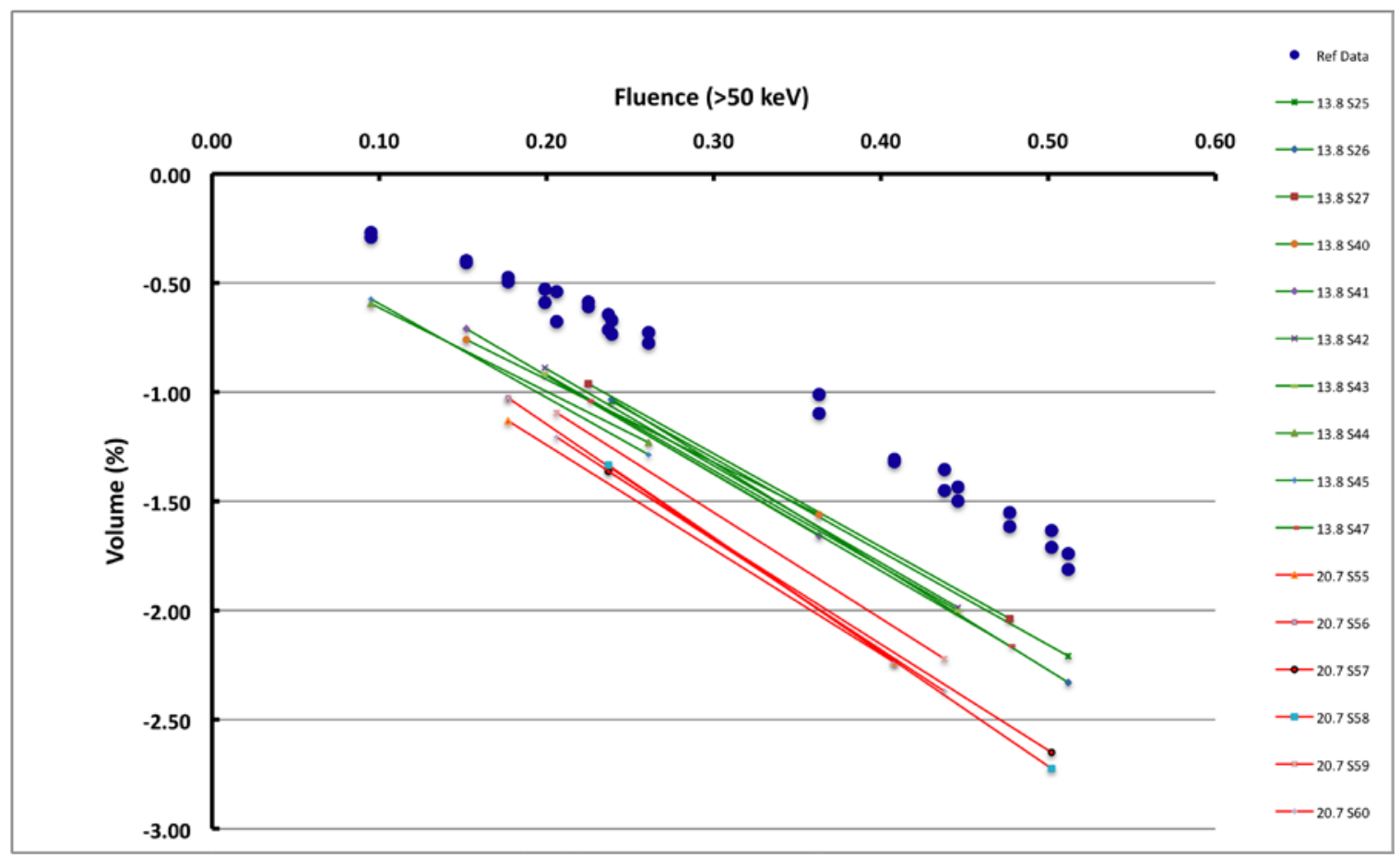

Figure 23: $600^{\circ} \mathrm{C}$ Volume Change Data

\section{Irradiation Induced Creep}

The quoted creep data have been derived by the difference between the stressed and reference specimens at the same position (axial height) within the column. No corrections appear to have been made to the measured data, however, it is not known which reference specimens were used in the original analysis.

The creep strain has been calculated assuming the German KFA correction for the changes in CTE and Modulus (See Appendix C). The modification resulted in a 5\% correction to the data. The lateral creep data is presented uncorrected as there is insufficient data to make the corrections.

The direct and lateral data are presented in Figures 24 and 25 and as expected from the dimensional change data show a very coherent picture with clear separation in the creep strain between 13.8 MPa and 20.7 MPa. 
ORNL/TM-2009/176

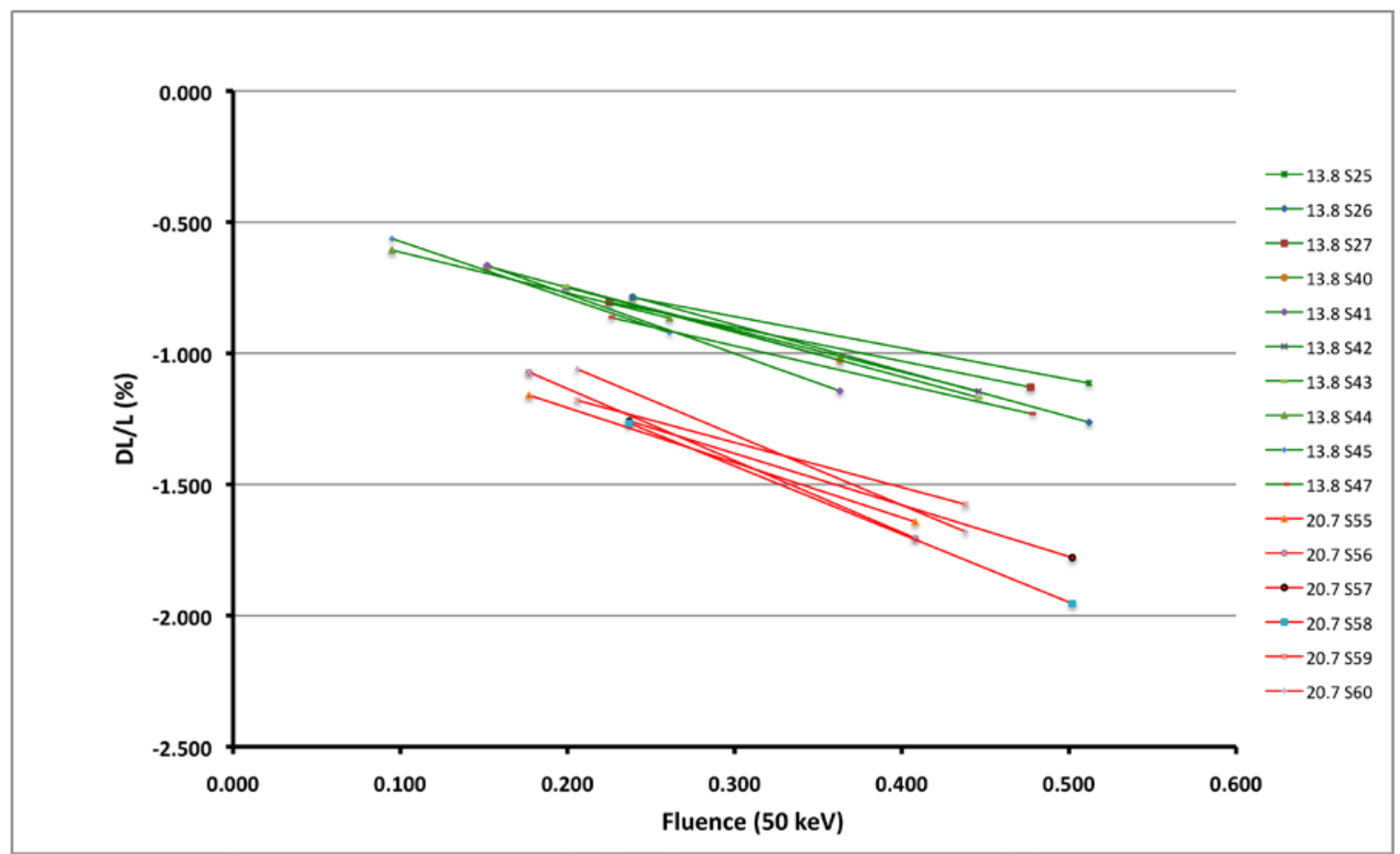

Figure 24: $600^{\circ} \mathrm{C}$ Direct Creep Data

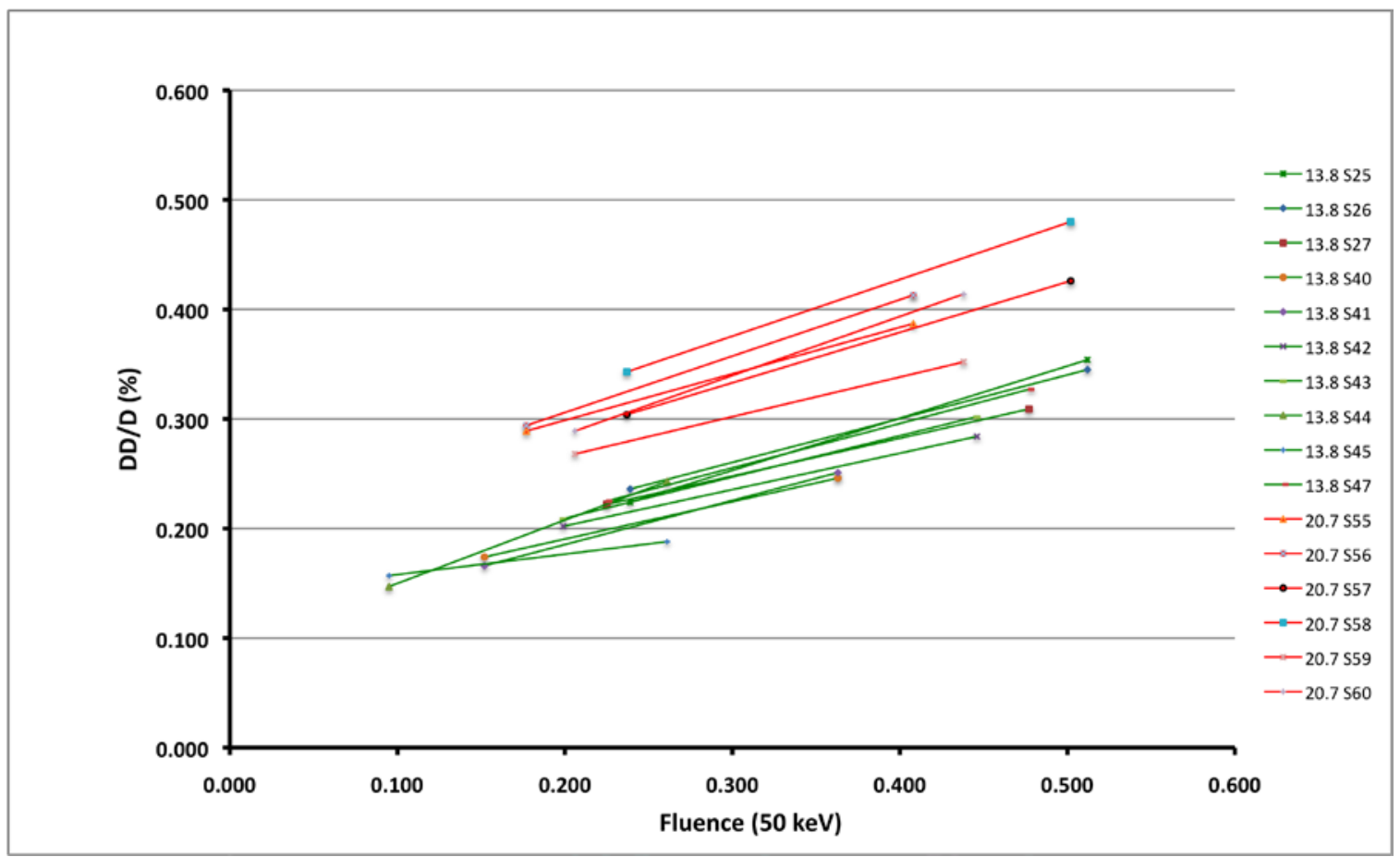

Figure 25: $600^{\circ} \mathrm{C}$ Lateral Creep Data

The direct creep data have been normalized by initial esu and presented in Figure 26. The normalization has used the initial dynamic Modulus. The two stress values collapse to a single dataset. 
ORNL/TM-2009/176

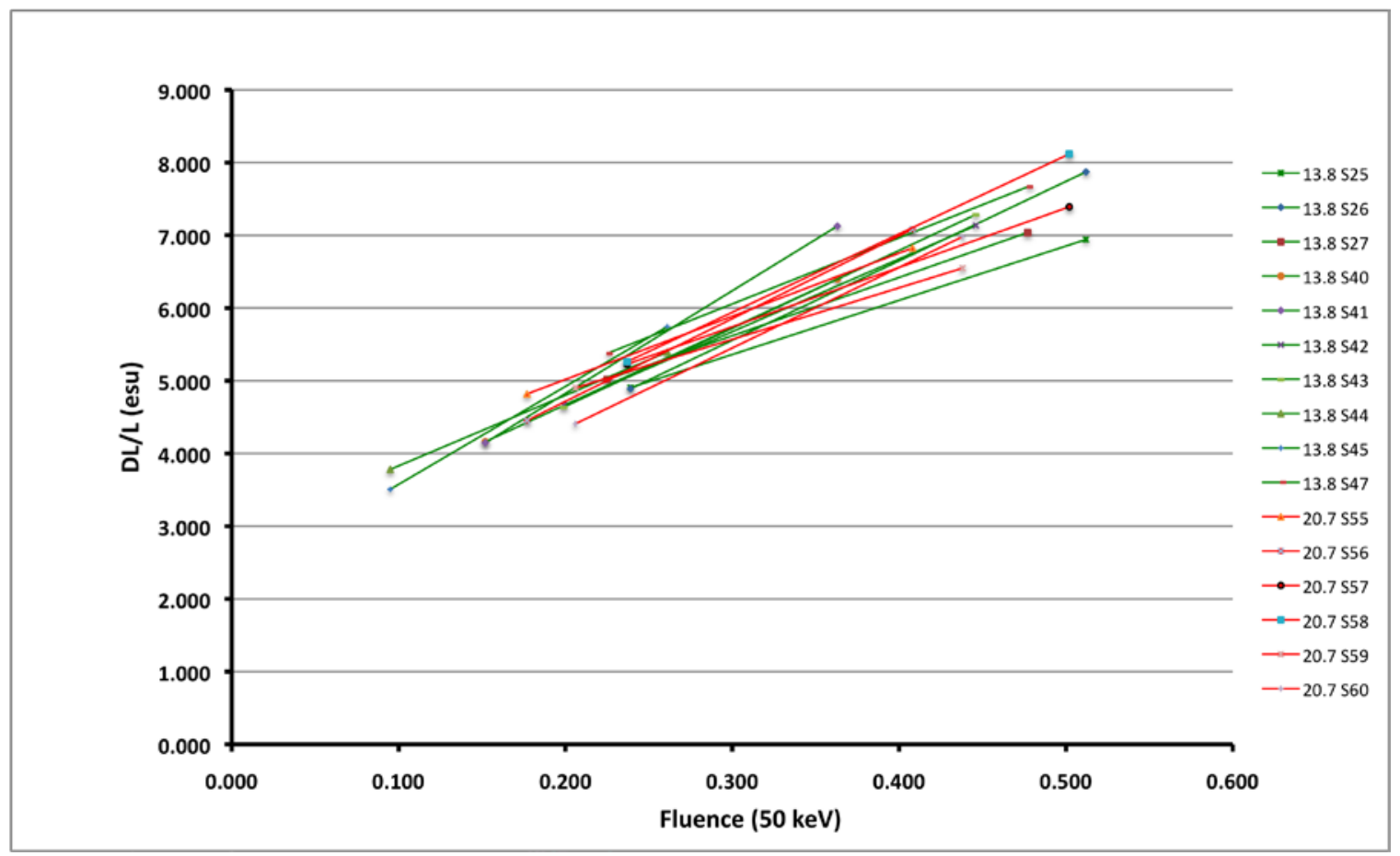

Figure 26: $600^{\circ} \mathrm{C}$ Normalised Direct Creep Data

\section{Modulus}

At the present time no pre-irradiated data has been discovered and so factorial changes cannot be assessed. The irradiated Young's Modulus data at $600^{\circ} \mathrm{C}$ are presented in Figure 27. The data are separated into Reference and Stressed specimens and indicate a modest dependence of Modulus on applied external load (at these levels of load and fluences). Figure 28 shows the equivalent plot for Shear Modulus and would appear to indicate a smaller dependence on applied external load at these levels of load and fluence. 


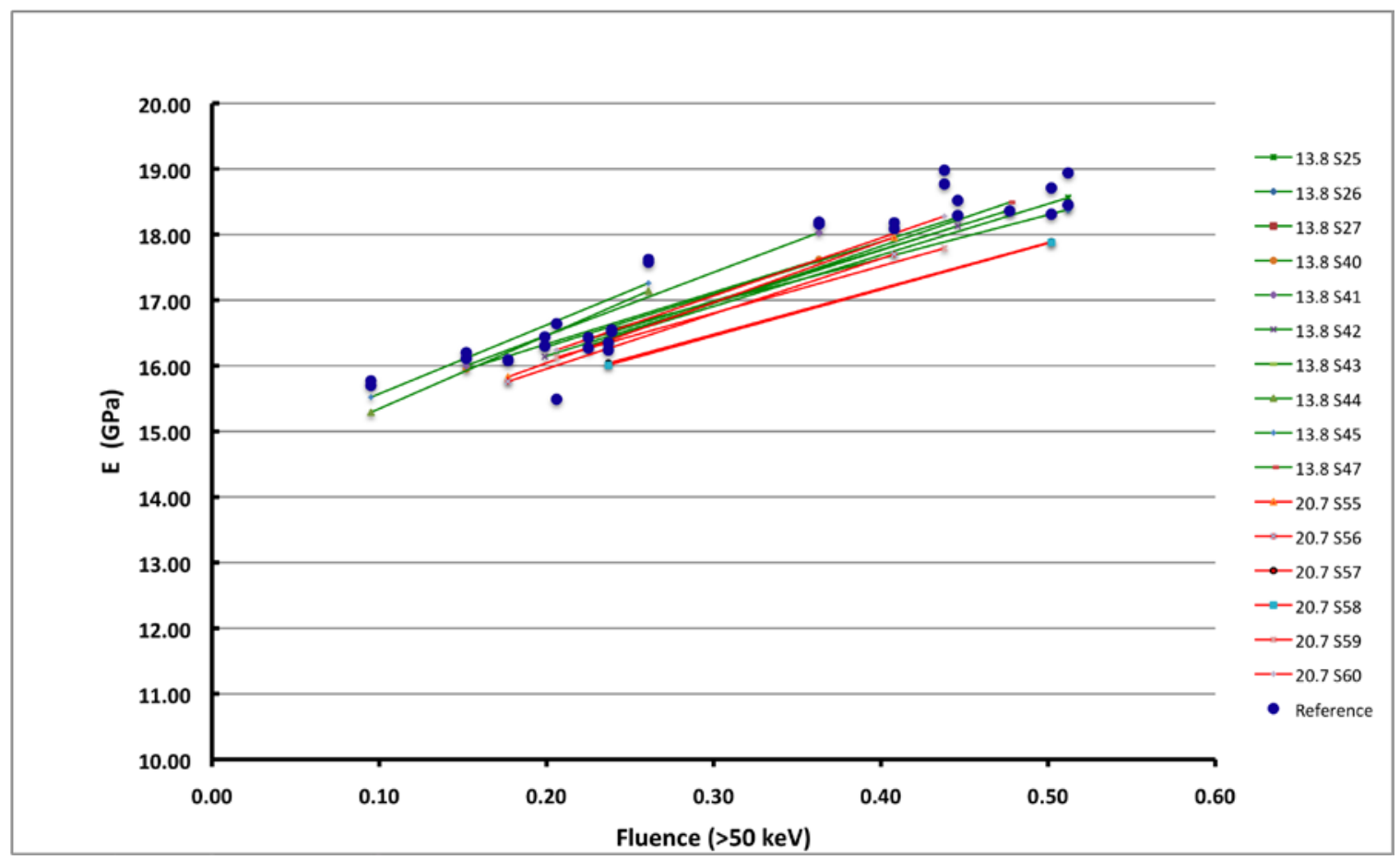

Figure 27: Young's Modulus at $600^{\circ} \mathrm{C}$

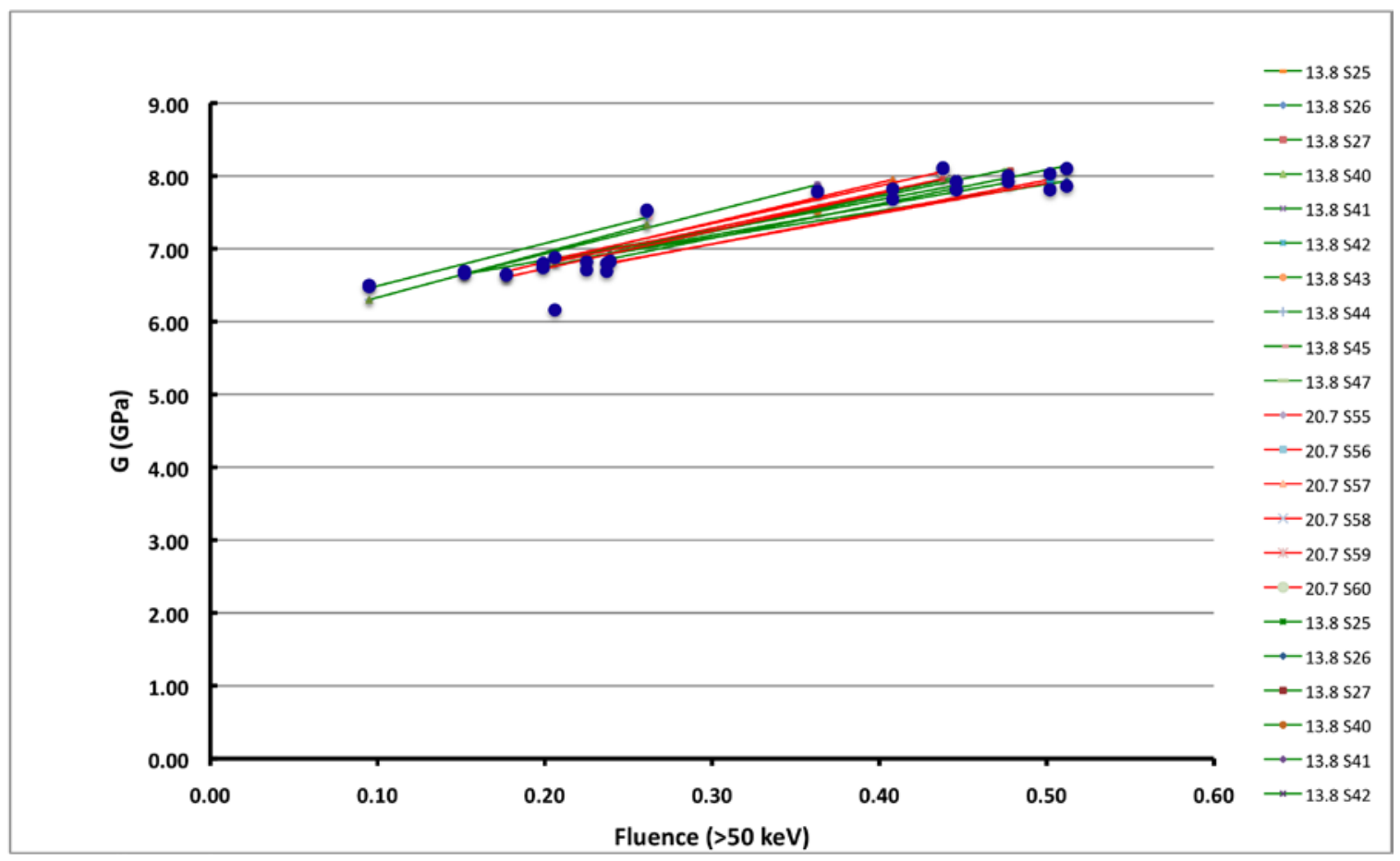

Figure 28: Shear Modulus at $600^{\circ} \mathrm{C}$

\section{Poisson's Ratio}

The $600^{\circ} \mathrm{C} \mathrm{H-451} \mathrm{data} \mathrm{presented} \mathrm{in} \mathrm{the} \mathrm{US/UK} \mathrm{exchange} \mathrm{is} \mathrm{shown} \mathrm{in} \mathrm{Figure} 29$ as a function of creep strain. The $900^{\circ} \mathrm{C}$ decrease in Poisson's ratio is also plotted in Figure 29 and indicates that the decrease in Poisson's ratio at $600^{\circ} \mathrm{C}$ is similar to that at $900^{\circ} \mathrm{C}$, 
which has previously been called into question casting some doubt on the validity of Figure 29.

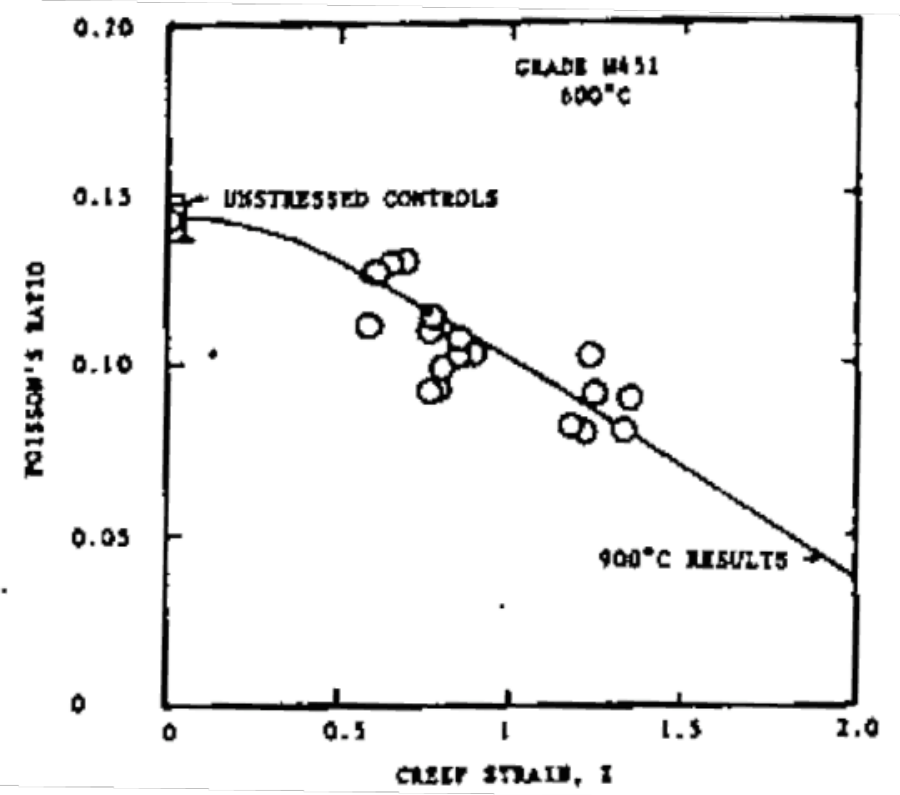

Figure 29: Effect of Creep strain on Poisson's ratio at $600^{\circ} \mathrm{C}$ (Kennedy and Eatherly, 1979)

Far more Poisson's Ratio data are reported in the $600^{\circ} \mathrm{C}$ computer print out and the effect of applied external load on Poisson's ratio is shown in Figure 30 for $\mathrm{H}-451$ and indicates an initial rise in Poisson ratio (based on $900^{\circ} \mathrm{C}$ unirradiated data as no unirradiated $600^{\circ} \mathrm{C}$ data has been found) followed by a subsequent decrease. This initial rise in Poisson's ratio is not represented in the data presented by Kennedy in Figure 29. Also the subsequent reduction is not as severe as that presented by Kennedy. Additionally, inspection of the available $600^{\circ} \mathrm{C}$ data indicates no Poisson's ratio data below 0.10 suggesting that Figure 29 is incorrect and should be ignored. 
ORNL/TM-2009/176

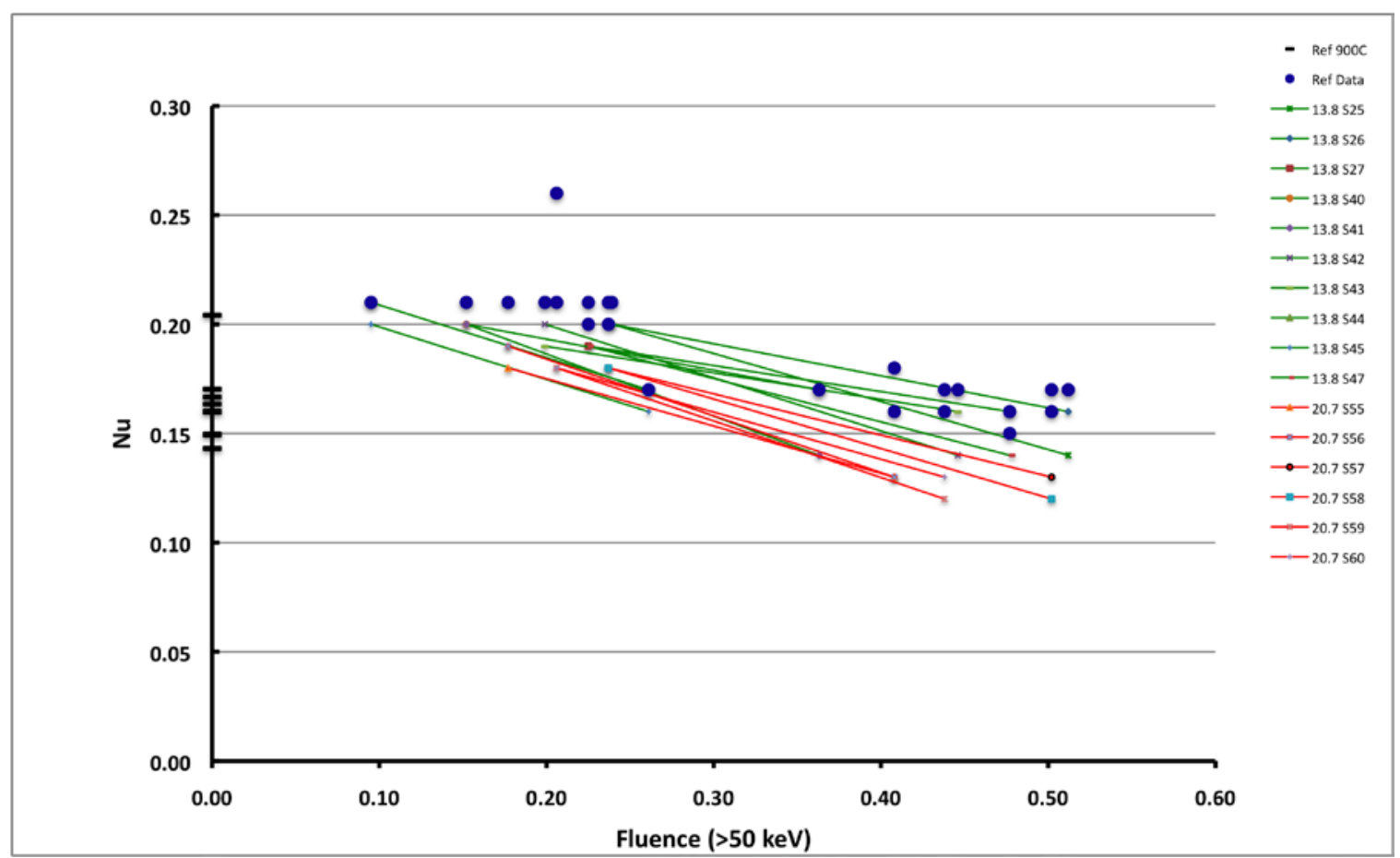

Figure 30: Effect of external load on Poisson's ratio at $600^{\circ} \mathrm{C}$

\section{Coefficient of Thermal Expansion}

Figure 31 shows the representation of the CTE dilatometer data from the OC-2 and OC-4 $600^{\circ} \mathrm{C}$ series of experiments presented at the 1979 carbon conference (Kennedy and Eatherly, 1979). As with the $900^{\circ} \mathrm{C}$ data, Kennedy evaluated by eye the dilatometer data as a linear function of temperature with $\mathrm{A}$ the intercept (i.e. the mean $\mathrm{CTE}$ at $0^{\circ} \mathrm{C}$ ) and $\mathrm{B}$ the slope. In Figure 31, $\mathrm{A}$ is presented as $\mathrm{A} / \mathrm{A}_{0}$ which is believed to be ratio of the stressed CTE to the unstressed CTE and B is presented as the slope determined from the stressed specimen. As stated earlier this approach is considered questionable as the evaluation is subjective and the data are non-linear.

$\mathrm{A} / \mathrm{A}_{0}$ and $\mathrm{B}$ are relatively flat for all grades up to $1.5 \%$ creep strain and the interpretation of these results is significantly influenced by the quoted pre-irradiated values. The preirradiation characterization data for the OC-2 and OC-4 experiments have not been found. It is recommended that these data are found. 
ORNL/TM-2009/176

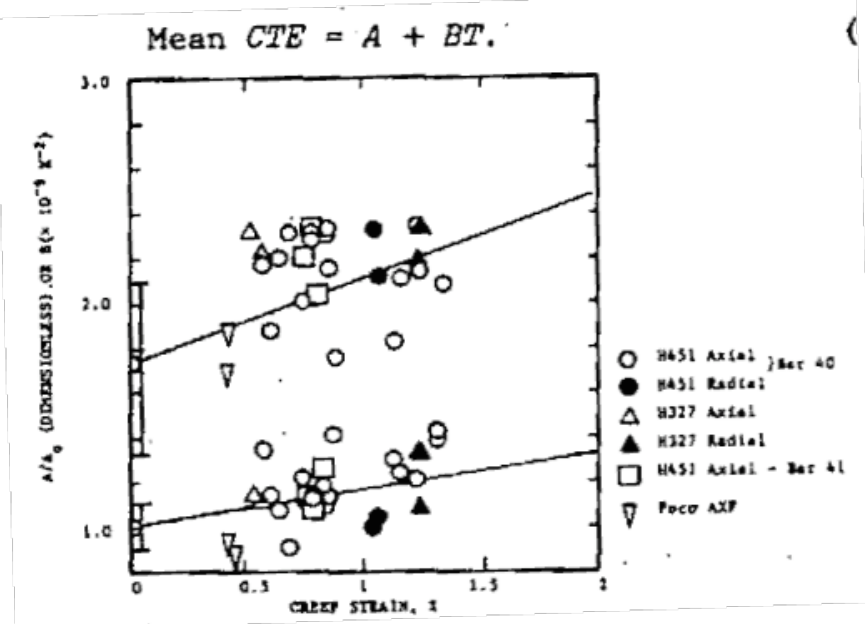

Figure 31: OC2 \& OCC4 - Effect of Creep Strain on the Mean Coefficient of thermal Expansion $(\alpha=\mathrm{A}+\mathrm{BT})$ (Kennedy)

The available dilatometer data from OC-2 and OC-4 have been re-assessed from the available extension data presented in Appendix B. As for OC-1 and OC-3 the determination of the mean $\mathrm{CTE}$ at $500^{\circ} \mathrm{C}$ from the extension data were similar in trend to previously quoted values but systematically offset by $0.5 \mathrm{E}-6 /{ }^{\circ} \mathrm{C}$. This represents a correction for the thermal expansion of quartz and is a consequence of the experimental apparatus and technique. For the purposes of this reassessment it has been included in the OC-2 and OC-4 analyses.

Figure 32 shows the post-irradiated dilatometer data for $\mathrm{H}-451$ specimens from the OC-2 experiment. The data are split into reference and stressed specimens. The OC-2 reference and stressed data are well represented by the UK theory.

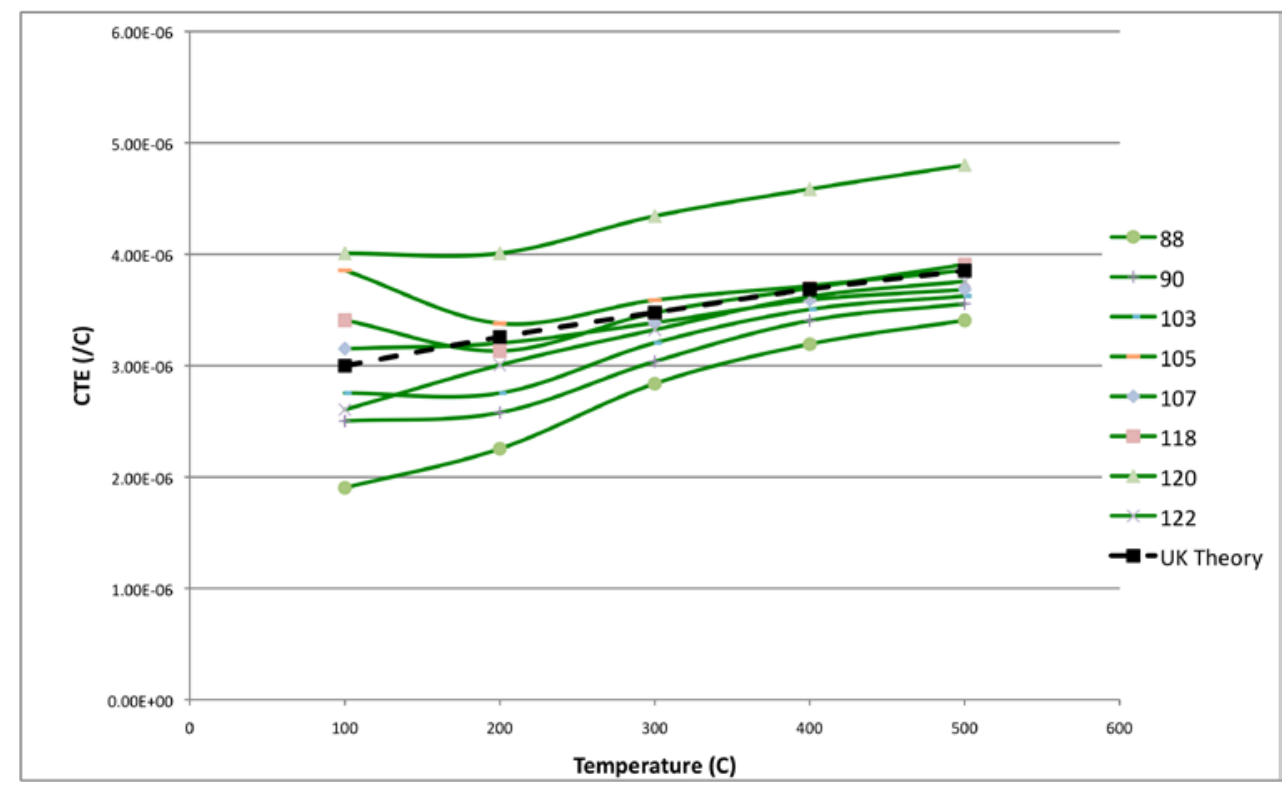

a) Reference 
ORNL/TM-2009/176

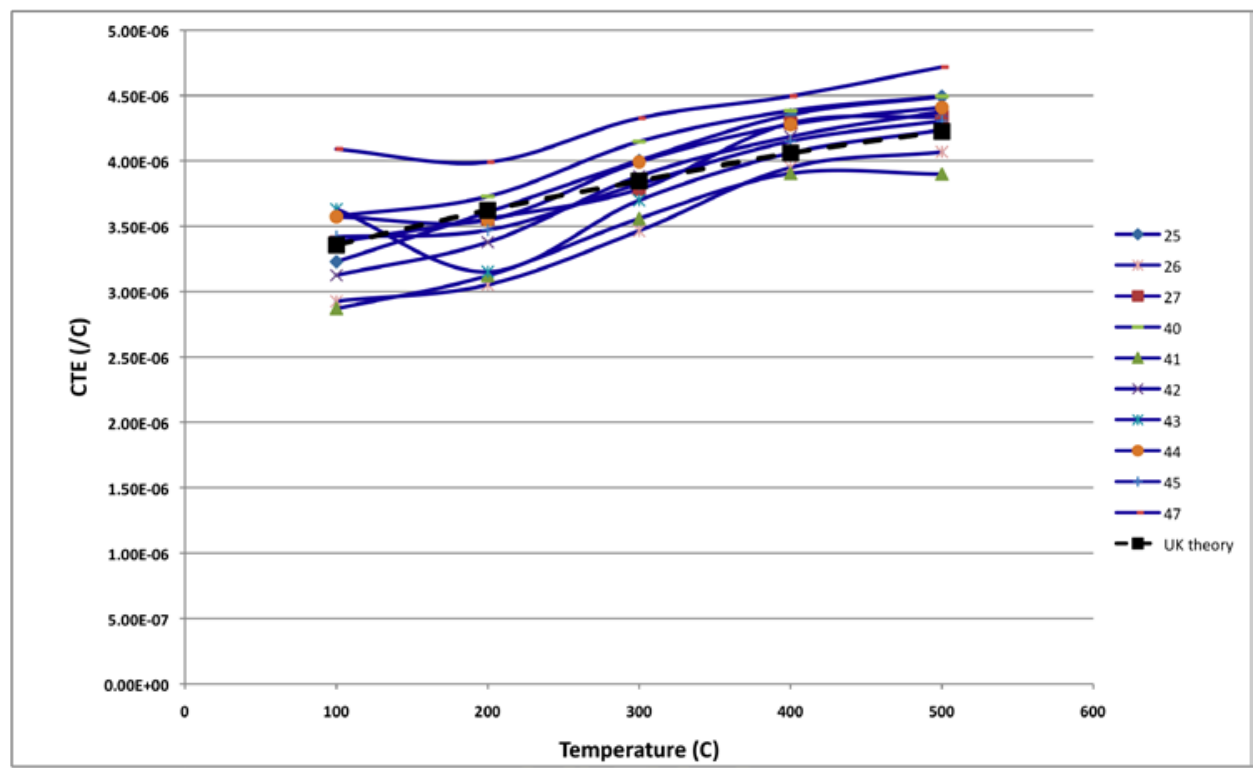

b) $13.8 \mathrm{MPa}$

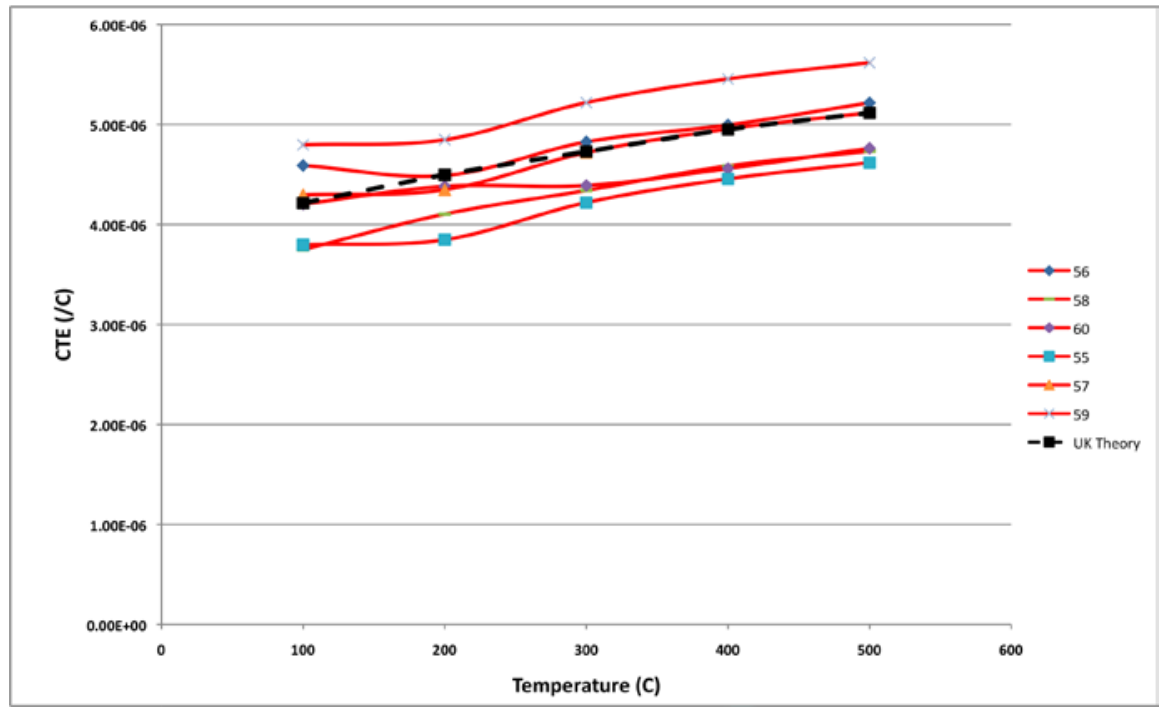

c) $20.7 \mathrm{MPa}$

Figure 32: Post-Irradiated Dilatometer Data from OC-2

Figure 33 shows the post-irradiated dilatometer data for $\mathrm{H}-451$ specimens from the OC-4 experiment. The UK theory prediction does not represent the reference or 13.8 MPa data particularly well although it does predict the $20.7 \mathrm{MPa}$ very well. This requires further consideration. 
ORNL/TM-2009/176

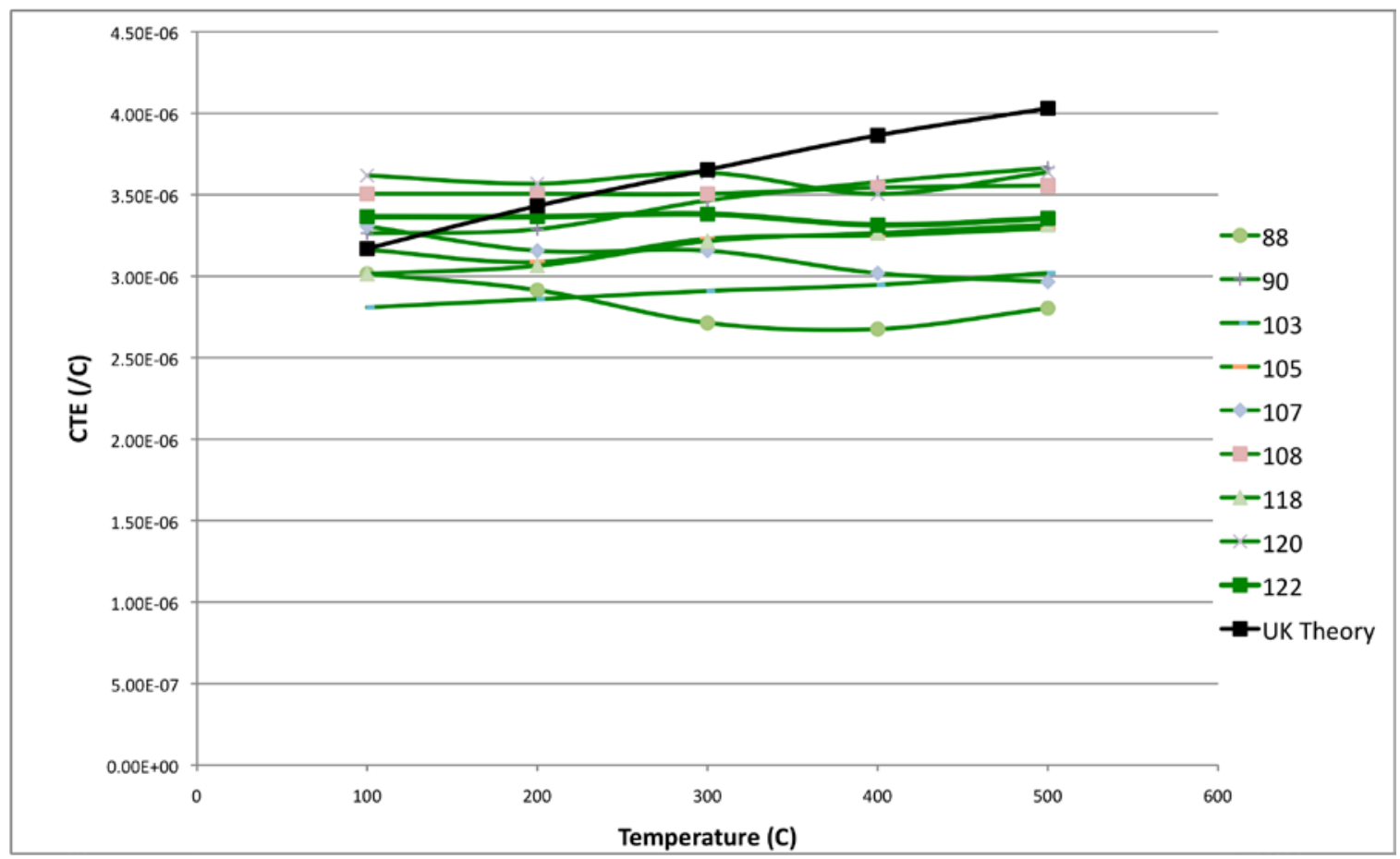

a) Reference

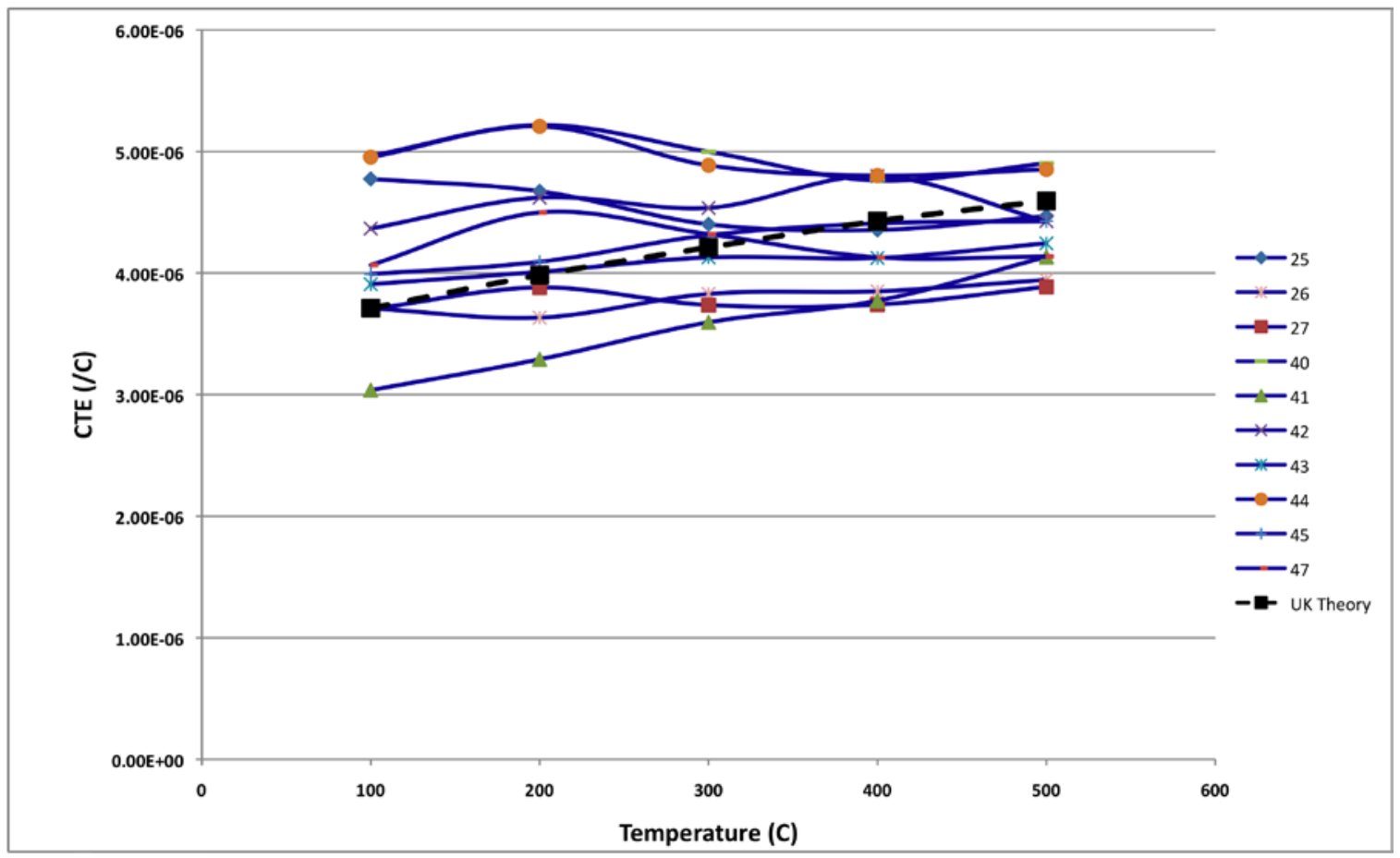

b) $13.8 \mathrm{MPa}$ 
ORNL/TM-2009/176

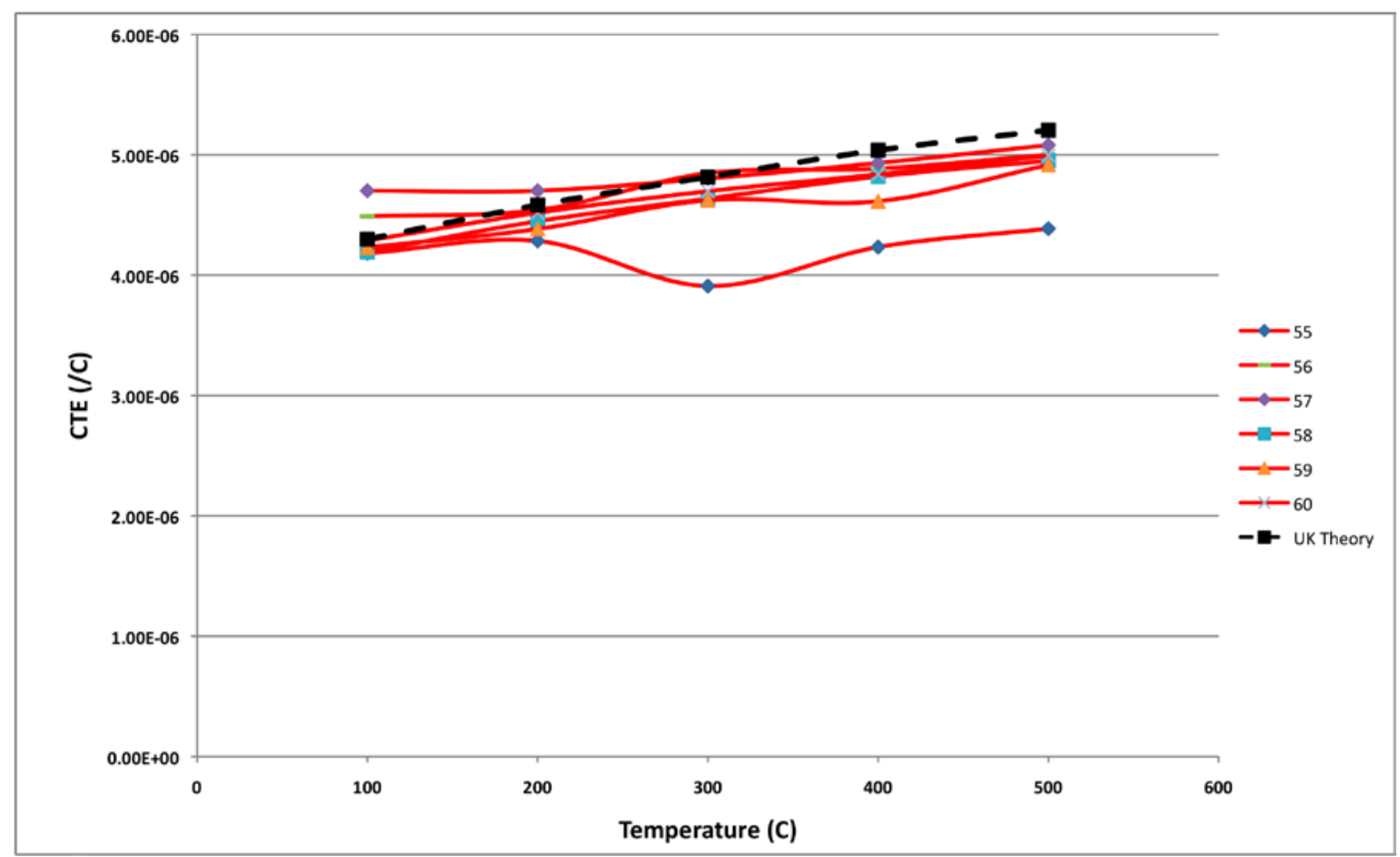

c) $20.7 \mathrm{MPa}$

Figure 33: Post-Irradiated Dilatometer Data from OC-4

Figure 34 shows the effect of the external load on the mean CTE measured at $500^{\circ} \mathrm{C}$. There is scatter in the unstressed and stressed CTE clear separation between the datasets at quite low fluence. The effect of the external load on CTE of irradiated $\mathrm{H}-541$ at $600^{\circ} \mathrm{C}$ appear to have saturated which does not appear to be the case for specimens irradiated at $900^{\circ} \mathrm{C}$. Further work is required to improve the available data if possible and calculate the fractional change in CTE. 
ORNL/TM-2009/176

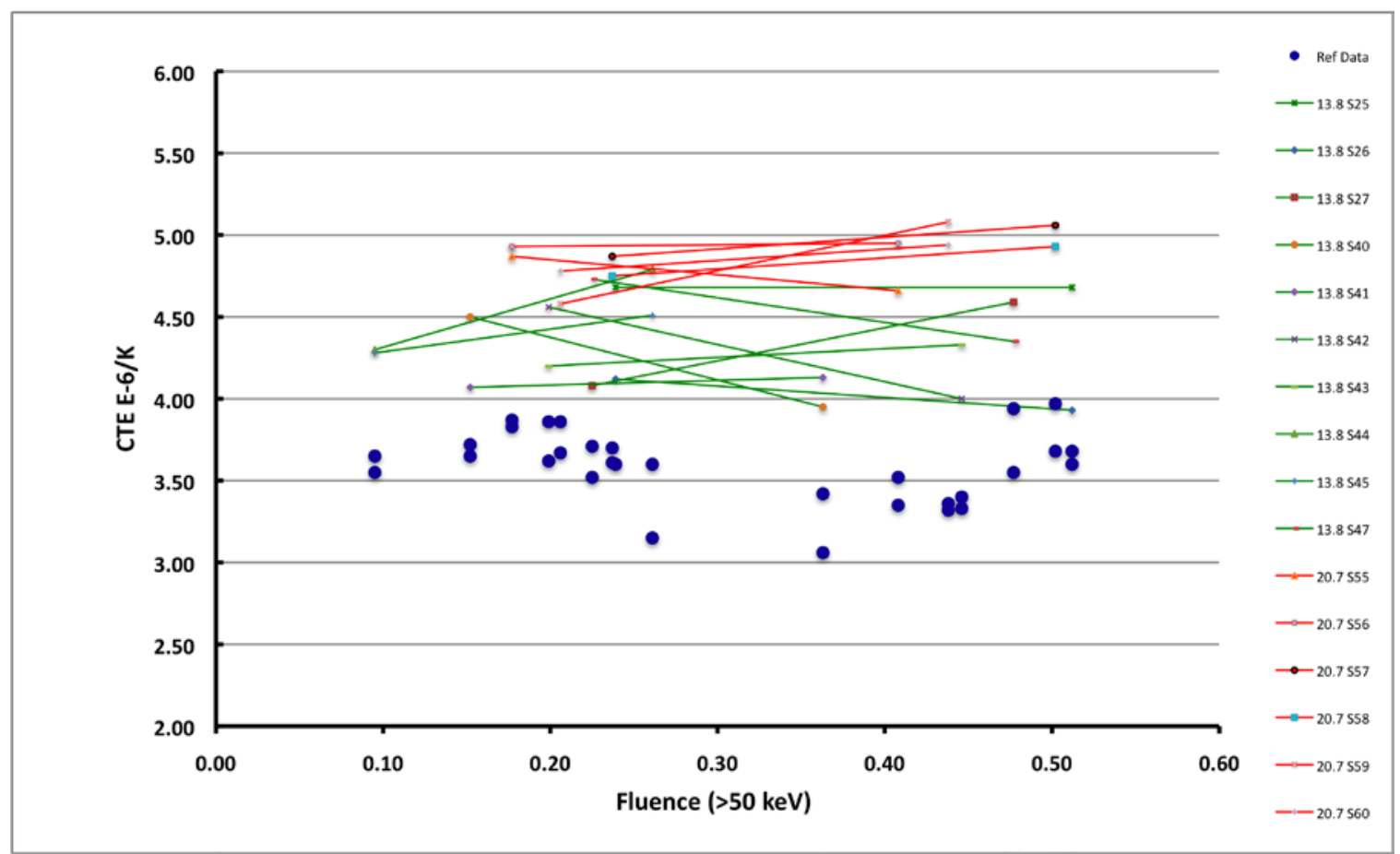

Figure 34: Effect of External Load on CTE (500) 


\section{Discussion}

The original OC-Series experiments planned to irradiate 3 sets of 28 stressed specimens along with their reference specimens to incremental fluences of 1,2 , 4 and $8\left(10^{25} \mathrm{n} / \mathrm{m}^{2}\right.$ $\mathrm{E}>0.18 \mathrm{MeV}$ ) at $600^{\circ} \mathrm{C}, 900^{\circ} \mathrm{C}$ and $1250^{\circ} \mathrm{C}$. Due to funding constraints the OC-Series was cut short and 3 sets of 28 stressed specimens were irradiated at $900^{\circ} \mathrm{C}(\mathrm{OC}-1,-3,-5)$ and 2 sets of 28 stressed specimens were irradiated at $600^{\circ} \mathrm{C}(\mathrm{OC}-2,-4$,$) .$

26 thermocouples fixed to the specimen holders were provided to monitor the Capsule temperature and the reference specimens were hollow to facilitate the translation of a movable thermocouple down the complete stack of specimens within the capsule.

Within the Capsule there was a factor of two variation in gamma heating along the column. To manage this variation five discrete gaps were used between the specimen holder and the Capsule with 20 heaters distributed along the column for fine control. Only 17 of the 20 heaters survived the total irradiation. At present it is not known which heaters failed or when they failed but this may be deduced from the temperature history. It is recommended that the temperature history of all the experiments is revisited.

For the duration of OC-1 it was difficult to maintain the Capsule temperature at $900 \pm 10$ ${ }^{\circ} \mathrm{C}$ down the length of Capsule. It was found that that the thermal design was erroneous and that the Capsule temperature was high $\left(930^{\circ} \mathrm{C}\right)$ in the top two positions and low (unknown) in positions 4 and 5 within the Capsule. There is also a $20^{\circ} \mathrm{C}$ systematic difference in irradiation temperature between the reference columns in the North and South of the Capsule.

It is not known if this is linked to the loss of heaters of if a similar variation in temperature is observed between the stressed columns. Irradiation creep is sensitive to irradiation temperature at $900^{\circ} \mathrm{C}$ and these variations should be investigated further.

Pre and Post irradiation radiographs were taken of the Capsule OC-1 and revealed that no obvious defects were found except that one of the reference columns had moved up by $12.7 \mathrm{~mm}$ and was attributed to movement of the thermocouple. It was not thought that this would have a significant effect on the results however given the discussion above this should be investigated further.

Additionally stressed specimens were solid and had different diameters to obtain different stresses within the same column. Therefore different thermal designs were required for the stressed specimens within a stressed column and for the reference column. If the thermal design were re-analysed with modern finite element analysis would this reveal differences in specimen temperatures between the stressed specimens and reference specimens especially at the higher irradiation temperature where radiation effects may be important. 
The bellows of Column 4 of OC-1 was subject to binding and potentially overloaded the column during the early phases of the experiment due to the thermal expansion of the column. The exact nature of this abnormal load configuration is not known but presumably leads to overstress in some specimens. Additionally during PIE it was observed that the spacers interfered with the stressed specimens and considerable force was required to remove the spacers in one column (it was not specified which). This would set up unusual stress patterns within the specimens (i.e. restrained shrinkage) and may have affected the results.

The results from the specimens within this column lead to some interesting but uncertain observations and require further analysis beyond the scope of this report.

No experimental reports were found for OC-2 through OC-5. Additional efforts should be made to extract details of these experiments.

From a data perspective it was also observed that some difference in fluence at various positions throughout all the Capsules were recorded between the reference and stressed specimens. This requires further information to resolve.

For the $900^{\circ} \mathrm{C}$ series of experiments there is considerable variability in the results, which may be due to the temperature variations above and could be investigated further.

In contrast to direct creep data (Figure 6) the lateral creep data (Figure 7) indicates little or no lateral creep at low fluences. Also shown in Figure 7 is the Column 4 data indicating that lateral creep has saturated earlier or at a lower level, which would not be expected if the specimens were significantly overloaded. Both these aspects require further consideration.

Of considerable importance is the discovery of annealed specimens. The analyses clearly illustrate the total recovery of creep strain in excess of 3.5 esu, which is substantially in excess of 1 esu normally attributed to primary creep strain. It should also be noted that these annealing results indicate there is apparently no secondary or "irrecoverable" creep established at these low fluences.

Unfortunately no data on the material property measurements of annealed samples was found. It is recommended that efforts are maintained to uncover this data if it available.

These anneal experiments are at very low fluence and it is recommended that irradiation anneal experiments should be included as part of future experiments. Any future anneals should commence at higher starting fluences to determine the full extent of "recoverable" creep and whether it saturates or not.

Some of the annealed data exhibit considerable recovery beyond the reference data set. This “over recovery" has been observed in thermal annealing experiments of unstressed PGA graphite and has also been observed in UK unstressed experiments where 
specimens have been re-irradiated at different irradiation temperatures and overshoot in the dimensional change has been observed.

In contrast to the $900^{\circ} \mathrm{C}$ data the $600^{\circ} \mathrm{C}$ data are subject to significantly less variability. This may be due to improved temperature and load control. This is illustrated by the volume behaviour (Figures 5 and 23). The $900^{\circ} \mathrm{C}$ data exhibit considerable scatter and show little separation at low fluence followed by subsequent increase in volume change at higher fluence (may be due to problems associated with OC-1 discussed above). This is in stark contrast to the $600^{\circ} \mathrm{C}$ irradiations where the data are well ordered and indicate significant volume change at low fluence (or low creep strain) followed by a period of relatively low volume change.

When the $900{ }^{\circ} \mathrm{C}$ Modulus are plotted as absolute values little or no dependence on stress levels is apparent. A small dependence on stress is apparent from the absolute Modulus values of the irradiations carried out at $600^{\circ} \mathrm{C}$.

When the $900{ }^{\circ} \mathrm{C}$ data are plotted showing specimen history and evaluated as fractional change in Modulii they appear to indicate a different behaviour with the Modulus obtained from stressed samples increasing at a different rate to reference specimens. This is an unexpected result and requires further investigation. No unirradiated results were uncovered for irradiations at $600{ }^{\circ} \mathrm{C}$ so fractional changes cannot be analysed at present.

The previously reported graphical H-451 Poisson's ratio data reported by Kennedy et al (1979) and reported by Brocklehurst and Kelly (1989) has been shown to be erroneous and should not be considered further.

Only OC-1 irradiated Poisson data could be found at $900^{\circ} \mathrm{C}$ and this showed a modest increase in Poisson ratio at low fluence with little or no difference between stressed and reference specimens. The initial rise is consistent with irradiated data obtained at $600^{\circ} \mathrm{C}$, however the data at $600^{\circ} \mathrm{C}$ indicate a dependence of Poisson ratio on stressed level with increasing stress decreasing Poisson ratio. This raises the question does tension induce the opposite effect?

The previously presented graphical H-451 CTE temperature dependence data presented by Kennedy has been shown to be questionable. The trend lines through the data appear to be misleading due to the unstressed data not being presented and the data at high creep strain were obtained from the overstressed data. Although the data from the overstressed specimens has not been considered as part of this work it may reveal important information for future experiments and should be investigated further.

The dependence of CTE on external load was re-evaluated from dilatometer data. The evaluation required an additional systematic offset due to the thermal expansion of quartz to reproduce the previously quoted CTE (800) values. This correction is consequence of the experimental technique and apparatus. 
There is considerable variability exhibited in the CTE obtained from both the preirradiated and post-irradiated extensometer data. The CTE (800) show considerable scatter but indicate that compression increases CTE (800) and the increase occurs at low fluence. However due to the scatter it is difficult to determine whether the change in CTE (800) has saturated at these fluences. The CTE(500) data also show considerable scatter and indicate that compression increases CTE(500) at very low fluences. The data may also indicate that the increase in $\mathrm{CTE}(500)$ has saturated at these low fluences.

A UK theory of CTE was applied to predict the effects of stress. The UK theory assumes that the temperature dependence of CTE is not affected by the applied external load, i.e. the effects of an external load on CTE can be measured over a relatively low temperature range and extrapolated to higher temperatures using the temperature dependence of crystal CTEs. This assumes that the effect of the external load on crystal CTEs is negligible.

As discussed above there is significant scatter in the dilatometer data but the UK theory is considered to predict the temperature dependence of CTE reasonably well for all available Capsules for both unstressed and stressed specimens with the exception of OC4 unstressed specimens. The OC-4 unstressed specimens appear out of trend with all the other results and this should be investigated further. It is not known whether significant structural changes have taken place at these modest fluences so this analysis only provides limited support for the UK approach. It is known that there is higher fluence data on $\mathrm{H}-451$ irradiated at $900^{\circ} \mathrm{C}$ in Petten. Some correspondence between KFA and ORNL was uncovered as part of the search of the ORNL archive. This correspondence should be investigated further.

During the course of this work two additional dilatometer measurement sets at 20.7 MPa were found. It is not known where these came from. Whilst they exhibit the same trend in temperature dependence the absolute CTE value is higher. These specimens need to be investigated further. 
ORNL/TM-2009/176

\section{Conclusions and Recommendations}

1. The archive associated with the OC-Series graphite irradiation creep experiments has been reviewed and a number of significant pieces of data have been uncovered.

2. Previously quoted Poisson's ratio dependence on creep has been demonstrated to be ill founded and as a result should not be considered further.

3. The CTE analysis methodology was found to be physically unsound and as a consequence published relationships between CTE and creep strain were found to be questionable.

4. Anneal data from the OC-Series indicates that there is substantial recovery of dimensional change well in excess of values normally attributed to primary creep. Some over recovery in dimensional change was observed and it is recommended that this is investigated further.

5. The $900^{\circ} \mathrm{C}$ Modulus data revealed some unexpected stressed specimen history behaviour when compared to reference history behaviour. It was not possible to examine the $600^{\circ} \mathrm{C}$ in the same way as there were only two irradiation Capsules spanning the fluences where the changes at $900^{\circ} \mathrm{C}$ become apparent. It is recommended that this is investigated further.

6. Whilst subject to significant scatter, the CTE data appear to have saturated at $600^{\circ} \mathrm{C}$ but not necessarily at $900^{\circ} \mathrm{C}$. It is recommended that the CTE data are investigated further.

7. The CTE data appear to be well represented by the UK theory.

8. During the archive search substantial data were found on other experiments such as the ORR series and Petten (US/FRG exchange - H-451 \& ATR-2E). It is recommended that these are examined in more detail. 


\section{Acknowledgements}

This work is sponsored by the U.S. Department of Energy, Office of Nuclear Energy Science and Technology under contract DE-AC05-00OR22725 with Oak Ridge National Laboratory, managed by UT-Battelle, LLC. 
ORNL/TM-2009/176

\section{References}

Brocklehurst J.E. and Kelly B.T. (1989), A review of irradiation induced creep in graphite under CAGR conditions. ND-R-1406(S), Pub. United Kingdom Atomic Energy Authority.

Burchell T.D. (2008), Irradiation induced creep behavior of H-451 graphite. Journal of Nuclear Materials 381 (2008) 46-54.

Davies M. and Bradford M. (2008), A revised description of graphite irradiation induced creep, Journal of Nuclear Materials 382, (2008) 39-45.

Kelly B.T. and Burchell T.D. (1994), The Analysis of Irradiation Creep Experiments on Nuclear Reactor Graphite. Carbon 32 (1994) 119-125.

Kennedy C.R., Cook W. H., and Eatherly W.P. (1977), Results of Irradiation Creep Testing at $900^{\circ} \mathrm{C}$. Ext. Abs. $13^{\text {th }}$ Biennial Conf. on Carbon, pp. 342-343, July 18-22, 1977. Irvine, CA. Pub. American Carbon Society.

Kennedy C.R. and Eatherly W.P. (1979), Results of the $600^{\circ} \mathrm{C}$ Compressive Creep Irradiation Experiment. Ext. Abs. $14^{\text {th }}$ Biennial Conf. on Carbon, pp. 453-454, June 2529, 1979. The Pennsylvania State University, PA. Pub. American Carbon Society.

Mobasheran A.S. (1990), Examination of experimental data for irradiation-induced creep in nuclear graphite. Ph.D Thesis, University Of Tennessee, Knoxville, TN.

Senn R.L., Conlin J.A., Cook W.H. and Eatherly W.P. (1977), Design, Operation and Initial results from Capsule OC-1, the First of a Series of Graphite Creep Irradiation Experiments. ORNL/TM-5798. Pub. Oak Ridge National Laboratory.

Tsang D.K.L., Marsden B.J., Fok S.L. and Hall G., Graphite thermal expansion relationship for different temperatute ranges, Carbon 43 (2005) 2902-2906. 


\section{Appendix A $900^{\circ} \mathrm{C}$ Specimen Characterisation Archive Data}

Hand written sheets of data were discovered in the archive for the OC1, OC3 and OC5 $900^{\circ} \mathrm{C}$ experiments. Specimen pre-characterised were also included. Similar information for OC2 and OC $4600^{\circ} \mathrm{C}$ experiments has not been uncovered yet. The datasheets are reproduced here for future reference. 


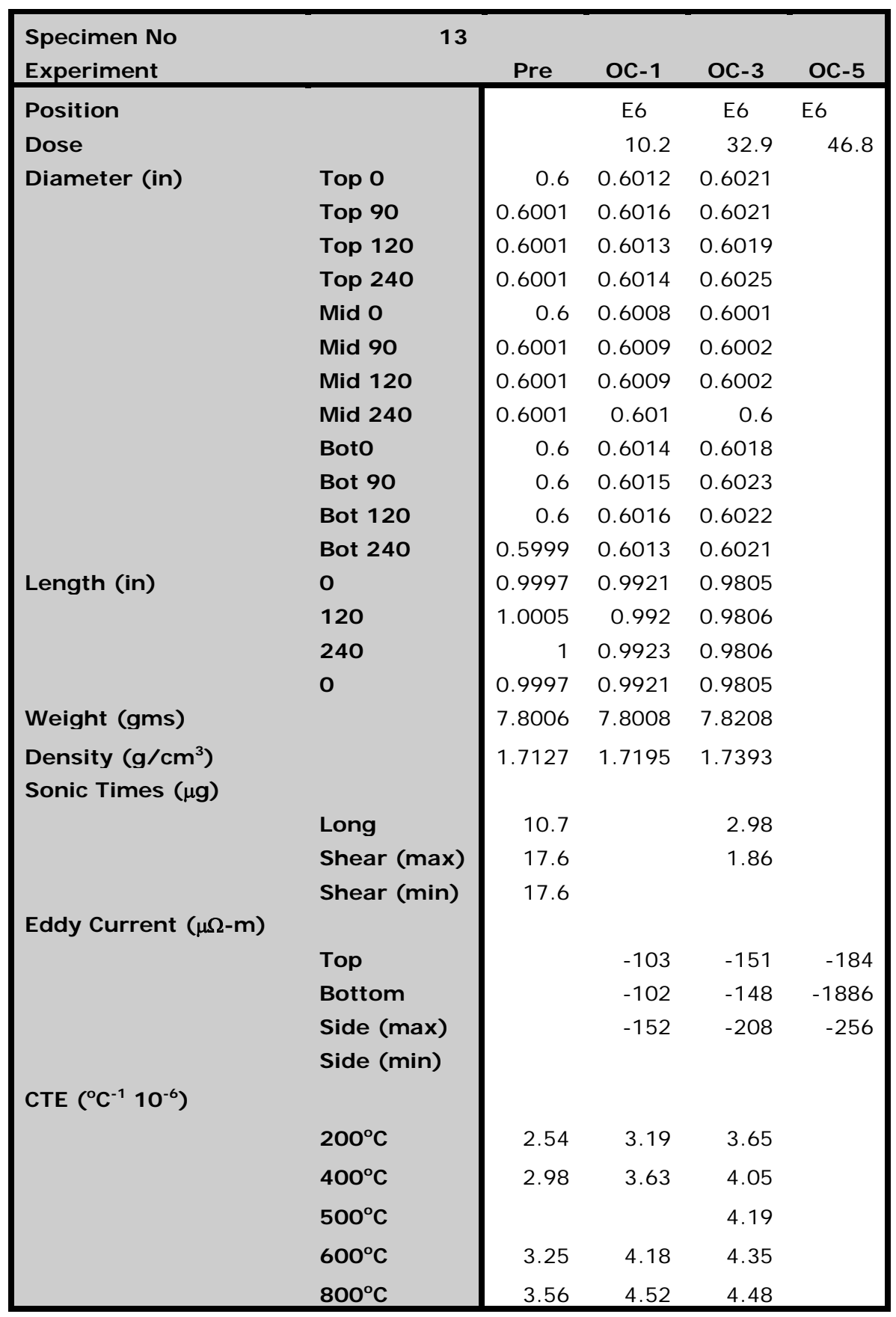




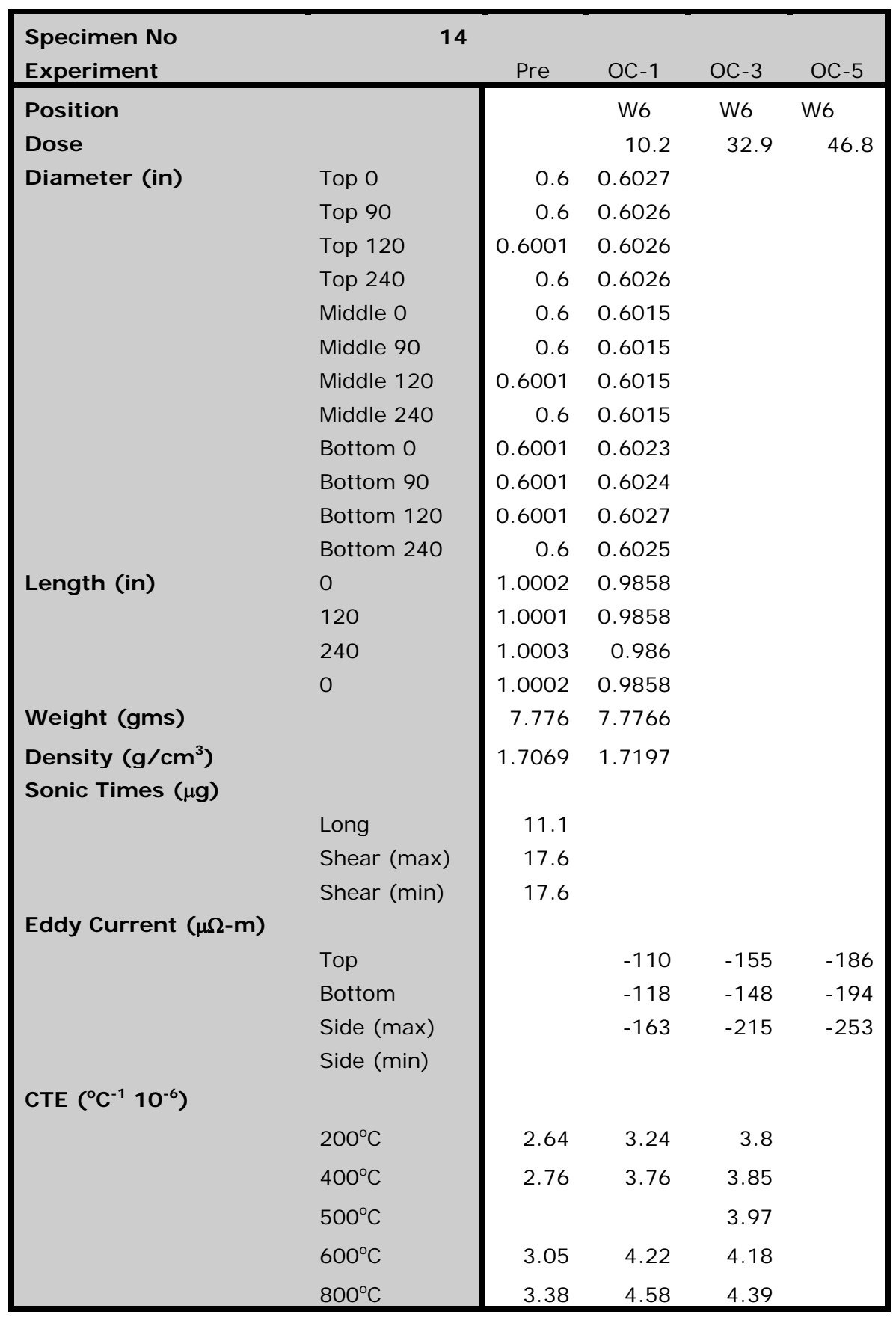




\begin{tabular}{|c|c|c|c|c|c|}
\hline $\begin{array}{l}\text { Specimen No } \\
\text { Experiment }\end{array}$ & 18 & Pre & OC-1 & $\mathrm{OC}-3$ & OC-5 \\
\hline $\begin{array}{l}\text { Position } \\
\text { Dose }\end{array}$ & & & $\begin{array}{l}\text { E7 } \\
10.5\end{array}$ & $\begin{array}{l}\text { E7 } \\
33.7\end{array}$ & $\begin{array}{l}\text { E7 } \\
47.9\end{array}$ \\
\hline \multirow[t]{12}{*}{ Diameter (in) } & Top 0 & 0.6 & 0.6014 & 0.6022 & \\
\hline & Top 90 & 0.5999 & 0.6014 & 0.6017 & \\
\hline & Top 120 & 0.5999 & 0.6015 & 0.602 & \\
\hline & Top 240 & 0.6 & 0.6014 & 0.6018 & \\
\hline & Middle 0 & 0.5999 & 0.6006 & 0.5999 & \\
\hline & Middle 90 & 0.5999 & 0.6006 & 0.5999 & \\
\hline & Middle 120 & 0.5999 & 0.6008 & 0.6 & \\
\hline & Middle 240 & 0.5999 & 0.6006 & 0.5997 & \\
\hline & Bottom 0 & 0.6 & 0.6015 & 0.6018 & \\
\hline & Bottom 90 & 0.5999 & 0.6011 & 0.602 & \\
\hline & Bottom 120 & 0.5999 & 0.6013 & 0.6017 & \\
\hline & Bottom 240 & 0.6 & 0.6011 & 0.6021 & \\
\hline \multirow[t]{4}{*}{ Length (in) } & 0 & 1.0004 & 0.9936 & 0.9827 & \\
\hline & 120 & 1.0001 & 0.9932 & 0.9827 & \\
\hline & 240 & 1.0002 & 0.9933 & 0.9826 & \\
\hline & 0 & 1.0004 & 0.9935 & 0.9827 & \\
\hline Weight (gms) & & 7.8901 & 7.8904 & 7.8911 & \\
\hline Density $\left(\mathrm{g} / \mathrm{cm}^{3}\right)$ & & 1.7324 & 1.7378 & 1.7565 & \\
\hline \multicolumn{6}{|l|}{ Sonic Times $(\mu \mathbf{g})$} \\
\hline & Long & 10.6 & & 3.05 & \\
\hline & Shear (max) & 17.3 & & 1.88 & \\
\hline & Shear (min) & 17 & & & \\
\hline \multicolumn{6}{|c|}{ Eddy Current ( $\mu \Omega-m)$} \\
\hline & Top & & -98 & -159 & -174 \\
\hline & Bottom & & -98 & -156 & -178 \\
\hline & Side (max) & & -143 & -193 & -236 \\
\hline & Side (min) & & & & \\
\hline \multicolumn{6}{|l|}{ CTE $\left({ }^{\circ} \mathrm{C}^{-1} 10^{-6}\right)$} \\
\hline & $200^{\circ} \mathrm{C}$ & 2.44 & 3.19 & 3.2 & \\
\hline & $400^{\circ} \mathrm{C}$ & 2.93 & 3.73 & 3.35 & \\
\hline & $500^{\circ} \mathrm{C}$ & & & 3.55 & \\
\hline & $600^{\circ} \mathrm{C}$ & 3.27 & 4.37 & 3.7 & \\
\hline & $800^{\circ} \mathrm{C}$ & 3.54 & 4.59 & 3.88 & \\
\hline
\end{tabular}




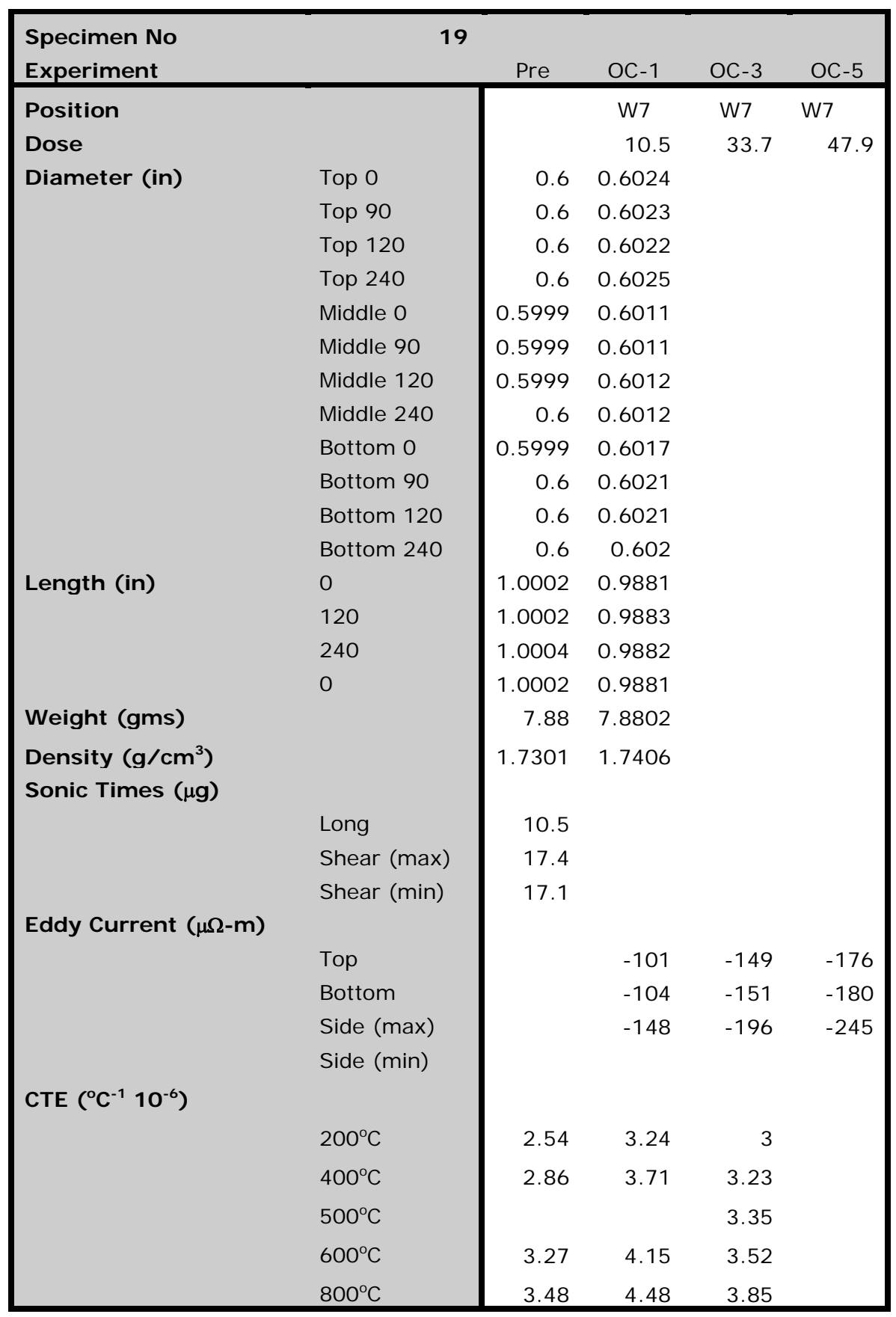




\begin{tabular}{|c|c|c|c|c|c|}
\hline $\begin{array}{l}\text { Specimen No } \\
\text { Experiment }\end{array}$ & 23 & Pre & OC-1 & $O C-3$ & OC-5 \\
\hline $\begin{array}{l}\text { Position } \\
\text { Dose }\end{array}$ & & & $\begin{array}{l}\text { E8 } \\
10.5\end{array}$ & $\begin{array}{l}\mathrm{E} 8 \\
23.3\end{array}$ & $\begin{array}{l}\text { E8 } \\
33.8\end{array}$ \\
\hline \multirow[t]{12}{*}{ Diameter (in) } & Top 0 & 0.6 & 0.6014 & 0.6026 & \\
\hline & Top 90 & 0.6001 & 0.6014 & 0.6023 & \\
\hline & Top 120 & 0.6001 & 0.6014 & 0.6023 & \\
\hline & Top 240 & 0.6 & 0.6015 & 0.6021 & \\
\hline & Mid 0 & 0.6 & 0.6008 & 0.6 & \\
\hline & Mid 90 & 0.6 & 0.6008 & 0.5999 & \\
\hline & Mid 120 & 0.6 & 0.6008 & 0.5999 & \\
\hline & Mid 240 & 0.6 & 0.6008 & 0.5999 & \\
\hline & Boto & 0.6 & 0.6015 & 0.6024 & \\
\hline & Bot 90 & 0.6 & 0.6014 & 0.6022 & \\
\hline & Bot 120 & 0.6 & 0.6014 & 0.6021 & \\
\hline & Bot 240 & 0.6 & 0.6014 & 0.6023 & \\
\hline \multirow[t]{4}{*}{ Length (in) } & 0 & 1.0001 & 0.992 & 0.9802 & \\
\hline & 120 & 1.0002 & 0.9926 & 0.9803 & \\
\hline & 240 & 1.0007 & 0.9924 & 0.9803 & \\
\hline & 0 & 1.0001 & 0.992 & 0.9802 & \\
\hline Weight (gms) & & 7.8158 & 7.8161 & 7.8185 & \\
\hline Density $\left(\mathrm{g} / \mathrm{cm}^{3}\right)$ & & 1.7156 & 1.7228 & 1.7431 & \\
\hline \multicolumn{6}{|l|}{ Sonic Times $(\mu \mathrm{g})$} \\
\hline & Long & 10.7 & & 2.98 & \\
\hline & Shear (max) & 17.7 & & 1.82 & \\
\hline & Shear (min) & 17.4 & & & \\
\hline \multicolumn{6}{|c|}{ Eddy Current ( $\mu \Omega-m)$} \\
\hline & Top & & -114 & -145 & -189 \\
\hline & Bottom & & -112 & -143 & -189 \\
\hline & Side (max) & & -168 & -198 & -255 \\
\hline & Side (min) & & & & \\
\hline \multicolumn{6}{|l|}{ CTE $\left({ }^{\circ} \mathrm{C}^{-1} 10^{-6}\right)$} \\
\hline & $200^{\circ} \mathrm{C}$ & 2.9 & 3.08 & 3.05 & \\
\hline & $400^{\circ} \mathrm{C}$ & 3.36 & 3.53 & 3.13 & \\
\hline & $500^{\circ} \mathrm{C}$ & & & 3.37 & \\
\hline & $600^{\circ} \mathrm{C}$ & 3.69 & 4.05 & 3.5 & \\
\hline & $800^{\circ} \mathrm{C}$ & 3.97 & 4.56 & 3.74 & \\
\hline
\end{tabular}




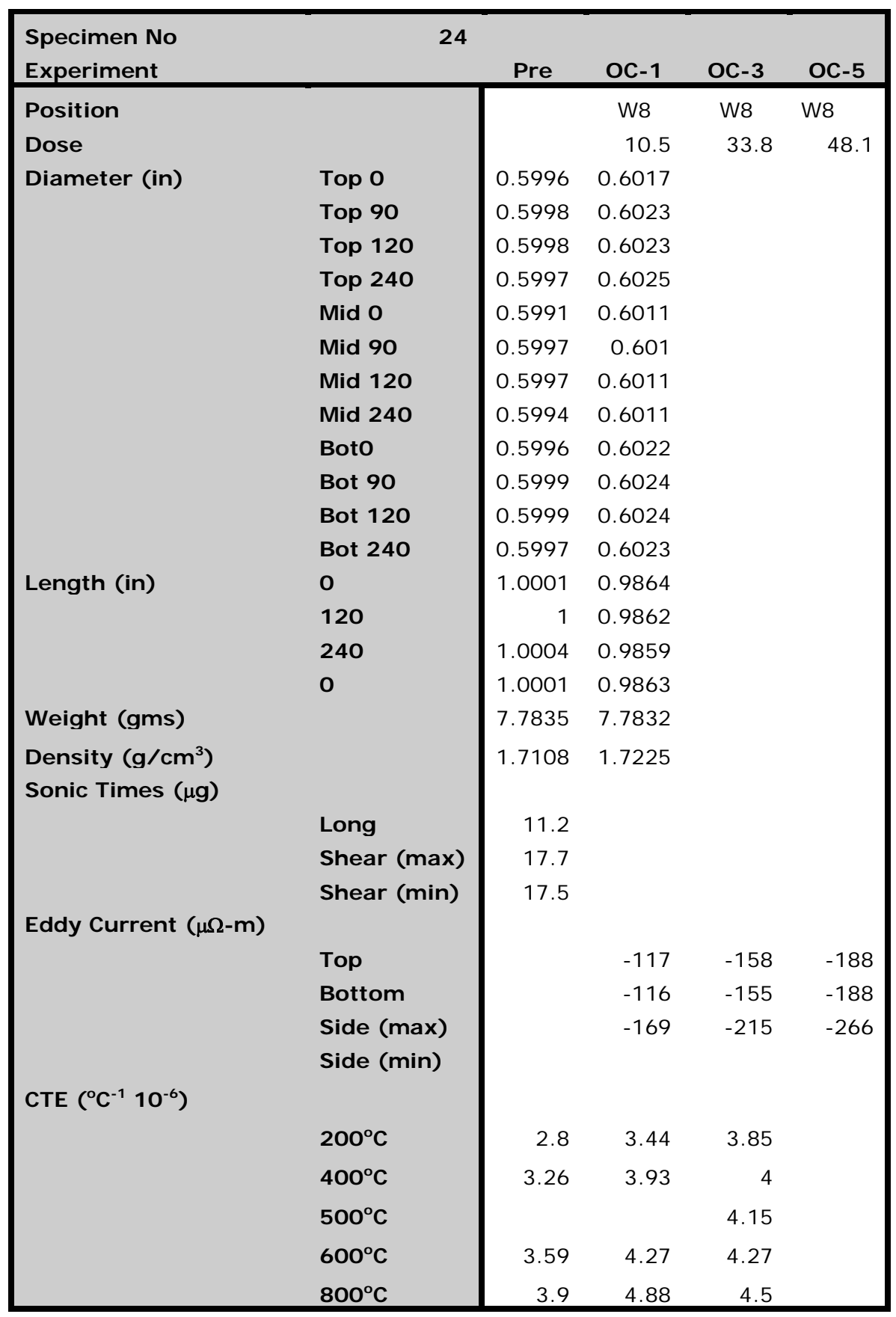




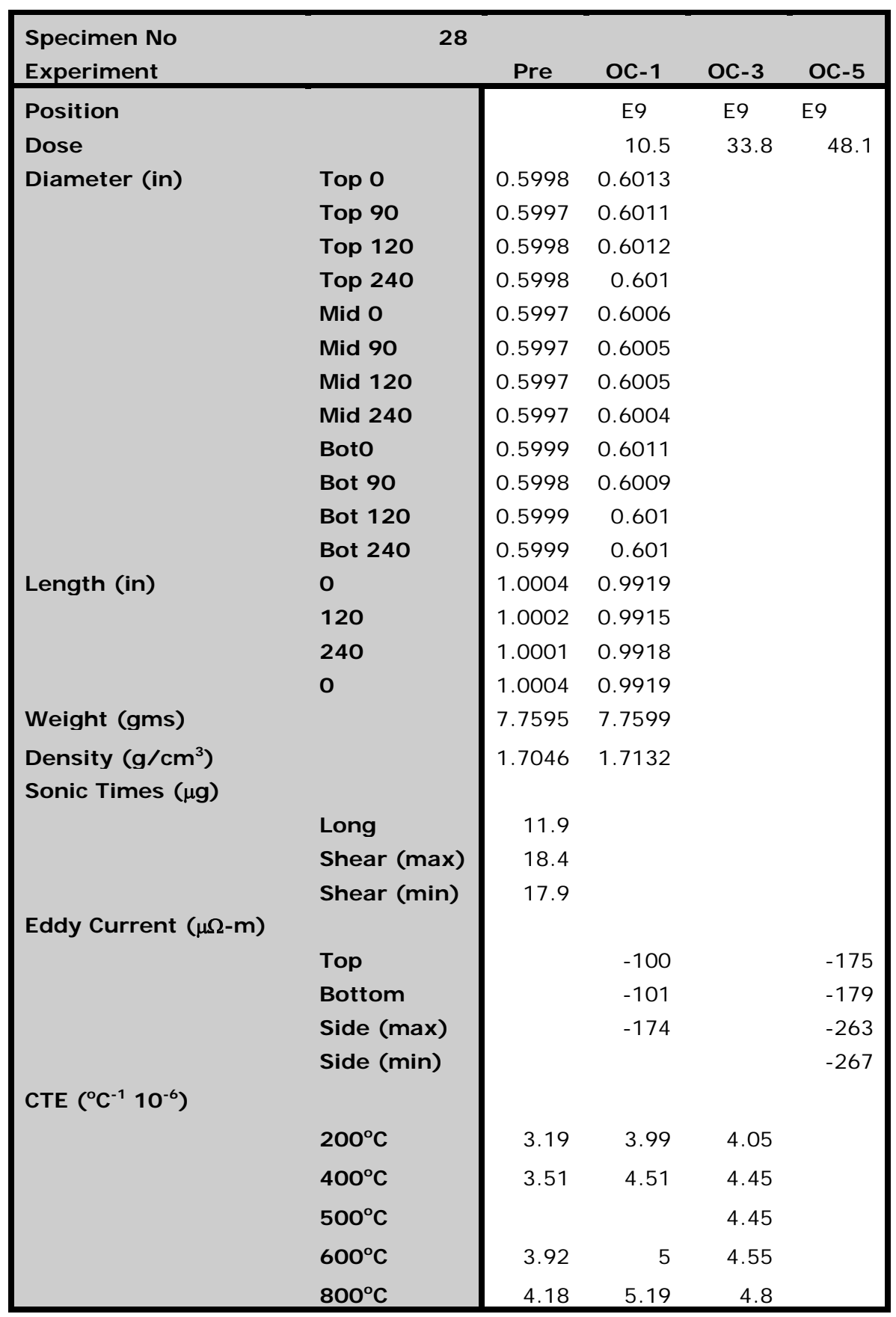




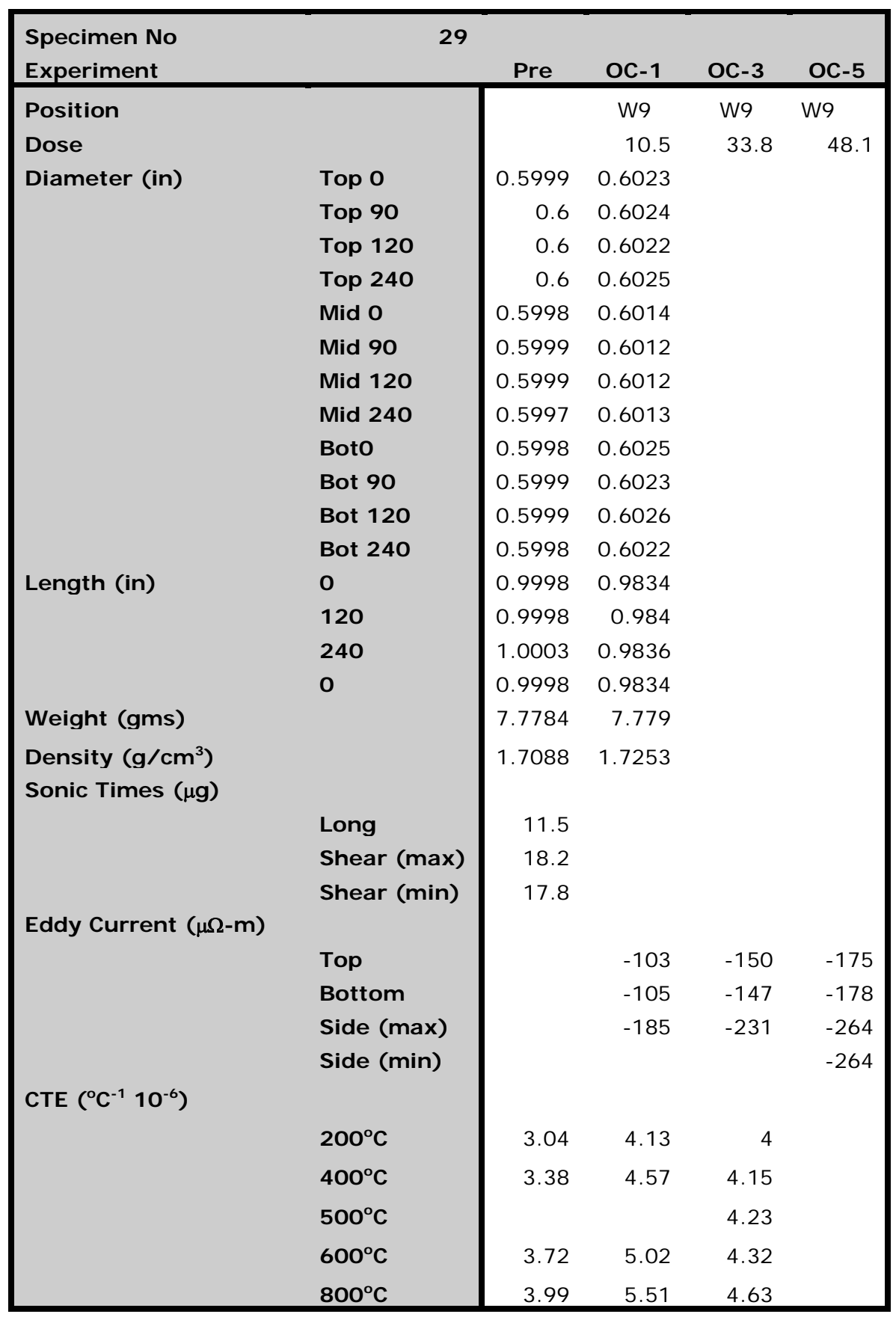




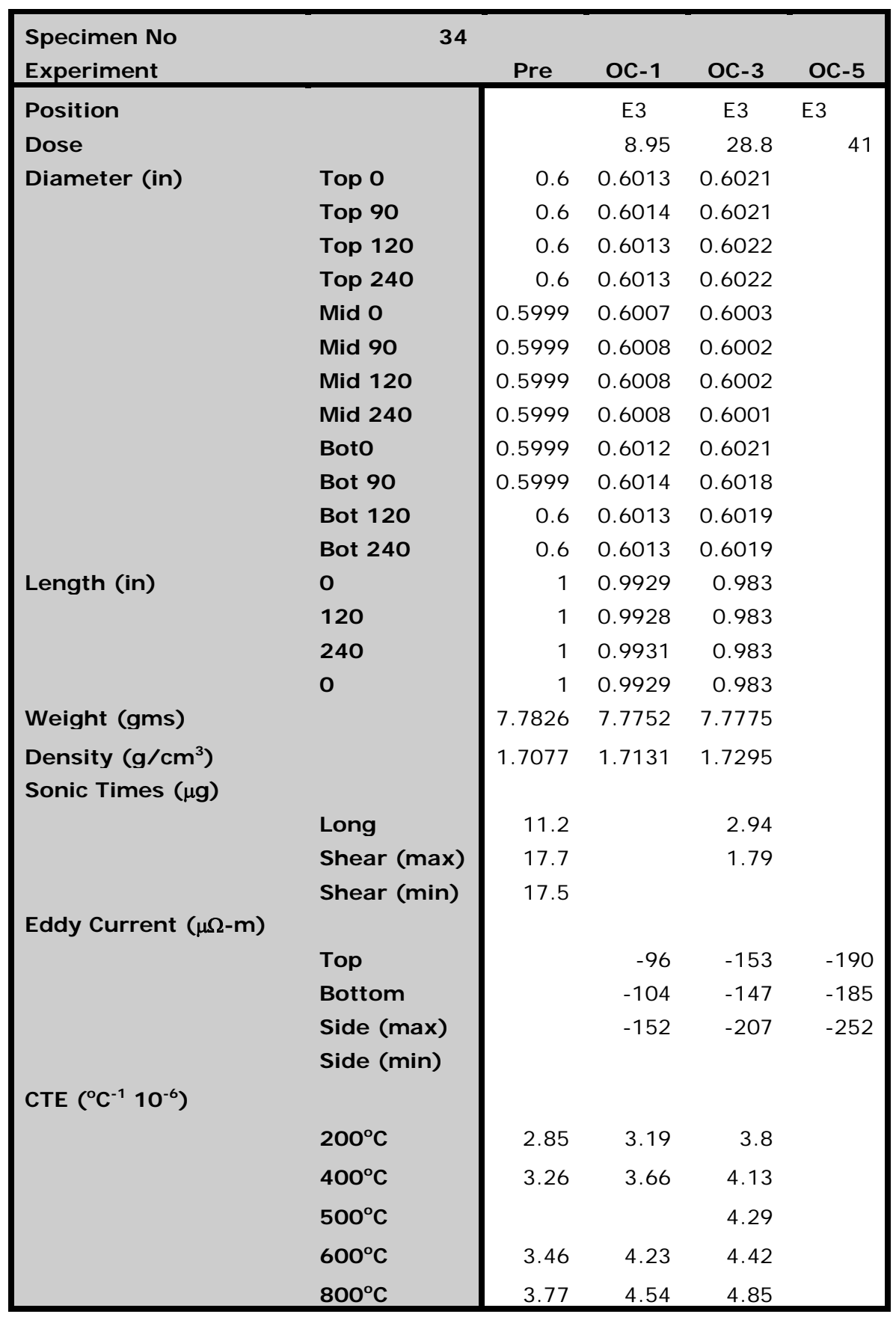




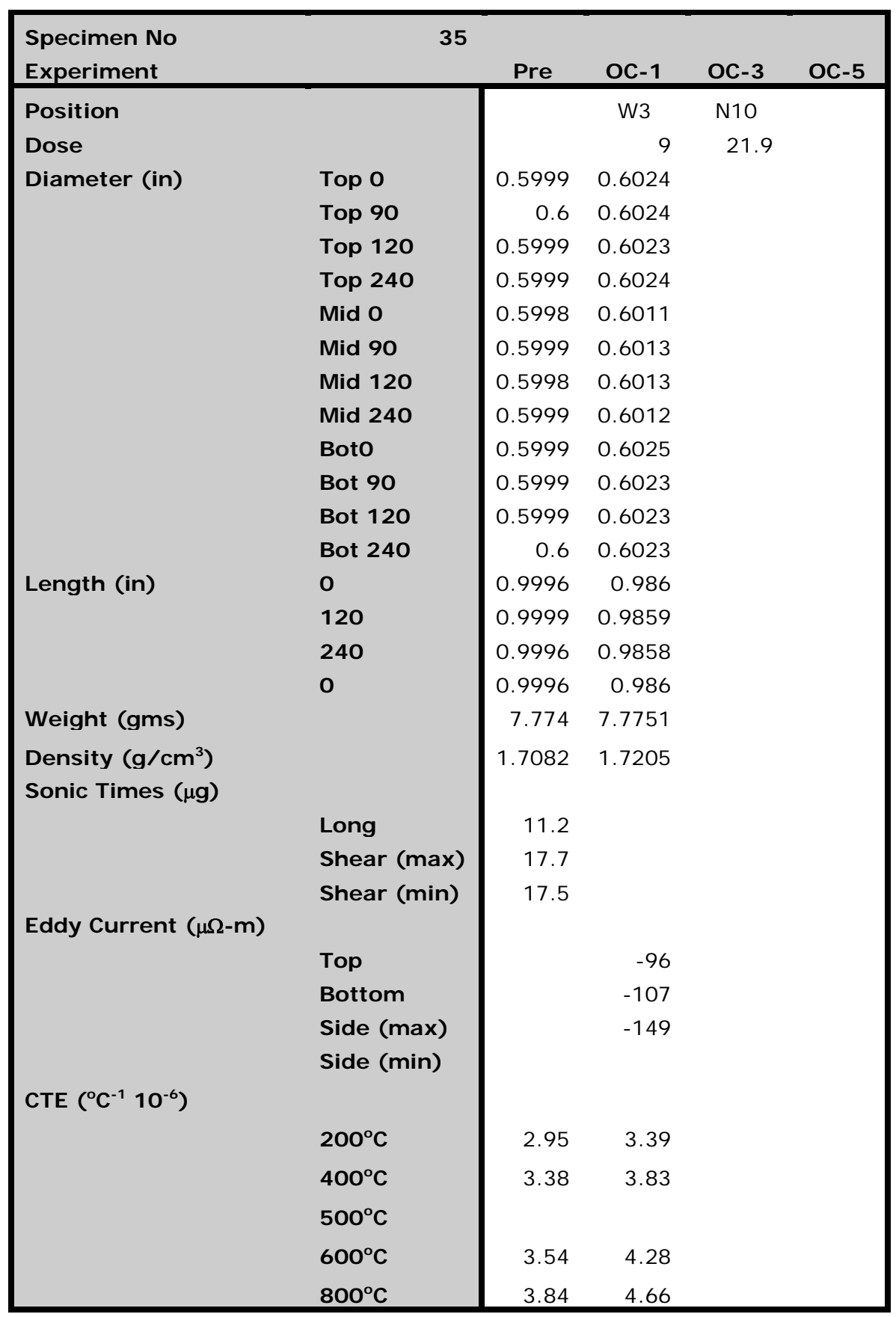




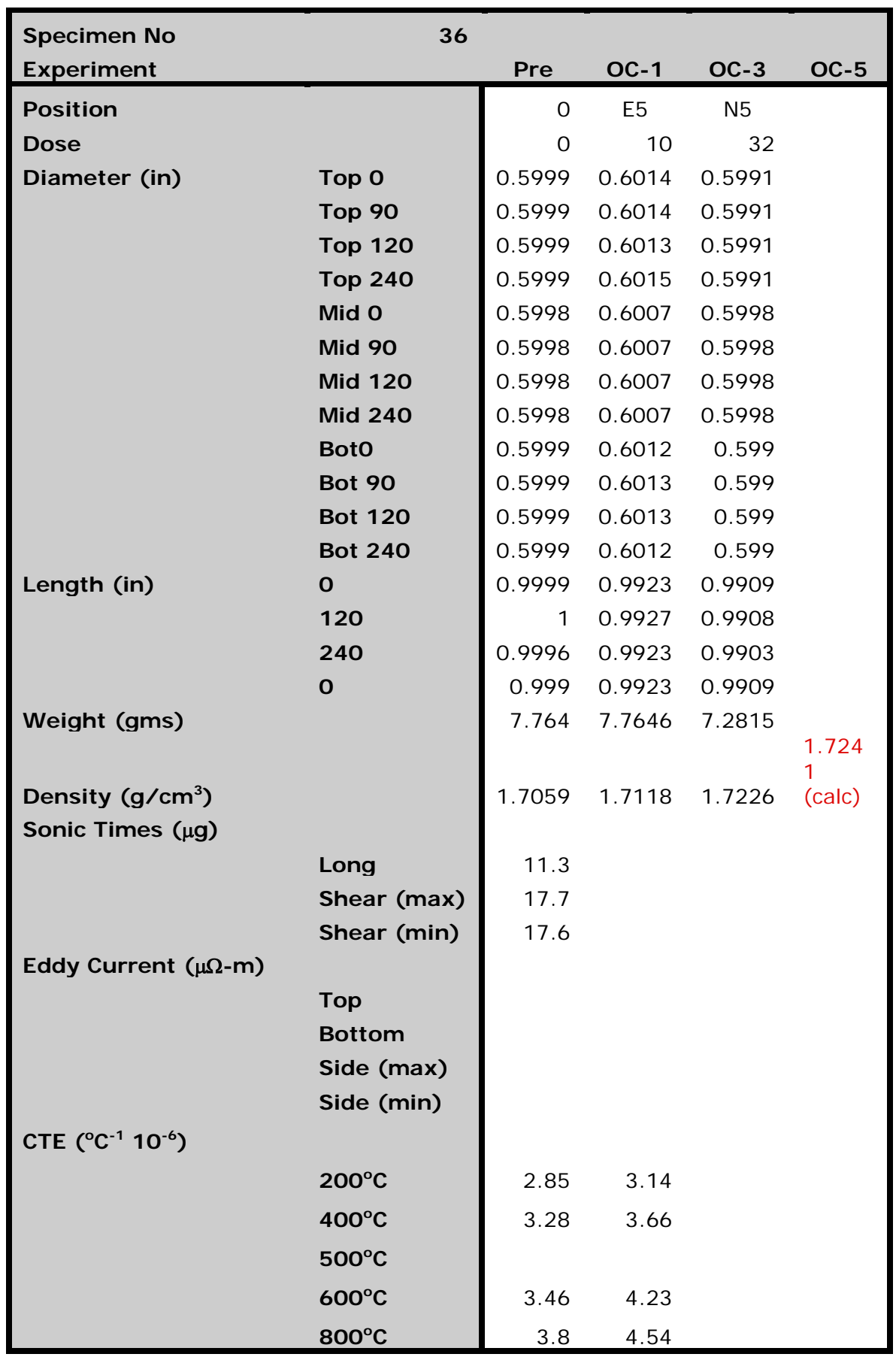




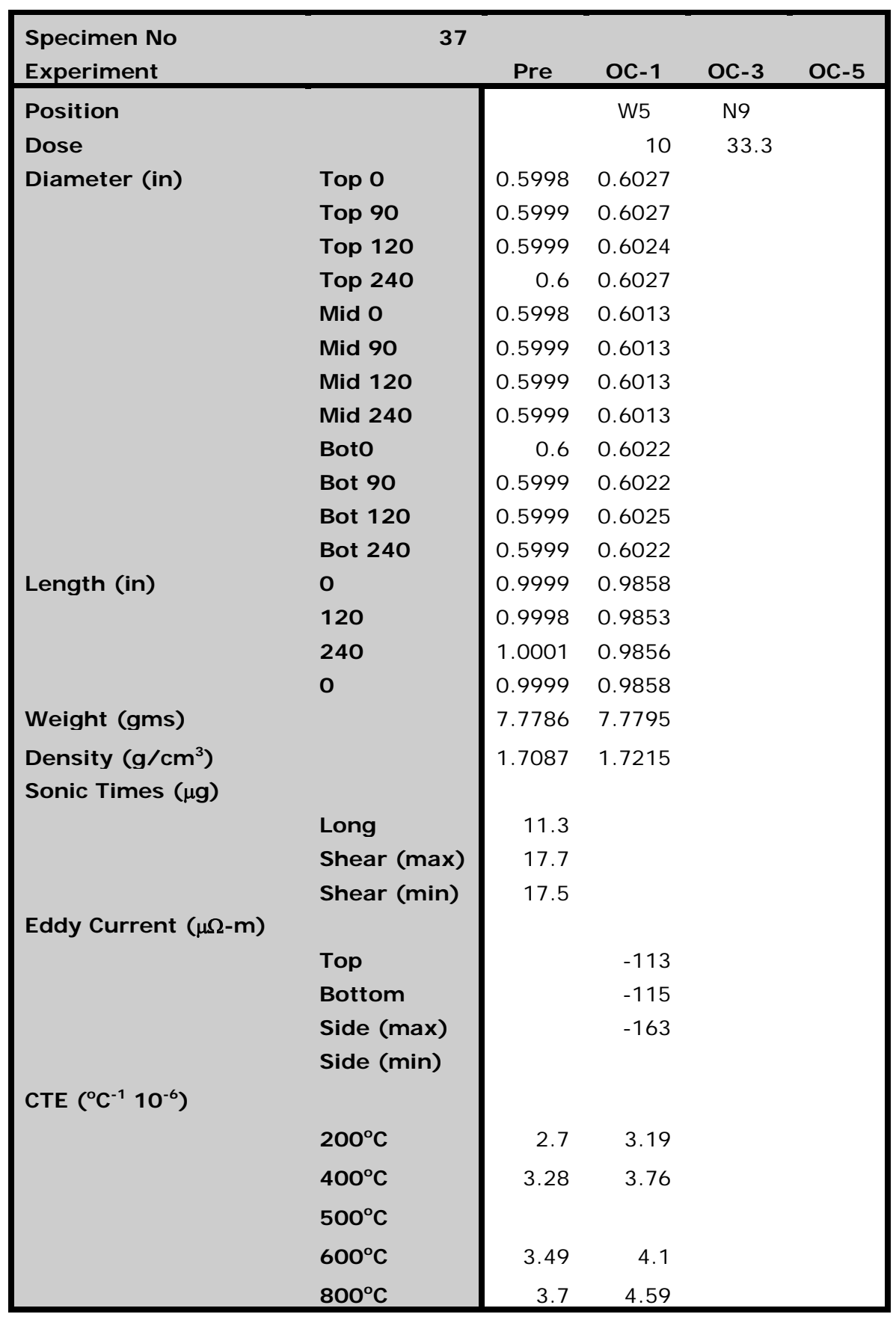




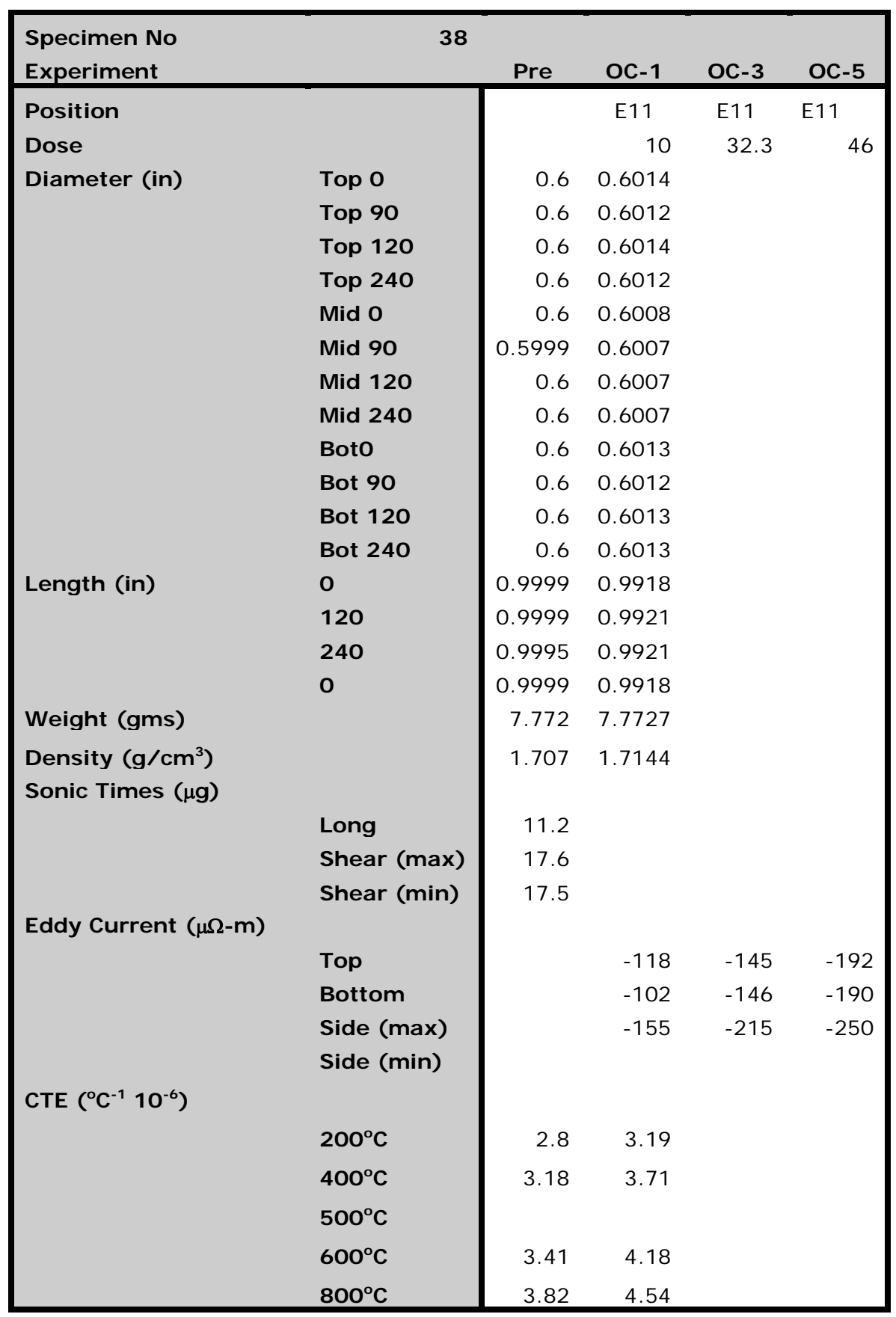




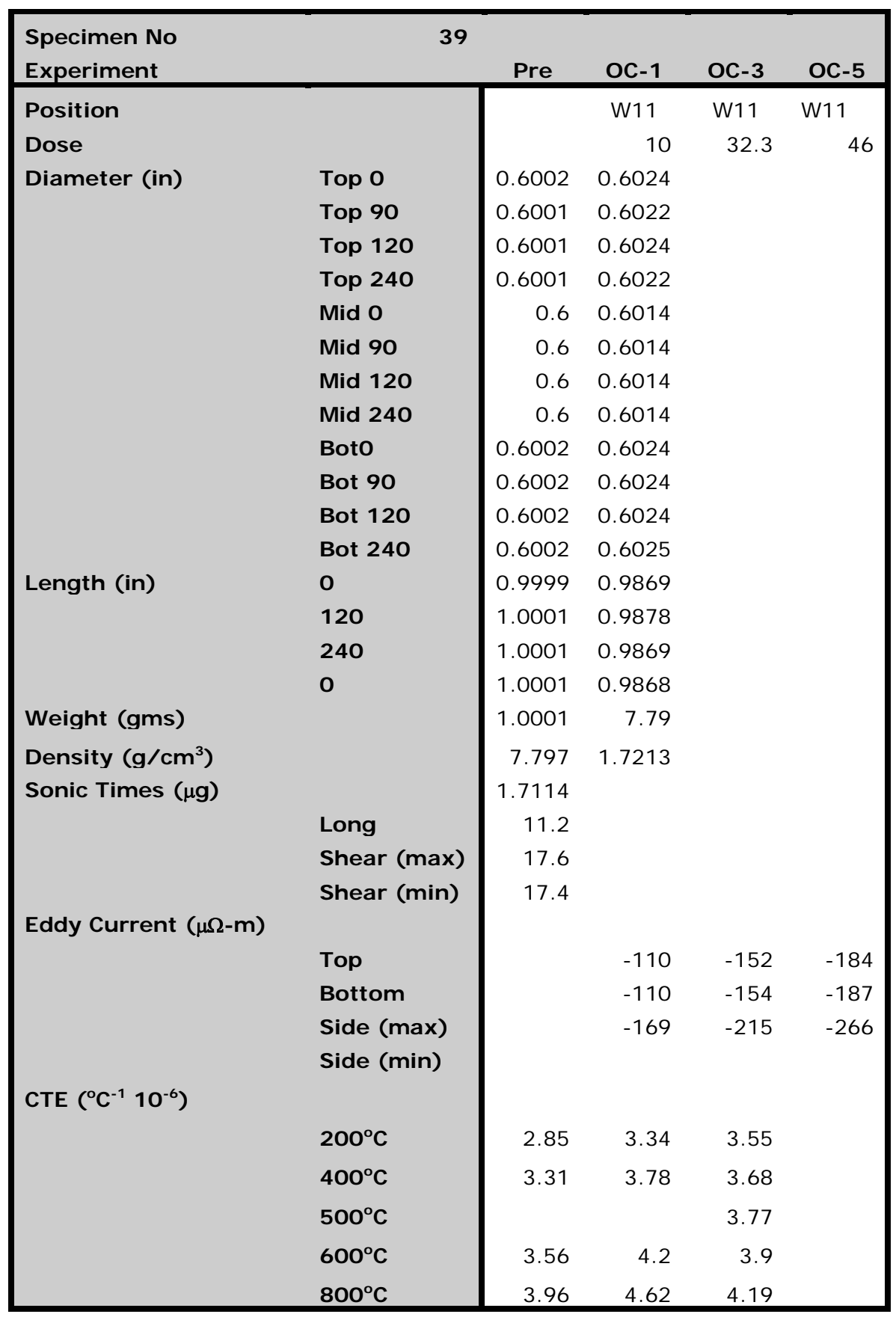




\begin{tabular}{|c|c|c|c|c|c|}
\hline $\begin{array}{l}\text { Specimen No } \\
\text { Experiment }\end{array}$ & 46 & Pre & OC- 1 & OC-3 & OC-5 \\
\hline $\begin{array}{l}\text { Position } \\
\text { Dose }\end{array}$ & & & $\begin{array}{l}\text { E1 } \\
7.52\end{array}$ & $\begin{array}{l}\mathrm{E} 1 \\
21.3\end{array}$ & $\begin{array}{l}\text { E1 } \\
34.4\end{array}$ \\
\hline \multirow[t]{12}{*}{ Diameter (in) } & Top 0 & 0.6002 & 0.6012 & 0.6017 & \\
\hline & Top 90 & 0.6002 & 0.6013 & 0.602 & \\
\hline & Top 120 & 0.6003 & 0.6014 & 0.6019 & \\
\hline & Top 240 & 0.6002 & 0.6013 & 0.6019 & \\
\hline & Mid 0 & 0.6002 & 0.6008 & 0.6005 & \\
\hline & Mid 90 & 0.6003 & 0.6009 & 0.6006 & \\
\hline & Mid 120 & 0.6004 & 0.6009 & 0.6005 & \\
\hline & Mid 240 & 0.6004 & 0.6009 & 0.6005 & \\
\hline & Boto & 0.6001 & 0.6013 & 0.602 & \\
\hline & Bot 90 & 0.6002 & 0.6013 & 0.6021 & \\
\hline & Bot 120 & 0.6004 & 0.6012 & 0.602 & \\
\hline & Bot 240 & 0.6004 & 0.6014 & 0.6022 & \\
\hline \multirow[t]{4}{*}{ Length (in) } & $\mathbf{0}$ & 0.9995 & 0.9938 & 0.9852 & \\
\hline & 120 & 1.0002 & 0.9928 & 0.9855 & \\
\hline & 240 & 1 & 0.9935 & 0.9853 & \\
\hline & 0 & 0.9995 & 0.9935 & 0.9852 & \\
\hline Weight (gms) & & 7.7826 & 7.7826 & 7.7859 & \\
\hline Density $\left(\mathrm{g} / \mathrm{cm}^{3}\right)$ & & 1.7077 & 1.7137 & 1.7268 & \\
\hline \multirow[t]{4}{*}{ Sonic Times $(\mu \mathbf{g})$} & & 11.3 & & 2.93 & \\
\hline & Long & 17.7 & & 1.81 & \\
\hline & Shear (max) & 17.5 & & & \\
\hline & Shear (min) & & -68 & -156 & -180 \\
\hline \multirow[t]{5}{*}{ Eddy Current ( $\mu \Omega-m)$} & & & -72 & -157 & -179 \\
\hline & Top & & -122 & -210 & -229 \\
\hline & Bottom & & & & \\
\hline & Side (max) & & & & \\
\hline & Side ( $\min )$ & & & & \\
\hline \multicolumn{6}{|l|}{ CTE $\left({ }^{\circ} \mathrm{C}^{-1} 10^{-6}\right)$} \\
\hline & $200^{\circ} \mathrm{C}$ & 2.95 & 3.44 & 2.95 & \\
\hline & $400^{\circ} \mathrm{C}$ & 3.38 & 3.71 & 3.55 & \\
\hline & $500^{\circ} \mathrm{C}$ & & & 3.81 & \\
\hline & $600^{\circ} \mathrm{C}$ & 3.54 & 4.23 & 4.03 & \\
\hline & $800^{\circ} \mathrm{C}$ & 3.9 & 4.59 & 4.39 & \\
\hline
\end{tabular}




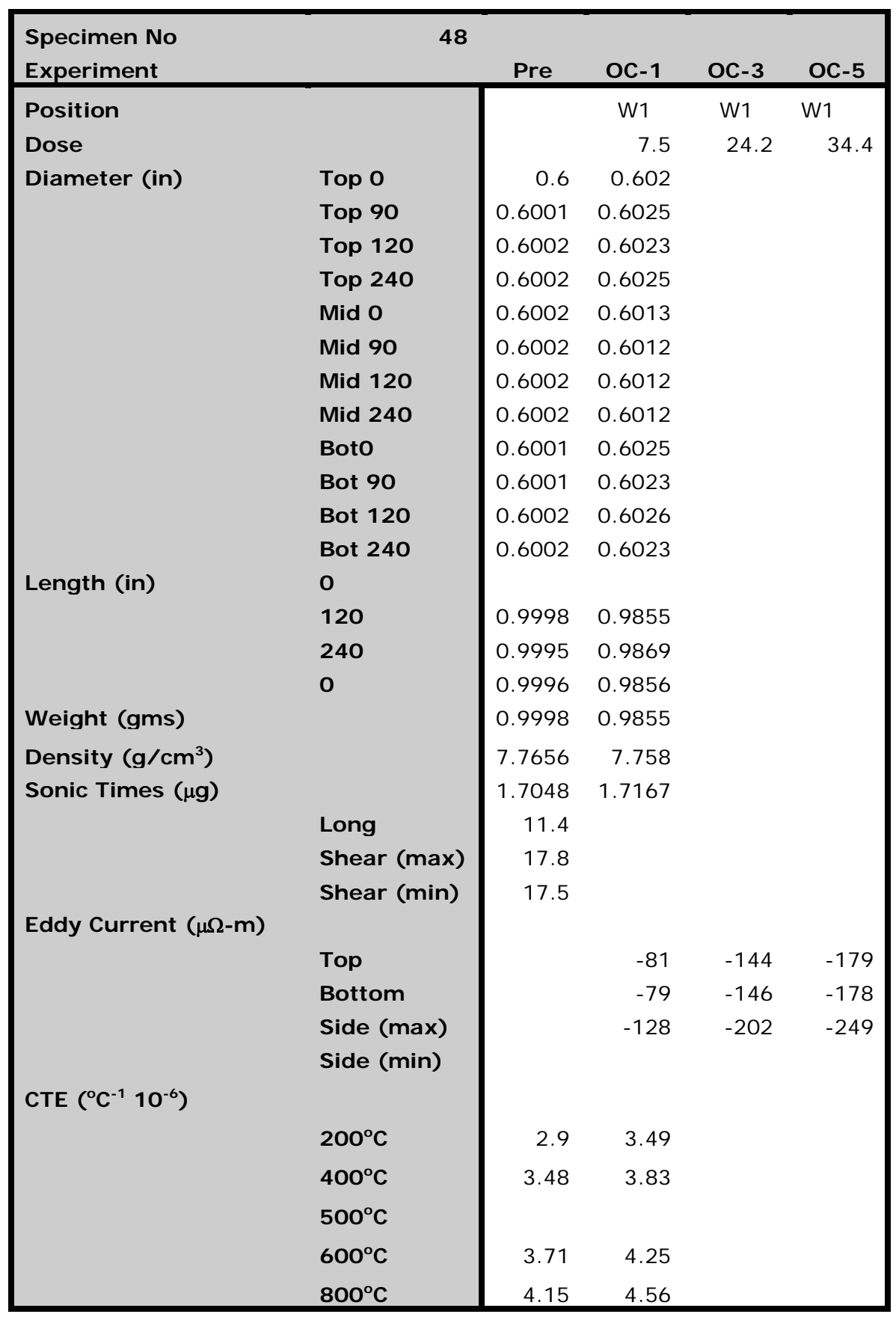




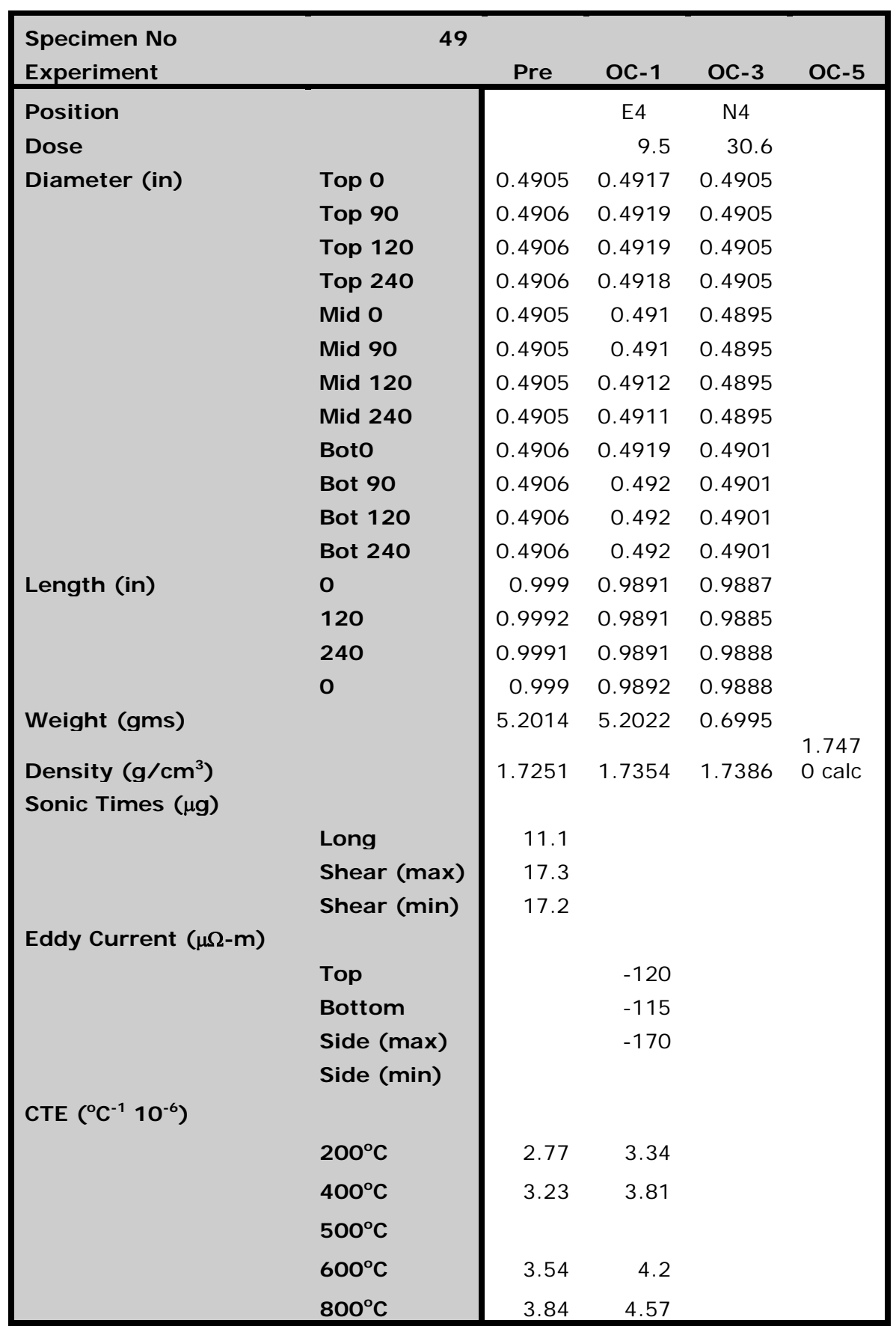




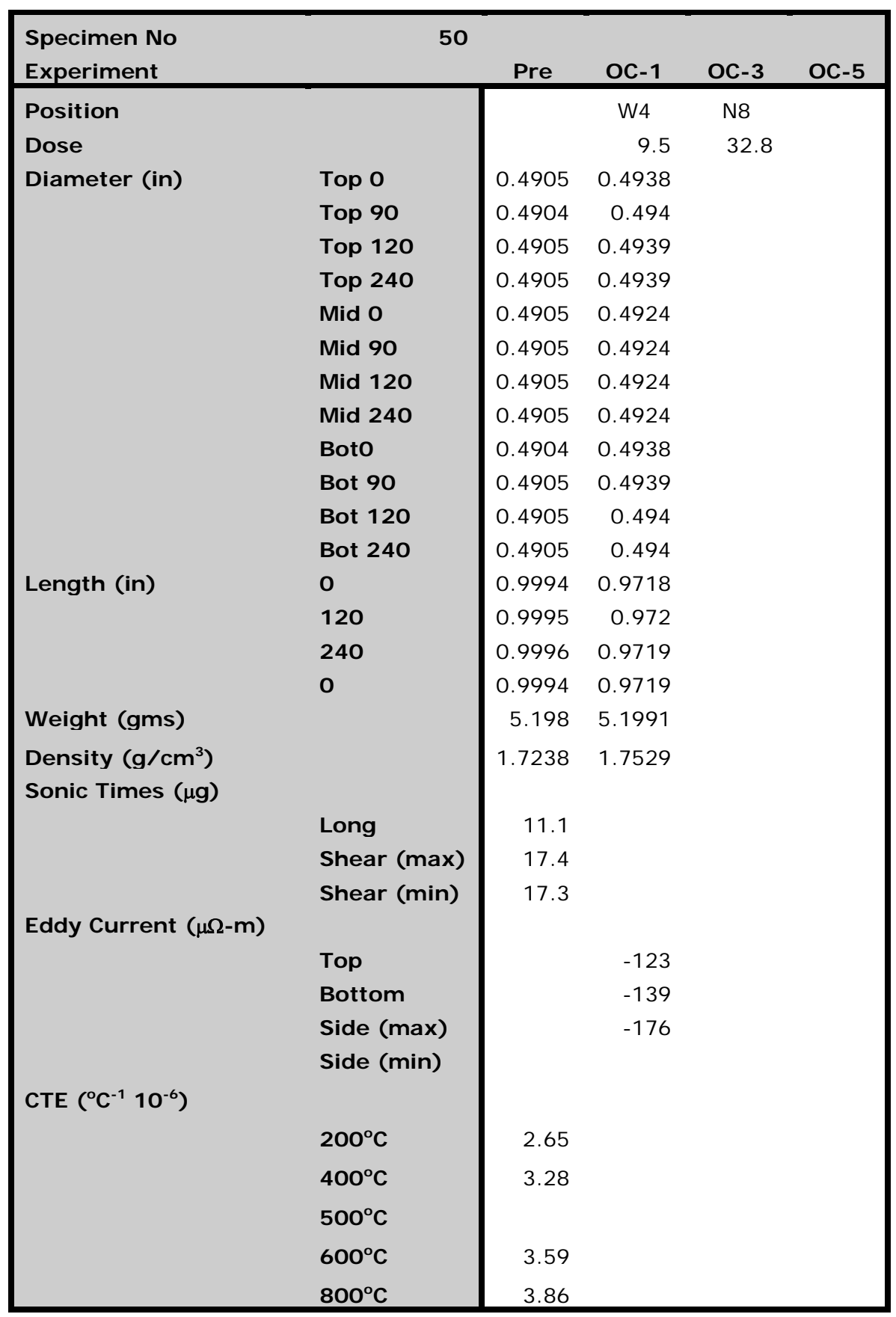




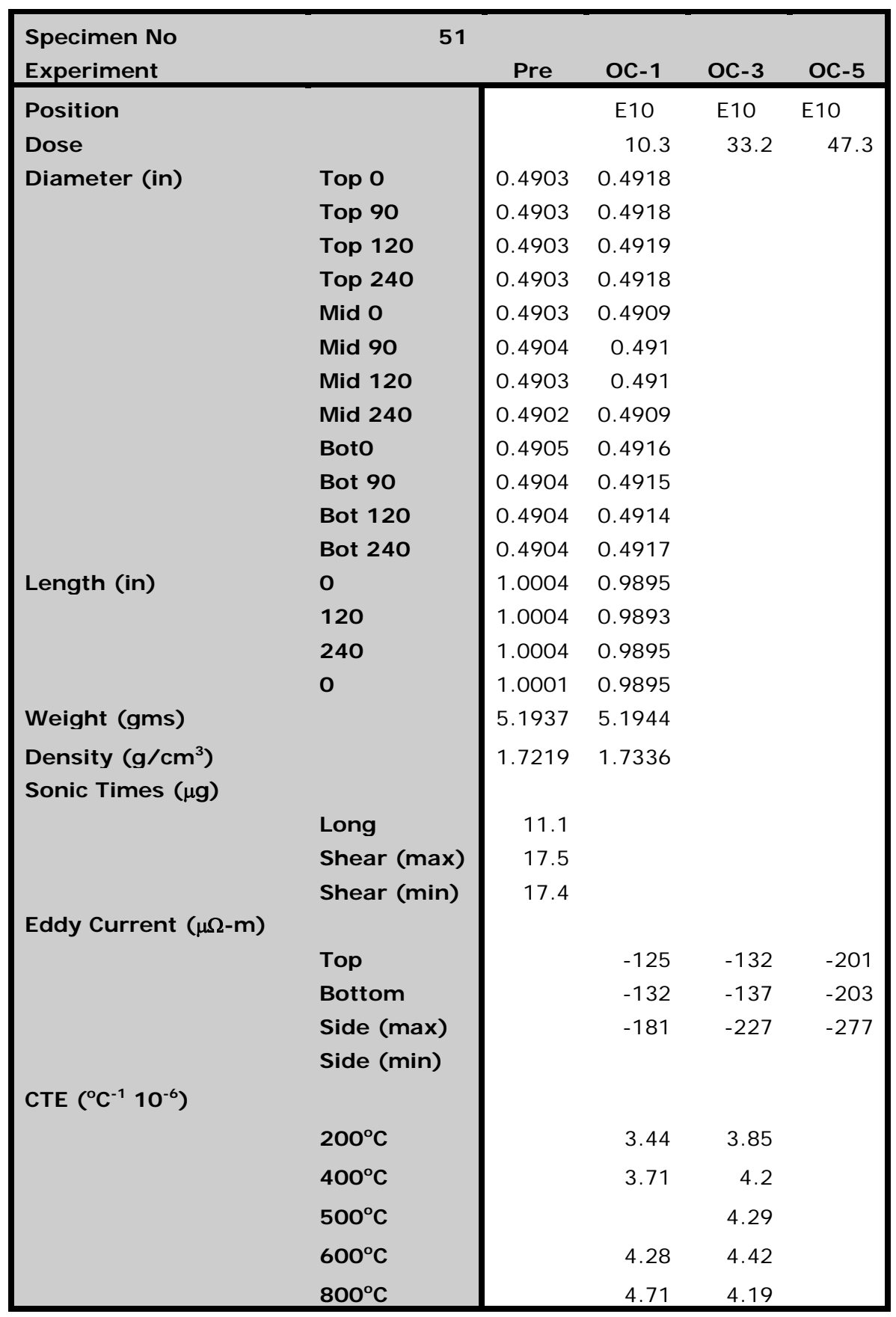




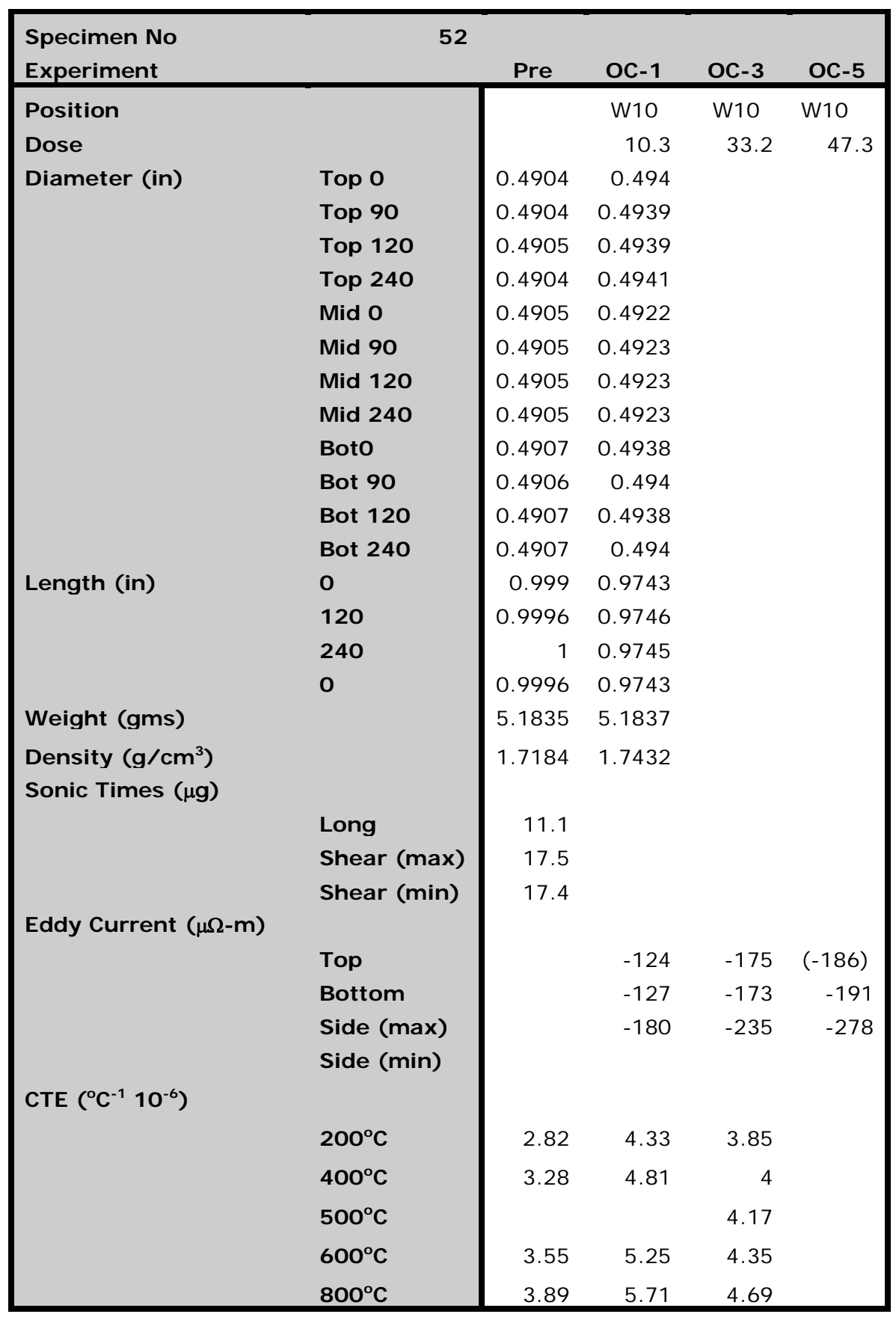




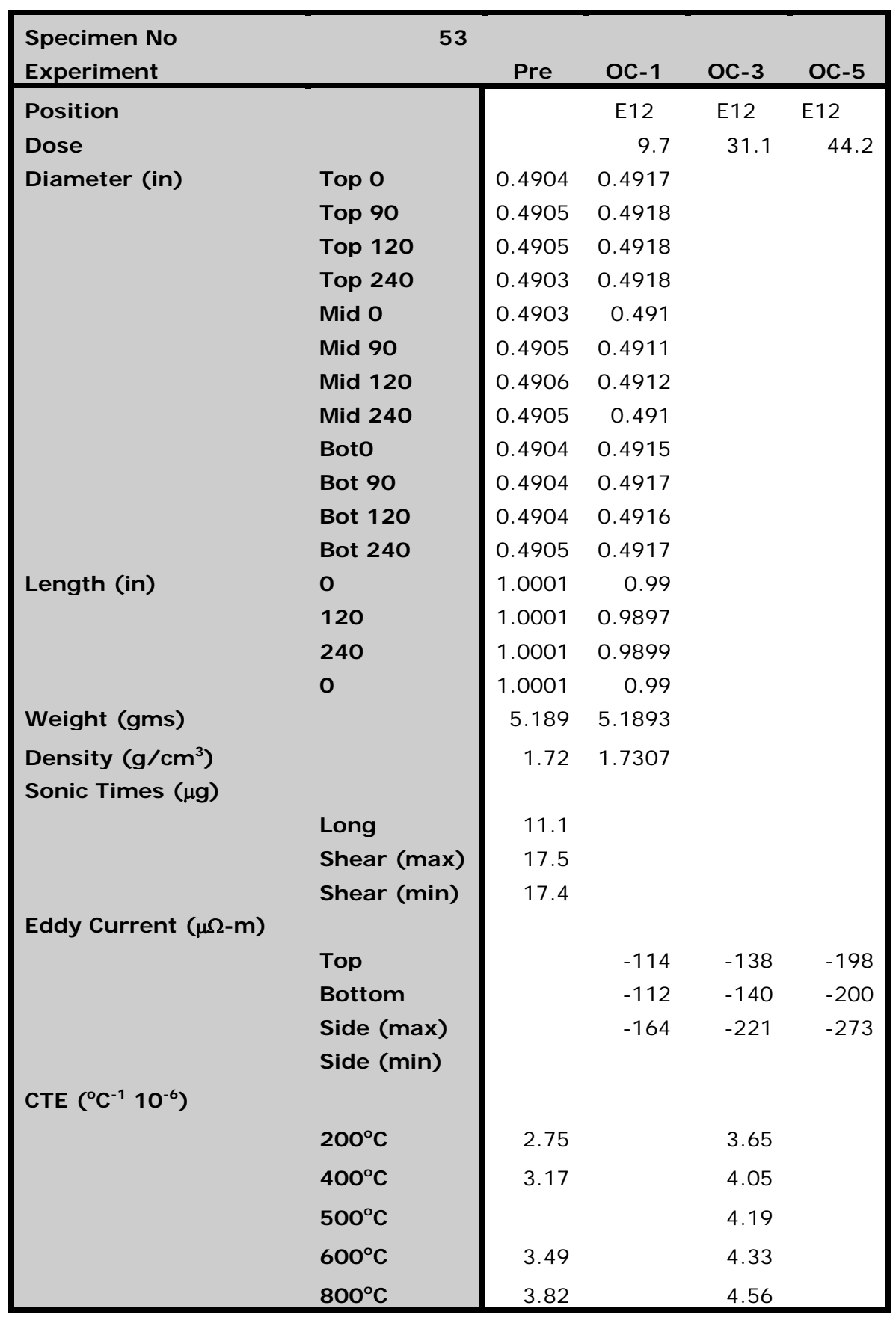




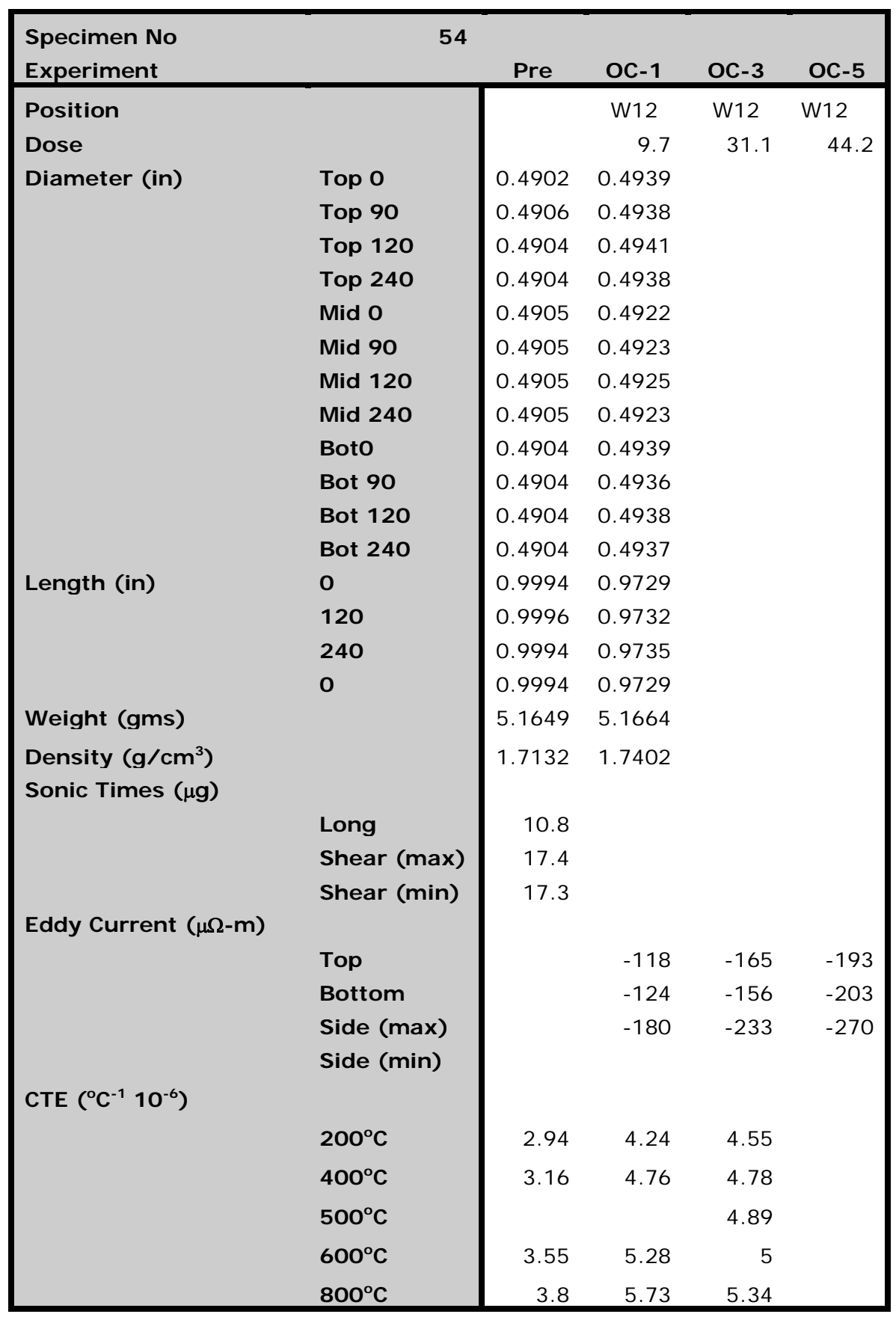




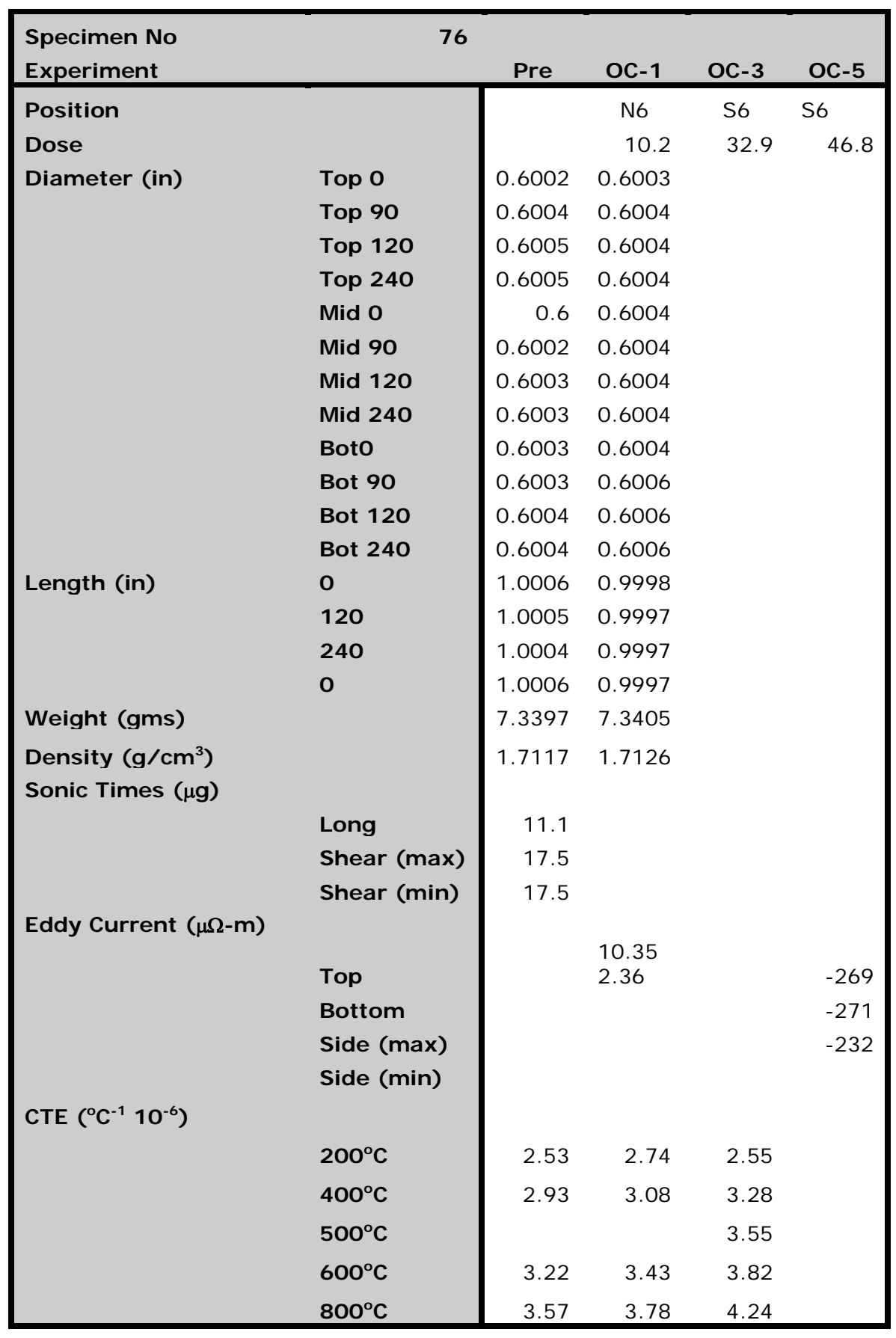




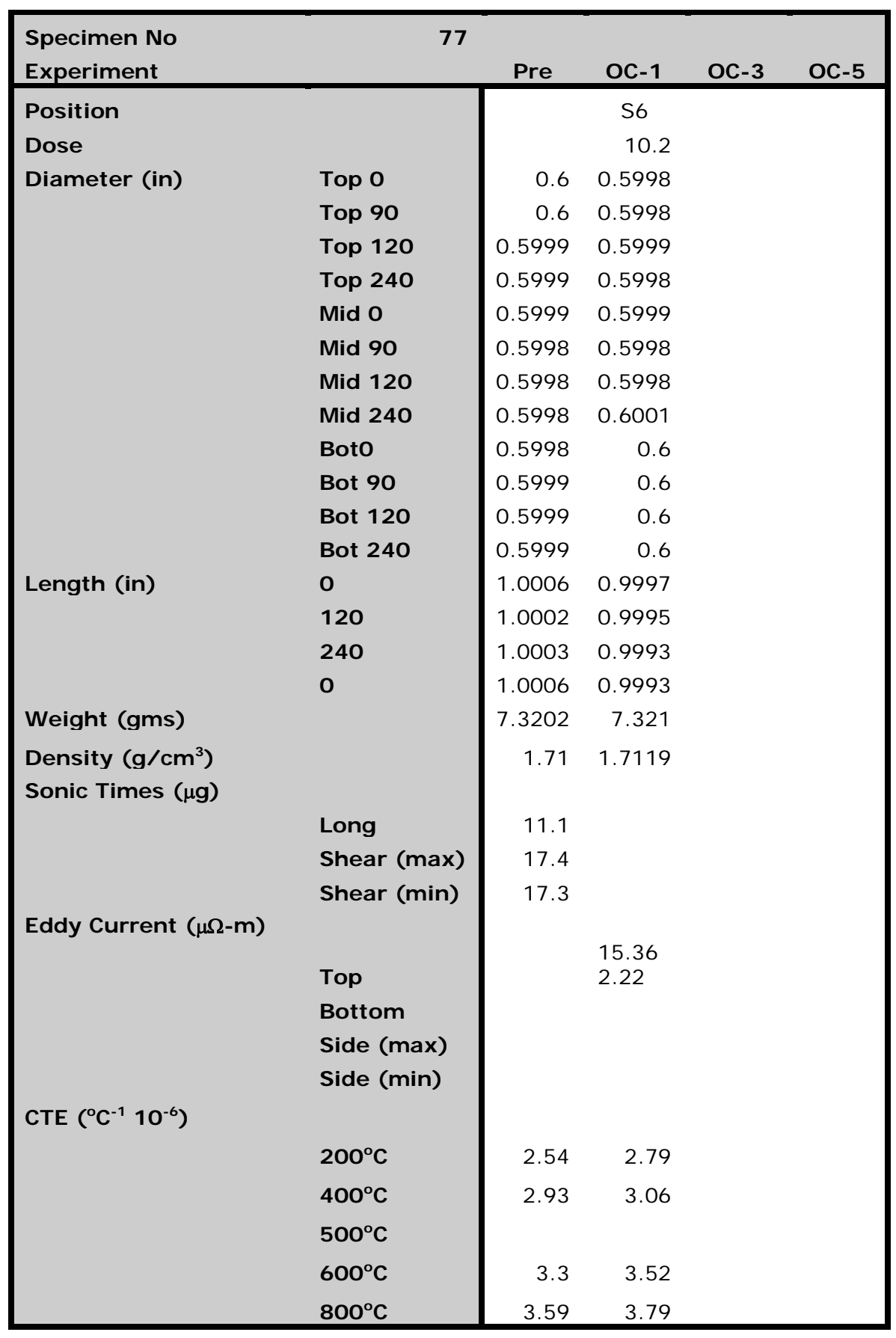




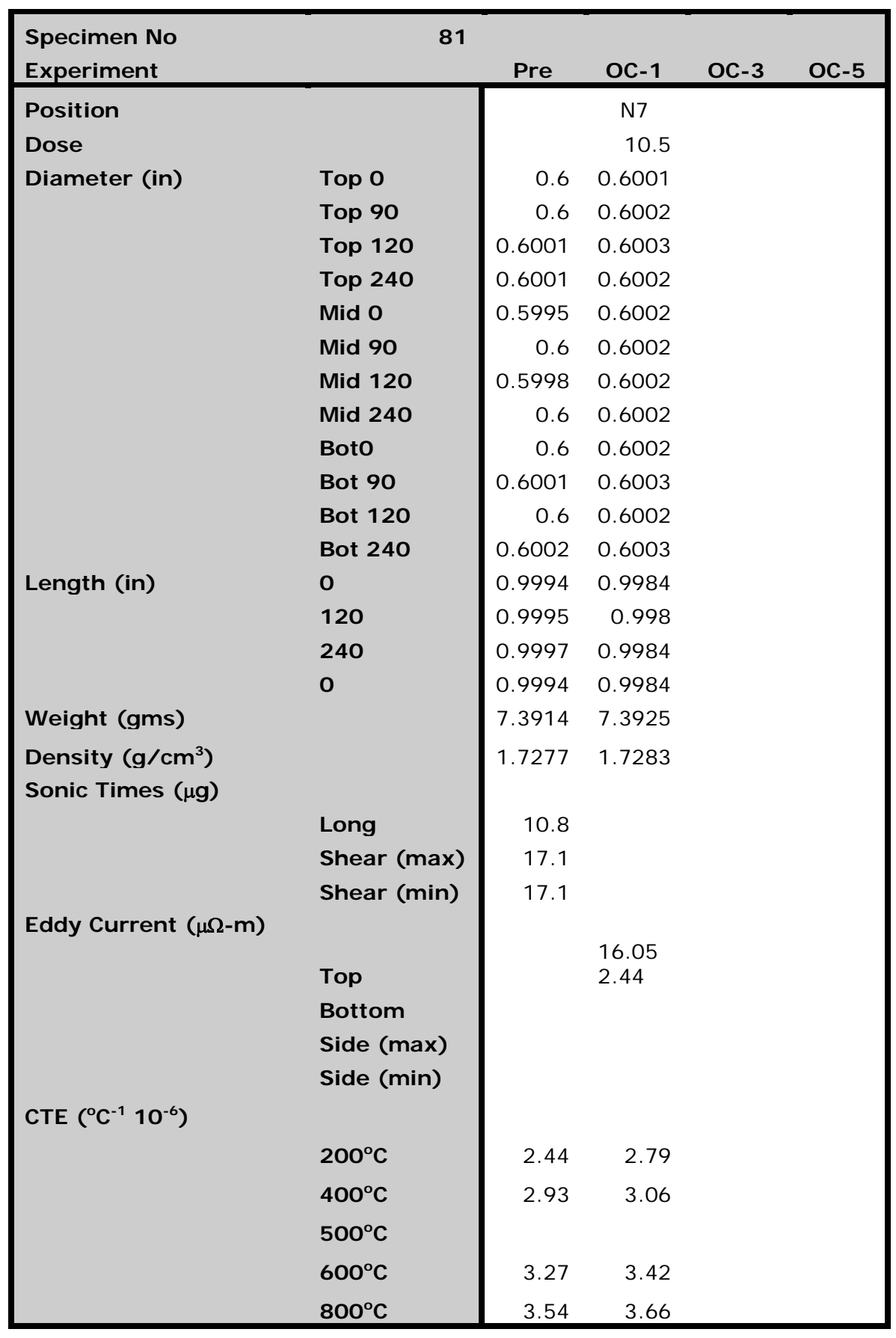




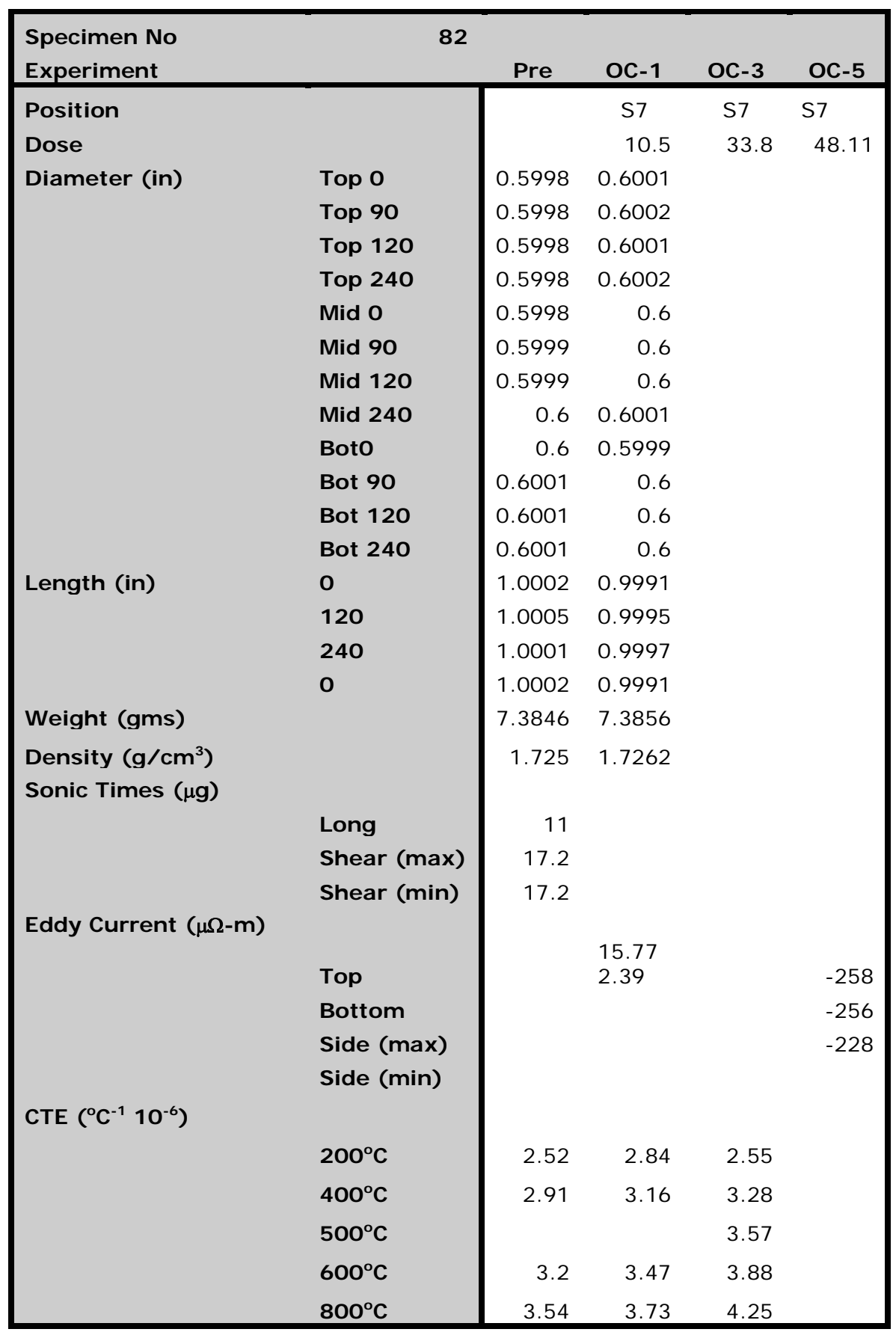




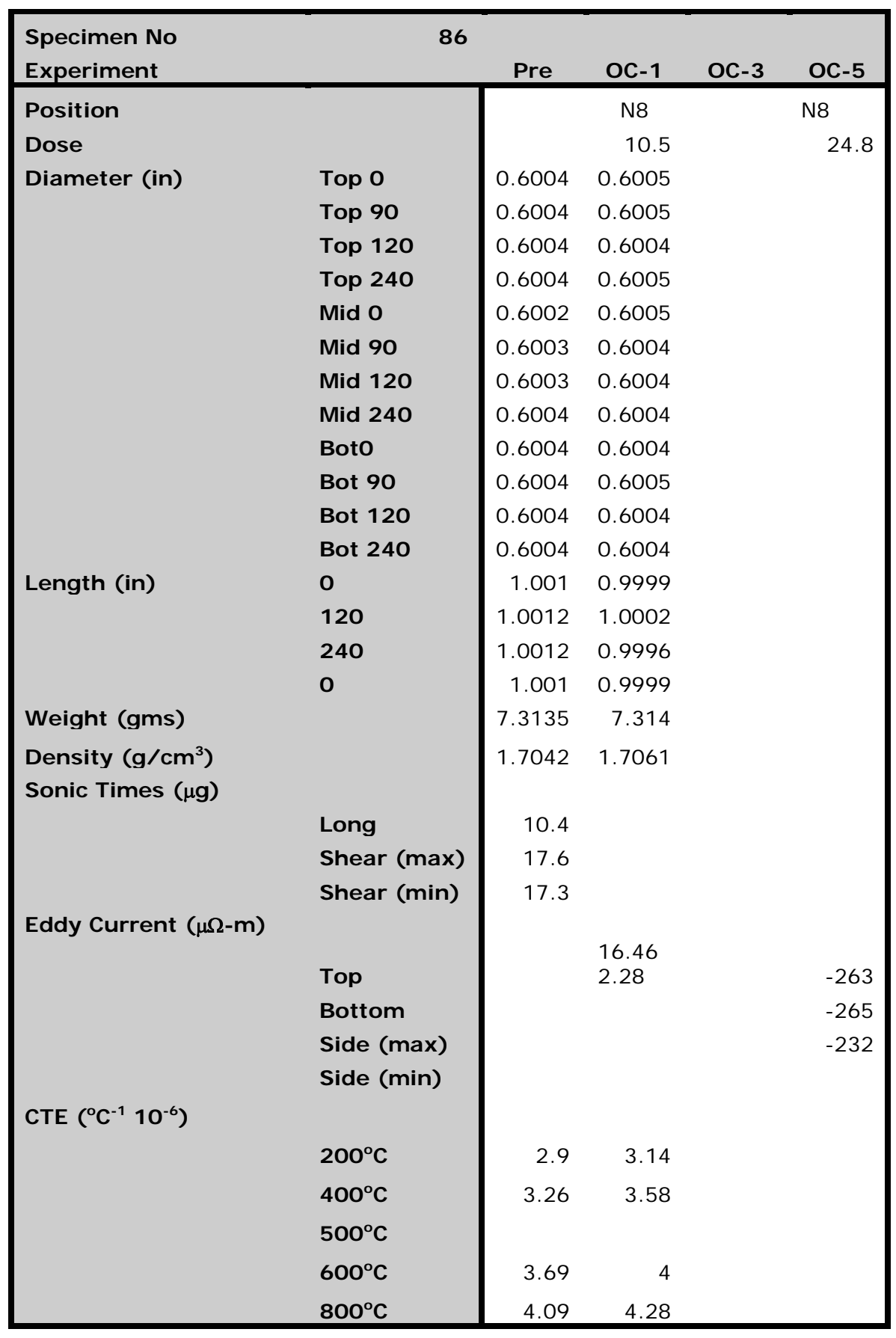




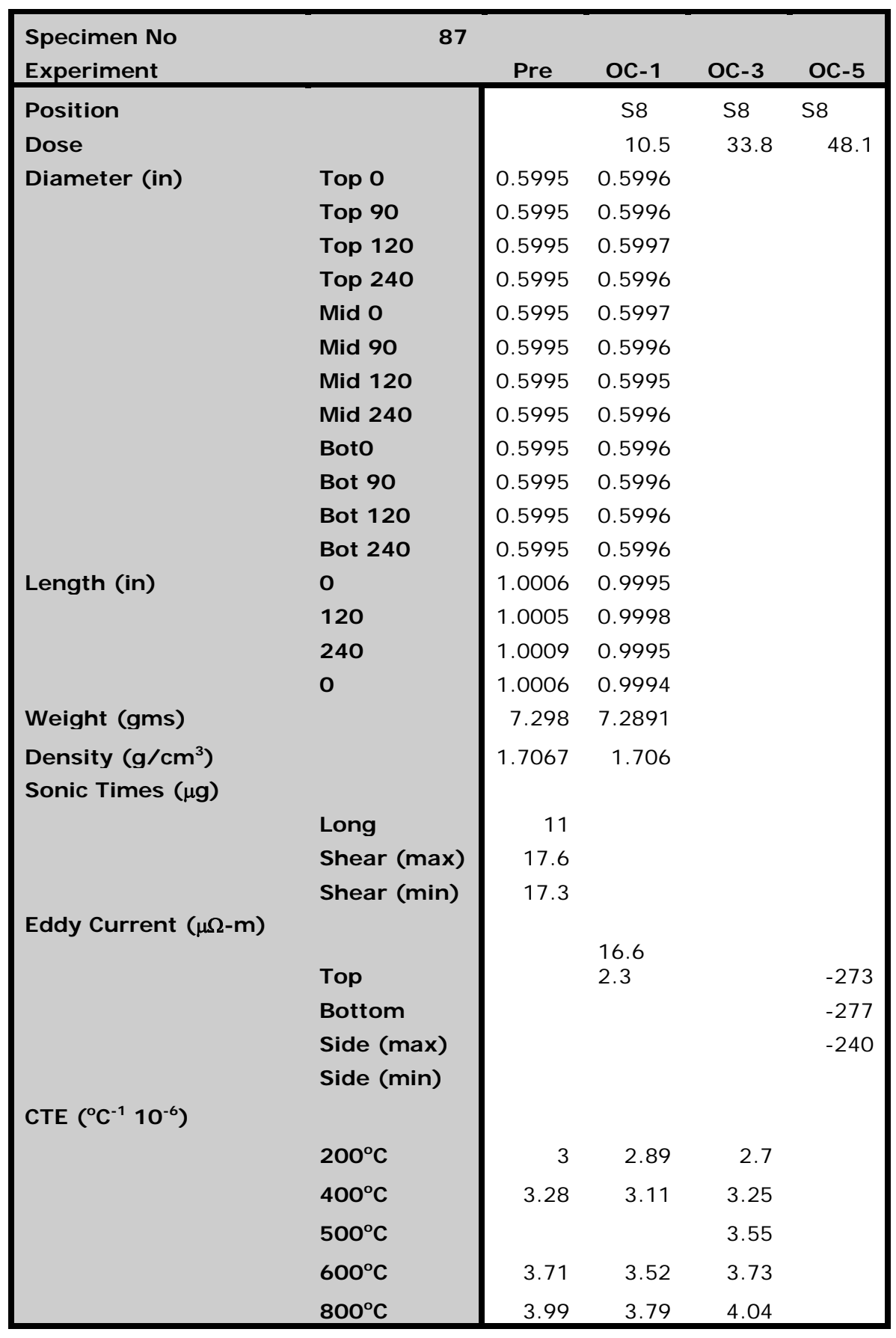




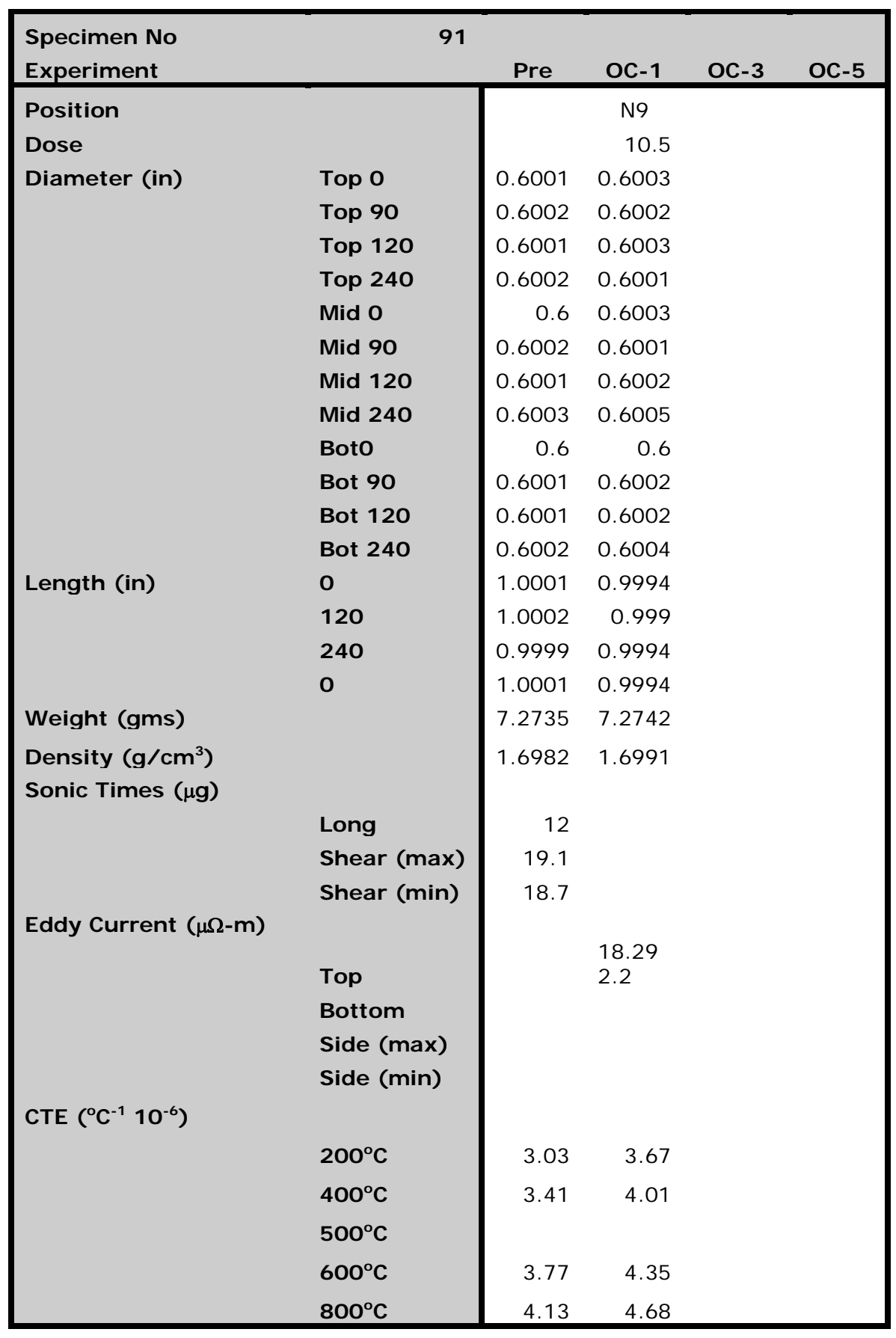




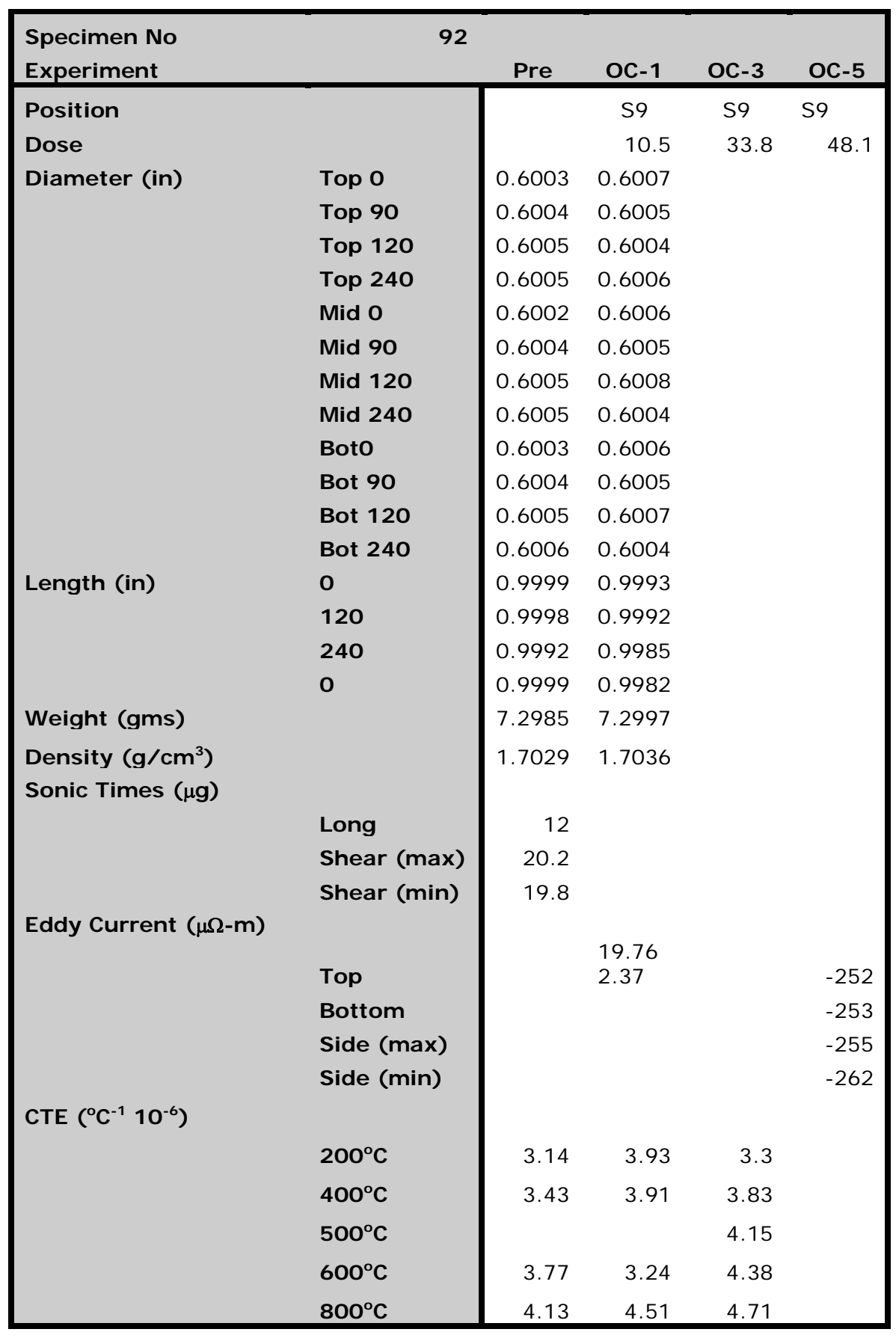




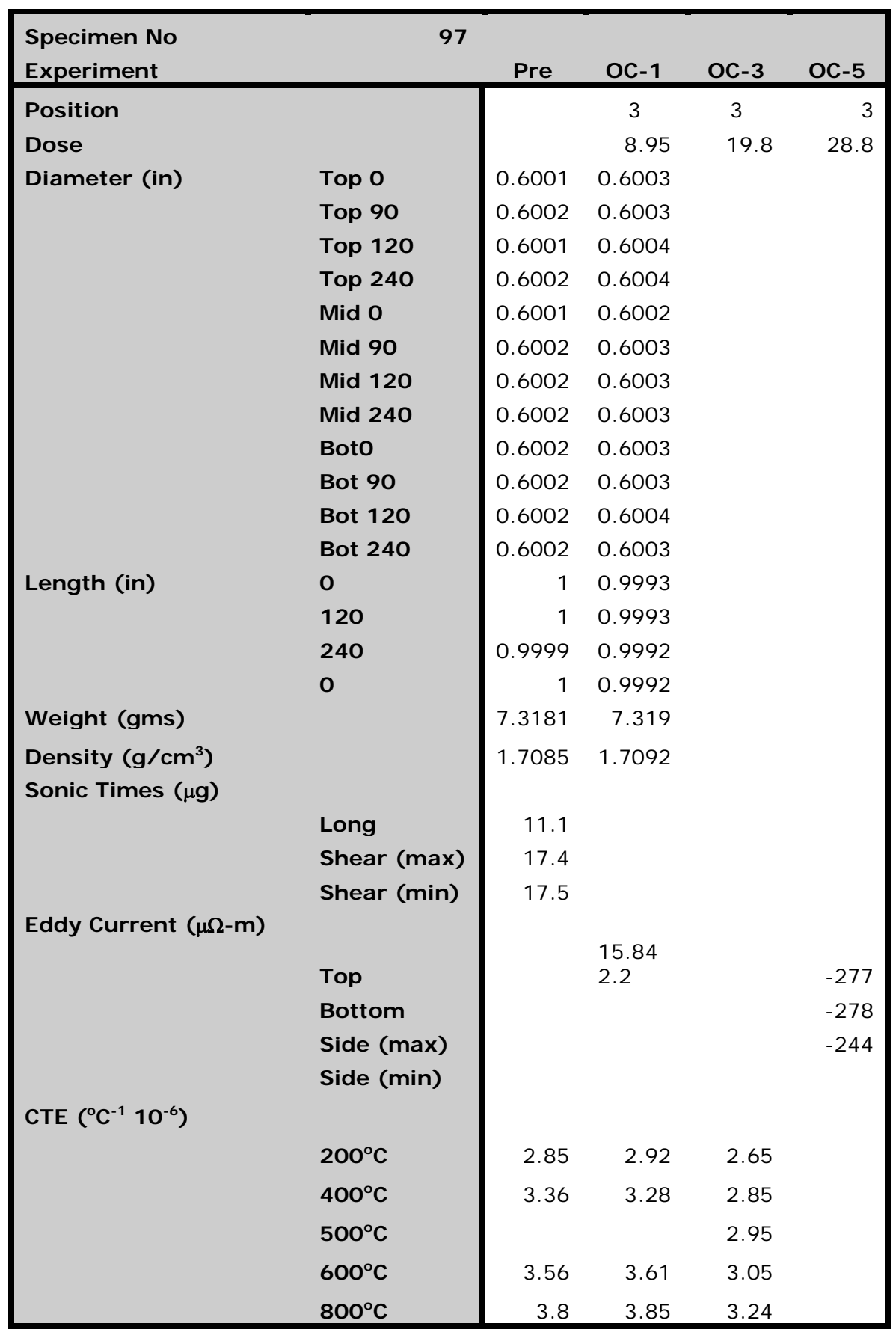




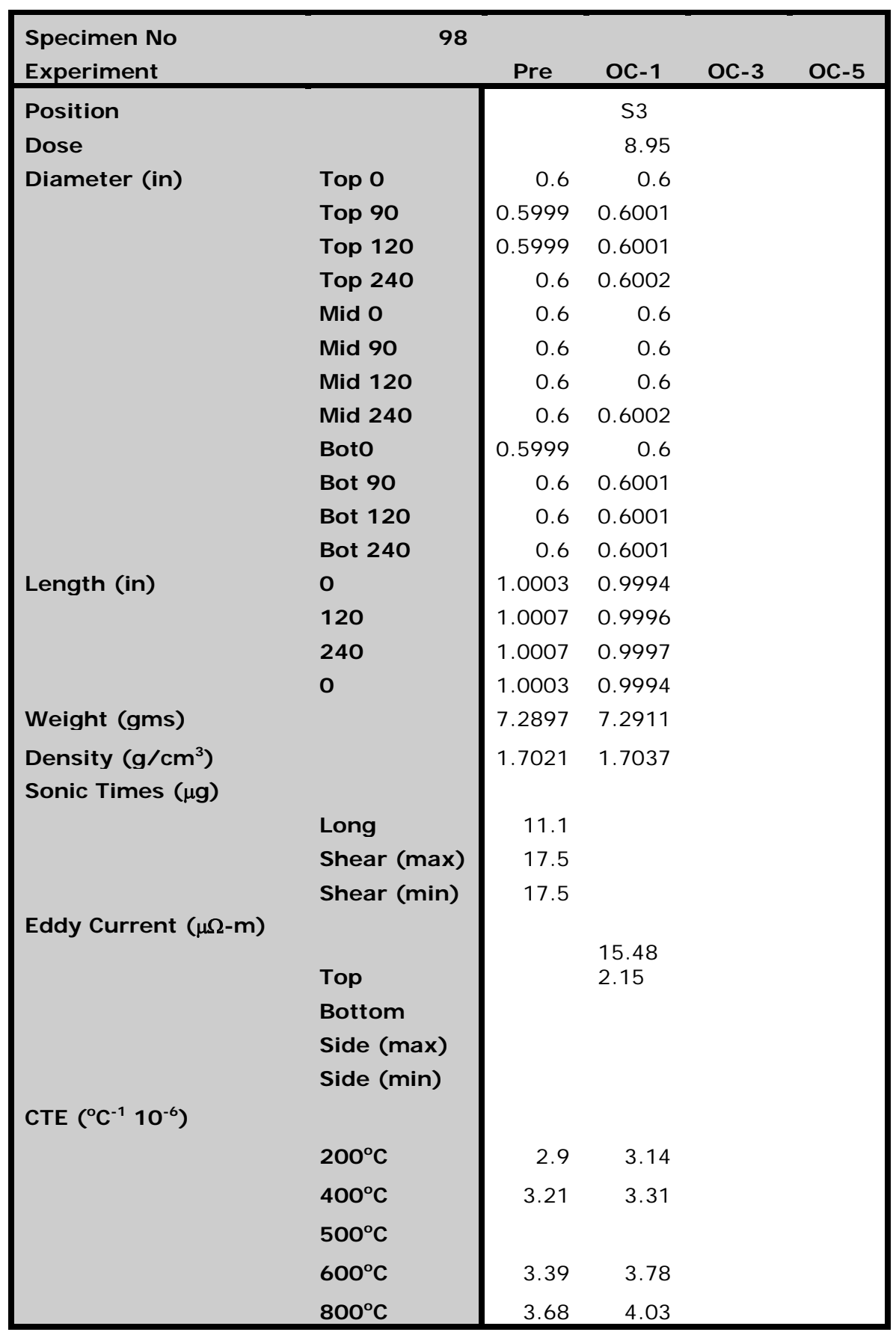




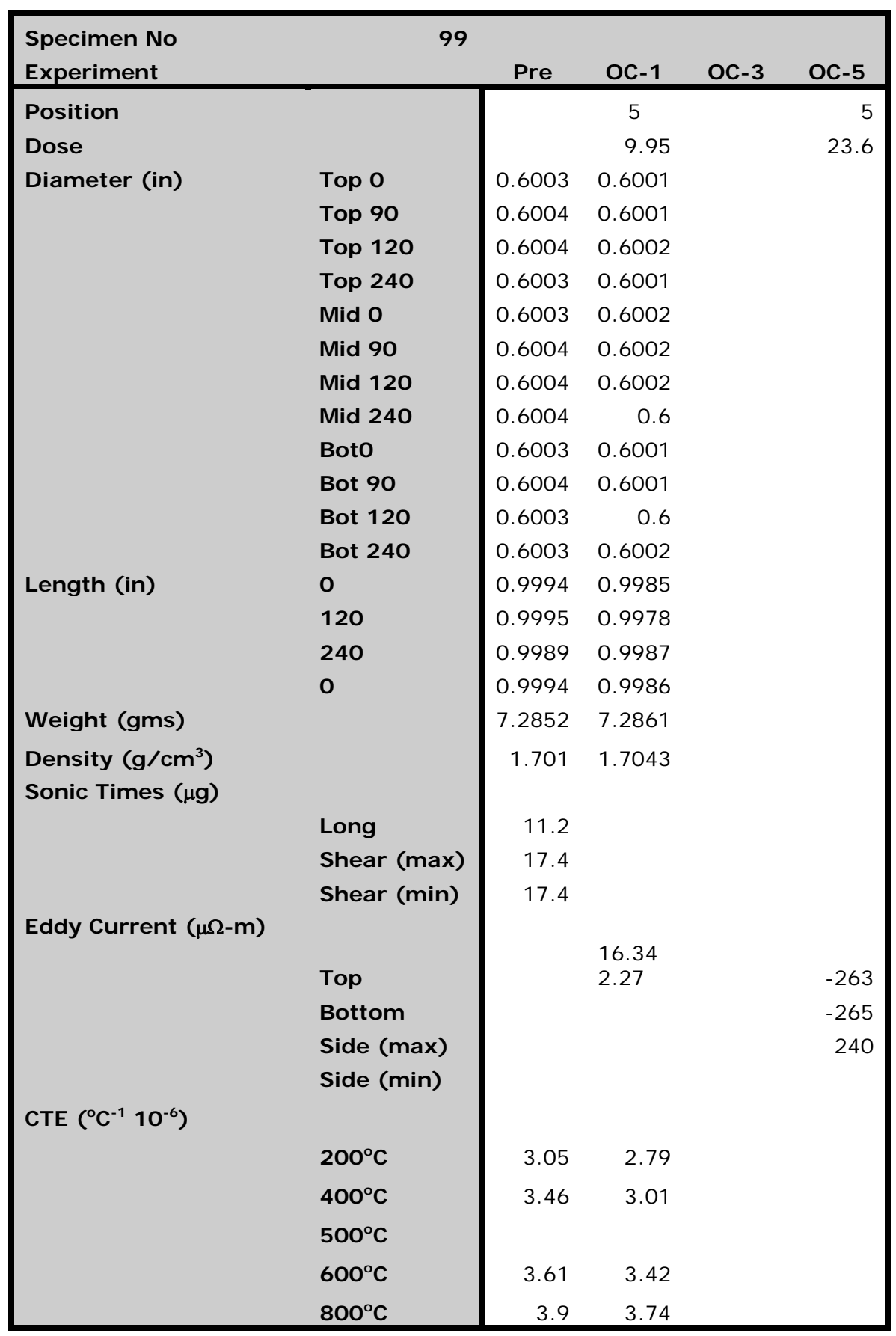




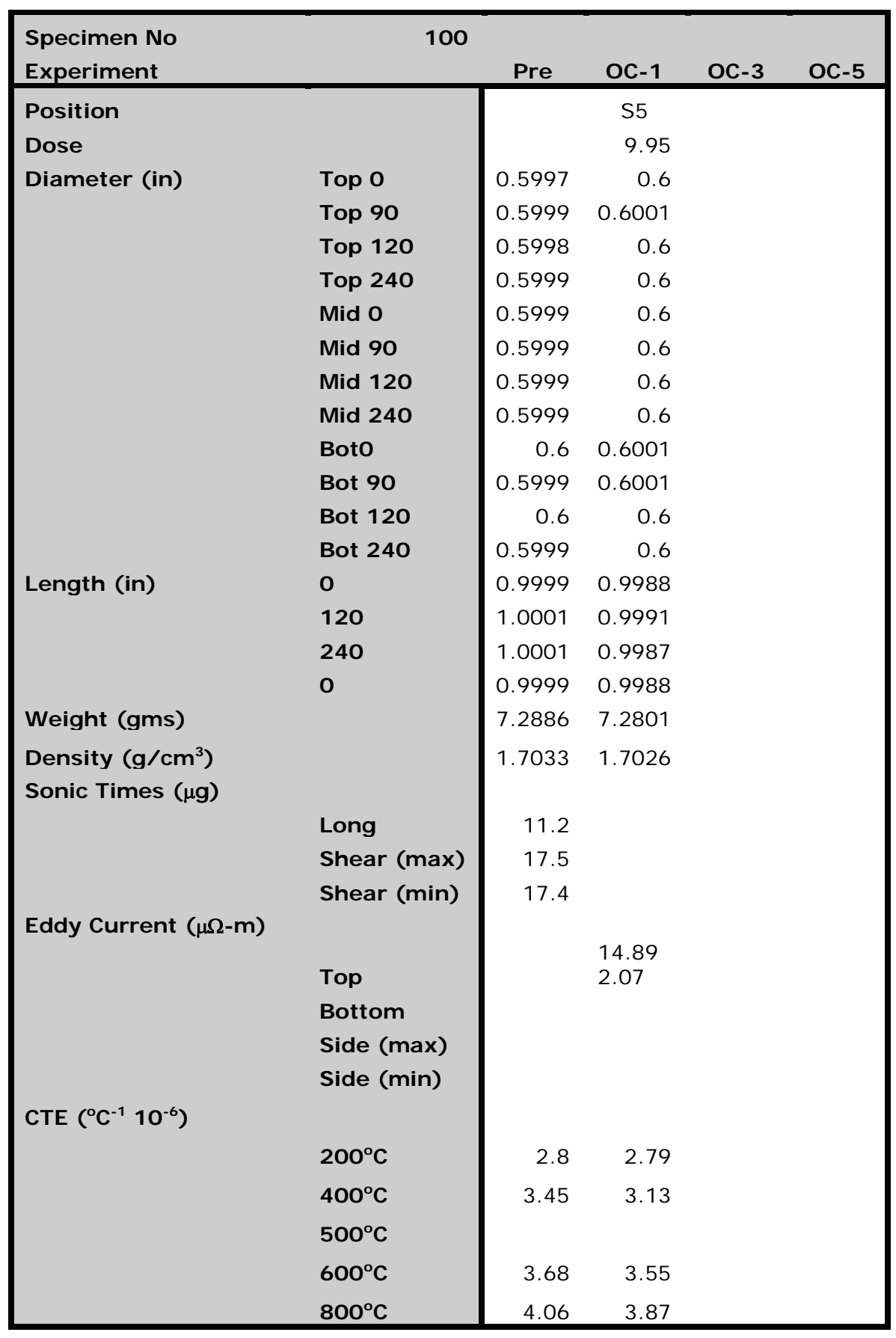




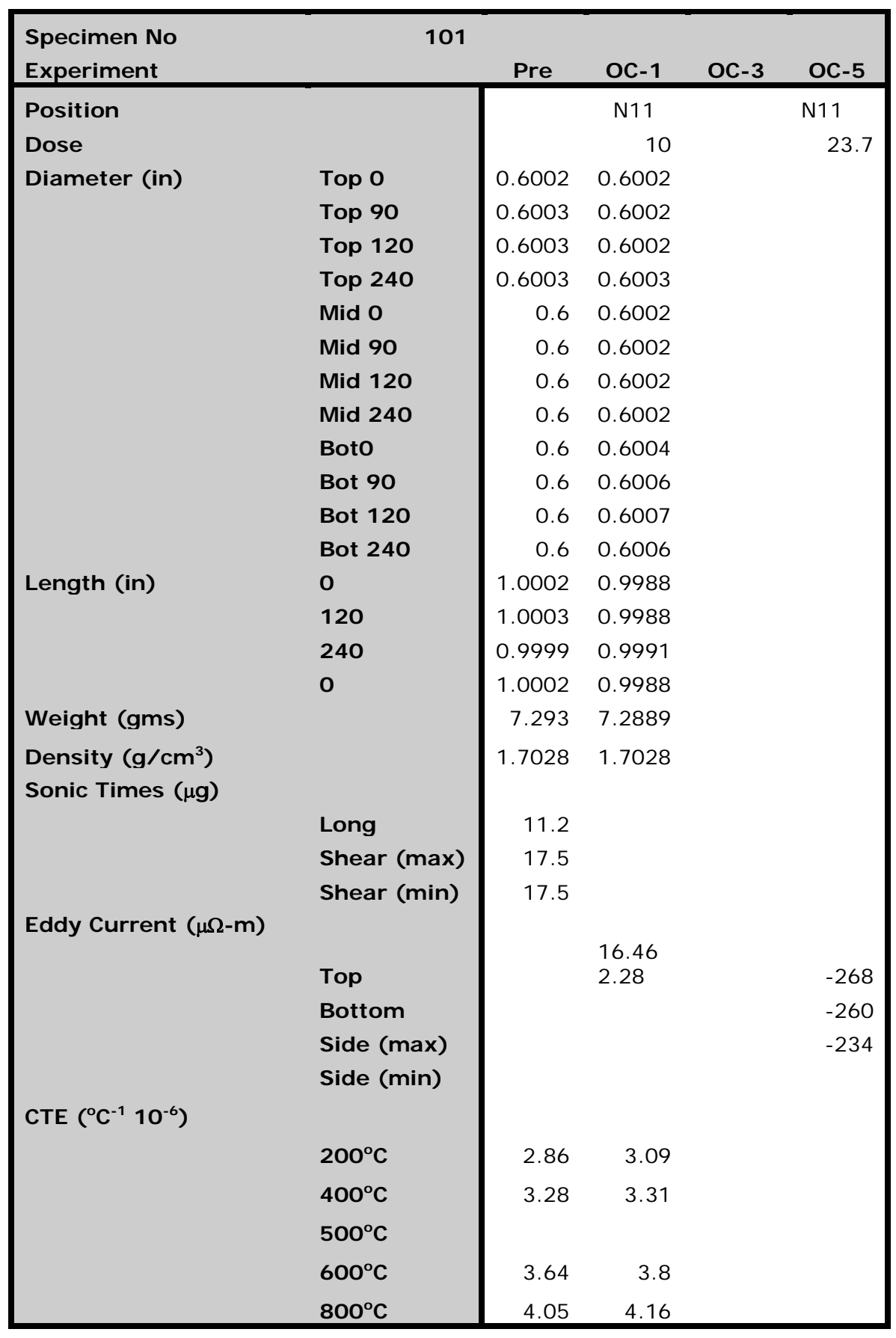




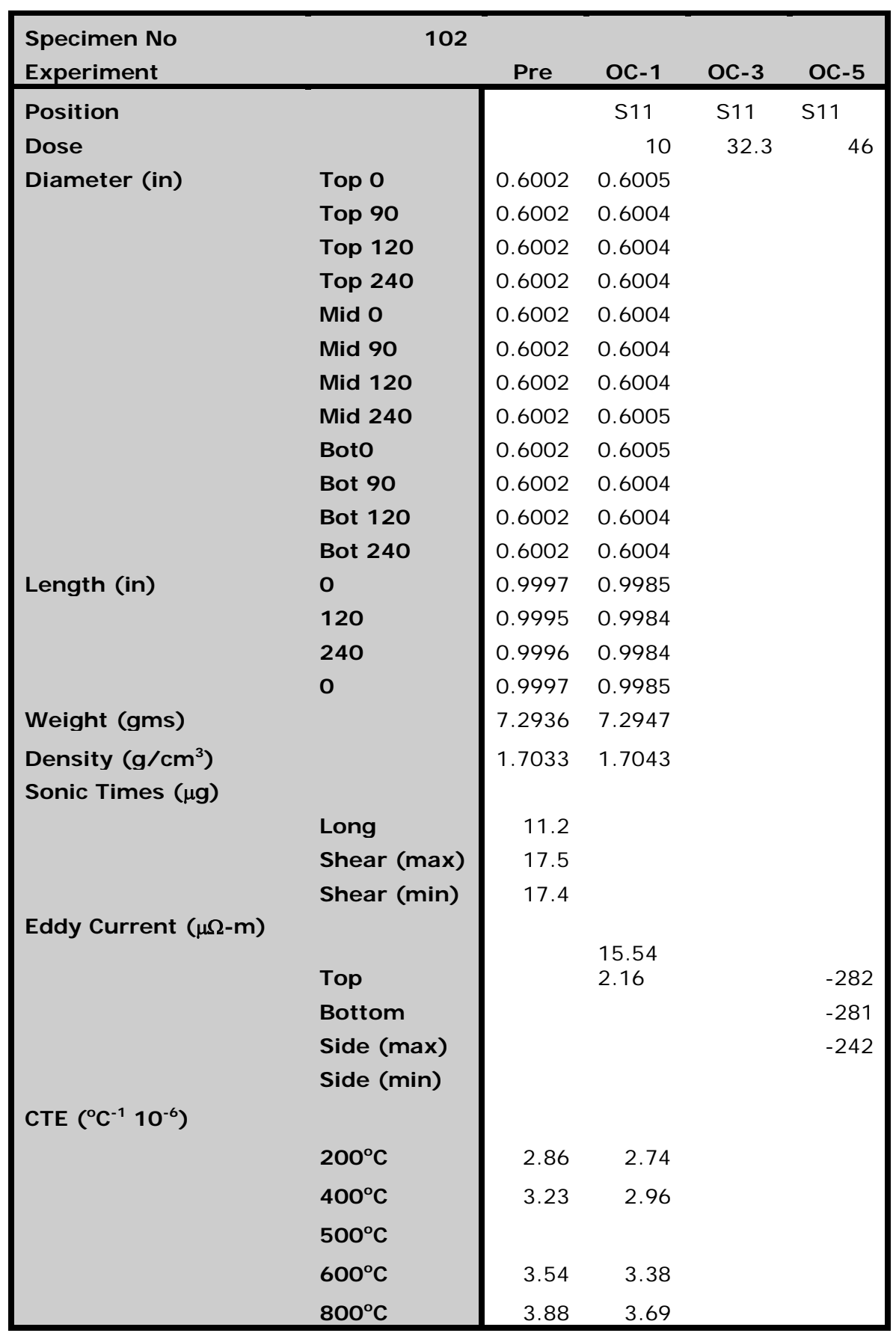




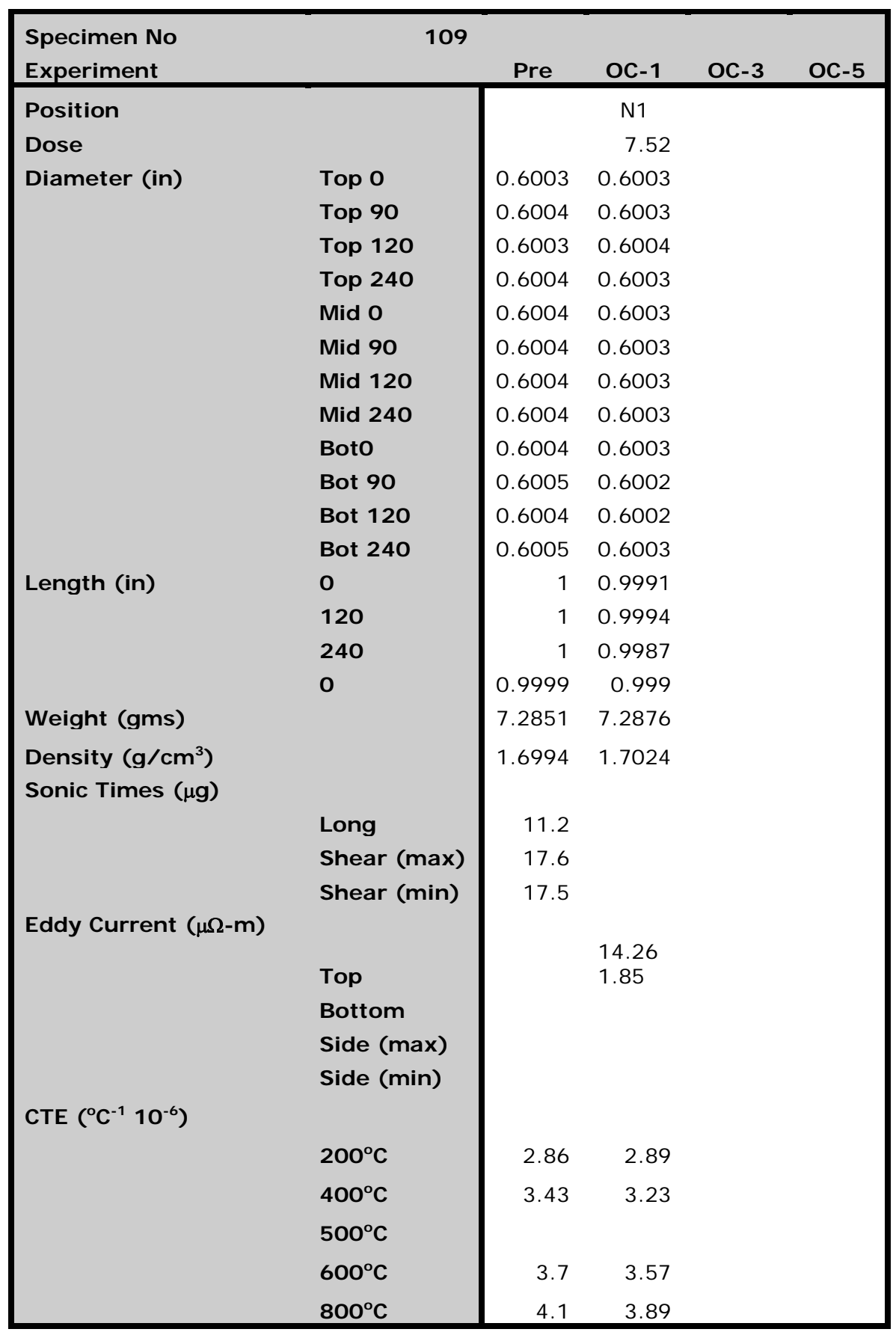




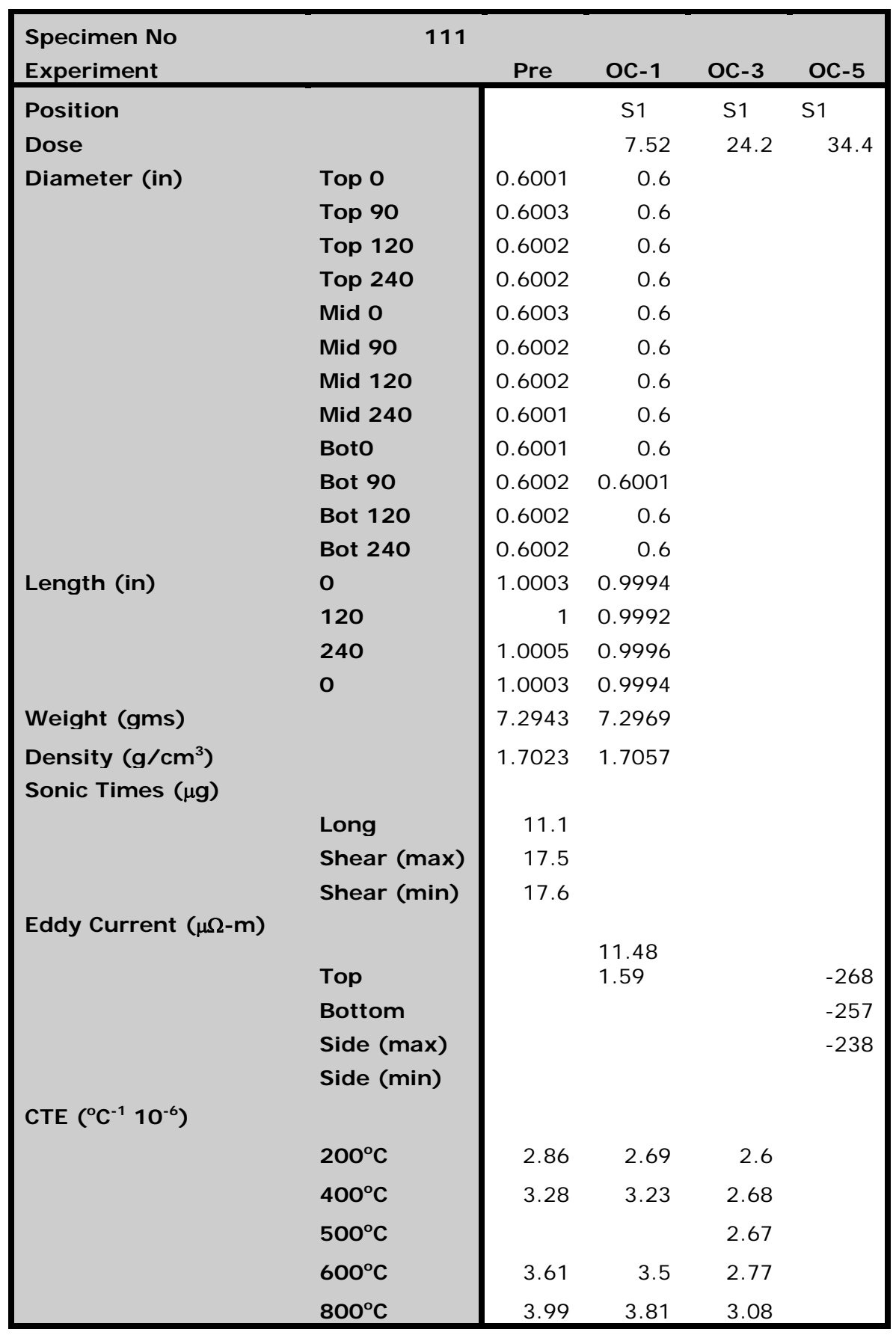




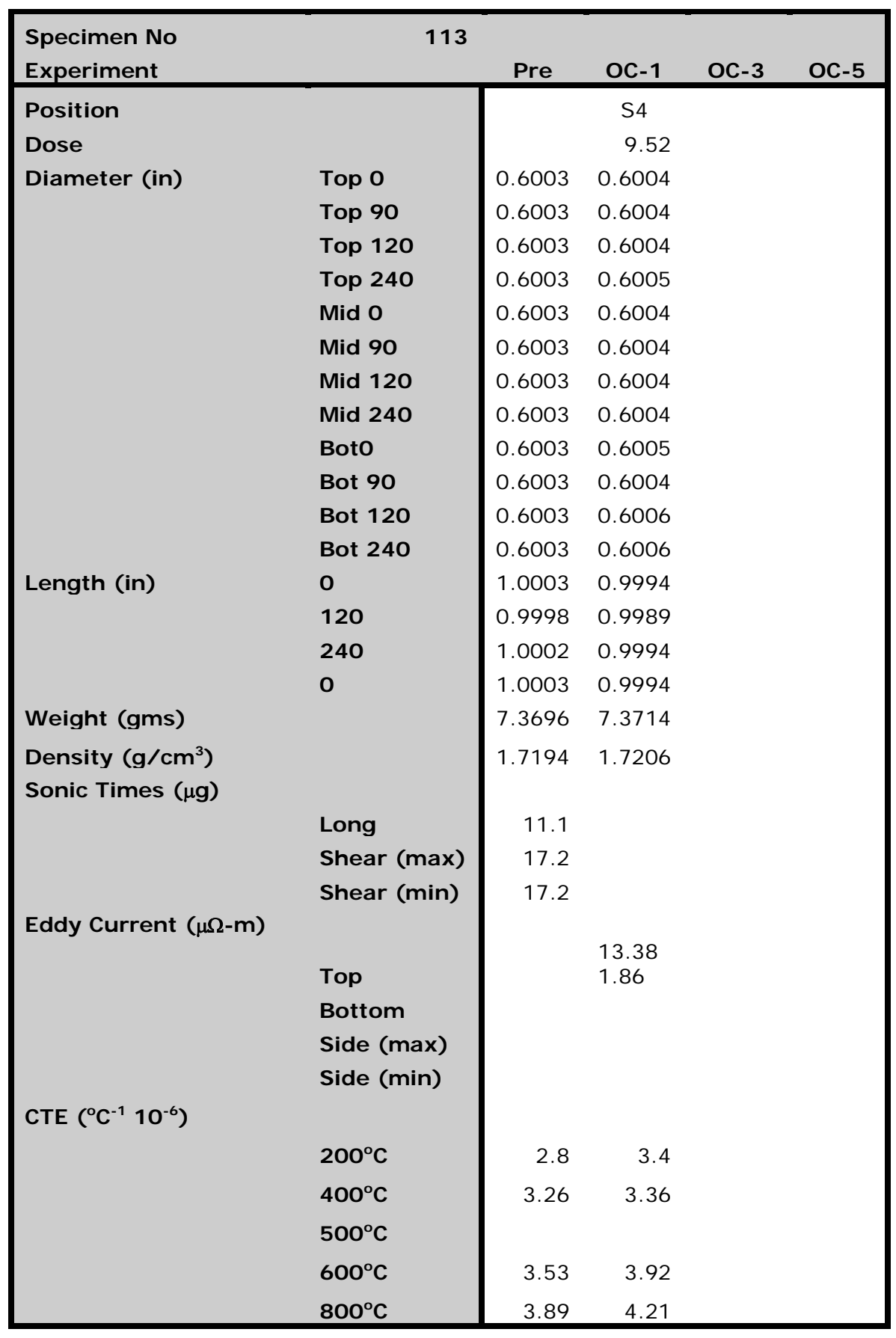




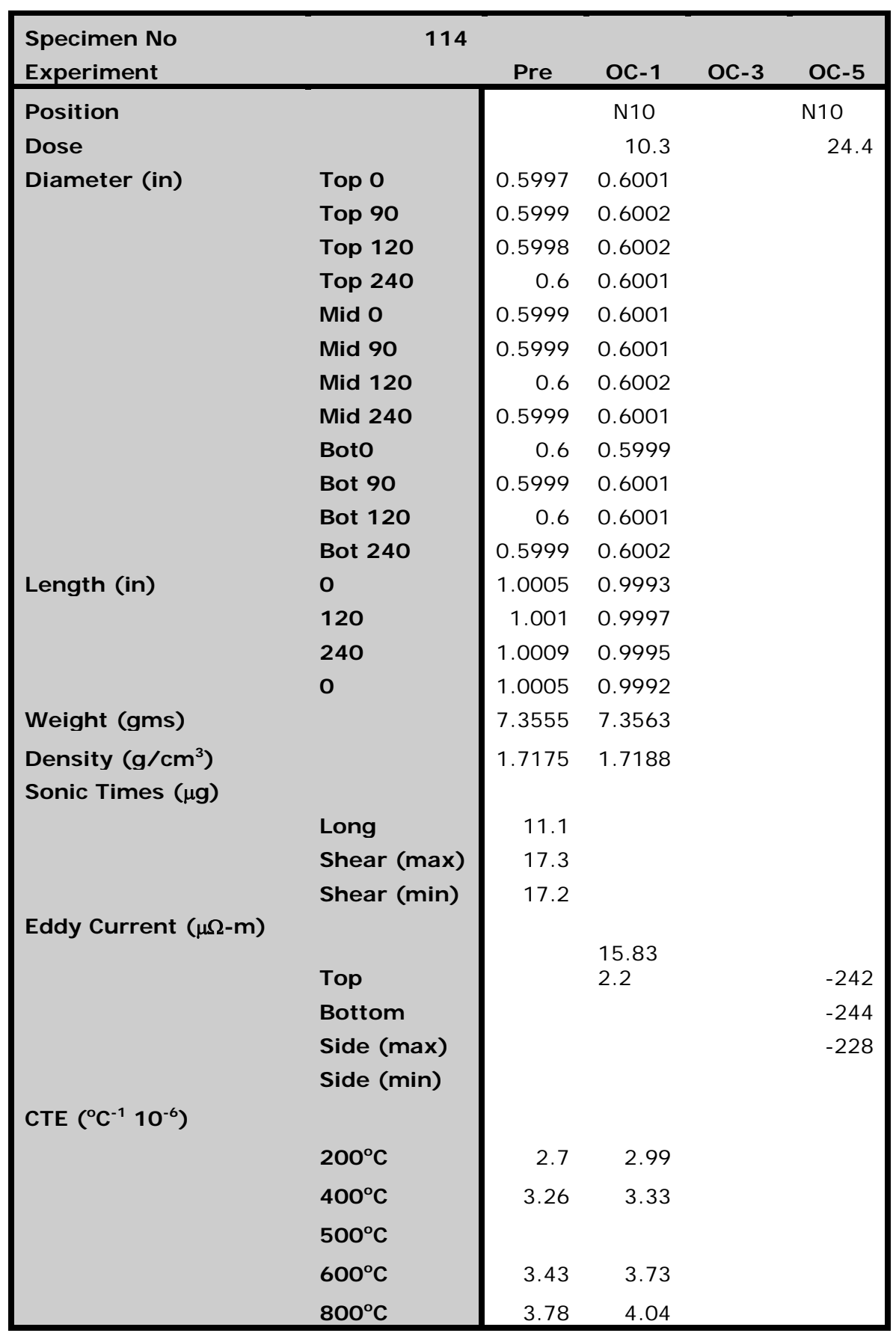




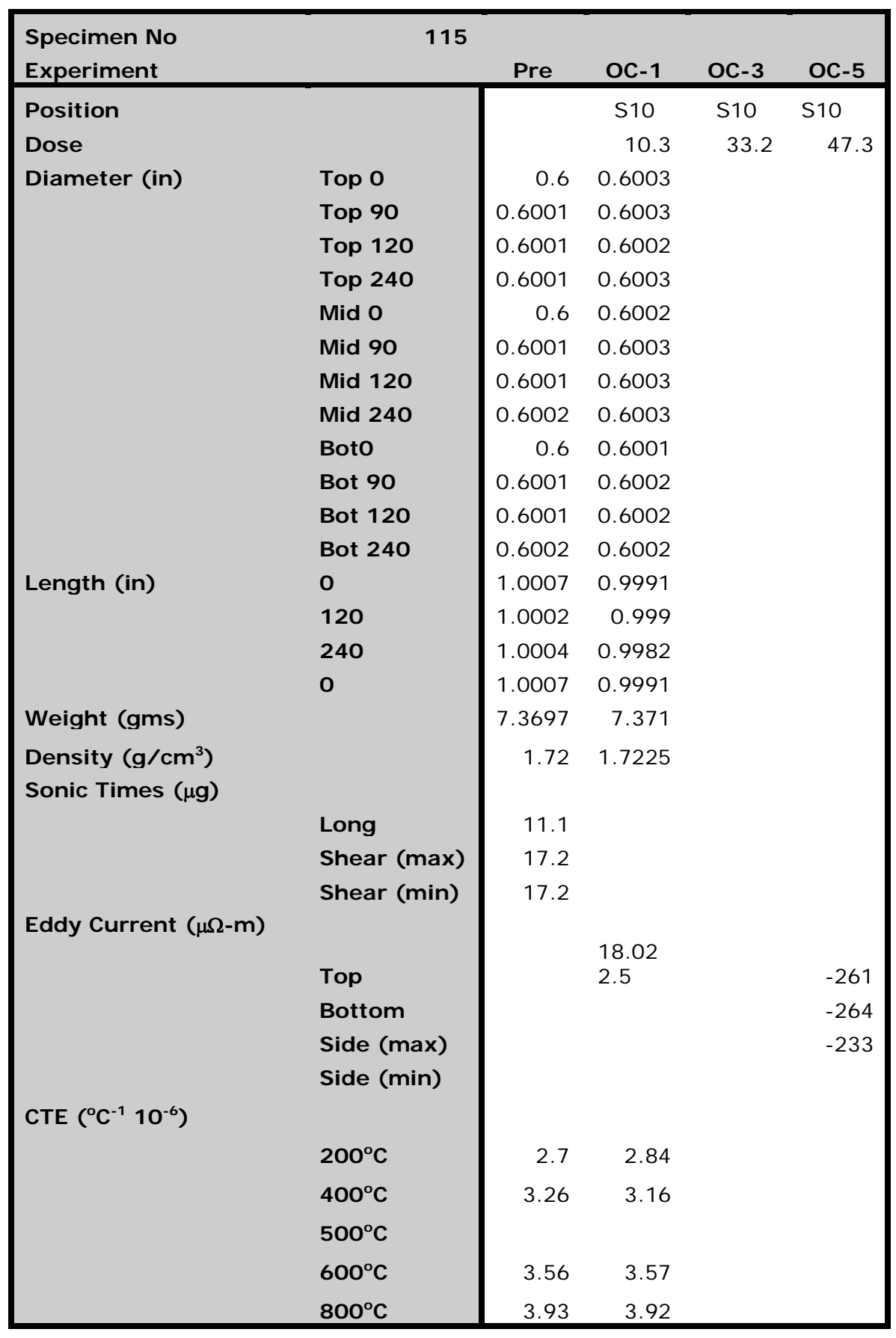




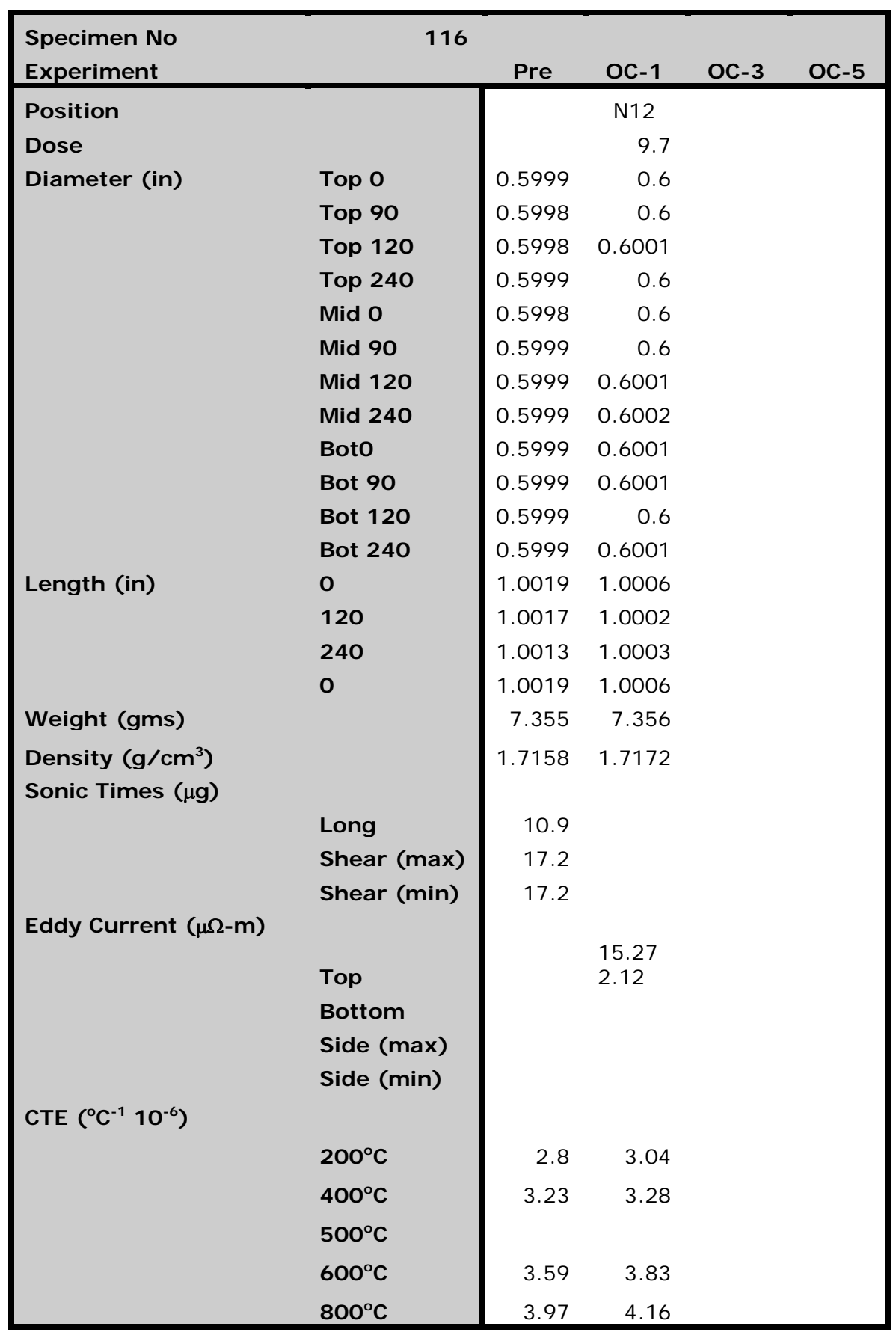




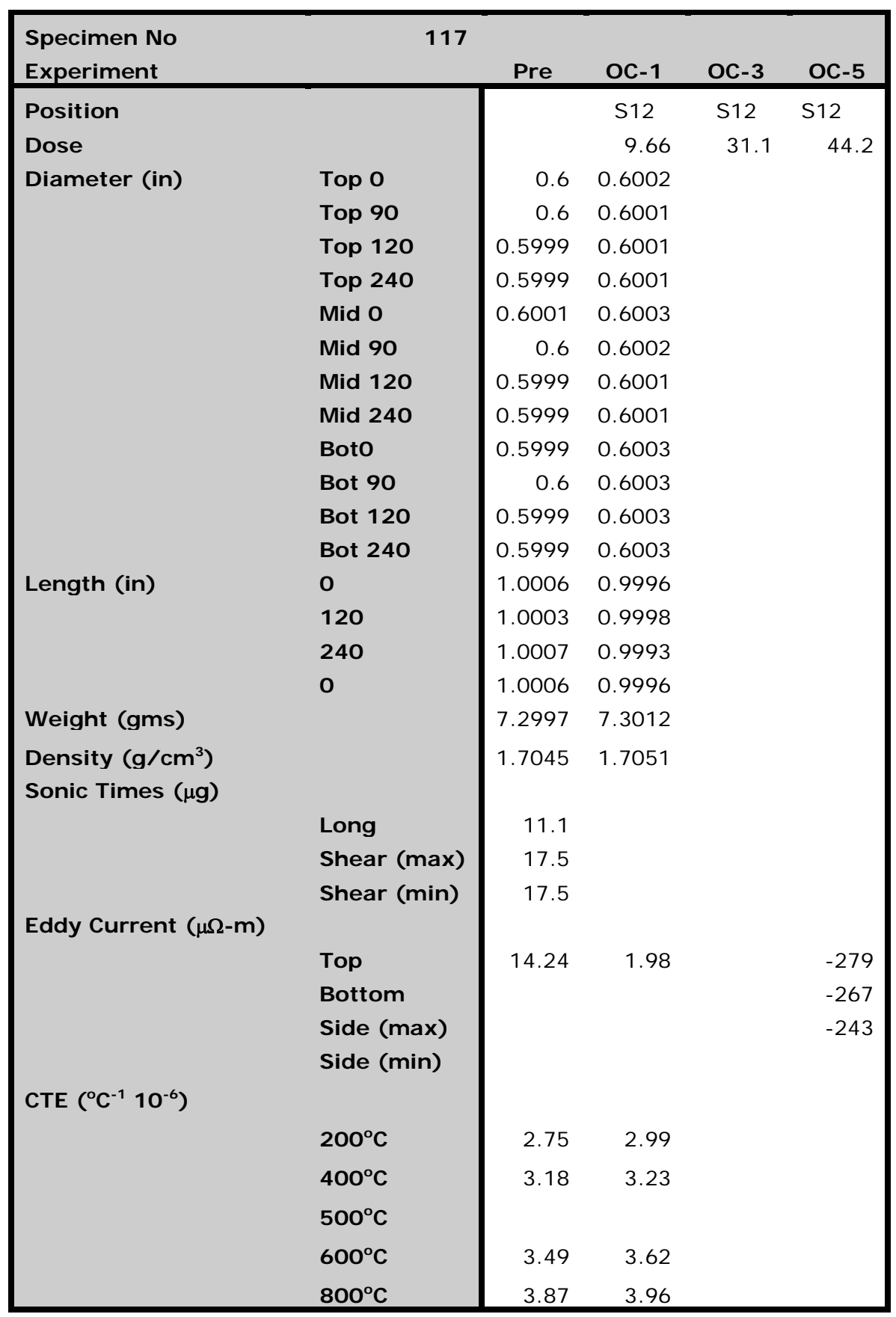




\section{Appendix B Dilatometer Archive Data}

\section{OC3 Dilatometer Data}

\begin{tabular}{|c|c|c|c|c|c|c|c|c|c|c|c|c|c|c|}
\hline Capsule & OC3 & $\begin{array}{l}\text { Specimen } \\
\text { Length }\end{array}$ & $\begin{array}{c}3 \\
0.9784\end{array}$ & Scale & 1" & $1.00 \mathrm{E}-03$ & & & & & & & & \\
\hline $\begin{array}{l}\text { Temperature } \\
\left({ }^{\circ} \mathrm{C}\right)\end{array}$ & Up & Down & $\begin{array}{l}\text { Delta } \\
\text { Down }\end{array}$ & $\begin{array}{l}\text { Down } \\
\text { length }\end{array}$ & CTE & CTEK & $\begin{array}{l}\text { Delta } \\
\text { CTEK }\end{array}$ & $\begin{array}{c}\text { CTE } \\
\text { adjusted }\end{array}$ & $\begin{array}{l}\text { Delta } \\
\text { Up }\end{array}$ & $\begin{array}{c}\text { Up } \\
\text { length }\end{array}$ & CTE & CTEK & $\begin{array}{l}\text { Delta } \\
\text { CTEK }\end{array}$ & $\begin{array}{c}\text { CTE } \\
\text { adjusted }\end{array}$ \\
\hline 0 & 0.98 & 1.20 & 0 & 0.9784 & & & & & 0 & 0.9784 & & & & \\
\hline 100 & 1.07 & 1.20 & 0 & 0.9784 & $0.00 \mathrm{E}+00$ & 5.50E-07 & 5.50E-07 & $5.00 \mathrm{E}-07$ & 0.09 & 0.9785 & $9.20 \mathrm{E}-07$ & $1.45 E-06$ & $5.30 \mathrm{E}-07$ & $1.42 \mathrm{E}-06$ \\
\hline 200 & 1.18 & 1.22 & 0.02 & 0.9784 & $1.02 \mathrm{E}-07$ & $6.50 E-07$ & $5.48 \mathrm{E}-07$ & $6.02 \mathrm{E}-07$ & 0.2 & 0.9786 & $1.02 \mathrm{E}-06$ & $1.55 E-06$ & $5.28 \mathrm{E}-07$ & $1.52 \mathrm{E}-06$ \\
\hline 300 & 1.27 & 1.23 & 0.03 & 0.9784 & $1.02 \mathrm{E}-07$ & $6.50 E-07$ & $5.48 \mathrm{E}-07$ & $6.02 \mathrm{E}-07$ & 0.29 & 0.9787 & $9.88 \mathrm{E}-07$ & $1.52 E-06$ & $5.28 \mathrm{E}-07$ & $1.49 \mathrm{E}-06$ \\
\hline 400 & 1.36 & 1.32 & 0.12 & 0.9785 & 3.07E-07 & $8.50 E-07$ & $5.43 \mathrm{E}-07$ & 8.07E-07 & 0.38 & 0.9788 & $9.71 \mathrm{E}-07$ & 1.50E-06 & $5.29 \mathrm{E}-07$ & 1.47E-06 \\
\hline 500 & 1.50 & 1.50 & 0.3 & 0.9787 & $6.13 \mathrm{E}-07$ & $1.15 E-06$ & 5.37E-07 & $1.11 \mathrm{E}-06$ & 0.52 & 0.9789 & $1.06 \mathrm{E}-06$ & $1.59 E-06$ & $5.27 \mathrm{E}-07$ & $1.56 \mathrm{E}-06$ \\
\hline 600 & 1.64 & 1.68 & 0.48 & 0.9789 & $8.18 \mathrm{E}-07$ & $1.35 E-06$ & $5.32 \mathrm{E}-07$ & $1.32 \mathrm{E}-06$ & 0.66 & 0.9791 & $1.12 \mathrm{E}-06$ & $1.65 E-06$ & $5.26 \mathrm{E}-07$ & $1.62 \mathrm{E}-06$ \\
\hline 700 & 1.80 & 1.89 & 0.69 & 0.9791 & $1.01 \mathrm{E}-06$ & $1.54 E-06$ & $5.28 \mathrm{E}-07$ & $1.51 \mathrm{E}-06$ & 0.82 & 0.9792 & $1.20 \mathrm{E}-06$ & $1.72 E-06$ & $5.24 \mathrm{E}-07$ & 1.70E-06 \\
\hline 800 & 2.08 & 2.11 & 0.91 & 0.9793 & 1.16E-06 & 1.69E-06 & $5.24 \mathrm{E}-07$ & $1.66 \mathrm{E}-06$ & 1.1 & 0.9795 & $1.41 \mathrm{E}-06$ & $1.93 E-06$ & $5.20 \mathrm{E}-07$ & $1.91 \mathrm{E}-06$ \\
\hline
\end{tabular}

\begin{tabular}{|c|c|c|c|c|c|c|c|c|c|c|c|c|c|c|}
\hline Capsule & OC3 & $\begin{array}{c}\text { Specimen } \\
\text { Length }\end{array}$ & $\begin{array}{c}3 \mathbf{G} \\
0.9899\end{array}$ & Scale & $1^{\prime \prime}$ & $1.00 \mathrm{E}-03$ & & & & & & & & \\
\hline $\begin{array}{c}\text { Temperature } \\
\left({ }^{\circ} \mathrm{C}\right)\end{array}$ & Up & Down & $\begin{array}{l}\text { Delta } \\
\text { Down }\end{array}$ & $\begin{array}{l}\text { Down } \\
\text { length }\end{array}$ & CTE & CTEK & $\begin{array}{l}\text { Delta } \\
\text { CTEK }\end{array}$ & $\begin{array}{c}\text { CTE } \\
\text { adjusted }\end{array}$ & $\begin{array}{c}\text { Delta } \\
\text { Up }\end{array}$ & $\begin{array}{c}\text { Up } \\
\text { length }\end{array}$ & CTE & CTEK & $\begin{array}{l}\text { Delta } \\
\text { CTEK }\end{array}$ & $\begin{array}{c}\text { CTE } \\
\text { adjusted }\end{array}$ \\
\hline 0 & 0.90 & -0.60 & 0 & 0.9899 & & & & & 0 & 0.9899 & & & & \\
\hline 100 & 1.05 & 0.79 & 1.39 & 0.9913 & $1.40 \mathrm{E}-05$ & $2.45 E-06$ & $-1.16 \mathrm{E}-05$ & $-1.16 \mathrm{E}-05$ & 0.15 & 0.9901 & $1.52 \mathrm{E}-06$ & $2.05 E-06$ & $5.35 \mathrm{E}-07$ & $2.02 \mathrm{E}-06$ \\
\hline 200 & 1.20 & 1.00 & 1.6 & 0.9915 & $8.08 \mathrm{E}-06$ & $2.55 E-06$ & $-5.53 E-06$ & $-5.53 E-06$ & 0.3 & 0.9902 & $1.52 \mathrm{E}-06$ & $2.05 E-06$ & $5.35 \mathrm{E}-07$ & $2.02 \mathrm{E}-06$ \\
\hline 300 & 1.32 & 1.20 & 1.8 & 0.9917 & $6.06 \mathrm{E}-06$ & $2.55 E-06$ & $-3.51 E-06$ & $-3.51 \mathrm{E}-06$ & 0.42 & 0.9903 & $1.41 \mathrm{E}-06$ & $1.95 E-06$ & $5.36 \mathrm{E}-07$ & $1.91 \mathrm{E}-06$ \\
\hline 400 & 1.53 & 1.45 & 2.05 & 0.9920 & $5.18 \mathrm{E}-06$ & $2.68 E-06$ & $-2.50 \mathrm{E}-06$ & $-2.50 \mathrm{E}-06$ & 0.63 & 0.9905 & 1.59E-06 & 2.13E-06 & $5.34 \mathrm{E}-07$ & 2.09E-06 \\
\hline 500 & 1.78 & 1.70 & 2.3 & 0.9922 & $4.65 \mathrm{E}-06$ & $2.75 E-06$ & $-1.90 \mathrm{E}-06$ & $-1.90 \mathrm{E}-06$ & 0.88 & 0.9908 & $1.78 \mathrm{E}-06$ & 2.31E-06 & $5.32 \mathrm{E}-07$ & $2.28 \mathrm{E}-06$ \\
\hline 600 & 2.04 & 1.96 & 2.56 & 0.9925 & 4.31E-06 & $2.82 E-06$ & $-1.49 \mathrm{E}-06$ & $-1.49 \mathrm{E}-06$ & 1.14 & 0.9910 & $1.92 \mathrm{E}-06$ & $2.45 E-06$ & $5.31 \mathrm{E}-07$ & $2.42 \mathrm{E}-06$ \\
\hline 700 & 2.32 & 2.25 & 2.85 & 0.9928 & 4.11E-06 & $2.91 E-06$ & $-1.21 \mathrm{E}-06$ & $-1.21 \mathrm{E}-06$ & 1.42 & 0.9913 & 2.05E-06 & $2.58 E-06$ & $5.29 \mathrm{E}-07$ & $2.55 \mathrm{E}-06$ \\
\hline 800 & 2.63 & 2.54 & 3.14 & 0.9930 & $3.97 \mathrm{E}-06$ & $2.98 E-06$ & $-9.90 \mathrm{E}-07$ & $-9.90 \mathrm{E}-07$ & 1.73 & 0.9916 & $2.18 \mathrm{E}-06$ & $2.71 E-06$ & $5.27 \mathrm{E}-07$ & $2.68 \mathrm{E}-06$ \\
\hline
\end{tabular}




\begin{tabular}{|c|c|c|c|c|c|c|c|c|c|c|c|c|c|c|}
\hline Capsule & OC3 & $\begin{array}{l}\text { Specimen } \\
\text { Length }\end{array}$ & $\begin{array}{c}\text { 4G } \\
0.9934\end{array}$ & Scale & 1" & $1.00 \mathrm{E}-03$ & & & & & & & & \\
\hline $\begin{array}{c}\text { Temperature } \\
\left({ }^{\circ} \mathrm{C}\right)\end{array}$ & Up & Down & $\begin{array}{l}\text { Delta } \\
\text { Down }\end{array}$ & $\begin{array}{l}\text { Down } \\
\text { length }\end{array}$ & CTE & CTEK & $\begin{array}{l}\text { Delta } \\
\text { CTEK }\end{array}$ & $\begin{array}{c}\text { CTE } \\
\text { adjusted }\end{array}$ & $\begin{array}{l}\text { Delta } \\
\text { Up }\end{array}$ & $\begin{array}{c}\text { Up } \\
\text { length }\end{array}$ & CTE & CTEK & $\begin{array}{l}\text { Delta } \\
\text { CTEK }\end{array}$ & $\begin{array}{c}\text { CTE } \\
\text { adjusted }\end{array}$ \\
\hline 0 & 1.09 & 0.90 & 0 & 0.9934 & & & & & 0 & 0.9934 & & & & \\
\hline 100 & 1.15 & 0.99 & 0.09 & 0.9935 & $9.06 \mathrm{E}-07$ & $1.45 E-06$ & $5.44 \mathrm{E}-07$ & $1.41 \mathrm{E}-06$ & 0.06 & 0.9935 & $6.04 \mathrm{E}-07$ & $1.15 E-06$ & $5.46 \mathrm{E}-07$ & 1.10E-06 \\
\hline 200 & 1.22 & 1.06 & 0.16 & 0.9936 & $8.05 E-07$ & $1.35 E-06$ & $5.45 \mathrm{E}-07$ & $1.31 \mathrm{E}-06$ & 0.13 & 0.9935 & $6.54 \mathrm{E}-07$ & $1.20 E-06$ & $5.46 \mathrm{E}-07$ & $1.15 \mathrm{E}-06$ \\
\hline 300 & 1.29 & 1.16 & 0.26 & 0.9937 & 8.72E-07 & $1.42 E-06$ & $5.44 \mathrm{E}-07$ & $1.37 \mathrm{E}-06$ & 0.2 & 0.9936 & $6.71 \mathrm{E}-07$ & $1.22 E-06$ & $5.45 \mathrm{E}-07$ & 1.17E-06 \\
\hline 400 & 1.38 & 1.27 & 0.37 & 0.9938 & $9.31 \mathrm{E}-07$ & $1.48 E-06$ & $5.44 \mathrm{E}-07$ & $1.43 \mathrm{E}-06$ & 0.29 & 0.9937 & $7.30 \mathrm{E}-07$ & $1.28 E-06$ & $5.45 \mathrm{E}-07$ & $1.23 \mathrm{E}-06$ \\
\hline 500 & 1.50 & 1.40 & 0.5 & 0.9939 & $1.01 \mathrm{E}-06$ & $1.55 E-06$ & $5.43 \mathrm{E}-07$ & $1.51 \mathrm{E}-06$ & 0.41 & 0.9938 & $8.25 \mathrm{E}-07$ & 1.37E-06 & $5.45 \mathrm{E}-07$ & $1.33 \mathrm{E}-06$ \\
\hline 600 & 1.62 & 1.53 & 0.63 & 0.9940 & $1.06 \mathrm{E}-06$ & $1.60 E-06$ & $5.43 \mathrm{E}-07$ & $1.56 \mathrm{E}-06$ & 0.53 & 0.9939 & 8.89E-07 & $1.43 E-06$ & $5.44 \mathrm{E}-07$ & $1.39 \mathrm{E}-06$ \\
\hline 700 & 1.78 & 1.72 & 0.82 & 0.9942 & $1.18 \mathrm{E}-06$ & $1.72 E-06$ & $5.42 \mathrm{E}-07$ & $1.68 \mathrm{E}-06$ & 0.69 & 0.9941 & $9.92 \mathrm{E}-07$ & $1.54 E-06$ & $5.43 \mathrm{E}-07$ & $1.49 \mathrm{E}-06$ \\
\hline 800 & 2.00 & 1.94 & 1.04 & 0.9944 & $1.31 \mathrm{E}-06$ & $1.85 E-06$ & $5.41 \mathrm{E}-07$ & $1.81 \mathrm{E}-06$ & 0.91 & 0.9943 & $1.15 \mathrm{E}-06$ & $1.69 E-06$ & $5.42 \mathrm{E}-07$ & $1.65 \mathrm{E}-06$ \\
\hline
\end{tabular}

\begin{tabular}{|c|c|c|c|c|c|c|c|c|c|c|c|c|c|c|}
\hline Capsule & OC3 & $\begin{array}{l}\text { Specimen } \\
\text { Length }\end{array}$ & $\begin{array}{c}5 \\
0.9868\end{array}$ & Scale & 1" & $1.00 \mathrm{E}-03$ & & & & & & & & \\
\hline $\begin{array}{c}\text { Temperature } \\
\left({ }^{\circ} \mathrm{C}\right)\end{array}$ & Up & Down & $\begin{array}{l}\text { Delta } \\
\text { Down }\end{array}$ & $\begin{array}{l}\text { Down } \\
\text { length }\end{array}$ & CTE & CTEK & $\begin{array}{l}\text { Delta } \\
\text { CTEK }\end{array}$ & $\begin{array}{c}\text { CTE } \\
\text { adjusted }\end{array}$ & $\begin{array}{l}\text { Delta } \\
\text { Up }\end{array}$ & $\begin{array}{c}\text { Up } \\
\text { length }\end{array}$ & CTE & CTEK & $\begin{array}{l}\text { Delta } \\
\text { CTEK }\end{array}$ & $\begin{array}{c}\text { CTE } \\
\text { adjusted }\end{array}$ \\
\hline 0 & 0.90 & 1.02 & 0 & 0.9868 & & & & & 0 & 0.9868 & & & & \\
\hline 100 & 1.00 & 1.09 & 0.07 & 0.9869 & $7.09 \mathrm{E}-07$ & $1.25 E-06$ & $5.41 \mathrm{E}-07$ & $1.21 \mathrm{E}-06$ & 0.1 & 0.9869 & $1.01 \mathrm{E}-06$ & $1.55 E-06$ & 5.37E-07 & $1.51 \mathrm{E}-06$ \\
\hline 200 & 1.14 & 1.20 & 0.18 & 0.9870 & $9.12 \mathrm{E}-07$ & $1.45 E-06$ & $5.38 \mathrm{E}-07$ & $1.41 \mathrm{E}-06$ & 0.24 & 0.9870 & $1.22 \mathrm{E}-06$ & $1.75 E-06$ & $5.34 \mathrm{E}-07$ & $1.72 \mathrm{E}-06$ \\
\hline 300 & 1.30 & 1.33 & 0.31 & 0.9871 & $1.05 \mathrm{E}-06$ & $1.58 E-06$ & 5.36E-07 & $1.55 \mathrm{E}-06$ & 0.4 & 0.9872 & $1.35 \mathrm{E}-06$ & $1.88 E-06$ & $5.32 \mathrm{E}-07$ & $1.85 \mathrm{E}-06$ \\
\hline 400 & 1.45 & 1.51 & 0.49 & 0.9873 & $1.24 \mathrm{E}-06$ & $1.78 E-06$ & 5.34E-07 & $1.74 \mathrm{E}-06$ & 0.55 & 0.9874 & $1.39 \mathrm{E}-06$ & $1.93 E-06$ & $5.32 \mathrm{E}-07$ & $1.89 \mathrm{E}-06$ \\
\hline 500 & 1.61 & 1.73 & 0.71 & 0.9875 & $1.44 \mathrm{E}-06$ & $1.97 E-06$ & 5.31E-07 & $1.94 \mathrm{E}-06$ & 0.71 & 0.9875 & $1.44 \mathrm{E}-06$ & 1.97E-06 & $5.31 \mathrm{E}-07$ & $1.94 \mathrm{E}-06$ \\
\hline 600 & 1.84 & 1.98 & 0.96 & 0.9878 & $1.62 \mathrm{E}-06$ & $2.15 E-06$ & 5.29E-07 & $2.12 \mathrm{E}-06$ & 0.94 & 0.9877 & $1.59 \mathrm{E}-06$ & $2.12 E-06$ & $5.28 \mathrm{E}-07$ & $2.09 \mathrm{E}-06$ \\
\hline 700 & 2.17 & 2.22 & 1.2 & 0.9880 & $1.74 \mathrm{E}-06$ & $2.26 E-06$ & $5.27 \mathrm{E}-07$ & $2.24 \mathrm{E}-06$ & 1.27 & 0.9881 & $1.84 \mathrm{E}-06$ & $2.36 E-06$ & $5.25 \mathrm{E}-07$ & $2.34 \mathrm{E}-06$ \\
\hline 800 & 2.52 & 2.57 & 1.55 & 0.9884 & $1.96 \mathrm{E}-06$ & 2.49E-06 & $5.24 \mathrm{E}-07$ & $2.46 \mathrm{E}-06$ & 1.62 & 0.9884 & $2.05 \mathrm{E}-06$ & $2.58 E-06$ & $5.23 \mathrm{E}-07$ & $2.55 \mathrm{E}-06$ \\
\hline
\end{tabular}




\begin{tabular}{|c|c|c|c|c|c|c|c|c|c|c|c|c|c|c|}
\hline Capsule & OC3 & $\begin{array}{l}\text { Specimen } \\
\text { Length }\end{array}$ & $\begin{array}{c}7 \\
0.9801\end{array}$ & Scale & 1" & $1.00 \mathrm{E}-03$ & & & & & & & & \\
\hline $\begin{array}{c}\text { Temperature } \\
\left({ }^{\circ} \mathrm{C}\right)\end{array}$ & Up & Down & $\begin{array}{l}\text { Delta } \\
\text { Down }\end{array}$ & $\begin{array}{l}\text { Down } \\
\text { length }\end{array}$ & CTE & CTEK & $\begin{array}{l}\text { Delta } \\
\text { CTEK }\end{array}$ & $\begin{array}{c}\text { CTE } \\
\text { adjusted }\end{array}$ & $\begin{array}{l}\text { Delta } \\
\text { Up }\end{array}$ & $\begin{array}{c}\text { Up } \\
\text { length }\end{array}$ & CTE & CTEK & $\begin{array}{l}\text { Delta } \\
\text { CTEK }\end{array}$ & $\begin{array}{c}\text { CTE } \\
\text { adjusted }\end{array}$ \\
\hline 0 & 1.00 & 1.35 & 0 & 0.9801 & & & & & 0 & 0.9801 & & & & \\
\hline 100 & 1.30 & 1.57 & 0.22 & 0.9803 & $2.24 \mathrm{E}-06$ & $2.45 E-06$ & $2.75 \mathrm{E}-06$ & $2.74 \mathrm{E}-06$ & 0.3 & 0.9804 & $3.06 \mathrm{E}-06$ & $3.55 E-06$ & 4.89E-07 & 3.56E-06 \\
\hline 200 & 1.60 & 1.78 & 0.43 & 0.9805 & $2.19 \mathrm{E}-06$ & $2.55 E-06$ & $2.70 \mathrm{E}-06$ & $2.69 \mathrm{E}-06$ & 0.6 & 0.9807 & $3.06 \mathrm{E}-06$ & $3.55 E-06$ & 4.89E-07 & $3.56 \mathrm{E}-06$ \\
\hline 300 & 1.95 & 2.01 & 0.66 & 0.9808 & $2.24 \mathrm{E}-06$ & $2.55 E-06$ & $2.75 \mathrm{E}-06$ & $2.74 \mathrm{E}-06$ & 0.95 & 0.9811 & $3.23 \mathrm{E}-06$ & $3.72 E-06$ & 4.85E-07 & $3.73 \mathrm{E}-06$ \\
\hline 400 & 2.30 & 2.30 & 0.95 & 0.9811 & $2.42 \mathrm{E}-06$ & $2.68 E-06$ & $2.93 \mathrm{E}-06$ & $2.92 \mathrm{E}-06$ & 1.3 & 0.9814 & $3.32 \mathrm{E}-06$ & $3.80 E-06$ & $4.84 \mathrm{E}-07$ & $3.82 \mathrm{E}-06$ \\
\hline 500 & 2.64 & 2.68 & 1.33 & 0.9814 & $2.71 \mathrm{E}-06$ & $2.75 E-06$ & $3.21 \mathrm{E}-06$ & $3.21 \mathrm{E}-06$ & 1.64 & 0.9817 & $3.35 \mathrm{E}-06$ & $3.83 E-06$ & $4.83 \mathrm{E}-07$ & $3.85 \mathrm{E}-06$ \\
\hline 600 & 3.01 & 3.08 & 1.73 & 0.9818 & $2.94 \mathrm{E}-06$ & $2.82 E-06$ & $3.43 \mathrm{E}-06$ & $3.44 \mathrm{E}-06$ & 2.01 & 0.9821 & $3.42 \mathrm{E}-06$ & $3.90 E-06$ & $4.82 \mathrm{E}-07$ & $3.92 \mathrm{E}-06$ \\
\hline 700 & 3.54 & 3.53 & 2.18 & 0.9823 & $3.18 \mathrm{E}-06$ & $2.91 E-06$ & 3.66E-06 & $3.68 \mathrm{E}-06$ & 2.54 & 0.9826 & 3.70E-06 & 4.18E-06 & $4.76 \mathrm{E}-07$ & 4.20E-06 \\
\hline 800 & 4.01 & 4.07 & 2.72 & 0.9828 & $3.47 \mathrm{E}-06$ & $2.98 E-06$ & $3.95 \mathrm{E}-06$ & $3.97 \mathrm{E}-06$ & 3.01 & 0.9831 & $3.84 \mathrm{E}-06$ & $4.31 E-06$ & $4.73 \mathrm{E}-07$ & 4.34E-06 \\
\hline
\end{tabular}

\begin{tabular}{|c|c|c|c|c|c|c|c|c|c|c|c|c|c|c|}
\hline Capsule & OC3 & $\begin{array}{l}\text { Specimen } \\
\text { Length }\end{array}$ & $\begin{array}{c}8 \\
0.9705\end{array}$ & Scale & $1 "$ & $1.00 \mathrm{E}-03$ & & & & & & & & \\
\hline $\begin{array}{c}\text { Temperature } \\
\left({ }^{\circ} \mathrm{C}\right)\end{array}$ & Up & Down & $\begin{array}{l}\text { Delta } \\
\text { Down }\end{array}$ & $\begin{array}{l}\text { Down } \\
\text { length }\end{array}$ & CTE & CTEK & $\begin{array}{l}\text { Delta } \\
\text { CTEK }\end{array}$ & $\begin{array}{c}\text { CTE } \\
\text { adjusted } \\
\end{array}$ & $\begin{array}{l}\text { Delta } \\
\text { Up }\end{array}$ & $\begin{array}{c}\text { Up } \\
\text { length }\end{array}$ & CTE & CTEK & $\begin{array}{l}\text { Delta } \\
\text { CTEK }\end{array}$ & $\begin{array}{c}\text { CTE } \\
\text { adjusted }\end{array}$ \\
\hline 0 & 0.79 & 0.59 & 0 & 0.9705 & & & & & 0 & 0.9705 & & & & \\
\hline 100 & 1.04 & 0.86 & 0.27 & 0.9708 & $2.78 \mathrm{E}-06$ & $4.45 E-06$ & 1.67E-06 & $3.28 \mathrm{E}-06$ & 0.25 & 0.9708 & $2.58 \mathrm{E}-06$ & $4.55 E-06$ & $1.97 \mathrm{E}-06$ & $3.08 \mathrm{E}-06$ \\
\hline 200 & 1.33 & 1.19 & 0.6 & 0.9711 & $3.09 \mathrm{E}-06$ & $4.60 E-06$ & $1.51 \mathrm{E}-06$ & 3.59E-06 & 0.54 & 0.9710 & $2.78 \mathrm{E}-06$ & $4.65 E-06$ & 1.87E-06 & $3.28 \mathrm{E}-06$ \\
\hline 300 & 1.64 & 1.51 & 0.92 & 0.9714 & $3.16 \mathrm{E}-06$ & $4.65 E-06$ & 1.49E-06 & $3.66 \mathrm{E}-06$ & 0.85 & 0.9714 & $2.92 \mathrm{E}-06$ & $4.72 E-06$ & $1.80 \mathrm{E}-06$ & $3.42 \mathrm{E}-06$ \\
\hline 400 & 1.98 & 1.90 & 1.31 & 0.9718 & $3.37 \mathrm{E}-06$ & 4.73E-06 & 1.35E-06 & 3.87E-06 & 1.19 & 0.9717 & $3.07 \mathrm{E}-06$ & $4.78 E-06$ & $1.71 \mathrm{E}-06$ & 3.57E-06 \\
\hline 500 & 2.40 & 2.31 & 1.72 & 0.9722 & $3.54 \mathrm{E}-06$ & $4.85 E-06$ & $1.31 \mathrm{E}-06$ & 4.04E-06 & 1.61 & 0.9721 & $3.32 \mathrm{E}-06$ & 4.89E-06 & 1.57E-06 & $3.82 \mathrm{E}-06$ \\
\hline 600 & 2.89 & 2.81 & 2.22 & 0.9727 & $3.81 \mathrm{E}-06$ & $4.98 E-06$ & 1.17E-06 & 4.31E-06 & 2.1 & 0.9726 & $3.61 \mathrm{E}-06$ & $5.00 E-06$ & 1.39E-06 & 4.11E-06 \\
\hline 700 & 3.40 & 3.40 & 2.81 & 0.9733 & 4.14E-06 & $5.15 E-06$ & $1.01 \mathrm{E}-06$ & 4.64E-06 & 2.61 & 0.9731 & $3.84 \mathrm{E}-06$ & $5.16 E-06$ & $1.32 \mathrm{E}-06$ & 4.34E-06 \\
\hline 800 & 4.02 & 4.07 & 3.48 & 0.9740 & $4.48 \mathrm{E}-06$ & $5.33 E-06$ & $8.43 \mathrm{E}-07$ & $4.98 \mathrm{E}-06$ & 3.23 & 0.9737 & 4.16E-06 & $5.34 E-06$ & $1.18 \mathrm{E}-06$ & 4.66E-06 \\
\hline
\end{tabular}




\begin{tabular}{|c|c|c|c|c|c|c|c|c|c|c|c|c|c|c|}
\hline Capsule & OC3 & $\begin{array}{l}\text { Specimen } \\
\text { Length }\end{array}$ & $\begin{array}{c}13 \\
0.9805\end{array}$ & Scale & 1" & $1.00 \mathrm{E}-03$ & & & & & & & & \\
\hline $\begin{array}{c}\text { Temperature } \\
\left({ }^{\circ} \mathrm{C}\right)\end{array}$ & Up & Down & $\begin{array}{l}\text { Delta } \\
\text { Down }\end{array}$ & $\begin{array}{l}\text { Down } \\
\text { length }\end{array}$ & CTE & CTEK & $\begin{array}{l}\text { Delta } \\
\text { CTEK }\end{array}$ & $\begin{array}{c}\text { CTE } \\
\text { adjusted }\end{array}$ & $\begin{array}{l}\text { Delta } \\
\text { Up }\end{array}$ & $\begin{array}{c}\text { Up } \\
\text { length }\end{array}$ & CTE & CTEK & $\begin{array}{l}\text { Delta } \\
\text { CTEK }\end{array}$ & $\begin{array}{c}\text { CTE } \\
\text { adjusted }\end{array}$ \\
\hline 0 & 1.08 & 1.19 & 0 & 0.9805 & & & & & 0 & 0.9805 & & & & \\
\hline 100 & 1.33 & 1.44 & 0.25 & 0.9808 & $2.55 \mathrm{E}-06$ & $3.05 E-06$ & $5.00 \mathrm{E}-07$ & $3.05 \mathrm{E}-06$ & 0.25 & 0.9808 & $2.55 \mathrm{E}-06$ & $3.05 E-06$ & $5.00 \mathrm{E}-07$ & $3.05 \mathrm{E}-06$ \\
\hline 200 & 1.60 & 1.72 & 0.53 & 0.9810 & $2.70 \mathrm{E}-06$ & $3.20 E-06$ & 4.97E-07 & $3.20 \mathrm{E}-06$ & 0.52 & 0.9810 & $2.65 \mathrm{E}-06$ & $3.15 E-06$ & $4.98 \mathrm{E}-07$ & $3.15 \mathrm{E}-06$ \\
\hline 300 & 1.89 & 2.00 & 0.81 & 0.9813 & $2.75 \mathrm{E}-06$ & $3.25 E-06$ & $4.96 \mathrm{E}-07$ & $3.25 \mathrm{E}-06$ & 0.81 & 0.9813 & $2.75 \mathrm{E}-06$ & $3.25 E-06$ & 4.96E-07 & $3.25 \mathrm{E}-06$ \\
\hline 400 & 2.20 & 2.31 & 1.12 & 0.9816 & $2.86 \mathrm{E}-06$ & $3.35 E-06$ & 4.94E-07 & $3.36 \mathrm{E}-06$ & 1.12 & 0.9816 & $2.86 \mathrm{E}-06$ & $3.35 E-06$ & $4.94 \mathrm{E}-07$ & 3.36E-06 \\
\hline 500 & 2.53 & 2.69 & 1.5 & 0.9820 & $3.06 \mathrm{E}-06$ & $3.55 E-06$ & $4.90 \mathrm{E}-07$ & 3.56E-06 & 1.45 & 0.9820 & $2.96 \mathrm{E}-06$ & $3.45 E-06$ & $4.92 \mathrm{E}-07$ & $3.46 \mathrm{E}-06$ \\
\hline 600 & 2.89 & 3.08 & 1.89 & 0.9824 & $3.21 \mathrm{E}-06$ & 3.70E-06 & 4.87E-07 & $3.71 \mathrm{E}-06$ & 1.81 & 0.9823 & $3.08 \mathrm{E}-06$ & 3.57E-06 & 4.89E-07 & $3.58 \mathrm{E}-06$ \\
\hline 700 & 3.29 & 3.47 & 2.28 & 0.9828 & $3.32 \mathrm{E}-06$ & $3.81 E-06$ & $4.85 \mathrm{E}-07$ & $3.82 \mathrm{E}-06$ & 2.21 & 0.9827 & $3.22 \mathrm{E}-06$ & $3.71 E-06$ & $4.87 \mathrm{E}-07$ & $3.72 \mathrm{E}-06$ \\
\hline 800 & 3.70 & 3.85 & 2.66 & 0.9832 & $3.39 \mathrm{E}-06$ & $3.88 E-06$ & 4.84E-07 & $3.89 \mathrm{E}-06$ & 2.62 & 0.9831 & $3.34 \mathrm{E}-06$ & $3.83 E-06$ & $4.85 \mathrm{E}-07$ & $3.84 \mathrm{E}-06$ \\
\hline
\end{tabular}

\begin{tabular}{|c|c|c|c|c|c|c|c|c|c|c|c|c|c|c|}
\hline Capsule & OC3 & $\begin{array}{l}\text { Specimen } \\
\text { Length }\end{array}$ & $\begin{array}{c}14 \\
0.9747\end{array}$ & $\begin{array}{l}\text { (check) } \\
\text { Scale }\end{array}$ & $1 "$ & $1.00 \mathrm{E}-03$ & & & & & & & & \\
\hline $\begin{array}{c}\text { Temperature } \\
\left({ }^{\circ} \mathrm{C}\right)\end{array}$ & Up & Down & $\begin{array}{l}\text { Delta } \\
\text { Down }\end{array}$ & $\begin{array}{l}\text { Down } \\
\text { length }\end{array}$ & CTE & CTEK & $\begin{array}{l}\text { Delta } \\
\text { CTEK }\end{array}$ & $\begin{array}{c}\text { CTE } \\
\text { adjusted }\end{array}$ & $\begin{array}{l}\text { Delta } \\
\text { Up }\end{array}$ & $\begin{array}{c}\text { Up } \\
\text { length }\end{array}$ & CTE & CTEK & $\begin{array}{l}\text { Delta } \\
\text { CTEK }\end{array}$ & $\begin{array}{c}\text { CTE } \\
\text { adjusted }\end{array}$ \\
\hline 0 & 0.71 & 1.62 & 0 & 0.9747 & & & & & 0 & 0.9747 & & & & \\
\hline 100 & 1.03 & 1.28 & -0.34 & 0.9744 & $-3.49 \mathrm{E}-06$ & $3.15 E-06$ & $6.64 \mathrm{E}-06$ & $-2.99 \mathrm{E}-06$ & 0.32 & 0.9750 & $3.28 \mathrm{E}-06$ & $3.75 E-06$ & 4.67E-07 & $3.78 \mathrm{E}-06$ \\
\hline 200 & 1.38 & 1.57 & -0.05 & 0.9747 & $-2.56 \mathrm{E}-07$ & $3.30 E-06$ & $3.56 \mathrm{E}-06$ & $2.44 \mathrm{E}-07$ & 0.67 & 0.9754 & $3.44 \mathrm{E}-06$ & $3.90 E-06$ & 4.63E-07 & $3.94 \mathrm{E}-06$ \\
\hline 300 & 1.73 & 1.88 & 0.26 & 0.9750 & $8.89 \mathrm{E}-07$ & $3.42 E-06$ & $2.53 \mathrm{E}-06$ & $1.39 \mathrm{E}-06$ & 1.02 & 0.9757 & $3.49 \mathrm{E}-06$ & $3.95 E-06$ & $4.62 \mathrm{E}-07$ & $3.99 \mathrm{E}-06$ \\
\hline 400 & 2.03 & 2.21 & 0.59 & 0.9753 & $1.51 \mathrm{E}-06$ & 3.53E-06 & $2.01 \mathrm{E}-06$ & $2.01 \mathrm{E}-06$ & 1.32 & 0.9760 & $3.39 \mathrm{E}-06$ & $3.85 E-06$ & $4.64 \mathrm{E}-07$ & $3.89 \mathrm{E}-06$ \\
\hline 500 & 2.42 & 2.61 & 0.99 & 0.9757 & $2.03 \mathrm{E}-06$ & $3.73 E-06$ & $1.70 \mathrm{E}-06$ & $2.53 \mathrm{E}-06$ & 1.71 & 0.9764 & $3.51 \mathrm{E}-06$ & 3.97E-06 & $4.61 \mathrm{E}-07$ & $4.01 \mathrm{E}-06$ \\
\hline 600 & 2.89 & 3.02 & 1.4 & 0.9761 & $2.39 \mathrm{E}-06$ & $3.88 E-06$ & $1.49 \mathrm{E}-06$ & $2.89 \mathrm{E}-06$ & 2.18 & 0.9769 & $3.73 \mathrm{E}-06$ & 4.18E-06 & 4.55E-07 & 4.23E-06 \\
\hline 700 & 3.32 & 3.49 & 1.87 & 0.9766 & $2.74 \mathrm{E}-06$ & $4.08 E-06$ & $1.34 \mathrm{E}-06$ & $3.24 \mathrm{E}-06$ & 2.61 & 0.9773 & $3.83 \mathrm{E}-06$ & $4.28 E-06$ & $4.53 \mathrm{E}-07$ & 4.33E-06 \\
\hline 800 & 3.78 & 3.93 & 2.31 & 0.9770 & $2.96 \mathrm{E}-06$ & 4.19E-06 & $1.22 \mathrm{E}-06$ & $3.46 \mathrm{E}-06$ & 3.07 & 0.9778 & $3.94 \mathrm{E}-06$ & 4.39E-06 & 4.50E-07 & 4.44E-06 \\
\hline
\end{tabular}




\begin{tabular}{|c|c|c|c|c|c|c|c|c|c|c|c|c|c|c|}
\hline Capsule & OC3 & $\begin{array}{c}\text { Specimen } \\
\text { Length }\end{array}$ & $\begin{array}{c}18 \\
0.9827\end{array}$ & Scale & 1" & $1.00 \mathrm{E}-03$ & & & & & & & & \\
\hline $\begin{array}{c}\text { Temperature } \\
\left({ }^{\circ} \mathrm{C}\right)\end{array}$ & Up & Down & $\begin{array}{l}\text { Delta } \\
\text { Down }\end{array}$ & $\begin{array}{l}\text { Down } \\
\text { length }\end{array}$ & CTE & CTEK & $\begin{array}{l}\text { Delta } \\
\text { CTEK }\end{array}$ & $\begin{array}{c}\text { CTE } \\
\text { adjusted } \\
\end{array}$ & $\begin{array}{l}\text { Delta } \\
\text { Up }\end{array}$ & $\begin{array}{l}\text { Up } \\
\text { length }\end{array}$ & CTE & CTEK & $\begin{array}{l}\text { Delta } \\
\text { CTEK }\end{array}$ & $\begin{array}{c}\text { CTE } \\
\text { adjusted }\end{array}$ \\
\hline 0 & 0.95 & 1.28 & 0 & 0.9827 & & & & & 0 & 0.9827 & & & & \\
\hline 100 & 1.26 & 1.53 & 0.25 & 0.9830 & $2.54 \mathrm{E}-06$ & $3.05 E-06$ & $5.06 \mathrm{E}-07$ & $3.04 \mathrm{E}-06$ & 0.31 & 0.9830 & $3.15 \mathrm{E}-06$ & $3.65 E-06$ & 4.95E-07 & $3.65 \mathrm{E}-06$ \\
\hline 200 & 1.55 & 1.78 & 0.5 & 0.9832 & $2.54 \mathrm{E}-06$ & $3.05 E-06$ & $5.06 \mathrm{E}-07$ & $3.04 \mathrm{E}-06$ & 0.6 & 0.9833 & $3.05 E-06$ & $3.55 E-06$ & 4.97E-07 & $3.55 \mathrm{E}-06$ \\
\hline 300 & 1.91 & 2.03 & 0.75 & 0.9835 & $2.54 \mathrm{E}-06$ & $3.05 E-06$ & $5.06 \mathrm{E}-07$ & $3.04 \mathrm{E}-06$ & 0.96 & 0.9837 & $3.26 \mathrm{E}-06$ & $3.75 E-06$ & 4.94E-07 & $3.76 \mathrm{E}-06$ \\
\hline 400 & 2.25 & 2.31 & 1.03 & 0.9837 & $2.62 \mathrm{E}-06$ & 3.13E-06 & $5.05 \mathrm{E}-07$ & $3.12 \mathrm{E}-06$ & 1.3 & 0.9840 & $3.31 \mathrm{E}-06$ & 3.80E-06 & 4.93E-07 & $3.81 \mathrm{E}-06$ \\
\hline 500 & 2.60 & 2.69 & 1.41 & 0.9841 & $2.87 \mathrm{E}-06$ & $3.37 E-06$ & $5.00 \mathrm{E}-07$ & $3.37 \mathrm{E}-06$ & 1.65 & 0.9844 & $3.36 \mathrm{E}-06$ & $3.85 E-06$ & $4.92 \mathrm{E}-07$ & $3.86 \mathrm{E}-06$ \\
\hline 600 & 2.97 & 3.05 & 1.77 & 0.9845 & $3.00 \mathrm{E}-06$ & $3.50 E-06$ & $4.98 \mathrm{E}-07$ & $3.50 \mathrm{E}-06$ & 2.02 & 0.9847 & $3.43 \mathrm{E}-06$ & $3.92 E-06$ & $4.90 \mathrm{E}-07$ & $3.93 \mathrm{E}-06$ \\
\hline 700 & 3.33 & 3.43 & 2.15 & 0.9849 & $3.13 E-06$ & $3.62 E-06$ & $4.96 \mathrm{E}-07$ & $3.63 \mathrm{E}-06$ & 2.38 & 0.9851 & $3.46 \mathrm{E}-06$ & $3.95 E-06$ & 4.90E-07 & $3.96 \mathrm{E}-06$ \\
\hline 800 & 3.73 & 3.83 & 2.55 & 0.9853 & $3.24 \mathrm{E}-06$ & $3.74 E-06$ & 4.93E-07 & 3.74E-06 & 2.78 & 0.9855 & $3.54 \mathrm{E}-06$ & 4.03E-06 & 4.89E-07 & 4.04E-06 \\
\hline
\end{tabular}

\begin{tabular}{|c|c|c|c|c|c|c|c|c|c|c|c|c|c|c|}
\hline Capsule & OC3 & $\begin{array}{l}\text { Specimen } \\
\text { Length }\end{array}$ & $\begin{array}{c}19 \\
0.98781\end{array}$ & Scale & 1" & $1.00 \mathrm{E}-03$ & & & & & & & & \\
\hline $\begin{array}{c}\text { Temperature } \\
\left({ }^{\circ} \mathrm{C}\right)\end{array}$ & Up & Down & $\begin{array}{l}\text { Delta } \\
\text { Down }\end{array}$ & $\begin{array}{l}\text { Down } \\
\text { length }\end{array}$ & CTE & CTEK & $\begin{array}{l}\text { Delta } \\
\text { CTEK }\end{array}$ & $\begin{array}{c}\text { CTE } \\
\text { adjusted }\end{array}$ & $\begin{array}{l}\text { Delta } \\
\text { Up }\end{array}$ & $\begin{array}{c}\text { Up } \\
\text { length }\end{array}$ & CTE & CTEK & $\begin{array}{l}\text { Delta } \\
\text { CTEK }\end{array}$ & $\begin{array}{c}\text { CTE } \\
\text { adjusted }\end{array}$ \\
\hline 0 & 0.86 & 0.86 & 0 & 0.9878 & & & & & 0 & 0.9878 & & & & \\
\hline 100 & 1.09 & 1.09 & 0.23 & 0.9880 & $2.33 \mathrm{E}-06$ & $2.85 E-06$ & $5.22 \mathrm{E}-07$ & $2.83 \mathrm{E}-06$ & 0.23 & 0.9880 & $2.33 \mathrm{E}-06$ & $2.85 E-06$ & $5.22 \mathrm{E}-07$ & $2.83 \mathrm{E}-06$ \\
\hline 200 & 1.35 & 1.37 & 0.51 & 0.9883 & $2.58 \mathrm{E}-06$ & 3.10E-06 & 5.19E-07 & $3.08 \mathrm{E}-06$ & 0.49 & 0.9883 & $2.48 \mathrm{E}-06$ & $3.00 E-06$ & $5.20 \mathrm{E}-07$ & $2.98 \mathrm{E}-06$ \\
\hline 300 & 1.63 & 1.69 & 0.83 & 0.9886 & $2.80 \mathrm{E}-06$ & 3.32E-06 & $5.15 \mathrm{E}-07$ & $3.30 \mathrm{E}-06$ & 0.77 & 0.9886 & $2.60 \mathrm{E}-06$ & $3.12 E-06$ & $5.18 \mathrm{E}-07$ & 3.10E-06 \\
\hline 400 & 1.93 & 2.00 & 1.14 & 0.9890 & $2.89 \mathrm{E}-06$ & $3.40 E-06$ & $5.15 \mathrm{E}-07$ & 3.39E-06 & 1.07 & 0.9889 & $2.71 \mathrm{E}-06$ & $3.23 E-06$ & 5.17E-07 & $3.21 \mathrm{E}-06$ \\
\hline 500 & 2.26 & 2.38 & 1.52 & 0.9893 & $3.08 \mathrm{E}-06$ & $3.59 E-06$ & 5.12E-07 & $3.58 \mathrm{E}-06$ & 1.4 & 0.9892 & $2.83 \mathrm{E}-06$ & $3.35 E-06$ & $5.15 \mathrm{E}-07$ & $3.33 \mathrm{E}-06$ \\
\hline 600 & 2.64 & 2.79 & 1.93 & 0.9897 & $3.26 \mathrm{E}-06$ & 3.77E-06 & $5.10 \mathrm{E}-07$ & $3.76 \mathrm{E}-06$ & 1.78 & 0.9896 & $3.00 \mathrm{E}-06$ & $3.52 E-06$ & $5.13 \mathrm{E}-07$ & 3.50E-06 \\
\hline 700 & 3.02 & 3.18 & 2.32 & 0.9901 & $3.36 \mathrm{E}-06$ & $3.86 E-06$ & 5.09E-07 & $3.86 \mathrm{E}-06$ & 2.16 & 0.9900 & $3.12 \mathrm{E}-06$ & $3.64 E-06$ & $5.11 \mathrm{E}-07$ & $3.62 \mathrm{E}-06$ \\
\hline 800 & 3.50 & 3.57 & 2.71 & 0.9905 & $3.43 \mathrm{E}-06$ & $3.94 E-06$ & $5.08 \mathrm{E}-07$ & $3.93 \mathrm{E}-06$ & 2.64 & 0.9905 & $3.34 \mathrm{E}-06$ & $3.85 E-06$ & $5.09 \mathrm{E}-07$ & 3.84E-06 \\
\hline
\end{tabular}




\begin{tabular}{|c|c|c|c|c|c|c|c|c|c|c|c|c|c|c|}
\hline Capsule & OC3 & $\begin{array}{l}\text { Specimen } \\
\text { Length }\end{array}$ & $\begin{array}{c}23 \\
0.9802\end{array}$ & Scale & 1" & $1.00 \mathrm{E}-03$ & & & & & & & & \\
\hline $\begin{array}{c}\text { Temperature } \\
\left({ }^{\circ} \mathrm{C}\right)\end{array}$ & Up & Down & $\begin{array}{l}\text { Delta } \\
\text { Down }\end{array}$ & $\begin{array}{l}\text { Down } \\
\text { length }\end{array}$ & CTE & CTEK & $\begin{array}{l}\text { Delta } \\
\text { CTEK }\end{array}$ & $\begin{array}{c}\text { CTE } \\
\text { adjusted }\end{array}$ & $\begin{array}{l}\text { Delta } \\
\text { Up }\end{array}$ & $\begin{array}{c}\text { Up } \\
\text { length }\end{array}$ & CTE & CTEK & $\begin{array}{l}\text { Delta } \\
\text { CTEK }\end{array}$ & $\begin{array}{c}\text { CTE } \\
\text { adjusted }\end{array}$ \\
\hline 0 & 1.15 & 1.45 & 0 & 0.9802 & & & & & 0 & 0.9802 & & & & \\
\hline 100 & 1.40 & 1.69 & 0.24 & 0.9804 & $2.45 \mathrm{E}-06$ & $2.95 E-06$ & $5.02 \mathrm{E}-07$ & $2.95 \mathrm{E}-06$ & 0.25 & 0.9805 & $2.55 \mathrm{E}-06$ & $3.05 E-06$ & $5.00 \mathrm{E}-07$ & $3.05 \mathrm{E}-06$ \\
\hline 200 & 1.69 & 1.91 & 0.46 & 0.9807 & $2.35 \mathrm{E}-06$ & $2.85 E-06$ & $5.04 \mathrm{E}-07$ & $2.85 \mathrm{E}-06$ & 0.54 & 0.9807 & $2.75 \mathrm{E}-06$ & $3.25 E-06$ & 4.95E-07 & $3.25 \mathrm{E}-06$ \\
\hline 300 & 2.04 & 2.20 & 0.75 & 0.9810 & $2.55 \mathrm{E}-06$ & $3.05 E-06$ & $5.00 \mathrm{E}-07$ & $3.05 \mathrm{E}-06$ & 0.89 & 0.9811 & $3.03 \mathrm{E}-06$ & $3.52 E-06$ & 4.89E-07 & $3.53 \mathrm{E}-06$ \\
\hline 400 & 2.31 & 2.46 & 1.01 & 0.9812 & $2.58 \mathrm{E}-06$ & $3.08 E-06$ & 4.99E-07 & $3.08 \mathrm{E}-06$ & 1.16 & 0.9814 & $2.96 \mathrm{E}-06$ & $3.45 E-06$ & 4.91E-07 & $3.46 \mathrm{E}-06$ \\
\hline 500 & 2.64 & 2.79 & 1.34 & 0.9815 & $2.73 \mathrm{E}-06$ & $3.23 E-06$ & 4.96E-07 & $3.23 \mathrm{E}-06$ & 1.49 & 0.9817 & $3.04 \mathrm{E}-06$ & $3.53 E-06$ & $4.90 \mathrm{E}-07$ & $3.54 \mathrm{E}-06$ \\
\hline 600 & 2.95 & 3.12 & 1.67 & 0.9819 & $2.84 \mathrm{E}-06$ & $3.33 E-06$ & 4.93E-07 & $3.34 \mathrm{E}-06$ & 1.8 & 0.9820 & $3.06 \mathrm{E}-06$ & $3.55 E-06$ & 4.89E-07 & 3.56E-06 \\
\hline 700 & 3.36 & 3.50 & 2.05 & 0.9823 & $2.99 \mathrm{E}-06$ & $3.48 E-06$ & $4.90 \mathrm{E}-07$ & $3.49 \mathrm{E}-06$ & 2.21 & 0.9824 & $3.22 \mathrm{E}-06$ & $3.71 E-06$ & $4.86 \mathrm{E}-07$ & $3.72 \mathrm{E}-06$ \\
\hline 800 & 3.70 & 3.83 & 2.38 & 0.9826 & $3.04 \mathrm{E}-06$ & $3.53 E-06$ & 4.90E-07 & $3.54 \mathrm{E}-06$ & 2.55 & 0.9828 & $3.25 \mathrm{E}-06$ & $3.74 E-06$ & $4.85 \mathrm{E}-07$ & $3.75 \mathrm{E}-06$ \\
\hline
\end{tabular}

\begin{tabular}{|c|c|c|c|c|c|c|c|c|c|c|c|c|c|c|}
\hline Capsule & OC3 & $\begin{array}{c}\text { Specimen } \\
\text { Length }\end{array}$ & $\begin{array}{c}24 \\
0.9748\end{array}$ & Scale & 1" & $1.00 \mathrm{E}-03$ & & & & & & & & \\
\hline $\begin{array}{c}\text { Temperature } \\
\left({ }^{\circ} \mathrm{C}\right)\end{array}$ & Up & Down & $\begin{array}{l}\text { Delta } \\
\text { Down }\end{array}$ & $\begin{array}{l}\text { Down } \\
\text { length }\end{array}$ & CTE & CTEK & $\begin{array}{l}\text { Delta } \\
\text { CTEK }\end{array}$ & $\begin{array}{c}\text { CTE } \\
\text { adjusted }\end{array}$ & $\begin{array}{c}\text { Delta } \\
\text { Up }\end{array}$ & $\begin{array}{c}\text { Up } \\
\text { length }\end{array}$ & CTE & CTEK & $\begin{array}{l}\text { Delta } \\
\text { CTEK }\end{array}$ & $\begin{array}{c}\text { CTE } \\
\text { adjusted }\end{array}$ \\
\hline 0 & 0.92 & 1.10 & 0 & 0.9748 & & & & & 0 & 0.9748 & & & & \\
\hline 100 & 1.20 & 1.40 & 0.3 & 0.9751 & $3.08 \mathrm{E}-06$ & 3.55E-06 & 4.72E-07 & $3.58 \mathrm{E}-06$ & 0.28 & 0.9751 & $2.87 \mathrm{E}-06$ & $3.35 E-06$ & $4.78 \mathrm{E}-07$ & 3.37E-06 \\
\hline 200 & 1.60 & 1.71 & 0.61 & 0.9754 & $3.13 \mathrm{E}-06$ & $3.60 E-06$ & $4.71 \mathrm{E}-07$ & $3.63 \mathrm{E}-06$ & 0.68 & 0.9755 & $3.49 \mathrm{E}-06$ & $3.95 E-06$ & $4.62 \mathrm{E}-07$ & $3.99 \mathrm{E}-06$ \\
\hline 300 & 1.98 & 2.08 & 0.98 & 0.9758 & $3.35 \mathrm{E}-06$ & $3.82 E-06$ & $4.65 \mathrm{E}-07$ & $3.85 \mathrm{E}-06$ & 1.06 & 0.9759 & $3.62 \mathrm{E}-06$ & $4.08 E-06$ & $4.58 \mathrm{E}-07$ & $4.12 \mathrm{E}-06$ \\
\hline 400 & 2.30 & 2.43 & 1.33 & 0.9761 & $3.41 \mathrm{E}-06$ & $3.88 E-06$ & $4.64 \mathrm{E}-07$ & $3.91 \mathrm{E}-06$ & 1.38 & 0.9762 & $3.54 \mathrm{E}-06$ & $4.00 E-06$ & 4.61E-07 & 4.04E-06 \\
\hline 500 & 2.72 & 2.83 & 1.73 & 0.9765 & $3.55 \mathrm{E}-06$ & $4.01 E-06$ & $4.61 \mathrm{E}-07$ & 4.05E-06 & 1.8 & 0.9766 & 3.69E-06 & $4.15 E-06$ & 4.57E-07 & 4.19E-06 \\
\hline 600 & 3.15 & 3.28 & 2.18 & 0.9770 & $3.73 \mathrm{E}-06$ & $4.18 E-06$ & $4.56 \mathrm{E}-07$ & $4.23 \mathrm{E}-06$ & 2.23 & 0.9770 & $3.81 \mathrm{E}-06$ & 4.27E-06 & $4.53 \mathrm{E}-07$ & $4.31 \mathrm{E}-06$ \\
\hline 700 & 3.58 & 3.70 & 2.6 & 0.9774 & $3.81 \mathrm{E}-06$ & $4.26 E-06$ & $4.54 \mathrm{E}-07$ & 4.31E-06 & 2.66 & 0.9775 & $3.90 \mathrm{E}-06$ & $4.35 E-06$ & $4.52 \mathrm{E}-07$ & 4.40E-06 \\
\hline 800 & 4.08 & 4.12 & 3.02 & 0.9778 & $3.87 \mathrm{E}-06$ & 4.33E-06 & $4.52 \mathrm{E}-07$ & 4.37E-06 & 3.16 & 0.9780 & 4.05E-06 & 4.50E-06 & 4.48E-07 & 4.55E-06 \\
\hline
\end{tabular}




\begin{tabular}{|c|c|c|c|c|c|c|c|c|c|c|c|c|c|c|}
\hline Capsule & OC3 & $\begin{array}{c}\text { Specimen } \\
\text { Length }\end{array}$ & $\begin{array}{c}25 \\
0.9834\end{array}$ & Scale & $1 "$ & $1.00 \mathrm{E}-03$ & & & & & & & & \\
\hline $\begin{array}{c}\text { Temperature } \\
\left({ }^{\circ} \mathrm{C}\right)\end{array}$ & Up & Down & $\begin{array}{l}\text { Delta } \\
\text { Down }\end{array}$ & $\begin{array}{l}\text { Down } \\
\text { length }\end{array}$ & CTE & CTEK & $\begin{array}{l}\text { Delta } \\
\text { CTEK }\end{array}$ & $\begin{array}{c}\text { CTE } \\
\text { adjusted }\end{array}$ & $\begin{array}{l}\text { Delta } \\
\text { Up }\end{array}$ & $\begin{array}{c}\text { Up } \\
\text { length }\end{array}$ & CTE & CTEK & $\begin{array}{l}\text { Delta } \\
\text { CTEK }\end{array}$ & $\begin{array}{c}\text { CTE } \\
\text { adjusted }\end{array}$ \\
\hline 0 & 0.47 & 0.47 & 0 & 0.9834 & & & & & 0 & 0.9834 & & & & \\
\hline 100 & 0.70 & 0.66 & 0.19 & 0.9836 & 1.93E-06 & $1.75 E-06$ & $-1.82 \mathrm{E}-07$ & $2.43 \mathrm{E}-06$ & 0.23 & 0.9836 & $2.34 \mathrm{E}-06$ & $2.45 E-06$ & $1.11 \mathrm{E}-07$ & $2.84 \mathrm{E}-06$ \\
\hline 200 & 0.92 & 0.87 & 0.4 & 0.9838 & $2.03 \mathrm{E}-06$ & $1.75 E-06$ & $-2.84 \mathrm{E}-07$ & $2.53 \mathrm{E}-06$ & 0.45 & 0.9839 & $2.29 \mathrm{E}-06$ & $2.35 E-06$ & $6.20 \mathrm{E}-08$ & 2.79E-06 \\
\hline 300 & 1.19 & 1.10 & 0.63 & 0.9840 & $2.14 \mathrm{E}-06$ & $1.82 E-06$ & $-3.19 E-07$ & $2.64 \mathrm{E}-06$ & 0.72 & 0.9841 & $2.44 \mathrm{E}-06$ & $2.42 E-06$ & $-2.45 \mathrm{E}-08$ & 2.94E-06 \\
\hline 400 & 1.45 & 1.36 & 0.89 & 0.9843 & $2.26 \mathrm{E}-06$ & $1.95 E-06$ & $-3.13 E-07$ & $2.76 \mathrm{E}-06$ & 0.98 & 0.9844 & 2.49E-06 & $2.45 E-06$ & $-4.14 \mathrm{E}-08$ & 2.99E-06 \\
\hline 500 & 1.76 & 1.67 & 1.2 & 0.9846 & $2.44 \mathrm{E}-06$ & $2.13 E-06$ & $-3.11 \mathrm{E}-07$ & $2.94 \mathrm{E}-06$ & 1.29 & 0.9847 & $2.62 \mathrm{E}-06$ & $2.51 E-06$ & $-1.14 \mathrm{E}-07$ & $3.12 \mathrm{E}-06$ \\
\hline 600 & 2.09 & 2.00 & 1.53 & 0.9849 & $2.59 \mathrm{E}-06$ & $2.33 E-06$ & $-2.60 \mathrm{E}-07$ & $3.09 \mathrm{E}-06$ & 1.62 & 0.9850 & $2.75 \mathrm{E}-06$ & $2.63 E-06$ & $-1.13 E-07$ & $3.25 \mathrm{E}-06$ \\
\hline 700 & 2.45 & 2.40 & 1.93 & 0.9853 & $2.80 \mathrm{E}-06$ & 2.49E-06 & $-3.12 \mathrm{E}-07$ & 3.30E-06 & 1.98 & 0.9854 & $2.88 \mathrm{E}-06$ & $2.74 E-06$ & $-1.41 \mathrm{E}-07$ & $3.38 \mathrm{E}-06$ \\
\hline 800 & 2.88 & 2.88 & 2.41 & 0.9858 & $3.06 \mathrm{E}-06$ & $2.74 E-06$ & $-3.26 \mathrm{E}-07$ & $3.56 \mathrm{E}-06$ & 2.41 & 0.9858 & $3.06 \mathrm{E}-06$ & $2.85 E-06$ & $-2.13 E-07$ & $3.56 \mathrm{E}-06$ \\
\hline
\end{tabular}

\begin{tabular}{|c|c|c|c|c|c|c|c|c|c|c|c|c|c|c|}
\hline Capsule & OC3 & $\begin{array}{l}\text { Specimen } \\
\text { Length }\end{array}$ & $\begin{array}{c}28 \\
0.9795\end{array}$ & Scale & 1" & $1.00 \mathrm{E}-03$ & & & & & & & & \\
\hline $\begin{array}{c}\text { Temperature } \\
\left({ }^{\circ} \mathrm{C}\right)\end{array}$ & Up & Down & $\begin{array}{l}\text { Delta } \\
\text { Down }\end{array}$ & $\begin{array}{l}\text { Down } \\
\text { length }\end{array}$ & CTE & CTEK & $\begin{array}{l}\text { Delta } \\
\text { CTEK }\end{array}$ & $\begin{array}{c}\text { CTE } \\
\text { adjusted }\end{array}$ & $\begin{array}{l}\text { Delta } \\
\text { Up }\end{array}$ & $\begin{array}{c}\text { Up } \\
\text { length }\end{array}$ & CTE & CTEK & $\begin{array}{l}\text { Delta } \\
\text { CTEK }\end{array}$ & $\begin{array}{c}\text { CTE } \\
\text { adjusted }\end{array}$ \\
\hline 0 & 1.20 & 1.38 & 0 & 0.9795 & & & & & 0 & 0.9795 & & & & \\
\hline 100 & 1.50 & 1.69 & 0.31 & 0.9798 & $3.16 \mathrm{E}-06$ & $3.65 E-06$ & 4.85E-07 & $3.66 \mathrm{E}-06$ & 0.3 & 0.9798 & $3.06 \mathrm{E}-06$ & $3.55 E-06$ & 4.87E-07 & 3.56E-06 \\
\hline 200 & 1.97 & 2.02 & 0.64 & 0.9801 & $3.27 \mathrm{E}-06$ & $3.75 E-06$ & $4.83 \mathrm{E}-07$ & 3.77E-06 & 0.77 & 0.9803 & $3.93 \mathrm{E}-06$ & 4.40E-06 & 4.69E-07 & 4.43E-06 \\
\hline 300 & 2.35 & 2.38 & 1 & 0.9805 & $3.40 \mathrm{E}-06$ & $3.88 E-06$ & $4.80 \mathrm{E}-07$ & $3.90 \mathrm{E}-06$ & 1.15 & 0.9807 & $3.91 \mathrm{E}-06$ & $4.38 E-06$ & 4.69E-07 & 4.41E-06 \\
\hline 400 & 2.76 & 2.76 & 1.38 & 0.9809 & $3.52 \mathrm{E}-06$ & $4.00 E-06$ & $4.78 \mathrm{E}-07$ & 4.02E-06 & 1.56 & 0.9811 & $3.98 \mathrm{E}-06$ & 4.45E-06 & $4.68 \mathrm{E}-07$ & 4.48E-06 \\
\hline 500 & 3.15 & 3.20 & 1.82 & 0.9813 & $3.72 \mathrm{E}-06$ & 4.19E-06 & 4.74E-07 & $4.22 \mathrm{E}-06$ & 1.95 & 0.9815 & $3.98 \mathrm{E}-06$ & $4.45 E-06$ & $4.68 \mathrm{E}-07$ & $4.48 \mathrm{E}-06$ \\
\hline 600 & 3.60 & 3.67 & 2.29 & 0.9818 & $3.90 \mathrm{E}-06$ & 4.37E-06 & 4.69E-07 & 4.40E-06 & 2.4 & 0.9819 & $4.08 \mathrm{E}-06$ & $4.55 E-06$ & 4.66E-07 & $4.58 \mathrm{E}-06$ \\
\hline 700 & 4.09 & 4.16 & 2.78 & 0.9823 & 4.05E-06 & $4.52 E-06$ & 4.66E-07 & 4.55E-06 & 2.89 & 0.9824 & $4.21 \mathrm{E}-06$ & $4.68 E-06$ & 4.63E-07 & 4.71E-06 \\
\hline 800 & 4.60 & 4.67 & 3.29 & 0.9828 & 4.20E-06 & 4.66E-06 & 4.63E-07 & 4.70E-06 & 3.4 & 0.9829 & $4.34 \mathrm{E}-06$ & $4.80 E-06$ & 4.61E-07 & 4.84E-06 \\
\hline
\end{tabular}




\begin{tabular}{|c|c|c|c|c|c|c|c|c|c|c|c|c|c|c|}
\hline Capsule & OC3 & $\begin{array}{c}\text { Specimen } \\
\text { Length }\end{array}$ & $\begin{array}{c}29 \\
0.9830 \\
\end{array}$ & Scale & 1" & $1.00 \mathrm{E}-03$ & & & & & & & & \\
\hline $\begin{array}{c}\text { Temperature } \\
\left({ }^{\circ} \mathrm{C}\right)\end{array}$ & Up & Down & $\begin{array}{l}\text { Delta } \\
\text { Down }\end{array}$ & $\begin{array}{l}\text { Down } \\
\text { length }\end{array}$ & CTE & CTEK & $\begin{array}{l}\text { Delta } \\
\text { CTEK }\end{array}$ & $\begin{array}{c}\text { CTE } \\
\text { adjusted } \\
\end{array}$ & $\begin{array}{l}\text { Delta } \\
\text { Up }\end{array}$ & $\begin{array}{l}\text { Up } \\
\text { length }\end{array}$ & CTE & CTEK & $\begin{array}{l}\text { Delta } \\
\text { CTEK }\end{array}$ & $\begin{array}{c}\text { CTE } \\
\text { adjusted }\end{array}$ \\
\hline 0 & 0.84 & 0.65 & 0 & 0.9830 & & & & & 0 & 0.9830 & & & & \\
\hline 100 & 1.19 & 0.99 & 0.34 & 0.9833 & $3.46 \mathrm{E}-06$ & $3.95 E-06$ & $4.91 \mathrm{E}-07$ & $3.96 \mathrm{E}-06$ & 0.35 & 0.9834 & $3.56 \mathrm{E}-06$ & $4.05 E-06$ & $4.89 \mathrm{E}-07$ & 4.06E-06 \\
\hline 200 & 1.53 & 1.34 & 0.69 & 0.9837 & $3.51 \mathrm{E}-06$ & $4.00 E-06$ & 4.90E-07 & 4.01E-06 & 0.69 & 0.9837 & $3.51 \mathrm{E}-06$ & $4.00 E-06$ & $4.90 \mathrm{E}-07$ & $4.01 \mathrm{E}-06$ \\
\hline 300 & 1.89 & 1.71 & 1.06 & 0.9841 & $3.59 \mathrm{E}-06$ & $4.08 E-06$ & 4.89E-07 & 4.09E-06 & 1.05 & 0.9841 & $3.56 \mathrm{E}-06$ & $4.05 E-06$ & 4.89E-07 & 4.06E-06 \\
\hline 400 & 2.28 & 2.12 & 1.47 & 0.9845 & $3.74 \mathrm{E}-06$ & $4.23 E-06$ & 4.86E-07 & $4.24 \mathrm{E}-06$ & 1.44 & 0.9844 & $3.66 \mathrm{E}-06$ & 4.15E-06 & $4.88 \mathrm{E}-07$ & 4.16E-06 \\
\hline 500 & 2.68 & 2.60 & 1.95 & 0.9850 & 3.97E-06 & $4.45 E-06$ & $4.83 \mathrm{E}-07$ & 4.47E-06 & 1.84 & 0.9848 & $3.74 \mathrm{E}-06$ & $4.23 E-06$ & 4.86E-07 & $4.24 \mathrm{E}-06$ \\
\hline 600 & 3.10 & 3.11 & 2.46 & 0.9855 & 4.17E-06 & $4.65 E-06$ & 4.79E-07 & 4.67E-06 & 2.26 & 0.9853 & $3.83 \mathrm{E}-06$ & $4.32 E-06$ & $4.84 \mathrm{E}-07$ & $4.33 \mathrm{E}-06$ \\
\hline 700 & 3.56 & 3.60 & 2.95 & 0.9860 & $4.29 \mathrm{E}-06$ & $4.76 E-06$ & 4.77E-07 & 4.79E-06 & 2.72 & 0.9857 & $3.95 \mathrm{E}-06$ & 4.44E-06 & $4.82 \mathrm{E}-07$ & 4.45E-06 \\
\hline 800 & 4.10 & 4.18 & 3.53 & 0.9865 & 4.49E-06 & $4.96 E-06$ & $4.73 \mathrm{E}-07$ & 4.99E-06 & 3.26 & 0.9863 & 4.15E-06 & 4.63E-06 & $4.80 \mathrm{E}-07$ & 4.65E-06 \\
\hline
\end{tabular}

\begin{tabular}{|c|c|c|c|c|c|c|c|c|c|c|c|c|c|c|}
\hline Capsule & OC3 & $\begin{array}{l}\text { Specimen } \\
\text { Length }\end{array}$ & $\begin{array}{c}34 \\
0.9830\end{array}$ & Scale & $1 "$ & $1.00 \mathrm{E}-03$ & & & & & & & & \\
\hline $\begin{array}{c}\text { Temperature } \\
\left({ }^{\circ} \mathrm{C}\right)\end{array}$ & Up & Down & $\begin{array}{l}\text { Delta } \\
\text { Down }\end{array}$ & $\begin{array}{l}\text { Down } \\
\text { length }\end{array}$ & CTE & CTEK & $\begin{array}{l}\text { Delta } \\
\text { CTEK }\end{array}$ & $\begin{array}{c}\text { CTE } \\
\text { adjusted }\end{array}$ & $\begin{array}{l}\text { Delta } \\
\text { Up }\end{array}$ & $\begin{array}{c}\text { Up } \\
\text { length }\end{array}$ & CTE & CTEK & $\begin{array}{l}\text { Delta } \\
\text { CTEK }\end{array}$ & $\begin{array}{c}\text { CTE } \\
\text { adjusted }\end{array}$ \\
\hline 0 & 0.96 & 1.29 & 0 & 0.9830 & & & & & 0 & 0.9830 & & & & \\
\hline 100 & 1.27 & 1.59 & 0.3 & 0.9833 & $3.05 \mathrm{E}-06$ & $3.55 E-06$ & $4.98 \mathrm{E}-07$ & $3.55 \mathrm{E}-06$ & 0.31 & 0.9833 & $3.15 \mathrm{E}-06$ & $3.65 E-06$ & 4.96E-07 & $3.65 \mathrm{E}-06$ \\
\hline 200 & 1.61 & 1.91 & 0.62 & 0.9836 & $3.15 \mathrm{E}-06$ & $3.65 E-06$ & $4.96 \mathrm{E}-07$ & $3.65 \mathrm{E}-06$ & 0.65 & 0.9837 & $3.31 \mathrm{E}-06$ & $3.80 E-06$ & 4.94E-07 & $3.81 \mathrm{E}-06$ \\
\hline 300 & 2.00 & 2.27 & 0.98 & 0.9840 & $3.32 \mathrm{E}-06$ & $3.82 E-06$ & 4.93E-07 & $3.82 \mathrm{E}-06$ & 1.04 & 0.9840 & $3.53 \mathrm{E}-06$ & $4.02 E-06$ & 4.89E-07 & 4.03E-06 \\
\hline 400 & 2.39 & 2.70 & 1.41 & 0.9844 & $3.59 \mathrm{E}-06$ & $4.08 E-06$ & 4.89E-07 & 4.09E-06 & 1.43 & 0.9844 & $3.64 \mathrm{E}-06$ & 4.13E-06 & $4.88 \mathrm{E}-07$ & 4.14E-06 \\
\hline 500 & 2.83 & 3.14 & 1.85 & 0.9849 & $3.76 \mathrm{E}-06$ & $4.25 E-06$ & $4.86 \mathrm{E}-07$ & 4.26E-06 & 1.87 & 0.9849 & $3.80 \mathrm{E}-06$ & 4.29E-06 & $4.85 \mathrm{E}-07$ & 4.30E-06 \\
\hline 600 & 3.28 & 3.60 & 2.31 & 0.9853 & $3.92 \mathrm{E}-06$ & $4.40 E-06$ & $4.83 \mathrm{E}-07$ & 4.42E-06 & 2.32 & 0.9853 & 3.93E-06 & 4.42E-06 & $4.82 \mathrm{E}-07$ & 4.43E-06 \\
\hline 700 & 3.81 & 4.03 & 2.74 & 0.9857 & $3.98 \mathrm{E}-06$ & $4.46 E-06$ & $4.82 \mathrm{E}-07$ & $4.48 \mathrm{E}-06$ & 2.85 & 0.9859 & 4.14E-06 & $4.62 E-06$ & 4.79E-07 & 4.64E-06 \\
\hline 800 & 4.40 & 4.52 & 3.23 & 0.9862 & 4.11E-06 & 4.59E-06 & $4.80 \mathrm{E}-07$ & 4.61E-06 & 3.44 & 0.9864 & 4.37E-06 & $4.85 E-06$ & 4.76E-07 & 4.87E-06 \\
\hline
\end{tabular}




\begin{tabular}{|c|c|c|c|c|c|c|c|c|c|c|c|c|c|c|}
\hline Capsule & OC3 & $\begin{array}{l}\text { Specimen } \\
\text { Length }\end{array}$ & $\begin{array}{c}38 \\
0.9807\end{array}$ & Scale & 1" & $1.00 \mathrm{E}-03$ & & & & & & & & \\
\hline $\begin{array}{c}\text { Temperature } \\
\left({ }^{\circ} \mathrm{C}\right)\end{array}$ & Up & Down & $\begin{array}{l}\text { Delta } \\
\text { Down }\end{array}$ & $\begin{array}{l}\text { Down } \\
\text { length }\end{array}$ & CTE & CTEK & $\begin{array}{l}\text { Delta } \\
\text { CTEK }\end{array}$ & $\begin{array}{c}\text { CTE } \\
\text { adjusted }\end{array}$ & $\begin{array}{l}\text { Delta } \\
\text { Up }\end{array}$ & $\begin{array}{c}\text { Up } \\
\text { length }\end{array}$ & CTE & CTEK & $\begin{array}{l}\text { Delta } \\
\text { CTEK }\end{array}$ & $\begin{array}{c}\text { CTE } \\
\text { adjusted }\end{array}$ \\
\hline 0 & 0.90 & 1.19 & 0 & 0.9807 & & & & & 0 & 0.9807 & & & & \\
\hline 100 & 1.20 & 1.46 & 0.27 & 0.9810 & $2.75 \mathrm{E}-06$ & $3.25 E-06$ & 4.97E-07 & $3.25 \mathrm{E}-06$ & 0.3 & 0.9810 & $3.06 \mathrm{E}-06$ & $3.55 E-06$ & 4.91E-07 & 3.56E-06 \\
\hline 200 & 1.53 & 1.72 & 0.53 & 0.9812 & $2.70 \mathrm{E}-06$ & $3.20 E-06$ & $4.98 \mathrm{E}-07$ & $3.20 \mathrm{E}-06$ & 0.63 & 0.9813 & $3.21 \mathrm{E}-06$ & $3.70 E-06$ & $4.88 \mathrm{E}-07$ & $3.71 \mathrm{E}-06$ \\
\hline 300 & 1.86 & 2.00 & 0.81 & 0.9815 & $2.75 \mathrm{E}-06$ & $3.25 E-06$ & 4.97E-07 & $3.25 \mathrm{E}-06$ & 0.96 & 0.9817 & $3.26 \mathrm{E}-06$ & $3.75 E-06$ & 4.87E-07 & 3.76E-06 \\
\hline 400 & 2.16 & 2.32 & 1.13 & 0.9818 & $2.88 \mathrm{E}-06$ & $3.38 E-06$ & 4.94E-07 & $3.38 \mathrm{E}-06$ & 1.26 & 0.9820 & $3.21 \mathrm{E}-06$ & 3.70E-06 & $4.88 \mathrm{E}-07$ & 3.71E-06 \\
\hline 500 & 2.49 & 2.69 & 1.5 & 0.9822 & $3.06 \mathrm{E}-06$ & $3.55 E-06$ & 4.91E-07 & 3.56E-06 & 1.59 & 0.9823 & $3.24 \mathrm{E}-06$ & 3.73E-06 & $4.87 \mathrm{E}-07$ & $3.74 \mathrm{E}-06$ \\
\hline 600 & 2.93 & 3.04 & 1.85 & 0.9826 & $3.14 \mathrm{E}-06$ & $3.63 E-06$ & 4.89E-07 & $3.64 \mathrm{E}-06$ & 2.03 & 0.9827 & $3.45 \mathrm{E}-06$ & $3.93 E-06$ & $4.83 \mathrm{E}-07$ & $3.95 \mathrm{E}-06$ \\
\hline 700 & 3.40 & 3.45 & 2.26 & 0.9830 & $3.29 \mathrm{E}-06$ & 3.78E-06 & $4.86 \mathrm{E}-07$ & $3.79 \mathrm{E}-06$ & 2.5 & 0.9832 & 3.64E-06 & 4.12E-06 & $4.79 \mathrm{E}-07$ & 4.14E-06 \\
\hline 800 & 3.83 & 3.87 & 2.68 & 0.9834 & $3.42 \mathrm{E}-06$ & $3.90 E-06$ & 4.84E-07 & $3.92 \mathrm{E}-06$ & 2.93 & 0.9836 & $3.73 \mathrm{E}-06$ & $4.21 E-06$ & 4.77E-07 & 4.23E-06 \\
\hline
\end{tabular}

\begin{tabular}{|c|c|c|c|c|c|c|c|c|c|c|c|c|c|c|}
\hline Capsule & OC3 & $\begin{array}{l}\text { Specimen } \\
\text { Length }\end{array}$ & $\begin{array}{l}39 \\
0.977\end{array}$ & Scale & 1" & $1.00 \mathrm{E}-03$ & & & & & & & & \\
\hline $\begin{array}{c}\text { Temperature } \\
\left({ }^{\circ} \mathrm{C}\right)\end{array}$ & Up & Down & $\begin{array}{l}\text { Delta } \\
\text { Down }\end{array}$ & $\begin{array}{l}\text { Down } \\
\text { length }\end{array}$ & CTE & CTEK & $\begin{array}{l}\text { Delta } \\
\text { CTEK }\end{array}$ & $\begin{array}{c}\text { CTE } \\
\text { adjusted }\end{array}$ & $\begin{array}{l}\text { Delta } \\
\text { Up }\end{array}$ & $\begin{array}{c}\text { Up } \\
\text { length }\end{array}$ & CTE & CTEK & $\begin{array}{l}\text { Delta } \\
\text { CTEK }\end{array}$ & $\begin{array}{c}\text { CTE } \\
\text { adjusted }\end{array}$ \\
\hline 0 & 0.98 & 1.15 & 0 & 0.9770 & & & & & 0 & 0.9770 & & & & \\
\hline 100 & 1.28 & 1.47 & 0.32 & 0.9773 & $3.28 \mathrm{E}-06$ & $3.75 E-06$ & 4.75E-07 & $3.78 \mathrm{E}-06$ & 0.3 & 0.9773 & $3.07 \mathrm{E}-06$ & $3.55 E-06$ & 4.79E-07 & 3.57E-06 \\
\hline 200 & 1.58 & 1.79 & 0.64 & 0.9776 & $3.28 \mathrm{E}-06$ & $3.75 E-06$ & $4.75 \mathrm{E}-07$ & $3.78 \mathrm{E}-06$ & 0.6 & 0.9776 & $3.07 \mathrm{E}-06$ & $3.55 E-06$ & 4.79E-07 & 3.57E-06 \\
\hline 300 & 1.90 & 2.11 & 0.96 & 0.9780 & $3.28 \mathrm{E}-06$ & $3.75 E-06$ & 4.75E-07 & $3.78 \mathrm{E}-06$ & 0.92 & 0.9779 & $3.14 \mathrm{E}-06$ & $3.62 E-06$ & 4.77E-07 & $3.64 \mathrm{E}-06$ \\
\hline 400 & 2.23 & 2.45 & 1.3 & 0.9783 & $3.33 \mathrm{E}-06$ & $3.80 E-06$ & 4.73E-07 & $3.83 \mathrm{E}-06$ & 1.25 & 0.9783 & $3.20 \mathrm{E}-06$ & $3.68 E-06$ & 4.76E-07 & 3.70E-06 \\
\hline 500 & 2.59 & 2.80 & 1.65 & 0.9787 & $3.38 \mathrm{E}-06$ & $3.85 E-06$ & 4.72E-07 & $3.88 \mathrm{E}-06$ & 1.61 & 0.9786 & 3.30E-06 & $3.77 E-06$ & 4.74E-07 & $3.80 \mathrm{E}-06$ \\
\hline 600 & 2.99 & 3.19 & 2.04 & 0.9790 & $3.48 \mathrm{E}-06$ & $3.95 E-06$ & 4.70E-07 & $3.98 \mathrm{E}-06$ & 2.01 & 0.9790 & $3.43 \mathrm{E}-06$ & $3.90 E-06$ & $4.71 \mathrm{E}-07$ & $3.93 \mathrm{E}-06$ \\
\hline 700 & 3.40 & 3.59 & 2.44 & 0.9794 & $3.57 \mathrm{E}-06$ & $4.04 E-06$ & 4.67E-07 & 4.07E-06 & 2.42 & 0.9794 & $3.54 \mathrm{E}-06$ & $4.01 E-06$ & $4.68 \mathrm{E}-07$ & 4.04E-06 \\
\hline 800 & 3.89 & 4.00 & 2.85 & 0.9799 & $3.65 \mathrm{E}-06$ & 4.11E-06 & 4.66E-07 & 4.15E-06 & 2.91 & 0.9799 & $3.72 \mathrm{E}-06$ & 4.19E-06 & 4.64E-07 & 4.22E-06 \\
\hline
\end{tabular}




\begin{tabular}{|c|c|c|c|c|c|c|c|c|c|c|c|c|c|c|}
\hline Capsule & OC3 & $\begin{array}{l}\text { Specimen } \\
\text { Length }\end{array}$ & $\begin{array}{c}46 \\
0.9852\end{array}$ & Scale & 1" & $1.00 \mathrm{E}-03$ & & & & & & & & \\
\hline $\begin{array}{c}\text { Temperature } \\
\left({ }^{\circ} \mathrm{C}\right)\end{array}$ & Up & Down & $\begin{array}{l}\text { Delta } \\
\text { Down }\end{array}$ & $\begin{array}{l}\text { Down } \\
\text { length }\end{array}$ & CTE & CTEK & $\begin{array}{l}\text { Delta } \\
\text { CTEK }\end{array}$ & $\begin{array}{c}\text { CTE } \\
\text { adjusted }\end{array}$ & $\begin{array}{l}\text { Delta } \\
\text { Up }\end{array}$ & $\begin{array}{c}\text { Up } \\
\text { length }\end{array}$ & CTE & CTEK & $\begin{array}{l}\text { Delta } \\
\text { CTEK }\end{array}$ & $\begin{array}{c}\text { CTE } \\
\text { adjusted }\end{array}$ \\
\hline 0 & 0.77 & 1.17 & 0 & 0.9852 & & & & & 0 & 0.9852 & & & & \\
\hline 100 & 0.96 & 1.30 & 0.13 & 0.9853 & $1.32 \mathrm{E}-06$ & $1.85 E-06$ & $5.30 \mathrm{E}-07$ & $1.82 \mathrm{E}-06$ & 0.19 & 0.9854 & $1.93 \mathrm{E}-06$ & $2.45 E-06$ & $5.21 \mathrm{E}-07$ & $2.43 \mathrm{E}-06$ \\
\hline 200 & 1.25 & 1.50 & 0.33 & 0.9855 & 1.67E-06 & $2.20 E-06$ & $5.25 \mathrm{E}-07$ & $2.17 \mathrm{E}-06$ & 0.48 & 0.9857 & $2.44 \mathrm{E}-06$ & $2.95 E-06$ & $5.14 \mathrm{E}-07$ & $2.94 \mathrm{E}-06$ \\
\hline 300 & 1.59 & 1.91 & 0.74 & 0.9859 & $2.50 \mathrm{E}-06$ & $3.02 E-06$ & $5.12 \mathrm{E}-07$ & $3.00 \mathrm{E}-06$ & 0.82 & 0.9860 & 2.77E-06 & $3.28 E-06$ & 5.09E-07 & 3.27E-06 \\
\hline 400 & 1.97 & 2.30 & 1.13 & 0.9863 & $2.87 \mathrm{E}-06$ & $3.38 E-06$ & $5.08 \mathrm{E}-07$ & $3.37 E-06$ & 1.2 & 0.9864 & $3.05 \mathrm{E}-06$ & $3.55 E-06$ & 5.05E-07 & $3.55 \mathrm{E}-06$ \\
\hline 500 & 2.40 & 2.73 & 1.56 & 0.9868 & 3.17E-06 & $3.67 E-06$ & 5.03E-07 & 3.67E-06 & 1.63 & 0.9868 & $3.31 \mathrm{E}-06$ & $3.81 E-06$ & $5.01 \mathrm{E}-07$ & $3.81 \mathrm{E}-06$ \\
\hline 600 & 2.86 & 3.18 & 2.01 & 0.9872 & $3.40 \mathrm{E}-06$ & $3.90 E-06$ & $5.00 \mathrm{E}-07$ & $3.90 \mathrm{E}-06$ & 2.09 & 0.9873 & $3.54 \mathrm{E}-06$ & $4.03 E-06$ & 4.97E-07 & $4.04 \mathrm{E}-06$ \\
\hline 700 & 3.35 & 3.59 & 2.42 & 0.9876 & $3.51 \mathrm{E}-06$ & $4.01 E-06$ & $4.98 \mathrm{E}-07$ & $4.01 \mathrm{E}-06$ & 2.58 & 0.9878 & $3.74 \mathrm{E}-06$ & 4.24E-06 & $4.94 \mathrm{E}-07$ & 4.24E-06 \\
\hline 800 & 3.84 & 4.12 & 2.95 & 0.9882 & $3.74 \mathrm{E}-06$ & 4.24E-06 & 4.94E-07 & 4.24E-06 & 3.07 & 0.9883 & $3.90 \mathrm{E}-06$ & $4.39 E-06$ & $4.92 \mathrm{E}-07$ & 4.40E-06 \\
\hline
\end{tabular}

\begin{tabular}{|c|c|c|c|c|c|c|c|c|c|c|c|c|c|c|}
\hline Capsule & OC3 & $\begin{array}{l}\text { Specimen } \\
\text { Length }\end{array}$ & $\begin{array}{l}48 \\
0.989\end{array}$ & Scale & $1 "$ & $1.00 \mathrm{E}-03$ & & & & & & & & \\
\hline $\begin{array}{c}\text { Temperature } \\
\left({ }^{\circ} \mathrm{C}\right)\end{array}$ & Up & Down & $\begin{array}{l}\text { Delta } \\
\text { Down }\end{array}$ & $\begin{array}{l}\text { Down } \\
\text { length }\end{array}$ & CTE & CTEK & $\begin{array}{l}\text { Delta } \\
\text { CTEK }\end{array}$ & $\begin{array}{c}\text { CTE } \\
\text { adjusted }\end{array}$ & $\begin{array}{l}\text { Delta } \\
\text { Up }\end{array}$ & $\begin{array}{c}\text { Up } \\
\text { length }\end{array}$ & CTE & CTEK & $\begin{array}{l}\text { Delta } \\
\text { CTEK }\end{array}$ & $\begin{array}{c}\text { CTE } \\
\text { adjusted }\end{array}$ \\
\hline 0 & 0.88 & 1.32 & 0 & 0.9890 & & & & & 0 & 0.9890 & & & & \\
\hline 100 & 1.16 & 1.59 & 0.27 & 0.9893 & $2.73 \mathrm{E}-06$ & $1.75 E-06$ & $-9.80 \mathrm{E}-07$ & $3.23 \mathrm{E}-06$ & 0.28 & 0.9893 & $2.83 \mathrm{E}-06$ & $2.45 E-06$ & $-3.81 \mathrm{E}-07$ & 3.33E-06 \\
\hline 200 & 1.48 & 1.88 & 0.56 & 0.9896 & $2.83 \mathrm{E}-06$ & $1.75 E-06$ & $-1.08 \mathrm{E}-06$ & $3.33 \mathrm{E}-06$ & 0.6 & 0.9896 & $3.03 \mathrm{E}-06$ & $2.35 E-06$ & $-6.83 \mathrm{E}-07$ & 3.53E-06 \\
\hline 300 & 1.86 & 2.16 & 0.84 & 0.9898 & $2.83 \mathrm{E}-06$ & $1.82 E-06$ & $-1.02 \mathrm{E}-06$ & 3.33E-06 & 0.98 & 0.9900 & $3.30 \mathrm{E}-06$ & $2.42 E-06$ & $-8.87 \mathrm{E}-07$ & 3.80E-06 \\
\hline 400 & 2.13 & 2.48 & 1.16 & 0.9902 & $2.93 \mathrm{E}-06$ & $1.95 E-06$ & $-9.82 \mathrm{E}-07$ & $3.43 \mathrm{E}-06$ & 1.25 & 0.9903 & $3.16 \mathrm{E}-06$ & $2.45 E-06$ & $-7.10 \mathrm{E}-07$ & $3.66 \mathrm{E}-06$ \\
\hline 500 & 2.56 & 2.81 & 1.49 & 0.9905 & $3.01 \mathrm{E}-06$ & $2.13 E-06$ & $-8.83 E-07$ & $3.51 \mathrm{E}-06$ & 1.68 & 0.9907 & $3.40 \mathrm{E}-06$ & $2.51 E-06$ & $-8.87 \mathrm{E}-07$ & 3.90E-06 \\
\hline 600 & 3.06 & 3.20 & 1.88 & 0.9909 & $3.17 \mathrm{E}-06$ & $2.33 E-06$ & $-8.35 \mathrm{E}-07$ & 3.67E-06 & 2.18 & 0.9912 & 3.67E-06 & $2.63 E-06$ & $-1.04 \mathrm{E}-06$ & 4.17E-06 \\
\hline 700 & 3.53 & 3.63 & 2.31 & 0.9913 & $3.34 \mathrm{E}-06$ & $2.49 E-06$ & $-8.45 E-07$ & 3.84E-06 & 2.65 & 0.9917 & $3.83 \mathrm{E}-06$ & 2.74E-06 & $-1.09 \mathrm{E}-06$ & 4.33E-06 \\
\hline 800 & 4.01 & 4.05 & 2.73 & 0.9917 & $3.45 \mathrm{E}-06$ & $2.74 E-06$ & $-7.13 \mathrm{E}-07$ & $3.95 \mathrm{E}-06$ & 3.13 & 0.9921 & $3.96 \mathrm{E}-06$ & $2.85 E-06$ & $-1.11 \mathrm{E}-06$ & 4.46E-06 \\
\hline
\end{tabular}




\begin{tabular}{|c|c|c|c|c|c|c|c|c|c|c|c|c|c|c|}
\hline Capsule & OC3 & $\begin{array}{l}\text { Specimen } \\
\text { Length }\end{array}$ & $\begin{array}{c}51 \\
0.9733\end{array}$ & Scale & 1" & $1.00 \mathrm{E}-03$ & & & & & & & & \\
\hline $\begin{array}{c}\text { Temperature } \\
\left({ }^{\circ} \mathrm{C}\right)\end{array}$ & Up & Down & $\begin{array}{l}\text { Delta } \\
\text { Down }\end{array}$ & $\begin{array}{l}\text { Down } \\
\text { length }\end{array}$ & CTE & CTEK & $\begin{array}{l}\text { Delta } \\
\text { CTEK }\end{array}$ & $\begin{array}{c}\text { CTE } \\
\text { adjusted }\end{array}$ & $\begin{array}{l}\text { Delta } \\
\text { Up }\end{array}$ & $\begin{array}{c}\text { Up } \\
\text { length }\end{array}$ & CTE & CTEK & $\begin{array}{l}\text { Delta } \\
\text { CTEK }\end{array}$ & $\begin{array}{c}\text { CTE } \\
\text { adjusted }\end{array}$ \\
\hline 0 & 0.80 & 0.71 & 0 & 0.9733 & & & & & 0 & 0.9733 & & & & \\
\hline 100 & 1.12 & 1.07 & 0.36 & 0.9737 & 3.70E-06 & $4.15 E-06$ & $4.51 \mathrm{E}-07$ & $4.20 \mathrm{E}-06$ & 0.32 & 0.9736 & $3.29 \mathrm{E}-06$ & $3.75 E-06$ & 4.62E-07 & 3.79E-06 \\
\hline 200 & 1.46 & 1.43 & 0.72 & 0.9740 & 3.70E-06 & $4.15 E-06$ & 4.51E-07 & $4.20 \mathrm{E}-06$ & 0.66 & 0.9740 & $3.39 \mathrm{E}-06$ & $3.85 E-06$ & 4.59E-07 & 3.89E-06 \\
\hline 300 & 1.90 & 1.80 & 1.09 & 0.9744 & $3.73 \mathrm{E}-06$ & 4.18E-06 & $4.50 \mathrm{E}-07$ & $4.23 \mathrm{E}-06$ & 1.1 & 0.9744 & 3.77E-06 & $4.22 E-06$ & 4.49E-07 & 4.27E-06 \\
\hline 400 & 2.26 & 2.20 & 1.49 & 0.9748 & $3.83 \mathrm{E}-06$ & $4.28 E-06$ & $4.48 \mathrm{E}-07$ & $4.33 \mathrm{E}-06$ & 1.46 & 0.9748 & $3.75 \mathrm{E}-06$ & 4.20E-06 & $4.50 \mathrm{E}-07$ & $4.25 \mathrm{E}-06$ \\
\hline 500 & 12.67 & 2.63 & 1.92 & 0.9752 & $3.95 \mathrm{E}-06$ & 4.39E-06 & $4.45 \mathrm{E}-07$ & $4.45 \mathrm{E}-06$ & 11.87 & 0.9852 & $2.44 \mathrm{E}-05$ & 4.29E-06 & $-2.01 E-05$ & $2.49 \mathrm{E}-05$ \\
\hline 600 & 3.12 & 3.13 & 2.42 & 0.9757 & 4.14E-06 & $4.58 E-06$ & 4.39E-07 & $4.64 \mathrm{E}-06$ & 2.32 & 0.9756 & $3.97 \mathrm{E}-06$ & $4.42 E-06$ & $4.43 \mathrm{E}-07$ & 4.47E-06 \\
\hline 700 & 3.61 & 3.61 & 2.9 & 0.9762 & 4.26E-06 & 4.69E-06 & 4.35E-07 & $4.76 \mathrm{E}-06$ & 2.81 & 0.9761 & $4.12 \mathrm{E}-06$ & 4.56E-06 & $4.40 \mathrm{E}-07$ & $4.62 \mathrm{E}-06$ \\
\hline 800 & 4.11 & 4.16 & 3.45 & 0.9768 & 4.43E-06 & $4.86 E-06$ & $4.31 \mathrm{E}-07$ & 4.93E-06 & 3.31 & 0.9766 & $4.25 \mathrm{E}-06$ & $4.69 E-06$ & $4.36 \mathrm{E}-07$ & 4.75E-06 \\
\hline
\end{tabular}

\begin{tabular}{|c|c|c|c|c|c|c|c|c|c|c|c|c|c|c|}
\hline Capsule & OC3 & $\begin{array}{l}\text { Specimen } \\
\text { Length }\end{array}$ & $\begin{array}{c}52 \\
0.9607\end{array}$ & Scale & 1" & $1.00 \mathrm{E}-03$ & & & & & & & & \\
\hline $\begin{array}{c}\text { Temperature } \\
\left({ }^{\circ} \mathrm{C}\right)\end{array}$ & Up & Down & $\begin{array}{l}\text { Delta } \\
\text { Down }\end{array}$ & $\begin{array}{l}\text { Down } \\
\text { length }\end{array}$ & CTE & CTEK & $\begin{array}{l}\text { Delta } \\
\text { CTEK }\end{array}$ & $\begin{array}{c}\text { CTE } \\
\text { adjusted }\end{array}$ & $\begin{array}{l}\text { Delta } \\
\text { Up }\end{array}$ & $\begin{array}{c}\text { Up } \\
\text { length }\end{array}$ & CTE & CTEK & $\begin{array}{l}\text { Delta } \\
\text { CTEK }\end{array}$ & $\begin{array}{c}\text { CTE } \\
\text { adjusted }\end{array}$ \\
\hline 0 & 0.92 & 0.65 & 0 & 0.9607 & & & & & 0 & 0.9607 & & & & \\
\hline 100 & 1.23 & 1.01 & 0.36 & 0.9611 & $3.75 \mathrm{E}-06$ & 4.15E-06 & $4.03 \mathrm{E}-07$ & 4.25E-06 & 0.31 & 0.9610 & $3.23 \mathrm{E}-06$ & $3.65 E-06$ & 4.23E-07 & 3.73E-06 \\
\hline 200 & 1.58 & 1.40 & 0.75 & 0.9615 & 3.90E-06 & 4.30E-06 & 3.97E-07 & 4.40E-06 & 0.66 & 0.9614 & $3.43 \mathrm{E}-06$ & $3.85 E-06$ & 4.15E-07 & 3.93E-06 \\
\hline 300 & 1.93 & 1.78 & 1.13 & 0.9618 & $3.92 \mathrm{E}-06$ & $4.32 E-06$ & $3.95 \mathrm{E}-07$ & 4.42E-06 & 1.01 & 0.9617 & $3.50 \mathrm{E}-06$ & $3.92 E-06$ & $4.12 \mathrm{E}-07$ & 4.00E-06 \\
\hline 400 & 2.30 & 2.16 & 1.51 & 0.9622 & $3.93 \mathrm{E}-06$ & $4.33 E-06$ & 3.96E-07 & 4.43E-06 & 1.38 & 0.9621 & 3.59E-06 & 4.00E-06 & 4.09E-07 & 4.09E-06 \\
\hline 500 & 2.73 & 2.62 & 1.97 & 0.9627 & 4.10E-06 & 4.49E-06 & 3.89E-07 & 4.60E-06 & 1.81 & 0.9625 & 3.77E-06 & 4.17E-06 & $4.02 \mathrm{E}-07$ & 4.27E-06 \\
\hline 600 & 3.19 & 3.13 & 2.48 & 0.9632 & 4.30E-06 & $4.68 E-06$ & $3.81 \mathrm{E}-07$ & $4.80 \mathrm{E}-06$ & 2.27 & 0.9630 & $3.94 \mathrm{E}-06$ & $4.33 E-06$ & $3.95 \mathrm{E}-07$ & 4.44E-06 \\
\hline 700 & 3.69 & 3.65 & 3 & 0.9637 & 4.46E-06 & $4.84 E-06$ & $3.74 \mathrm{E}-07$ & 4.96E-06 & 2.77 & 0.9635 & $4.12 \mathrm{E}-06$ & $4.51 E-06$ & $3.88 \mathrm{E}-07$ & 4.62E-06 \\
\hline 800 & 4.23 & 4.23 & 3.58 & 0.9643 & 4.66E-06 & $5.03 E-06$ & 3.67E-07 & $5.16 \mathrm{E}-06$ & 3.31 & 0.9640 & $4.31 \mathrm{E}-06$ & $4.69 E-06$ & $3.80 \mathrm{E}-07$ & $4.81 \mathrm{E}-06$ \\
\hline
\end{tabular}




\begin{tabular}{|c|c|c|c|c|c|c|c|c|c|c|c|c|c|c|}
\hline Capsule & OC3 & $\begin{array}{l}\text { Specimen } \\
\text { Length }\end{array}$ & $\begin{array}{c}53 \\
0.9761\end{array}$ & Scale & 1" & $1.00 \mathrm{E}-03$ & & & & & & & & \\
\hline $\begin{array}{c}\text { Temperature } \\
\left({ }^{\circ} \mathrm{C}\right)\end{array}$ & Up & Down & $\begin{array}{l}\text { Delta } \\
\text { Down }\end{array}$ & $\begin{array}{l}\text { Down } \\
\text { length }\end{array}$ & CTE & CTEK & $\begin{array}{l}\text { Delta } \\
\text { CTEK }\end{array}$ & $\begin{array}{c}\text { CTE } \\
\text { adjusted }\end{array}$ & $\begin{array}{l}\text { Delta } \\
\text { Up }\end{array}$ & $\begin{array}{c}\text { Up } \\
\text { length }\end{array}$ & CTE & CTEK & $\begin{array}{l}\text { Delta } \\
\text { CTEK }\end{array}$ & $\begin{array}{c}\text { CTE } \\
\text { adjusted }\end{array}$ \\
\hline 0 & 0.88 & 1.00 & 0 & 0.9761 & & & & & 0 & 0.9761 & & & & \\
\hline 100 & 1.17 & 1.29 & 0.29 & 0.9764 & 2.97E-06 & $3.45 E-06$ & 4.79E-07 & $3.47 \mathrm{E}-06$ & 0.29 & 0.9764 & 2.97E-06 & $3.45 E-06$ & 4.79E-07 & $3.47 \mathrm{E}-06$ \\
\hline 200 & 1.50 & 1.59 & 0.59 & 0.9767 & $3.02 E-06$ & $3.50 E-06$ & $4.78 \mathrm{E}-07$ & $3.52 \mathrm{E}-06$ & 0.62 & 0.9767 & $3.18 \mathrm{E}-06$ & $3.65 E-06$ & 4.74E-07 & $3.68 \mathrm{E}-06$ \\
\hline 300 & 1.90 & 1.88 & 0.88 & 0.9770 & $3.01 \mathrm{E}-06$ & $3.48 E-06$ & $4.78 \mathrm{E}-07$ & $3.51 \mathrm{E}-06$ & 1.02 & 0.9771 & $3.48 \mathrm{E}-06$ & $3.95 E-06$ & 4.67E-07 & $3.98 \mathrm{E}-06$ \\
\hline 400 & 2.28 & 2.23 & 1.23 & 0.9773 & $3.15 \mathrm{E}-06$ & $3.63 E-06$ & $4.75 \mathrm{E}-07$ & $3.65 \mathrm{E}-06$ & 1.4 & 0.9775 & 3.59E-06 & $4.05 E-06$ & 4.64E-07 & 4.09E-06 \\
\hline 500 & 2.70 & 2.65 & 1.65 & 0.9778 & $3.38 \mathrm{E}-06$ & $3.85 E-06$ & 4.69E-07 & $3.88 \mathrm{E}-06$ & 1.82 & 0.9779 & $3.73 \mathrm{E}-06$ & 4.19E-06 & $4.61 \mathrm{E}-07$ & 4.23E-06 \\
\hline 600 & 3.15 & 3.10 & 2.1 & 0.9782 & 3.59E-06 & $4.05 E-06$ & 4.64E-07 & 4.09E-06 & 2.27 & 0.9784 & $3.88 \mathrm{E}-06$ & $4.33 E-06$ & 4.57E-07 & $4.38 \mathrm{E}-06$ \\
\hline 700 & 3.60 & 3.54 & 2.54 & 0.9786 & $3.72 \mathrm{E}-06$ & 4.18E-06 & 4.61E-07 & $4.22 \mathrm{E}-06$ & 2.72 & 0.9788 & $3.98 \mathrm{E}-06$ & 4.44E-06 & $4.54 \mathrm{E}-07$ & $4.48 \mathrm{E}-06$ \\
\hline 800 & 4.09 & 4.09 & 3.09 & 0.9792 & $3.96 \mathrm{E}-06$ & $4.41 E-06$ & 4.55E-07 & 4.46E-06 & 3.21 & 0.9793 & $4.11 \mathrm{E}-06$ & $4.56 E-06$ & $4.51 \mathrm{E}-07$ & 4.61E-06 \\
\hline
\end{tabular}

\begin{tabular}{|c|c|c|c|c|c|c|c|c|c|c|c|c|c|c|}
\hline Capsule & OC3 & $\begin{array}{l}\text { Specimen } \\
\text { Length }\end{array}$ & $\begin{array}{c}\mathbf{5 4} \\
0.9605\end{array}$ & Scale & 1" & $1.00 \mathrm{E}-03$ & & & & & & & & \\
\hline $\begin{array}{c}\text { Temperature } \\
\left({ }^{\circ} \mathrm{C}\right)\end{array}$ & Up & Down & $\begin{array}{l}\text { Delta } \\
\text { Down }\end{array}$ & $\begin{array}{l}\text { Down } \\
\text { length }\end{array}$ & CTE & CTEK & $\begin{array}{l}\text { Delta } \\
\text { CTEK }\end{array}$ & $\begin{array}{c}\text { CTE } \\
\text { adjusted }\end{array}$ & $\begin{array}{l}\text { Delta } \\
\text { Up }\end{array}$ & $\begin{array}{c}\text { Up } \\
\text { length }\end{array}$ & CTE & CTEK & $\begin{array}{l}\text { Delta } \\
\text { CTEK }\end{array}$ & $\begin{array}{c}\text { CTE } \\
\text { adjusted }\end{array}$ \\
\hline 0 & 0.83 & 0.92 & 0 & 0.9605 & & & & & 0 & 0.9605 & & & & \\
\hline 100 & 1.23 & 1.31 & 0.39 & 0.9609 & 4.06E-06 & 4.45E-06 & $3.90 \mathrm{E}-07$ & 4.56E-06 & 0.4 & 0.9609 & $4.16 \mathrm{E}-06$ & $4.55 E-06$ & 3.86E-07 & 4.66E-06 \\
\hline 200 & 1.65 & 1.73 & 0.81 & 0.9613 & $4.22 \mathrm{E}-06$ & 4.60E-06 & $3.83 \mathrm{E}-07$ & $4.72 \mathrm{E}-06$ & 0.82 & 0.9613 & $4.27 \mathrm{E}-06$ & $4.65 E-06$ & $3.81 \mathrm{E}-07$ & 4.77E-06 \\
\hline 300 & 2.08 & 2.15 & 1.23 & 0.9617 & 4.27E-06 & $4.65 E-06$ & $3.81 \mathrm{E}-07$ & 4.77E-06 & 1.25 & 0.9618 & $4.34 \mathrm{E}-06$ & $4.72 E-06$ & $3.78 \mathrm{E}-07$ & 4.84E-06 \\
\hline 400 & 2.52 & 2.59 & 1.67 & 0.9622 & $4.35 \mathrm{E}-06$ & $4.73 E-06$ & $3.78 \mathrm{E}-07$ & $4.85 \mathrm{E}-06$ & 1.69 & 0.9622 & $4.40 \mathrm{E}-06$ & $4.78 E-06$ & 3.76E-07 & 4.90E-06 \\
\hline 500 & 3.00 & 3.07 & 2.15 & 0.9627 & $4.48 \mathrm{E}-06$ & $4.85 E-06$ & $3.73 \mathrm{E}-07$ & 4.98E-06 & 2.17 & 0.9627 & $4.52 \mathrm{E}-06$ & 4.89E-06 & $3.72 \mathrm{E}-07$ & $5.02 \mathrm{E}-06$ \\
\hline 600 & 3.50 & 3.58 & 2.66 & 0.9632 & $4.62 \mathrm{E}-06$ & $4.98 E-06$ & 3.67E-07 & $5.12 \mathrm{E}-06$ & 2.67 & 0.9632 & $4.63 \mathrm{E}-06$ & $5.00 E-06$ & 3.67E-07 & 5.13E-06 \\
\hline 700 & 4.06 & 4.14 & 3.22 & 0.9637 & 4.79E-06 & 5.15E-06 & $3.61 \mathrm{E}-07$ & 5.29E-06 & 3.23 & 0.9637 & $4.80 \mathrm{E}-06$ & 5.16E-06 & $3.60 \mathrm{E}-07$ & 5.30E-06 \\
\hline 800 & 4.66 & 4.74 & 3.82 & 0.9643 & 4.97E-06 & $5.33 E-06$ & $3.54 \mathrm{E}-07$ & $5.47 \mathrm{E}-06$ & 3.83 & 0.9643 & $4.98 \mathrm{E}-06$ & $5.34 E-06$ & 3.53E-07 & $5.48 \mathrm{E}-06$ \\
\hline
\end{tabular}




\begin{tabular}{|c|c|c|c|c|c|c|c|c|c|c|c|c|c|c|}
\hline Capsule & OC3 & $\begin{array}{l}\text { Specimen } \\
\text { Length }\end{array}$ & $\begin{array}{c}61 \\
0.9823\end{array}$ & Scale & 1" & $1.00 \mathrm{E}-03$ & & & & & & & & \\
\hline $\begin{array}{c}\text { Temperature } \\
\left({ }^{\circ} \mathrm{C}\right)\end{array}$ & Up & Down & $\begin{array}{l}\text { Delta } \\
\text { Down }\end{array}$ & $\begin{array}{l}\text { Down } \\
\text { length }\end{array}$ & CTE & CTEK & $\begin{array}{l}\text { Delta } \\
\text { CTEK }\end{array}$ & $\begin{array}{c}\text { CTE } \\
\text { adjusted }\end{array}$ & $\begin{array}{l}\text { Delta } \\
\text { Up }\end{array}$ & $\begin{array}{c}\text { Up } \\
\text { length }\end{array}$ & CTE & CTEK & $\begin{array}{l}\text { Delta } \\
\text { CTEK }\end{array}$ & $\begin{array}{c}\text { CTE } \\
\text { adjusted }\end{array}$ \\
\hline 0 & 0.75 & 0.95 & 0 & 0.9823 & & & & & 0 & 0.9823 & & & & \\
\hline 100 & 1.08 & 1.29 & 0.34 & 0.9826 & $3.46 \mathrm{E}-06$ & $3.95 E-06$ & 4.89E-07 & $3.96 \mathrm{E}-06$ & 0.33 & 0.9826 & $3.36 \mathrm{E}-06$ & $3.85 E-06$ & 4.91E-07 & $3.86 \mathrm{E}-06$ \\
\hline 200 & 1.47 & 1.63 & 0.68 & 0.9830 & $3.46 \mathrm{E}-06$ & $3.95 E-06$ & 4.89E-07 & $3.96 \mathrm{E}-06$ & 0.72 & 0.9830 & $3.66 \mathrm{E}-06$ & 4.15E-06 & $4.85 \mathrm{E}-07$ & 4.16E-06 \\
\hline 300 & 1.86 & 2.01 & 1.06 & 0.9834 & 3.60E-06 & $4.08 E-06$ & $4.86 \mathrm{E}-07$ & 4.10E-06 & 1.11 & 0.9834 & 3.77E-06 & $4.25 E-06$ & 4.83E-07 & 4.27E-06 \\
\hline 400 & 2.28 & 2.43 & 1.48 & 0.9838 & 3.77E-06 & $4.25 E-06$ & $4.83 \mathrm{E}-07$ & 4.27E-06 & 1.53 & 0.9838 & 3.89E-06 & $4.38 E-06$ & $4.81 \mathrm{E}-07$ & 4.39E-06 \\
\hline 500 & 2.79 & 2.90 & 1.95 & 0.9843 & $3.97 \mathrm{E}-06$ & 4.45E-06 & $4.80 \mathrm{E}-07$ & 4.47E-06 & 2.04 & 0.9843 & 4.15E-06 & 4.63E-06 & 4.76E-07 & 4.65E-06 \\
\hline 600 & 3.30 & 3.40 & 2.45 & 0.9848 & 4.16E-06 & $4.63 E-06$ & $4.76 \mathrm{E}-07$ & $4.66 \mathrm{E}-06$ & 2.55 & 0.9849 & 4.33E-06 & 4.80E-06 & $4.73 E-07$ & $4.83 \mathrm{E}-06$ \\
\hline 700 & 3.87 & 3.90 & 2.95 & 0.9853 & $4.29 \mathrm{E}-06$ & 4.76E-06 & $4.74 \mathrm{E}-07$ & 4.79E-06 & 3.12 & 0.9854 & $4.54 \mathrm{E}-06$ & $5.01 E-06$ & $4.70 \mathrm{E}-07$ & $5.04 \mathrm{E}-06$ \\
\hline 800 & 4.46 & 4.50 & 3.55 & 0.9859 & $4.52 \mathrm{E}-06$ & 4.99E-06 & 4.70E-07 & $5.02 \mathrm{E}-06$ & 3.71 & 0.9860 & $4.72 \mathrm{E}-06$ & $5.19 E-06$ & 4.66E-07 & $5.22 \mathrm{E}-06$ \\
\hline
\end{tabular}

\begin{tabular}{|c|c|c|c|c|c|c|c|c|c|c|c|c|c|c|}
\hline Capsule & OC3 & $\begin{array}{l}\text { Specimen } \\
\text { Length }\end{array}$ & $\begin{array}{c}62 \\
0.9810\end{array}$ & Scale & 1" & $1.00 \mathrm{E}-03$ & & & & & & & & \\
\hline $\begin{array}{c}\text { Temperature } \\
\left({ }^{\circ} \mathrm{C}\right)\end{array}$ & Up & Down & $\begin{array}{l}\text { Delta } \\
\text { Down }\end{array}$ & $\begin{array}{l}\text { Down } \\
\text { length }\end{array}$ & CTE & CTEK & $\begin{array}{l}\text { Delta } \\
\text { CTEK }\end{array}$ & $\begin{array}{c}\text { CTE } \\
\text { adjusted }\end{array}$ & $\begin{array}{l}\text { Delta } \\
\text { Up }\end{array}$ & $\begin{array}{c}\text { Up } \\
\text { length }\end{array}$ & CTE & CTEK & $\begin{array}{l}\text { Delta } \\
\text { CTEK }\end{array}$ & $\begin{array}{c}\text { CTE } \\
\text { adjusted }\end{array}$ \\
\hline 0 & 1.00 & 1.00 & 0 & 0.9810 & & & & & 0 & 0.9810 & & & & \\
\hline 100 & 1.30 & 1.30 & 0.3 & 0.9813 & $3.06 \mathrm{E}-06$ & $3.55 E-06$ & $4.92 \mathrm{E}-07$ & $3.56 \mathrm{E}-06$ & 0.3 & 0.9813 & $3.06 \mathrm{E}-06$ & $3.55 E-06$ & $4.92 \mathrm{E}-07$ & 3.56E-06 \\
\hline 200 & 1.65 & 1.61 & 0.61 & 0.9816 & $3.11 \mathrm{E}-06$ & $3.60 E-06$ & 4.91E-07 & $3.61 \mathrm{E}-06$ & 0.65 & 0.9817 & $3.31 \mathrm{E}-06$ & $3.80 E-06$ & 4.87E-07 & $3.81 \mathrm{E}-06$ \\
\hline 300 & 2.05 & 1.97 & 0.97 & 0.9820 & $3.30 \mathrm{E}-06$ & $3.78 E-06$ & 4.87E-07 & $3.80 \mathrm{E}-06$ & 1.05 & 0.9821 & 3.57E-06 & $4.05 E-06$ & $4.82 \mathrm{E}-07$ & 4.07E-06 \\
\hline 400 & 2.38 & 2.31 & 1.31 & 0.9823 & $3.34 \mathrm{E}-06$ & $3.83 E-06$ & 4.87E-07 & $3.84 \mathrm{E}-06$ & 1.38 & 0.9824 & $3.52 \mathrm{E}-06$ & 4.00E-06 & 4.83E-07 & 4.02E-06 \\
\hline 500 & 2.75 & 2.71 & 1.71 & 0.9827 & $3.49 \mathrm{E}-06$ & $3.97 E-06$ & 4.84E-07 & 3.99E-06 & 1.75 & 0.9828 & 3.57E-06 & $4.05 E-06$ & 4.82E-07 & 4.07E-06 \\
\hline 600 & 3.17 & 3.17 & 2.17 & 0.9832 & $3.69 \mathrm{E}-06$ & $4.17 E-06$ & 4.79E-07 & 4.19E-06 & 2.17 & 0.9832 & 3.69E-06 & 4.17E-06 & 4.79E-07 & 4.19E-06 \\
\hline 700 & 3.60 & 3.63 & 2.63 & 0.9836 & $3.83 \mathrm{E}-06$ & $4.31 E-06$ & 4.77E-07 & 4.33E-06 & 2.6 & 0.9836 & $3.79 \mathrm{E}-06$ & 4.26E-06 & $4.78 \mathrm{E}-07$ & 4.29E-06 \\
\hline 800 & 4.07 & 4.12 & 3.12 & 0.9841 & $3.98 \mathrm{E}-06$ & 4.45E-06 & $4.74 \mathrm{E}-07$ & 4.48E-06 & 3.07 & 0.9841 & $3.91 \mathrm{E}-06$ & $4.39 E-06$ & 4.75E-07 & 4.41E-06 \\
\hline
\end{tabular}




\begin{tabular}{|c|c|c|c|c|c|c|c|c|c|c|c|c|c|c|}
\hline Capsule & OC3 & $\begin{array}{l}\text { Specimen } \\
\text { Length }\end{array}$ & $\begin{array}{c}66 \\
0.9959\end{array}$ & Scale & 1" & $1.00 \mathrm{E}-03$ & & & & & & & & \\
\hline $\begin{array}{c}\text { Temperature } \\
\left({ }^{\circ} \mathrm{C}\right)\end{array}$ & Up & Down & $\begin{array}{l}\text { Delta } \\
\text { Down }\end{array}$ & $\begin{array}{l}\text { Down } \\
\text { length }\end{array}$ & CTE & CTEK & $\begin{array}{l}\text { Delta } \\
\text { CTEK }\end{array}$ & $\begin{array}{c}\text { CTE } \\
\text { adjusted }\end{array}$ & $\begin{array}{l}\text { Delta } \\
\text { Up }\end{array}$ & $\begin{array}{c}\text { Up } \\
\text { length }\end{array}$ & CTE & CTEK & $\begin{array}{l}\text { Delta } \\
\text { CTEK }\end{array}$ & $\begin{array}{c}\text { CTE } \\
\text { adjusted }\end{array}$ \\
\hline 0 & 0.92 & 0.45 & 0 & 0.9959 & & & & & 0 & 0.9959 & & & & \\
\hline 100 & 1.13 & 0.61 & 0.16 & 0.9961 & $1.61 \mathrm{E}-06$ & $3.55 E-06$ & $1.94 \mathrm{E}-06$ & $2.11 \mathrm{E}-06$ & 0.21 & 0.9961 & $2.11 \mathrm{E}-06$ & $3.65 E-06$ & $1.54 \mathrm{E}-06$ & $2.61 \mathrm{E}-06$ \\
\hline 200 & 1.40 & 0.83 & 0.38 & 0.9963 & $1.91 \mathrm{E}-06$ & $3.65 E-06$ & $1.74 \mathrm{E}-06$ & $2.41 \mathrm{E}-06$ & 0.48 & 0.9964 & $2.41 \mathrm{E}-06$ & 3.80E-06 & 1.39E-06 & 2.91E-06 \\
\hline 300 & 1.94 & 1.20 & 0.75 & 0.9967 & $2.51 \mathrm{E}-06$ & $3.82 E-06$ & $1.31 \mathrm{E}-06$ & $3.01 \mathrm{E}-06$ & 1.02 & 0.9969 & $3.41 \mathrm{E}-06$ & $4.02 E-06$ & $6.02 \mathrm{E}-07$ & 3.91E-06 \\
\hline 400 & 2.69 & 1.79 & 1.34 & 0.9972 & 3.36E-06 & $4.08 E-06$ & 7.11E-07 & $3.86 \mathrm{E}-06$ & 1.77 & 0.9977 & 4.44E-06 & 4.13E-06 & $-3.18 \mathrm{E}-07$ & $4.94 \mathrm{E}-06$ \\
\hline 500 & 3.60 & 2.69 & 2.24 & 0.9981 & 4.50E-06 & $4.25 E-06$ & $-2.48 \mathrm{E}-07$ & $5.00 \mathrm{E}-06$ & 2.68 & 0.9986 & $5.38 \mathrm{E}-06$ & 4.29E-06 & $-1.09 E-06$ & $5.88 \mathrm{E}-06$ \\
\hline 600 & 4.63 & 3.71 & 3.26 & 0.9992 & $5.46 \mathrm{E}-06$ & 4.40E-06 & $-1.06 \mathrm{E}-06$ & $5.96 \mathrm{E}-06$ & 3.71 & 0.9996 & $6.21 \mathrm{E}-06$ & $4.42 E-06$ & $-1.79 \mathrm{E}-06$ & $6.71 E-06$ \\
\hline 700 & 5.78 & 5.05 & 4.6 & 1.0005 & $6.60 \mathrm{E}-06$ & 4.46E-06 & $-2.13 E-06$ & $7.10 \mathrm{E}-06$ & 4.86 & 1.0008 & 6.97E-06 & $4.62 E-06$ & $-2.35 E-06$ & 7.47E-06 \\
\hline 800 & 7.08 & 7.15 & 6.7 & 1.0026 & $8.41 \mathrm{E}-06$ & 4.59E-06 & $-3.82 \mathrm{E}-06$ & 8.91E-06 & 6.16 & 1.0021 & $7.73 \mathrm{E}-06$ & $4.85 E-06$ & $-2.88 \mathrm{E}-06$ & $8.23 \mathrm{E}-06$ \\
\hline
\end{tabular}

\begin{tabular}{|c|c|c|c|c|c|c|c|c|c|c|c|c|c|c|}
\hline Capsule & OC3 & $\begin{array}{l}\text { Specimen } \\
\text { Length }\end{array}$ & $\begin{array}{c}69 \\
0.9979\end{array}$ & Scale & $1 "$ & $1.00 \mathrm{E}-03$ & & & & & & & & \\
\hline $\begin{array}{c}\text { Temperature } \\
\left({ }^{\circ} \mathrm{C}\right)\end{array}$ & Up & Down & $\begin{array}{l}\text { Delta } \\
\text { Down }\end{array}$ & $\begin{array}{l}\text { Down } \\
\text { length }\end{array}$ & CTE & CTEK & $\begin{array}{l}\text { Delta } \\
\text { CTEK }\end{array}$ & $\begin{array}{c}\text { CTE } \\
\text { adjusted }\end{array}$ & $\begin{array}{l}\text { Delta } \\
\text { Up }\end{array}$ & $\begin{array}{c}\text { Up } \\
\text { length }\end{array}$ & CTE & CTEK & $\begin{array}{l}\text { Delta } \\
\text { CTEK }\end{array}$ & $\begin{array}{c}\text { CTE } \\
\text { adjusted }\end{array}$ \\
\hline 0 & 0.99 & 1.11 & 0 & 0.9979 & & & & & 0 & 0.9979 & & & & \\
\hline 100 & 1.01 & 1.06 & -0.05 & 0.9979 & $-5.01 E-07$ & 4.50E-07 & $9.51 \mathrm{E}-07$ & $-1.05 E-09$ & 0.02 & 0.9979 & $2.00 \mathrm{E}-07$ & $5.50 E-07$ & $3.50 \mathrm{E}-07$ & $7.00 \mathrm{E}-07$ \\
\hline 200 & 1.10 & 1.05 & -0.06 & 0.9978 & $-3.01 \mathrm{E}-07$ & $5.00 E-07$ & $8.01 \mathrm{E}-07$ & 1.99E-07 & 0.11 & 0.9980 & $5.51 \mathrm{E}-07$ & $6.50 E-07$ & $9.88 \mathrm{E}-08$ & $1.05 \mathrm{E}-06$ \\
\hline 300 & 1.18 & 1.10 & -0.01 & 0.9979 & $-3.34 \mathrm{E}-08$ & $5.50 E-07$ & $5.83 \mathrm{E}-07$ & 4.67E-07 & 0.19 & 0.9981 & $6.35 \mathrm{E}-07$ & $6.83 E-07$ & $4.83 \mathrm{E}-08$ & 1.13E-06 \\
\hline 400 & 1.33 & 1.21 & 0.1 & 0.9980 & $2.51 \mathrm{E}-07$ & $6.00 E-07$ & $3.49 \mathrm{E}-07$ & 7.51E-07 & 0.34 & 0.9982 & $8.52 \mathrm{E}-07$ & $7.25 E-07$ & $-1.27 \mathrm{E}-07$ & 1.35E-06 \\
\hline 500 & 1.57 & 1.46 & 0.35 & 0.9983 & 7.01E-07 & $6.90 E-07$ & $-1.15 E-08$ & $1.20 \mathrm{E}-06$ & 0.58 & 0.9985 & $1.16 \mathrm{E}-06$ & 7.70E-07 & $-3.92 \mathrm{E}-07$ & $1.66 \mathrm{E}-06$ \\
\hline 600 & 1.90 & 1.76 & 0.65 & 0.9986 & $1.09 \mathrm{E}-06$ & $7.66 E-07$ & $-3.20 \mathrm{E}-07$ & $1.59 \mathrm{E}-06$ & 0.91 & 0.9988 & $1.52 \mathrm{E}-06$ & $8.50 E-07$ & $-6.70 \mathrm{E}-07$ & $2.02 \mathrm{E}-06$ \\
\hline 700 & 2.29 & 2.16 & 1.05 & 0.9990 & $1.50 \mathrm{E}-06$ & $8.50 E-07$ & $-6.53 E-07$ & $2.00 \mathrm{E}-06$ & 1.3 & 0.9992 & $1.86 \mathrm{E}-06$ & 9.21E-07 & $-9.40 \mathrm{E}-07$ & $2.36 \mathrm{E}-06$ \\
\hline 800 & 2.78 & 2.78 & 1.67 & 0.9996 & $2.09 \mathrm{E}-06$ & $9.75 E-07$ & $-1.12 \mathrm{E}-06$ & $2.59 \mathrm{E}-06$ & 1.79 & 0.9997 & $2.24 \mathrm{E}-06$ & $1.00 E-06$ & $-1.24 \mathrm{E}-06$ & $2.74 \mathrm{E}-06$ \\
\hline
\end{tabular}




\begin{tabular}{|c|c|c|c|c|c|c|c|c|c|c|c|c|c|c|}
\hline Capsule & OC3 & $\begin{array}{l}\text { Specimen } \\
\text { Length }\end{array}$ & $\begin{array}{c}71 \\
0.9976\end{array}$ & Scale & 1" & $1.00 \mathrm{E}-03$ & & & & & & & & \\
\hline $\begin{array}{c}\text { Temperature } \\
\left({ }^{\circ} \mathrm{C}\right)\end{array}$ & Up & Down & $\begin{array}{l}\text { Delta } \\
\text { Down }\end{array}$ & $\begin{array}{l}\text { Down } \\
\text { length }\end{array}$ & CTE & CTEK & $\begin{array}{l}\text { Delta } \\
\text { CTEK }\end{array}$ & $\begin{array}{c}\text { CTE } \\
\text { adjusted }\end{array}$ & $\begin{array}{l}\text { Delta } \\
\text { Up }\end{array}$ & $\begin{array}{c}\text { Up } \\
\text { length }\end{array}$ & CTE & CTEK & $\begin{array}{l}\text { Delta } \\
\text { CTEK }\end{array}$ & $\begin{array}{c}\text { CTE } \\
\text { adjusted }\end{array}$ \\
\hline 0 & 0.88 & 1.08 & 0 & 0.9976 & & & & & 0 & 0.9976 & & & & \\
\hline 100 & 0.96 & 1.17 & 0.09 & 0.9977 & $9.02 \mathrm{E}-07$ & $3.55 E-06$ & $2.65 \mathrm{E}-06$ & $1.40 \mathrm{E}-06$ & 0.08 & 0.9977 & $8.02 \mathrm{E}-07$ & $3.65 E-06$ & $2.85 \mathrm{E}-06$ & $1.30 \mathrm{E}-06$ \\
\hline 200 & 1.17 & 1.33 & 0.25 & 0.9979 & $1.25 \mathrm{E}-06$ & $3.65 E-06$ & $2.40 \mathrm{E}-06$ & $1.75 \mathrm{E}-06$ & 0.29 & 0.9979 & $1.45 \mathrm{E}-06$ & $3.80 E-06$ & $2.35 \mathrm{E}-06$ & $1.95 \mathrm{E}-06$ \\
\hline 300 & 1.41 & 1.53 & 0.45 & 0.9981 & $1.50 \mathrm{E}-06$ & $3.82 E-06$ & $2.31 \mathrm{E}-06$ & $2.00 \mathrm{E}-06$ & 0.53 & 0.9981 & 1.77E-06 & $4.02 E-06$ & $2.25 \mathrm{E}-06$ & 2.27E-06 \\
\hline 400 & 1.74 & 1.81 & 0.73 & 0.9983 & $1.83 \mathrm{E}-06$ & $4.08 E-06$ & $2.25 \mathrm{E}-06$ & $2.33 \mathrm{E}-06$ & 0.86 & 0.9985 & $2.16 \mathrm{E}-06$ & 4.13E-06 & $1.97 \mathrm{E}-06$ & $2.66 \mathrm{E}-06$ \\
\hline 500 & 2.08 & 2.12 & 1.04 & 0.9986 & $2.09 \mathrm{E}-06$ & $4.25 E-06$ & $2.16 \mathrm{E}-06$ & $2.59 \mathrm{E}-06$ & 1.2 & 0.9988 & $2.41 \mathrm{E}-06$ & 4.29E-06 & $1.88 \mathrm{E}-06$ & $2.91 \mathrm{E}-06$ \\
\hline 600 & 2.45 & 2.50 & 1.42 & 0.9990 & $2.37 \mathrm{E}-06$ & 4.40E-06 & $2.03 \mathrm{E}-06$ & 2.87E-06 & 1.57 & 0.9992 & $2.62 \mathrm{E}-06$ & $4.42 E-06$ & 1.79E-06 & $3.12 \mathrm{E}-06$ \\
\hline 700 & 2.89 & 2.95 & 1.87 & 0.9995 & $2.68 \mathrm{E}-06$ & 4.46E-06 & 1.79E-06 & $3.18 \mathrm{E}-06$ & 2.01 & 0.9996 & $2.88 \mathrm{E}-06$ & $4.62 E-06$ & $1.74 \mathrm{E}-06$ & $3.38 \mathrm{E}-06$ \\
\hline 800 & 3.32 & 3.49 & 2.41 & 1.0000 & $3.02 \mathrm{E}-06$ & 4.59E-06 & $1.57 \mathrm{E}-06$ & $3.52 \mathrm{E}-06$ & 2.44 & 1.0000 & $3.06 \mathrm{E}-06$ & $4.85 E-06$ & $1.79 \mathrm{E}-06$ & $3.56 \mathrm{E}-06$ \\
\hline
\end{tabular}

\begin{tabular}{|c|c|c|c|c|c|c|c|c|c|c|c|c|c|c|}
\hline Capsule & OC3 & $\begin{array}{l}\text { Specimen } \\
\text { Length }\end{array}$ & $\begin{array}{c}74 \\
0.9981\end{array}$ & $\begin{array}{l}\text { check } \\
\text { Scale }\end{array}$ & $1 "$ & $1.00 \mathrm{E}-03$ & & & & & & & & \\
\hline $\begin{array}{c}\text { Temperature } \\
\left({ }^{\circ} \mathrm{C}\right)\end{array}$ & Up & Down & $\begin{array}{l}\text { Delta } \\
\text { Down }\end{array}$ & $\begin{array}{l}\text { Down } \\
\text { length }\end{array}$ & CTE & CTEK & $\begin{array}{l}\text { Delta } \\
\text { CTEK }\end{array}$ & $\begin{array}{c}\text { CTE } \\
\text { adjusted } \\
\end{array}$ & $\begin{array}{l}\text { Delta } \\
\text { Up }\end{array}$ & $\begin{array}{c}\text { Up } \\
\text { length }\end{array}$ & CTE & CTEK & $\begin{array}{l}\text { Delta } \\
\text { CTEK }\end{array}$ & $\begin{array}{c}\text { CTE } \\
\text { adjusted }\end{array}$ \\
\hline 0 & 0.40 & 0.64 & 0 & 0.9981 & & & & & 0 & 0.9981 & & & & \\
\hline 100 & 0.77 & 0.88 & 0.24 & 0.9983 & $2.40 \mathrm{E}-06$ & $1.75 E-06$ & $-6.55 E-07$ & $2.90 \mathrm{E}-06$ & 0.37 & 0.9985 & 3.71E-06 & $2.45 E-06$ & $-1.26 \mathrm{E}-06$ & $4.21 \mathrm{E}-06$ \\
\hline 200 & 1.12 & 1.12 & 0.48 & 0.9986 & $2.40 \mathrm{E}-06$ & $1.75 E-06$ & $-6.55 E-07$ & $2.90 \mathrm{E}-06$ & 0.72 & 0.9988 & $3.61 \mathrm{E}-06$ & $2.35 E-06$ & $-1.26 \mathrm{E}-06$ & 4.11E-06 \\
\hline 300 & 1.51 & 1.39 & 0.75 & 0.9989 & $2.50 \mathrm{E}-06$ & $1.82 E-06$ & $-6.89 \mathrm{E}-07$ & $3.00 \mathrm{E}-06$ & 1.11 & 0.9992 & $3.71 \mathrm{E}-06$ & $2.42 E-06$ & $-1.29 \mathrm{E}-06$ & $4.21 \mathrm{E}-06$ \\
\hline 400 & 1.91 & 1.76 & 1.12 & 0.9992 & $2.81 \mathrm{E}-06$ & $1.95 E-06$ & $-8.55 \mathrm{E}-07$ & $3.31 E-06$ & 1.51 & 0.9996 & $3.78 \mathrm{E}-06$ & $2.45 E-06$ & $-1.33 \mathrm{E}-06$ & $4.28 \mathrm{E}-06$ \\
\hline 500 & 2.36 & 2.22 & 1.58 & 0.9997 & 3.17E-06 & $2.13 E-06$ & $-1.04 \mathrm{E}-06$ & 3.67E-06 & 1.96 & 1.0001 & 3.93E-06 & $2.51 E-06$ & $-1.42 \mathrm{E}-06$ & 4.43E-06 \\
\hline 600 & 2.87 & 2.77 & 2.13 & 1.0002 & $3.56 \mathrm{E}-06$ & $2.33 E-06$ & $-1.22 \mathrm{E}-06$ & 4.06E-06 & 2.47 & 1.0006 & 4.12E-06 & $2.63 E-06$ & $-1.49 \mathrm{E}-06$ & 4.62E-06 \\
\hline 700 & 3.45 & 3.36 & 2.72 & 1.0008 & $3.89 \mathrm{E}-06$ & $2.49 E-06$ & $-1.40 \mathrm{E}-06$ & 4.39E-06 & 3.05 & 1.0012 & 4.37E-06 & $2.74 E-06$ & $-1.63 E-06$ & 4.87E-06 \\
\hline 800 & 4.08 & 4.14 & 3.5 & 1.0016 & $4.38 \mathrm{E}-06$ & $2.74 E-06$ & $-1.65 \mathrm{E}-06$ & $4.88 \mathrm{E}-06$ & 3.68 & 1.0018 & $4.61 \mathrm{E}-06$ & $2.85 E-06$ & $-1.76 \mathrm{E}-06$ & $5.11 \mathrm{E}-06$ \\
\hline
\end{tabular}




\begin{tabular}{|c|c|c|c|c|c|c|c|c|c|c|c|c|c|c|}
\hline Capsule & OC3 & $\begin{array}{l}\text { Specimen } \\
\text { Length }\end{array}$ & $\begin{array}{c}76 \\
0.9981\end{array}$ & Scale & 1" & $1.00 \mathrm{E}-03$ & & & & & & & & \\
\hline $\begin{array}{c}\text { Temperature } \\
\left({ }^{\circ} \mathrm{C}\right)\end{array}$ & Up & Down & $\begin{array}{l}\text { Delta } \\
\text { Down }\end{array}$ & $\begin{array}{l}\text { Down } \\
\text { length }\end{array}$ & CTE & CTEK & $\begin{array}{l}\text { Delta } \\
\text { CTEK }\end{array}$ & $\begin{array}{c}\text { CTE } \\
\text { adjusted }\end{array}$ & $\begin{array}{l}\text { Delta } \\
\text { Up }\end{array}$ & $\begin{array}{c}\text { Up } \\
\text { length }\end{array}$ & CTE & CTEK & $\begin{array}{l}\text { Delta } \\
\text { CTEK }\end{array}$ & $\begin{array}{c}\text { CTE } \\
\text { adjusted }\end{array}$ \\
\hline 0 & 1.00 & 1.07 & 0 & 0.9961 & & & & & 0 & 0.9961 & & & & \\
\hline 100 & 1.15 & 1.21 & 0.14 & 0.9962 & $1.41 \mathrm{E}-06$ & $3.55 E-06$ & $2.14 \mathrm{E}-06$ & $1.91 \mathrm{E}-06$ & 0.15 & 0.9963 & $1.51 \mathrm{E}-06$ & $3.65 E-06$ & $2.14 \mathrm{E}-06$ & $2.01 \mathrm{E}-06$ \\
\hline 200 & 1.40 & 1.43 & 0.36 & 0.9965 & $1.81 \mathrm{E}-06$ & $3.65 E-06$ & $1.84 \mathrm{E}-06$ & $2.31 \mathrm{E}-06$ & 0.4 & 0.9965 & $2.01 \mathrm{E}-06$ & $3.80 E-06$ & $1.79 \mathrm{E}-06$ & $2.51 \mathrm{E}-06$ \\
\hline 300 & 1.72 & 1.69 & 0.62 & 0.9967 & 2.07E-06 & $3.82 E-06$ & $1.74 \mathrm{E}-06$ & 2.57E-06 & 0.72 & 0.9968 & $2.41 \mathrm{E}-06$ & $4.02 E-06$ & $1.61 \mathrm{E}-06$ & 2.91E-06 \\
\hline 400 & 2.09 & 2.00 & 0.93 & 0.9970 & $2.33 \mathrm{E}-06$ & $4.08 E-06$ & $1.74 \mathrm{E}-06$ & $2.83 \mathrm{E}-06$ & 1.09 & 0.9972 & $2.74 \mathrm{E}-06$ & 4.13E-06 & 1.39E-06 & $3.24 \mathrm{E}-06$ \\
\hline 500 & 2.50 & 2.39 & 1.32 & 0.9974 & $2.65 \mathrm{E}-06$ & $4.25 E-06$ & 1.60E-06 & $3.15 \mathrm{E}-06$ & 1.5 & 0.9976 & $3.01 \mathrm{E}-06$ & 4.29E-06 & $1.28 \mathrm{E}-06$ & $3.51 \mathrm{E}-06$ \\
\hline 600 & 2.96 & 2.82 & 1.75 & 0.9979 & $2.93 \mathrm{E}-06$ & 4.40E-06 & $1.47 \mathrm{E}-06$ & $3.43 \mathrm{E}-06$ & 1.96 & 0.9981 & $3.28 \mathrm{E}-06$ & $4.42 E-06$ & 1.14E-06 & $3.78 \mathrm{E}-06$ \\
\hline 700 & 3.45 & 3.32 & 2.25 & 0.9984 & $3.23 \mathrm{E}-06$ & 4.46E-06 & $1.24 \mathrm{E}-06$ & $3.73 \mathrm{E}-06$ & 2.45 & 0.9986 & 3.51E-06 & $4.62 E-06$ & $1.11 \mathrm{E}-06$ & $4.01 \mathrm{E}-06$ \\
\hline 800 & 3.95 & 3.98 & 2.91 & 0.9990 & $3.65 \mathrm{E}-06$ & 4.59E-06 & $9.35 \mathrm{E}-07$ & 4.15E-06 & 2.95 & 0.9991 & $3.70 \mathrm{E}-06$ & $4.85 E-06$ & $1.15 \mathrm{E}-06$ & 4.20E-06 \\
\hline
\end{tabular}

\begin{tabular}{|c|c|c|c|c|c|c|c|c|c|c|c|c|c|c|}
\hline Capsule & OC3 & $\begin{array}{l}\text { Specimen } \\
\text { Length }\end{array}$ & $\begin{array}{c}80 \\
0.9978\end{array}$ & $\begin{array}{l}\text { check } \\
\text { Scale }\end{array}$ & $1 "$ & $1.00 \mathrm{E}-03$ & & & & & & & & \\
\hline $\begin{array}{c}\text { Temperature } \\
\left({ }^{\circ} \mathrm{C}\right)\end{array}$ & Up & Down & $\begin{array}{l}\text { Delta } \\
\text { Down }\end{array}$ & $\begin{array}{l}\text { Down } \\
\text { length }\end{array}$ & CTE & CTEK & $\begin{array}{l}\text { Delta } \\
\text { CTEK }\end{array}$ & $\begin{array}{c}\text { CTE } \\
\text { adjusted } \\
\end{array}$ & $\begin{array}{l}\text { Delta } \\
\text { Up }\end{array}$ & $\begin{array}{c}\text { Up } \\
\text { length }\end{array}$ & CTE & CTEK & $\begin{array}{l}\text { Delta } \\
\text { CTEK }\end{array}$ & $\begin{array}{c}\text { CTE } \\
\text { adjusted }\end{array}$ \\
\hline 0 & 0.93 & 1.22 & 0 & 0.9978 & & & & & 0 & 0.9978 & & & & \\
\hline 100 & 1.39 & 1.62 & 0.4 & 0.9982 & 4.01E-06 & $2.55 E-06$ & $-1.46 \mathrm{E}-06$ & 4.51E-06 & 0.46 & 0.9983 & 4.61E-06 & $2.85 E-06$ & $-1.76 \mathrm{E}-06$ & $5.11 \mathrm{E}-06$ \\
\hline 200 & 1.81 & 2.04 & 0.82 & 0.9986 & 4.11E-06 & $2.60 E-06$ & $-1.51 \mathrm{E}-06$ & 4.61E-06 & 0.88 & 0.9987 & 4.41E-06 & $2.75 E-06$ & $-1.66 \mathrm{E}-06$ & 4.91E-06 \\
\hline 300 & 2.28 & 2.48 & 1.26 & 0.9991 & $4.21 \mathrm{E}-06$ & $2.65 E-06$ & $-1.56 \mathrm{E}-06$ & $4.71 \mathrm{E}-06$ & 1.35 & 0.9992 & 4.51E-06 & $2.78 E-06$ & $-1.73 E-06$ & $5.01 \mathrm{E}-06$ \\
\hline 400 & 2.78 & 2.95 & 1.73 & 0.9995 & $4.33 \mathrm{E}-06$ & 2.73E-06 & $-1.61 E-06$ & 4.83E-06 & 1.85 & 0.9997 & 4.64E-06 & $2.85 E-06$ & $-1.79 \mathrm{E}-06$ & $5.14 \mathrm{E}-06$ \\
\hline 500 & 3.30 & 3.50 & 2.28 & 1.0001 & 4.57E-06 & $2.83 E-06$ & $-1.74 \mathrm{E}-06$ & 5.07E-06 & 2.37 & 1.0002 & $4.75 \mathrm{E}-06$ & $2.91 E-06$ & $-1.84 \mathrm{E}-06$ & $5.25 \mathrm{E}-06$ \\
\hline 600 & 3.88 & 4.09 & 2.87 & 1.0007 & 4.79E-06 & $2.95 E-06$ & $-1.84 \mathrm{E}-06$ & 5.29E-06 & 2.95 & 1.0008 & 4.93E-06 & $3.00 E-06$ & $-1.93 E-06$ & $5.43 \mathrm{E}-06$ \\
\hline 700 & 4.50 & 4.72 & 3.5 & 1.0013 & $5.01 \mathrm{E}-06$ & $3.05 E-06$ & $-1.96 \mathrm{E}-06$ & 5.51E-06 & 3.57 & 1.0014 & 5.11E-06 & $3.09 E-06$ & $-2.02 \mathrm{E}-06$ & 5.61E-06 \\
\hline 800 & 5.24 & 5.47 & 4.25 & 1.0021 & $5.32 \mathrm{E}-06$ & $3.21 E-06$ & $-2.11 \mathrm{E}-06$ & $5.82 \mathrm{E}-06$ & 4.31 & 1.0021 & $5.40 \mathrm{E}-06$ & $3.24 E-06$ & $-2.16 \mathrm{E}-06$ & 5.90E-06 \\
\hline
\end{tabular}




\begin{tabular}{|c|c|c|c|c|c|c|c|c|c|c|c|c|c|c|}
\hline Capsule & OC3 & $\begin{array}{l}\text { Specimen } \\
\text { Length }\end{array}$ & $\begin{array}{c}82 \\
0.9956\end{array}$ & Scale & 1" & $1.00 \mathrm{E}-03$ & & & & & & & & \\
\hline $\begin{array}{c}\text { Temperature } \\
\left({ }^{\circ} \mathrm{C}\right)\end{array}$ & Up & Down & $\begin{array}{l}\text { Delta } \\
\text { Down }\end{array}$ & $\begin{array}{l}\text { Down } \\
\text { length }\end{array}$ & CTE & CTEK & $\begin{array}{l}\text { Delta } \\
\text { CTEK }\end{array}$ & $\begin{array}{c}\text { CTE } \\
\text { adjusted }\end{array}$ & $\begin{array}{l}\text { Delta } \\
\text { Up }\end{array}$ & $\begin{array}{c}\text { Up } \\
\text { length }\end{array}$ & CTE & CTEK & $\begin{array}{l}\text { Delta } \\
\text { CTEK }\end{array}$ & $\begin{array}{c}\text { CTE } \\
\text { adjusted }\end{array}$ \\
\hline 0 & 0.90 & 1.03 & 0 & 0.9956 & & & & & 0 & 0.9956 & & & & \\
\hline 100 & 1.06 & 1.18 & 0.15 & 0.9958 & $1.51 \mathrm{E}-06$ & $3.55 E-06$ & $2.04 \mathrm{E}-06$ & $2.01 \mathrm{E}-06$ & 0.16 & 0.9958 & $1.61 \mathrm{E}-06$ & $3.65 E-06$ & $2.04 \mathrm{E}-06$ & $2.11 \mathrm{E}-06$ \\
\hline 200 & 1.30 & 1.40 & 0.37 & 0.9960 & $1.86 \mathrm{E}-06$ & $3.65 E-06$ & $1.79 \mathrm{E}-06$ & $2.36 \mathrm{E}-06$ & 0.4 & 0.9960 & $2.01 \mathrm{E}-06$ & 3.80E-06 & 1.79E-06 & $2.51 \mathrm{E}-06$ \\
\hline 300 & 1.61 & 1.64 & 0.61 & 0.9962 & $2.04 \mathrm{E}-06$ & $3.82 E-06$ & $1.77 \mathrm{E}-06$ & $2.54 \mathrm{E}-06$ & 0.71 & 0.9963 & $2.38 \mathrm{E}-06$ & $4.02 E-06$ & $1.64 \mathrm{E}-06$ & $2.88 \mathrm{E}-06$ \\
\hline 400 & 1.99 & 1.99 & 0.96 & 0.9966 & $2.41 \mathrm{E}-06$ & $4.08 E-06$ & $1.66 \mathrm{E}-06$ & $2.91 \mathrm{E}-06$ & 1.09 & 0.9967 & $2.74 \mathrm{E}-06$ & 4.13E-06 & 1.39E-06 & $3.24 \mathrm{E}-06$ \\
\hline 500 & 2.41 & 2.34 & 1.31 & 0.9969 & $2.63 \mathrm{E}-06$ & $4.25 E-06$ & $1.62 \mathrm{E}-06$ & $3.13 \mathrm{E}-06$ & 1.51 & 0.9971 & 3.03E-06 & 4.29E-06 & $1.26 \mathrm{E}-06$ & $3.53 \mathrm{E}-06$ \\
\hline 600 & 2.90 & 2.78 & 1.75 & 0.9974 & $2.93 \mathrm{E}-06$ & 4.40E-06 & $1.47 \mathrm{E}-06$ & $3.43 \mathrm{E}-06$ & 2 & 0.9976 & 3.35E-06 & $4.42 E-06$ & 1.07E-06 & $3.85 \mathrm{E}-06$ \\
\hline 700 & 3.38 & 3.25 & 2.22 & 0.9978 & 3.19E-06 & 4.46E-06 & $1.28 \mathrm{E}-06$ & $3.69 \mathrm{E}-06$ & 2.48 & 0.9981 & $3.56 \mathrm{E}-06$ & $4.62 E-06$ & $1.06 \mathrm{E}-06$ & 4.06E-06 \\
\hline 800 & 3.86 & 3.85 & 2.82 & 0.9984 & $3.54 \mathrm{E}-06$ & 4.59E-06 & $1.05 \mathrm{E}-06$ & 4.04E-06 & 2.96 & 0.9986 & $3.72 \mathrm{E}-06$ & $4.85 E-06$ & $1.13 \mathrm{E}-06$ & 4.22E-06 \\
\hline
\end{tabular}

\begin{tabular}{|c|c|c|c|c|c|c|c|c|c|c|c|c|c|c|}
\hline Capsule & OC3 & $\begin{array}{l}\text { Specimen } \\
\text { Length }\end{array}$ & $\begin{array}{c}85 \\
0.9703\end{array}$ & $\begin{array}{l}\text { check } \\
\text { Scale }\end{array}$ & $1 "$ & $1.00 \mathrm{E}-03$ & & & & & & & & \\
\hline $\begin{array}{c}\text { Temperature } \\
\left({ }^{\circ} \mathrm{C}\right)\end{array}$ & Up & Down & $\begin{array}{l}\text { Delta } \\
\text { Down }\end{array}$ & $\begin{array}{l}\text { Down } \\
\text { length }\end{array}$ & CTE & CTEK & $\begin{array}{l}\text { Delta } \\
\text { CTEK }\end{array}$ & $\begin{array}{c}\text { CTE } \\
\text { adjusted } \\
\end{array}$ & $\begin{array}{l}\text { Delta } \\
\text { Up }\end{array}$ & $\begin{array}{c}\text { Up } \\
\text { length }\end{array}$ & CTE & CTEK & $\begin{array}{l}\text { Delta } \\
\text { CTEK }\end{array}$ & $\begin{array}{c}\text { CTE } \\
\text { adjusted }\end{array}$ \\
\hline 0 & 0.96 & 1.07 & 0 & 0.9703 & & & & & 0 & 0.9703 & & & & \\
\hline 100 & 1.44 & 1.41 & 0.34 & 0.9706 & $3.50 \mathrm{E}-06$ & $2.25 E-06$ & $-1.25 \mathrm{E}-06$ & 4.00E-06 & 0.48 & 0.9708 & 4.95E-06 & $2.95 E-06$ & $-2.00 \mathrm{E}-06$ & $5.45 \mathrm{E}-06$ \\
\hline 200 & 1.90 & 1.83 & 0.76 & 0.9711 & $3.92 \mathrm{E}-06$ & $2.45 E-06$ & $-1.47 \mathrm{E}-06$ & 4.42E-06 & 0.94 & 0.9712 & 4.84E-06 & 2.90E-06 & $-1.94 \mathrm{E}-06$ & 5.34E-06 \\
\hline 300 & 2.29 & 2.23 & 1.16 & 0.9715 & $3.99 \mathrm{E}-06$ & $2.48 E-06$ & $-1.50 \mathrm{E}-06$ & 4.49E-06 & 1.33 & 0.9716 & 4.57E-06 & $2.78 E-06$ & $-1.79 \mathrm{E}-06$ & 5.07E-06 \\
\hline 400 & 2.72 & 2.77 & 1.7 & 0.9720 & $4.38 \mathrm{E}-06$ & $2.68 E-06$ & $-1.71 E-06$ & $4.88 \mathrm{E}-06$ & 1.76 & 0.9721 & 4.53E-06 & $2.75 E-06$ & $-1.78 \mathrm{E}-06$ & $5.03 \mathrm{E}-06$ \\
\hline 500 & 3.22 & 3.32 & 2.25 & 0.9726 & $4.64 \mathrm{E}-06$ & 2.79E-06 & $-1.85 \mathrm{E}-06$ & $5.14 \mathrm{E}-06$ & 2.26 & 0.9726 & $4.66 \mathrm{E}-06$ & $2.81 E-06$ & $-1.85 \mathrm{E}-06$ & 5.16E-06 \\
\hline 600 & 3.83 & 3.93 & 2.86 & 0.9732 & 4.91E-06 & $2.93 E-06$ & $-1.98 \mathrm{E}-06$ & 5.41E-06 & 2.87 & 0.9732 & 4.93E-06 & 2.95E-06 & $-1.98 \mathrm{E}-06$ & $5.43 \mathrm{E}-06$ \\
\hline 700 & 4.45 & 4.52 & 3.45 & 0.9738 & $5.08 \mathrm{E}-06$ & $3.01 E-06$ & $-2.07 E-06$ & $5.58 \mathrm{E}-06$ & 3.49 & 0.9738 & $5.14 \mathrm{E}-06$ & $3.05 E-06$ & $-2.09 \mathrm{E}-06$ & $5.64 \mathrm{E}-06$ \\
\hline 800 & 5.13 & 5.20 & 4.13 & 0.9744 & $5.32 \mathrm{E}-06$ & 3.13E-06 & $-2.20 \mathrm{E}-06$ & $5.82 \mathrm{E}-06$ & 4.17 & 0.9745 & $5.37 \mathrm{E}-06$ & $3.16 E-06$ & $-2.21 \mathrm{E}-06$ & 5.87E-06 \\
\hline
\end{tabular}




\begin{tabular}{|c|c|c|c|c|c|c|c|c|c|c|c|c|c|c|}
\hline Capsule & OC3 & $\begin{array}{c}\text { Specimen } \\
\text { Length }\end{array}$ & $\begin{array}{c}87 \\
0.9955\end{array}$ & Scale & 1" & $1.00 \mathrm{E}-03$ & & & & & & & & \\
\hline $\begin{array}{c}\text { Temperature } \\
\left({ }^{\circ} \mathrm{C}\right)\end{array}$ & Up & Down & $\begin{array}{l}\text { Delta } \\
\text { Down }\end{array}$ & $\begin{array}{l}\text { Down } \\
\text { length }\end{array}$ & CTE & CTEK & $\begin{array}{l}\text { Delta } \\
\text { CTEK }\end{array}$ & $\begin{array}{c}\text { CTE } \\
\text { adjusted } \\
\end{array}$ & $\begin{array}{l}\text { Delta } \\
\text { Up }\end{array}$ & $\begin{array}{l}\text { Up } \\
\text { length }\end{array}$ & CTE & CTEK & $\begin{array}{l}\text { Delta } \\
\text { CTEK }\end{array}$ & $\begin{array}{c}\text { CTE } \\
\text { adjusted }\end{array}$ \\
\hline 0 & 0.94 & 1.02 & 0 & 0.9955 & & & & & 0 & 0.9955 & & & & \\
\hline 100 & 1.10 & 1.14 & 0.12 & 0.9956 & $1.21 \mathrm{E}-06$ & $1.71 E-06$ & $5.00 \mathrm{E}-07$ & $2.75 \mathrm{E}-06$ & 0.16 & 0.9957 & $1.61 \mathrm{E}-06$ & $2.11 E-06$ & $5.00 \mathrm{E}-07$ & $-1.11 \mathrm{E}-06$ \\
\hline 200 & 1.37 & 1.37 & 0.35 & 0.9959 & $1.76 \mathrm{E}-06$ & $2.26 E-06$ & $5.00 \mathrm{E}-07$ & $2.70 \mathrm{E}-06$ & 0.43 & 0.9959 & $2.16 \mathrm{E}-06$ & 2.66E-06 & $5.00 \mathrm{E}-07$ & $-1.66 \mathrm{E}-06$ \\
\hline 300 & 1.68 & 1.60 & 0.58 & 0.9961 & $1.94 \mathrm{E}-06$ & $2.44 E-06$ & $5.00 \mathrm{E}-07$ & $2.75 \mathrm{E}-06$ & 0.74 & 0.9962 & $2.48 \mathrm{E}-06$ & $2.98 E-06$ & $5.00 \mathrm{E}-07$ & $-1.98 \mathrm{E}-06$ \\
\hline 400 & 2.02 & 1.91 & 0.89 & 0.9964 & $2.24 \mathrm{E}-06$ & $2.74 E-06$ & $5.00 \mathrm{E}-07$ & $2.93 \mathrm{E}-06$ & 1.08 & 0.9966 & $2.71 E-06$ & $3.21 E-06$ & $5.00 \mathrm{E}-07$ & $-2.21 E-06$ \\
\hline 500 & 2.42 & 2.28 & 1.26 & 0.9968 & $2.53 \mathrm{E}-06$ & $3.03 E-06$ & $5.00 \mathrm{E}-07$ & $3.21 \mathrm{E}-06$ & 1.48 & 0.9970 & 2.97E-06 & $3.47 E-06$ & $5.00 \mathrm{E}-07$ & $-2.47 \mathrm{E}-06$ \\
\hline 600 & 2.85 & 2.69 & 1.67 & 0.9972 & $2.80 \mathrm{E}-06$ & $3.30 E-06$ & $5.00 \mathrm{E}-07$ & $3.43 \mathrm{E}-06$ & 1.91 & 0.9974 & $3.20 \mathrm{E}-06$ & 3.70E-06 & $5.00 \mathrm{E}-07$ & $-2.70 \mathrm{E}-06$ \\
\hline 700 & 3.27 & 3.16 & 2.14 & 0.9976 & 3.07E-06 & $3.57 E-06$ & $5.00 \mathrm{E}-07$ & $3.66 \mathrm{E}-06$ & 2.33 & 0.9978 & $3.34 \mathrm{E}-06$ & $3.84 E-06$ & $5.00 \mathrm{E}-07$ & $-2.84 \mathrm{E}-06$ \\
\hline 800 & 3.73 & 3.74 & 2.72 & 0.9982 & $3.42 \mathrm{E}-06$ & $3.92 E-06$ & $5.00 \mathrm{E}-07$ & 3.95E-06 & 2.79 & 0.9983 & $3.50 \mathrm{E}-06$ & 4.00E-06 & $5.00 \mathrm{E}-07$ & $-3.00 \mathrm{E}-06$ \\
\hline
\end{tabular}

\begin{tabular}{|c|c|c|c|c|c|c|c|c|c|c|c|c|c|c|}
\hline Capsule & OC3 & $\begin{array}{l}\text { Specimen } \\
\text { Length }\end{array}$ & $\begin{array}{c}92 \\
0.9965\end{array}$ & Scale & 1" & $1.00 \mathrm{E}-03$ & & & & & & & & \\
\hline $\begin{array}{c}\text { Temperature } \\
\left({ }^{\circ} \mathrm{C}\right)\end{array}$ & Up & Down & $\begin{array}{l}\text { Delta } \\
\text { Down }\end{array}$ & $\begin{array}{l}\text { Down } \\
\text { length }\end{array}$ & CTE & CTEK & $\begin{array}{l}\text { Delta } \\
\text { CTEK }\end{array}$ & $\begin{array}{c}\text { CTE } \\
\text { adjusted }\end{array}$ & $\begin{array}{l}\text { Delta } \\
\text { Up }\end{array}$ & $\begin{array}{c}\text { Up } \\
\text { length }\end{array}$ & CTE & CTEK & $\begin{array}{l}\text { Delta } \\
\text { CTEK }\end{array}$ & $\begin{array}{c}\text { CTE } \\
\text { adjusted }\end{array}$ \\
\hline 0 & 1.00 & 1.20 & 0 & 0.9965 & & & & & 0 & 0.9965 & & & & \\
\hline 100 & 1.22 & 1.41 & 0.21 & 0.9967 & $2.11 \mathrm{E}-06$ & $3.55 E-06$ & 1.44E-06 & $2.61 \mathrm{E}-06$ & 0.22 & 0.9967 & $2.21 \mathrm{E}-06$ & $3.65 E-06$ & 1.44E-06 & $2.71 \mathrm{E}-06$ \\
\hline 200 & 1.55 & 1.70 & 0.5 & 0.9970 & $2.51 \mathrm{E}-06$ & $3.65 E-06$ & $1.14 \mathrm{E}-06$ & $3.01 \mathrm{E}-06$ & 0.55 & 0.9971 & $2.76 \mathrm{E}-06$ & $3.80 E-06$ & $1.04 \mathrm{E}-06$ & $3.26 \mathrm{E}-06$ \\
\hline 300 & 1.88 & 1.96 & 0.76 & 0.9973 & $2.54 \mathrm{E}-06$ & $3.82 E-06$ & $1.27 \mathrm{E}-06$ & $3.04 \mathrm{E}-06$ & 0.88 & 0.9974 & $2.94 \mathrm{E}-06$ & $4.02 E-06$ & 1.07E-06 & 3.44E-06 \\
\hline 400 & 2.31 & 2.31 & 1.11 & 0.9976 & $2.78 \mathrm{E}-06$ & $4.08 E-06$ & $1.29 \mathrm{E}-06$ & $3.28 \mathrm{E}-06$ & 1.31 & 0.9978 & $3.29 \mathrm{E}-06$ & 4.13E-06 & $8.38 \mathrm{E}-07$ & 3.79E-06 \\
\hline 500 & 2.80 & 2.72 & 1.52 & 0.9980 & $3.05 \mathrm{E}-06$ & $4.25 E-06$ & $1.20 \mathrm{E}-06$ & 3.55E-06 & 1.8 & 0.9983 & 3.61E-06 & 4.29E-06 & $6.77 \mathrm{E}-07$ & 4.11E-06 \\
\hline 600 & 3.30 & 3.18 & 1.98 & 0.9985 & $3.31 \mathrm{E}-06$ & 4.40E-06 & 1.09E-06 & $3.81 \mathrm{E}-06$ & 2.3 & 0.9988 & $3.85 \mathrm{E}-06$ & 4.42E-06 & $5.69 \mathrm{E}-07$ & 4.35E-06 \\
\hline 700 & 3.81 & 3.70 & 2.5 & 0.9990 & $3.58 \mathrm{E}-06$ & $4.46 E-06$ & $8.80 \mathrm{E}-07$ & 4.08E-06 & 2.81 & 0.9993 & 4.03E-06 & $4.62 E-06$ & $5.93 \mathrm{E}-07$ & 4.53E-06 \\
\hline 800 & 4.33 & 4.35 & 3.15 & 0.9997 & $3.95 \mathrm{E}-06$ & 4.59E-06 & $6.36 \mathrm{E}-07$ & 4.45E-06 & 3.33 & 0.9998 & $4.18 \mathrm{E}-06$ & $4.85 E-06$ & $6.73 \mathrm{E}-07$ & $4.68 \mathrm{E}-06$ \\
\hline
\end{tabular}




\begin{tabular}{|c|c|c|c|c|c|c|c|c|c|c|c|c|c|c|}
\hline Capsule & OC3 & $\begin{array}{l}\text { Specimen } \\
\text { Length }\end{array}$ & $\begin{array}{c}95 \\
0.9985\end{array}$ & $\begin{array}{l}\text { check } \\
\text { Scale }\end{array}$ & $1 "$ & $1.00 \mathrm{E}-03$ & & & & & & & & \\
\hline $\begin{array}{c}\text { Temperature } \\
\left({ }^{\circ} \mathrm{C}\right)\end{array}$ & Up & Down & $\begin{array}{l}\text { Delta } \\
\text { Down }\end{array}$ & $\begin{array}{l}\text { Down } \\
\text { length }\end{array}$ & CTE & CTEK & $\begin{array}{l}\text { Delta } \\
\text { CTEK }\end{array}$ & $\begin{array}{c}\text { CTE } \\
\text { adjusted }\end{array}$ & $\begin{array}{l}\text { Delta } \\
\text { Up }\end{array}$ & $\begin{array}{c}\text { Up } \\
\text { length }\end{array}$ & CTE & CTEK & $\begin{array}{l}\text { Delta } \\
\text { CTEK }\end{array}$ & $\begin{array}{c}\text { CTE } \\
\text { adjusted }\end{array}$ \\
\hline 0 & 0.95 & 1.28 & 0 & 0.9827 & & & & & 0 & 0.9827 & & & & \\
\hline 100 & 1.26 & 1.53 & 0.25 & 0.9830 & $2.54 \mathrm{E}-06$ & $3.05 E-06$ & $5.06 \mathrm{E}-07$ & $3.04 \mathrm{E}-06$ & 0.31 & 0.9830 & $3.15 \mathrm{E}-06$ & $3.65 E-06$ & 4.95E-07 & $3.65 \mathrm{E}-06$ \\
\hline 200 & 1.55 & 1.78 & 0.5 & 0.9832 & $2.54 \mathrm{E}-06$ & $3.05 E-06$ & $5.06 \mathrm{E}-07$ & $3.04 \mathrm{E}-06$ & 0.6 & 0.9833 & $3.05 \mathrm{E}-06$ & $3.55 E-06$ & 4.97E-07 & $3.55 \mathrm{E}-06$ \\
\hline 300 & 1.91 & 2.03 & 0.75 & 0.9835 & $2.54 \mathrm{E}-06$ & $3.05 E-06$ & $5.06 \mathrm{E}-07$ & $3.04 \mathrm{E}-06$ & 0.96 & 0.9837 & $3.26 \mathrm{E}-06$ & $3.75 E-06$ & 4.94E-07 & 3.76E-06 \\
\hline 400 & 2.25 & 2.31 & 1.03 & 0.9837 & $2.62 \mathrm{E}-06$ & $3.13 E-06$ & $5.05 \mathrm{E}-07$ & $3.12 \mathrm{E}-06$ & 1.3 & 0.9840 & 3.31E-06 & 3.80E-06 & 4.93E-07 & $3.81 \mathrm{E}-06$ \\
\hline 500 & 2.60 & 2.69 & 1.41 & 0.9841 & $2.87 \mathrm{E}-06$ & $3.37 E-06$ & $5.00 \mathrm{E}-07$ & 3.37E-06 & 1.65 & 0.9844 & $3.36 \mathrm{E}-06$ & $3.85 E-06$ & $4.92 \mathrm{E}-07$ & $3.86 \mathrm{E}-06$ \\
\hline 600 & 2.97 & 3.05 & 1.77 & 0.9845 & $3.00 \mathrm{E}-06$ & $3.50 E-06$ & $4.98 \mathrm{E}-07$ & $3.50 \mathrm{E}-06$ & 2.02 & 0.9847 & $3.43 \mathrm{E}-06$ & $3.92 E-06$ & 4.90E-07 & $3.93 \mathrm{E}-06$ \\
\hline 700 & 3.33 & 3.43 & 2.15 & 0.9849 & 3.13E-06 & $3.62 E-06$ & $4.96 \mathrm{E}-07$ & $3.63 \mathrm{E}-06$ & 2.38 & 0.9851 & $3.46 \mathrm{E}-06$ & $3.95 E-06$ & 4.90E-07 & 3.96E-06 \\
\hline 800 & 3.73 & 3.83 & 2.55 & 0.9853 & $3.24 \mathrm{E}-06$ & $3.74 E-06$ & $4.93 \mathrm{E}-07$ & $3.74 \mathrm{E}-06$ & 2.78 & 0.9855 & $3.54 \mathrm{E}-06$ & 4.03E-06 & $4.89 \mathrm{E}-07$ & 4.04E-06 \\
\hline
\end{tabular}

\begin{tabular}{|c|c|c|c|c|c|c|c|c|c|c|c|c|c|c|}
\hline Capsule & OC3 & $\begin{array}{l}\text { Specimen } \\
\text { Length }\end{array}$ & $\begin{array}{l}97 \\
0.996\end{array}$ & Scale & 1" & $1.00 \mathrm{E}-03$ & & & & & & & & \\
\hline $\begin{array}{c}\text { Temperature } \\
\left({ }^{\circ} \mathrm{C}\right)\end{array}$ & Up & Down & $\begin{array}{l}\text { Delta } \\
\text { Down }\end{array}$ & $\begin{array}{l}\text { Down } \\
\text { length }\end{array}$ & CTE & CTEK & $\begin{array}{l}\text { Delta } \\
\text { CTEK }\end{array}$ & $\begin{array}{c}\text { CTE } \\
\text { adjusted }\end{array}$ & $\begin{array}{l}\text { Delta } \\
\text { Up }\end{array}$ & $\begin{array}{c}\text { Up } \\
\text { length }\end{array}$ & CTE & CTEK & $\begin{array}{l}\text { Delta } \\
\text { CTEK }\end{array}$ & $\begin{array}{c}\text { CTE } \\
\text { adjusted }\end{array}$ \\
\hline 0 & 0.94 & 0.41 & 0 & 0.9960 & & & & & 0 & 0.9960 & & & & \\
\hline 100 & 1.03 & 0.61 & 0.2 & 0.9962 & $2.01 \mathrm{E}-06$ & $2.55 E-06$ & $5.42 \mathrm{E}-07$ & $2.51 \mathrm{E}-06$ & 0.09 & 0.9961 & $9.04 \mathrm{E}-07$ & $1.45 E-06$ & $5.46 \mathrm{E}-07$ & $1.40 \mathrm{E}-06$ \\
\hline 200 & 1.14 & 0.83 & 0.42 & 0.9964 & $2.11 \mathrm{E}-06$ & $2.65 E-06$ & $5.42 \mathrm{E}-07$ & $2.61 \mathrm{E}-06$ & 0.2 & 0.9962 & $1.00 \mathrm{E}-06$ & $1.55 E-06$ & $5.46 \mathrm{E}-07$ & $1.50 \mathrm{E}-06$ \\
\hline 300 & 1.27 & 1.08 & 0.67 & 0.9967 & $2.24 \mathrm{E}-06$ & $2.78 E-06$ & $5.41 \mathrm{E}-07$ & $2.74 \mathrm{E}-06$ & 0.33 & 0.9963 & $1.10 \mathrm{E}-06$ & $1.65 E-06$ & 5.46E-07 & 1.60E-06 \\
\hline 400 & 1.43 & 1.33 & 0.92 & 0.9969 & $2.31 \mathrm{E}-06$ & $2.85 E-06$ & $5.41 \mathrm{E}-07$ & $2.81 \mathrm{E}-06$ & 0.49 & 0.9965 & $1.23 \mathrm{E}-06$ & $1.78 E-06$ & $5.45 \mathrm{E}-07$ & $1.73 \mathrm{E}-06$ \\
\hline 500 & 1.61 & 1.61 & 1.2 & 0.9972 & $2.41 \mathrm{E}-06$ & $2.95 E-06$ & $5.40 \mathrm{E}-07$ & 2.91E-06 & 0.67 & 0.9967 & $1.35 \mathrm{E}-06$ & $1.89 E-06$ & $5.45 \mathrm{E}-07$ & $1.85 \mathrm{E}-06$ \\
\hline 600 & 1.84 & 1.91 & 1.5 & 0.9975 & $2.51 \mathrm{E}-06$ & $3.05 E-06$ & $5.40 \mathrm{E}-07$ & 3.01E-06 & 0.9 & 0.9969 & $1.51 \mathrm{E}-06$ & $2.05 E-06$ & $5.44 \mathrm{E}-07$ & $2.01 \mathrm{E}-06$ \\
\hline 700 & 2.13 & 2.21 & 1.8 & 0.9978 & $2.58 \mathrm{E}-06$ & $3.12 E-06$ & 5.39E-07 & $3.08 \mathrm{E}-06$ & 1.19 & 0.9972 & $1.71 \mathrm{E}-06$ & $2.25 E-06$ & $5.43 \mathrm{E}-07$ & $2.21 \mathrm{E}-06$ \\
\hline 800 & 2.46 & 2.56 & 2.15 & 0.9982 & $2.70 \mathrm{E}-06$ & $3.24 E-06$ & 5.39E-07 & $3.20 \mathrm{E}-06$ & 1.52 & 0.9975 & $1.91 \mathrm{E}-06$ & $2.45 E-06$ & $5.42 \mathrm{E}-07$ & $2.41 \mathrm{E}-06$ \\
\hline
\end{tabular}




\begin{tabular}{|c|c|c|c|c|c|c|c|c|c|c|c|c|c|c|}
\hline Capsule & OC3 & $\begin{array}{l}\text { Specimen } \\
\text { Length }\end{array}$ & $\begin{array}{c}102 \\
0.995\end{array}$ & Scale & 1" & $1.00 \mathrm{E}-03$ & & & & & & & & \\
\hline $\begin{array}{c}\text { Temperature } \\
\left({ }^{\circ} \mathrm{C}\right)\end{array}$ & Up & Down & $\begin{array}{l}\text { Delta } \\
\text { Down }\end{array}$ & $\begin{array}{l}\text { Down } \\
\text { length }\end{array}$ & CTE & CTEK & $\begin{array}{l}\text { Delta } \\
\text { CTEK }\end{array}$ & $\begin{array}{c}\text { CTE } \\
\text { adjusted }\end{array}$ & $\begin{array}{l}\text { Delta } \\
\text { Up }\end{array}$ & $\begin{array}{c}\text { Up } \\
\text { length }\end{array}$ & CTE & CTEK & $\begin{array}{l}\text { Delta } \\
\text { CTEK }\end{array}$ & $\begin{array}{c}\text { CTE } \\
\text { adjusted }\end{array}$ \\
\hline 0 & 1.03 & 1.14 & 0 & 0.9950 & & & & & 0 & 0.9950 & & & & \\
\hline 100 & 1.22 & 1.32 & 0.18 & 0.9952 & $1.81 \mathrm{E}-06$ & $3.55 E-06$ & $1.74 \mathrm{E}-06$ & $2.31 \mathrm{E}-06$ & 0.19 & 0.9952 & $1.91 \mathrm{E}-06$ & $3.65 E-06$ & $1.74 \mathrm{E}-06$ & $2.41 \mathrm{E}-06$ \\
\hline 200 & 1.50 & 1.54 & 0.4 & 0.9954 & $2.01 \mathrm{E}-06$ & $3.65 E-06$ & $1.64 \mathrm{E}-06$ & $2.51 \mathrm{E}-06$ & 0.47 & 0.9955 & $2.36 \mathrm{E}-06$ & $3.80 E-06$ & $1.44 \mathrm{E}-06$ & $2.86 \mathrm{E}-06$ \\
\hline 300 & 1.81 & 1.76 & 0.62 & 0.9956 & $2.08 \mathrm{E}-06$ & $3.82 E-06$ & $1.74 \mathrm{E}-06$ & $2.58 \mathrm{E}-06$ & 0.78 & 0.9958 & $2.61 \mathrm{E}-06$ & $4.02 E-06$ & $1.40 \mathrm{E}-06$ & 3.11E-06 \\
\hline 400 & 2.20 & 2.10 & 0.96 & 0.9960 & $2.41 \mathrm{E}-06$ & $4.08 E-06$ & $1.66 \mathrm{E}-06$ & $2.91 \mathrm{E}-06$ & 1.17 & 0.9962 & $2.94 \mathrm{E}-06$ & $4.13 E-06$ & 1.19E-06 & $3.44 \mathrm{E}-06$ \\
\hline 500 & 2.60 & 2.49 & 1.35 & 0.9964 & $2.71 \mathrm{E}-06$ & $4.25 E-06$ & $1.54 \mathrm{E}-06$ & $3.21 \mathrm{E}-06$ & 1.57 & 0.9966 & $3.16 \mathrm{E}-06$ & 4.29E-06 & $1.13 \mathrm{E}-06$ & $3.66 \mathrm{E}-06$ \\
\hline 600 & 3.03 & 2.92 & 1.78 & 0.9968 & $2.98 \mathrm{E}-06$ & 4.40E-06 & $1.42 \mathrm{E}-06$ & $3.48 \mathrm{E}-06$ & 2 & 0.9970 & 3.35E-06 & $4.42 E-06$ & 1.07E-06 & $3.85 \mathrm{E}-06$ \\
\hline 700 & 3.49 & 2.42 & 1.28 & 0.9963 & $1.84 \mathrm{E}-06$ & 4.46E-06 & $2.63 \mathrm{E}-06$ & $2.34 \mathrm{E}-06$ & 2.46 & 0.9975 & 3.53E-06 & $4.62 E-06$ & $1.09 \mathrm{E}-06$ & 4.03E-06 \\
\hline 800 & 3.98 & 4.01 & 2.87 & 0.9979 & $3.61 \mathrm{E}-06$ & 4.59E-06 & $9.81 \mathrm{E}-07$ & 4.11E-06 & 2.95 & 0.9980 & $3.71 \mathrm{E}-06$ & $4.85 E-06$ & $1.14 \mathrm{E}-06$ & 4.21E-06 \\
\hline
\end{tabular}

\begin{tabular}{|c|c|c|c|c|c|c|c|c|c|c|c|c|c|c|}
\hline Capsule & OC3 & $\begin{array}{c}\text { Specimen } \\
\text { Length }\end{array}$ & $\begin{array}{l}111 \\
0.9972\end{array}$ & Scale & $1^{\prime \prime}$ & $1.00 \mathrm{E}-03$ & & & & & & & & \\
\hline $\begin{array}{c}\text { Temperature } \\
\left({ }^{\circ} \mathrm{C}\right)\end{array}$ & Up & Down & $\begin{array}{l}\text { Delta } \\
\text { Down }\end{array}$ & $\begin{array}{l}\text { Down } \\
\text { length }\end{array}$ & CTE & CTEK & $\begin{array}{l}\text { Delta } \\
\text { CTEK }\end{array}$ & $\begin{array}{c}\text { CTE } \\
\text { adjusted }\end{array}$ & $\begin{array}{c}\text { Delta } \\
\text { Up }\end{array}$ & $\begin{array}{c}\text { Up } \\
\text { length }\end{array}$ & CTE & CTEK & $\begin{array}{l}\text { Delta } \\
\text { CTEK } \\
\end{array}$ & $\begin{array}{c}\text { CTE } \\
\text { adjusted }\end{array}$ \\
\hline 0 & 1.00 & 1.15 & 0 & 0.9972 & & & & & 0 & 0.9972 & & & & \\
\hline 100 & 1.30 & 1.50 & 0.35 & 0.9976 & $3.51 \mathrm{E}-06$ & $3.55 E-06$ & 4.02E-08 & 4.01E-06 & 0.3 & 0.9975 & $3.01 \mathrm{E}-06$ & $3.65 E-06$ & $6.42 \mathrm{E}-07$ & $3.51 \mathrm{E}-06$ \\
\hline 200 & 1.65 & 1.76 & 0.61 & 0.9978 & $3.06 \mathrm{E}-06$ & $3.65 E-06$ & $5.91 \mathrm{E}-07$ & $3.56 \mathrm{E}-06$ & 0.65 & 0.9979 & $3.26 \mathrm{E}-06$ & $3.80 E-06$ & $5.41 \mathrm{E}-07$ & $3.76 \mathrm{E}-06$ \\
\hline 300 & 2.02 & 2.27 & 1.12 & 0.9983 & $3.74 \mathrm{E}-06$ & $3.82 E-06$ & $7.22 \mathrm{E}-08$ & $4.24 \mathrm{E}-06$ & 1.02 & 0.9982 & $3.41 \mathrm{E}-06$ & $4.02 E-06$ & $6.06 \mathrm{E}-07$ & $3.91 \mathrm{E}-06$ \\
\hline 400 & 2.45 & 2.72 & 1.57 & 0.9988 & $3.94 \mathrm{E}-06$ & $4.08 E-06$ & $1.39 \mathrm{E}-07$ & 4.44E-06 & 1.45 & 0.9987 & $3.64 \mathrm{E}-06$ & $4.13 E-06$ & $4.90 \mathrm{E}-07$ & 4.14E-06 \\
\hline 500 & 3.12 & 3.26 & 2.11 & 0.9993 & $4.23 \mathrm{E}-06$ & $4.25 E-06$ & $1.82 \mathrm{E}-08$ & $4.73 \mathrm{E}-06$ & 2.12 & 0.9993 & $4.25 \mathrm{E}-06$ & $4.29 E-06$ & $3.81 \mathrm{E}-08$ & 4.75E-06 \\
\hline 600 & 3.85 & 3.85 & 2.7 & 0.9999 & 4.51E-06 & 4.40E-06 & $-1.13 \mathrm{E}-07$ & $5.01 \mathrm{E}-06$ & 2.85 & 1.0001 & $4.76 \mathrm{E}-06$ & 4.42E-06 & $-3.47 \mathrm{E}-07$ & $5.26 \mathrm{E}-06$ \\
\hline 700 & 4.61 & 4.50 & 3.35 & 1.0006 & $4.80 \mathrm{E}-06$ & $4.46 E-06$ & $-3.35 E-07$ & $5.30 \mathrm{E}-06$ & 3.61 & 1.0008 & 5.17E-06 & $4.62 E-06$ & $-5.51 E-07$ & 5.67E-06 \\
\hline 800 & 5.39 & 5.29 & 4.14 & 1.0013 & $5.19 \mathrm{E}-06$ & $4.59 E-06$ & $-6.03 E-07$ & $5.69 \mathrm{E}-06$ & 4.39 & 1.0016 & $5.50 \mathrm{E}-06$ & $4.85 E-06$ & $-6.53 \mathrm{E}-07$ & $6.00 \mathrm{E}-06$ \\
\hline
\end{tabular}




\begin{tabular}{|c|c|c|c|c|c|c|c|c|c|c|c|c|c|c|}
\hline Capsule & OC3 & $\begin{array}{l}\text { Specimen } \\
\text { Length }\end{array}$ & $\begin{array}{c}115 \\
0.9953\end{array}$ & Scale & 1" & $1.00 \mathrm{E}-03$ & & & & & & & & \\
\hline $\begin{array}{c}\text { Temperature } \\
\left({ }^{\circ} \mathrm{C}\right)\end{array}$ & Up & Down & $\begin{array}{l}\text { Delta } \\
\text { Down }\end{array}$ & $\begin{array}{l}\text { Down } \\
\text { length }\end{array}$ & CTE & CTEK & $\begin{array}{l}\text { Delta } \\
\text { CTEK }\end{array}$ & $\begin{array}{c}\text { CTE } \\
\text { adjusted }\end{array}$ & $\begin{array}{l}\text { Delta } \\
\text { Up }\end{array}$ & $\begin{array}{c}\text { Up } \\
\text { length }\end{array}$ & CTE & CTEK & $\begin{array}{l}\text { Delta } \\
\text { CTEK }\end{array}$ & $\begin{array}{c}\text { CTE } \\
\text { adjusted }\end{array}$ \\
\hline 0 & 0.94 & 1.00 & 0 & 0.9953 & & & & & 0 & 0.9953 & & & & \\
\hline 100 & 1.08 & 1.68 & 0.68 & 0.9960 & $6.83 \mathrm{E}-06$ & $3.55 E-06$ & $-3.28 \mathrm{E}-06$ & 7.33E-06 & 0.14 & 0.9954 & $1.41 \mathrm{E}-06$ & $3.65 E-06$ & $2.24 \mathrm{E}-06$ & $1.91 \mathrm{E}-06$ \\
\hline 200 & 1.30 & 1.26 & 0.26 & 0.9956 & $1.31 \mathrm{E}-06$ & $3.65 E-06$ & $2.34 \mathrm{E}-06$ & $1.81 \mathrm{E}-06$ & 0.36 & 0.9957 & $1.81 \mathrm{E}-06$ & $3.80 E-06$ & $1.99 \mathrm{E}-06$ & $2.31 \mathrm{E}-06$ \\
\hline 300 & 1.61 & 1.53 & 0.53 & 0.9958 & $1.78 \mathrm{E}-06$ & $3.82 E-06$ & $2.04 \mathrm{E}-06$ & $2.28 \mathrm{E}-06$ & 0.67 & 0.9960 & $2.24 \mathrm{E}-06$ & $4.02 E-06$ & 1.77E-06 & $2.74 \mathrm{E}-06$ \\
\hline 400 & 1.94 & 1.87 & 0.87 & 0.9962 & 2.19E-06 & $4.08 E-06$ & $1.89 \mathrm{E}-06$ & 2.69E-06 & 1 & 0.9963 & $2.51 \mathrm{E}-06$ & 4.13E-06 & $1.61 \mathrm{E}-06$ & $3.01 E-06$ \\
\hline 500 & 2.35 & 2.24 & 1.24 & 0.9965 & $2.49 \mathrm{E}-06$ & $4.25 E-06$ & $1.76 \mathrm{E}-06$ & $2.99 \mathrm{E}-06$ & 1.41 & 0.9967 & $2.83 \mathrm{E}-06$ & 4.29E-06 & $1.46 \mathrm{E}-06$ & 3.33E-06 \\
\hline 600 & 2.77 & 2.67 & 1.67 & 0.9970 & $2.80 \mathrm{E}-06$ & 4.40E-06 & $1.60 \mathrm{E}-06$ & $3.30 \mathrm{E}-06$ & 1.83 & 0.9971 & $3.06 \mathrm{E}-06$ & $4.42 E-06$ & $1.35 \mathrm{E}-06$ & 3.56E-06 \\
\hline 700 & 3.22 & 3.14 & 2.14 & 0.9974 & $3.07 \mathrm{E}-06$ & 4.46E-06 & 1.39E-06 & $3.57 \mathrm{E}-06$ & 2.28 & 0.9976 & $3.27 \mathrm{E}-06$ & $4.62 E-06$ & $1.35 \mathrm{E}-06$ & 3.77E-06 \\
\hline 800 & 3.69 & 3.75 & 2.75 & 0.9981 & $3.45 \mathrm{E}-06$ & 4.59E-06 & $1.13 \mathrm{E}-06$ & $3.95 \mathrm{E}-06$ & 2.75 & 0.9981 & $3.45 \mathrm{E}-06$ & $4.85 E-06$ & $1.40 \mathrm{E}-06$ & $3.95 \mathrm{E}-06$ \\
\hline
\end{tabular}

\begin{tabular}{|c|c|c|c|c|c|c|c|c|c|c|c|c|c|c|}
\hline Capsule & OC3 & $\begin{array}{l}\text { Specimen } \\
\text { Length }\end{array}$ & $\begin{array}{c}117 \\
0.9966\end{array}$ & Scale & 1" & $1.00 \mathrm{E}-03$ & & & & & & & & \\
\hline $\begin{array}{c}\text { Temperature } \\
\left({ }^{\circ} \mathrm{C}\right)\end{array}$ & Up & Down & $\begin{array}{l}\text { Delta } \\
\text { Down }\end{array}$ & $\begin{array}{l}\text { Down } \\
\text { length }\end{array}$ & CTE & CTEK & $\begin{array}{l}\text { Delta } \\
\text { CTEK }\end{array}$ & $\begin{array}{c}\text { CTE } \\
\text { adjusted }\end{array}$ & $\begin{array}{l}\text { Delta } \\
\text { Up }\end{array}$ & $\begin{array}{c}\text { Up } \\
\text { length }\end{array}$ & CTE & CTEK & $\begin{array}{l}\text { Delta } \\
\text { CTEK }\end{array}$ & $\begin{array}{c}\text { CTE } \\
\text { adjusted }\end{array}$ \\
\hline 0 & 0.84 & 1.14 & 0 & 0.9966 & & & & & 0 & 0.9966 & & & & \\
\hline 100 & 1.02 & 1.32 & 0.18 & 0.9968 & $1.81 \mathrm{E}-06$ & $3.55 E-06$ & $1.74 \mathrm{E}-06$ & $2.31 \mathrm{E}-06$ & 0.18 & 0.9968 & $1.81 \mathrm{E}-06$ & $3.65 E-06$ & $1.84 \mathrm{E}-06$ & $2.31 \mathrm{E}-06$ \\
\hline 200 & 1.29 & 1.57 & 0.43 & 0.9970 & $2.16 \mathrm{E}-06$ & $3.65 E-06$ & 1.49E-06 & $2.66 \mathrm{E}-06$ & 0.45 & 0.9971 & $2.26 \mathrm{E}-06$ & $3.80 E-06$ & $1.54 \mathrm{E}-06$ & $2.76 \mathrm{E}-06$ \\
\hline 300 & 1.61 & 1.85 & 0.71 & 0.9973 & $2.37 \mathrm{E}-06$ & $3.82 E-06$ & $1.44 \mathrm{E}-06$ & 2.87E-06 & 0.77 & 0.9974 & $2.58 \mathrm{E}-06$ & $4.02 E-06$ & $1.44 \mathrm{E}-06$ & $3.08 \mathrm{E}-06$ \\
\hline 400 & 1.98 & 2.16 & 1.02 & 0.9976 & $2.56 \mathrm{E}-06$ & $4.08 E-06$ & $1.52 \mathrm{E}-06$ & $3.06 \mathrm{E}-06$ & 1.14 & 0.9977 & $2.86 \mathrm{E}-06$ & 4.13E-06 & $1.27 \mathrm{E}-06$ & 3.36E-06 \\
\hline 500 & 2.38 & 2.51 & 1.37 & 0.9980 & $2.75 \mathrm{E}-06$ & $4.25 E-06$ & $1.50 \mathrm{E}-06$ & $3.25 \mathrm{E}-06$ & 1.54 & 0.9981 & 3.09E-06 & 4.29E-06 & $1.20 \mathrm{E}-06$ & 3.59E-06 \\
\hline 600 & 2.83 & 2.95 & 1.81 & 0.9984 & $3.03 \mathrm{E}-06$ & $4.40 E-06$ & $1.37 \mathrm{E}-06$ & 3.53E-06 & 1.99 & 0.9986 & $3.33 \mathrm{E}-06$ & 4.42E-06 & 1.09E-06 & $3.83 \mathrm{E}-06$ \\
\hline 700 & 3.30 & 3.40 & 2.26 & 0.9989 & $3.24 \mathrm{E}-06$ & $4.46 E-06$ & $1.22 \mathrm{E}-06$ & 3.74E-06 & 2.46 & 0.9991 & $3.53 \mathrm{E}-06$ & $4.62 E-06$ & $1.09 \mathrm{E}-06$ & 4.03E-06 \\
\hline 800 & 3.79 & 3.81 & 2.67 & 0.9993 & $3.35 \mathrm{E}-06$ & 4.59E-06 & $1.24 \mathrm{E}-06$ & $3.85 \mathrm{E}-06$ & 2.95 & 0.9996 & $3.70 \mathrm{E}-06$ & $4.85 E-06$ & 1.15E-06 & 4.20E-06 \\
\hline
\end{tabular}




\begin{tabular}{|c|c|c|c|c|c|c|c|c|c|c|c|c|c|c|}
\hline Capsule & OC3 & $\begin{array}{l}\text { Specimen } \\
\text { Length }\end{array}$ & $\begin{array}{c}124 \\
0.9972\end{array}$ & Scale & 1" & $1.00 \mathrm{E}-03$ & & & & & & & & \\
\hline $\begin{array}{c}\text { Temperature } \\
\left({ }^{\circ} \mathrm{C}\right)\end{array}$ & Up & Down & $\begin{array}{l}\text { Delta } \\
\text { Down }\end{array}$ & $\begin{array}{l}\text { Down } \\
\text { length }\end{array}$ & CTE & CTEK & $\begin{array}{l}\text { Delta } \\
\text { CTEK }\end{array}$ & $\begin{array}{c}\text { CTE } \\
\text { adjusted }\end{array}$ & $\begin{array}{l}\text { Delta } \\
\text { Up }\end{array}$ & $\begin{array}{c}\text { Up } \\
\text { length }\end{array}$ & CTE & CTEK & $\begin{array}{l}\text { Delta } \\
\text { CTEK }\end{array}$ & $\begin{array}{c}\text { CTE } \\
\text { adjusted }\end{array}$ \\
\hline 0 & 0.97 & 1.03 & 0 & 0.9972 & & & & & 0 & 0.9972 & & & & \\
\hline 100 & 1.16 & 1.20 & 0.17 & 0.9974 & 1.70E-06 & $2.25 E-06$ & $5.45 \mathrm{E}-07$ & $2.20 \mathrm{E}-06$ & 0.19 & 0.9974 & $1.91 \mathrm{E}-06$ & $2.45 E-06$ & $5.45 \mathrm{E}-07$ & $2.41 \mathrm{E}-06$ \\
\hline 200 & 1.36 & 1.40 & 0.37 & 0.9976 & $1.86 \mathrm{E}-06$ & $2.40 E-06$ & $5.45 \mathrm{E}-07$ & $2.36 \mathrm{E}-06$ & 0.39 & 0.9976 & $1.96 \mathrm{E}-06$ & $2.50 E-06$ & $5.45 \mathrm{E}-07$ & $2.46 \mathrm{E}-06$ \\
\hline 300 & 1.56 & 1.64 & 0.61 & 0.9978 & $2.04 \mathrm{E}-06$ & $2.58 E-06$ & $5.44 \mathrm{E}-07$ & $2.54 \mathrm{E}-06$ & 0.59 & 0.9978 & $1.97 \mathrm{E}-06$ & $2.52 E-06$ & $5.44 \mathrm{E}-07$ & 2.47E-06 \\
\hline 400 & 1.79 & 1.89 & 0.86 & 0.9981 & $2.16 \mathrm{E}-06$ & 2.70E-06 & $5.44 \mathrm{E}-07$ & $2.66 \mathrm{E}-06$ & 0.82 & 0.9980 & $2.06 \mathrm{E}-06$ & 2.60E-06 & $5.44 \mathrm{E}-07$ & $2.56 \mathrm{E}-06$ \\
\hline 500 & 2.01 & 2.17 & 1.14 & 0.9983 & $2.29 \mathrm{E}-06$ & $2.83 E-06$ & $5.44 \mathrm{E}-07$ & $2.79 \mathrm{E}-06$ & 1.04 & 0.9982 & 2.09E-06 & 2.63E-06 & $5.44 \mathrm{E}-07$ & $2.59 \mathrm{E}-06$ \\
\hline 600 & 2.30 & 2.50 & 1.47 & 0.9987 & $2.46 \mathrm{E}-06$ & $3.00 E-06$ & $5.43 \mathrm{E}-07$ & $2.96 \mathrm{E}-06$ & 1.33 & 0.9985 & $2.22 \mathrm{E}-06$ & 2.77E-06 & $5.43 \mathrm{E}-07$ & $2.72 \mathrm{E}-06$ \\
\hline 700 & 2.59 & 2.80 & 1.77 & 0.9990 & $2.54 \mathrm{E}-06$ & $3.08 E-06$ & $5.42 \mathrm{E}-07$ & $3.04 \mathrm{E}-06$ & 1.62 & 0.9988 & $2.32 \mathrm{E}-06$ & $2.86 E-06$ & $5.43 \mathrm{E}-07$ & $2.82 \mathrm{E}-06$ \\
\hline 800 & 2.90 & 3.15 & 2.12 & 0.9993 & $2.66 \mathrm{E}-06$ & $3.20 E-06$ & $5.43 \mathrm{E}-07$ & $3.16 \mathrm{E}-06$ & 1.93 & 0.9991 & $2.42 \mathrm{E}-06$ & $2.96 E-06$ & $5.43 \mathrm{E}-07$ & $2.92 \mathrm{E}-06$ \\
\hline
\end{tabular}

\begin{tabular}{|c|c|c|c|c|c|c|c|c|c|c|c|c|c|c|}
\hline Capsule & OC3 & $\begin{array}{l}\text { Specimen } \\
\text { Length }\end{array}$ & $\begin{array}{l}125 \\
0.998\end{array}$ & Scale & 1" & $1.00 \mathrm{E}-03$ & & & & & & & & \\
\hline $\begin{array}{c}\text { Temperature } \\
\left({ }^{\circ} \mathrm{C}\right)\end{array}$ & Up & Down & $\begin{array}{l}\text { Delta } \\
\text { Down }\end{array}$ & $\begin{array}{l}\text { Down } \\
\text { length }\end{array}$ & CTE & CTEK & $\begin{array}{l}\text { Delta } \\
\text { CTEK }\end{array}$ & $\begin{array}{c}\text { CTE } \\
\text { adjusted }\end{array}$ & $\begin{array}{l}\text { Delta } \\
\text { Up }\end{array}$ & $\begin{array}{c}\text { Up } \\
\text { length }\end{array}$ & CTE & CTEK & $\begin{array}{l}\text { Delta } \\
\text { CTEK }\end{array}$ & $\begin{array}{c}\text { CTE } \\
\text { adjusted }\end{array}$ \\
\hline 0 & 0.80 & 0.90 & 0 & 0.9980 & & & & & 0 & 0.9980 & & & & \\
\hline 100 & 1.00 & 1.10 & 0.2 & 0.9982 & $2.00 \mathrm{E}-06$ & $3.55 E-06$ & 1.55E-06 & $2.50 \mathrm{E}-06$ & 0.2 & 0.9982 & $2.00 \mathrm{E}-06$ & $3.65 E-06$ & $1.65 \mathrm{E}-06$ & $2.50 \mathrm{E}-06$ \\
\hline 200 & 1.27 & 1.32 & 0.42 & 0.9984 & $2.10 \mathrm{E}-06$ & $3.65 E-06$ & 1.55E-06 & $2.60 \mathrm{E}-06$ & 0.47 & 0.9985 & $2.35 \mathrm{E}-06$ & $3.80 E-06$ & $1.45 \mathrm{E}-06$ & $2.85 \mathrm{E}-06$ \\
\hline 300 & 1.60 & 1.60 & 0.7 & 0.9987 & $2.34 \mathrm{E}-06$ & $3.82 E-06$ & $1.48 \mathrm{E}-06$ & $2.84 \mathrm{E}-06$ & 0.8 & 0.9988 & 2.67E-06 & $4.02 E-06$ & $1.34 \mathrm{E}-06$ & 3.17E-06 \\
\hline 400 & 1.95 & 1.90 & 1 & 0.9990 & $2.51 \mathrm{E}-06$ & $4.08 E-06$ & 1.57E-06 & $3.01 \mathrm{E}-06$ & 1.15 & 0.9992 & $2.88 \mathrm{E}-06$ & 4.13E-06 & $1.24 \mathrm{E}-06$ & $3.38 \mathrm{E}-06$ \\
\hline 500 & 2.40 & 2.28 & 1.38 & 0.9994 & 2.77E-06 & $4.25 E-06$ & $1.48 \mathrm{E}-06$ & $3.27 \mathrm{E}-06$ & 1.6 & 0.9996 & $3.21 \mathrm{E}-06$ & 4.29E-06 & $1.08 \mathrm{E}-06$ & $3.71 \mathrm{E}-06$ \\
\hline 600 & 2.84 & 2.72 & 1.82 & 0.9998 & $3.04 \mathrm{E}-06$ & $4.40 E-06$ & $1.36 \mathrm{E}-06$ & $3.54 \mathrm{E}-06$ & 2.04 & 1.0000 & $3.41 \mathrm{E}-06$ & 4.42E-06 & $1.01 \mathrm{E}-06$ & $3.91 \mathrm{E}-06$ \\
\hline 700 & 3.30 & 3.22 & 2.32 & 1.0003 & $3.32 \mathrm{E}-06$ & $4.46 E-06$ & $1.14 \mathrm{E}-06$ & $3.82 \mathrm{E}-06$ & 2.5 & 1.0005 & $3.58 \mathrm{E}-06$ & $4.62 E-06$ & $1.04 \mathrm{E}-06$ & $4.08 \mathrm{E}-06$ \\
\hline 800 & 3.76 & 3.81 & 2.91 & 1.0009 & $3.64 \mathrm{E}-06$ & 4.59E-06 & $9.42 \mathrm{E}-07$ & 4.14E-06 & 2.96 & 1.0010 & $3.71 \mathrm{E}-06$ & $4.85 E-06$ & $1.14 \mathrm{E}-06$ & 4.21E-06 \\
\hline
\end{tabular}




\begin{tabular}{|c|c|c|c|c|c|c|c|c|c|c|c|c|c|c|}
\hline Capsule & OC3 & $\begin{array}{c}\text { Specimen } \\
\text { Length }\end{array}$ & $\begin{array}{c}126 \\
0.9976\end{array}$ & $\begin{array}{l}\text { check } \\
\text { Scale }\end{array}$ & $1 "$ & $1.00 \mathrm{E}-03$ & & & & & & & & \\
\hline $\begin{array}{c}\text { Temperature } \\
\left({ }^{\circ} \mathrm{C}\right)\end{array}$ & Up & Down & $\begin{array}{l}\text { Delta } \\
\text { Down }\end{array}$ & $\begin{array}{l}\text { Down } \\
\text { length }\end{array}$ & CTE & CTEK & $\begin{array}{l}\text { Delta } \\
\text { CTEK }\end{array}$ & $\begin{array}{c}\text { CTE } \\
\text { adjusted }\end{array}$ & $\begin{array}{l}\text { Delta } \\
\text { Up }\end{array}$ & $\begin{array}{c}\text { Up } \\
\text { length }\end{array}$ & CTE & CTEK & $\begin{array}{l}\text { Delta } \\
\text { CTEK }\end{array}$ & $\begin{array}{c}\text { CTE } \\
\text { adjusted }\end{array}$ \\
\hline 0 & 0.43 & 0.50 & 0 & 0.9976 & & & & & 0 & 0.9976 & & & & \\
\hline 100 & 0.80 & 0.86 & 0.36 & 0.9980 & 3.61E-06 & $2.35 E-06$ & $-1.26 \mathrm{E}-06$ & 4.11E-06 & 0.37 & 0.9980 & $3.71 \mathrm{E}-06$ & $2.35 E-06$ & $-1.36 \mathrm{E}-06$ & $4.21 \mathrm{E}-06$ \\
\hline 200 & 1.19 & 1.25 & 0.75 & 0.9984 & $3.76 \mathrm{E}-06$ & $2.45 E-06$ & $-1.31 E-06$ & $4.26 \mathrm{E}-06$ & 0.76 & 0.9984 & $3.81 \mathrm{E}-06$ & $2.45 E-06$ & $-1.36 \mathrm{E}-06$ & 4.31E-06 \\
\hline 300 & 1.59 & 1.66 & 1.16 & 0.9988 & $3.88 \mathrm{E}-06$ & $2.48 E-06$ & $-1.39 E-06$ & $4.38 \mathrm{E}-06$ & 1.16 & 0.9988 & $3.88 \mathrm{E}-06$ & $2.48 E-06$ & $-1.39 E-06$ & $4.38 \mathrm{E}-06$ \\
\hline 400 & 2.03 & 2.16 & 1.66 & 0.9993 & 4.16E-06 & $2.63 E-06$ & $-1.53 \mathrm{E}-06$ & $4.66 \mathrm{E}-06$ & 1.6 & 0.9992 & $4.01 \mathrm{E}-06$ & $2.55 E-06$ & $-1.46 \mathrm{E}-06$ & $4.51 \mathrm{E}-06$ \\
\hline 500 & 2.56 & 2.72 & 2.22 & 0.9998 & 4.45E-06 & 2.77E-06 & $-1.68 \mathrm{E}-06$ & 4.95E-06 & 2.13 & 0.9997 & 4.27E-06 & 2.67E-06 & $-1.60 \mathrm{E}-06$ & 4.77E-06 \\
\hline 600 & 3.18 & 3.28 & 2.78 & 1.0004 & 4.64E-06 & $2.87 E-06$ & $-1.78 \mathrm{E}-06$ & $5.14 \mathrm{E}-06$ & 2.75 & 1.0004 & 4.59E-06 & $2.83 E-06$ & $-1.76 \mathrm{E}-06$ & 5.09E-06 \\
\hline 700 & 3.83 & 3.92 & 3.42 & 1.0010 & 4.90E-06 & 2.99E-06 & $-1.91 \mathrm{E}-06$ & $5.40 \mathrm{E}-06$ & 3.4 & 1.0010 & 4.87E-06 & 2.98E-06 & $-1.89 \mathrm{E}-06$ & 5.37E-06 \\
\hline 800 & 4.55 & 4.65 & 4.15 & 1.0018 & $5.20 \mathrm{E}-06$ & $3.15 E-06$ & $-2.05 E-06$ & $5.70 \mathrm{E}-06$ & 4.12 & 1.0017 & $5.16 \mathrm{E}-06$ & $3.13 E-06$ & $-2.04 E-06$ & $5.66 \mathrm{E}-06$ \\
\hline
\end{tabular}

\begin{tabular}{|c|c|c|c|c|c|c|c|c|c|c|c|c|c|c|}
\hline Capsule & OC3 & $\begin{array}{l}\text { Specimen } \\
\text { Length }\end{array}$ & $\begin{array}{c}171 \\
0.9879\end{array}$ & Scale & 1" & $1.00 \mathrm{E}-03$ & & & & & & & & \\
\hline $\begin{array}{c}\text { Temperature } \\
\left({ }^{\circ} \mathrm{C}\right)\end{array}$ & Up & Down & $\begin{array}{l}\text { Delta } \\
\text { Down }\end{array}$ & $\begin{array}{l}\text { Down } \\
\text { length }\end{array}$ & CTE & CTEK & $\begin{array}{l}\text { Delta } \\
\text { CTEK }\end{array}$ & $\begin{array}{c}\text { CTE } \\
\text { adjusted }\end{array}$ & $\begin{array}{l}\text { Delta } \\
\text { Up }\end{array}$ & $\begin{array}{c}\text { Up } \\
\text { length }\end{array}$ & CTE & CTEK & $\begin{array}{l}\text { Delta } \\
\text { CTEK }\end{array}$ & $\begin{array}{c}\text { CTE } \\
\text { adjusted }\end{array}$ \\
\hline 0 & 0.90 & 1.00 & 0 & 0.9879 & & & & & 0 & 0.9879 & & & & \\
\hline 100 & 1.14 & 1.20 & 0.2 & 0.9881 & $2.02 \mathrm{E}-06$ & $2.55 E-06$ & $5.26 \mathrm{E}-07$ & $2.52 \mathrm{E}-06$ & 0.24 & 0.9881 & $2.43 \mathrm{E}-06$ & $2.95 E-06$ & $5.21 \mathrm{E}-07$ & 2.93E-06 \\
\hline 200 & 1.40 & 1.42 & 0.42 & 0.9883 & $2.13 \mathrm{E}-06$ & $2.65 E-06$ & $5.24 \mathrm{E}-07$ & $2.63 \mathrm{E}-06$ & 0.5 & 0.9884 & $2.53 \mathrm{E}-06$ & $3.05 E-06$ & 5.19E-07 & 3.03E-06 \\
\hline 300 & 1.72 & 1.69 & 0.69 & 0.9886 & $2.33 \mathrm{E}-06$ & $2.85 E-06$ & $5.22 \mathrm{E}-07$ & $2.83 \mathrm{E}-06$ & 0.82 & 0.9887 & $2.77 \mathrm{E}-06$ & $3.28 E-06$ & 5.16E-07 & 3.27E-06 \\
\hline 400 & 2.00 & 1.97 & 0.97 & 0.9889 & $2.45 \mathrm{E}-06$ & $2.98 E-06$ & $5.20 \mathrm{E}-07$ & $2.95 \mathrm{E}-06$ & 1.1 & 0.9890 & $2.78 \mathrm{E}-06$ & $3.30 E-06$ & 5.16E-07 & $3.28 \mathrm{E}-06$ \\
\hline 500 & 2.32 & 2.30 & 1.3 & 0.9892 & $2.63 \mathrm{E}-06$ & $3.15 E-06$ & $5.18 \mathrm{E}-07$ & $3.13 \mathrm{E}-06$ & 1.42 & 0.9893 & 2.87E-06 & 3.39E-06 & $5.15 \mathrm{E}-07$ & 3.37E-06 \\
\hline 600 & 2.70 & 2.70 & 1.7 & 0.9896 & $2.87 \mathrm{E}-06$ & $3.38 E-06$ & 5.15E-07 & 3.37E-06 & 1.8 & 0.9897 & $3.04 \mathrm{E}-06$ & $3.55 E-06$ & $5.13 \mathrm{E}-07$ & 3.54E-06 \\
\hline 700 & 3.10 & 3.08 & 2.08 & 0.9900 & $3.01 \mathrm{E}-06$ & $3.52 E-06$ & 5.13E-07 & $3.51 \mathrm{E}-06$ & 2.2 & 0.9901 & $3.18 \mathrm{E}-06$ & $3.69 E-06$ & $5.11 \mathrm{E}-07$ & $3.68 \mathrm{E}-06$ \\
\hline 800 & 3.55 & 3.57 & 2.57 & 0.9905 & $3.25 \mathrm{E}-06$ & $3.76 E-06$ & $5.10 \mathrm{E}-07$ & $3.75 \mathrm{E}-06$ & 2.65 & 0.9906 & $3.35 \mathrm{E}-06$ & $3.86 E-06$ & $5.09 \mathrm{E}-07$ & $3.85 \mathrm{E}-06$ \\
\hline
\end{tabular}




\begin{tabular}{|c|c|c|c|c|c|c|c|c|c|c|c|c|c|c|}
\hline Capsule & OC3 & $\begin{array}{l}\text { Specimen } \\
\text { Length }\end{array}$ & $\begin{array}{c}172 \\
0.9871\end{array}$ & Scale & 1" & $1.00 \mathrm{E}-03$ & & & & & & & & \\
\hline $\begin{array}{c}\text { Temperature } \\
\left({ }^{\circ} \mathrm{C}\right)\end{array}$ & Up & Down & $\begin{array}{l}\text { Delta } \\
\text { Down }\end{array}$ & $\begin{array}{l}\text { Down } \\
\text { length }\end{array}$ & CTE & CTEK & $\begin{array}{l}\text { Delta } \\
\text { CTEK }\end{array}$ & $\begin{array}{c}\text { CTE } \\
\text { adjusted }\end{array}$ & $\begin{array}{l}\text { Delta } \\
\text { Up }\end{array}$ & $\begin{array}{c}\text { Up } \\
\text { length }\end{array}$ & CTE & CTEK & $\begin{array}{l}\text { Delta } \\
\text { CTEK }\end{array}$ & $\begin{array}{c}\text { CTE } \\
\text { adjusted }\end{array}$ \\
\hline 0 & 1.43 & 1.98 & 0 & 0.9871 & & & & & 0 & 0.9871 & & & & \\
\hline 100 & 1.75 & 2.25 & 0.27 & 0.9874 & $2.74 \mathrm{E}-06$ & $3.25 E-06$ & $5.15 \mathrm{E}-07$ & $3.24 \mathrm{E}-06$ & 0.32 & 0.9874 & $3.24 \mathrm{E}-06$ & $3.75 E-06$ & $5.08 \mathrm{E}-07$ & 3.74E-06 \\
\hline 200 & 2.10 & 2.60 & 0.62 & 0.9877 & $3.14 \mathrm{E}-06$ & $3.65 E-06$ & 5.09E-07 & $3.64 \mathrm{E}-06$ & 0.67 & 0.9878 & 3.39E-06 & $3.90 E-06$ & $5.06 \mathrm{E}-07$ & 3.89E-06 \\
\hline 300 & 2.46 & 2.97 & 0.99 & 0.9881 & 3.34E-06 & $3.85 E-06$ & 5.07E-07 & $3.84 \mathrm{E}-06$ & 1.03 & 0.9881 & $3.48 \mathrm{E}-06$ & $3.98 E-06$ & $5.05 \mathrm{E}-07$ & $3.98 \mathrm{E}-06$ \\
\hline 400 & 2.85 & 3.38 & 1.4 & 0.9885 & $3.55 \mathrm{E}-06$ & $4.05 E-06$ & $5.04 \mathrm{E}-07$ & $4.05 E-06$ & 1.42 & 0.9885 & $3.60 \mathrm{E}-06$ & 4.10E-06 & $5.04 \mathrm{E}-07$ & 4.10E-06 \\
\hline 500 & 3.28 & 3.80 & 1.82 & 0.9889 & $3.69 \mathrm{E}-06$ & 4.19E-06 & $5.02 \mathrm{E}-07$ & 4.19E-06 & 1.85 & 0.9890 & $3.75 \mathrm{E}-06$ & $4.25 E-06$ & $5.02 \mathrm{E}-07$ & 4.25E-06 \\
\hline 600 & 3.72 & 4.26 & 2.28 & 0.9894 & $3.85 \mathrm{E}-06$ & $4.35 E-06$ & $5.00 \mathrm{E}-07$ & $4.35 \mathrm{E}-06$ & 2.29 & 0.9894 & 3.87E-06 & 4.37E-06 & 4.99E-07 & 4.37E-06 \\
\hline 700 & 4.17 & 4.69 & 2.71 & 0.9898 & $3.92 \mathrm{E}-06$ & 4.42E-06 & 4.99E-07 & $4.42 \mathrm{E}-06$ & 2.74 & 0.9898 & 3.97E-06 & 4.46E-06 & $4.99 \mathrm{E}-07$ & 4.47E-06 \\
\hline 800 & 4.60 & 5.12 & 3.14 & 0.9902 & $3.98 \mathrm{E}-06$ & $4.48 E-06$ & 4.99E-07 & $4.48 \mathrm{E}-06$ & 3.17 & 0.9903 & $4.01 \mathrm{E}-06$ & $4.51 E-06$ & $4.98 \mathrm{E}-07$ & 4.51E-06 \\
\hline
\end{tabular}

\begin{tabular}{|c|c|c|c|c|c|c|c|c|c|c|c|c|c|c|}
\hline Capsule & OC3 & $\begin{array}{l}\text { Specimen } \\
\text { Length }\end{array}$ & $\begin{array}{c}173 \\
0.9858\end{array}$ & Scale & 1" & $1.00 \mathrm{E}-03$ & & & & & & & & \\
\hline $\begin{array}{c}\text { Temperature } \\
\left({ }^{\circ} \mathrm{C}\right)\end{array}$ & Up & Down & $\begin{array}{l}\text { Delta } \\
\text { Down }\end{array}$ & $\begin{array}{l}\text { Down } \\
\text { length }\end{array}$ & CTE & CTEK & $\begin{array}{l}\text { Delta } \\
\text { CTEK }\end{array}$ & $\begin{array}{c}\text { CTE } \\
\text { adjusted }\end{array}$ & $\begin{array}{l}\text { Delta } \\
\text { Up }\end{array}$ & $\begin{array}{c}\text { Up } \\
\text { length }\end{array}$ & CTE & CTEK & $\begin{array}{l}\text { Delta } \\
\text { CTEK }\end{array}$ & $\begin{array}{c}\text { CTE } \\
\text { adjusted }\end{array}$ \\
\hline 0 & 1.56 & 1.77 & 0 & 0.9858 & & & & & 0 & 0.9858 & & & & \\
\hline 100 & 1.79 & 1.95 & 0.18 & 0.9860 & $1.83 \mathrm{E}-06$ & $2.35 E-06$ & $5.24 \mathrm{E}-07$ & $2.33 \mathrm{E}-06$ & 0.23 & 0.9860 & $2.33 \mathrm{E}-06$ & $2.85 E-06$ & 5.17E-07 & $2.83 \mathrm{E}-06$ \\
\hline 200 & 2.00 & 2.18 & 0.41 & 0.9862 & $2.08 \mathrm{E}-06$ & $2.60 E-06$ & $5.20 \mathrm{E}-07$ & $2.58 \mathrm{E}-06$ & 0.44 & 0.9862 & $2.23 \mathrm{E}-06$ & $2.75 E-06$ & $5.18 \mathrm{E}-07$ & 2.73E-06 \\
\hline 300 & 2.30 & 2.41 & 0.64 & 0.9864 & $2.16 \mathrm{E}-06$ & $2.68 E-06$ & 5.19E-07 & $2.66 \mathrm{E}-06$ & 0.74 & 0.9865 & $2.50 \mathrm{E}-06$ & $3.02 E-06$ & $5.14 \mathrm{E}-07$ & 3.00E-06 \\
\hline 400 & 2.58 & 2.66 & 0.89 & 0.9867 & $2.26 \mathrm{E}-06$ & $2.78 E-06$ & $5.18 \mathrm{E}-07$ & $2.76 \mathrm{E}-06$ & 1.02 & 0.9868 & $2.59 \mathrm{E}-06$ & $3.10 E-06$ & 5.13E-07 & 3.09E-06 \\
\hline 500 & 2.89 & 2.97 & 1.2 & 0.9870 & $2.43 \mathrm{E}-06$ & 2.95E-06 & 5.15E-07 & 2.93E-06 & 1.33 & 0.9871 & $2.70 \mathrm{E}-06$ & 3.21E-06 & $5.12 \mathrm{E}-07$ & 3.20E-06 \\
\hline 600 & 3.19 & 3.29 & 1.52 & 0.9873 & $2.57 \mathrm{E}-06$ & $3.08 E-06$ & 5.13E-07 & 3.07E-06 & 1.63 & 0.9874 & $2.76 \mathrm{E}-06$ & $3.27 E-06$ & $5.10 \mathrm{E}-07$ & $3.26 \mathrm{E}-06$ \\
\hline 700 & 3.57 & 3.62 & 1.85 & 0.9877 & $2.68 \mathrm{E}-06$ & $3.19 E-06$ & 5.11E-07 & $3.18 \mathrm{E}-06$ & 2.01 & 0.9878 & $2.91 \mathrm{E}-06$ & $3.42 E-06$ & $5.08 \mathrm{E}-07$ & $3.41 \mathrm{E}-06$ \\
\hline 800 & 3.99 & 3.99 & 2.22 & 0.9880 & $2.81 \mathrm{E}-06$ & $3.33 E-06$ & $5.10 \mathrm{E}-07$ & $3.31 \mathrm{E}-06$ & 2.43 & 0.9882 & $3.08 \mathrm{E}-06$ & $3.59 E-06$ & $5.06 \mathrm{E}-07$ & $3.58 \mathrm{E}-06$ \\
\hline
\end{tabular}




\begin{tabular}{|c|c|c|c|c|c|c|c|c|c|c|c|c|c|c|}
\hline Capsule & OC3 & $\begin{array}{l}\text { Specimen } \\
\text { Length }\end{array}$ & $\begin{array}{c}206 \\
0.998\end{array}$ & Scale & 1" & $1.00 \mathrm{E}-03$ & & & & & & & & \\
\hline $\begin{array}{c}\text { Temperature } \\
\left({ }^{\circ} \mathrm{C}\right)\end{array}$ & Up & Down & $\begin{array}{l}\text { Delta } \\
\text { Down }\end{array}$ & $\begin{array}{l}\text { Down } \\
\text { length }\end{array}$ & CTE & CTEK & $\begin{array}{l}\text { Delta } \\
\text { CTEK }\end{array}$ & $\begin{array}{c}\text { CTE } \\
\text { adjusted }\end{array}$ & $\begin{array}{l}\text { Delta } \\
\text { Up }\end{array}$ & $\begin{array}{c}\text { Up } \\
\text { length }\end{array}$ & CTE & CTEK & $\begin{array}{l}\text { Delta } \\
\text { CTEK }\end{array}$ & $\begin{array}{c}\text { CTE } \\
\text { adjusted }\end{array}$ \\
\hline 0 & 0.90 & 1.20 & 0 & 0.9980 & & & & & 0 & 0.9980 & & & & \\
\hline 100 & 1.25 & 1.51 & 0.31 & 0.9983 & 3.11E-06 & $3.55 E-06$ & 4.44E-07 & $3.61 \mathrm{E}-06$ & 0.35 & 0.9984 & $3.51 \mathrm{E}-06$ & $3.65 E-06$ & $1.43 \mathrm{E}-07$ & 4.01E-06 \\
\hline 200 & 1.75 & 1.99 & 0.79 & 0.9988 & 3.96E-06 & $3.65 E-06$ & $-3.08 \mathrm{E}-07$ & 4.46E-06 & 0.85 & 0.9989 & 4.26E-06 & $3.80 E-06$ & $-4.59 \mathrm{E}-07$ & 4.76E-06 \\
\hline 300 & 2.38 & 2.50 & 1.3 & 0.9993 & 4.34E-06 & $3.82 E-06$ & $-5.26 \mathrm{E}-07$ & 4.84E-06 & 1.48 & 0.9995 & 4.94E-06 & $4.02 E-06$ & $-9.27 \mathrm{E}-07$ & $5.44 \mathrm{E}-06$ \\
\hline 400 & 3.12 & 3.23 & 2.03 & 1.0000 & 5.09E-06 & $4.08 E-06$ & $-1.01 E-06$ & 5.59E-06 & 2.22 & 1.0002 & $5.56 \mathrm{E}-06$ & 4.13E-06 & $-1.44 \mathrm{E}-06$ & $6.06 \mathrm{E}-06$ \\
\hline 500 & 3.97 & 4.02 & 2.82 & 1.0008 & $5.65 \mathrm{E}-06$ & $4.25 E-06$ & $-1.40 \mathrm{E}-06$ & $6.15 \mathrm{E}-06$ & 3.07 & 1.0011 & $6.15 \mathrm{E}-06$ & 4.29E-06 & $-1.86 \mathrm{E}-06$ & $6.65 \mathrm{E}-06$ \\
\hline 600 & 4.82 & 4.82 & 3.62 & 1.0016 & $6.05 E-06$ & 4.40E-06 & $-1.65 E-06$ & $6.55 \mathrm{E}-06$ & 3.92 & 1.0019 & $6.55 \mathrm{E}-06$ & $4.42 E-06$ & $-2.13 E-06$ & $7.05 E-06$ \\
\hline 700 & 5.78 & 5.81 & 4.61 & 1.0026 & $6.60 \mathrm{E}-06$ & 4.46E-06 & $-2.13 E-06$ & $7.10 \mathrm{E}-06$ & 4.88 & 1.0029 & $6.99 \mathrm{E}-06$ & $4.62 E-06$ & $-2.36 \mathrm{E}-06$ & 7.49E-06 \\
\hline 800 & 6.80 & 6.89 & 5.69 & 1.0037 & 7.13E-06 & 4.59E-06 & $-2.54 \mathrm{E}-06$ & 7.63E-06 & 5.9 & 1.0039 & 7.39E-06 & $4.85 E-06$ & $-2.54 \mathrm{E}-06$ & $7.89 \mathrm{E}-06$ \\
\hline
\end{tabular}

\begin{tabular}{|c|c|c|c|c|c|c|c|c|c|c|c|c|c|c|}
\hline Capsule & OC3 & $\begin{array}{l}\text { Specimen } \\
\text { Length }\end{array}$ & $\begin{array}{l}207 \\
0.9976\end{array}$ & Scale & 1" & $1.00 \mathrm{E}-03$ & & & & & & & & \\
\hline $\begin{array}{c}\text { Temperature } \\
\left({ }^{\circ} \mathrm{C}\right)\end{array}$ & Up & Down & $\begin{array}{l}\text { Delta } \\
\text { Down }\end{array}$ & $\begin{array}{l}\text { Down } \\
\text { length }\end{array}$ & CTE & CTEK & $\begin{array}{l}\text { Delta } \\
\text { CTEK }\end{array}$ & $\begin{array}{c}\text { CTE } \\
\text { adjusted }\end{array}$ & $\begin{array}{l}\text { Delta } \\
\text { Up }\end{array}$ & $\begin{array}{c}\text { Up } \\
\text { length }\end{array}$ & CTE & CTEK & $\begin{array}{l}\text { Delta } \\
\text { CTEK }\end{array}$ & $\begin{array}{c}\text { CTE } \\
\text { adjusted }\end{array}$ \\
\hline 0 & 0.88 & 1.12 & 0 & 0.9976 & & & & & 0 & 0.9976 & & & & \\
\hline 100 & 1.06 & 1.31 & 0.19 & 0.9978 & $1.90 \mathrm{E}-06$ & $3.55 E-06$ & 1.65E-06 & $2.40 \mathrm{E}-06$ & 0.18 & 0.9978 & $1.80 \mathrm{E}-06$ & $3.65 E-06$ & $1.85 \mathrm{E}-06$ & 2.30E-06 \\
\hline 200 & 1.30 & 1.53 & 0.41 & 0.9980 & $2.05 \mathrm{E}-06$ & $3.65 E-06$ & $1.60 \mathrm{E}-06$ & $2.55 \mathrm{E}-06$ & 0.42 & 0.9980 & $2.11 \mathrm{E}-06$ & $3.80 E-06$ & $1.69 \mathrm{E}-06$ & $2.61 \mathrm{E}-06$ \\
\hline 300 & 1.59 & 1.78 & 0.66 & 0.9983 & $2.21 \mathrm{E}-06$ & $3.82 E-06$ & $1.61 \mathrm{E}-06$ & $2.71 \mathrm{E}-06$ & 0.71 & 0.9983 & $2.37 \mathrm{E}-06$ & $4.02 E-06$ & $1.64 \mathrm{E}-06$ & 2.87E-06 \\
\hline 400 & 1.90 & 2.09 & 0.97 & 0.9986 & $2.43 \mathrm{E}-06$ & $4.08 E-06$ & $1.64 \mathrm{E}-06$ & $2.93 \mathrm{E}-06$ & 1.02 & 0.9986 & $2.56 \mathrm{E}-06$ & 4.13E-06 & 1.57E-06 & 3.06E-06 \\
\hline 500 & 2.45 & 2.45 & 1.33 & 0.9989 & 2.67E-06 & $4.25 E-06$ & $1.58 \mathrm{E}-06$ & 3.17E-06 & 1.57 & 0.9992 & $3.15 \mathrm{E}-06$ & 4.29E-06 & 1.14E-06 & $3.65 \mathrm{E}-06$ \\
\hline 600 & 2.93 & 2.87 & 1.75 & 0.9994 & $2.92 \mathrm{E}-06$ & $4.40 E-06$ & $1.48 \mathrm{E}-06$ & $3.42 \mathrm{E}-06$ & 2.05 & 0.9997 & $3.42 \mathrm{E}-06$ & 4.42E-06 & $9.91 \mathrm{E}-07$ & $3.92 \mathrm{E}-06$ \\
\hline 700 & 3.45 & 3.38 & 2.26 & 0.9999 & $3.24 \mathrm{E}-06$ & $4.46 E-06$ & $1.23 \mathrm{E}-06$ & 3.74E-06 & 2.57 & 1.0002 & $3.68 \mathrm{E}-06$ & $4.62 E-06$ & $9.41 \mathrm{E}-07$ & $4.18 \mathrm{E}-06$ \\
\hline 800 & 3.99 & 4.02 & 2.9 & 1.0005 & $3.63 \mathrm{E}-06$ & 4.59E-06 & $9.53 \mathrm{E}-07$ & 4.13E-06 & 3.11 & 1.0007 & $3.90 \mathrm{E}-06$ & $4.85 E-06$ & $9.53 \mathrm{E}-07$ & 4.40E-06 \\
\hline
\end{tabular}




\section{OC2 Dilatometer Data}

\begin{tabular}{|c|c|c|c|c|c|c|c|c|c|c|c|c|c|c|}
\hline $\begin{array}{l}\text { Capsule } \\
\text { Column }\end{array}$ & $\begin{array}{c}\text { OC2 } \\
\text { East }\end{array}$ & $\begin{array}{c}\text { Specimen } \\
\text { Length }\end{array}$ & $\begin{array}{c}15 \\
0.9902\end{array}$ & $\begin{array}{c}\text { Material } \\
\text { Scale }\end{array}$ & $\begin{array}{c}\text { H-451 } \\
1 "\end{array}$ & $5.00 \mathrm{E}-04$ & & & & & & & & \\
\hline $\begin{array}{c}\text { Temperature } \\
\left({ }^{\circ} \mathrm{C}\right) \\
\end{array}$ & Up & Down & $\begin{array}{l}\text { Delta } \\
\text { Down }\end{array}$ & $\begin{array}{l}\text { Down } \\
\text { length }\end{array}$ & CTE & CTEK & $\begin{array}{l}\text { Delta } \\
\text { CTEK }\end{array}$ & $\begin{array}{c}\text { CTE } \\
\text { adjusted }\end{array}$ & $\begin{array}{l}\text { Delta } \\
\text { Up }\end{array}$ & $\begin{array}{c}\text { Up } \\
\text { length }\end{array}$ & CTE & CTEK & $\begin{array}{l}\text { Delta } \\
\text { CTEK }\end{array}$ & $\begin{array}{c}\text { CTE } \\
\text { adjusted }\end{array}$ \\
\hline 0 & 0.78 & 0.80 & 0 & 0.9902 & & & & & 0 & 0.9902 & & & & \\
\hline 100 & 1.41 & 1.52 & 0.72 & 0.9906 & $3.64 \mathrm{E}-06$ & & & 4.14E-06 & 0.63 & 0.9905 & $3.18 \mathrm{E}-06$ & & & $3.68 \mathrm{E}-06$ \\
\hline 200 & 2.21 & 2.28 & 1.48 & 0.9909 & $3.74 \mathrm{E}-06$ & & & 4.24E-06 & 1.43 & 0.9909 & $3.61 \mathrm{E}-06$ & & & 4.11E-06 \\
\hline 300 & 2.98 & 3.12 & 2.32 & 0.9914 & $3.90 \mathrm{E}-06$ & & & 4.40E-06 & 2.2 & 0.9913 & $3.70 \mathrm{E}-06$ & & & 4.20E-06 \\
\hline 400 & 3.75 & 4.10 & 3.3 & 0.9919 & 4.17E-06 & & & 4.67E-06 & 2.97 & 0.9917 & $3.75 \mathrm{E}-06$ & & & $4.25 \mathrm{E}-06$ \\
\hline 500 & 4.80 & 5.00 & 4.2 & 0.9923 & $4.24 \mathrm{E}-06$ & $4.50 E-06$ & $2.42 \mathrm{E}-07$ & 4.74E-06 & 4.02 & 0.9922 & $4.06 \mathrm{E}-06$ & $4.50 E-06$ & $5.98 \mathrm{E}-08$ & 4.56E-06 \\
\hline
\end{tabular}

\begin{tabular}{|c|c|c|c|c|c|c|c|c|c|c|c|c|c|c|}
\hline $\begin{array}{l}\text { Capsule } \\
\text { Column }\end{array}$ & $\begin{array}{c}\text { OC2 } \\
\text { East }\end{array}$ & $\begin{array}{c}\text { Specimen } \\
\text { Length }\end{array}$ & $\begin{array}{c}20 \\
0.9903 \\
\end{array}$ & $\begin{array}{c}\text { Material } \\
\text { Scale }\end{array}$ & $\begin{array}{c}\text { H-451 } \\
1 "\end{array}$ & $5.00 \mathrm{E}-04$ & & & & & & & & \\
\hline $\begin{array}{c}\text { Temperature } \\
\left({ }^{\circ} \mathrm{C}\right)\end{array}$ & Up & Down & $\begin{array}{l}\text { Delta } \\
\text { Down }\end{array}$ & $\begin{array}{l}\text { Down } \\
\text { length }\end{array}$ & CTE & CTEK & $\begin{array}{l}\text { Delta } \\
\text { CTEK }\end{array}$ & $\begin{array}{c}\text { CTE } \\
\text { adjusted }\end{array}$ & $\begin{array}{l}\text { Delta } \\
\text { Up }\end{array}$ & $\begin{array}{c}\text { Up } \\
\text { length }\end{array}$ & CTE & CTEK & $\begin{array}{l}\text { Delta } \\
\text { CTEK }\end{array}$ & $\begin{array}{c}\text { CTE } \\
\text { adjusted }\end{array}$ \\
\hline 0 & 1.10 & 1.45 & 0 & 0.9903 & & & & & 0 & 0.9903 & & & & \\
\hline 100 & 1.77 & 2.02 & 0.57 & 0.9906 & $2.88 \mathrm{E}-06$ & & $-2.88 \mathrm{E}-06$ & $3.38 \mathrm{E}-06$ & 0.67 & 0.9906 & $3.38 \mathrm{E}-06$ & & $-3.38 \mathrm{E}-06$ & $3.88 \mathrm{E}-06$ \\
\hline 200 & 2.50 & 2.70 & 1.25 & 0.9909 & $3.16 \mathrm{E}-06$ & & $-3.16 \mathrm{E}-06$ & $3.66 \mathrm{E}-06$ & 1.4 & 0.9910 & 3.53E-06 & & $-3.53 \mathrm{E}-06$ & 4.03E-06 \\
\hline 300 & 3.30 & 3.52 & 2.07 & 0.9913 & $3.48 \mathrm{E}-06$ & & $-3.48 \mathrm{E}-06$ & 3.98E-06 & 2.2 & 0.9914 & 3.70E-06 & & $-3.70 \mathrm{E}-06$ & 4.20E-06 \\
\hline 400 & 4.21 & 4.50 & 3.05 & 0.9918 & $3.85 \mathrm{E}-06$ & & $-3.85 \mathrm{E}-06$ & 4.35E-06 & 3.11 & 0.9919 & 3.93E-06 & & $-3.93 \mathrm{E}-06$ & 4.43E-06 \\
\hline 500 & 5.22 & 5.39 & 3.94 & 0.9923 & $3.98 \mathrm{E}-06$ & 4.57E-06 & $5.91 \mathrm{E}-07$ & $4.48 \mathrm{E}-06$ & 4.12 & 0.9924 & 4.16E-06 & 4.57E-06 & $4.10 \mathrm{E}-07$ & 4.66E-06 \\
\hline
\end{tabular}

\begin{tabular}{|c|c|c|c|c|c|c|c|c|c|c|c|c|c|c|}
\hline $\begin{array}{l}\text { Capsule } \\
\text { Column }\end{array}$ & $\begin{array}{l}\text { OC2 } \\
\text { East }\end{array}$ & $\begin{array}{c}\text { Specimen } \\
\text { Length }\end{array}$ & $\begin{array}{c}25 \\
0.9896\end{array}$ & $\begin{array}{c}\text { Material } \\
\text { Scale }\end{array}$ & $\begin{array}{c}\text { H-451 } \\
1 "\end{array}$ & $5.00 \mathrm{E}-04$ & & & & & & & & \\
\hline $\begin{array}{c}\text { Temperature } \\
\left({ }^{\circ} \mathrm{C}\right)\end{array}$ & Up & Down & $\begin{array}{l}\text { Delta } \\
\text { Down }\end{array}$ & $\begin{array}{l}\text { Down } \\
\text { length }\end{array}$ & CTE & CTEK & $\begin{array}{l}\text { Delta } \\
\text { CTEK }\end{array}$ & $\begin{array}{c}\text { CTE } \\
\text { adjusted }\end{array}$ & $\begin{array}{l}\text { Delta } \\
\text { Up }\end{array}$ & $\begin{array}{c}\text { Up } \\
\text { length }\end{array}$ & CTE & CTEK & $\begin{array}{l}\text { Delta } \\
\text { CTEK }\end{array}$ & $\begin{array}{c}\text { CTE } \\
\text { adjusted }\end{array}$ \\
\hline 0 & 0.90 & 1.27 & 0 & 0.9896 & & & & & 0 & 0.9896 & & & & \\
\hline 100 & 1.60 & 1.81 & 0.54 & 0.9899 & $2.73 \mathrm{E}-06$ & & $-2.73 E-06$ & $3.23 \mathrm{E}-06$ & 0.7 & 0.9900 & $3.54 \mathrm{E}-06$ & & $-3.54 \mathrm{E}-06$ & 4.04E-06 \\
\hline 200 & 2.35 & 2.50 & 1.23 & 0.9902 & $3.11 \mathrm{E}-06$ & & $-3.11 \mathrm{E}-06$ & 3.61E-06 & 1.45 & 0.9903 & $3.66 \mathrm{E}-06$ & & $-3.66 \mathrm{E}-06$ & 4.16E-06 \\
\hline 300 & 3.22 & 3.35 & 2.08 & 0.9906 & $3.50 \mathrm{E}-06$ & & $-3.50 \mathrm{E}-06$ & 4.00E-06 & 2.32 & 0.9908 & 3.91E-06 & & $-3.91 \mathrm{E}-06$ & 4.41E-06 \\
\hline
\end{tabular}


ORNL/TM-2009/176

\begin{tabular}{|c|c|c|c|c|c|c|c|c|c|c|c|c|c|c|}
\hline 400 & 4.11 & 4.32 & 3.05 & 0.9911 & $3.85 \mathrm{E}-06$ & & $-3.85 E-06$ & 4.35E-06 & 3.21 & 0.9912 & $4.05 \mathrm{E}-06$ & & $-4.05 E-06$ & $4.55 \mathrm{E}-06$ \\
\hline 500 & 5.12 & 5.22 & 3.95 & 0.9916 & $3.99 \mathrm{E}-06$ & $4.68 E-06$ & $6.88 \mathrm{E}-07$ & 4.49E-06 & 4.22 & 0.9917 & 4.26E-06 & $4.68 E-06$ & $-4.26 \mathrm{E}-06$ & 4.76E-06 \\
\hline
\end{tabular}




\begin{tabular}{|c|c|c|c|c|c|c|c|c|c|c|c|c|c|c|}
\hline $\begin{array}{l}\text { Capsule } \\
\text { Column }\end{array}$ & $\begin{array}{l}\text { OC2 } \\
\text { East }\end{array}$ & $\begin{array}{c}\text { Specimen } \\
\text { Length }\end{array}$ & $\begin{array}{c}27 \\
0.9898\end{array}$ & $\begin{array}{c}\text { Material } \\
\text { Scale }\end{array}$ & $\begin{array}{c}\text { H-451 } \\
1 "\end{array}$ & $5.00 \mathrm{E}-04$ & & & & & & & & \\
\hline $\begin{array}{c}\text { Temperature } \\
\left({ }^{\circ} \mathrm{C}\right) \\
\end{array}$ & Up & Down & $\begin{array}{l}\text { Delta } \\
\text { Down }\end{array}$ & $\begin{array}{l}\text { Down } \\
\text { length }\end{array}$ & CTE & CTEK & $\begin{array}{l}\text { Delta } \\
\text { CTEK }\end{array}$ & $\begin{array}{c}\text { CTE } \\
\text { adjusted }\end{array}$ & $\begin{array}{l}\text { Delta } \\
\text { Up }\end{array}$ & $\begin{array}{c}\text { Up } \\
\text { length }\end{array}$ & CTE & CTEK & $\begin{array}{l}\text { Delta } \\
\text { CTEK }\end{array}$ & $\begin{array}{c}\text { CTE } \\
\text { adjusted }\end{array}$ \\
\hline 0 & 1.00 & 1.10 & 0 & 0.9898 & & & & & 0 & 0.9898 & & & & \\
\hline 100 & 1.52 & 1.67 & 0.57 & 0.9901 & $2.88 \mathrm{E}-06$ & & $-2.88 \mathrm{E}-06$ & $3.38 \mathrm{E}-06$ & 0.52 & 0.9901 & $2.63 \mathrm{E}-06$ & & $-2.63 \mathrm{E}-06$ & $3.13 \mathrm{E}-06$ \\
\hline 200 & 2.20 & 2.31 & 1.21 & 0.9904 & 3.06E-06 & & $-3.06 \mathrm{E}-06$ & $3.56 \mathrm{E}-06$ & 1.2 & 0.9904 & $3.03 \mathrm{E}-06$ & & $-3.03 E-06$ & 3.53E-06 \\
\hline 300 & 3.02 & 3.05 & 1.95 & 0.9908 & $3.28 \mathrm{E}-06$ & & $-3.28 \mathrm{E}-06$ & $3.78 \mathrm{E}-06$ & 2.02 & 0.9908 & $3.40 \mathrm{E}-06$ & & $-3.40 \mathrm{E}-06$ & $3.90 \mathrm{E}-06$ \\
\hline 400 & 4.00 & 4.10 & 3 & 0.9913 & $3.79 \mathrm{E}-06$ & & $-3.79 \mathrm{E}-06$ & 4.29E-06 & 3 & 0.9913 & $3.79 \mathrm{E}-06$ & & $-3.79 \mathrm{E}-06$ & 4.29E-06 \\
\hline 500 & 4.72 & 4.90 & 3.8 & 0.9917 & $3.84 \mathrm{E}-06$ & $4.08 E-06$ & $2.41 \mathrm{E}-07$ & $4.34 \mathrm{E}-06$ & 3.72 & 0.9917 & $3.76 \mathrm{E}-06$ & $4.08 E-06$ & $3.22 \mathrm{E}-07$ & $4.26 \mathrm{E}-06$ \\
\hline
\end{tabular}

\begin{tabular}{|c|c|c|c|c|c|c|c|c|c|c|c|c|c|c|}
\hline $\begin{array}{l}\text { Capsule } \\
\text { Column }\end{array}$ & $\begin{array}{l}\text { OC2 } \\
\text { East }\end{array}$ & $\begin{array}{l}\text { Specimen } \\
\text { Length }\end{array}$ & $\begin{array}{c}30 \\
0.988\end{array}$ & $\begin{array}{l}\text { Material } \\
\text { Scale }\end{array}$ & $\begin{array}{c}\text { H-451 } \\
1 "\end{array}$ & $5.00 \mathrm{E}-04$ & & & & & & & & \\
\hline $\begin{array}{c}\text { Temperature } \\
\left({ }^{\circ} \mathrm{C}\right)\end{array}$ & Up & Down & $\begin{array}{l}\text { Delta } \\
\text { Down }\end{array}$ & $\begin{array}{l}\text { Down } \\
\text { length }\end{array}$ & CTE & CTEK & $\begin{array}{l}\text { Delta } \\
\text { CTEK }\end{array}$ & $\begin{array}{c}\text { CTE } \\
\text { adjusted }\end{array}$ & $\begin{array}{l}\text { Delta } \\
\text { Up }\end{array}$ & $\begin{array}{c}\text { Up } \\
\text { length }\end{array}$ & CTE & CTEK & $\begin{array}{l}\text { Delta } \\
\text { CTEK }\end{array}$ & $\begin{array}{c}\text { CTE } \\
\text { adjusted }\end{array}$ \\
\hline 0 & 0.82 & 0.95 & 0 & 0.9880 & & & & & 0 & 0.9880 & & & & \\
\hline 100 & 1.68 & 1.70 & 0.75 & 0.9884 & $3.80 \mathrm{E}-06$ & & $-3.80 \mathrm{E}-06$ & $4.30 \mathrm{E}-06$ & 0.86 & 0.9884 & $4.35 \mathrm{E}-06$ & & $-4.35 \mathrm{E}-06$ & $4.85 \mathrm{E}-06$ \\
\hline 200 & 2.49 & 2.52 & 1.57 & 0.9888 & 3.97E-06 & & $-3.97 \mathrm{E}-06$ & 4.47E-06 & 1.67 & 0.9888 & $4.23 \mathrm{E}-06$ & & $-4.23 E-06$ & 4.73E-06 \\
\hline 300 & 3.40 & 3.58 & 2.63 & 0.9893 & 4.44E-06 & & $-4.44 \mathrm{E}-06$ & $4.94 \mathrm{E}-06$ & 2.58 & 0.9893 & $4.35 \mathrm{E}-06$ & & $-4.35 E-06$ & $4.85 \mathrm{E}-06$ \\
\hline 400 & 4.41 & 4.70 & 3.75 & 0.9899 & $4.74 \mathrm{E}-06$ & & $-4.74 \mathrm{E}-06$ & $5.24 \mathrm{E}-06$ & 3.59 & 0.9898 & $4.54 \mathrm{E}-06$ & & $-4.54 \mathrm{E}-06$ & $5.04 \mathrm{E}-06$ \\
\hline 500 & 5.60 & 5.72 & 4.77 & 0.9904 & $4.83 \mathrm{E}-06$ & $5.24 E-06$ & $4.12 \mathrm{E}-07$ & $5.33 \mathrm{E}-06$ & 4.78 & 0.9904 & $4.84 \mathrm{E}-06$ & $5.24 E-06$ & $4.02 \mathrm{E}-07$ & $5.34 \mathrm{E}-06$ \\
\hline
\end{tabular}

\begin{tabular}{|c|c|c|c|c|c|c|c|c|c|c|c|c|c|c|}
\hline $\begin{array}{l}\text { Capsule } \\
\text { Column }\end{array}$ & $\begin{array}{c}\text { OC2 } \\
\text { East }\end{array}$ & $\begin{array}{c}\text { Specimen } \\
\text { Length }\end{array}$ & $\begin{array}{c}40 \\
0.9916 \\
\end{array}$ & $\begin{array}{c}\text { Material } \\
\text { Scale }\end{array}$ & $\begin{array}{c}\text { H-451 } \\
1 " \\
\end{array}$ & $5.00 \mathrm{E}-04$ & & & & & & & & \\
\hline $\begin{array}{c}\text { Temperature } \\
\left({ }^{\circ} \mathrm{C}\right)\end{array}$ & Up & Down & $\begin{array}{l}\text { Delta } \\
\text { Down }\end{array}$ & $\begin{array}{l}\text { Down } \\
\text { length }\end{array}$ & CTE & CTEK & $\begin{array}{l}\text { Delta } \\
\text { CTEK }\end{array}$ & $\begin{array}{c}\text { CTE } \\
\text { adjusted }\end{array}$ & $\begin{array}{l}\text { Delta } \\
\text { Up }\end{array}$ & $\begin{array}{c}\text { Up } \\
\text { length }\end{array}$ & CTE & CTEK & $\begin{array}{l}\text { Delta } \\
\text { CTEK }\end{array}$ & $\begin{array}{c}\text { CTE } \\
\text { adjusted }\end{array}$ \\
\hline 0 & 0.81 & 1.02 & 0 & 0.9916 & & & & & 0 & 0.9916 & & & & \\
\hline 100 & 1.48 & 1.63 & 0.61 & 0.9919 & $3.08 \mathrm{E}-06$ & & $-3.08 \mathrm{E}-06$ & $3.58 \mathrm{E}-06$ & 0.67 & 0.9919 & $3.38 \mathrm{E}-06$ & & $-3.38 \mathrm{E}-06$ & $3.88 \mathrm{E}-06$ \\
\hline 200 & 2.19 & 2.30 & 1.28 & 0.9922 & $3.23 \mathrm{E}-06$ & & $-3.23 \mathrm{E}-06$ & 3.73E-06 & 1.38 & 0.9923 & $3.48 \mathrm{E}-06$ & & $-3.48 \mathrm{E}-06$ & $3.98 \mathrm{E}-06$ \\
\hline 300 & 2.95 & 3.19 & 2.17 & 0.9927 & $3.65 \mathrm{E}-06$ & & $-3.65 E-06$ & 4.15E-06 & 2.14 & 0.9927 & $3.60 \mathrm{E}-06$ & & $-3.60 \mathrm{E}-06$ & 4.10E-06 \\
\hline 400 & 3.88 & 4.10 & 3.08 & 0.9931 & $3.88 \mathrm{E}-06$ & & $-3.88 \mathrm{E}-06$ & $4.38 \mathrm{E}-06$ & 3.07 & 0.9931 & $3.87 \mathrm{E}-06$ & & $-3.87 \mathrm{E}-06$ & 4.37E-06 \\
\hline 500 & 4.88 & 4.98 & 3.96 & 0.9936 & 3.99E-06 & 4.50E-06 & $5.06 \mathrm{E}-07$ & 4.49E-06 & 4.07 & 0.9936 & 4.10E-06 & 4.50E-06 & 3.96E-07 & 4.60E-06 \\
\hline
\end{tabular}




\begin{tabular}{|c|c|c|c|c|c|c|c|c|c|c|c|c|c|c|}
\hline $\begin{array}{l}\text { Capsule } \\
\text { Column }\end{array}$ & $\begin{array}{l}\text { OC2 } \\
\text { East }\end{array}$ & $\begin{array}{c}\text { Specimen } \\
\text { Length }\end{array}$ & $\begin{array}{c}42 \\
0.9904 \\
\end{array}$ & $\begin{array}{c}\text { Material } \\
\text { Scale }\end{array}$ & $\begin{array}{c}\text { H-451 } \\
1 "\end{array}$ & $5.00 \mathrm{E}-04$ & & & & & & & & \\
\hline $\begin{array}{c}\text { Temperature } \\
\left({ }^{\circ} \mathrm{C}\right) \\
\end{array}$ & Up & Down & $\begin{array}{l}\text { Delta } \\
\text { Down }\end{array}$ & $\begin{array}{l}\text { Down } \\
\text { length }\end{array}$ & CTE & CTEK & $\begin{array}{l}\text { Delta } \\
\text { CTEK }\end{array}$ & $\begin{array}{c}\text { CTE } \\
\text { adjusted }\end{array}$ & $\begin{array}{l}\text { Delta } \\
\text { Up }\end{array}$ & $\begin{array}{c}\text { Up } \\
\text { length }\end{array}$ & CTE & CTEK & $\begin{array}{l}\text { Delta } \\
\text { CTEK }\end{array}$ & $\begin{array}{c}\text { CTE } \\
\text { adjusted }\end{array}$ \\
\hline 0 & 0.92 & 1.38 & 0 & 0.9904 & & & & & 0 & 0.9904 & & & & \\
\hline 100 & 1.55 & 1.90 & 0.52 & 0.9907 & $2.63 \mathrm{E}-06$ & & $-2.63 \mathrm{E}-06$ & $3.13 \mathrm{E}-06$ & 0.63 & 0.9907 & $3.18 \mathrm{E}-06$ & & $-3.18 \mathrm{E}-06$ & $3.68 \mathrm{E}-06$ \\
\hline 200 & 2.28 & 2.52 & 1.14 & 0.9910 & $2.88 \mathrm{E}-06$ & & $-2.88 \mathrm{E}-06$ & $3.38 \mathrm{E}-06$ & 1.36 & 0.9911 & $3.43 \mathrm{E}-06$ & & $-3.43 E-06$ & 3.93E-06 \\
\hline 300 & 3.12 & 3.39 & 2.01 & 0.9914 & $3.38 \mathrm{E}-06$ & & $-3.38 \mathrm{E}-06$ & $3.88 \mathrm{E}-06$ & 2.2 & 0.9915 & $3.70 \mathrm{E}-06$ & & $-3.70 \mathrm{E}-06$ & $4.20 \mathrm{E}-06$ \\
\hline 400 & 4.00 & 4.30 & 2.92 & 0.9919 & $3.69 \mathrm{E}-06$ & & $-3.69 \mathrm{E}-06$ & 4.19E-06 & 3.08 & 0.9919 & $3.89 \mathrm{E}-06$ & & $-3.89 \mathrm{E}-06$ & 4.39E-06 \\
\hline 500 & 5.00 & 5.22 & 3.84 & 0.9923 & $3.88 \mathrm{E}-06$ & $4.56 E+00$ & $4.56 \mathrm{E}+00$ & $4.38 \mathrm{E}-06$ & 4.08 & 0.9924 & $4.12 \mathrm{E}-06$ & $4.56 E+00$ & $4.56 \mathrm{E}+00$ & 4.62E-06 \\
\hline
\end{tabular}

\begin{tabular}{|c|c|c|c|c|c|c|c|c|c|c|c|c|c|c|}
\hline $\begin{array}{l}\text { Capsule } \\
\text { Column }\end{array}$ & $\begin{array}{l}\text { OC2 } \\
\text { East }\end{array}$ & $\begin{array}{l}\text { Specimen } \\
\text { Length }\end{array}$ & $\begin{array}{c}44 \\
0.9927\end{array}$ & $\begin{array}{l}\text { Material } \\
\text { Scale }\end{array}$ & $\begin{array}{c}\text { H-451 } \\
1 "\end{array}$ & $5.00 \mathrm{E}-04$ & & & & & & & & \\
\hline $\begin{array}{c}\text { Temperature } \\
\left({ }^{\circ} \mathrm{C}\right)\end{array}$ & Up & Down & $\begin{array}{l}\text { Delta } \\
\text { Down }\end{array}$ & $\begin{array}{l}\text { Down } \\
\text { length }\end{array}$ & CTE & CTEK & $\begin{array}{l}\text { Delta } \\
\text { CTEK }\end{array}$ & $\begin{array}{c}\text { CTE } \\
\text { adjusted }\end{array}$ & $\begin{array}{l}\text { Delta } \\
\text { Up }\end{array}$ & $\begin{array}{c}\text { Up } \\
\text { length }\end{array}$ & CTE & CTEK & $\begin{array}{l}\text { Delta } \\
\text { CTEK }\end{array}$ & $\begin{array}{c}\text { CTE } \\
\text { adjusted }\end{array}$ \\
\hline 0 & 0.78 & 0.90 & 0 & 0.9927 & & & & & 0 & 0.9927 & & & & \\
\hline 100 & 1.40 & 1.51 & 0.61 & 0.9930 & 3.07E-06 & & $-3.07 E-06$ & $3.57 \mathrm{E}-06$ & 0.62 & 0.9930 & $3.12 \mathrm{E}-06$ & & $-3.12 \mathrm{E}-06$ & $3.62 \mathrm{E}-06$ \\
\hline 200 & 2.02 & 2.11 & 1.21 & 0.9933 & $3.05 \mathrm{E}-06$ & & $-3.05 E-06$ & $3.55 \mathrm{E}-06$ & 1.24 & 0.9933 & $3.12 \mathrm{E}-06$ & & $-3.12 \mathrm{E}-06$ & $3.62 \mathrm{E}-06$ \\
\hline 300 & 2.80 & 2.98 & 2.08 & 0.9937 & $3.49 \mathrm{E}-06$ & & $-3.49 \mathrm{E}-06$ & $3.99 \mathrm{E}-06$ & 2.02 & 0.9937 & $3.39 \mathrm{E}-06$ & & $-3.39 E-06$ & $3.89 \mathrm{E}-06$ \\
\hline 400 & 3.62 & 3.90 & 3 & 0.9942 & $3.78 \mathrm{E}-06$ & & $-3.78 \mathrm{E}-06$ & $4.28 \mathrm{E}-06$ & 2.84 & 0.9941 & $3.58 \mathrm{E}-06$ & & $-3.58 \mathrm{E}-06$ & $4.08 \mathrm{E}-06$ \\
\hline 500 & 4.60 & 4.78 & 3.88 & 0.9946 & $3.91 \mathrm{E}-06$ & $4.30 E-06$ & $3.91 \mathrm{E}-07$ & $4.41 \mathrm{E}-06$ & 3.82 & 0.9946 & $3.85 \mathrm{E}-06$ & 4.30E-06 & $4.52 \mathrm{E}-07$ & 4.35E-06 \\
\hline
\end{tabular}

\begin{tabular}{|c|c|c|c|c|c|c|c|c|c|c|c|c|c|c|}
\hline $\begin{array}{l}\text { Capsule } \\
\text { Column }\end{array}$ & $\begin{array}{c}\text { OC2 } \\
\text { East }\end{array}$ & $\begin{array}{c}\text { Specimen } \\
\text { Length }\end{array}$ & $\begin{array}{c}55 \\
0.9857 \\
\end{array}$ & $\begin{array}{c}\text { Material } \\
\text { Scale }\end{array}$ & $\begin{array}{c}\text { H-451 } \\
1 " \\
\end{array}$ & $5.00 \mathrm{E}-04$ & & & & & & & & \\
\hline $\begin{array}{c}\text { Temperature } \\
\left({ }^{\circ} \mathrm{C}\right)\end{array}$ & Up & Down & $\begin{array}{l}\text { Delta } \\
\text { Down }\end{array}$ & $\begin{array}{l}\text { Down } \\
\text { length }\end{array}$ & CTE & CTEK & $\begin{array}{l}\text { Delta } \\
\text { CTEK }\end{array}$ & $\begin{array}{c}\text { CTE } \\
\text { adjusted }\end{array}$ & $\begin{array}{l}\text { Delta } \\
\text { Up }\end{array}$ & $\begin{array}{c}\text { Up } \\
\text { length }\end{array}$ & CTE & CTEK & $\begin{array}{l}\text { Delta } \\
\text { CTEK }\end{array}$ & $\begin{array}{c}\text { CTE } \\
\text { adjusted }\end{array}$ \\
\hline 0 & 0.70 & 1.40 & 0 & 0.9857 & & & & & 0 & 0.9857 & & & & \\
\hline 100 & 1.40 & 2.05 & 0.65 & 0.9860 & $3.30 \mathrm{E}-06$ & & $-3.30 \mathrm{E}-06$ & $3.80 \mathrm{E}-06$ & 0.7 & 0.9861 & $3.55 \mathrm{E}-06$ & & $-3.55 E-06$ & $4.05 \mathrm{E}-06$ \\
\hline 200 & 2.20 & 2.72 & 1.32 & 0.9864 & 3.35E-06 & & $-3.35 \mathrm{E}-06$ & 3.85E-06 & 1.5 & 0.9865 & $3.80 \mathrm{E}-06$ & & $-3.80 \mathrm{E}-06$ & 4.30E-06 \\
\hline 300 & 3.10 & 3.60 & 2.2 & 0.9868 & $3.72 \mathrm{E}-06$ & & $-3.72 E-06$ & $4.22 \mathrm{E}-06$ & 2.4 & 0.9869 & $4.06 \mathrm{E}-06$ & & $-4.06 E-06$ & 4.56E-06 \\
\hline 400 & 4.08 & 4.52 & 3.12 & 0.9873 & $3.96 \mathrm{E}-06$ & & $-3.96 \mathrm{E}-06$ & 4.46E-06 & 3.38 & 0.9874 & $4.29 \mathrm{E}-06$ & & $-4.29 \mathrm{E}-06$ & 4.79E-06 \\
\hline 500 & 5.10 & 5.46 & 4.06 & 0.9877 & 4.12E-06 & 4.87E-06 & $7.51 \mathrm{E}-07$ & 4.62E-06 & 4.4 & 0.9879 & 4.46E-06 & 4.87E-06 & 4.06E-07 & 4.96E-06 \\
\hline
\end{tabular}




\begin{tabular}{|c|c|c|c|c|c|c|c|c|c|c|c|c|c|c|}
\hline $\begin{array}{l}\text { Capsule } \\
\text { Column }\end{array}$ & $\begin{array}{l}\text { OC2 } \\
\text { East }\end{array}$ & $\begin{array}{c}\text { Specimen } \\
\text { Length }\end{array}$ & $\begin{array}{c}57 \\
0.9845\end{array}$ & $\begin{array}{c}\text { Material } \\
\text { Scale }\end{array}$ & $\begin{array}{c}\text { H-451 } \\
1 "\end{array}$ & $5.00 \mathrm{E}-04$ & & & & & & & & \\
\hline $\begin{array}{c}\text { Temperature } \\
\left({ }^{\circ} \mathrm{C}\right) \\
\end{array}$ & Up & Down & $\begin{array}{l}\text { Delta } \\
\text { Down }\end{array}$ & $\begin{array}{l}\text { Down } \\
\text { length }\end{array}$ & CTE & CTEK & $\begin{array}{l}\text { Delta } \\
\text { CTEK }\end{array}$ & $\begin{array}{c}\text { CTE } \\
\text { adjusted }\end{array}$ & $\begin{array}{l}\text { Delta } \\
\text { Up }\end{array}$ & $\begin{array}{c}\text { Up } \\
\text { length }\end{array}$ & CTE & CTEK & $\begin{array}{l}\text { Delta } \\
\text { CTEK }\end{array}$ & $\begin{array}{c}\text { CTE } \\
\text { adjusted }\end{array}$ \\
\hline 0 & 0.80 & 1.10 & 0 & 0.9845 & & & & & 0 & 0.9845 & & & & \\
\hline 100 & 1.57 & 1.80 & 0.7 & 0.9849 & 3.56E-06 & & $-3.56 \mathrm{E}-06$ & 4.06E-06 & 0.77 & 0.9849 & $3.91 \mathrm{E}-06$ & & $-3.91 \mathrm{E}-06$ & $4.41 \mathrm{E}-06$ \\
\hline 200 & 2.32 & 2.50 & 1.4 & 0.9852 & 3.56E-06 & & $-3.56 \mathrm{E}-06$ & 4.06E-06 & 1.52 & 0.9853 & $3.86 \mathrm{E}-06$ & & $-3.86 \mathrm{E}-06$ & 4.36E-06 \\
\hline 300 & 3.22 & 3.40 & 2.3 & 0.9857 & $3.89 \mathrm{E}-06$ & & $-3.89 E-06$ & $4.39 \mathrm{E}-06$ & 2.42 & 0.9857 & $4.10 \mathrm{E}-06$ & & $-4.10 \mathrm{E}-06$ & 4.60E-06 \\
\hline 400 & 4.17 & 4.36 & 3.26 & 0.9861 & 4.14E-06 & & $-4.14 \mathrm{E}-06$ & 4.64E-06 & 3.37 & 0.9862 & $4.28 \mathrm{E}-06$ & & $-4.28 \mathrm{E}-06$ & $4.78 \mathrm{E}-06$ \\
\hline 500 & 5.20 & 5.39 & 4.29 & 0.9866 & 4.36E-06 & 4.87E-06 & $5.12 \mathrm{E}-07$ & $4.86 \mathrm{E}-06$ & 4.4 & 0.9867 & 4.47E-06 & 4.87E-06 & $4.01 \mathrm{E}-07$ & 4.97E-06 \\
\hline
\end{tabular}

\begin{tabular}{|c|c|c|c|c|c|c|c|c|c|c|c|c|c|c|}
\hline $\begin{array}{l}\text { Capsule } \\
\text { Column }\end{array}$ & $\begin{array}{l}\text { OC2 } \\
\text { East }\end{array}$ & $\begin{array}{l}\text { Specimen } \\
\text { Length }\end{array}$ & $\begin{array}{c}59 \\
0.9862\end{array}$ & $\begin{array}{l}\text { Material } \\
\text { Scale }\end{array}$ & $\begin{array}{c}\text { H-451 } \\
1 "\end{array}$ & $5.00 \mathrm{E}-04$ & & & & & & & & \\
\hline $\begin{array}{c}\text { Temperature } \\
\left({ }^{\circ} \mathrm{C}\right)\end{array}$ & Up & Down & $\begin{array}{l}\text { Delta } \\
\text { Down }\end{array}$ & $\begin{array}{l}\text { Down } \\
\text { length }\end{array}$ & CTE & CTEK & $\begin{array}{l}\text { Delta } \\
\text { CTEK }\end{array}$ & $\begin{array}{c}\text { CTE } \\
\text { adjusted }\end{array}$ & $\begin{array}{l}\text { Delta } \\
\text { Up }\end{array}$ & $\begin{array}{c}\text { Up } \\
\text { length }\end{array}$ & CTE & CTEK & $\begin{array}{l}\text { Delta } \\
\text { CTEK }\end{array}$ & $\begin{array}{c}\text { CTE } \\
\text { adjusted }\end{array}$ \\
\hline 0 & 0.60 & 1.12 & 0 & 0.9862 & & & & & 0 & 0.9862 & & & & \\
\hline 100 & 1.45 & 1.78 & 0.66 & 0.9865 & $3.35 \mathrm{E}-06$ & & $-3.35 E-06$ & $3.85 \mathrm{E}-06$ & 0.85 & 0.9866 & $4.31 \mathrm{E}-06$ & & $-4.31 \mathrm{E}-06$ & 4.81E-06 \\
\hline 200 & 2.20 & 2.48 & 1.36 & 0.9869 & $3.45 \mathrm{E}-06$ & & $-3.45 E-06$ & $3.95 \mathrm{E}-06$ & 1.6 & 0.9870 & 4.06E-06 & & $-4.06 \mathrm{E}-06$ & 4.56E-06 \\
\hline 300 & 2.92 & 2.31 & 1.19 & 0.9868 & $2.01 \mathrm{E}-06$ & & $-2.01 \mathrm{E}-06$ & $2.51 \mathrm{E}-06$ & 2.32 & 0.9874 & $3.92 \mathrm{E}-06$ & & $-3.92 \mathrm{E}-06$ & $4.42 \mathrm{E}-06$ \\
\hline 400 & 3.88 & 4.22 & 3.1 & 0.9878 & $3.93 \mathrm{E}-06$ & & $-3.93 E-06$ & $4.43 \mathrm{E}-06$ & 3.28 & 0.9878 & $4.16 \mathrm{E}-06$ & & $-4.16 \mathrm{E}-06$ & 4.66E-06 \\
\hline 500 & 4.95 & 5.21 & 4.09 & 0.9882 & 4.15E-06 & $3.58 \mathrm{E}-06$ & $-5.67 \mathrm{E}-07$ & $4.65 \mathrm{E}-06$ & 4.35 & 0.9884 & $4.41 \mathrm{E}-06$ & $3.58 E-06$ & $-8.31 \mathrm{E}-07$ & 4.91E-06 \\
\hline
\end{tabular}

\begin{tabular}{|c|c|c|c|c|c|c|c|c|c|c|c|c|c|c|}
\hline $\begin{array}{l}\text { Capsule } \\
\text { Column }\end{array}$ & $\begin{array}{c}\text { OC2 } \\
\text { East }\end{array}$ & $\begin{array}{c}\text { Specimen } \\
\text { Length }\end{array}$ & $\begin{array}{c}2 \\
0.9934 \\
\end{array}$ & $\begin{array}{c}\text { Material } \\
\text { Scale }\end{array}$ & $\begin{array}{c}\text { H-327 } \\
1 "\end{array}$ & $5.00 \mathrm{E}-04$ & & & & & & & & \\
\hline $\begin{array}{c}\text { Temperature } \\
\left({ }^{\circ} \mathrm{C}\right)\end{array}$ & Up & Down & $\begin{array}{l}\text { Delta } \\
\text { Down }\end{array}$ & $\begin{array}{l}\text { Down } \\
\text { length }\end{array}$ & CTE & CTEK & $\begin{array}{l}\text { Delta } \\
\text { CTEK }\end{array}$ & $\begin{array}{c}\text { CTE } \\
\text { adjusted }\end{array}$ & $\begin{array}{l}\text { Delta } \\
\text { Up }\end{array}$ & $\begin{array}{c}\text { Up } \\
\text { length }\end{array}$ & CTE & CTEK & $\begin{array}{l}\text { Delta } \\
\text { CTEK }\end{array}$ & $\begin{array}{c}\text { CTE } \\
\text { adjusted }\end{array}$ \\
\hline 0 & 0.95 & 1.08 & 0 & 0.9934 & & & & & 0 & 0.9934 & & & & \\
\hline 100 & 1.10 & 1.18 & 0.1 & 0.9935 & 5.03E-07 & & $-5.03 E-07$ & $1.00 \mathrm{E}-06$ & 0.15 & 0.9935 & 7.55E-07 & & $-7.55 E-07$ & $1.25 \mathrm{E}-06$ \\
\hline 200 & 1.30 & 1.40 & 0.32 & 0.9936 & $8.05 E-07$ & & $-8.05 E-07$ & $1.31 \mathrm{E}-06$ & 0.35 & 0.9936 & 8.81E-07 & & $-8.81 E-07$ & $1.38 \mathrm{E}-06$ \\
\hline 300 & 1.60 & 1.70 & 0.62 & 0.9937 & $1.04 \mathrm{E}-06$ & & $-1.04 \mathrm{E}-06$ & $1.54 \mathrm{E}-06$ & 0.65 & 0.9937 & $1.09 \mathrm{E}-06$ & & $-1.09 E-06$ & $1.59 \mathrm{E}-06$ \\
\hline 400 & 1.98 & 2.13 & 1.05 & 0.9939 & $1.32 \mathrm{E}-06$ & & $-1.32 \mathrm{E}-06$ & $1.82 \mathrm{E}-06$ & 1.03 & 0.9939 & $1.30 \mathrm{E}-06$ & & $-1.30 \mathrm{E}-06$ & $1.80 \mathrm{E}-06$ \\
\hline 500 & 2.50 & 2.62 & 1.54 & 0.9942 & $1.55 \mathrm{E}-06$ & 2.00E-06 & 4.50E-07 & 2.05E-06 & 1.55 & 0.9942 & 1.56E-06 & $2.00 E-06$ & 4.40E-07 & 2.06E-06 \\
\hline
\end{tabular}




\begin{tabular}{|c|c|c|c|c|c|c|c|c|c|c|c|c|c|c|}
\hline $\begin{array}{l}\text { Capsule } \\
\text { Column }\end{array}$ & $\begin{array}{l}\text { OC2 } \\
\text { East }\end{array}$ & $\begin{array}{c}\text { Specimen } \\
\text { Length }\end{array}$ & $\begin{array}{c}4 \\
0.9934\end{array}$ & $\begin{array}{c}\text { Material } \\
\text { Scale }\end{array}$ & $\begin{array}{c}\text { H-327 } \\
1 "\end{array}$ & $5.00 \mathrm{E}-04$ & & & & & & & & \\
\hline $\begin{array}{c}\text { Temperature } \\
\left({ }^{\circ} \mathrm{C}\right) \\
\end{array}$ & Up & Down & $\begin{array}{l}\text { Delta } \\
\text { Down }\end{array}$ & $\begin{array}{l}\text { Down } \\
\text { length }\end{array}$ & CTE & CTEK & $\begin{array}{l}\text { Delta } \\
\text { CTEK }\end{array}$ & $\begin{array}{c}\text { CTE } \\
\text { adjusted }\end{array}$ & $\begin{array}{l}\text { Delta } \\
\text { Up }\end{array}$ & $\begin{array}{c}\text { Up } \\
\text { length }\end{array}$ & CTE & CTEK & $\begin{array}{l}\text { Delta } \\
\text { CTEK }\end{array}$ & $\begin{array}{c}\text { CTE } \\
\text { adjusted }\end{array}$ \\
\hline 0 & 0.70 & 0.88 & 0 & 0.9934 & & & & & 0 & 0.9934 & & & & \\
\hline 100 & 0.78 & 0.98 & 0.1 & 0.9935 & $5.03 \mathrm{E}-07$ & & $-5.03 E-07$ & $1.00 \mathrm{E}-06$ & 0.08 & 0.9934 & $4.03 \mathrm{E}-07$ & & $-4.03 E-07$ & $9.03 \mathrm{E}-07$ \\
\hline 200 & 0.90 & 1.04 & 0.16 & 0.9935 & 4.03E-07 & & $-4.03 \mathrm{E}-07$ & $9.03 \mathrm{E}-07$ & 0.2 & 0.9935 & $5.03 \mathrm{E}-07$ & & $-5.03 E-07$ & $1.00 \mathrm{E}-06$ \\
\hline 300 & 1.20 & 1.34 & 0.46 & 0.9936 & 7.72E-07 & & $-7.72 E-07$ & $1.27 \mathrm{E}-06$ & 0.5 & 0.9937 & 8.39E-07 & & $-8.39 \mathrm{E}-07$ & $1.34 \mathrm{E}-06$ \\
\hline 400 & 1.60 & 1.72 & 0.84 & 0.9938 & $1.06 \mathrm{E}-06$ & & $-1.06 \mathrm{E}-06$ & $1.56 \mathrm{E}-06$ & 0.9 & 0.9939 & $1.13 \mathrm{E}-06$ & & $-1.13 \mathrm{E}-06$ & $1.63 \mathrm{E}-06$ \\
\hline 500 & 2.00 & 2.18 & 1.3 & 0.9941 & $1.31 \mathrm{E}-06$ & $1.85 E-06$ & $5.41 \mathrm{E}-07$ & $1.81 \mathrm{E}-06$ & 1.3 & 0.9941 & $1.31 \mathrm{E}-06$ & $1.85 E-06$ & $5.41 \mathrm{E}-07$ & $1.81 \mathrm{E}-06$ \\
\hline
\end{tabular}

\begin{tabular}{|c|c|c|c|c|c|c|c|c|c|c|c|c|c|c|}
\hline $\begin{array}{l}\text { Capsule } \\
\text { Column }\end{array}$ & $\begin{array}{l}\text { OC2 } \\
\text { East }\end{array}$ & $\begin{array}{l}\text { Specimen } \\
\text { Length }\end{array}$ & $\begin{array}{c}9 \\
0.9864\end{array}$ & $\begin{array}{l}\text { Material } \\
\text { Scale }\end{array}$ & $\begin{array}{c}\text { H-327 } \\
1 "\end{array}$ & $5.00 \mathrm{E}-04$ & & & & & & & & \\
\hline $\begin{array}{c}\text { Temperature } \\
\left({ }^{\circ} \mathrm{C}\right)\end{array}$ & Up & Down & $\begin{array}{l}\text { Delta } \\
\text { Down }\end{array}$ & $\begin{array}{l}\text { Down } \\
\text { length }\end{array}$ & CTE & CTEK & $\begin{array}{l}\text { Delta } \\
\text { CTEK }\end{array}$ & $\begin{array}{c}\text { CTE } \\
\text { adjusted }\end{array}$ & $\begin{array}{l}\text { Delta } \\
\text { Up }\end{array}$ & $\begin{array}{c}\text { Up } \\
\text { length }\end{array}$ & CTE & CTEK & $\begin{array}{l}\text { Delta } \\
\text { CTEK }\end{array}$ & $\begin{array}{c}\text { CTE } \\
\text { adjusted }\end{array}$ \\
\hline 0 & 0.90 & 1.25 & 0 & 0.9864 & & & & & 0 & 0.9864 & & & & \\
\hline 100 & 1.55 & 1.78 & 0.53 & 0.9867 & 2.69E-06 & & $-2.69 \mathrm{E}-06$ & 3.19E-06 & 0.65 & 0.9867 & $3.29 \mathrm{E}-06$ & & $-3.29 E-06$ & 3.79E-06 \\
\hline 200 & 2.22 & 2.33 & 1.08 & 0.9869 & $2.74 \mathrm{E}-06$ & & $-2.74 \mathrm{E}-06$ & $3.24 \mathrm{E}-06$ & 1.32 & 0.9871 & $3.35 \mathrm{E}-06$ & & $-3.35 E-06$ & $3.85 \mathrm{E}-06$ \\
\hline 300 & 2.93 & 3.20 & 1.95 & 0.9874 & $3.29 \mathrm{E}-06$ & & $-3.29 E-06$ & $3.79 \mathrm{E}-06$ & 2.03 & 0.9874 & $3.43 \mathrm{E}-06$ & & $-3.43 E-06$ & $3.93 \mathrm{E}-06$ \\
\hline 400 & 3.72 & 4.22 & 2.97 & 0.9879 & $3.76 \mathrm{E}-06$ & & $-3.76 E-06$ & $4.26 \mathrm{E}-06$ & 2.82 & 0.9878 & $3.57 \mathrm{E}-06$ & & $-3.57 \mathrm{E}-06$ & 4.07E-06 \\
\hline 500 & 4.70 & 5.08 & 3.83 & 0.9883 & $3.88 \mathrm{E}-06$ & $4.28 \mathrm{E}-06$ & 3.97E-07 & $4.38 \mathrm{E}-06$ & 3.8 & 0.9883 & $3.85 \mathrm{E}-06$ & $4.28 E-06$ & $4.28 \mathrm{E}-07$ & 4.35E-06 \\
\hline
\end{tabular}

\begin{tabular}{|c|c|c|c|c|c|c|c|c|c|c|c|c|c|c|}
\hline $\begin{array}{l}\text { Capsule } \\
\text { Column }\end{array}$ & $\begin{array}{c}\text { OC2 } \\
\text { East }\end{array}$ & $\begin{array}{c}\text { Specimen } \\
\text { Length }\end{array}$ & $\begin{array}{c}1 \mathrm{G} \\
0.9961 \\
\end{array}$ & $\begin{array}{c}\text { Material } \\
\text { Scale }\end{array}$ & $\begin{array}{c}\text { H-327 } \\
1 " \\
\end{array}$ & $5.00 \mathrm{E}-04$ & & & & & & & & \\
\hline $\begin{array}{c}\text { Temperature } \\
\left({ }^{\circ} \mathrm{C}\right)\end{array}$ & Up & Down & $\begin{array}{l}\text { Delta } \\
\text { Down }\end{array}$ & $\begin{array}{l}\text { Down } \\
\text { length }\end{array}$ & CTE & CTEK & $\begin{array}{l}\text { Delta } \\
\text { CTEK }\end{array}$ & $\begin{array}{c}\text { CTE } \\
\text { adjusted }\end{array}$ & $\begin{array}{l}\text { Delta } \\
\text { Up }\end{array}$ & $\begin{array}{c}\text { Up } \\
\text { length }\end{array}$ & CTE & CTEK & $\begin{array}{l}\text { Delta } \\
\text { CTEK }\end{array}$ & $\begin{array}{c}\text { CTE } \\
\text { adjusted }\end{array}$ \\
\hline 0 & 0.10 & 1.12 & 0 & 0.9961 & & & & & 0 & 0.9961 & & & & \\
\hline 100 & 1.28 & 2.18 & 1.06 & 0.9966 & $5.32 \mathrm{E}-06$ & & $-5.32 E-06$ & $5.82 \mathrm{E}-06$ & 1.18 & 0.9967 & $5.92 \mathrm{E}-06$ & & $-5.92 E-06$ & $6.42 \mathrm{E}-06$ \\
\hline 200 & 2.48 & 3.22 & 2.1 & 0.9972 & $5.27 \mathrm{E}-06$ & & $-5.27 \mathrm{E}-06$ & 5.77E-06 & 2.38 & 0.9973 & 5.97E-06 & & $-5.97 \mathrm{E}-06$ & 6.47E-06 \\
\hline 300 & 3.78 & 4.60 & 3.48 & 0.9978 & $5.82 \mathrm{E}-06$ & & $-5.82 E-06$ & $6.32 \mathrm{E}-06$ & 3.68 & 0.9979 & $6.16 \mathrm{E}-06$ & & $-6.16 E-06$ & $6.66 \mathrm{E}-06$ \\
\hline 400 & 5.12 & 5.90 & 4.78 & 0.9985 & $6.00 \mathrm{E}-06$ & & $-6.00 \mathrm{E}-06$ & $6.50 \mathrm{E}-06$ & 5.02 & 0.9986 & $6.30 \mathrm{E}-06$ & & $-6.30 \mathrm{E}-06$ & $6.80 \mathrm{E}-06$ \\
\hline 500 & 6.55 & 7.10 & 5.98 & 0.9991 & $6.00 \mathrm{E}-06$ & $6.92 E-06$ & $9.17 \mathrm{E}-07$ & $6.50 \mathrm{E}-06$ & 6.45 & 0.9993 & $6.48 \mathrm{E}-06$ & $6.92 E-06$ & 4.45E-07 & $6.98 \mathrm{E}-06$ \\
\hline
\end{tabular}




\begin{tabular}{|c|c|c|c|c|c|c|c|c|c|c|c|c|c|c|}
\hline $\begin{array}{l}\text { Capsule } \\
\text { Column }\end{array}$ & $\begin{array}{l}\text { OC2 } \\
\text { West }\end{array}$ & $\begin{array}{c}\text { Specimen } \\
\text { Length }\end{array}$ & $\begin{array}{c}16 \\
0.9901\end{array}$ & $\begin{array}{c}\text { Material } \\
\text { Scale }\end{array}$ & $\begin{array}{c}\text { H-451 } \\
1 "\end{array}$ & $5.00 \mathrm{E}-04$ & & & & & & & & \\
\hline $\begin{array}{c}\text { Temperature } \\
\left({ }^{\circ} \mathrm{C}\right) \\
\end{array}$ & Up & Down & $\begin{array}{l}\text { Delta } \\
\text { Down }\end{array}$ & $\begin{array}{l}\text { Down } \\
\text { length }\end{array}$ & CTE & CTEK & $\begin{array}{l}\text { Delta } \\
\text { CTEK }\end{array}$ & $\begin{array}{c}\text { CTE } \\
\text { adjusted }\end{array}$ & $\begin{array}{l}\text { Delta } \\
\text { Up }\end{array}$ & $\begin{array}{c}\text { Up } \\
\text { length }\end{array}$ & CTE & CTEK & $\begin{array}{l}\text { Delta } \\
\text { CTEK }\end{array}$ & $\begin{array}{c}\text { CTE } \\
\text { adjusted }\end{array}$ \\
\hline 0 & 1.01 & 1.50 & 0 & 0.9901 & & & & & 0 & 0.9901 & & & & \\
\hline 100 & 1.70 & 2.10 & 0.6 & 0.9904 & 3.03E-06 & & $-3.03 E-06$ & 3.53E-06 & 0.69 & 0.9904 & $3.48 \mathrm{E}-06$ & & $-3.48 \mathrm{E}-06$ & $3.98 \mathrm{E}-06$ \\
\hline 200 & 2.40 & 2.72 & 1.22 & 0.9907 & $3.08 \mathrm{E}-06$ & & $-3.08 \mathrm{E}-06$ & $3.58 \mathrm{E}-06$ & 1.39 & 0.9908 & $3.51 \mathrm{E}-06$ & & $-3.51 \mathrm{E}-06$ & 4.01E-06 \\
\hline 300 & 3.22 & 3.60 & 2.1 & 0.9912 & $3.53 \mathrm{E}-06$ & & $-3.53 \mathrm{E}-06$ & $4.03 E-06$ & 2.21 & 0.9912 & $3.72 \mathrm{E}-06$ & & $-3.72 \mathrm{E}-06$ & $4.22 \mathrm{E}-06$ \\
\hline 400 & 4.08 & 4.50 & 3 & 0.9916 & $3.79 \mathrm{E}-06$ & & $-3.79 \mathrm{E}-06$ & 4.29E-06 & 3.07 & 0.9916 & $3.88 \mathrm{E}-06$ & & $-3.88 \mathrm{E}-06$ & $4.38 \mathrm{E}-06$ \\
\hline 500 & 5.02 & 5.31 & 3.81 & 0.9920 & $3.85 \mathrm{E}-06$ & 4.50E-06 & $6.52 \mathrm{E}-07$ & $4.35 \mathrm{E}-06$ & 4.01 & 0.9921 & $4.05 \mathrm{E}-06$ & $4.50 E-06$ & 4.50E-07 & $4.55 \mathrm{E}-06$ \\
\hline
\end{tabular}

\begin{tabular}{|c|c|c|c|c|c|c|c|c|c|c|c|c|c|c|}
\hline $\begin{array}{l}\text { Capsule } \\
\text { Column }\end{array}$ & $\begin{array}{l}\text { OC2 } \\
\text { West }\end{array}$ & $\begin{array}{l}\text { Specimen } \\
\text { Length }\end{array}$ & $\begin{array}{c}21 \\
0.9905\end{array}$ & $\begin{array}{c}\text { Material } \\
\text { Scale }\end{array}$ & $\begin{array}{c}\text { H-451 } \\
1 "\end{array}$ & $5.00 \mathrm{E}-04$ & & & & & & & & \\
\hline $\begin{array}{c}\text { Temperature } \\
\left({ }^{\circ} \mathrm{C}\right)\end{array}$ & Up & Down & $\begin{array}{l}\text { Delta } \\
\text { Down }\end{array}$ & $\begin{array}{l}\text { Down } \\
\text { length }\end{array}$ & CTE & CTEK & $\begin{array}{l}\text { Delta } \\
\text { CTEK }\end{array}$ & $\begin{array}{c}\text { CTE } \\
\text { adjusted }\end{array}$ & $\begin{array}{l}\text { Delta } \\
\text { Up }\end{array}$ & $\begin{array}{l}\text { Up } \\
\text { length }\end{array}$ & CTE & CTEK & $\begin{array}{l}\text { Delta } \\
\text { CTEK }\end{array}$ & $\begin{array}{c}\text { CTE } \\
\text { adjusted }\end{array}$ \\
\hline 0 & 1.32 & 1.88 & 0 & 0.9905 & & & & & 0 & 0.9905 & & & & \\
\hline 100 & 1.92 & 2.38 & 0.5 & 0.9908 & $2.52 \mathrm{E}-06$ & & $-2.52 E-06$ & $3.02 \mathrm{E}-06$ & 0.6 & 0.9908 & $3.03 E-06$ & & $-3.03 E-06$ & $3.53 \mathrm{E}-06$ \\
\hline 200 & 2.68 & 2.85 & 0.97 & 0.9910 & $2.45 \mathrm{E}-06$ & & $-2.45 \mathrm{E}-06$ & 2.95E-06 & 1.36 & 0.9912 & $3.43 \mathrm{E}-06$ & & $-3.43 E-06$ & 3.93E-06 \\
\hline 300 & 3.32 & 3.60 & 1.72 & 0.9914 & $2.89 \mathrm{E}-06$ & & $-2.89 \mathrm{E}-06$ & 3.39E-06 & 2 & 0.9915 & 3.37E-06 & & $-3.37 \mathrm{E}-06$ & 3.87E-06 \\
\hline 400 & 4.20 & 4.57 & 2.69 & 0.9918 & $3.39 \mathrm{E}-06$ & & $-3.39 \mathrm{E}-06$ & $3.89 \mathrm{E}-06$ & 2.88 & 0.9919 & $3.63 \mathrm{E}-06$ & & $-3.63 \mathrm{E}-06$ & 4.13E-06 \\
\hline 500 & 5.20 & 5.38 & 3.5 & 0.9923 & $3.53 \mathrm{E}-06$ & 4.50E-06 & $9.66 \mathrm{E}-07$ & 4.03E-06 & 3.88 & 0.9924 & $3.92 \mathrm{E}-06$ & 4.50E-06 & $5.83 \mathrm{E}-07$ & 4.42E-06 \\
\hline
\end{tabular}

\begin{tabular}{|c|c|c|c|c|c|c|c|c|c|c|c|c|c|c|}
\hline $\begin{array}{l}\text { Capsule } \\
\text { Column }\end{array}$ & $\begin{array}{c}\text { OC2 } \\
\text { West }\end{array}$ & $\begin{array}{c}\text { Specimen } \\
\text { Length }\end{array}$ & $\begin{array}{c}26 \\
0.9894 \\
\end{array}$ & $\begin{array}{c}\text { Material } \\
\text { Scale }\end{array}$ & $\begin{array}{c}\text { H-451 } \\
1 " \\
\end{array}$ & $5.00 \mathrm{E}-04$ & & & & & & & & \\
\hline $\begin{array}{c}\text { Temperature } \\
\left({ }^{\circ} \mathrm{C}\right)\end{array}$ & Up & Down & $\begin{array}{l}\text { Delta } \\
\text { Down }\end{array}$ & $\begin{array}{l}\text { Down } \\
\text { length }\end{array}$ & CTE & CTEK & $\begin{array}{l}\text { Delta } \\
\text { CTEK }\end{array}$ & $\begin{array}{c}\text { CTE } \\
\text { adjusted }\end{array}$ & $\begin{array}{l}\text { Delta } \\
\text { Up }\end{array}$ & $\begin{array}{c}\text { Up } \\
\text { length }\end{array}$ & CTE & CTEK & $\begin{array}{l}\text { Delta } \\
\text { CTEK }\end{array}$ & $\begin{array}{c}\text { CTE } \\
\text { adjusted }\end{array}$ \\
\hline 0 & 0.79 & 0.79 & 0 & 0.9894 & & & & & 0 & 0.9894 & & & & \\
\hline 100 & 1.27 & 1.27 & 0.48 & 0.9896 & $2.43 \mathrm{E}-06$ & & $-2.43 E-06$ & $2.93 \mathrm{E}-06$ & 0.48 & 0.9896 & $2.43 \mathrm{E}-06$ & & $-2.43 E-06$ & $2.93 \mathrm{E}-06$ \\
\hline 200 & 1.88 & 1.80 & 1.01 & 0.9899 & $2.55 \mathrm{E}-06$ & & $-2.55 \mathrm{E}-06$ & 3.05E-06 & 1.09 & 0.9899 & $2.75 \mathrm{E}-06$ & & $-2.75 E-06$ & $3.25 \mathrm{E}-06$ \\
\hline 300 & 2.42 & 2.55 & 1.76 & 0.9903 & $2.96 \mathrm{E}-06$ & & $-2.96 \mathrm{E}-06$ & $3.46 \mathrm{E}-06$ & 1.63 & 0.9902 & $2.75 \mathrm{E}-06$ & & $-2.75 E-06$ & $3.25 \mathrm{E}-06$ \\
\hline 400 & 3.22 & 3.52 & 2.73 & 0.9908 & $3.45 \mathrm{E}-06$ & & $-3.45 E-06$ & $3.95 \mathrm{E}-06$ & 2.43 & 0.9906 & $3.07 \mathrm{E}-06$ & & $-3.07 E-06$ & 3.57E-06 \\
\hline 500 & 4.15 & 4.32 & 3.53 & 0.9912 & $3.57 \mathrm{E}-06$ & 4.50E-06 & $9.32 \mathrm{E}-07$ & 4.07E-06 & 3.36 & 0.9911 & 3.40E-06 & 4.50E-06 & 1.10E-06 & $3.90 \mathrm{E}-06$ \\
\hline
\end{tabular}




\begin{tabular}{|c|c|c|c|c|c|c|c|c|c|c|c|c|c|c|}
\hline $\begin{array}{l}\text { Capsule } \\
\text { Column }\end{array}$ & $\begin{array}{l}\text { OC2 } \\
\text { West }\end{array}$ & $\begin{array}{c}\text { Specimen } \\
\text { Length }\end{array}$ & $\begin{array}{c}31 \\
0.9874\end{array}$ & $\begin{array}{c}\text { Material } \\
\text { Scale }\end{array}$ & $\begin{array}{c}\text { H-451 } \\
1 "\end{array}$ & $5.00 \mathrm{E}-04$ & & & & & & & & \\
\hline $\begin{array}{c}\text { Temperature } \\
\left({ }^{\circ} \mathrm{C}\right) \\
\end{array}$ & Up & Down & $\begin{array}{l}\text { Delta } \\
\text { Down }\end{array}$ & $\begin{array}{l}\text { Down } \\
\text { length }\end{array}$ & CTE & CTEK & $\begin{array}{l}\text { Delta } \\
\text { CTEK }\end{array}$ & $\begin{array}{c}\text { CTE } \\
\text { adjusted }\end{array}$ & $\begin{array}{l}\text { Delta } \\
\text { Up }\end{array}$ & $\begin{array}{c}\text { Up } \\
\text { length }\end{array}$ & CTE & CTEK & $\begin{array}{l}\text { Delta } \\
\text { CTEK }\end{array}$ & $\begin{array}{c}\text { CTE } \\
\text { adjusted }\end{array}$ \\
\hline 0 & 0.80 & 1.50 & 0 & 0.9874 & & & & & 0 & 0.9874 & & & & \\
\hline 100 & 1.62 & 2.10 & 0.6 & 0.9877 & $3.04 \mathrm{E}-06$ & & $-3.04 \mathrm{E}-06$ & $3.54 \mathrm{E}-06$ & 0.82 & 0.9878 & 4.15E-06 & & $-4.15 \mathrm{E}-06$ & 4.65E-06 \\
\hline 200 & 2.51 & 2.83 & 1.33 & 0.9881 & 3.37E-06 & & $-3.37 \mathrm{E}-06$ & $3.87 \mathrm{E}-06$ & 1.71 & 0.9883 & $4.33 \mathrm{E}-06$ & & $-4.33 \mathrm{E}-06$ & 4.83E-06 \\
\hline 300 & 3.50 & 3.80 & 2.3 & 0.9886 & $3.88 \mathrm{E}-06$ & & $-3.88 \mathrm{E}-06$ & $4.38 \mathrm{E}-06$ & 2.7 & 0.9888 & $4.56 \mathrm{E}-06$ & & $-4.56 \mathrm{E}-06$ & 5.06E-06 \\
\hline 400 & 4.50 & 4.90 & 3.4 & 0.9891 & $4.30 \mathrm{E}-06$ & & $-4.30 \mathrm{E}-06$ & $4.80 \mathrm{E}-06$ & 3.7 & 0.9893 & $4.68 \mathrm{E}-06$ & & $-4.68 \mathrm{E}-06$ & $5.18 \mathrm{E}-06$ \\
\hline 500 & 5.62 & 6.14 & 4.64 & 0.9897 & 4.70E-06 & 4.50E-06 & $-1.99 \mathrm{E}-07$ & $5.20 \mathrm{E}-06$ & 4.82 & 0.9898 & $4.88 \mathrm{E}-06$ & $4.50 E-06$ & $-3.82 \mathrm{E}-07$ & $5.38 \mathrm{E}-06$ \\
\hline
\end{tabular}

\begin{tabular}{|c|c|c|c|c|c|c|c|c|c|c|c|c|c|c|}
\hline $\begin{array}{l}\text { Capsule } \\
\text { Column }\end{array}$ & $\begin{array}{l}\text { OC2 } \\
\text { West }\end{array}$ & $\begin{array}{l}\text { Specimen } \\
\text { Length }\end{array}$ & $\begin{array}{c}41 \\
0.9916\end{array}$ & $\begin{array}{l}\text { Material } \\
\text { Scale }\end{array}$ & $\begin{array}{c}\text { H-451 } \\
1 "\end{array}$ & $5.00 \mathrm{E}-04$ & & & & & & & & \\
\hline $\begin{array}{c}\text { Temperature } \\
\left({ }^{\circ} \mathrm{C}\right)\end{array}$ & Up & Down & $\begin{array}{l}\text { Delta } \\
\text { Down }\end{array}$ & $\begin{array}{l}\text { Down } \\
\text { length }\end{array}$ & CTE & CTEK & $\begin{array}{l}\text { Delta } \\
\text { CTEK }\end{array}$ & $\begin{array}{c}\text { CTE } \\
\text { adjusted }\end{array}$ & $\begin{array}{l}\text { Delta } \\
\text { Up }\end{array}$ & $\begin{array}{c}\text { Up } \\
\text { length }\end{array}$ & CTE & CTEK & $\begin{array}{l}\text { Delta } \\
\text { CTEK }\end{array}$ & $\begin{array}{c}\text { CTE } \\
\text { adjusted }\end{array}$ \\
\hline 0 & 0.95 & 1.38 & 0 & 0.9916 & & & & & 0 & 0.9916 & & & & \\
\hline 100 & 1.43 & 1.85 & 0.47 & 0.9918 & $2.37 \mathrm{E}-06$ & & $-2.37 \mathrm{E}-06$ & $2.87 \mathrm{E}-06$ & 0.48 & 0.9918 & $2.42 \mathrm{E}-06$ & & $-2.42 \mathrm{E}-06$ & $2.92 \mathrm{E}-06$ \\
\hline 200 & 2.05 & 2.42 & 1.04 & 0.9921 & $2.62 \mathrm{E}-06$ & & $-2.62 \mathrm{E}-06$ & $3.12 \mathrm{E}-06$ & 1.1 & 0.9922 & $2.77 \mathrm{E}-06$ & & $-2.77 \mathrm{E}-06$ & 3.27E-06 \\
\hline 300 & 2.81 & 3.20 & 1.82 & 0.9925 & $3.06 \mathrm{E}-06$ & & $-3.06 E-06$ & $3.56 \mathrm{E}-06$ & 1.86 & 0.9925 & $3.13 \mathrm{E}-06$ & & $-3.13 E-06$ & $3.63 \mathrm{E}-06$ \\
\hline 400 & 3.68 & 4.08 & 2.7 & 0.9930 & $3.40 \mathrm{E}-06$ & & $-3.40 \mathrm{E}-06$ & $3.90 \mathrm{E}-06$ & 2.73 & 0.9930 & $3.44 \mathrm{E}-06$ & & $-3.44 \mathrm{E}-06$ & $3.94 \mathrm{E}-06$ \\
\hline 500 & 4.55 & 4.75 & 3.37 & 0.9933 & $3.40 \mathrm{E}-06$ & $4.50 E-06$ & $1.10 \mathrm{E}-06$ & $3.90 \mathrm{E}-06$ & 3.6 & 0.9934 & $3.63 \mathrm{E}-06$ & 4.50E-06 & $8.70 \mathrm{E}-07$ & 4.13E-06 \\
\hline
\end{tabular}

\begin{tabular}{|c|c|c|c|c|c|c|c|c|c|c|c|c|c|c|}
\hline $\begin{array}{l}\text { Capsule } \\
\text { Column }\end{array}$ & $\begin{array}{c}\text { OC2 } \\
\text { West }\end{array}$ & $\begin{array}{c}\text { Specimen } \\
\text { Length }\end{array}$ & $\begin{array}{c}43 \\
0.9901 \\
\end{array}$ & $\begin{array}{c}\text { Material } \\
\text { Scale }\end{array}$ & $\begin{array}{c}\text { H-451 } \\
1 " \\
\end{array}$ & $5.00 \mathrm{E}-04$ & & & & & & & & \\
\hline $\begin{array}{c}\text { Temperature } \\
\left({ }^{\circ} \mathrm{C}\right)\end{array}$ & Up & Down & $\begin{array}{l}\text { Delta } \\
\text { Down }\end{array}$ & $\begin{array}{l}\text { Down } \\
\text { length }\end{array}$ & CTE & CTEK & $\begin{array}{l}\text { Delta } \\
\text { CTEK }\end{array}$ & $\begin{array}{c}\text { CTE } \\
\text { adjusted }\end{array}$ & $\begin{array}{l}\text { Delta } \\
\text { Up }\end{array}$ & $\begin{array}{c}\text { Up } \\
\text { length }\end{array}$ & CTE & CTEK & $\begin{array}{l}\text { Delta } \\
\text { CTEK }\end{array}$ & $\begin{array}{c}\text { CTE } \\
\text { adjusted }\end{array}$ \\
\hline 0 & 1.10 & 1.00 & 0 & 0.9901 & & & & & 0 & 0.9901 & & & & \\
\hline 100 & 1.25 & 1.62 & 0.62 & 0.9904 & $3.13 \mathrm{E}-06$ & & $-3.13 E-06$ & $3.63 \mathrm{E}-06$ & 0.15 & 0.9902 & 7.57E-07 & & $-7.57 \mathrm{E}-07$ & $1.26 \mathrm{E}-06$ \\
\hline 200 & 1.91 & 2.05 & 1.05 & 0.9906 & $2.65 \mathrm{E}-06$ & & $-2.65 E-06$ & $3.15 E-06$ & 0.81 & 0.9905 & $2.05 \mathrm{E}-06$ & & $-2.05 E-06$ & $2.55 \mathrm{E}-06$ \\
\hline 300 & 2.70 & 2.90 & 1.9 & 0.9911 & $3.20 \mathrm{E}-06$ & & $-3.20 \mathrm{E}-06$ & $3.70 \mathrm{E}-06$ & 1.6 & 0.9909 & $2.69 \mathrm{E}-06$ & & $-2.69 \mathrm{E}-06$ & 3.19E-06 \\
\hline 400 & 3.55 & 3.82 & 2.82 & 0.9915 & $3.56 \mathrm{E}-06$ & & $-3.56 \mathrm{E}-06$ & 4.06E-06 & 2.45 & 0.9913 & $3.09 \mathrm{E}-06$ & & $-3.09 \mathrm{E}-06$ & $3.59 \mathrm{E}-06$ \\
\hline 500 & 4.52 & 4.70 & 3.7 & 0.9920 & 3.74E-06 & 4.50E-06 & $7.63 \mathrm{E}-07$ & 4.24E-06 & 3.42 & 0.9918 & 3.45E-06 & 4.50E-06 & $1.05 \mathrm{E}-06$ & $3.95 \mathrm{E}-06$ \\
\hline
\end{tabular}




\begin{tabular}{|c|c|c|c|c|c|c|c|c|c|c|c|c|c|c|}
\hline $\begin{array}{l}\text { Capsule } \\
\text { Column }\end{array}$ & $\begin{array}{l}\text { OC2 } \\
\text { West }\end{array}$ & $\begin{array}{c}\text { Specimen } \\
\text { Length }\end{array}$ & $\begin{array}{c}45 \\
0.9931 \\
\end{array}$ & $\begin{array}{c}\text { Material } \\
\text { Scale }\end{array}$ & $\begin{array}{c}\text { H-451 } \\
1 "\end{array}$ & $5.00 \mathrm{E}-04$ & & & & & & & & \\
\hline $\begin{array}{c}\text { Temperature } \\
\left({ }^{\circ} \mathrm{C}\right) \\
\end{array}$ & Up & Down & $\begin{array}{l}\text { Delta } \\
\text { Down }\end{array}$ & $\begin{array}{l}\text { Down } \\
\text { length }\end{array}$ & CTE & CTEK & $\begin{array}{l}\text { Delta } \\
\text { CTEK }\end{array}$ & $\begin{array}{c}\text { CTE } \\
\text { adjusted }\end{array}$ & $\begin{array}{l}\text { Delta } \\
\text { Up }\end{array}$ & $\begin{array}{c}\text { Up } \\
\text { length }\end{array}$ & CTE & CTEK & $\begin{array}{l}\text { Delta } \\
\text { CTEK }\end{array}$ & $\begin{array}{c}\text { CTE } \\
\text { adjusted }\end{array}$ \\
\hline 0 & 0.87 & 1.12 & 0 & 0.9931 & & & & & 0 & 0.9931 & & & & \\
\hline 100 & 1.50 & 1.70 & 0.58 & 0.9934 & $2.92 \mathrm{E}-06$ & & $-2.92 \mathrm{E}-06$ & $3.42 \mathrm{E}-06$ & 0.63 & 0.9934 & 3.17E-06 & & $-3.17 \mathrm{E}-06$ & 3.67E-06 \\
\hline 200 & 2.18 & 2.30 & 1.18 & 0.9937 & 2.97E-06 & & $-2.97 \mathrm{E}-06$ & $3.47 \mathrm{E}-06$ & 1.31 & 0.9938 & $3.30 \mathrm{E}-06$ & & $-3.30 \mathrm{E}-06$ & $3.80 \mathrm{E}-06$ \\
\hline 300 & 2.93 & 3.10 & 1.98 & 0.9941 & $3.32 \mathrm{E}-06$ & & $-3.32 \mathrm{E}-06$ & $3.82 \mathrm{E}-06$ & 2.06 & 0.9941 & $3.46 \mathrm{E}-06$ & & $-3.46 \mathrm{E}-06$ & $3.96 \mathrm{E}-06$ \\
\hline 400 & 3.78 & 4.02 & 2.9 & 0.9946 & $3.65 \mathrm{E}-06$ & & $-3.65 E-06$ & 4.15E-06 & 2.91 & 0.9946 & $3.66 \mathrm{E}-06$ & & $-3.66 \mathrm{E}-06$ & 4.16E-06 \\
\hline 500 & 4.70 & 4.90 & 3.78 & 0.9950 & $3.81 \mathrm{E}-06$ & 4.50E-06 & $6.94 \mathrm{E}-07$ & $4.31 \mathrm{E}-06$ & 3.83 & 0.9950 & $3.86 \mathrm{E}-06$ & $4.50 E-06$ & $6.43 \mathrm{E}-07$ & 4.36E-06 \\
\hline
\end{tabular}

\begin{tabular}{|c|c|c|c|c|c|c|c|c|c|c|c|c|c|c|}
\hline $\begin{array}{l}\text { Capsule } \\
\text { Column }\end{array}$ & $\begin{array}{l}\text { OC2 } \\
\text { West }\end{array}$ & $\begin{array}{l}\text { Specimen } \\
\text { Length }\end{array}$ & $\begin{array}{c}47 \\
0.9887\end{array}$ & $\begin{array}{l}\text { Material } \\
\text { Scale }\end{array}$ & $\begin{array}{c}\text { H-451 } \\
1 "\end{array}$ & $5.00 \mathrm{E}-04$ & & & & & & & & \\
\hline $\begin{array}{c}\text { Temperature } \\
\left({ }^{\circ} \mathrm{C}\right)\end{array}$ & Up & Down & $\begin{array}{l}\text { Delta } \\
\text { Down }\end{array}$ & $\begin{array}{l}\text { Down } \\
\text { length }\end{array}$ & CTE & CTEK & $\begin{array}{l}\text { Delta } \\
\text { CTEK }\end{array}$ & $\begin{array}{c}\text { CTE } \\
\text { adjusted }\end{array}$ & $\begin{array}{l}\text { Delta } \\
\text { Up }\end{array}$ & $\begin{array}{c}\text { Up } \\
\text { length }\end{array}$ & CTE & CTEK & $\begin{array}{l}\text { Delta } \\
\text { CTEK }\end{array}$ & $\begin{array}{c}\text { CTE } \\
\text { adjusted }\end{array}$ \\
\hline 0 & 0.22 & 0.74 & 0 & 0.9887 & & & & & 0 & 0.9887 & & & & \\
\hline 100 & 1.00 & 1.45 & 0.71 & 0.9891 & $3.59 \mathrm{E}-06$ & & $-3.59 E-06$ & 4.09E-06 & 0.78 & 0.9891 & $3.94 \mathrm{E}-06$ & & $-3.94 \mathrm{E}-06$ & 4.44E-06 \\
\hline 200 & 1.75 & 2.12 & 1.38 & 0.9894 & $3.49 \mathrm{E}-06$ & & $-3.49 \mathrm{E}-06$ & 3.99E-06 & 1.53 & 0.9895 & $3.87 \mathrm{E}-06$ & & $-3.87 \mathrm{E}-06$ & 4.37E-06 \\
\hline 300 & 2.60 & 3.01 & 2.27 & 0.9898 & $3.83 \mathrm{E}-06$ & & $-3.83 E-06$ & $4.33 \mathrm{E}-06$ & 2.38 & 0.9899 & 4.01E-06 & & $-4.01 \mathrm{E}-06$ & 4.51E-06 \\
\hline 400 & 3.48 & 3.90 & 3.16 & 0.9903 & $4.00 \mathrm{E}-06$ & & $-4.00 E-06$ & $4.50 \mathrm{E}-06$ & 3.26 & 0.9903 & $4.12 \mathrm{E}-06$ & & $-4.12 \mathrm{E}-06$ & $4.62 \mathrm{E}-06$ \\
\hline 500 & 4.45 & 4.91 & 4.17 & 0.9908 & $4.22 \mathrm{E}-06$ & $4.50 E-06$ & $2.82 \mathrm{E}-07$ & $4.72 \mathrm{E}-06$ & 4.23 & 0.9908 & $4.28 \mathrm{E}-06$ & 4.50E-06 & $2.22 \mathrm{E}-07$ & 4.78E-06 \\
\hline
\end{tabular}

\begin{tabular}{|c|c|c|c|c|c|c|c|c|c|c|c|c|c|c|}
\hline $\begin{array}{l}\text { Capsule } \\
\text { Column }\end{array}$ & $\begin{array}{c}\text { OC2 } \\
\text { West }\end{array}$ & $\begin{array}{c}\text { Specimen } \\
\text { Length }\end{array}$ & $\begin{array}{c}56 \\
0.9776 \\
\end{array}$ & $\begin{array}{c}\text { Material } \\
\text { Scale }\end{array}$ & $\begin{array}{c}\text { H-451 } \\
1 " \\
\end{array}$ & $5.00 \mathrm{E}-04$ & & & & & & & & \\
\hline $\begin{array}{c}\text { Temperature } \\
\left({ }^{\circ} \mathrm{C}\right)\end{array}$ & Up & Down & $\begin{array}{l}\text { Delta } \\
\text { Down }\end{array}$ & $\begin{array}{l}\text { Down } \\
\text { length }\end{array}$ & CTE & CTEK & $\begin{array}{l}\text { Delta } \\
\text { CTEK }\end{array}$ & $\begin{array}{c}\text { CTE } \\
\text { adjusted }\end{array}$ & $\begin{array}{l}\text { Delta } \\
\text { Up }\end{array}$ & $\begin{array}{c}\text { Up } \\
\text { length }\end{array}$ & CTE & CTEK & $\begin{array}{l}\text { Delta } \\
\text { CTEK }\end{array}$ & $\begin{array}{c}\text { CTE } \\
\text { adjusted }\end{array}$ \\
\hline 0 & 0.15 & 1.07 & 0 & 0.9776 & & & & & 0 & 0.9776 & & & & \\
\hline 100 & 0.90 & 1.74 & 0.67 & 0.9779 & $3.43 \mathrm{E}-06$ & & $-3.43 E-06$ & $3.93 \mathrm{E}-06$ & 0.75 & 0.9780 & $3.84 \mathrm{E}-06$ & & $-3.84 \mathrm{E}-06$ & $4.34 \mathrm{E}-06$ \\
\hline 200 & 1.70 & 2.40 & 1.33 & 0.9783 & $3.40 \mathrm{E}-06$ & & $-3.40 \mathrm{E}-06$ & 3.90E-06 & 1.55 & 0.9784 & $3.96 \mathrm{E}-06$ & & $-3.96 \mathrm{E}-06$ & 4.46E-06 \\
\hline 300 & 2.63 & 3.20 & 2.13 & 0.9787 & $3.63 \mathrm{E}-06$ & & $-3.63 E-06$ & 4.13E-06 & 2.48 & 0.9788 & $4.23 \mathrm{E}-06$ & & $-4.23 E-06$ & 4.73E-06 \\
\hline 400 & 3.60 & 4.10 & 3.03 & 0.9791 & $3.87 \mathrm{E}-06$ & & $-3.87 \mathrm{E}-06$ & 4.37E-06 & 3.45 & 0.9793 & 4.41E-06 & & $-4.41 \mathrm{E}-06$ & $4.91 \mathrm{E}-06$ \\
\hline 500 & 4.60 & 4.99 & 3.92 & 0.9796 & 4.01E-06 & 4.50E-06 & 4.90E-07 & 4.51E-06 & 4.45 & 0.9798 & 4.55E-06 & 4.50E-06 & $-5.20 \mathrm{E}-08$ & 5.05E-06 \\
\hline
\end{tabular}




\begin{tabular}{|c|c|c|c|c|c|c|c|c|c|c|c|c|c|c|}
\hline $\begin{array}{l}\text { Capsule } \\
\text { Column }\end{array}$ & $\begin{array}{l}\text { OC2 } \\
\text { West }\end{array}$ & $\begin{array}{c}\text { Specimen } \\
\text { Length }\end{array}$ & $\begin{array}{c}58 \\
0.985\end{array}$ & $\begin{array}{c}\text { Material } \\
\text { Scale }\end{array}$ & $\begin{array}{c}\text { H-451 } \\
1 "\end{array}$ & $5.00 \mathrm{E}-04$ & & & & & & & & \\
\hline $\begin{array}{c}\text { Temperature } \\
\left({ }^{\circ} \mathrm{C}\right) \\
\end{array}$ & Up & Down & $\begin{array}{l}\text { Delta } \\
\text { Down }\end{array}$ & $\begin{array}{l}\text { Down } \\
\text { length }\end{array}$ & CTE & CTEK & $\begin{array}{l}\text { Delta } \\
\text { CTEK }\end{array}$ & $\begin{array}{c}\text { CTE } \\
\text { adjusted }\end{array}$ & $\begin{array}{l}\text { Delta } \\
\text { Up }\end{array}$ & $\begin{array}{c}\text { Up } \\
\text { length }\end{array}$ & CTE & CTEK & $\begin{array}{l}\text { Delta } \\
\text { CTEK }\end{array}$ & $\begin{array}{c}\text { CTE } \\
\text { adjusted }\end{array}$ \\
\hline 0 & 0.55 & 1.08 & 0 & 0.9850 & & & & & 0 & 0.9850 & & & & \\
\hline 100 & 1.25 & 1.72 & 0.64 & 0.9853 & $3.25 \mathrm{E}-06$ & & $-3.25 \mathrm{E}-06$ & $3.75 \mathrm{E}-06$ & 0.7 & 0.9854 & $3.55 \mathrm{E}-06$ & & $-3.55 \mathrm{E}-06$ & 4.05E-06 \\
\hline 200 & 2.07 & 2.50 & 1.42 & 0.9857 & 3.60E-06 & & $-3.60 \mathrm{E}-06$ & 4.10E-06 & 1.52 & 0.9858 & $3.86 \mathrm{E}-06$ & & $-3.86 \mathrm{E}-06$ & 4.36E-06 \\
\hline 300 & 2.92 & 3.35 & 2.27 & 0.9861 & $3.84 \mathrm{E}-06$ & & $-3.84 \mathrm{E}-06$ & $4.34 \mathrm{E}-06$ & 2.37 & 0.9862 & $4.01 \mathrm{E}-06$ & & $-4.01 \mathrm{E}-06$ & 4.51E-06 \\
\hline 400 & 3.85 & 4.30 & 3.22 & 0.9866 & 4.09E-06 & & $-4.09 \mathrm{E}-06$ & 4.59E-06 & 3.3 & 0.9867 & 4.19E-06 & & $-4.19 \mathrm{E}-06$ & 4.69E-06 \\
\hline 500 & 4.88 & 5.25 & 4.17 & 0.9871 & 4.23E-06 & 4.50E-06 & $2.66 \mathrm{E}-07$ & $4.73 \mathrm{E}-06$ & 4.33 & 0.9872 & $4.40 \mathrm{E}-06$ & $4.50 E-06$ & $1.04 \mathrm{E}-07$ & 4.90E-06 \\
\hline
\end{tabular}

\begin{tabular}{|c|c|c|c|c|c|c|c|c|c|c|c|c|c|c|}
\hline $\begin{array}{l}\text { Capsule } \\
\text { Column }\end{array}$ & $\begin{array}{l}\text { OC2 } \\
\text { West }\end{array}$ & $\begin{array}{l}\text { Specimen } \\
\text { Length }\end{array}$ & $\begin{array}{c}60 \\
0.9853\end{array}$ & $\begin{array}{l}\text { Material } \\
\text { Scale }\end{array}$ & $\begin{array}{c}\text { H-451 } \\
1 "\end{array}$ & $5.00 \mathrm{E}-04$ & & & & & & & & \\
\hline $\begin{array}{c}\text { Temperature } \\
\left({ }^{\circ} \mathrm{C}\right)\end{array}$ & Up & Down & $\begin{array}{l}\text { Delta } \\
\text { Down }\end{array}$ & $\begin{array}{l}\text { Down } \\
\text { length }\end{array}$ & CTE & CTEK & $\begin{array}{l}\text { Delta } \\
\text { CTEK }\end{array}$ & $\begin{array}{c}\text { CTE } \\
\text { adjusted }\end{array}$ & $\begin{array}{l}\text { Delta } \\
\text { Up }\end{array}$ & $\begin{array}{c}\text { Up } \\
\text { length }\end{array}$ & CTE & CTEK & $\begin{array}{l}\text { Delta } \\
\text { CTEK }\end{array}$ & $\begin{array}{c}\text { CTE } \\
\text { adjusted }\end{array}$ \\
\hline 0 & 2.78 & 3.22 & 0 & 0.9853 & & & & & 0 & 0.9853 & & & & \\
\hline 100 & 3.52 & 3.95 & 0.73 & 0.9857 & $3.70 \mathrm{E}-06$ & & $-3.70 \mathrm{E}-06$ & $4.20 \mathrm{E}-06$ & 0.74 & 0.9857 & $3.76 \mathrm{E}-06$ & & $-3.76 \mathrm{E}-06$ & 4.26E-06 \\
\hline 200 & 4.32 & 4.75 & 1.53 & 0.9861 & $3.88 \mathrm{E}-06$ & & $-3.88 \mathrm{E}-06$ & $4.38 \mathrm{E}-06$ & 1.54 & 0.9861 & $3.91 \mathrm{E}-06$ & & $-3.91 \mathrm{E}-06$ & 4.41E-06 \\
\hline 300 & 5.21 & 5.52 & 2.3 & 0.9865 & 3.89E-06 & & $-3.89 \mathrm{E}-06$ & 4.39E-06 & 2.43 & 0.9865 & 4.11E-06 & & $-4.11 \mathrm{E}-06$ & 4.61E-06 \\
\hline 400 & 6.12 & 6.42 & 3.2 & 0.9869 & $4.06 \mathrm{E}-06$ & & $-4.06 E-06$ & $4.56 \mathrm{E}-06$ & 3.34 & 0.9870 & $4.24 \mathrm{E}-06$ & & $-4.24 \mathrm{E}-06$ & 4.74E-06 \\
\hline 500 & 7.18 & 7.42 & 4.2 & 0.9874 & $4.26 \mathrm{E}-06$ & $4.50 E-06$ & $2.37 \mathrm{E}-07$ & $4.76 \mathrm{E}-06$ & 4.4 & 0.9875 & 4.47E-06 & 4.50E-06 & $3.44 \mathrm{E}-08$ & 4.97E-06 \\
\hline
\end{tabular}

\begin{tabular}{|c|c|c|c|c|c|c|c|c|c|c|c|c|c|c|}
\hline $\begin{array}{l}\text { Capsule } \\
\text { Column }\end{array}$ & $\begin{array}{c}\text { OC2 } \\
\text { West }\end{array}$ & $\begin{array}{c}\text { Specimen } \\
\text { Length }\end{array}$ & $\begin{array}{c}4 \\
0.9938 \\
\end{array}$ & $\begin{array}{c}\text { Material } \\
\text { Scale }\end{array}$ & $\begin{array}{c}\text { H-327 } \\
1 " \\
\end{array}$ & $5.00 \mathrm{E}-04$ & & & & & & & & \\
\hline $\begin{array}{c}\text { Temperature } \\
\left({ }^{\circ} \mathrm{C}\right)\end{array}$ & Up & Down & $\begin{array}{l}\text { Delta } \\
\text { Down }\end{array}$ & $\begin{array}{l}\text { Down } \\
\text { length }\end{array}$ & CTE & CTEK & $\begin{array}{l}\text { Delta } \\
\text { CTEK }\end{array}$ & $\begin{array}{c}\text { CTE } \\
\text { adjusted }\end{array}$ & $\begin{array}{l}\text { Delta } \\
\text { Up }\end{array}$ & $\begin{array}{c}\text { Up } \\
\text { length }\end{array}$ & CTE & CTEK & $\begin{array}{l}\text { Delta } \\
\text { CTEK }\end{array}$ & $\begin{array}{c}\text { CTE } \\
\text { adjusted }\end{array}$ \\
\hline 0 & 0.90 & 0.90 & 0 & 0.9938 & & & & & 0 & 0.9938 & & & & \\
\hline 100 & 0.92 & 1.00 & 0.1 & 0.9939 & $5.03 \mathrm{E}-07$ & & $-5.03 E-07$ & $1.00 \mathrm{E}-06$ & 0.02 & 0.9938 & $1.01 \mathrm{E}-07$ & & $-1.01 \mathrm{E}-07$ & $6.01 \mathrm{E}-07$ \\
\hline 200 & 1.18 & 1.20 & 0.3 & 0.9940 & $7.55 \mathrm{E}-07$ & & $-7.55 E-07$ & $1.25 \mathrm{E}-06$ & 0.28 & 0.9939 & $7.04 \mathrm{E}-07$ & & $-7.04 \mathrm{E}-07$ & $1.20 \mathrm{E}-06$ \\
\hline 300 & 1.40 & 1.52 & 0.62 & 0.9941 & $1.04 \mathrm{E}-06$ & & $-1.04 \mathrm{E}-06$ & $1.54 \mathrm{E}-06$ & 0.5 & 0.9941 & 8.39E-07 & & $-8.39 E-07$ & $1.34 \mathrm{E}-06$ \\
\hline 400 & 1.77 & 1.95 & 1.05 & 0.9943 & $1.32 \mathrm{E}-06$ & & $-1.32 \mathrm{E}-06$ & $1.82 \mathrm{E}-06$ & 0.87 & 0.9942 & $1.09 \mathrm{E}-06$ & & $-1.09 \mathrm{E}-06$ & $1.59 \mathrm{E}-06$ \\
\hline 500 & 2.20 & 2.30 & 1.4 & 0.9945 & $1.41 \mathrm{E}-06$ & 4.50E-06 & 3.09E-06 & 1.91E-06 & 1.3 & 0.9945 & $1.31 \mathrm{E}-06$ & 4.50E-06 & $3.19 \mathrm{E}-06$ & $1.81 \mathrm{E}-06$ \\
\hline
\end{tabular}




\begin{tabular}{|c|c|c|c|c|c|c|c|c|c|c|c|c|c|c|}
\hline $\begin{array}{l}\text { Capsule } \\
\text { Column }\end{array}$ & $\begin{array}{l}\text { OC2 } \\
\text { West }\end{array}$ & $\begin{array}{c}\text { Specimen } \\
\text { Length }\end{array}$ & $\begin{array}{c}10 \\
0.9862\end{array}$ & $\begin{array}{c}\text { Material } \\
\text { Scale }\end{array}$ & $\begin{array}{c}\mathrm{H}-327 \\
1 "\end{array}$ & $5.00 \mathrm{E}-04$ & & & & & & & & \\
\hline $\begin{array}{c}\text { Temperature } \\
\left({ }^{\circ} \mathrm{C}\right) \\
\end{array}$ & Up & Down & $\begin{array}{l}\text { Delta } \\
\text { Down }\end{array}$ & $\begin{array}{l}\text { Down } \\
\text { length }\end{array}$ & CTE & CTEK & $\begin{array}{l}\text { Delta } \\
\text { CTEK }\end{array}$ & $\begin{array}{c}\text { CTE } \\
\text { adjusted }\end{array}$ & $\begin{array}{l}\text { Delta } \\
\text { Up }\end{array}$ & $\begin{array}{c}\text { Up } \\
\text { length }\end{array}$ & CTE & CTEK & $\begin{array}{l}\text { Delta } \\
\text { CTEK }\end{array}$ & $\begin{array}{c}\text { CTE } \\
\text { adjusted }\end{array}$ \\
\hline 0 & 0.91 & 1.17 & 0 & 0.9862 & & & & & 0 & 0.9862 & & & & \\
\hline 100 & 1.52 & 1.87 & 0.7 & 0.9866 & $3.55 \mathrm{E}-06$ & & $-3.55 \mathrm{E}-06$ & $4.05 \mathrm{E}-06$ & 0.61 & 0.9865 & $3.09 \mathrm{E}-06$ & & $-3.09 \mathrm{E}-06$ & $3.59 \mathrm{E}-06$ \\
\hline 200 & 2.22 & 2.40 & 1.23 & 0.9868 & $3.12 \mathrm{E}-06$ & & $-3.12 \mathrm{E}-06$ & $3.62 \mathrm{E}-06$ & 1.31 & 0.9869 & $3.32 \mathrm{E}-06$ & & $-3.32 \mathrm{E}-06$ & $3.82 \mathrm{E}-06$ \\
\hline 300 & 2.98 & 3.12 & 1.95 & 0.9872 & $3.30 \mathrm{E}-06$ & & $-3.30 \mathrm{E}-06$ & $3.80 \mathrm{E}-06$ & 2.07 & 0.9872 & $3.50 \mathrm{E}-06$ & & $-3.50 \mathrm{E}-06$ & 4.00E-06 \\
\hline 400 & 3.71 & 4.00 & 2.83 & 0.9876 & $3.59 \mathrm{E}-06$ & & $-3.59 \mathrm{E}-06$ & 4.09E-06 & 2.8 & 0.9876 & $3.55 \mathrm{E}-06$ & & $-3.55 E-06$ & 4.05E-06 \\
\hline 500 & 4.69 & 4.90 & 3.73 & 0.9881 & $3.78 \mathrm{E}-06$ & 4.50E-06 & $7.18 \mathrm{E}-07$ & $4.28 \mathrm{E}-06$ & 3.78 & 0.9881 & $3.83 \mathrm{E}-06$ & 4.50E-06 & $6.67 \mathrm{E}-07$ & 4.33E-06 \\
\hline
\end{tabular}

\begin{tabular}{|c|c|c|c|c|c|c|c|c|c|c|c|c|c|c|}
\hline $\begin{array}{l}\text { Capsule } \\
\text { Column }\end{array}$ & $\begin{array}{l}\text { OC2 } \\
\text { West }\end{array}$ & $\begin{array}{l}\text { Specimen } \\
\text { Length }\end{array}$ & $\begin{array}{c}2 G \\
0.9956\end{array}$ & $\begin{array}{l}\text { Material } \\
\text { Scale }\end{array}$ & $\begin{array}{c}\text { H-327 } \\
1 "\end{array}$ & $5.00 \mathrm{E}-04$ & & & & & & & & \\
\hline $\begin{array}{c}\text { Temperature } \\
\left({ }^{\circ} \mathrm{C}\right)\end{array}$ & Up & Down & $\begin{array}{l}\text { Delta } \\
\text { Down }\end{array}$ & $\begin{array}{l}\text { Down } \\
\text { length }\end{array}$ & CTE & CTEK & $\begin{array}{l}\text { Delta } \\
\text { CTEK }\end{array}$ & $\begin{array}{c}\text { CTE } \\
\text { adjusted }\end{array}$ & $\begin{array}{l}\text { Delta } \\
\text { Up }\end{array}$ & $\begin{array}{c}\text { Up } \\
\text { length }\end{array}$ & CTE & CTEK & $\begin{array}{l}\text { Delta } \\
\text { CTEK }\end{array}$ & $\begin{array}{c}\text { CTE } \\
\text { adjusted }\end{array}$ \\
\hline 0 & 0.50 & 1.22 & 0 & 0.9956 & & & & & 0 & 0.9956 & & & & \\
\hline 100 & 1.52 & 2.20 & 0.98 & 0.9961 & $4.92 \mathrm{E}-06$ & & $-4.92 E-06$ & $5.42 \mathrm{E}-06$ & 1.02 & 0.9961 & $5.12 \mathrm{E}-06$ & & $-5.12 \mathrm{E}-06$ & $5.62 \mathrm{E}-06$ \\
\hline 200 & 2.65 & 3.21 & 1.99 & 0.9966 & $5.00 \mathrm{E}-06$ & & $-5.00 \mathrm{E}-06$ & $5.50 \mathrm{E}-06$ & 2.15 & 0.9967 & $5.40 \mathrm{E}-06$ & & $-5.40 E-06$ & $5.90 \mathrm{E}-06$ \\
\hline 300 & 3.83 & 4.40 & 3.18 & 0.9972 & $5.32 \mathrm{E}-06$ & & $-5.32 E-06$ & $5.82 \mathrm{E}-06$ & 3.33 & 0.9973 & 5.57E-06 & & $-5.57 \mathrm{E}-06$ & 6.07E-06 \\
\hline 400 & 5.10 & 5.60 & 4.38 & 0.9978 & $5.50 \mathrm{E}-06$ & & $-5.50 E-06$ & $6.00 \mathrm{E}-06$ & 4.6 & 0.9979 & $5.78 \mathrm{E}-06$ & & $-5.78 \mathrm{E}-06$ & $6.28 \mathrm{E}-06$ \\
\hline 500 & 6.32 & 6.88 & 5.66 & 0.9984 & 5.69E-06 & $4.50 E-06$ & $-1.19 \mathrm{E}-06$ & $6.19 \mathrm{E}-06$ & 5.82 & 0.9985 & $5.85 \mathrm{E}-06$ & 4.50E-06 & $-1.35 \mathrm{E}-06$ & $6.35 \mathrm{E}-06$ \\
\hline
\end{tabular}

\begin{tabular}{|c|c|c|c|c|c|c|c|c|c|c|c|c|c|c|}
\hline $\begin{array}{l}\text { Capsule } \\
\text { Column }\end{array}$ & $\begin{array}{c}\text { OC2 } \\
\text { North }\end{array}$ & $\begin{array}{c}\text { Specimen } \\
\text { Length }\end{array}$ & $\begin{array}{c}78 \\
0.9991 \\
\end{array}$ & $\begin{array}{c}\text { Material } \\
\text { Scale }\end{array}$ & $\begin{array}{c}\text { H-451 } \\
1 " \\
\end{array}$ & $5.00 \mathrm{E}-04$ & & & & & & & & \\
\hline $\begin{array}{c}\text { Temperature } \\
\left({ }^{\circ} \mathrm{C}\right)\end{array}$ & Up & Down & $\begin{array}{l}\text { Delta } \\
\text { Down }\end{array}$ & $\begin{array}{l}\text { Down } \\
\text { length }\end{array}$ & CTE & CTEK & $\begin{array}{l}\text { Delta } \\
\text { CTEK }\end{array}$ & $\begin{array}{c}\text { CTE } \\
\text { adjusted }\end{array}$ & $\begin{array}{l}\text { Delta } \\
\text { Up }\end{array}$ & $\begin{array}{c}\text { Up } \\
\text { length }\end{array}$ & CTE & CTEK & $\begin{array}{l}\text { Delta } \\
\text { CTEK }\end{array}$ & $\begin{array}{c}\text { CTE } \\
\text { adjusted }\end{array}$ \\
\hline 0 & 0.75 & 1.85 & 0 & 0.9991 & & & & & 0 & 0.9991 & & & & \\
\hline 100 & 1.38 & 2.10 & 0.25 & 0.9992 & $1.25 \mathrm{E}-06$ & $1.75 E-06$ & 4.99E-07 & $1.75 \mathrm{E}-06$ & 0.63 & 0.9994 & 3.15E-06 & $3.65 E-06$ & 4.97E-07 & $3.65 \mathrm{E}-06$ \\
\hline 200 & 1.90 & 2.60 & 0.75 & 0.9995 & $1.88 \mathrm{E}-06$ & $2.34 E-06$ & 4.58E-07 & $2.38 \mathrm{E}-06$ & 1.15 & 0.9997 & $2.88 \mathrm{E}-06$ & $3.34 E-06$ & 4.57E-07 & $3.38 \mathrm{E}-06$ \\
\hline 300 & 2.61 & 3.10 & 1.25 & 0.9997 & 2.09E-06 & $2.56 E-06$ & $4.78 \mathrm{E}-07$ & $2.59 \mathrm{E}-06$ & 1.86 & 1.0000 & $3.10 \mathrm{E}-06$ & $3.58 E-06$ & 4.77E-07 & $3.60 \mathrm{E}-06$ \\
\hline 400 & 3.32 & 3.80 & 1.95 & 1.0001 & $2.44 \mathrm{E}-06$ & $2.91 E-06$ & 4.67E-07 & $2.94 \mathrm{E}-06$ & 2.57 & 1.0004 & $3.22 \mathrm{E}-06$ & $3.68 E-06$ & 4.67E-07 & $3.72 \mathrm{E}-06$ \\
\hline 500 & 4.09 & 4.45 & 2.6 & 1.0004 & $2.60 \mathrm{E}-06$ & 3.07E-06 & $1.21 \mathrm{E}-06$ & 3.10E-06 & 3.34 & 1.0008 & 3.34E-06 & $3.81 E-06$ & 4.67E-07 & $3.84 \mathrm{E}-06$ \\
\hline
\end{tabular}




\begin{tabular}{|c|c|c|c|c|c|c|c|c|c|c|c|c|c|c|}
\hline $\begin{array}{l}\text { Capsule } \\
\text { Column }\end{array}$ & $\begin{array}{l}\text { OC2 } \\
\text { North }\end{array}$ & $\begin{array}{l}\text { Specimen } \\
\text { Length }\end{array}$ & $\begin{array}{c}83 \\
0.9992\end{array}$ & $\begin{array}{c}\text { Material } \\
\text { Scale }\end{array}$ & $\begin{array}{c}\text { H-451 } \\
1 "\end{array}$ & $5.00 \mathrm{E}-04$ & & & & & & & & \\
\hline $\begin{array}{c}\text { Temperature } \\
\left({ }^{\circ} \mathrm{C}\right) \\
\end{array}$ & Up & Down & $\begin{array}{l}\text { Delta } \\
\text { Down }\end{array}$ & $\begin{array}{l}\text { Down } \\
\text { length }\end{array}$ & CTE & CTEK & $\begin{array}{l}\text { Delta } \\
\text { CTEK }\end{array}$ & $\begin{array}{c}\text { CTE } \\
\text { adjusted }\end{array}$ & $\begin{array}{l}\text { Delta } \\
\text { Up }\end{array}$ & $\begin{array}{c}\text { Up } \\
\text { length }\end{array}$ & CTE & CTEK & $\begin{array}{l}\text { Delta } \\
\text { CTEK }\end{array}$ & $\begin{array}{c}\text { CTE } \\
\text { adjusted }\end{array}$ \\
\hline 0 & 0.73 & 1.30 & 0 & 0.9992 & & & & & 0 & 0.9992 & & & & \\
\hline 100 & 1.27 & 1.76 & 0.46 & 0.9994 & $2.30 \mathrm{E}-06$ & 2.80E-06 & $4.98 \mathrm{E}-07$ & $2.80 \mathrm{E}-06$ & 0.54 & 0.9995 & $2.70 \mathrm{E}-06$ & $2.70 E+00$ & $2.70 E+00$ & $3.20 \mathrm{E}-06$ \\
\hline 200 & 1.80 & 2.20 & 0.9 & 0.9997 & $2.25 \mathrm{E}-06$ & 2.71E-06 & $4.58 \mathrm{E}-07$ & $2.75 \mathrm{E}-06$ & 1.07 & 0.9997 & $2.68 \mathrm{E}-06$ & $2.94 E+00$ & $2.93 \mathrm{E}+00$ & $3.18 \mathrm{E}-06$ \\
\hline 300 & 2.45 & 2.89 & 1.59 & 1.0000 & $2.65 \mathrm{E}-06$ & $3.13 E-06$ & $4.78 \mathrm{E}-07$ & $3.15 \mathrm{E}-06$ & 1.72 & 1.0001 & 2.87E-06 & $3.18 E+00$ & $3.18 \mathrm{E}+00$ & 3.37E-06 \\
\hline 400 & 3.20 & 3.63 & 2.33 & 1.0004 & $2.91 \mathrm{E}-06$ & $3.38 E-06$ & 4.67E-07 & $3.41 \mathrm{E}-06$ & 2.47 & 1.0004 & 3.09E-06 & $3.40 E+00$ & $3.39 \mathrm{E}+00$ & $3.59 \mathrm{E}-06$ \\
\hline 500 & 4.00 & 4.32 & 3.02 & 1.0007 & $3.02 \mathrm{E}-06$ & $3.49 E-06$ & $6.98 \mathrm{E}-07$ & $3.52 \mathrm{E}-06$ & 3.27 & 1.0008 & 3.27E-06 & $3.61 E+00$ & $3.61 \mathrm{E}+00$ & 3.77E-06 \\
\hline
\end{tabular}

\begin{tabular}{|c|c|c|c|c|c|c|c|c|c|c|c|c|c|c|}
\hline $\begin{array}{l}\text { Capsule } \\
\text { Column }\end{array}$ & $\begin{array}{l}\text { OC2 } \\
\text { North }\end{array}$ & $\begin{array}{c}\text { Specimen } \\
\text { Length }\end{array}$ & $\begin{array}{c}88 \\
0.9977\end{array}$ & $\begin{array}{c}\text { Material } \\
\text { Scale }\end{array}$ & $\begin{array}{c}\text { H-451 } \\
1 "\end{array}$ & $5.00 \mathrm{E}-04$ & & & & & & & & \\
\hline $\begin{array}{c}\text { Temperature } \\
\left({ }^{\circ} \mathrm{C}\right)\end{array}$ & Up & Down & $\begin{array}{l}\text { Delta } \\
\text { Down }\end{array}$ & $\begin{array}{l}\text { Down } \\
\text { length }\end{array}$ & CTE & CTEK & $\begin{array}{l}\text { Delta } \\
\text { CTEK }\end{array}$ & $\begin{array}{c}\text { CTE } \\
\text { adjusted }\end{array}$ & $\begin{array}{l}\text { Delta } \\
\text { Up }\end{array}$ & $\begin{array}{c}\text { Up } \\
\text { length }\end{array}$ & CTE & CTEK & $\begin{array}{l}\text { Delta } \\
\text { CTEK }\end{array}$ & $\begin{array}{c}\text { CTE } \\
\text { adjusted }\end{array}$ \\
\hline 0 & 0.66 & 1.20 & 0 & 0.9977 & & & & & 0 & 0.9977 & & & & \\
\hline 100 & 1.10 & 1.48 & 0.28 & 0.9978 & $1.40 \mathrm{E}-06$ & $1.90 E-06$ & 4.97E-07 & $1.90 \mathrm{E}-06$ & 0.44 & 0.9979 & $2.21 \mathrm{E}-06$ & $2.70 E-06$ & 4.95E-07 & $2.71 \mathrm{E}-06$ \\
\hline 200 & 1.65 & 1.90 & 0.7 & 0.9981 & $1.75 \mathrm{E}-06$ & $2.21 E-06$ & 4.56E-07 & $2.25 \mathrm{E}-06$ & 0.99 & 0.9982 & $2.48 \mathrm{E}-06$ & $2.94 E-06$ & 4.54E-07 & $2.98 \mathrm{E}-06$ \\
\hline 300 & 2.28 & 2.60 & 1.4 & 0.9984 & $2.34 \mathrm{E}-06$ & $2.81 E-06$ & 4.74E-07 & $2.84 \mathrm{E}-06$ & 1.62 & 0.9985 & $2.71 \mathrm{E}-06$ & $3.18 E-06$ & 4.74E-07 & $3.21 \mathrm{E}-06$ \\
\hline 400 & 3.00 & 3.35 & 2.15 & 0.9988 & $2.69 \mathrm{E}-06$ & $3.16 E-06$ & 4.63E-07 & 3.19E-06 & 2.34 & 0.9989 & 2.93E-06 & $3.40 E-06$ & 4.63E-07 & $3.43 \mathrm{E}-06$ \\
\hline 500 & 3.80 & 4.10 & 2.9 & 0.9992 & $2.91 \mathrm{E}-06$ & $3.37 E-06$ & $5.73 \mathrm{E}-07$ & $3.41 \mathrm{E}-06$ & 3.14 & 0.9993 & $3.15 \mathrm{E}-06$ & $3.61 E-06$ & 3.33E-07 & 3.65E-06 \\
\hline
\end{tabular}

\begin{tabular}{|c|c|c|c|c|c|c|c|c|c|c|c|c|c|c|}
\hline $\begin{array}{l}\text { Capsule } \\
\text { Column }\end{array}$ & $\begin{array}{c}\text { OC2 } \\
\text { North }\end{array}$ & $\begin{array}{c}\text { Specimen } \\
\text { Length }\end{array}$ & $\begin{array}{c}90 \\
0.9983\end{array}$ & $\begin{array}{c}\text { Material } \\
\text { Scale }\end{array}$ & $\begin{array}{c}\text { H-451 } \\
1 "\end{array}$ & $5.00 \mathrm{E}-04$ & & & & & & & & \\
\hline $\begin{array}{c}\text { Temperature } \\
\left({ }^{\circ} \mathrm{C}\right)\end{array}$ & Up & Down & $\begin{array}{l}\text { Delta } \\
\text { Down }\end{array}$ & $\begin{array}{l}\text { Down } \\
\text { length }\end{array}$ & CTE & CTEK & $\begin{array}{l}\text { Delta } \\
\text { CTEK }\end{array}$ & $\begin{array}{c}\text { CTE } \\
\text { adjusted }\end{array}$ & $\begin{array}{l}\text { Delta } \\
\text { Up }\end{array}$ & $\begin{array}{c}\text { Up } \\
\text { length }\end{array}$ & CTE & CTEK & $\begin{array}{l}\text { Delta } \\
\text { CTEK }\end{array}$ & $\begin{array}{c}\text { CTE } \\
\text { adjusted }\end{array}$ \\
\hline 0 & 0.40 & 0.98 & 0 & 0.9983 & & & & & 0 & 0.9983 & & & & \\
\hline 100 & 1.00 & 1.38 & 0.4 & 0.9985 & $2.00 \mathrm{E}-06$ & & $-2.00 \mathrm{E}-06$ & $2.50 \mathrm{E}-06$ & 0.6 & 0.9986 & $3.01 \mathrm{E}-06$ & & $-3.01 E-06$ & $3.51 \mathrm{E}-06$ \\
\hline 200 & 1.45 & 1.81 & 0.83 & 0.9987 & $2.08 \mathrm{E}-06$ & & $-2.08 \mathrm{E}-06$ & $2.58 \mathrm{E}-06$ & 1.05 & 0.9988 & $2.63 \mathrm{E}-06$ & & $-2.63 E-06$ & $3.13 \mathrm{E}-06$ \\
\hline 300 & 2.10 & 2.50 & 1.52 & 0.9991 & $2.54 \mathrm{E}-06$ & & $-2.54 \mathrm{E}-06$ & $3.04 \mathrm{E}-06$ & 1.7 & 0.9992 & $2.84 \mathrm{E}-06$ & & $-2.84 \mathrm{E}-06$ & 3.34E-06 \\
\hline 400 & 2.80 & 3.30 & 2.32 & 0.9995 & $2.90 \mathrm{E}-06$ & & $-2.90 \mathrm{E}-06$ & $3.40 \mathrm{E}-06$ & 2.4 & 0.9995 & 3.01E-06 & & $-3.01 \mathrm{E}-06$ & $3.51 \mathrm{E}-06$ \\
\hline 500 & 3.60 & 4.03 & 3.05 & 0.9998 & $3.06 \mathrm{E}-06$ & $5.24 E-06$ & $2.18 \mathrm{E}-06$ & $3.56 \mathrm{E}-06$ & 3.2 & 0.9999 & $3.21 \mathrm{E}-06$ & $5.24 E-06$ & $2.03 \mathrm{E}-06$ & $3.71 \mathrm{E}-06$ \\
\hline
\end{tabular}




\begin{tabular}{|c|c|c|c|c|c|c|c|c|c|c|c|c|c|c|}
\hline $\begin{array}{l}\text { Capsule } \\
\text { Column }\end{array}$ & $\begin{array}{c}\text { OC2 } \\
\text { North }\end{array}$ & $\begin{array}{c}\text { Specimen } \\
\text { Length }\end{array}$ & $\begin{array}{c}93 \\
0.9983 \\
\end{array}$ & $\begin{array}{c}\text { Material } \\
\text { Scale }\end{array}$ & $\begin{array}{c}\text { H-451 } \\
1 "\end{array}$ & $5.00 \mathrm{E}-04$ & & & & & & & & \\
\hline $\begin{array}{c}\text { Temperature } \\
\left({ }^{\circ} \mathrm{C}\right) \\
\end{array}$ & Up & Down & $\begin{array}{l}\text { Delta } \\
\text { Down }\end{array}$ & $\begin{array}{l}\text { Down } \\
\text { length }\end{array}$ & CTE & CTEK & $\begin{array}{l}\text { Delta } \\
\text { CTEK }\end{array}$ & $\begin{array}{c}\text { CTE } \\
\text { adjusted }\end{array}$ & $\begin{array}{l}\text { Delta } \\
\text { Up }\end{array}$ & $\begin{array}{c}\text { Up } \\
\text { length }\end{array}$ & CTE & CTEK & $\begin{array}{l}\text { Delta } \\
\text { CTEK }\end{array}$ & $\begin{array}{c}\text { CTE } \\
\text { adjusted }\end{array}$ \\
\hline 0 & 0.92 & 1.35 & 0 & 0.9983 & & & & & 0 & 0.9983 & & & & \\
\hline 100 & 1.50 & 2.00 & 0.65 & 0.9986 & $3.26 \mathrm{E}-06$ & $3.00 E+00$ & $3.00 \mathrm{E}+00$ & $3.76 \mathrm{E}-06$ & 0.58 & 0.9986 & $2.90 \mathrm{E}-06$ & & $-2.90 \mathrm{E}-06$ & $3.40 \mathrm{E}-06$ \\
\hline 200 & 2.18 & 2.62 & 1.27 & 0.9989 & $3.18 \mathrm{E}-06$ & & $-3.18 \mathrm{E}-06$ & $3.68 \mathrm{E}-06$ & 1.26 & 0.9989 & $3.16 \mathrm{E}-06$ & & $-3.16 \mathrm{E}-06$ & $3.66 \mathrm{E}-06$ \\
\hline 300 & 2.98 & 3.43 & 2.08 & 0.9993 & $3.47 \mathrm{E}-06$ & & $-3.47 \mathrm{E}-06$ & $3.97 \mathrm{E}-06$ & 2.06 & 0.9993 & $3.44 \mathrm{E}-06$ & & $-3.44 \mathrm{E}-06$ & $3.94 \mathrm{E}-06$ \\
\hline 400 & 3.80 & 4.30 & 2.95 & 0.9998 & $3.69 \mathrm{E}-06$ & & $-3.69 \mathrm{E}-06$ & 4.19E-06 & 2.88 & 0.9997 & $3.61 \mathrm{E}-06$ & & $-3.61 \mathrm{E}-06$ & 4.11E-06 \\
\hline 500 & 4.62 & 5.10 & 3.75 & 1.0002 & $3.76 \mathrm{E}-06$ & $4.08 E-06$ & $3.24 \mathrm{E}-07$ & $4.26 \mathrm{E}-06$ & 3.7 & 1.0002 & $3.71 \mathrm{E}-06$ & $4.08 E-06$ & $3.74 \mathrm{E}-07$ & $4.21 \mathrm{E}-06$ \\
\hline
\end{tabular}

\begin{tabular}{|c|c|c|c|c|c|c|c|c|c|c|c|c|c|c|}
\hline $\begin{array}{l}\text { Capsule } \\
\text { Column }\end{array}$ & $\begin{array}{l}\text { OC2 } \\
\text { North }\end{array}$ & $\begin{array}{l}\text { Specimen } \\
\text { Length }\end{array}$ & $\begin{array}{c}103 \\
0.9984\end{array}$ & $\begin{array}{l}\text { Material } \\
\text { Scale }\end{array}$ & $\begin{array}{c}\text { H-451 } \\
1 "\end{array}$ & $5.00 \mathrm{E}-04$ & & & & & & & & \\
\hline $\begin{array}{c}\text { Temperature } \\
\left({ }^{\circ} \mathrm{C}\right)\end{array}$ & Up & Down & $\begin{array}{l}\text { Delta } \\
\text { Down }\end{array}$ & $\begin{array}{l}\text { Down } \\
\text { length }\end{array}$ & CTE & CTEK & $\begin{array}{l}\text { Delta } \\
\text { CTEK }\end{array}$ & $\begin{array}{c}\text { CTE } \\
\text { adjusted }\end{array}$ & $\begin{array}{l}\text { Delta } \\
\text { Up }\end{array}$ & $\begin{array}{c}\text { Up } \\
\text { length }\end{array}$ & CTE & CTEK & $\begin{array}{l}\text { Delta } \\
\text { CTEK }\end{array}$ & $\begin{array}{c}\text { CTE } \\
\text { adjusted }\end{array}$ \\
\hline 0 & 0.91 & 1.70 & 0 & 0.9984 & & & & & 0 & 0.9984 & & & & \\
\hline 100 & 1.52 & 2.15 & 0.45 & 0.9986 & $2.25 \mathrm{E}-06$ & & $-2.25 \mathrm{E}-06$ & $2.75 \mathrm{E}-06$ & 0.61 & 0.9987 & $3.05 E-06$ & & $-3.05 E-06$ & $3.55 \mathrm{E}-06$ \\
\hline 200 & 2.12 & 2.60 & 0.9 & 0.9989 & $2.25 \mathrm{E}-06$ & & $-2.25 \mathrm{E}-06$ & $2.75 \mathrm{E}-06$ & 1.21 & 0.9990 & $3.03 \mathrm{E}-06$ & & $-3.03 E-06$ & $3.53 \mathrm{E}-06$ \\
\hline 300 & 2.80 & 3.32 & 1.62 & 0.9992 & $2.70 \mathrm{E}-06$ & & $-2.70 \mathrm{E}-06$ & $3.20 \mathrm{E}-06$ & 1.89 & 0.9993 & $3.16 \mathrm{E}-06$ & & $-3.16 E-06$ & 3.66E-06 \\
\hline 400 & 3.52 & 4.10 & 2.4 & 0.9996 & $3.00 \mathrm{E}-06$ & & $-3.00 E-06$ & $3.50 \mathrm{E}-06$ & 2.61 & 0.9997 & $3.27 \mathrm{E}-06$ & & $-3.27 \mathrm{E}-06$ & 3.77E-06 \\
\hline 500 & 4.31 & 4.82 & 3.12 & 1.0000 & $3.12 \mathrm{E}-06$ & $4.50 E-06$ & $1.38 \mathrm{E}-06$ & $3.62 \mathrm{E}-06$ & 3.4 & 1.0001 & $3.41 \mathrm{E}-06$ & 4.50E-06 & $1.09 \mathrm{E}-06$ & $3.91 \mathrm{E}-06$ \\
\hline
\end{tabular}

\begin{tabular}{|c|c|c|c|c|c|c|c|c|c|c|c|c|c|c|}
\hline $\begin{array}{l}\text { Capsule } \\
\text { Column }\end{array}$ & $\begin{array}{c}\text { OC2 } \\
\text { North }\end{array}$ & $\begin{array}{c}\text { Specimen } \\
\text { Length }\end{array}$ & $\begin{array}{c}105 \\
0.9984 \\
\end{array}$ & $\begin{array}{c}\text { Material } \\
\text { Scale }\end{array}$ & $\begin{array}{c}\text { H-451 } \\
1 " \\
\end{array}$ & $5.00 \mathrm{E}-04$ & & & & & & & & \\
\hline $\begin{array}{c}\text { Temperature } \\
\left({ }^{\circ} \mathrm{C}\right)\end{array}$ & Up & Down & $\begin{array}{l}\text { Delta } \\
\text { Down }\end{array}$ & $\begin{array}{l}\text { Down } \\
\text { length }\end{array}$ & CTE & CTEK & $\begin{array}{l}\text { Delta } \\
\text { CTEK }\end{array}$ & $\begin{array}{c}\text { CTE } \\
\text { adjusted }\end{array}$ & $\begin{array}{l}\text { Delta } \\
\text { Up }\end{array}$ & $\begin{array}{c}\text { Up } \\
\text { length }\end{array}$ & CTE & CTEK & $\begin{array}{l}\text { Delta } \\
\text { CTEK }\end{array}$ & $\begin{array}{c}\text { CTE } \\
\text { adjusted }\end{array}$ \\
\hline 0 & 1.05 & 1.55 & 0 & 0.9984 & & & & & 0 & 0.9984 & & & & \\
\hline 100 & 1.71 & 2.22 & 0.67 & 0.9987 & $3.36 \mathrm{E}-06$ & & $-3.36 \mathrm{E}-06$ & $3.86 \mathrm{E}-06$ & 0.66 & 0.9987 & 3.31E-06 & & $-3.31 \mathrm{E}-06$ & $3.81 \mathrm{E}-06$ \\
\hline 200 & 2.32 & 2.70 & 1.15 & 0.9990 & $2.88 \mathrm{E}-06$ & & $-2.88 \mathrm{E}-06$ & $3.38 \mathrm{E}-06$ & 1.27 & 0.9990 & $3.18 \mathrm{E}-06$ & & $-3.18 \mathrm{E}-06$ & $3.68 \mathrm{E}-06$ \\
\hline 300 & 3.03 & 3.40 & 1.85 & 0.9993 & $3.09 \mathrm{E}-06$ & & $-3.09 E-06$ & $3.59 \mathrm{E}-06$ & 1.98 & 0.9994 & $3.31 \mathrm{E}-06$ & & $-3.31 \mathrm{E}-06$ & $3.81 \mathrm{E}-06$ \\
\hline 400 & 3.76 & 4.12 & 2.57 & 0.9997 & $3.22 \mathrm{E}-06$ & & $-3.22 \mathrm{E}-06$ & $3.72 \mathrm{E}-06$ & 2.71 & 0.9998 & $3.39 \mathrm{E}-06$ & & $-3.39 \mathrm{E}-06$ & $3.89 \mathrm{E}-06$ \\
\hline 500 & 4.50 & 4.90 & 3.35 & 1.0001 & 3.36E-06 & $4.56 E+00$ & $4.56 \mathrm{E}+00$ & 3.86E-06 & 3.45 & 1.0001 & 3.46E-06 & $4.56 E+00$ & $4.56 \mathrm{E}+00$ & $3.96 \mathrm{E}-06$ \\
\hline
\end{tabular}




\begin{tabular}{|c|c|c|c|c|c|c|c|c|c|c|c|c|c|c|}
\hline $\begin{array}{l}\text { Capsule } \\
\text { Column }\end{array}$ & $\begin{array}{l}\text { OC2 } \\
\text { North }\end{array}$ & $\begin{array}{l}\text { Specimen } \\
\text { Length }\end{array}$ & $\begin{array}{c}107 \\
0.9992\end{array}$ & $\begin{array}{l}\text { Material } \\
\text { Scale }\end{array}$ & $\begin{array}{l}\text { H-451 } \\
1 "\end{array}$ & $5.00 \mathrm{E}-04$ & & & & & & & & \\
\hline $\begin{array}{c}\text { Temperature } \\
\left({ }^{\circ} \mathrm{C}\right)\end{array}$ & Up & Down & $\begin{array}{l}\text { Delta } \\
\text { Down }\end{array}$ & $\begin{array}{l}\text { Down } \\
\text { length }\end{array}$ & CTE & CTEK & $\begin{array}{l}\text { Delta } \\
\text { CTEK }\end{array}$ & $\begin{array}{c}\text { CTE } \\
\text { adjusted }\end{array}$ & $\begin{array}{l}\text { Delta } \\
\text { Up }\end{array}$ & $\begin{array}{c}\text { Up } \\
\text { length }\end{array}$ & CTE & CTEK & $\begin{array}{l}\text { Delta } \\
\text { CTEK }\end{array}$ & $\begin{array}{c}\text { CTE } \\
\text { adjusted }\end{array}$ \\
\hline 0 & 0.75 & 1.02 & 0 & 0.9992 & & & & & 0 & 0.9992 & & & & \\
\hline 100 & 1.30 & 1.55 & 0.53 & 0.9995 & $2.65 \mathrm{E}-06$ & & $-2.65 E-06$ & 3.15E-06 & 0.55 & 0.9995 & $2.75 \mathrm{E}-06$ & & $-2.75 \mathrm{E}-06$ & $3.25 \mathrm{E}-06$ \\
\hline 200 & 1.82 & 2.10 & 1.08 & 0.9997 & $2.70 \mathrm{E}-06$ & & $-2.70 \mathrm{E}-06$ & $3.20 \mathrm{E}-06$ & 1.07 & 0.9997 & $2.68 \mathrm{E}-06$ & & $-2.68 \mathrm{E}-06$ & $3.18 \mathrm{E}-06$ \\
\hline 300 & 2.50 & 2.75 & 1.73 & 1.0001 & $2.89 \mathrm{E}-06$ & & $-2.89 \mathrm{E}-06$ & 3.39E-06 & 1.75 & 1.0001 & $2.92 \mathrm{E}-06$ & & $-2.92 \mathrm{E}-06$ & $3.42 \mathrm{E}-06$ \\
\hline 400 & 3.20 & 3.49 & 2.47 & 1.0004 & $3.09 \mathrm{E}-06$ & & $-3.09 \mathrm{E}-06$ & 3.59E-06 & 2.45 & 1.0004 & $3.06 \mathrm{E}-06$ & & $-3.06 \mathrm{E}-06$ & 3.56E-06 \\
\hline 500 & 3.92 & 4.20 & 3.18 & 1.0008 & $3.18 \mathrm{E}-06$ & 4.30E-06 & $1.12 \mathrm{E}-06$ & $3.68 \mathrm{E}-06$ & 3.17 & 1.0008 & 3.17E-06 & 4.30E-06 & $1.13 \mathrm{E}-06$ & 3.67E-06 \\
\hline
\end{tabular}

\begin{tabular}{|c|c|c|c|c|c|c|c|c|c|c|c|c|c|c|}
\hline $\begin{array}{l}\text { Capsule } \\
\text { Column }\end{array}$ & $\begin{array}{l}\text { OC2 } \\
\text { North }\end{array}$ & $\begin{array}{l}\text { Specimen } \\
\text { Length }\end{array}$ & $\begin{array}{c}118 \\
0.9974\end{array}$ & $\begin{array}{c}\text { Material } \\
\text { Scale }\end{array}$ & $\begin{array}{c}\text { H-451 } \\
1 "\end{array}$ & $5.00 \mathrm{E}-04$ & & & & & & & & \\
\hline $\begin{array}{c}\text { Temperature } \\
\left({ }^{\circ} \mathrm{C}\right)\end{array}$ & Up & Down & $\begin{array}{l}\text { Delta } \\
\text { Down }\end{array}$ & $\begin{array}{l}\text { Down } \\
\text { length }\end{array}$ & CTE & CTEK & $\begin{array}{l}\text { Delta } \\
\text { CTEK }\end{array}$ & $\begin{array}{c}\text { CTE } \\
\text { adjusted }\end{array}$ & $\begin{array}{c}\text { Delta } \\
\text { Up }\end{array}$ & $\begin{array}{c}\text { Up } \\
\text { length }\end{array}$ & CTE & CTEK & $\begin{array}{l}\text { Delta } \\
\text { CTEK }\end{array}$ & $\begin{array}{c}\text { CTE } \\
\text { adjusted }\end{array}$ \\
\hline 0 & 0.32 & 0.60 & 0 & 0.9974 & & & & & 0 & 0.9974 & & & & \\
\hline 100 & 0.88 & 1.18 & 0.58 & 0.9977 & $2.91 \mathrm{E}-06$ & & $-2.91 \mathrm{E}-06$ & $3.41 \mathrm{E}-06$ & 0.56 & 0.9977 & $2.81 \mathrm{E}-06$ & & $-2.81 \mathrm{E}-06$ & $3.31 \mathrm{E}-06$ \\
\hline 200 & 1.48 & 1.65 & 1.05 & 0.9979 & 2.63E-06 & & $-2.63 \mathrm{E}-06$ & 3.13E-06 & 1.16 & 0.9980 & $2.91 \mathrm{E}-06$ & & $-2.91 \mathrm{E}-06$ & $3.41 \mathrm{E}-06$ \\
\hline 300 & 2.18 & 2.38 & 1.78 & 0.9983 & 2.97E-06 & & $-2.97 \mathrm{E}-06$ & 3.47E-06 & 1.86 & 0.9983 & $3.11 \mathrm{E}-06$ & & $-3.11 \mathrm{E}-06$ & $3.61 \mathrm{E}-06$ \\
\hline 400 & 2.90 & 3.15 & 2.55 & 0.9987 & $3.20 \mathrm{E}-06$ & & $-3.20 \mathrm{E}-06$ & 3.70E-06 & 2.58 & 0.9987 & $3.23 \mathrm{E}-06$ & & $-3.23 E-06$ & $3.73 E-06$ \\
\hline 500 & 3.70 & 4.00 & 3.4 & 0.9991 & $3.41 \mathrm{E}-06$ & 4.87E-06 & $1.46 \mathrm{E}-06$ & $3.91 \mathrm{E}-06$ & 3.38 & 0.9991 & 3.39E-06 & 4.87E-06 & $1.48 \mathrm{E}-06$ & $3.89 \mathrm{E}-06$ \\
\hline
\end{tabular}

\begin{tabular}{|c|c|c|c|c|c|c|c|c|c|c|c|c|c|c|}
\hline $\begin{array}{l}\text { Capsule } \\
\text { Column }\end{array}$ & $\begin{array}{l}\text { OC2 } \\
\text { North }\end{array}$ & $\begin{array}{l}\text { Specimen } \\
\text { Length }\end{array}$ & $\begin{array}{c}1200 \\
0.9976\end{array}$ & $\begin{array}{l}\text { Material } \\
\text { Scale }\end{array}$ & $\begin{array}{l}\text { H-451 } \\
1 "\end{array}$ & $5.00 \mathrm{E}-04$ & & & & & & & & \\
\hline $\begin{array}{c}\text { Temperature } \\
\left({ }^{\circ} \mathrm{C}\right)\end{array}$ & Up & Down & $\begin{array}{l}\text { Delta } \\
\text { Down }\end{array}$ & $\begin{array}{l}\text { Down } \\
\text { length }\end{array}$ & CTE & CTEK & $\begin{array}{l}\text { Delta } \\
\text { CTEK }\end{array}$ & $\begin{array}{c}\text { CTE } \\
\text { adjusted }\end{array}$ & $\begin{array}{l}\text { Delta } \\
\text { Up }\end{array}$ & $\begin{array}{c}\text { Up } \\
\text { length }\end{array}$ & CTE & CTEK & $\begin{array}{l}\text { Delta } \\
\text { CTEK }\end{array}$ & $\begin{array}{c}\text { CTE } \\
\text { adjusted }\end{array}$ \\
\hline 0 & 0.80 & 1.10 & 0 & 0.9976 & & & & & 0 & 0.9976 & & & & \\
\hline 100 & 1.57 & 1.80 & 0.7 & 0.9980 & $3.51 \mathrm{E}-06$ & & $-3.51 \mathrm{E}-06$ & 4.01E-06 & 0.77 & 0.9980 & $3.86 \mathrm{E}-06$ & & $-3.86 \mathrm{E}-06$ & 4.36E-06 \\
\hline 200 & 2.32 & 2.50 & 1.4 & 0.9983 & $3.51 \mathrm{E}-06$ & & $-3.51 \mathrm{E}-06$ & 4.01E-06 & 1.52 & 0.9984 & $3.81 \mathrm{E}-06$ & & $-3.81 \mathrm{E}-06$ & $4.31 \mathrm{E}-06$ \\
\hline 300 & 3.22 & 3.40 & 2.3 & 0.9988 & $3.84 \mathrm{E}-06$ & & $-3.84 \mathrm{E}-06$ & 4.34E-06 & 2.42 & 0.9988 & 4.04E-06 & & $-4.04 \mathrm{E}-06$ & 4.54E-06 \\
\hline 400 & 4.17 & 4.36 & 3.26 & 0.9992 & $4.08 \mathrm{E}-06$ & & $-4.08 \mathrm{E}-06$ & $4.58 \mathrm{E}-06$ & 3.37 & 0.9993 & $4.22 \mathrm{E}-06$ & & $-4.22 \mathrm{E}-06$ & $4.72 \mathrm{E}-06$ \\
\hline 500 & 5.20 & 5.39 & 4.29 & 0.9997 & 4.30E-06 & 4.87E-06 & $5.70 \mathrm{E}-07$ & 4.80E-06 & 4.4 & 0.9998 & $4.41 \mathrm{E}-06$ & 4.87E-06 & 4.59E-07 & 4.91E-06 \\
\hline
\end{tabular}




\begin{tabular}{|c|c|c|c|c|c|c|c|c|c|c|c|c|c|c|}
\hline $\begin{array}{l}\text { Capsule } \\
\text { Column }\end{array}$ & $\begin{array}{c}\text { OC2 } \\
\text { North }\end{array}$ & $\begin{array}{c}\text { Specimen } \\
\text { Length }\end{array}$ & $\begin{array}{c}122 \\
0.9978\end{array}$ & $\begin{array}{c}\text { Material } \\
\text { Scale }\end{array}$ & $\begin{array}{c}\text { H-451 } \\
1 "\end{array}$ & $5.00 \mathrm{E}-04$ & & & & & & & & \\
\hline $\begin{array}{c}\text { Temperature } \\
\left({ }^{\circ} \mathrm{C}\right) \\
\end{array}$ & Up & Down & $\begin{array}{l}\text { Delta } \\
\text { Down }\end{array}$ & $\begin{array}{l}\text { Down } \\
\text { length }\end{array}$ & CTE & CTEK & $\begin{array}{l}\text { Delta } \\
\text { CTEK }\end{array}$ & $\begin{array}{c}\text { CTE } \\
\text { adjusted }\end{array}$ & $\begin{array}{l}\text { Delta } \\
\text { Up }\end{array}$ & $\begin{array}{c}\text { Up } \\
\text { length }\end{array}$ & CTE & CTEK & $\begin{array}{l}\text { Delta } \\
\text { CTEK }\end{array}$ & $\begin{array}{c}\text { CTE } \\
\text { adjusted }\end{array}$ \\
\hline 0 & 0.60 & 1.10 & 0 & 0.9978 & & & & & 0 & 0.9978 & & & & \\
\hline 100 & 1.17 & 1.52 & 0.42 & 0.9980 & $2.10 \mathrm{E}-06$ & & $-2.10 \mathrm{E}-06$ & $2.60 \mathrm{E}-06$ & 0.57 & 0.9981 & $2.86 \mathrm{E}-06$ & & $-2.86 \mathrm{E}-06$ & $3.36 \mathrm{E}-06$ \\
\hline 200 & 1.70 & 2.10 & 1 & 0.9983 & $2.51 \mathrm{E}-06$ & & $-2.51 \mathrm{E}-06$ & $3.01 \mathrm{E}-06$ & 1.1 & 0.9984 & $2.76 \mathrm{E}-06$ & & $-2.76 \mathrm{E}-06$ & $3.26 \mathrm{E}-06$ \\
\hline 300 & 2.40 & 2.79 & 1.69 & 0.9986 & $2.82 \mathrm{E}-06$ & & $-2.82 \mathrm{E}-06$ & $3.32 \mathrm{E}-06$ & 1.8 & 0.9987 & $3.01 \mathrm{E}-06$ & & $-3.01 \mathrm{E}-06$ & $3.51 \mathrm{E}-06$ \\
\hline 400 & 3.20 & 3.59 & 2.49 & 0.9990 & $3.12 \mathrm{E}-06$ & & $-3.12 \mathrm{E}-06$ & $3.62 \mathrm{E}-06$ & 2.6 & 0.9991 & $3.26 \mathrm{E}-06$ & & $-3.26 \mathrm{E}-06$ & $3.76 \mathrm{E}-06$ \\
\hline 500 & 4.02 & 4.35 & 3.25 & 0.9994 & $3.26 \mathrm{E}-06$ & $3.58 E-06$ & $3.23 \mathrm{E}-07$ & $3.76 \mathrm{E}-06$ & 3.42 & 0.9995 & $3.43 \mathrm{E}-06$ & $3.58 E-06$ & $1.52 \mathrm{E}-07$ & $3.93 \mathrm{E}-06$ \\
\hline
\end{tabular}

\begin{tabular}{|c|c|c|c|c|c|c|c|c|c|c|c|c|c|c|}
\hline $\begin{array}{l}\text { Capsule } \\
\text { Column }\end{array}$ & $\begin{array}{l}\text { OC2 } \\
\text { North }\end{array}$ & $\begin{array}{l}\text { Specimen } \\
\text { Length }\end{array}$ & $\begin{array}{c}65 \\
0.9989\end{array}$ & $\begin{array}{l}\text { Material } \\
\text { Scale }\end{array}$ & $\begin{array}{c}\text { H-327 } \\
1 "\end{array}$ & $5.00 \mathrm{E}-04$ & & & & & & & & \\
\hline $\begin{array}{c}\text { Temperature } \\
\left({ }^{\circ} \mathrm{C}\right)\end{array}$ & Up & Down & $\begin{array}{l}\text { Delta } \\
\text { Down }\end{array}$ & $\begin{array}{l}\text { Down } \\
\text { length }\end{array}$ & CTE & CTEK & $\begin{array}{l}\text { Delta } \\
\text { CTEK }\end{array}$ & $\begin{array}{c}\text { CTE } \\
\text { adjusted }\end{array}$ & $\begin{array}{l}\text { Delta } \\
\text { Up }\end{array}$ & $\begin{array}{c}\text { Up } \\
\text { length }\end{array}$ & CTE & CTEK & $\begin{array}{l}\text { Delta } \\
\text { CTEK }\end{array}$ & $\begin{array}{c}\text { CTE } \\
\text { adjusted }\end{array}$ \\
\hline 0 & 1.05 & 1.15 & 0 & 0.9989 & & & & & 0 & 0.9989 & & & & \\
\hline 100 & 1.10 & 1.21 & 0.06 & 0.9989 & $3.00 \mathrm{E}-07$ & & $-3.00 E-07$ & $8.00 \mathrm{E}-07$ & 0.05 & 0.9989 & $2.50 \mathrm{E}-07$ & & $-2.50 \mathrm{E}-07$ & 7.50E-07 \\
\hline 200 & 1.22 & 1.31 & 0.16 & 0.9990 & $4.00 \mathrm{E}-07$ & & $-4.00 E-07$ & $9.00 \mathrm{E}-07$ & 0.17 & 0.9990 & $4.25 \mathrm{E}-07$ & & $-4.25 E-07$ & $9.25 \mathrm{E}-07$ \\
\hline 300 & 1.42 & 1.58 & 0.43 & 0.9991 & 7.17E-07 & & $-7.17 \mathrm{E}-07$ & $1.22 \mathrm{E}-06$ & 0.37 & 0.9991 & $6.17 E-07$ & & $-6.17 \mathrm{E}-07$ & $1.12 \mathrm{E}-06$ \\
\hline 400 & 1.70 & 1.92 & 0.77 & 0.9993 & $9.64 \mathrm{E}-07$ & & $-9.64 \mathrm{E}-07$ & 1.46E-06 & 0.65 & 0.9992 & $8.13 \mathrm{E}-07$ & & $-8.13 \mathrm{E}-07$ & $1.31 \mathrm{E}-06$ \\
\hline 500 & 2.02 & 2.18 & 1.03 & 0.9994 & $1.03 \mathrm{E}-06$ & $2.00 E-06$ & $9.69 \mathrm{E}-07$ & $1.53 \mathrm{E}-06$ & 0.97 & 0.9994 & $9.71 \mathrm{E}-07$ & $2.00 E-06$ & $1.03 \mathrm{E}-06$ & 1.47E-06 \\
\hline
\end{tabular}

\begin{tabular}{|c|c|c|c|c|c|c|c|c|c|c|c|c|c|c|}
\hline $\begin{array}{l}\text { Capsule } \\
\text { Column }\end{array}$ & $\begin{array}{c}\text { OC2 } \\
\text { North }\end{array}$ & $\begin{array}{c}\text { Specimen } \\
\text { Length }\end{array}$ & $\begin{array}{c}72 \\
0.9994 \\
\end{array}$ & $\begin{array}{c}\text { Material } \\
\text { Scale }\end{array}$ & $\begin{array}{c}\text { H-327 } \\
1 " \\
\end{array}$ & $5.00 \mathrm{E}-04$ & & & & & & & & \\
\hline $\begin{array}{c}\text { Temperature } \\
\left({ }^{\circ} \mathrm{C}\right)\end{array}$ & Up & Down & $\begin{array}{l}\text { Delta } \\
\text { Down }\end{array}$ & $\begin{array}{l}\text { Down } \\
\text { length }\end{array}$ & CTE & CTEK & $\begin{array}{l}\text { Delta } \\
\text { CTEK }\end{array}$ & $\begin{array}{c}\text { CTE } \\
\text { adjusted }\end{array}$ & $\begin{array}{l}\text { Delta } \\
\text { Up }\end{array}$ & $\begin{array}{c}\text { Up } \\
\text { length }\end{array}$ & CTE & CTEK & $\begin{array}{l}\text { Delta } \\
\text { CTEK }\end{array}$ & $\begin{array}{c}\text { CTE } \\
\text { adjusted }\end{array}$ \\
\hline 0 & 0.93 & 1.30 & 0 & 0.9994 & & & & & 0 & 0.9994 & & & & \\
\hline 100 & 1.43 & 1.72 & 0.42 & 0.9996 & $2.10 \mathrm{E}-06$ & & $-2.10 \mathrm{E}-06$ & $2.60 \mathrm{E}-06$ & 0.5 & 0.9997 & $2.50 \mathrm{E}-06$ & & $-2.50 \mathrm{E}-06$ & $3.00 \mathrm{E}-06$ \\
\hline 200 & 1.90 & 2.20 & 0.9 & 0.9999 & $2.25 \mathrm{E}-06$ & & $-2.25 \mathrm{E}-06$ & $2.75 \mathrm{E}-06$ & 0.97 & 0.9999 & $2.43 \mathrm{E}-06$ & & $-2.43 E-06$ & $2.93 \mathrm{E}-06$ \\
\hline 300 & 2.45 & 2.72 & 1.42 & 1.0001 & $2.37 \mathrm{E}-06$ & & $-2.37 \mathrm{E}-06$ & $2.87 \mathrm{E}-06$ & 1.52 & 1.0002 & $2.53 \mathrm{E}-06$ & & $-2.53 \mathrm{E}-06$ & $3.03 E-06$ \\
\hline 400 & 3.10 & 3.38 & 2.08 & 1.0004 & $2.60 \mathrm{E}-06$ & & $-2.60 \mathrm{E}-06$ & 3.10E-06 & 2.17 & 1.0005 & $2.71 \mathrm{E}-06$ & & $-2.71 \mathrm{E}-06$ & $3.21 \mathrm{E}-06$ \\
\hline 500 & 3.25 & 4.00 & 2.7 & 1.0008 & $2.70 \mathrm{E}-06$ & $1.85 E-06$ & $-8.52 \mathrm{E}-07$ & $3.20 \mathrm{E}-06$ & 2.32 & 1.0006 & $2.32 \mathrm{E}-06$ & $1.85 E-06$ & $-4.71 \mathrm{E}-07$ & $2.82 \mathrm{E}-06$ \\
\hline
\end{tabular}




\begin{tabular}{|c|c|c|c|c|c|c|c|c|c|c|c|c|c|c|}
\hline $\begin{array}{l}\text { Capsule } \\
\text { Column }\end{array}$ & $\begin{array}{l}\text { OC2 } \\
\text { North }\end{array}$ & $\begin{array}{c}\text { Specimen } \\
\text { Length }\end{array}$ & $\begin{array}{c}12 \\
0.9823 \\
\end{array}$ & $\begin{array}{c}\text { Material } \\
\text { Scale }\end{array}$ & $\begin{array}{c}\text { H-327 } \\
1 "\end{array}$ & $5.00 \mathrm{E}-04$ & & & & & & & & \\
\hline $\begin{array}{c}\text { Temperature } \\
\left({ }^{\circ} \mathrm{C}\right)\end{array}$ & Up & Down & $\begin{array}{l}\text { Delta } \\
\text { Down }\end{array}$ & $\begin{array}{l}\text { Down } \\
\text { length }\end{array}$ & CTE & CTEK & $\begin{array}{l}\text { Delta } \\
\text { CTEK }\end{array}$ & $\begin{array}{c}\text { CTE } \\
\text { adjusted }\end{array}$ & $\begin{array}{l}\text { Delta } \\
\text { Up }\end{array}$ & $\begin{array}{c}\text { Up } \\
\text { length }\end{array}$ & CTE & CTEK & $\begin{array}{l}\text { Delta } \\
\text { CTEK }\end{array}$ & $\begin{array}{c}\text { CTE } \\
\text { adjusted }\end{array}$ \\
\hline 0 & 0.75 & 1.18 & 0 & 0.9823 & & & & & 0 & 0.9823 & & & & \\
\hline 100 & 1.72 & 2.15 & 0.97 & 0.9828 & 4.94E-06 & & $-4.94 \mathrm{E}-06$ & $5.44 \mathrm{E}-06$ & 0.97 & 0.9828 & $4.94 \mathrm{E}-06$ & & $-4.94 \mathrm{E}-06$ & $5.44 \mathrm{E}-06$ \\
\hline 200 & 2.82 & 3.20 & 2.02 & 0.9833 & $5.14 \mathrm{E}-06$ & & $-5.14 \mathrm{E}-06$ & $5.64 \mathrm{E}-06$ & 2.07 & 0.9833 & $5.27 \mathrm{E}-06$ & & $-5.27 \mathrm{E}-06$ & 5.77E-06 \\
\hline 300 & 4.00 & 4.38 & 3.2 & 0.9839 & $5.43 \mathrm{E}-06$ & & $-5.43 E-06$ & 5.93E-06 & 3.25 & 0.9839 & 5.51E-06 & & $-5.51 E-06$ & $6.01 \mathrm{E}-06$ \\
\hline 400 & 5.22 & 5.58 & 4.4 & 0.9845 & $5.60 \mathrm{E}-06$ & & $-5.60 \mathrm{E}-06$ & $6.10 \mathrm{E}-06$ & 4.47 & 0.9845 & 5.69E-06 & & $-5.69 \mathrm{E}-06$ & 6.19E-06 \\
\hline 500 & 6.49 & 6.70 & 5.52 & 0.9851 & $5.62 \mathrm{E}-06$ & $4.28 E-06$ & $-1.34 \mathrm{E}-06$ & $6.12 \mathrm{E}-06$ & 5.74 & 0.9852 & $5.84 \mathrm{E}-06$ & $4.28 E-06$ & $-1.56 \mathrm{E}-06$ & $6.34 \mathrm{E}-06$ \\
\hline
\end{tabular}

\begin{tabular}{|c|c|c|c|c|c|c|c|c|c|c|c|c|c|c|}
\hline $\begin{array}{l}\text { Capsule } \\
\text { Column }\end{array}$ & $\begin{array}{l}\text { OC2 } \\
\text { North }\end{array}$ & $\begin{array}{l}\text { Specimen } \\
\text { Length }\end{array}$ & $\begin{array}{c}13 \\
0.9843\end{array}$ & $\begin{array}{c}\text { Material } \\
\text { Scale }\end{array}$ & $\begin{array}{c}\mathrm{H}-327 \\
1 "\end{array}$ & $5.00 \mathrm{E}-04$ & & & & & & & & \\
\hline $\begin{array}{c}\begin{array}{c}\text { Temperature } \\
\left({ }^{\circ} \mathrm{C}\right)\end{array} \\
\end{array}$ & Up & Down & $\begin{array}{l}\text { Delta } \\
\text { Down }\end{array}$ & $\begin{array}{l}\text { Down } \\
\text { length }\end{array}$ & CTE & CTEK & $\begin{array}{l}\text { Delta } \\
\text { CTEK }\end{array}$ & $\begin{array}{c}\text { CTE } \\
\text { adjusted }\end{array}$ & $\begin{array}{l}\text { Delta } \\
\text { Up }\end{array}$ & $\begin{array}{c}\text { Up } \\
\text { length }\end{array}$ & CTE & CTEK & $\begin{array}{l}\text { Delta } \\
\text { CTEK }\end{array}$ & $\begin{array}{c}\text { CTE } \\
\text { adjusted }\end{array}$ \\
\hline 0 & 0.13 & 0.58 & 0 & 0.9843 & & & & & 0 & 0.9843 & & & & \\
\hline 100 & 1.10 & 1.60 & 1.02 & 0.9848 & $5.18 \mathrm{E}-06$ & & $-5.18 \mathrm{E}-06$ & $5.68 \mathrm{E}-06$ & 0.97 & 0.9848 & 4.93E-06 & & $-4.93 \mathrm{E}-06$ & 5.43E-06 \\
\hline 200 & 2.18 & 2.58 & 2 & 0.9853 & $5.08 \mathrm{E}-06$ & & $-5.08 \mathrm{E}-06$ & $5.58 \mathrm{E}-06$ & 2.05 & 0.9853 & $5.21 \mathrm{E}-06$ & & $-5.21 \mathrm{E}-06$ & 5.71E-06 \\
\hline 300 & 3.35 & 3.70 & 3.12 & 0.9859 & $5.28 \mathrm{E}-06$ & & $-5.28 \mathrm{E}-06$ & $5.78 \mathrm{E}-06$ & 3.22 & 0.9859 & $5.45 \mathrm{E}-06$ & & $-5.45 E-06$ & 5.95E-06 \\
\hline 400 & 4.55 & 4.88 & 4.3 & 0.9865 & $5.46 \mathrm{E}-06$ & & $-5.46 \mathrm{E}-06$ & $5.96 \mathrm{E}-06$ & 4.42 & 0.9865 & $5.61 \mathrm{E}-06$ & & $-5.61 \mathrm{E}-06$ & $6.11 \mathrm{E}-06$ \\
\hline 500 & 5.78 & 6.00 & 5.42 & 0.9870 & $5.51 \mathrm{E}-06$ & $6.92 E-06$ & $1.41 \mathrm{E}-06$ & $6.01 \mathrm{E}-06$ & 5.65 & 0.9871 & 5.74E-06 & $6.92 E-06$ & $1.18 \mathrm{E}-06$ & $6.24 \mathrm{E}-06$ \\
\hline
\end{tabular}

\begin{tabular}{|c|c|c|c|c|c|c|c|c|c|c|c|c|c|c|}
\hline $\begin{array}{l}\text { Capsule } \\
\text { Column }\end{array}$ & $\begin{array}{l}\text { OC2 } \\
\text { North }\end{array}$ & $\begin{array}{l}\text { Specimen } \\
\text { Length }\end{array}$ & $\begin{array}{c}17 \\
0.9994\end{array}$ & $\begin{array}{c}\text { Material } \\
\text { Scale }\end{array}$ & $\begin{array}{c}\text { POCO } \\
1 "\end{array}$ & 5.00E-04 & & & & & & & & \\
\hline $\begin{array}{c}\text { Temperature } \\
\left({ }^{\circ} \mathrm{C}\right)\end{array}$ & Up & Down & $\begin{array}{l}\text { Delta } \\
\text { Down }\end{array}$ & $\begin{array}{l}\text { Down } \\
\text { length }\end{array}$ & CTE & CTEK & $\begin{array}{l}\text { Delta } \\
\text { CTEK }\end{array}$ & $\begin{array}{c}\text { CTE } \\
\text { adjusted }\end{array}$ & $\begin{array}{l}\text { Delta } \\
\text { Up }\end{array}$ & $\begin{array}{c}\text { Up } \\
\text { length }\end{array}$ & CTE & CTEK & $\begin{array}{l}\text { Delta } \\
\text { CTEK }\end{array}$ & $\begin{array}{c}\text { CTE } \\
\text { adjusted }\end{array}$ \\
\hline 0 & 0.18 & 0.50 & 0 & 0.9836 & & & & & 0 & 0.9836 & & & & \\
\hline 100 & 1.10 & 1.40 & 0.9 & 0.9841 & $4.58 \mathrm{E}-06$ & & $-4.58 \mathrm{E}-06$ & $5.08 \mathrm{E}-06$ & 0.92 & 0.9841 & $4.68 \mathrm{E}-06$ & & $-4.68 \mathrm{E}-06$ & $5.18 \mathrm{E}-06$ \\
\hline 200 & 2.08 & 2.32 & 1.82 & 0.9845 & 4.63E-06 & & $-4.63 \mathrm{E}-06$ & $5.13 \mathrm{E}-06$ & 1.9 & 0.9846 & 4.83E-06 & & $-4.83 \mathrm{E}-06$ & 5.33E-06 \\
\hline 300 & 3.15 & 3.42 & 2.92 & 0.9851 & 4.95E-06 & & $-4.95 E-06$ & $5.45 \mathrm{E}-06$ & 2.97 & 0.9851 & 5.03E-06 & & $-5.03 E-06$ & $5.53 \mathrm{E}-06$ \\
\hline 400 & 4.25 & 4.50 & 4 & 0.9856 & $5.08 \mathrm{E}-06$ & & $-5.08 \mathrm{E}-06$ & $5.58 \mathrm{E}-06$ & 4.07 & 0.9856 & 5.17E-06 & & $-5.17 \mathrm{E}-06$ & 5.67E-06 \\
\hline 500 & 5.42 & 5.60 & 5.1 & 0.9862 & $5.19 \mathrm{E}-06$ & $6.92 E-06$ & $1.73 \mathrm{E}-06$ & $5.69 \mathrm{E}-06$ & 5.24 & 0.9862 & $5.33 \mathrm{E}-06$ & $6.92 E-06$ & $1.59 \mathrm{E}-06$ & $5.83 \mathrm{E}-06$ \\
\hline
\end{tabular}




\begin{tabular}{|c|c|c|c|c|c|c|c|c|c|c|c|c|c|c|}
\hline $\begin{array}{l}\text { Capsule } \\
\text { Column }\end{array}$ & $\begin{array}{l}\text { OC2 } \\
\text { South }\end{array}$ & $\begin{array}{c}\text { Specimen } \\
\text { Length }\end{array}$ & $\begin{array}{c}67 \\
0.1000\end{array}$ & $\begin{array}{c}\text { Material } \\
\text { Scale }\end{array}$ & $\begin{array}{c}\text { H-451 } \\
1 "\end{array}$ & $5.00 \mathrm{E}-04$ & & & & & & & & \\
\hline $\begin{array}{c}\text { Temperature } \\
\left({ }^{\circ} \mathrm{C}\right) \\
\end{array}$ & Up & Down & $\begin{array}{l}\text { Delta } \\
\text { Down }\end{array}$ & $\begin{array}{l}\text { Down } \\
\text { length }\end{array}$ & CTE & CTEK & $\begin{array}{l}\text { Delta } \\
\text { CTEK }\end{array}$ & $\begin{array}{c}\text { CTE } \\
\text { adjusted }\end{array}$ & $\begin{array}{l}\text { Delta } \\
\text { Up }\end{array}$ & $\begin{array}{c}\text { Up } \\
\text { length }\end{array}$ & CTE & CTEK & $\begin{array}{l}\text { Delta } \\
\text { CTEK }\end{array}$ & $\begin{array}{c}\text { CTE } \\
\text { adjusted }\end{array}$ \\
\hline 0 & 0.10 & 0.35 & 0 & 0.1000 & & & & & 0 & 0.1000 & & & & \\
\hline 100 & 1.01 & 1.30 & 0.95 & 0.1005 & 4.75E-05 & & $-4.75 \mathrm{E}-05$ & $4.80 \mathrm{E}-05$ & 0.91 & 0.1005 & 4.55E-05 & & $-4.55 \mathrm{E}-05$ & 4.60E-05 \\
\hline 200 & 1.98 & 2.22 & 1.87 & 0.1009 & 4.67E-05 & & $-4.67 E-05$ & $4.72 \mathrm{E}-05$ & 1.88 & 0.1009 & $4.70 \mathrm{E}-05$ & & $-4.70 \mathrm{E}-05$ & 4.75E-05 \\
\hline 300 & 3.05 & 3.20 & 2.85 & 0.1014 & $4.75 \mathrm{E}-05$ & & $-4.75 E-05$ & $4.80 \mathrm{E}-05$ & 2.95 & 0.1015 & $4.92 \mathrm{E}-05$ & & $-4.92 \mathrm{E}-05$ & 4.97E-05 \\
\hline 400 & 4.02 & 4.22 & 3.87 & 0.1019 & 4.84E-05 & & $-4.84 \mathrm{E}-05$ & 4.89E-05 & 3.92 & 0.1020 & 4.90E-05 & & $-4.90 \mathrm{E}-05$ & 4.95E-05 \\
\hline 500 & 5.12 & 5.30 & 4.95 & 0.1025 & $4.95 \mathrm{E}-05$ & 4.87E-06 & $-4.46 \mathrm{E}-05$ & $5.00 \mathrm{E}-05$ & 5.02 & 0.1025 & $5.02 \mathrm{E}-05$ & 4.87E-06 & $-4.53 \mathrm{E}-05$ & 5.07E-05 \\
\hline
\end{tabular}

\begin{tabular}{|c|c|c|c|c|c|c|c|c|c|c|c|c|c|c|}
\hline $\begin{array}{l}\text { Capsule } \\
\text { Column }\end{array}$ & $\begin{array}{l}\text { OC2 } \\
\text { South }\end{array}$ & $\begin{array}{l}\text { Specimen } \\
\text { Length }\end{array}$ & $\begin{array}{c}73 \\
0.1000\end{array}$ & $\begin{array}{l}\text { Material } \\
\text { Scale }\end{array}$ & $\begin{array}{c}\text { H-451 } \\
1 "\end{array}$ & $5.00 \mathrm{E}-04$ & & & & & & & & \\
\hline $\begin{array}{c}\text { Temperature } \\
\left({ }^{\circ} \mathrm{C}\right)\end{array}$ & Up & Down & $\begin{array}{l}\text { Delta } \\
\text { Down }\end{array}$ & $\begin{array}{l}\text { Down } \\
\text { length }\end{array}$ & CTE & CTEK & $\begin{array}{l}\text { Delta } \\
\text { CTEK }\end{array}$ & $\begin{array}{c}\text { CTE } \\
\text { adjusted }\end{array}$ & $\begin{array}{l}\text { Delta } \\
\text { Up }\end{array}$ & $\begin{array}{c}\text { Up } \\
\text { length }\end{array}$ & CTE & CTEK & $\begin{array}{l}\text { Delta } \\
\text { CTEK }\end{array}$ & $\begin{array}{c}\text { CTE } \\
\text { adjusted }\end{array}$ \\
\hline 0 & 0.49 & 0.97 & 0 & 0.1000 & & & & & 0 & 0.1000 & & & & \\
\hline 100 & 0.75 & 1.22 & 0.25 & 0.1001 & $1.25 \mathrm{E}-05$ & & $-1.25 \mathrm{E}-05$ & $1.30 \mathrm{E}-05$ & 0.26 & 0.1001 & $1.30 \mathrm{E}-05$ & & $-1.30 \mathrm{E}-05$ & $1.35 \mathrm{E}-05$ \\
\hline 200 & 1.05 & 1.70 & 0.73 & 0.1004 & $1.82 \mathrm{E}-05$ & & $-1.82 \mathrm{E}-05$ & $1.87 \mathrm{E}-05$ & 0.56 & 0.1003 & $1.40 \mathrm{E}-05$ & & $-1.40 \mathrm{E}-05$ & $1.45 \mathrm{E}-05$ \\
\hline 300 & 1.58 & 2.02 & 1.05 & 0.1005 & $1.75 \mathrm{E}-05$ & & $-1.75 E-05$ & $1.80 \mathrm{E}-05$ & 1.09 & 0.1005 & $1.82 \mathrm{E}-05$ & & $-1.82 \mathrm{E}-05$ & $1.87 \mathrm{E}-05$ \\
\hline 400 & 2.12 & 2.60 & 1.63 & 0.1008 & $2.04 \mathrm{E}-05$ & & $-2.04 \mathrm{E}-05$ & $2.09 E-05$ & 1.63 & 0.1008 & $2.04 \mathrm{E}-05$ & & $-2.04 \mathrm{E}-05$ & 2.09E-05 \\
\hline 500 & 2.78 & 3.20 & 2.23 & 0.1011 & $2.23 \mathrm{E}-05$ & 4.87E-06 & $-1.74 \mathrm{E}-05$ & $2.28 \mathrm{E}-05$ & 2.29 & 0.1011 & $2.29 \mathrm{E}-05$ & $4.87 E-06$ & $-1.80 \mathrm{E}-05$ & $2.34 \mathrm{E}-05$ \\
\hline
\end{tabular}

\begin{tabular}{|c|c|c|c|c|c|c|c|c|c|c|c|c|c|c|}
\hline $\begin{array}{l}\text { Capsule } \\
\text { Column }\end{array}$ & $\begin{array}{c}\text { OC2 } \\
\text { South }\end{array}$ & $\begin{array}{c}\text { Specimen } \\
\text { Length }\end{array}$ & $\begin{array}{c}79 \\
0.1000 \\
\end{array}$ & $\begin{array}{c}\text { Material } \\
\text { Scale }\end{array}$ & $\begin{array}{c}\text { H-451 } \\
1 " \\
\end{array}$ & $5.00 \mathrm{E}-04$ & & & & & & & & \\
\hline $\begin{array}{c}\text { Temperature } \\
\left({ }^{\circ} \mathrm{C}\right)\end{array}$ & $U p$ & Down & $\begin{array}{l}\text { Delta } \\
\text { Down }\end{array}$ & $\begin{array}{l}\text { Down } \\
\text { length }\end{array}$ & CTE & CTEK & $\begin{array}{l}\text { Delta } \\
\text { CTEK }\end{array}$ & $\begin{array}{c}\text { CTE } \\
\text { adjusted }\end{array}$ & $\begin{array}{l}\text { Delta } \\
\text { Up }\end{array}$ & $\begin{array}{c}\text { Up } \\
\text { length }\end{array}$ & CTE & CTEK & $\begin{array}{l}\text { Delta } \\
\text { CTEK }\end{array}$ & $\begin{array}{c}\text { CTE } \\
\text { adjusted }\end{array}$ \\
\hline 0 & 1.00 & 1.55 & 0 & 0.1000 & & & & & 0 & 0.1000 & & & & \\
\hline 100 & 1.50 & 2.00 & 0.45 & 0.1002 & $2.25 \mathrm{E}-05$ & & $-2.25 E-05$ & $2.30 \mathrm{E}-05$ & 0.5 & 0.1003 & $2.50 \mathrm{E}-05$ & & $-2.50 \mathrm{E}-05$ & $2.55 \mathrm{E}-05$ \\
\hline 200 & 2.02 & 2.52 & 0.97 & 0.1005 & $2.43 \mathrm{E}-05$ & & $-2.43 E-05$ & $2.48 \mathrm{E}-05$ & 1.02 & 0.1005 & $2.55 \mathrm{E}-05$ & & $-2.55 \mathrm{E}-05$ & $2.60 \mathrm{E}-05$ \\
\hline 300 & 2.68 & 3.20 & 1.65 & 0.1008 & $2.75 \mathrm{E}-05$ & & $-2.75 E-05$ & $2.80 \mathrm{E}-05$ & 1.68 & 0.1008 & $2.80 \mathrm{E}-05$ & & $-2.80 \mathrm{E}-05$ & $2.85 \mathrm{E}-05$ \\
\hline 400 & 3.40 & 3.95 & 2.4 & 0.1012 & $3.00 \mathrm{E}-05$ & & $-3.00 \mathrm{E}-05$ & $3.05 \mathrm{E}-05$ & 2.4 & 0.1012 & $3.00 \mathrm{E}-05$ & & $-3.00 \mathrm{E}-05$ & 3.05E-05 \\
\hline 500 & 4.20 & 4.65 & 3.1 & 0.1016 & $3.10 \mathrm{E}-05$ & 4.87E-06 & $-2.61 \mathrm{E}-05$ & $3.15 \mathrm{E}-05$ & 3.2 & 0.1016 & $3.20 \mathrm{E}-05$ & 4.87E-06 & $-2.71 \mathrm{E}-05$ & $3.25 \mathrm{E}-05$ \\
\hline
\end{tabular}




\begin{tabular}{|c|c|c|c|c|c|c|c|c|c|c|c|c|c|c|}
\hline $\begin{array}{l}\text { Capsule } \\
\text { Column }\end{array}$ & $\begin{array}{l}\text { OC2 } \\
\text { South }\end{array}$ & $\begin{array}{c}\text { Specimen } \\
\text { Length }\end{array}$ & $\begin{array}{c}84 \\
0.1000\end{array}$ & $\begin{array}{c}\text { Material } \\
\text { Scale }\end{array}$ & $\begin{array}{c}\text { H-451 } \\
1 "\end{array}$ & $5.00 \mathrm{E}-04$ & & & & & & & & \\
\hline $\begin{array}{c}\text { Temperature } \\
\left({ }^{\circ} \mathrm{C}\right) \\
\end{array}$ & Up & Down & $\begin{array}{l}\text { Delta } \\
\text { Down }\end{array}$ & $\begin{array}{l}\text { Down } \\
\text { length }\end{array}$ & CTE & CTEK & $\begin{array}{l}\text { Delta } \\
\text { CTEK }\end{array}$ & $\begin{array}{c}\text { CTE } \\
\text { adjusted }\end{array}$ & $\begin{array}{l}\text { Delta } \\
\text { Up }\end{array}$ & $\begin{array}{c}\text { Up } \\
\text { length }\end{array}$ & CTE & CTEK & $\begin{array}{l}\text { Delta } \\
\text { CTEK }\end{array}$ & $\begin{array}{c}\text { CTE } \\
\text { adjusted }\end{array}$ \\
\hline 0 & 0.10 & 0.35 & 0 & 0.1000 & & & & & 0 & 0.1000 & & & & \\
\hline 100 & 1.01 & 1.30 & 0.95 & 0.1005 & 4.75E-05 & & $-4.75 \mathrm{E}-05$ & $4.80 \mathrm{E}-05$ & 0.91 & 0.1005 & 4.55E-05 & & $-4.55 \mathrm{E}-05$ & 4.60E-05 \\
\hline 200 & 1.98 & 2.22 & 1.87 & 0.1009 & 4.67E-05 & & $-4.67 E-05$ & $4.72 \mathrm{E}-05$ & 1.88 & 0.1009 & $4.70 \mathrm{E}-05$ & & $-4.70 \mathrm{E}-05$ & 4.75E-05 \\
\hline 300 & 3.05 & 3.20 & 2.85 & 0.1014 & $4.75 \mathrm{E}-05$ & & $-4.75 E-05$ & $4.80 \mathrm{E}-05$ & 2.95 & 0.1015 & $4.92 \mathrm{E}-05$ & & $-4.92 \mathrm{E}-05$ & 4.97E-05 \\
\hline 400 & 4.02 & 4.22 & 3.87 & 0.1019 & 4.84E-05 & & $-4.84 \mathrm{E}-05$ & 4.89E-05 & 3.92 & 0.1020 & 4.90E-05 & & $-4.90 \mathrm{E}-05$ & 4.95E-05 \\
\hline 500 & 5.12 & 5.30 & 4.95 & 0.1025 & $4.95 \mathrm{E}-05$ & 4.87E-06 & $-4.46 \mathrm{E}-05$ & $5.00 \mathrm{E}-05$ & 5.02 & 0.1025 & $5.02 \mathrm{E}-05$ & 4.87E-06 & $-4.53 \mathrm{E}-05$ & 5.07E-05 \\
\hline
\end{tabular}

\begin{tabular}{|c|c|c|c|c|c|c|c|c|c|c|c|c|c|c|}
\hline $\begin{array}{l}\text { Capsule } \\
\text { Column }\end{array}$ & $\begin{array}{l}\text { OC2 } \\
\text { South }\end{array}$ & $\begin{array}{l}\text { Specimen } \\
\text { Length }\end{array}$ & $\begin{array}{c}73 \\
0.1000\end{array}$ & $\begin{array}{l}\text { Material } \\
\text { Scale }\end{array}$ & $\begin{array}{c}\text { H-451 } \\
1 "\end{array}$ & $5.00 \mathrm{E}-04$ & & & & & & & & \\
\hline $\begin{array}{c}\text { Temperature } \\
\left({ }^{\circ} \mathrm{C}\right)\end{array}$ & Up & Down & $\begin{array}{l}\text { Delta } \\
\text { Down }\end{array}$ & $\begin{array}{l}\text { Down } \\
\text { length }\end{array}$ & CTE & CTEK & $\begin{array}{l}\text { Delta } \\
\text { CTEK }\end{array}$ & $\begin{array}{c}\text { CTE } \\
\text { adjusted }\end{array}$ & $\begin{array}{l}\text { Delta } \\
\text { Up }\end{array}$ & $\begin{array}{c}\text { Up } \\
\text { length }\end{array}$ & CTE & CTEK & $\begin{array}{l}\text { Delta } \\
\text { CTEK }\end{array}$ & $\begin{array}{c}\text { CTE } \\
\text { adjusted }\end{array}$ \\
\hline 0 & 0.49 & 0.97 & 0 & 0.1000 & & & & & 0 & 0.1000 & & & & \\
\hline 100 & 0.75 & 1.22 & 0.25 & 0.1001 & $1.25 \mathrm{E}-05$ & & $-1.25 \mathrm{E}-05$ & $1.30 \mathrm{E}-05$ & 0.26 & 0.1001 & $1.30 \mathrm{E}-05$ & & $-1.30 \mathrm{E}-05$ & $1.35 \mathrm{E}-05$ \\
\hline 200 & 1.05 & 1.70 & 0.73 & 0.1004 & $1.82 \mathrm{E}-05$ & & $-1.82 \mathrm{E}-05$ & $1.87 \mathrm{E}-05$ & 0.56 & 0.1003 & $1.40 \mathrm{E}-05$ & & $-1.40 \mathrm{E}-05$ & $1.45 \mathrm{E}-05$ \\
\hline 300 & 1.58 & 2.02 & 1.05 & 0.1005 & $1.75 \mathrm{E}-05$ & & $-1.75 E-05$ & $1.80 \mathrm{E}-05$ & 1.09 & 0.1005 & $1.82 \mathrm{E}-05$ & & $-1.82 \mathrm{E}-05$ & $1.87 \mathrm{E}-05$ \\
\hline 400 & 2.12 & 2.60 & 1.63 & 0.1008 & $2.04 \mathrm{E}-05$ & & $-2.04 \mathrm{E}-05$ & $2.09 E-05$ & 1.63 & 0.1008 & $2.04 \mathrm{E}-05$ & & $-2.04 \mathrm{E}-05$ & 2.09E-05 \\
\hline 500 & 2.78 & 3.20 & 2.23 & 0.1011 & $2.23 \mathrm{E}-05$ & 4.87E-06 & $-1.74 \mathrm{E}-05$ & $2.28 \mathrm{E}-05$ & 2.29 & 0.1011 & $2.29 \mathrm{E}-05$ & $4.87 E-06$ & $-1.80 \mathrm{E}-05$ & $2.34 \mathrm{E}-05$ \\
\hline
\end{tabular}

\begin{tabular}{|c|c|c|c|c|c|c|c|c|c|c|c|c|c|c|}
\hline $\begin{array}{l}\text { Capsule } \\
\text { Column }\end{array}$ & $\begin{array}{c}\text { OC2 } \\
\text { South }\end{array}$ & $\begin{array}{c}\text { Specimen } \\
\text { Length }\end{array}$ & $\begin{array}{c}79 \\
0.1000 \\
\end{array}$ & $\begin{array}{c}\text { Material } \\
\text { Scale }\end{array}$ & $\begin{array}{c}\text { H-451 } \\
1 " \\
\end{array}$ & $5.00 \mathrm{E}-04$ & & & & & & & & \\
\hline $\begin{array}{c}\text { Temperature } \\
\left({ }^{\circ} \mathrm{C}\right)\end{array}$ & $U p$ & Down & $\begin{array}{l}\text { Delta } \\
\text { Down }\end{array}$ & $\begin{array}{l}\text { Down } \\
\text { length }\end{array}$ & CTE & CTEK & $\begin{array}{l}\text { Delta } \\
\text { CTEK }\end{array}$ & $\begin{array}{c}\text { CTE } \\
\text { adjusted }\end{array}$ & $\begin{array}{l}\text { Delta } \\
\text { Up }\end{array}$ & $\begin{array}{c}\text { Up } \\
\text { length }\end{array}$ & CTE & CTEK & $\begin{array}{l}\text { Delta } \\
\text { CTEK }\end{array}$ & $\begin{array}{c}\text { CTE } \\
\text { adjusted }\end{array}$ \\
\hline 0 & 1.00 & 1.55 & 0 & 0.1000 & & & & & 0 & 0.1000 & & & & \\
\hline 100 & 1.50 & 2.00 & 0.45 & 0.1002 & $2.25 \mathrm{E}-05$ & & $-2.25 E-05$ & $2.30 \mathrm{E}-05$ & 0.5 & 0.1003 & $2.50 \mathrm{E}-05$ & & $-2.50 \mathrm{E}-05$ & $2.55 \mathrm{E}-05$ \\
\hline 200 & 2.02 & 2.52 & 0.97 & 0.1005 & $2.43 \mathrm{E}-05$ & & $-2.43 E-05$ & $2.48 \mathrm{E}-05$ & 1.02 & 0.1005 & $2.55 \mathrm{E}-05$ & & $-2.55 \mathrm{E}-05$ & $2.60 \mathrm{E}-05$ \\
\hline 300 & 2.68 & 3.20 & 1.65 & 0.1008 & $2.75 \mathrm{E}-05$ & & $-2.75 E-05$ & $2.80 \mathrm{E}-05$ & 1.68 & 0.1008 & $2.80 \mathrm{E}-05$ & & $-2.80 \mathrm{E}-05$ & $2.85 \mathrm{E}-05$ \\
\hline 400 & 3.40 & 3.95 & 2.4 & 0.1012 & $3.00 \mathrm{E}-05$ & & $-3.00 \mathrm{E}-05$ & $3.05 \mathrm{E}-05$ & 2.4 & 0.1012 & $3.00 \mathrm{E}-05$ & & $-3.00 \mathrm{E}-05$ & 3.05E-05 \\
\hline 500 & 4.20 & 4.65 & 3.1 & 0.1016 & $3.10 \mathrm{E}-05$ & 4.87E-06 & $-2.61 \mathrm{E}-05$ & $3.15 \mathrm{E}-05$ & 3.2 & 0.1016 & $3.20 \mathrm{E}-05$ & 4.87E-06 & $-2.71 \mathrm{E}-05$ & $3.25 \mathrm{E}-05$ \\
\hline
\end{tabular}




\begin{tabular}{|c|c|c|c|c|c|c|c|c|c|c|c|c|c|c|}
\hline $\begin{array}{l}\text { Capsule } \\
\text { Column }\end{array}$ & $\begin{array}{l}\text { OC2 } \\
\text { South }\end{array}$ & $\begin{array}{l}\text { Specimen } \\
\text { Length }\end{array}$ & $\begin{array}{c}67 \\
0.1000\end{array}$ & $\begin{array}{l}\text { Material } \\
\text { Scale }\end{array}$ & $\begin{array}{l}\text { H-451 } \\
1 "\end{array}$ & $5.00 \mathrm{E}-04$ & & & & & & & & \\
\hline $\begin{array}{c}\text { Temperature } \\
\left({ }^{\circ} \mathrm{C}\right)\end{array}$ & Up & Down & $\begin{array}{l}\text { Delta } \\
\text { Down }\end{array}$ & $\begin{array}{l}\text { Down } \\
\text { length }\end{array}$ & CTE & CTEK & $\begin{array}{l}\text { Delta } \\
\text { CTEK }\end{array}$ & $\begin{array}{c}\text { CTE } \\
\text { adjusted }\end{array}$ & $\begin{array}{l}\text { Delta } \\
\text { Up }\end{array}$ & $\begin{array}{c}\text { Up } \\
\text { length }\end{array}$ & CTE & CTEK & $\begin{array}{l}\text { Delta } \\
\text { CTEK }\end{array}$ & $\begin{array}{c}\text { CTE } \\
\text { adjusted }\end{array}$ \\
\hline 0 & 0.10 & 0.35 & 0 & 0.1000 & & & & & 0 & 0.1000 & & & & \\
\hline 100 & 1.01 & 1.30 & 0.95 & 0.1005 & $4.75 \mathrm{E}-05$ & & $-4.75 \mathrm{E}-05$ & $4.80 \mathrm{E}-05$ & 0.91 & 0.1005 & 4.55E-05 & & $-4.55 \mathrm{E}-05$ & 4.60E-05 \\
\hline 200 & 1.98 & 2.22 & 1.87 & 0.1009 & 4.67E-05 & & $-4.67 \mathrm{E}-05$ & $4.72 \mathrm{E}-05$ & 1.88 & 0.1009 & $4.70 \mathrm{E}-05$ & & $-4.70 \mathrm{E}-05$ & 4.75E-05 \\
\hline 300 & 3.05 & 3.20 & 2.85 & 0.1014 & $4.75 \mathrm{E}-05$ & & $-4.75 E-05$ & $4.80 \mathrm{E}-05$ & 2.95 & 0.1015 & 4.92E-05 & & $-4.92 \mathrm{E}-05$ & 4.97E-05 \\
\hline 400 & 4.02 & 4.22 & 3.87 & 0.1019 & $4.84 \mathrm{E}-05$ & & $-4.84 \mathrm{E}-05$ & 4.89E-05 & 3.92 & 0.1020 & 4.90E-05 & & $-4.90 \mathrm{E}-05$ & 4.95E-05 \\
\hline 500 & 5.12 & 5.30 & 4.95 & 0.1025 & $4.95 \mathrm{E}-05$ & 4.87E-06 & $-4.46 \mathrm{E}-05$ & $5.00 \mathrm{E}-05$ & 5.02 & 0.1025 & $5.02 \mathrm{E}-05$ & 4.87E-06 & $-4.53 \mathrm{E}-05$ & 5.07E-05 \\
\hline
\end{tabular}

\begin{tabular}{|c|c|c|c|c|c|c|c|c|c|c|c|c|c|c|}
\hline $\begin{array}{l}\text { Capsule } \\
\text { Column }\end{array}$ & $\begin{array}{l}\text { OC2 } \\
\text { South }\end{array}$ & $\begin{array}{l}\text { Specimen } \\
\text { Length }\end{array}$ & $\begin{array}{c}73 \\
0.1000\end{array}$ & $\begin{array}{c}\text { Material } \\
\text { Scale }\end{array}$ & $\begin{array}{c}\text { H-451 } \\
1 "\end{array}$ & $5.00 \mathrm{E}-04$ & & & & & & & & \\
\hline $\begin{array}{c}\text { Temperature } \\
\left({ }^{\circ} \mathrm{C}\right)\end{array}$ & Up & Down & $\begin{array}{l}\text { Delta } \\
\text { Down }\end{array}$ & $\begin{array}{l}\text { Down } \\
\text { length }\end{array}$ & CTE & CTEK & $\begin{array}{l}\text { Delta } \\
\text { CTEK }\end{array}$ & $\begin{array}{c}\text { CTE } \\
\text { adjusted }\end{array}$ & $\begin{array}{c}\text { Delta } \\
\text { Up }\end{array}$ & $\begin{array}{c}\text { Up } \\
\text { length }\end{array}$ & CTE & CTEK & $\begin{array}{l}\text { Delta } \\
\text { CTEK }\end{array}$ & $\begin{array}{c}\text { CTE } \\
\text { adjusted }\end{array}$ \\
\hline 0 & 0.49 & 0.97 & 0 & 0.1000 & & & & & 0 & 0.1000 & & & & \\
\hline 100 & 0.75 & 1.22 & 0.25 & 0.1001 & $1.25 \mathrm{E}-05$ & & $-1.25 \mathrm{E}-05$ & $1.30 \mathrm{E}-05$ & 0.26 & 0.1001 & $1.30 \mathrm{E}-05$ & & $-1.30 \mathrm{E}-05$ & $1.35 \mathrm{E}-05$ \\
\hline 200 & 1.05 & 1.70 & 0.73 & 0.1004 & $1.82 \mathrm{E}-05$ & & $-1.82 \mathrm{E}-05$ & $1.87 \mathrm{E}-05$ & 0.56 & 0.1003 & $1.40 \mathrm{E}-05$ & & $-1.40 \mathrm{E}-05$ & $1.45 \mathrm{E}-05$ \\
\hline 300 & 1.58 & 2.02 & 1.05 & 0.1005 & $1.75 \mathrm{E}-05$ & & $-1.75 \mathrm{E}-05$ & $1.80 \mathrm{E}-05$ & 1.09 & 0.1005 & $1.82 \mathrm{E}-05$ & & $-1.82 \mathrm{E}-05$ & $1.87 \mathrm{E}-05$ \\
\hline 400 & 2.12 & 2.60 & 1.63 & 0.1008 & $2.04 \mathrm{E}-05$ & & $-2.04 \mathrm{E}-05$ & 2.09E-05 & 1.63 & 0.1008 & $2.04 \mathrm{E}-05$ & & $-2.04 \mathrm{E}-05$ & $2.09 \mathrm{E}-05$ \\
\hline 500 & 2.78 & 3.20 & 2.23 & 0.1011 & $2.23 \mathrm{E}-05$ & 4.87E-06 & $-1.74 \mathrm{E}-05$ & $2.28 \mathrm{E}-05$ & 2.29 & 0.1011 & $2.29 \mathrm{E}-05$ & 4.87E-06 & $-1.80 \mathrm{E}-05$ & $2.34 \mathrm{E}-05$ \\
\hline
\end{tabular}

\begin{tabular}{|c|c|c|c|c|c|c|c|c|c|c|c|c|c|c|}
\hline $\begin{array}{l}\text { Capsule } \\
\text { Column }\end{array}$ & $\begin{array}{l}\text { OC2 } \\
\text { South }\end{array}$ & $\begin{array}{c}\text { Specimen } \\
\text { Length }\end{array}$ & $\begin{array}{c}79 \\
0.1000\end{array}$ & $\begin{array}{c}\text { Material } \\
\text { Scale }\end{array}$ & $\begin{array}{c}\text { H-451 } \\
1 "\end{array}$ & $5.00 \mathrm{E}-04$ & & & & & & & & \\
\hline $\begin{array}{c}\text { Temperature } \\
\left({ }^{\circ} \mathrm{C}\right)\end{array}$ & Up & Down & $\begin{array}{l}\text { Delta } \\
\text { Down }\end{array}$ & $\begin{array}{l}\text { Down } \\
\text { length }\end{array}$ & CTE & CTEK & $\begin{array}{l}\text { Delta } \\
\text { CTEK }\end{array}$ & $\begin{array}{c}\text { CTE } \\
\text { adjusted }\end{array}$ & $\begin{array}{l}\text { Delta } \\
\text { Up }\end{array}$ & $\begin{array}{c}\text { Up } \\
\text { length }\end{array}$ & CTE & CTEK & $\begin{array}{l}\text { Delta } \\
\text { CTEK }\end{array}$ & $\begin{array}{c}\text { CTE } \\
\text { adjusted }\end{array}$ \\
\hline 0 & 1.00 & 1.55 & 0 & 0.1000 & & & & & 0 & 0.1000 & & & & \\
\hline 100 & 1.50 & 2.00 & 0.45 & 0.1002 & $2.25 \mathrm{E}-05$ & & $-2.25 \mathrm{E}-05$ & $2.30 \mathrm{E}-05$ & 0.5 & 0.1003 & $2.50 \mathrm{E}-05$ & & $-2.50 \mathrm{E}-05$ & $2.55 \mathrm{E}-05$ \\
\hline 200 & 2.02 & 2.52 & 0.97 & 0.1005 & $2.43 \mathrm{E}-05$ & & $-2.43 E-05$ & $2.48 \mathrm{E}-05$ & 1.02 & 0.1005 & $2.55 \mathrm{E}-05$ & & $-2.55 \mathrm{E}-05$ & $2.60 \mathrm{E}-05$ \\
\hline 300 & 2.68 & 3.20 & 1.65 & 0.1008 & $2.75 \mathrm{E}-05$ & & $-2.75 \mathrm{E}-05$ & $2.80 \mathrm{E}-05$ & 1.68 & 0.1008 & $2.80 \mathrm{E}-05$ & & $-2.80 \mathrm{E}-05$ & 2.85E-05 \\
\hline 400 & 3.40 & 3.95 & 2.4 & 0.1012 & $3.00 \mathrm{E}-05$ & & $-3.00 \mathrm{E}-05$ & $3.05 E-05$ & 2.4 & 0.1012 & $3.00 \mathrm{E}-05$ & & $-3.00 \mathrm{E}-05$ & $3.05 \mathrm{E}-05$ \\
\hline 500 & 4.20 & 4.65 & 3.1 & 0.1016 & $3.10 \mathrm{E}-05$ & $4.87 E-06$ & $-2.61 \mathrm{E}-05$ & $3.15 \mathrm{E}-05$ & 3.2 & 0.1016 & $3.20 \mathrm{E}-05$ & $4.87 E-06$ & $-2.71 \mathrm{E}-05$ & $3.25 \mathrm{E}-05$ \\
\hline
\end{tabular}




\begin{tabular}{|c|c|c|c|c|c|c|c|c|c|c|c|c|c|c|}
\hline $\begin{array}{l}\text { Capsule } \\
\text { Column }\end{array}$ & $\begin{array}{l}\text { OC2 } \\
\text { South }\end{array}$ & $\begin{array}{c}\text { Specimen } \\
\text { Length }\end{array}$ & $\begin{array}{c}67 \\
0.1000\end{array}$ & $\begin{array}{c}\text { Material } \\
\text { Scale }\end{array}$ & $\begin{array}{c}\text { H-451 } \\
1 "\end{array}$ & $5.00 \mathrm{E}-04$ & & & & & & & & \\
\hline $\begin{array}{c}\text { Temperature } \\
\left({ }^{\circ} \mathrm{C}\right) \\
\end{array}$ & Up & Down & $\begin{array}{l}\text { Delta } \\
\text { Down }\end{array}$ & $\begin{array}{l}\text { Down } \\
\text { length }\end{array}$ & CTE & CTEK & $\begin{array}{l}\text { Delta } \\
\text { CTEK }\end{array}$ & $\begin{array}{c}\text { CTE } \\
\text { adjusted }\end{array}$ & $\begin{array}{l}\text { Delta } \\
\text { Up }\end{array}$ & $\begin{array}{c}\text { Up } \\
\text { length }\end{array}$ & CTE & CTEK & $\begin{array}{l}\text { Delta } \\
\text { CTEK }\end{array}$ & $\begin{array}{c}\text { CTE } \\
\text { adjusted }\end{array}$ \\
\hline 0 & 0.10 & 0.35 & 0 & 0.1000 & & & & & 0 & 0.1000 & & & & \\
\hline 100 & 1.01 & 1.30 & 0.95 & 0.1005 & 4.75E-05 & & $-4.75 \mathrm{E}-05$ & $4.80 \mathrm{E}-05$ & 0.91 & 0.1005 & 4.55E-05 & & $-4.55 \mathrm{E}-05$ & 4.60E-05 \\
\hline 200 & 1.98 & 2.22 & 1.87 & 0.1009 & 4.67E-05 & & $-4.67 E-05$ & $4.72 \mathrm{E}-05$ & 1.88 & 0.1009 & $4.70 \mathrm{E}-05$ & & $-4.70 \mathrm{E}-05$ & 4.75E-05 \\
\hline 300 & 3.05 & 3.20 & 2.85 & 0.1014 & $4.75 \mathrm{E}-05$ & & $-4.75 E-05$ & $4.80 \mathrm{E}-05$ & 2.95 & 0.1015 & $4.92 \mathrm{E}-05$ & & $-4.92 \mathrm{E}-05$ & 4.97E-05 \\
\hline 400 & 4.02 & 4.22 & 3.87 & 0.1019 & 4.84E-05 & & $-4.84 \mathrm{E}-05$ & 4.89E-05 & 3.92 & 0.1020 & 4.90E-05 & & $-4.90 \mathrm{E}-05$ & 4.95E-05 \\
\hline 500 & 5.12 & 5.30 & 4.95 & 0.1025 & $4.95 \mathrm{E}-05$ & 4.87E-06 & $-4.46 \mathrm{E}-05$ & $5.00 \mathrm{E}-05$ & 5.02 & 0.1025 & $5.02 \mathrm{E}-05$ & 4.87E-06 & $-4.53 \mathrm{E}-05$ & 5.07E-05 \\
\hline
\end{tabular}

\begin{tabular}{|c|c|c|c|c|c|c|c|c|c|c|c|c|c|c|}
\hline $\begin{array}{l}\text { Capsule } \\
\text { Column }\end{array}$ & $\begin{array}{l}\text { OC2 } \\
\text { South }\end{array}$ & $\begin{array}{l}\text { Specimen } \\
\text { Length }\end{array}$ & $\begin{array}{c}73 \\
0.1000\end{array}$ & $\begin{array}{l}\text { Material } \\
\text { Scale }\end{array}$ & $\begin{array}{c}\text { H-451 } \\
1 "\end{array}$ & $5.00 \mathrm{E}-04$ & & & & & & & & \\
\hline $\begin{array}{c}\text { Temperature } \\
\left({ }^{\circ} \mathrm{C}\right)\end{array}$ & Up & Down & $\begin{array}{l}\text { Delta } \\
\text { Down }\end{array}$ & $\begin{array}{l}\text { Down } \\
\text { length }\end{array}$ & CTE & CTEK & $\begin{array}{l}\text { Delta } \\
\text { CTEK }\end{array}$ & $\begin{array}{c}\text { CTE } \\
\text { adjusted }\end{array}$ & $\begin{array}{l}\text { Delta } \\
\text { Up }\end{array}$ & $\begin{array}{c}\text { Up } \\
\text { length }\end{array}$ & CTE & CTEK & $\begin{array}{l}\text { Delta } \\
\text { CTEK }\end{array}$ & $\begin{array}{c}\text { CTE } \\
\text { adjusted }\end{array}$ \\
\hline 0 & 0.49 & 0.97 & 0 & 0.1000 & & & & & 0 & 0.1000 & & & & \\
\hline 100 & 0.75 & 1.22 & 0.25 & 0.1001 & $1.25 \mathrm{E}-05$ & & $-1.25 \mathrm{E}-05$ & $1.30 \mathrm{E}-05$ & 0.26 & 0.1001 & $1.30 \mathrm{E}-05$ & & $-1.30 \mathrm{E}-05$ & $1.35 \mathrm{E}-05$ \\
\hline 200 & 1.05 & 1.70 & 0.73 & 0.1004 & $1.82 \mathrm{E}-05$ & & $-1.82 \mathrm{E}-05$ & $1.87 \mathrm{E}-05$ & 0.56 & 0.1003 & $1.40 \mathrm{E}-05$ & & $-1.40 \mathrm{E}-05$ & $1.45 \mathrm{E}-05$ \\
\hline 300 & 1.58 & 2.02 & 1.05 & 0.1005 & $1.75 \mathrm{E}-05$ & & $-1.75 E-05$ & $1.80 \mathrm{E}-05$ & 1.09 & 0.1005 & $1.82 \mathrm{E}-05$ & & $-1.82 \mathrm{E}-05$ & $1.87 \mathrm{E}-05$ \\
\hline 400 & 2.12 & 2.60 & 1.63 & 0.1008 & $2.04 \mathrm{E}-05$ & & $-2.04 \mathrm{E}-05$ & $2.09 E-05$ & 1.63 & 0.1008 & $2.04 \mathrm{E}-05$ & & $-2.04 \mathrm{E}-05$ & 2.09E-05 \\
\hline 500 & 2.78 & 3.20 & 2.23 & 0.1011 & $2.23 \mathrm{E}-05$ & 4.87E-06 & $-1.74 \mathrm{E}-05$ & $2.28 \mathrm{E}-05$ & 2.29 & 0.1011 & $2.29 \mathrm{E}-05$ & $4.87 E-06$ & $-1.80 \mathrm{E}-05$ & $2.34 \mathrm{E}-05$ \\
\hline
\end{tabular}

\begin{tabular}{|c|c|c|c|c|c|c|c|c|c|c|c|c|c|c|}
\hline $\begin{array}{l}\text { Capsule } \\
\text { Column }\end{array}$ & $\begin{array}{c}\text { OC2 } \\
\text { South }\end{array}$ & $\begin{array}{c}\text { Specimen } \\
\text { Length }\end{array}$ & $\begin{array}{c}79 \\
0.1000 \\
\end{array}$ & $\begin{array}{c}\text { Material } \\
\text { Scale }\end{array}$ & $\begin{array}{c}\text { H-451 } \\
1 " \\
\end{array}$ & $5.00 \mathrm{E}-04$ & & & & & & & & \\
\hline $\begin{array}{c}\text { Temperature } \\
\left({ }^{\circ} \mathrm{C}\right)\end{array}$ & $U p$ & Down & $\begin{array}{l}\text { Delta } \\
\text { Down }\end{array}$ & $\begin{array}{l}\text { Down } \\
\text { length }\end{array}$ & CTE & CTEK & $\begin{array}{l}\text { Delta } \\
\text { CTEK }\end{array}$ & $\begin{array}{c}\text { CTE } \\
\text { adjusted }\end{array}$ & $\begin{array}{l}\text { Delta } \\
\text { Up }\end{array}$ & $\begin{array}{c}\text { Up } \\
\text { length }\end{array}$ & CTE & CTEK & $\begin{array}{l}\text { Delta } \\
\text { CTEK }\end{array}$ & $\begin{array}{c}\text { CTE } \\
\text { adjusted }\end{array}$ \\
\hline 0 & 1.00 & 1.55 & 0 & 0.1000 & & & & & 0 & 0.1000 & & & & \\
\hline 100 & 1.50 & 2.00 & 0.45 & 0.1002 & $2.25 \mathrm{E}-05$ & & $-2.25 E-05$ & $2.30 \mathrm{E}-05$ & 0.5 & 0.1003 & $2.50 \mathrm{E}-05$ & & $-2.50 \mathrm{E}-05$ & $2.55 \mathrm{E}-05$ \\
\hline 200 & 2.02 & 2.52 & 0.97 & 0.1005 & $2.43 \mathrm{E}-05$ & & $-2.43 E-05$ & $2.48 \mathrm{E}-05$ & 1.02 & 0.1005 & $2.55 \mathrm{E}-05$ & & $-2.55 \mathrm{E}-05$ & $2.60 \mathrm{E}-05$ \\
\hline 300 & 2.68 & 3.20 & 1.65 & 0.1008 & $2.75 \mathrm{E}-05$ & & $-2.75 E-05$ & $2.80 \mathrm{E}-05$ & 1.68 & 0.1008 & $2.80 \mathrm{E}-05$ & & $-2.80 \mathrm{E}-05$ & $2.85 \mathrm{E}-05$ \\
\hline 400 & 3.40 & 3.95 & 2.4 & 0.1012 & $3.00 \mathrm{E}-05$ & & $-3.00 \mathrm{E}-05$ & $3.05 \mathrm{E}-05$ & 2.4 & 0.1012 & $3.00 \mathrm{E}-05$ & & $-3.00 \mathrm{E}-05$ & 3.05E-05 \\
\hline 500 & 4.20 & 4.65 & 3.1 & 0.1016 & $3.10 \mathrm{E}-05$ & 4.87E-06 & $-2.61 \mathrm{E}-05$ & $3.15 \mathrm{E}-05$ & 3.2 & 0.1016 & $3.20 \mathrm{E}-05$ & 4.87E-06 & $-2.71 \mathrm{E}-05$ & $3.25 \mathrm{E}-05$ \\
\hline
\end{tabular}




\begin{tabular}{|c|c|c|c|c|c|c|c|c|c|c|c|c|c|c|}
\hline $\begin{array}{l}\text { Capsule } \\
\text { Column }\end{array}$ & $\begin{array}{l}\text { OC2 } \\
\text { South }\end{array}$ & $\begin{array}{l}\text { Specimen } \\
\text { Length }\end{array}$ & $\begin{array}{c}67 \\
0.1000\end{array}$ & $\begin{array}{l}\text { Material } \\
\text { Scale }\end{array}$ & $\begin{array}{l}\text { H-451 } \\
1 "\end{array}$ & $5.00 \mathrm{E}-04$ & & & & & & & & \\
\hline $\begin{array}{c}\text { Temperature } \\
\left({ }^{\circ} \mathrm{C}\right)\end{array}$ & Up & Down & $\begin{array}{l}\text { Delta } \\
\text { Down }\end{array}$ & $\begin{array}{l}\text { Down } \\
\text { length }\end{array}$ & CTE & CTEK & $\begin{array}{l}\text { Delta } \\
\text { CTEK }\end{array}$ & $\begin{array}{c}\text { CTE } \\
\text { adjusted }\end{array}$ & $\begin{array}{l}\text { Delta } \\
\text { Up }\end{array}$ & $\begin{array}{c}\text { Up } \\
\text { length }\end{array}$ & CTE & CTEK & $\begin{array}{l}\text { Delta } \\
\text { CTEK }\end{array}$ & $\begin{array}{c}\text { CTE } \\
\text { adjusted }\end{array}$ \\
\hline 0 & 0.10 & 0.35 & 0 & 0.1000 & & & & & 0 & 0.1000 & & & & \\
\hline 100 & 1.01 & 1.30 & 0.95 & 0.1005 & $4.75 \mathrm{E}-05$ & & $-4.75 \mathrm{E}-05$ & $4.80 \mathrm{E}-05$ & 0.91 & 0.1005 & 4.55E-05 & & $-4.55 \mathrm{E}-05$ & 4.60E-05 \\
\hline 200 & 1.98 & 2.22 & 1.87 & 0.1009 & 4.67E-05 & & $-4.67 \mathrm{E}-05$ & $4.72 \mathrm{E}-05$ & 1.88 & 0.1009 & $4.70 \mathrm{E}-05$ & & $-4.70 \mathrm{E}-05$ & 4.75E-05 \\
\hline 300 & 3.05 & 3.20 & 2.85 & 0.1014 & $4.75 \mathrm{E}-05$ & & $-4.75 E-05$ & $4.80 \mathrm{E}-05$ & 2.95 & 0.1015 & 4.92E-05 & & $-4.92 \mathrm{E}-05$ & 4.97E-05 \\
\hline 400 & 4.02 & 4.22 & 3.87 & 0.1019 & $4.84 \mathrm{E}-05$ & & $-4.84 \mathrm{E}-05$ & 4.89E-05 & 3.92 & 0.1020 & 4.90E-05 & & $-4.90 \mathrm{E}-05$ & 4.95E-05 \\
\hline 500 & 5.12 & 5.30 & 4.95 & 0.1025 & $4.95 \mathrm{E}-05$ & 4.87E-06 & $-4.46 \mathrm{E}-05$ & $5.00 \mathrm{E}-05$ & 5.02 & 0.1025 & $5.02 \mathrm{E}-05$ & 4.87E-06 & $-4.53 \mathrm{E}-05$ & 5.07E-05 \\
\hline
\end{tabular}

\begin{tabular}{|c|c|c|c|c|c|c|c|c|c|c|c|c|c|c|}
\hline $\begin{array}{l}\text { Capsule } \\
\text { Column }\end{array}$ & $\begin{array}{l}\text { OC2 } \\
\text { South }\end{array}$ & $\begin{array}{l}\text { Specimen } \\
\text { Length }\end{array}$ & $\begin{array}{c}73 \\
0.1000\end{array}$ & $\begin{array}{c}\text { Material } \\
\text { Scale }\end{array}$ & $\begin{array}{c}\text { H-451 } \\
1 "\end{array}$ & $5.00 \mathrm{E}-04$ & & & & & & & & \\
\hline $\begin{array}{c}\text { Temperature } \\
\left({ }^{\circ} \mathrm{C}\right)\end{array}$ & Up & Down & $\begin{array}{l}\text { Delta } \\
\text { Down }\end{array}$ & $\begin{array}{l}\text { Down } \\
\text { length }\end{array}$ & CTE & CTEK & $\begin{array}{l}\text { Delta } \\
\text { CTEK }\end{array}$ & $\begin{array}{c}\text { CTE } \\
\text { adjusted }\end{array}$ & $\begin{array}{c}\text { Delta } \\
\text { Up }\end{array}$ & $\begin{array}{c}\text { Up } \\
\text { length }\end{array}$ & CTE & CTEK & $\begin{array}{l}\text { Delta } \\
\text { CTEK }\end{array}$ & $\begin{array}{c}\text { CTE } \\
\text { adjusted }\end{array}$ \\
\hline 0 & 0.49 & 0.97 & 0 & 0.1000 & & & & & 0 & 0.1000 & & & & \\
\hline 100 & 0.75 & 1.22 & 0.25 & 0.1001 & $1.25 \mathrm{E}-05$ & & $-1.25 \mathrm{E}-05$ & $1.30 \mathrm{E}-05$ & 0.26 & 0.1001 & $1.30 \mathrm{E}-05$ & & $-1.30 \mathrm{E}-05$ & $1.35 \mathrm{E}-05$ \\
\hline 200 & 1.05 & 1.70 & 0.73 & 0.1004 & $1.82 \mathrm{E}-05$ & & $-1.82 \mathrm{E}-05$ & $1.87 \mathrm{E}-05$ & 0.56 & 0.1003 & $1.40 \mathrm{E}-05$ & & $-1.40 \mathrm{E}-05$ & $1.45 \mathrm{E}-05$ \\
\hline 300 & 1.58 & 2.02 & 1.05 & 0.1005 & $1.75 \mathrm{E}-05$ & & $-1.75 \mathrm{E}-05$ & $1.80 \mathrm{E}-05$ & 1.09 & 0.1005 & $1.82 \mathrm{E}-05$ & & $-1.82 \mathrm{E}-05$ & $1.87 \mathrm{E}-05$ \\
\hline 400 & 2.12 & 2.60 & 1.63 & 0.1008 & $2.04 \mathrm{E}-05$ & & $-2.04 \mathrm{E}-05$ & 2.09E-05 & 1.63 & 0.1008 & $2.04 \mathrm{E}-05$ & & $-2.04 \mathrm{E}-05$ & $2.09 \mathrm{E}-05$ \\
\hline 500 & 2.78 & 3.20 & 2.23 & 0.1011 & $2.23 \mathrm{E}-05$ & 4.87E-06 & $-1.74 \mathrm{E}-05$ & $2.28 \mathrm{E}-05$ & 2.29 & 0.1011 & $2.29 \mathrm{E}-05$ & 4.87E-06 & $-1.80 \mathrm{E}-05$ & $2.34 \mathrm{E}-05$ \\
\hline
\end{tabular}

\begin{tabular}{|c|c|c|c|c|c|c|c|c|c|c|c|c|c|c|}
\hline $\begin{array}{l}\text { Capsule } \\
\text { Column }\end{array}$ & $\begin{array}{l}\text { OC2 } \\
\text { South }\end{array}$ & $\begin{array}{c}\text { Specimen } \\
\text { Length }\end{array}$ & $\begin{array}{c}79 \\
0.1000\end{array}$ & $\begin{array}{c}\text { Material } \\
\text { Scale }\end{array}$ & $\begin{array}{c}\text { H-451 } \\
1 "\end{array}$ & $5.00 \mathrm{E}-04$ & & & & & & & & \\
\hline $\begin{array}{c}\text { Temperature } \\
\left({ }^{\circ} \mathrm{C}\right)\end{array}$ & Up & Down & $\begin{array}{l}\text { Delta } \\
\text { Down }\end{array}$ & $\begin{array}{l}\text { Down } \\
\text { length }\end{array}$ & CTE & CTEK & $\begin{array}{l}\text { Delta } \\
\text { CTEK }\end{array}$ & $\begin{array}{c}\text { CTE } \\
\text { adjusted }\end{array}$ & $\begin{array}{l}\text { Delta } \\
\text { Up }\end{array}$ & $\begin{array}{c}\text { Up } \\
\text { length }\end{array}$ & CTE & CTEK & $\begin{array}{l}\text { Delta } \\
\text { CTEK }\end{array}$ & $\begin{array}{c}\text { CTE } \\
\text { adjusted }\end{array}$ \\
\hline 0 & 1.00 & 1.55 & 0 & 0.1000 & & & & & 0 & 0.1000 & & & & \\
\hline 100 & 1.50 & 2.00 & 0.45 & 0.1002 & $2.25 \mathrm{E}-05$ & & $-2.25 \mathrm{E}-05$ & $2.30 \mathrm{E}-05$ & 0.5 & 0.1003 & $2.50 \mathrm{E}-05$ & & $-2.50 \mathrm{E}-05$ & $2.55 \mathrm{E}-05$ \\
\hline 200 & 2.02 & 2.52 & 0.97 & 0.1005 & $2.43 \mathrm{E}-05$ & & $-2.43 E-05$ & $2.48 \mathrm{E}-05$ & 1.02 & 0.1005 & $2.55 \mathrm{E}-05$ & & $-2.55 \mathrm{E}-05$ & $2.60 \mathrm{E}-05$ \\
\hline 300 & 2.68 & 3.20 & 1.65 & 0.1008 & $2.75 \mathrm{E}-05$ & & $-2.75 \mathrm{E}-05$ & $2.80 \mathrm{E}-05$ & 1.68 & 0.1008 & $2.80 \mathrm{E}-05$ & & $-2.80 \mathrm{E}-05$ & 2.85E-05 \\
\hline 400 & 3.40 & 3.95 & 2.4 & 0.1012 & $3.00 \mathrm{E}-05$ & & $-3.00 \mathrm{E}-05$ & $3.05 E-05$ & 2.4 & 0.1012 & $3.00 \mathrm{E}-05$ & & $-3.00 \mathrm{E}-05$ & $3.05 \mathrm{E}-05$ \\
\hline 500 & 4.20 & 4.65 & 3.1 & 0.1016 & $3.10 \mathrm{E}-05$ & $4.87 E-06$ & $-2.61 \mathrm{E}-05$ & $3.15 \mathrm{E}-05$ & 3.2 & 0.1016 & $3.20 \mathrm{E}-05$ & $4.87 E-06$ & $-2.71 \mathrm{E}-05$ & $3.25 \mathrm{E}-05$ \\
\hline
\end{tabular}




\begin{tabular}{|c|c|c|c|c|c|c|c|c|c|c|c|c|c|c|}
\hline $\begin{array}{l}\text { Capsule } \\
\text { Column }\end{array}$ & $\begin{array}{l}\text { OC2 } \\
\text { South }\end{array}$ & $\begin{array}{c}\text { Specimen } \\
\text { Length }\end{array}$ & $\begin{array}{c}67 \\
0.1000\end{array}$ & $\begin{array}{c}\text { Material } \\
\text { Scale }\end{array}$ & $\begin{array}{c}\text { H-451 } \\
1 "\end{array}$ & $5.00 \mathrm{E}-04$ & & & & & & & & \\
\hline $\begin{array}{c}\text { Temperature } \\
\left({ }^{\circ} \mathrm{C}\right) \\
\end{array}$ & Up & Down & $\begin{array}{l}\text { Delta } \\
\text { Down }\end{array}$ & $\begin{array}{l}\text { Down } \\
\text { length }\end{array}$ & CTE & CTEK & $\begin{array}{l}\text { Delta } \\
\text { CTEK }\end{array}$ & $\begin{array}{c}\text { CTE } \\
\text { adjusted }\end{array}$ & $\begin{array}{l}\text { Delta } \\
\text { Up }\end{array}$ & $\begin{array}{c}\text { Up } \\
\text { length }\end{array}$ & CTE & CTEK & $\begin{array}{l}\text { Delta } \\
\text { CTEK }\end{array}$ & $\begin{array}{c}\text { CTE } \\
\text { adjusted }\end{array}$ \\
\hline 0 & 0.10 & 0.35 & 0 & 0.1000 & & & & & 0 & 0.1000 & & & & \\
\hline 100 & 1.01 & 1.30 & 0.95 & 0.1005 & 4.75E-05 & & $-4.75 \mathrm{E}-05$ & $4.80 \mathrm{E}-05$ & 0.91 & 0.1005 & 4.55E-05 & & $-4.55 \mathrm{E}-05$ & 4.60E-05 \\
\hline 200 & 1.98 & 2.22 & 1.87 & 0.1009 & 4.67E-05 & & $-4.67 E-05$ & $4.72 \mathrm{E}-05$ & 1.88 & 0.1009 & $4.70 \mathrm{E}-05$ & & $-4.70 \mathrm{E}-05$ & 4.75E-05 \\
\hline 300 & 3.05 & 3.20 & 2.85 & 0.1014 & $4.75 \mathrm{E}-05$ & & $-4.75 E-05$ & $4.80 \mathrm{E}-05$ & 2.95 & 0.1015 & $4.92 \mathrm{E}-05$ & & $-4.92 \mathrm{E}-05$ & 4.97E-05 \\
\hline 400 & 4.02 & 4.22 & 3.87 & 0.1019 & 4.84E-05 & & $-4.84 \mathrm{E}-05$ & 4.89E-05 & 3.92 & 0.1020 & 4.90E-05 & & $-4.90 \mathrm{E}-05$ & 4.95E-05 \\
\hline 500 & 5.12 & 5.30 & 4.95 & 0.1025 & $4.95 \mathrm{E}-05$ & 4.87E-06 & $-4.46 \mathrm{E}-05$ & $5.00 \mathrm{E}-05$ & 5.02 & 0.1025 & $5.02 \mathrm{E}-05$ & 4.87E-06 & $-4.53 \mathrm{E}-05$ & 5.07E-05 \\
\hline
\end{tabular}

\begin{tabular}{|c|c|c|c|c|c|c|c|c|c|c|c|c|c|c|}
\hline $\begin{array}{l}\text { Capsule } \\
\text { Column }\end{array}$ & $\begin{array}{l}\text { OC2 } \\
\text { South }\end{array}$ & $\begin{array}{l}\text { Specimen } \\
\text { Length }\end{array}$ & $\begin{array}{c}73 \\
0.1000\end{array}$ & $\begin{array}{l}\text { Material } \\
\text { Scale }\end{array}$ & $\begin{array}{c}\text { H-451 } \\
1 "\end{array}$ & $5.00 \mathrm{E}-04$ & & & & & & & & \\
\hline $\begin{array}{c}\text { Temperature } \\
\left({ }^{\circ} \mathrm{C}\right)\end{array}$ & Up & Down & $\begin{array}{l}\text { Delta } \\
\text { Down }\end{array}$ & $\begin{array}{l}\text { Down } \\
\text { length }\end{array}$ & CTE & CTEK & $\begin{array}{l}\text { Delta } \\
\text { CTEK }\end{array}$ & $\begin{array}{c}\text { CTE } \\
\text { adjusted }\end{array}$ & $\begin{array}{l}\text { Delta } \\
\text { Up }\end{array}$ & $\begin{array}{c}\text { Up } \\
\text { length }\end{array}$ & CTE & CTEK & $\begin{array}{l}\text { Delta } \\
\text { CTEK }\end{array}$ & $\begin{array}{c}\text { CTE } \\
\text { adjusted }\end{array}$ \\
\hline 0 & 0.49 & 0.97 & 0 & 0.1000 & & & & & 0 & 0.1000 & & & & \\
\hline 100 & 0.75 & 1.22 & 0.25 & 0.1001 & $1.25 \mathrm{E}-05$ & & $-1.25 \mathrm{E}-05$ & $1.30 \mathrm{E}-05$ & 0.26 & 0.1001 & $1.30 \mathrm{E}-05$ & & $-1.30 \mathrm{E}-05$ & $1.35 \mathrm{E}-05$ \\
\hline 200 & 1.05 & 1.70 & 0.73 & 0.1004 & $1.82 \mathrm{E}-05$ & & $-1.82 \mathrm{E}-05$ & $1.87 \mathrm{E}-05$ & 0.56 & 0.1003 & $1.40 \mathrm{E}-05$ & & $-1.40 \mathrm{E}-05$ & $1.45 \mathrm{E}-05$ \\
\hline 300 & 1.58 & 2.02 & 1.05 & 0.1005 & $1.75 \mathrm{E}-05$ & & $-1.75 E-05$ & $1.80 \mathrm{E}-05$ & 1.09 & 0.1005 & $1.82 \mathrm{E}-05$ & & $-1.82 \mathrm{E}-05$ & $1.87 \mathrm{E}-05$ \\
\hline 400 & 2.12 & 2.60 & 1.63 & 0.1008 & $2.04 \mathrm{E}-05$ & & $-2.04 \mathrm{E}-05$ & $2.09 E-05$ & 1.63 & 0.1008 & $2.04 \mathrm{E}-05$ & & $-2.04 \mathrm{E}-05$ & 2.09E-05 \\
\hline 500 & 2.78 & 3.20 & 2.23 & 0.1011 & $2.23 \mathrm{E}-05$ & 4.87E-06 & $-1.74 \mathrm{E}-05$ & $2.28 \mathrm{E}-05$ & 2.29 & 0.1011 & $2.29 \mathrm{E}-05$ & $4.87 E-06$ & $-1.80 \mathrm{E}-05$ & $2.34 \mathrm{E}-05$ \\
\hline
\end{tabular}

\begin{tabular}{|c|c|c|c|c|c|c|c|c|c|c|c|c|c|c|}
\hline $\begin{array}{l}\text { Capsule } \\
\text { Column }\end{array}$ & $\begin{array}{c}\text { OC2 } \\
\text { South }\end{array}$ & $\begin{array}{c}\text { Specimen } \\
\text { Length }\end{array}$ & $\begin{array}{c}79 \\
0.1000 \\
\end{array}$ & $\begin{array}{c}\text { Material } \\
\text { Scale }\end{array}$ & $\begin{array}{c}\text { H-451 } \\
1 " \\
\end{array}$ & $5.00 \mathrm{E}-04$ & & & & & & & & \\
\hline $\begin{array}{c}\text { Temperature } \\
\left({ }^{\circ} \mathrm{C}\right)\end{array}$ & $U p$ & Down & $\begin{array}{l}\text { Delta } \\
\text { Down }\end{array}$ & $\begin{array}{l}\text { Down } \\
\text { length }\end{array}$ & CTE & CTEK & $\begin{array}{l}\text { Delta } \\
\text { CTEK }\end{array}$ & $\begin{array}{c}\text { CTE } \\
\text { adjusted }\end{array}$ & $\begin{array}{l}\text { Delta } \\
\text { Up }\end{array}$ & $\begin{array}{c}\text { Up } \\
\text { length }\end{array}$ & CTE & CTEK & $\begin{array}{l}\text { Delta } \\
\text { CTEK }\end{array}$ & $\begin{array}{c}\text { CTE } \\
\text { adjusted }\end{array}$ \\
\hline 0 & 1.00 & 1.55 & 0 & 0.1000 & & & & & 0 & 0.1000 & & & & \\
\hline 100 & 1.50 & 2.00 & 0.45 & 0.1002 & $2.25 \mathrm{E}-05$ & & $-2.25 E-05$ & $2.30 \mathrm{E}-05$ & 0.5 & 0.1003 & $2.50 \mathrm{E}-05$ & & $-2.50 \mathrm{E}-05$ & $2.55 \mathrm{E}-05$ \\
\hline 200 & 2.02 & 2.52 & 0.97 & 0.1005 & $2.43 \mathrm{E}-05$ & & $-2.43 E-05$ & $2.48 \mathrm{E}-05$ & 1.02 & 0.1005 & $2.55 \mathrm{E}-05$ & & $-2.55 \mathrm{E}-05$ & $2.60 \mathrm{E}-05$ \\
\hline 300 & 2.68 & 3.20 & 1.65 & 0.1008 & $2.75 \mathrm{E}-05$ & & $-2.75 E-05$ & $2.80 \mathrm{E}-05$ & 1.68 & 0.1008 & $2.80 \mathrm{E}-05$ & & $-2.80 \mathrm{E}-05$ & $2.85 \mathrm{E}-05$ \\
\hline 400 & 3.40 & 3.95 & 2.4 & 0.1012 & $3.00 \mathrm{E}-05$ & & $-3.00 \mathrm{E}-05$ & $3.05 \mathrm{E}-05$ & 2.4 & 0.1012 & $3.00 \mathrm{E}-05$ & & $-3.00 \mathrm{E}-05$ & 3.05E-05 \\
\hline 500 & 4.20 & 4.65 & 3.1 & 0.1016 & $3.10 \mathrm{E}-05$ & 4.87E-06 & $-2.61 \mathrm{E}-05$ & $3.15 \mathrm{E}-05$ & 3.2 & 0.1016 & $3.20 \mathrm{E}-05$ & 4.87E-06 & $-2.71 \mathrm{E}-05$ & $3.25 \mathrm{E}-05$ \\
\hline
\end{tabular}




\begin{tabular}{|c|c|c|c|c|c|c|c|c|c|c|c|c|c|c|}
\hline $\begin{array}{l}\text { Capsule } \\
\text { Column }\end{array}$ & $\begin{array}{l}\text { OC2 } \\
\text { South }\end{array}$ & $\begin{array}{c}\text { Specimen } \\
\text { Length }\end{array}$ & $\begin{array}{c}67 \\
0.1000\end{array}$ & $\begin{array}{c}\text { Material } \\
\text { Scale }\end{array}$ & $\begin{array}{c}\text { H-451 } \\
1 "\end{array}$ & $5.00 \mathrm{E}-04$ & & & & & & & & \\
\hline $\begin{array}{c}\text { Temperature } \\
\left({ }^{\circ} \mathrm{C}\right) \\
\end{array}$ & Up & Down & $\begin{array}{l}\text { Delta } \\
\text { Down }\end{array}$ & $\begin{array}{l}\text { Down } \\
\text { length }\end{array}$ & CTE & CTEK & $\begin{array}{l}\text { Delta } \\
\text { CTEK }\end{array}$ & $\begin{array}{c}\text { CTE } \\
\text { adjusted }\end{array}$ & $\begin{array}{l}\text { Delta } \\
\text { Up }\end{array}$ & $\begin{array}{c}\text { Up } \\
\text { length }\end{array}$ & CTE & CTEK & $\begin{array}{l}\text { Delta } \\
\text { CTEK }\end{array}$ & $\begin{array}{c}\text { CTE } \\
\text { adjusted }\end{array}$ \\
\hline 0 & 0.10 & 0.35 & 0 & 0.1000 & & & & & 0 & 0.1000 & & & & \\
\hline 100 & 1.01 & 1.30 & 0.95 & 0.1005 & 4.75E-05 & & $-4.75 \mathrm{E}-05$ & $4.80 \mathrm{E}-05$ & 0.91 & 0.1005 & 4.55E-05 & & $-4.55 \mathrm{E}-05$ & 4.60E-05 \\
\hline 200 & 1.98 & 2.22 & 1.87 & 0.1009 & 4.67E-05 & & $-4.67 E-05$ & $4.72 \mathrm{E}-05$ & 1.88 & 0.1009 & $4.70 \mathrm{E}-05$ & & $-4.70 \mathrm{E}-05$ & 4.75E-05 \\
\hline 300 & 3.05 & 3.20 & 2.85 & 0.1014 & $4.75 \mathrm{E}-05$ & & $-4.75 E-05$ & $4.80 \mathrm{E}-05$ & 2.95 & 0.1015 & $4.92 \mathrm{E}-05$ & & $-4.92 \mathrm{E}-05$ & 4.97E-05 \\
\hline 400 & 4.02 & 4.22 & 3.87 & 0.1019 & 4.84E-05 & & $-4.84 \mathrm{E}-05$ & 4.89E-05 & 3.92 & 0.1020 & 4.90E-05 & & $-4.90 \mathrm{E}-05$ & 4.95E-05 \\
\hline 500 & 5.12 & 5.30 & 4.95 & 0.1025 & $4.95 \mathrm{E}-05$ & 4.87E-06 & $-4.46 \mathrm{E}-05$ & $5.00 \mathrm{E}-05$ & 5.02 & 0.1025 & $5.02 \mathrm{E}-05$ & 4.87E-06 & $-4.53 \mathrm{E}-05$ & 5.07E-05 \\
\hline
\end{tabular}

\begin{tabular}{|c|c|c|c|c|c|c|c|c|c|c|c|c|c|c|}
\hline $\begin{array}{l}\text { Capsule } \\
\text { Column }\end{array}$ & $\begin{array}{l}\text { OC2 } \\
\text { South }\end{array}$ & $\begin{array}{l}\text { Specimen } \\
\text { Length }\end{array}$ & $\begin{array}{c}73 \\
0.1000\end{array}$ & $\begin{array}{l}\text { Material } \\
\text { Scale }\end{array}$ & $\begin{array}{c}\text { H-451 } \\
1 "\end{array}$ & $5.00 \mathrm{E}-04$ & & & & & & & & \\
\hline $\begin{array}{c}\text { Temperature } \\
\left({ }^{\circ} \mathrm{C}\right)\end{array}$ & Up & Down & $\begin{array}{l}\text { Delta } \\
\text { Down }\end{array}$ & $\begin{array}{l}\text { Down } \\
\text { length }\end{array}$ & CTE & CTEK & $\begin{array}{l}\text { Delta } \\
\text { CTEK }\end{array}$ & $\begin{array}{c}\text { CTE } \\
\text { adjusted }\end{array}$ & $\begin{array}{l}\text { Delta } \\
\text { Up }\end{array}$ & $\begin{array}{c}\text { Up } \\
\text { length }\end{array}$ & CTE & CTEK & $\begin{array}{l}\text { Delta } \\
\text { CTEK }\end{array}$ & $\begin{array}{c}\text { CTE } \\
\text { adjusted }\end{array}$ \\
\hline 0 & 0.49 & 0.97 & 0 & 0.1000 & & & & & 0 & 0.1000 & & & & \\
\hline 100 & 0.75 & 1.22 & 0.25 & 0.1001 & $1.25 \mathrm{E}-05$ & & $-1.25 \mathrm{E}-05$ & $1.30 \mathrm{E}-05$ & 0.26 & 0.1001 & $1.30 \mathrm{E}-05$ & & $-1.30 \mathrm{E}-05$ & $1.35 \mathrm{E}-05$ \\
\hline 200 & 1.05 & 1.70 & 0.73 & 0.1004 & $1.82 \mathrm{E}-05$ & & $-1.82 \mathrm{E}-05$ & $1.87 \mathrm{E}-05$ & 0.56 & 0.1003 & $1.40 \mathrm{E}-05$ & & $-1.40 \mathrm{E}-05$ & $1.45 \mathrm{E}-05$ \\
\hline 300 & 1.58 & 2.02 & 1.05 & 0.1005 & $1.75 \mathrm{E}-05$ & & $-1.75 E-05$ & $1.80 \mathrm{E}-05$ & 1.09 & 0.1005 & $1.82 \mathrm{E}-05$ & & $-1.82 \mathrm{E}-05$ & $1.87 \mathrm{E}-05$ \\
\hline 400 & 2.12 & 2.60 & 1.63 & 0.1008 & $2.04 \mathrm{E}-05$ & & $-2.04 \mathrm{E}-05$ & $2.09 E-05$ & 1.63 & 0.1008 & $2.04 \mathrm{E}-05$ & & $-2.04 \mathrm{E}-05$ & 2.09E-05 \\
\hline 500 & 2.78 & 3.20 & 2.23 & 0.1011 & $2.23 \mathrm{E}-05$ & 4.87E-06 & $-1.74 \mathrm{E}-05$ & $2.28 \mathrm{E}-05$ & 2.29 & 0.1011 & $2.29 \mathrm{E}-05$ & $4.87 E-06$ & $-1.80 \mathrm{E}-05$ & $2.34 \mathrm{E}-05$ \\
\hline
\end{tabular}

\begin{tabular}{|c|c|c|c|c|c|c|c|c|c|c|c|c|c|c|}
\hline $\begin{array}{l}\text { Capsule } \\
\text { Column }\end{array}$ & $\begin{array}{c}\text { OC2 } \\
\text { South }\end{array}$ & $\begin{array}{c}\text { Specimen } \\
\text { Length }\end{array}$ & $\begin{array}{c}79 \\
0.1000 \\
\end{array}$ & $\begin{array}{c}\text { Material } \\
\text { Scale }\end{array}$ & $\begin{array}{c}\text { H-451 } \\
1 " \\
\end{array}$ & $5.00 \mathrm{E}-04$ & & & & & & & & \\
\hline $\begin{array}{c}\text { Temperature } \\
\left({ }^{\circ} \mathrm{C}\right)\end{array}$ & $U p$ & Down & $\begin{array}{l}\text { Delta } \\
\text { Down }\end{array}$ & $\begin{array}{l}\text { Down } \\
\text { length }\end{array}$ & CTE & CTEK & $\begin{array}{l}\text { Delta } \\
\text { CTEK }\end{array}$ & $\begin{array}{c}\text { CTE } \\
\text { adjusted }\end{array}$ & $\begin{array}{l}\text { Delta } \\
\text { Up }\end{array}$ & $\begin{array}{c}\text { Up } \\
\text { length }\end{array}$ & CTE & CTEK & $\begin{array}{l}\text { Delta } \\
\text { CTEK }\end{array}$ & $\begin{array}{c}\text { CTE } \\
\text { adjusted }\end{array}$ \\
\hline 0 & 1.00 & 1.55 & 0 & 0.1000 & & & & & 0 & 0.1000 & & & & \\
\hline 100 & 1.50 & 2.00 & 0.45 & 0.1002 & $2.25 \mathrm{E}-05$ & & $-2.25 E-05$ & $2.30 \mathrm{E}-05$ & 0.5 & 0.1003 & $2.50 \mathrm{E}-05$ & & $-2.50 \mathrm{E}-05$ & $2.55 \mathrm{E}-05$ \\
\hline 200 & 2.02 & 2.52 & 0.97 & 0.1005 & $2.43 \mathrm{E}-05$ & & $-2.43 E-05$ & $2.48 \mathrm{E}-05$ & 1.02 & 0.1005 & $2.55 \mathrm{E}-05$ & & $-2.55 \mathrm{E}-05$ & $2.60 \mathrm{E}-05$ \\
\hline 300 & 2.68 & 3.20 & 1.65 & 0.1008 & $2.75 \mathrm{E}-05$ & & $-2.75 E-05$ & $2.80 \mathrm{E}-05$ & 1.68 & 0.1008 & $2.80 \mathrm{E}-05$ & & $-2.80 \mathrm{E}-05$ & $2.85 \mathrm{E}-05$ \\
\hline 400 & 3.40 & 3.95 & 2.4 & 0.1012 & $3.00 \mathrm{E}-05$ & & $-3.00 \mathrm{E}-05$ & $3.05 \mathrm{E}-05$ & 2.4 & 0.1012 & $3.00 \mathrm{E}-05$ & & $-3.00 \mathrm{E}-05$ & 3.05E-05 \\
\hline 500 & 4.20 & 4.65 & 3.1 & 0.1016 & $3.10 \mathrm{E}-05$ & 4.87E-06 & $-2.61 \mathrm{E}-05$ & $3.15 \mathrm{E}-05$ & 3.2 & 0.1016 & $3.20 \mathrm{E}-05$ & 4.87E-06 & $-2.71 \mathrm{E}-05$ & $3.25 \mathrm{E}-05$ \\
\hline
\end{tabular}




\begin{tabular}{|c|c|c|c|c|c|c|c|c|c|c|c|c|c|c|}
\hline $\begin{array}{l}\text { Capsule } \\
\text { Column }\end{array}$ & $\begin{array}{l}\text { OC2 } \\
\text { South }\end{array}$ & $\begin{array}{c}\text { Specimen } \\
\text { Length }\end{array}$ & $\begin{array}{c}67 \\
0.1000\end{array}$ & $\begin{array}{c}\text { Material } \\
\text { Scale }\end{array}$ & $\begin{array}{c}\text { H-451 } \\
1 "\end{array}$ & $5.00 \mathrm{E}-04$ & & & & & & & & \\
\hline $\begin{array}{c}\text { Temperature } \\
\left({ }^{\circ} \mathrm{C}\right) \\
\end{array}$ & Up & Down & $\begin{array}{l}\text { Delta } \\
\text { Down }\end{array}$ & $\begin{array}{l}\text { Down } \\
\text { length }\end{array}$ & CTE & CTEK & $\begin{array}{l}\text { Delta } \\
\text { CTEK }\end{array}$ & $\begin{array}{c}\text { CTE } \\
\text { adjusted }\end{array}$ & $\begin{array}{l}\text { Delta } \\
\text { Up }\end{array}$ & $\begin{array}{c}\text { Up } \\
\text { length }\end{array}$ & CTE & CTEK & $\begin{array}{l}\text { Delta } \\
\text { CTEK }\end{array}$ & $\begin{array}{c}\text { CTE } \\
\text { adjusted }\end{array}$ \\
\hline 0 & 0.10 & 0.35 & 0 & 0.1000 & & & & & 0 & 0.1000 & & & & \\
\hline 100 & 1.01 & 1.30 & 0.95 & 0.1005 & 4.75E-05 & & $-4.75 \mathrm{E}-05$ & $4.80 \mathrm{E}-05$ & 0.91 & 0.1005 & 4.55E-05 & & $-4.55 \mathrm{E}-05$ & 4.60E-05 \\
\hline 200 & 1.98 & 2.22 & 1.87 & 0.1009 & 4.67E-05 & & $-4.67 E-05$ & $4.72 \mathrm{E}-05$ & 1.88 & 0.1009 & $4.70 \mathrm{E}-05$ & & $-4.70 \mathrm{E}-05$ & 4.75E-05 \\
\hline 300 & 3.05 & 3.20 & 2.85 & 0.1014 & $4.75 \mathrm{E}-05$ & & $-4.75 E-05$ & $4.80 \mathrm{E}-05$ & 2.95 & 0.1015 & $4.92 \mathrm{E}-05$ & & $-4.92 \mathrm{E}-05$ & 4.97E-05 \\
\hline 400 & 4.02 & 4.22 & 3.87 & 0.1019 & 4.84E-05 & & $-4.84 \mathrm{E}-05$ & 4.89E-05 & 3.92 & 0.1020 & 4.90E-05 & & $-4.90 \mathrm{E}-05$ & 4.95E-05 \\
\hline 500 & 5.12 & 5.30 & 4.95 & 0.1025 & $4.95 \mathrm{E}-05$ & 4.87E-06 & $-4.46 \mathrm{E}-05$ & $5.00 \mathrm{E}-05$ & 5.02 & 0.1025 & $5.02 \mathrm{E}-05$ & 4.87E-06 & $-4.53 \mathrm{E}-05$ & 5.07E-05 \\
\hline
\end{tabular}

\begin{tabular}{|c|c|c|c|c|c|c|c|c|c|c|c|c|c|c|}
\hline $\begin{array}{l}\text { Capsule } \\
\text { Column }\end{array}$ & $\begin{array}{l}\text { OC2 } \\
\text { South }\end{array}$ & $\begin{array}{l}\text { Specimen } \\
\text { Length }\end{array}$ & $\begin{array}{c}73 \\
0.1000\end{array}$ & $\begin{array}{l}\text { Material } \\
\text { Scale }\end{array}$ & $\begin{array}{c}\text { H-451 } \\
1 "\end{array}$ & $5.00 \mathrm{E}-04$ & & & & & & & & \\
\hline $\begin{array}{c}\text { Temperature } \\
\left({ }^{\circ} \mathrm{C}\right)\end{array}$ & Up & Down & $\begin{array}{l}\text { Delta } \\
\text { Down }\end{array}$ & $\begin{array}{l}\text { Down } \\
\text { length }\end{array}$ & CTE & CTEK & $\begin{array}{l}\text { Delta } \\
\text { CTEK }\end{array}$ & $\begin{array}{c}\text { CTE } \\
\text { adjusted }\end{array}$ & $\begin{array}{l}\text { Delta } \\
\text { Up }\end{array}$ & $\begin{array}{c}\text { Up } \\
\text { length }\end{array}$ & CTE & CTEK & $\begin{array}{l}\text { Delta } \\
\text { CTEK }\end{array}$ & $\begin{array}{c}\text { CTE } \\
\text { adjusted }\end{array}$ \\
\hline 0 & 0.49 & 0.97 & 0 & 0.1000 & & & & & 0 & 0.1000 & & & & \\
\hline 100 & 0.75 & 1.22 & 0.25 & 0.1001 & $1.25 \mathrm{E}-05$ & & $-1.25 \mathrm{E}-05$ & $1.30 \mathrm{E}-05$ & 0.26 & 0.1001 & $1.30 \mathrm{E}-05$ & & $-1.30 \mathrm{E}-05$ & $1.35 \mathrm{E}-05$ \\
\hline 200 & 1.05 & 1.70 & 0.73 & 0.1004 & $1.82 \mathrm{E}-05$ & & $-1.82 \mathrm{E}-05$ & $1.87 \mathrm{E}-05$ & 0.56 & 0.1003 & $1.40 \mathrm{E}-05$ & & $-1.40 \mathrm{E}-05$ & $1.45 \mathrm{E}-05$ \\
\hline 300 & 1.58 & 2.02 & 1.05 & 0.1005 & $1.75 \mathrm{E}-05$ & & $-1.75 E-05$ & $1.80 \mathrm{E}-05$ & 1.09 & 0.1005 & $1.82 \mathrm{E}-05$ & & $-1.82 \mathrm{E}-05$ & $1.87 \mathrm{E}-05$ \\
\hline 400 & 2.12 & 2.60 & 1.63 & 0.1008 & $2.04 \mathrm{E}-05$ & & $-2.04 \mathrm{E}-05$ & $2.09 E-05$ & 1.63 & 0.1008 & $2.04 \mathrm{E}-05$ & & $-2.04 \mathrm{E}-05$ & 2.09E-05 \\
\hline 500 & 2.78 & 3.20 & 2.23 & 0.1011 & $2.23 \mathrm{E}-05$ & 4.87E-06 & $-1.74 \mathrm{E}-05$ & $2.28 \mathrm{E}-05$ & 2.29 & 0.1011 & $2.29 \mathrm{E}-05$ & $4.87 E-06$ & $-1.80 \mathrm{E}-05$ & $2.34 \mathrm{E}-05$ \\
\hline
\end{tabular}

\begin{tabular}{|c|c|c|c|c|c|c|c|c|c|c|c|c|c|c|}
\hline $\begin{array}{l}\text { Capsule } \\
\text { Column }\end{array}$ & $\begin{array}{c}\text { OC2 } \\
\text { South }\end{array}$ & $\begin{array}{c}\text { Specimen } \\
\text { Length }\end{array}$ & $\begin{array}{c}79 \\
0.1000 \\
\end{array}$ & $\begin{array}{c}\text { Material } \\
\text { Scale }\end{array}$ & $\begin{array}{c}\text { H-451 } \\
1 " \\
\end{array}$ & $5.00 \mathrm{E}-04$ & & & & & & & & \\
\hline $\begin{array}{c}\text { Temperature } \\
\left({ }^{\circ} \mathrm{C}\right)\end{array}$ & $U p$ & Down & $\begin{array}{l}\text { Delta } \\
\text { Down }\end{array}$ & $\begin{array}{l}\text { Down } \\
\text { length }\end{array}$ & CTE & CTEK & $\begin{array}{l}\text { Delta } \\
\text { CTEK }\end{array}$ & $\begin{array}{c}\text { CTE } \\
\text { adjusted }\end{array}$ & $\begin{array}{l}\text { Delta } \\
\text { Up }\end{array}$ & $\begin{array}{c}\text { Up } \\
\text { length }\end{array}$ & CTE & CTEK & $\begin{array}{l}\text { Delta } \\
\text { CTEK }\end{array}$ & $\begin{array}{c}\text { CTE } \\
\text { adjusted }\end{array}$ \\
\hline 0 & 1.00 & 1.55 & 0 & 0.1000 & & & & & 0 & 0.1000 & & & & \\
\hline 100 & 1.50 & 2.00 & 0.45 & 0.1002 & $2.25 \mathrm{E}-05$ & & $-2.25 E-05$ & $2.30 \mathrm{E}-05$ & 0.5 & 0.1003 & $2.50 \mathrm{E}-05$ & & $-2.50 \mathrm{E}-05$ & $2.55 \mathrm{E}-05$ \\
\hline 200 & 2.02 & 2.52 & 0.97 & 0.1005 & $2.43 \mathrm{E}-05$ & & $-2.43 E-05$ & $2.48 \mathrm{E}-05$ & 1.02 & 0.1005 & $2.55 \mathrm{E}-05$ & & $-2.55 \mathrm{E}-05$ & $2.60 \mathrm{E}-05$ \\
\hline 300 & 2.68 & 3.20 & 1.65 & 0.1008 & $2.75 \mathrm{E}-05$ & & $-2.75 E-05$ & $2.80 \mathrm{E}-05$ & 1.68 & 0.1008 & $2.80 \mathrm{E}-05$ & & $-2.80 \mathrm{E}-05$ & $2.85 \mathrm{E}-05$ \\
\hline 400 & 3.40 & 3.95 & 2.4 & 0.1012 & $3.00 \mathrm{E}-05$ & & $-3.00 \mathrm{E}-05$ & $3.05 \mathrm{E}-05$ & 2.4 & 0.1012 & $3.00 \mathrm{E}-05$ & & $-3.00 \mathrm{E}-05$ & 3.05E-05 \\
\hline 500 & 4.20 & 4.65 & 3.1 & 0.1016 & $3.10 \mathrm{E}-05$ & 4.87E-06 & $-2.61 \mathrm{E}-05$ & $3.15 \mathrm{E}-05$ & 3.2 & 0.1016 & $3.20 \mathrm{E}-05$ & 4.87E-06 & $-2.71 \mathrm{E}-05$ & $3.25 \mathrm{E}-05$ \\
\hline
\end{tabular}




\begin{tabular}{|c|c|c|c|c|c|c|c|c|c|c|c|c|c|c|}
\hline $\begin{array}{l}\text { Capsule } \\
\text { Column }\end{array}$ & $\begin{array}{l}\text { OC2 } \\
\text { South }\end{array}$ & $\begin{array}{c}\text { Specimen } \\
\text { Length }\end{array}$ & $\begin{array}{c}67 \\
0.1000\end{array}$ & $\begin{array}{c}\text { Material } \\
\text { Scale }\end{array}$ & $\begin{array}{c}\text { H-451 } \\
1 "\end{array}$ & $5.00 \mathrm{E}-04$ & & & & & & & & \\
\hline $\begin{array}{c}\text { Temperature } \\
\left({ }^{\circ} \mathrm{C}\right) \\
\end{array}$ & Up & Down & $\begin{array}{l}\text { Delta } \\
\text { Down }\end{array}$ & $\begin{array}{l}\text { Down } \\
\text { length }\end{array}$ & CTE & CTEK & $\begin{array}{l}\text { Delta } \\
\text { CTEK }\end{array}$ & $\begin{array}{c}\text { CTE } \\
\text { adjusted }\end{array}$ & $\begin{array}{l}\text { Delta } \\
\text { Up }\end{array}$ & $\begin{array}{c}\text { Up } \\
\text { length }\end{array}$ & CTE & CTEK & $\begin{array}{l}\text { Delta } \\
\text { CTEK }\end{array}$ & $\begin{array}{c}\text { CTE } \\
\text { adjusted }\end{array}$ \\
\hline 0 & 0.10 & 0.35 & 0 & 0.1000 & & & & & 0 & 0.1000 & & & & \\
\hline 100 & 1.01 & 1.30 & 0.95 & 0.1005 & 4.75E-05 & & $-4.75 \mathrm{E}-05$ & $4.80 \mathrm{E}-05$ & 0.91 & 0.1005 & 4.55E-05 & & $-4.55 \mathrm{E}-05$ & 4.60E-05 \\
\hline 200 & 1.98 & 2.22 & 1.87 & 0.1009 & 4.67E-05 & & $-4.67 E-05$ & $4.72 \mathrm{E}-05$ & 1.88 & 0.1009 & $4.70 \mathrm{E}-05$ & & $-4.70 \mathrm{E}-05$ & 4.75E-05 \\
\hline 300 & 3.05 & 3.20 & 2.85 & 0.1014 & $4.75 \mathrm{E}-05$ & & $-4.75 E-05$ & $4.80 \mathrm{E}-05$ & 2.95 & 0.1015 & $4.92 \mathrm{E}-05$ & & $-4.92 \mathrm{E}-05$ & 4.97E-05 \\
\hline 400 & 4.02 & 4.22 & 3.87 & 0.1019 & 4.84E-05 & & $-4.84 \mathrm{E}-05$ & 4.89E-05 & 3.92 & 0.1020 & 4.90E-05 & & $-4.90 \mathrm{E}-05$ & 4.95E-05 \\
\hline 500 & 5.12 & 5.30 & 4.95 & 0.1025 & $4.95 \mathrm{E}-05$ & 4.87E-06 & $-4.46 \mathrm{E}-05$ & $5.00 \mathrm{E}-05$ & 5.02 & 0.1025 & $5.02 \mathrm{E}-05$ & 4.87E-06 & $-4.53 \mathrm{E}-05$ & 5.07E-05 \\
\hline
\end{tabular}

\begin{tabular}{|c|c|c|c|c|c|c|c|c|c|c|c|c|c|c|}
\hline $\begin{array}{l}\text { Capsule } \\
\text { Column }\end{array}$ & $\begin{array}{l}\text { OC2 } \\
\text { South }\end{array}$ & $\begin{array}{l}\text { Specimen } \\
\text { Length }\end{array}$ & $\begin{array}{c}73 \\
0.1000\end{array}$ & $\begin{array}{l}\text { Material } \\
\text { Scale }\end{array}$ & $\begin{array}{c}\text { H-451 } \\
1 "\end{array}$ & $5.00 \mathrm{E}-04$ & & & & & & & & \\
\hline $\begin{array}{c}\text { Temperature } \\
\left({ }^{\circ} \mathrm{C}\right)\end{array}$ & Up & Down & $\begin{array}{l}\text { Delta } \\
\text { Down }\end{array}$ & $\begin{array}{l}\text { Down } \\
\text { length }\end{array}$ & CTE & CTEK & $\begin{array}{l}\text { Delta } \\
\text { CTEK }\end{array}$ & $\begin{array}{c}\text { CTE } \\
\text { adjusted }\end{array}$ & $\begin{array}{l}\text { Delta } \\
\text { Up }\end{array}$ & $\begin{array}{c}\text { Up } \\
\text { length }\end{array}$ & CTE & CTEK & $\begin{array}{l}\text { Delta } \\
\text { CTEK }\end{array}$ & $\begin{array}{c}\text { CTE } \\
\text { adjusted }\end{array}$ \\
\hline 0 & 0.49 & 0.97 & 0 & 0.1000 & & & & & 0 & 0.1000 & & & & \\
\hline 100 & 0.75 & 1.22 & 0.25 & 0.1001 & $1.25 \mathrm{E}-05$ & & $-1.25 \mathrm{E}-05$ & $1.30 \mathrm{E}-05$ & 0.26 & 0.1001 & $1.30 \mathrm{E}-05$ & & $-1.30 \mathrm{E}-05$ & $1.35 \mathrm{E}-05$ \\
\hline 200 & 1.05 & 1.70 & 0.73 & 0.1004 & $1.82 \mathrm{E}-05$ & & $-1.82 \mathrm{E}-05$ & $1.87 \mathrm{E}-05$ & 0.56 & 0.1003 & $1.40 \mathrm{E}-05$ & & $-1.40 \mathrm{E}-05$ & $1.45 \mathrm{E}-05$ \\
\hline 300 & 1.58 & 2.02 & 1.05 & 0.1005 & $1.75 \mathrm{E}-05$ & & $-1.75 E-05$ & $1.80 \mathrm{E}-05$ & 1.09 & 0.1005 & $1.82 \mathrm{E}-05$ & & $-1.82 \mathrm{E}-05$ & $1.87 \mathrm{E}-05$ \\
\hline 400 & 2.12 & 2.60 & 1.63 & 0.1008 & $2.04 \mathrm{E}-05$ & & $-2.04 \mathrm{E}-05$ & $2.09 E-05$ & 1.63 & 0.1008 & $2.04 \mathrm{E}-05$ & & $-2.04 \mathrm{E}-05$ & 2.09E-05 \\
\hline 500 & 2.78 & 3.20 & 2.23 & 0.1011 & $2.23 \mathrm{E}-05$ & 4.87E-06 & $-1.74 \mathrm{E}-05$ & $2.28 \mathrm{E}-05$ & 2.29 & 0.1011 & $2.29 \mathrm{E}-05$ & $4.87 E-06$ & $-1.80 \mathrm{E}-05$ & $2.34 \mathrm{E}-05$ \\
\hline
\end{tabular}

\begin{tabular}{|c|c|c|c|c|c|c|c|c|c|c|c|c|c|c|}
\hline $\begin{array}{l}\text { Capsule } \\
\text { Column }\end{array}$ & $\begin{array}{c}\text { OC2 } \\
\text { South }\end{array}$ & $\begin{array}{c}\text { Specimen } \\
\text { Length }\end{array}$ & $\begin{array}{c}79 \\
0.1000 \\
\end{array}$ & $\begin{array}{c}\text { Material } \\
\text { Scale }\end{array}$ & $\begin{array}{c}\text { H-451 } \\
1 " \\
\end{array}$ & $5.00 \mathrm{E}-04$ & & & & & & & & \\
\hline $\begin{array}{c}\text { Temperature } \\
\left({ }^{\circ} \mathrm{C}\right)\end{array}$ & $U p$ & Down & $\begin{array}{l}\text { Delta } \\
\text { Down }\end{array}$ & $\begin{array}{l}\text { Down } \\
\text { length }\end{array}$ & CTE & CTEK & $\begin{array}{l}\text { Delta } \\
\text { CTEK }\end{array}$ & $\begin{array}{c}\text { CTE } \\
\text { adjusted }\end{array}$ & $\begin{array}{l}\text { Delta } \\
\text { Up }\end{array}$ & $\begin{array}{c}\text { Up } \\
\text { length }\end{array}$ & CTE & CTEK & $\begin{array}{l}\text { Delta } \\
\text { CTEK }\end{array}$ & $\begin{array}{c}\text { CTE } \\
\text { adjusted }\end{array}$ \\
\hline 0 & 1.00 & 1.55 & 0 & 0.1000 & & & & & 0 & 0.1000 & & & & \\
\hline 100 & 1.50 & 2.00 & 0.45 & 0.1002 & $2.25 \mathrm{E}-05$ & & $-2.25 E-05$ & $2.30 \mathrm{E}-05$ & 0.5 & 0.1003 & $2.50 \mathrm{E}-05$ & & $-2.50 \mathrm{E}-05$ & $2.55 \mathrm{E}-05$ \\
\hline 200 & 2.02 & 2.52 & 0.97 & 0.1005 & $2.43 \mathrm{E}-05$ & & $-2.43 E-05$ & $2.48 \mathrm{E}-05$ & 1.02 & 0.1005 & $2.55 \mathrm{E}-05$ & & $-2.55 \mathrm{E}-05$ & $2.60 \mathrm{E}-05$ \\
\hline 300 & 2.68 & 3.20 & 1.65 & 0.1008 & $2.75 \mathrm{E}-05$ & & $-2.75 E-05$ & $2.80 \mathrm{E}-05$ & 1.68 & 0.1008 & $2.80 \mathrm{E}-05$ & & $-2.80 \mathrm{E}-05$ & $2.85 \mathrm{E}-05$ \\
\hline 400 & 3.40 & 3.95 & 2.4 & 0.1012 & $3.00 \mathrm{E}-05$ & & $-3.00 \mathrm{E}-05$ & $3.05 \mathrm{E}-05$ & 2.4 & 0.1012 & $3.00 \mathrm{E}-05$ & & $-3.00 \mathrm{E}-05$ & 3.05E-05 \\
\hline 500 & 4.20 & 4.65 & 3.1 & 0.1016 & $3.10 \mathrm{E}-05$ & 4.87E-06 & $-2.61 \mathrm{E}-05$ & $3.15 \mathrm{E}-05$ & 3.2 & 0.1016 & $3.20 \mathrm{E}-05$ & 4.87E-06 & $-2.71 \mathrm{E}-05$ & $3.25 \mathrm{E}-05$ \\
\hline
\end{tabular}




\begin{tabular}{|c|c|c|c|c|c|c|c|c|c|c|c|c|c|c|}
\hline $\begin{array}{l}\text { Capsule } \\
\text { Column }\end{array}$ & $\begin{array}{l}\text { OC2 } \\
\text { North }\end{array}$ & $\begin{array}{l}\text { Specimen } \\
\text { Length }\end{array}$ & $\begin{array}{c}83 \\
0.9992\end{array}$ & $\begin{array}{c}\text { Material } \\
\text { Scale }\end{array}$ & $\begin{array}{c}\text { H-451 } \\
1 "\end{array}$ & $5.00 \mathrm{E}-04$ & & & & & & & & \\
\hline $\begin{array}{c}\text { Temperature } \\
\left({ }^{\circ} \mathrm{C}\right) \\
\end{array}$ & Up & Down & $\begin{array}{l}\text { Delta } \\
\text { Down }\end{array}$ & $\begin{array}{l}\text { Down } \\
\text { length }\end{array}$ & CTE & CTEK & $\begin{array}{l}\text { Delta } \\
\text { CTEK }\end{array}$ & $\begin{array}{c}\text { CTE } \\
\text { adjusted }\end{array}$ & $\begin{array}{l}\text { Delta } \\
\text { Up }\end{array}$ & $\begin{array}{c}\text { Up } \\
\text { length }\end{array}$ & CTE & CTEK & $\begin{array}{l}\text { Delta } \\
\text { CTEK }\end{array}$ & $\begin{array}{c}\text { CTE } \\
\text { adjusted }\end{array}$ \\
\hline 0 & 0.73 & 1.30 & 0 & 0.9992 & & & & & 0 & 0.9992 & & & & \\
\hline 100 & 1.27 & 1.76 & 0.46 & 0.9994 & $2.30 \mathrm{E}-06$ & 2.80E-06 & $4.98 \mathrm{E}-07$ & $2.80 \mathrm{E}-06$ & 0.54 & 0.9995 & $2.70 \mathrm{E}-06$ & $2.70 E+00$ & $2.70 E+00$ & $3.20 \mathrm{E}-06$ \\
\hline 200 & 1.80 & 2.20 & 0.9 & 0.9997 & $2.25 \mathrm{E}-06$ & 2.71E-06 & $4.58 \mathrm{E}-07$ & $2.75 \mathrm{E}-06$ & 1.07 & 0.9997 & $2.68 \mathrm{E}-06$ & $2.94 E+00$ & $2.93 \mathrm{E}+00$ & $3.18 \mathrm{E}-06$ \\
\hline 300 & 2.45 & 2.89 & 1.59 & 1.0000 & $2.65 \mathrm{E}-06$ & $3.13 E-06$ & $4.78 \mathrm{E}-07$ & $3.15 \mathrm{E}-06$ & 1.72 & 1.0001 & $2.87 \mathrm{E}-06$ & $3.18 E+00$ & $3.18 \mathrm{E}+00$ & 3.37E-06 \\
\hline 400 & 3.20 & 3.63 & 2.33 & 1.0004 & $2.91 \mathrm{E}-06$ & $3.38 E-06$ & 4.67E-07 & $3.41 \mathrm{E}-06$ & 2.47 & 1.0004 & $3.09 \mathrm{E}-06$ & $3.40 E+00$ & $3.39 \mathrm{E}+00$ & $3.59 \mathrm{E}-06$ \\
\hline 500 & 4.00 & 4.32 & 3.02 & 1.0007 & $3.02 \mathrm{E}-06$ & $3.49 E-06$ & $6.98 \mathrm{E}-07$ & $3.52 \mathrm{E}-06$ & 3.27 & 1.0008 & $3.27 \mathrm{E}-06$ & $3.61 E+00$ & $3.61 \mathrm{E}+00$ & 3.77E-06 \\
\hline
\end{tabular}

\begin{tabular}{|c|c|c|c|c|c|c|c|c|c|c|c|c|c|c|}
\hline $\begin{array}{l}\text { Capsule } \\
\text { Column }\end{array}$ & $\begin{array}{l}\text { OC2 } \\
\text { North }\end{array}$ & $\begin{array}{c}\text { Specimen } \\
\text { Length }\end{array}$ & $\begin{array}{c}88 \\
0.9977\end{array}$ & $\begin{array}{c}\text { Material } \\
\text { Scale }\end{array}$ & $\begin{array}{c}\text { H-451 } \\
1 "\end{array}$ & $5.00 \mathrm{E}-04$ & & & & & & & & \\
\hline $\begin{array}{c}\text { Temperature } \\
\left({ }^{\circ} \mathrm{C}\right)\end{array}$ & Up & Down & $\begin{array}{l}\text { Delta } \\
\text { Down }\end{array}$ & $\begin{array}{l}\text { Down } \\
\text { length }\end{array}$ & CTE & CTEK & $\begin{array}{l}\text { Delta } \\
\text { CTEK }\end{array}$ & $\begin{array}{c}\text { CTE } \\
\text { adjusted }\end{array}$ & $\begin{array}{l}\text { Delta } \\
\text { Up }\end{array}$ & $\begin{array}{c}\text { Up } \\
\text { length }\end{array}$ & CTE & CTEK & $\begin{array}{l}\text { Delta } \\
\text { CTEK }\end{array}$ & $\begin{array}{c}\text { CTE } \\
\text { adjusted }\end{array}$ \\
\hline 0 & 0.66 & 1.20 & 0 & 0.9977 & & & & & 0 & 0.9977 & & & & \\
\hline 100 & 1.10 & 1.48 & 0.28 & 0.9978 & $1.40 \mathrm{E}-06$ & $1.90 E-06$ & 4.97E-07 & $1.90 \mathrm{E}-06$ & 0.44 & 0.9979 & $2.21 \mathrm{E}-06$ & $2.70 E-06$ & 4.95E-07 & $2.71 \mathrm{E}-06$ \\
\hline 200 & 1.65 & 1.90 & 0.7 & 0.9981 & $1.75 \mathrm{E}-06$ & $2.21 E-06$ & 4.56E-07 & $2.25 \mathrm{E}-06$ & 0.99 & 0.9982 & $2.48 \mathrm{E}-06$ & $2.94 E-06$ & 4.54E-07 & $2.98 \mathrm{E}-06$ \\
\hline 300 & 2.28 & 2.60 & 1.4 & 0.9984 & $2.34 \mathrm{E}-06$ & $2.81 E-06$ & 4.74E-07 & $2.84 \mathrm{E}-06$ & 1.62 & 0.9985 & $2.71 \mathrm{E}-06$ & $3.18 E-06$ & 4.74E-07 & $3.21 \mathrm{E}-06$ \\
\hline 400 & 3.00 & 3.35 & 2.15 & 0.9988 & $2.69 \mathrm{E}-06$ & $3.16 E-06$ & 4.63E-07 & 3.19E-06 & 2.34 & 0.9989 & 2.93E-06 & $3.40 E-06$ & 4.63E-07 & $3.43 \mathrm{E}-06$ \\
\hline 500 & 3.80 & 4.10 & 2.9 & 0.9992 & $2.91 \mathrm{E}-06$ & $3.37 E-06$ & $5.73 \mathrm{E}-07$ & $3.41 \mathrm{E}-06$ & 3.14 & 0.9993 & $3.15 \mathrm{E}-06$ & $3.61 E-06$ & 3.33E-07 & 3.65E-06 \\
\hline
\end{tabular}

\begin{tabular}{|c|c|c|c|c|c|c|c|c|c|c|c|c|c|c|}
\hline $\begin{array}{l}\text { Capsule } \\
\text { Column }\end{array}$ & $\begin{array}{c}\text { OC2 } \\
\text { North }\end{array}$ & $\begin{array}{c}\text { Specimen } \\
\text { Length }\end{array}$ & $\begin{array}{c}90 \\
0.9983\end{array}$ & $\begin{array}{c}\text { Material } \\
\text { Scale }\end{array}$ & $\begin{array}{c}\text { H-451 } \\
1 "\end{array}$ & $5.00 \mathrm{E}-04$ & & & & & & & & \\
\hline $\begin{array}{c}\text { Temperature } \\
\left({ }^{\circ} \mathrm{C}\right)\end{array}$ & Up & Down & $\begin{array}{l}\text { Delta } \\
\text { Down }\end{array}$ & $\begin{array}{l}\text { Down } \\
\text { length }\end{array}$ & CTE & CTEK & $\begin{array}{l}\text { Delta } \\
\text { CTEK }\end{array}$ & $\begin{array}{c}\text { CTE } \\
\text { adjusted }\end{array}$ & $\begin{array}{l}\text { Delta } \\
\text { Up }\end{array}$ & $\begin{array}{c}\text { Up } \\
\text { length }\end{array}$ & CTE & CTEK & $\begin{array}{l}\text { Delta } \\
\text { CTEK }\end{array}$ & $\begin{array}{c}\text { CTE } \\
\text { adjusted }\end{array}$ \\
\hline 0 & 0.40 & 0.98 & 0 & 0.9983 & & & & & 0 & 0.9983 & & & & \\
\hline 100 & 1.00 & 1.38 & 0.4 & 0.9985 & $2.00 \mathrm{E}-06$ & & $-2.00 \mathrm{E}-06$ & $2.50 \mathrm{E}-06$ & 0.6 & 0.9986 & $3.01 \mathrm{E}-06$ & & $-3.01 E-06$ & $3.51 \mathrm{E}-06$ \\
\hline 200 & 1.45 & 1.81 & 0.83 & 0.9987 & $2.08 \mathrm{E}-06$ & & $-2.08 \mathrm{E}-06$ & $2.58 \mathrm{E}-06$ & 1.05 & 0.9988 & $2.63 \mathrm{E}-06$ & & $-2.63 E-06$ & $3.13 \mathrm{E}-06$ \\
\hline 300 & 2.10 & 2.50 & 1.52 & 0.9991 & $2.54 \mathrm{E}-06$ & & $-2.54 \mathrm{E}-06$ & $3.04 \mathrm{E}-06$ & 1.7 & 0.9992 & $2.84 \mathrm{E}-06$ & & $-2.84 \mathrm{E}-06$ & 3.34E-06 \\
\hline 400 & 2.80 & 3.30 & 2.32 & 0.9995 & $2.90 \mathrm{E}-06$ & & $-2.90 \mathrm{E}-06$ & $3.40 \mathrm{E}-06$ & 2.4 & 0.9995 & 3.01E-06 & & $-3.01 \mathrm{E}-06$ & $3.51 \mathrm{E}-06$ \\
\hline 500 & 3.60 & 4.03 & 3.05 & 0.9998 & $3.06 \mathrm{E}-06$ & $5.24 E-06$ & $2.18 \mathrm{E}-06$ & $3.56 \mathrm{E}-06$ & 3.2 & 0.9999 & $3.21 \mathrm{E}-06$ & $5.24 E-06$ & $2.03 \mathrm{E}-06$ & $3.71 \mathrm{E}-06$ \\
\hline
\end{tabular}


OC4 Dilatometer Data 


\begin{tabular}{|c|c|c|c|c|c|c|c|c|c|c|c|c|c|c|}
\hline $\begin{array}{l}\text { Capsule } \\
\text { Column }\end{array}$ & $\begin{array}{l}\text { OC4 } \\
\text { West }\end{array}$ & $\begin{array}{c}\text { Specimen } \\
\text { Length }\end{array}$ & $\begin{array}{c}10 \\
0.9883\end{array}$ & $\begin{array}{c}\text { Material } \\
\text { Scale }\end{array}$ & $\begin{array}{c}\text { H-451 } \\
1 "\end{array}$ & $5.00 \mathrm{E}-04$ & & & & & & & & \\
\hline $\begin{array}{c}\text { Temperature } \\
\left({ }^{\circ} \mathrm{C}\right) \\
\end{array}$ & Up & Down & $\begin{array}{l}\text { Delta } \\
\text { Down }\end{array}$ & $\begin{array}{l}\text { Down } \\
\text { length }\end{array}$ & CTE & CTEK & $\begin{array}{l}\text { Delta } \\
\text { CTEK }\end{array}$ & $\begin{array}{c}\text { CTE } \\
\text { adjusted }\end{array}$ & $\begin{array}{l}\text { Delta } \\
\text { Up }\end{array}$ & $\begin{array}{c}\text { Up } \\
\text { length }\end{array}$ & CTE & CTEK & $\begin{array}{l}\text { Delta } \\
\text { CTEK }\end{array}$ & $\begin{array}{c}\text { CTE } \\
\text { adjusted }\end{array}$ \\
\hline 0 & 1.13 & 1.50 & 0 & 0.9883 & & & & & 0 & 0.9883 & & & & \\
\hline 100 & 1.98 & 2.09 & 0.59 & 0.9886 & $2.98 \mathrm{E}-06$ & $3.50 E+00$ & $3.50 \mathrm{E}+00$ & $3.48 \mathrm{E}-06$ & 0.85 & 0.9887 & $4.30 \mathrm{E}-06$ & $4.80 E+00$ & $4.80 \mathrm{E}+00$ & 4.80E-06 \\
\hline 200 & 2.76 & 2.72 & 1.22 & 0.9889 & $3.09 \mathrm{E}-06$ & $3.60 E+00$ & $3.60 \mathrm{E}+00$ & $3.59 \mathrm{E}-06$ & 1.63 & 0.9891 & $4.12 \mathrm{E}-06$ & $4.62 E+00$ & $4.62 \mathrm{E}+00$ & 4.62E-06 \\
\hline 300 & 3.50 & 3.43 & 1.93 & 0.9893 & $3.25 \mathrm{E}-06$ & $3.77 E+00$ & $3.77 E+00$ & $3.75 \mathrm{E}-06$ & 2.37 & 0.9895 & $4.00 \mathrm{E}-06$ & $4.50 E+00$ & $4.50 \mathrm{E}+00$ & 4.50E-06 \\
\hline 400 & 4.21 & 4.28 & 2.78 & 0.9897 & $3.52 \mathrm{E}-06$ & $4.02 E+00$ & $4.02 \mathrm{E}+00$ & $4.02 \mathrm{E}-06$ & 3.08 & 0.9898 & $3.90 \mathrm{E}-06$ & $4.40 E+00$ & $4.40 \mathrm{E}+00$ & 4.40E-06 \\
\hline 500 & 5.17 & 5.34 & 3.84 & 0.9902 & $3.89 \mathrm{E}-06$ & $4.39 E+00$ & $4.39 \mathrm{E}+00$ & 4.39E-06 & 4.04 & 0.9903 & 4.09E-06 & $4.59 E+00$ & $4.59 \mathrm{E}+00$ & 4.59E-06 \\
\hline
\end{tabular}

\begin{tabular}{|c|c|c|c|c|c|c|c|c|c|c|c|c|c|c|}
\hline $\begin{array}{l}\text { Capsule } \\
\text { Column }\end{array}$ & $\begin{array}{l}\text { OC4 } \\
\text { West }\end{array}$ & $\begin{array}{l}\text { Specimen } \\
\text { Length }\end{array}$ & $\begin{array}{c}16 \\
0.9825\end{array}$ & $\begin{array}{l}\text { Material } \\
\text { Scale }\end{array}$ & $\begin{array}{l}\text { H-451 } \\
1 "\end{array}$ & $5.00 \mathrm{E}-04$ & & & & & & & & \\
\hline $\begin{array}{c}\text { Temperature } \\
\left({ }^{\circ} \mathrm{C}\right)\end{array}$ & Up & Down & $\begin{array}{l}\text { Delta } \\
\text { Down }\end{array}$ & $\begin{array}{l}\text { Down } \\
\text { length }\end{array}$ & CTE & CTEK & $\begin{array}{l}\text { Delta } \\
\text { CTEK }\end{array}$ & $\begin{array}{c}\text { CTE } \\
\text { adjusted }\end{array}$ & $\begin{array}{l}\text { Delta } \\
\text { Up }\end{array}$ & $\begin{array}{c}\text { Up } \\
\text { length }\end{array}$ & CTE & CTEK & $\begin{array}{l}\text { Delta } \\
\text { CTEK }\end{array}$ & $\begin{array}{c}\text { CTE } \\
\text { adjusted }\end{array}$ \\
\hline 0 & 1.08 & 0.93 & 0 & 0.9825 & & & & & 0 & 0.9825 & & & & \\
\hline 100 & 1.92 & 1.64 & 0.71 & 0.9829 & $3.61 \mathrm{E}-06$ & 4.10E-06 & 4.87E-07 & 4.11E-06 & 0.84 & 0.9829 & 4.27E-06 & $4.75 E-06$ & 4.75E-07 & 4.77E-06 \\
\hline 200 & 2.80 & 2.38 & 1.45 & 0.9832 & $3.69 \mathrm{E}-06$ & 4.17E-06 & $4.80 \mathrm{E}-07$ & 4.19E-06 & 1.72 & 0.9834 & $4.38 \mathrm{E}-06$ & $4.85 E-06$ & $4.73 \mathrm{E}-07$ & $4.88 \mathrm{E}-06$ \\
\hline 300 & 3.50 & 3.22 & 2.29 & 0.9836 & $3.88 \mathrm{E}-06$ & 4.37E-06 & $4.85 \mathrm{E}-07$ & $4.38 \mathrm{E}-06$ & 2.42 & 0.9837 & 4.11E-06 & $4.58 E-06$ & $4.75 \mathrm{E}-07$ & $4.61 \mathrm{E}-06$ \\
\hline 400 & 3.98 & 3.98 & 3.05 & 0.9840 & $3.88 \mathrm{E}-06$ & $4.36 E-06$ & $4.80 \mathrm{E}-07$ & $4.38 \mathrm{E}-06$ & 2.9 & 0.9840 & $3.69 \mathrm{E}-06$ & $4.18 E-06$ & 4.90E-07 & 4.19E-06 \\
\hline 500 & 4.75 & 4.88 & 3.95 & 0.9845 & $4.02 \mathrm{E}-06$ & $4.50 E-06$ & $4.80 \mathrm{E}-07$ & $4.52 \mathrm{E}-06$ & 3.67 & 0.9843 & $3.74 \mathrm{E}-06$ & $4.22 E-06$ & $4.85 \mathrm{E}-07$ & 4.24E-06 \\
\hline
\end{tabular}

\begin{tabular}{|c|c|c|c|c|c|c|c|c|c|c|c|c|c|c|}
\hline $\begin{array}{l}\text { Capsule } \\
\text { Column }\end{array}$ & $\begin{array}{r}\text { OC4 } \\
\text { West }\end{array}$ & $\begin{array}{c}\text { Specimen } \\
\text { Length }\end{array}$ & $\begin{array}{c}19 \\
0.9955 \\
\end{array}$ & $\begin{array}{c}\text { Material } \\
\text { Scale }\end{array}$ & $\begin{array}{c}\text { H-451 } \\
1 " \\
\end{array}$ & $5.00 \mathrm{E}-04$ & & & & & & & & \\
\hline $\begin{array}{c}\text { Temperature } \\
\left({ }^{\circ} \mathrm{C}\right)\end{array}$ & Up & Down & $\begin{array}{l}\text { Delta } \\
\text { Down }\end{array}$ & $\begin{array}{l}\text { Down } \\
\text { length }\end{array}$ & CTE & CTEK & $\begin{array}{l}\text { Delta } \\
\text { CTEK }\end{array}$ & $\begin{array}{c}\text { CTE } \\
\text { adjusted }\end{array}$ & $\begin{array}{l}\text { Delta } \\
\text { Up }\end{array}$ & $\begin{array}{c}\text { Up } \\
\text { length }\end{array}$ & CTE & CTEK & $\begin{array}{l}\text { Delta } \\
\text { CTEK }\end{array}$ & $\begin{array}{c}\text { CTE } \\
\text { adjusted }\end{array}$ \\
\hline 0 & 0.90 & 1.38 & 0 & 0.9955 & & & & & 0 & 0.9955 & & & & \\
\hline 100 & 1.59 & 1.90 & 0.52 & 0.9958 & $2.61 \mathrm{E}-06$ & $3.15 E-06$ & $5.38 \mathrm{E}-07$ & $3.11 \mathrm{E}-06$ & 0.69 & 0.9958 & 3.47E-06 & $4.00 E-06$ & $5.34 \mathrm{E}-07$ & 3.97E-06 \\
\hline 200 & 2.28 & 2.45 & 1.07 & 0.9960 & $2.69 \mathrm{E}-06$ & $3.22 E-06$ & 5.33E-07 & 3.19E-06 & 1.38 & 0.9962 & $3.47 \mathrm{E}-06$ & $4.00 E-06$ & $5.34 \mathrm{E}-07$ & 3.97E-06 \\
\hline 300 & 2.79 & 3.01 & 1.63 & 0.9963 & $2.73 \mathrm{E}-06$ & 3.27E-06 & $5.41 \mathrm{E}-07$ & $3.23 \mathrm{E}-06$ & 1.89 & 0.9964 & $3.16 \mathrm{E}-06$ & $3.70 E-06$ & $5.36 \mathrm{E}-07$ & $3.66 \mathrm{E}-06$ \\
\hline 400 & 3.42 & 3.57 & 2.19 & 0.9966 & $2.75 \mathrm{E}-06$ & $3.29 E-06$ & 5.40E-07 & $3.25 \mathrm{E}-06$ & 2.52 & 0.9968 & $3.16 \mathrm{E}-06$ & $3.70 E-06$ & $5.36 \mathrm{E}-07$ & $3.66 \mathrm{E}-06$ \\
\hline 500 & 4.03 & 4.15 & 2.77 & 0.9969 & $2.78 \mathrm{E}-06$ & $3.32 E-06$ & 5.37E-07 & $3.28 \mathrm{E}-06$ & 3.13 & 0.9971 & $3.14 \mathrm{E}-06$ & $3.68 E-06$ & $5.36 \mathrm{E}-07$ & $3.64 \mathrm{E}-06$ \\
\hline
\end{tabular}




\begin{tabular}{|c|c|c|c|c|c|c|c|c|c|c|c|c|c|c|}
\hline $\begin{array}{l}\text { Capsule } \\
\text { Column }\end{array}$ & $\begin{array}{l}\text { OC4 } \\
\text { West }\end{array}$ & $\begin{array}{c}\text { Specimen } \\
\text { Length }\end{array}$ & $\begin{array}{c}26 \\
0.9813\end{array}$ & $\begin{array}{c}\text { Material } \\
\text { Scale }\end{array}$ & $\begin{array}{c}\text { H-451 } \\
1 "\end{array}$ & $5.00 \mathrm{E}-04$ & & & & & & & & \\
\hline $\begin{array}{c}\text { Temperature } \\
\left({ }^{\circ} \mathrm{C}\right) \\
\end{array}$ & Up & Down & $\begin{array}{l}\text { Delta } \\
\text { Down }\end{array}$ & $\begin{array}{l}\text { Down } \\
\text { length }\end{array}$ & CTE & CTEK & $\begin{array}{l}\text { Delta } \\
\text { CTEK }\end{array}$ & $\begin{array}{c}\text { CTE } \\
\text { adjusted }\end{array}$ & $\begin{array}{l}\text { Delta } \\
\text { Up }\end{array}$ & $\begin{array}{c}\text { Up } \\
\text { length }\end{array}$ & CTE & CTEK & $\begin{array}{l}\text { Delta } \\
\text { CTEK }\end{array}$ & $\begin{array}{c}\text { CTE } \\
\text { adjusted }\end{array}$ \\
\hline 0 & 0.95 & 1.27 & 0 & 0.9813 & & & & & 0 & 0.9813 & & & & \\
\hline 100 & 1.75 & 1.90 & 0.63 & 0.9816 & $3.21 \mathrm{E}-06$ & $3.70 E-06$ & $4.90 \mathrm{E}-07$ & $3.71 \mathrm{E}-06$ & 0.8 & 0.9817 & $4.08 \mathrm{E}-06$ & $4.55 E-06$ & $4.74 \mathrm{E}-07$ & $4.58 \mathrm{E}-06$ \\
\hline 200 & 2.50 & 2.50 & 1.23 & 0.9819 & $3.13 \mathrm{E}-06$ & $3.62 E-06$ & $4.86 \mathrm{E}-07$ & $3.63 \mathrm{E}-06$ & 1.55 & 0.9821 & $3.95 \mathrm{E}-06$ & $4.42 E-06$ & $4.71 \mathrm{E}-07$ & 4.45E-06 \\
\hline 300 & 3.02 & 3.23 & 1.96 & 0.9823 & $3.33 \mathrm{E}-06$ & $3.82 E-06$ & $4.91 \mathrm{E}-07$ & $3.83 \mathrm{E}-06$ & 2.07 & 0.9823 & $3.52 \mathrm{E}-06$ & $4.00 E-06$ & $4.84 \mathrm{E}-07$ & $4.02 \mathrm{E}-06$ \\
\hline 400 & 3.75 & 3.90 & 2.63 & 0.9826 & $3.35 \mathrm{E}-06$ & $3.84 E-06$ & $4.90 \mathrm{E}-07$ & $3.85 \mathrm{E}-06$ & 2.8 & 0.9827 & $3.57 \mathrm{E}-06$ & $4.05 E-06$ & $4.83 \mathrm{E}-07$ & 4.07E-06 \\
\hline 500 & 4.50 & 4.65 & 3.38 & 0.9830 & $3.44 \mathrm{E}-06$ & $3.93 E-06$ & $4.86 \mathrm{E}-07$ & $3.94 \mathrm{E}-06$ & 3.55 & 0.9831 & $3.62 \mathrm{E}-06$ & 4.10E-06 & $4.82 \mathrm{E}-07$ & 4.12E-06 \\
\hline
\end{tabular}

\begin{tabular}{|c|c|c|c|c|c|c|c|c|c|c|c|c|c|c|}
\hline $\begin{array}{l}\text { Capsule } \\
\text { Column }\end{array}$ & $\begin{array}{l}\text { OC4 } \\
\text { West }\end{array}$ & $\begin{array}{l}\text { Specimen } \\
\text { Length }\end{array}$ & $\begin{array}{c}27 \\
0.9833\end{array}$ & $\begin{array}{l}\text { Material } \\
\text { Scale }\end{array}$ & $\begin{array}{l}\text { H-451 } \\
1 "\end{array}$ & $5.00 \mathrm{E}-04$ & & & & & & & & \\
\hline $\begin{array}{c}\text { Temperature } \\
\left({ }^{\circ} \mathrm{C}\right)\end{array}$ & Up & Down & $\begin{array}{l}\text { Delta } \\
\text { Down }\end{array}$ & $\begin{array}{l}\text { Down } \\
\text { length }\end{array}$ & CTE & CTEK & $\begin{array}{l}\text { Delta } \\
\text { CTEK }\end{array}$ & $\begin{array}{c}\text { CTE } \\
\text { adjusted }\end{array}$ & $\begin{array}{l}\text { Delta } \\
\text { Up }\end{array}$ & $\begin{array}{c}\text { Up } \\
\text { length }\end{array}$ & CTE & CTEK & $\begin{array}{l}\text { Delta } \\
\text { CTEK }\end{array}$ & $\begin{array}{c}\text { CTE } \\
\text { adjusted }\end{array}$ \\
\hline 0 & 1.51 & 1.87 & 0 & 0.9833 & & & & & 0 & 0.9833 & & & & \\
\hline 100 & 2.35 & 2.50 & 0.63 & 0.9836 & $3.20 \mathrm{E}-06$ & 3.70E-06 & 4.97E-07 & $3.70 \mathrm{E}-06$ & 0.84 & 0.9837 & 4.27E-06 & $4.75 E-06$ & 4.79E-07 & 4.77E-06 \\
\hline 200 & 3.10 & 3.20 & 1.33 & 0.9840 & $3.38 \mathrm{E}-06$ & $3.88 E-06$ & 4.99E-07 & $3.88 \mathrm{E}-06$ & 1.59 & 0.9841 & 4.04E-06 & $4.52 E-06$ & 4.77E-07 & 4.54E-06 \\
\hline 300 & 3.78 & 3.78 & 1.91 & 0.9843 & $3.24 \mathrm{E}-06$ & 3.73E-06 & 4.93E-07 & 3.74E-06 & 2.27 & 0.9844 & $3.85 \mathrm{E}-06$ & $4.33 E-06$ & $4.82 \mathrm{E}-07$ & 4.35E-06 \\
\hline 400 & 4.39 & 4.42 & 2.55 & 0.9846 & $3.24 \mathrm{E}-06$ & $3.73 E-06$ & $4.88 \mathrm{E}-07$ & $3.74 \mathrm{E}-06$ & 2.88 & 0.9847 & $3.66 \mathrm{E}-06$ & $4.15 E-06$ & 4.89E-07 & 4.16E-06 \\
\hline 500 & 5.10 & 5.20 & 3.33 & 0.9850 & 3.39E-06 & $3.88 E-06$ & $4.93 \mathrm{E}-07$ & $3.89 \mathrm{E}-06$ & 3.59 & 0.9851 & $3.65 \mathrm{E}-06$ & $4.14 E-06$ & $4.89 \mathrm{E}-07$ & 4.15E-06 \\
\hline
\end{tabular}

\begin{tabular}{|c|c|c|c|c|c|c|c|c|c|c|c|c|c|c|}
\hline $\begin{array}{l}\text { Capsule } \\
\text { Column }\end{array}$ & $\begin{array}{r}\text { OC4 } \\
\text { West }\end{array}$ & $\begin{array}{c}\text { Specimen } \\
\text { Length }\end{array}$ & $\begin{array}{c}31 \\
0.9795 \\
\end{array}$ & $\begin{array}{c}\text { Material } \\
\text { Scale }\end{array}$ & $\begin{array}{c}\text { H-451 } \\
1 " \\
\end{array}$ & $5.00 \mathrm{E}-04$ & & & & & & & & \\
\hline $\begin{array}{c}\text { Temperature } \\
\left({ }^{\circ} \mathrm{C}\right)\end{array}$ & Up & Down & $\begin{array}{l}\text { Delta } \\
\text { Down }\end{array}$ & $\begin{array}{l}\text { Down } \\
\text { length }\end{array}$ & CTE & CTEK & $\begin{array}{l}\text { Delta } \\
\text { CTEK }\end{array}$ & $\begin{array}{c}\text { CTE } \\
\text { adjusted }\end{array}$ & $\begin{array}{l}\text { Delta } \\
\text { Up }\end{array}$ & $\begin{array}{c}\text { Up } \\
\text { length }\end{array}$ & CTE & CTEK & $\begin{array}{l}\text { Delta } \\
\text { CTEK }\end{array}$ & $\begin{array}{c}\text { CTE } \\
\text { adjusted }\end{array}$ \\
\hline 0 & 0.83 & 1.24 & 0 & 0.9795 & & & & & 0 & 0.9795 & & & & \\
\hline 100 & 1.87 & 1.98 & 0.74 & 0.9799 & $3.78 \mathrm{E}-06$ & $4.25 E-06$ & 4.73E-07 & $4.28 \mathrm{E}-06$ & 1.04 & 0.9800 & 5.31E-06 & $5.75 E-06$ & 4.41E-07 & $5.81 \mathrm{E}-06$ \\
\hline 200 & 2.77 & 2.81 & 1.57 & 0.9803 & 4.01E-06 & 4.48E-06 & 4.73E-07 & 4.51E-06 & 1.94 & 0.9805 & 4.95E-06 & 5.40E-06 & 4.48E-07 & $5.45 \mathrm{E}-06$ \\
\hline 300 & 3.61 & 3.62 & 2.38 & 0.9807 & $4.05 \mathrm{E}-06$ & $4.52 E-06$ & 4.70E-07 & 4.55E-06 & 2.78 & 0.9809 & 4.73E-06 & $5.18 E-06$ & 4.50E-07 & $5.23 \mathrm{E}-06$ \\
\hline 400 & 4.45 & 4.45 & 3.21 & 0.9811 & 4.10E-06 & 4.56E-06 & 4.64E-07 & 4.60E-06 & 3.62 & 0.9813 & $4.62 \mathrm{E}-06$ & 5.07E-06 & 4.50E-07 & $5.12 \mathrm{E}-06$ \\
\hline 500 & 5.40 & 5.48 & 4.24 & 0.9816 & 4.33E-06 & 4.79E-06 & 4.61E-07 & 4.83E-06 & 4.57 & 0.9818 & 4.67E-06 & $5.20 E-06$ & $5.34 \mathrm{E}-07$ & 5.17E-06 \\
\hline
\end{tabular}




\begin{tabular}{|c|c|c|c|c|c|c|c|c|c|c|c|c|c|c|}
\hline $\begin{array}{l}\text { Capsule } \\
\text { Column }\end{array}$ & $\begin{array}{l}\text { OC4 } \\
\text { West }\end{array}$ & $\begin{array}{c}\text { Specimen } \\
\text { Length }\end{array}$ & $\begin{array}{c}41 \\
0.9854\end{array}$ & $\begin{array}{c}\text { Material } \\
\text { Scale }\end{array}$ & $\begin{array}{c}\text { H-451 } \\
1 "\end{array}$ & $5.00 \mathrm{E}-04$ & & & & & & & & \\
\hline $\begin{array}{c}\text { Temperature } \\
\left({ }^{\circ} \mathrm{C}\right) \\
\end{array}$ & Up & Down & $\begin{array}{l}\text { Delta } \\
\text { Down }\end{array}$ & $\begin{array}{l}\text { Down } \\
\text { length }\end{array}$ & CTE & CTEK & $\begin{array}{l}\text { Delta } \\
\text { CTEK }\end{array}$ & $\begin{array}{c}\text { CTE } \\
\text { adjusted }\end{array}$ & $\begin{array}{l}\text { Delta } \\
\text { Up }\end{array}$ & $\begin{array}{c}\text { Up } \\
\text { length }\end{array}$ & CTE & CTEK & $\begin{array}{l}\text { Delta } \\
\text { CTEK }\end{array}$ & $\begin{array}{c}\text { CTE } \\
\text { adjusted }\end{array}$ \\
\hline 0 & 1.17 & 1.92 & 0 & 0.9854 & & & & & 0 & 0.9854 & & & & \\
\hline 100 & 2.00 & 2.42 & 0.5 & 0.9857 & $2.54 \mathrm{E}-06$ & $3.05 E-06$ & $5.13 \mathrm{E}-07$ & $3.04 \mathrm{E}-06$ & 0.83 & 0.9858 & $4.21 \mathrm{E}-06$ & 4.70E-06 & 4.89E-07 & $4.71 \mathrm{E}-06$ \\
\hline 200 & 2.88 & 3.02 & 1.1 & 0.9860 & 2.79E-06 & $3.30 E-06$ & 5.09E-07 & $3.29 \mathrm{E}-06$ & 1.71 & 0.9863 & 4.34E-06 & $4.82 E-06$ & $4.82 \mathrm{E}-07$ & 4.84E-06 \\
\hline 300 & 3.67 & 3.75 & 1.83 & 0.9863 & $3.10 \mathrm{E}-06$ & $3.60 E-06$ & $5.05 \mathrm{E}-07$ & $3.60 \mathrm{E}-06$ & 2.5 & 0.9867 & 4.23E-06 & $4.72 E-06$ & $4.92 \mathrm{E}-07$ & 4.73E-06 \\
\hline 400 & 4.38 & 4.50 & 2.58 & 0.9867 & $3.27 \mathrm{E}-06$ & $3.78 E-06$ & 5.07E-07 & $3.77 \mathrm{E}-06$ & 3.21 & 0.9870 & 4.07E-06 & $4.56 E-06$ & $4.88 \mathrm{E}-07$ & 4.57E-06 \\
\hline 500 & 5.05 & 5.50 & 3.58 & 0.9872 & $3.63 \mathrm{E}-06$ & 4.13E-06 & 4.97E-07 & 4.13E-06 & 3.88 & 0.9873 & $3.94 \mathrm{E}-06$ & $4.43 E-06$ & $4.93 \mathrm{E}-07$ & 4.44E-06 \\
\hline
\end{tabular}

\begin{tabular}{|c|c|c|c|c|c|c|c|c|c|c|c|c|c|c|}
\hline $\begin{array}{l}\text { Capsule } \\
\text { Column }\end{array}$ & $\begin{array}{l}\text { OC4 } \\
\text { West }\end{array}$ & $\begin{array}{l}\text { Specimen } \\
\text { Length }\end{array}$ & $\begin{array}{c}43 \\
0.9828\end{array}$ & $\begin{array}{l}\text { Material } \\
\text { Scale }\end{array}$ & $\begin{array}{l}\text { H-451 } \\
1 "\end{array}$ & $5.00 \mathrm{E}-04$ & & & & & & & & \\
\hline $\begin{array}{c}\text { Temperature } \\
\left({ }^{\circ} \mathrm{C}\right)\end{array}$ & Up & Down & $\begin{array}{l}\text { Delta } \\
\text { Down }\end{array}$ & $\begin{array}{l}\text { Down } \\
\text { length }\end{array}$ & CTE & CTEK & $\begin{array}{l}\text { Delta } \\
\text { CTEK }\end{array}$ & $\begin{array}{c}\text { CTE } \\
\text { adjusted }\end{array}$ & $\begin{array}{l}\text { Delta } \\
\text { Up }\end{array}$ & $\begin{array}{c}\text { Up } \\
\text { length }\end{array}$ & CTE & CTEK & $\begin{array}{l}\text { Delta } \\
\text { CTEK }\end{array}$ & $\begin{array}{c}\text { CTE } \\
\text { adjusted }\end{array}$ \\
\hline 0 & 1.75 & 1.50 & 0 & 0.9828 & & & & & 0 & 0.9828 & & & & \\
\hline 100 & 2.37 & 2.17 & 0.67 & 0.9831 & $3.41 \mathrm{E}-06$ & $3.90 E-06$ & 4.91E-07 & $3.91 \mathrm{E}-06$ & 0.62 & 0.9831 & $3.15 \mathrm{E}-06$ & $3.65 E-06$ & 4.96E-07 & 3.65E-06 \\
\hline 200 & 3.00 & 2.88 & 1.38 & 0.9835 & $3.51 \mathrm{E}-06$ & $4.00 E-06$ & $4.90 \mathrm{E}-07$ & 4.01E-06 & 1.25 & 0.9834 & $3.18 \mathrm{E}-06$ & $3.68 E-06$ & $5.00 \mathrm{E}-07$ & $3.68 \mathrm{E}-06$ \\
\hline 300 & 3.64 & 3.64 & 2.14 & 0.9839 & 3.63E-06 & 4.12E-06 & $4.91 \mathrm{E}-07$ & 4.13E-06 & 1.89 & 0.9837 & $3.21 \mathrm{E}-06$ & 3.70E-06 & 4.95E-07 & $3.71 \mathrm{E}-06$ \\
\hline 400 & 4.29 & 4.35 & 2.85 & 0.9842 & $3.62 \mathrm{E}-06$ & 4.11E-06 & $4.85 \mathrm{E}-07$ & 4.12E-06 & 2.54 & 0.9841 & $3.23 \mathrm{E}-06$ & $3.72 E-06$ & 4.89E-07 & 3.73E-06 \\
\hline 500 & 5.18 & 5.18 & 3.68 & 0.9846 & $3.74 \mathrm{E}-06$ & $4.23 E-06$ & $4.86 \mathrm{E}-07$ & $4.24 \mathrm{E}-06$ & 3.43 & 0.9845 & $3.49 \mathrm{E}-06$ & $3.98 E-06$ & $4.90 \mathrm{E}-07$ & 3.99E-06 \\
\hline
\end{tabular}

\begin{tabular}{|c|c|c|c|c|c|c|c|c|c|c|c|c|c|c|}
\hline $\begin{array}{l}\text { Capsule } \\
\text { Column }\end{array}$ & $\begin{array}{r}\text { OC4 } \\
\text { West }\end{array}$ & $\begin{array}{c}\text { Specimen } \\
\text { Length }\end{array}$ & $\begin{array}{c}45 \\
0.9881 \\
\end{array}$ & $\begin{array}{c}\text { Material } \\
\text { Scale }\end{array}$ & $\begin{array}{c}\text { H-451 } \\
1 " \\
\end{array}$ & $5.00 \mathrm{E}-04$ & & & & & & & & \\
\hline $\begin{array}{c}\text { Temperature } \\
\left({ }^{\circ} \mathrm{C}\right)\end{array}$ & Up & Down & $\begin{array}{l}\text { Delta } \\
\text { Down }\end{array}$ & $\begin{array}{l}\text { Down } \\
\text { length }\end{array}$ & CTE & CTEK & $\begin{array}{l}\text { Delta } \\
\text { CTEK }\end{array}$ & $\begin{array}{c}\text { CTE } \\
\text { adjusted }\end{array}$ & $\begin{array}{l}\text { Delta } \\
\text { Up }\end{array}$ & $\begin{array}{c}\text { Up } \\
\text { length }\end{array}$ & CTE & CTEK & $\begin{array}{l}\text { Delta } \\
\text { CTEK }\end{array}$ & $\begin{array}{c}\text { CTE } \\
\text { adjusted }\end{array}$ \\
\hline 0 & 0.90 & 0.69 & 0 & 0.9881 & & & & & 0 & 0.9881 & & & & \\
\hline 100 & 1.65 & 1.38 & 0.69 & 0.9884 & $3.49 \mathrm{E}-06$ & 4.00E-06 & $5.08 \mathrm{E}-07$ & $3.99 \mathrm{E}-06$ & 0.75 & 0.9885 & $3.80 \mathrm{E}-06$ & 4.30E-06 & $5.05 \mathrm{E}-07$ & 4.30E-06 \\
\hline 200 & 2.40 & 2.11 & 1.42 & 0.9888 & $3.59 \mathrm{E}-06$ & 4.10E-06 & 5.07E-07 & 4.09E-06 & 1.5 & 0.9889 & $3.80 \mathrm{E}-06$ & 4.30E-06 & $5.05 \mathrm{E}-07$ & 4.30E-06 \\
\hline 300 & 3.05 & 2.95 & 2.26 & 0.9892 & $3.81 \mathrm{E}-06$ & $4.32 E-06$ & $5.08 \mathrm{E}-07$ & $4.31 \mathrm{E}-06$ & 2.15 & 0.9892 & $3.63 \mathrm{E}-06$ & $4.13 E-06$ & $5.04 \mathrm{E}-07$ & 4.13E-06 \\
\hline 400 & 3.78 & 3.78 & 3.09 & 0.9896 & $3.91 \mathrm{E}-06$ & $4.41 E-06$ & $5.01 \mathrm{E}-07$ & 4.41E-06 & 2.88 & 0.9895 & $3.64 \mathrm{E}-06$ & $4.15 E-06$ & 5.07E-07 & 4.14E-06 \\
\hline 500 & 4.57 & 4.57 & 3.88 & 0.9900 & 3.93E-06 & $4.43 E-06$ & 5.03E-07 & 4.43E-06 & 3.67 & 0.9899 & $3.71 \mathrm{E}-06$ & $4.22 E-06$ & 5.06E-07 & $4.21 \mathrm{E}-06$ \\
\hline
\end{tabular}




\begin{tabular}{|c|c|c|c|c|c|c|c|c|c|c|c|c|c|c|}
\hline $\begin{array}{l}\text { Capsule } \\
\text { Column }\end{array}$ & $\begin{array}{l}\text { OC4 } \\
\text { West }\end{array}$ & $\begin{array}{c}\text { Specimen } \\
\text { Length }\end{array}$ & $\begin{array}{c}47 \\
0.9815\end{array}$ & $\begin{array}{c}\text { Material } \\
\text { Scale }\end{array}$ & $\begin{array}{c}\text { H-451 } \\
1 "\end{array}$ & $5.00 \mathrm{E}-04$ & & & & & & & & \\
\hline $\begin{array}{c}\text { Temperature } \\
\left({ }^{\circ} \mathrm{C}\right) \\
\end{array}$ & Up & Down & $\begin{array}{l}\text { Delta } \\
\text { Down }\end{array}$ & $\begin{array}{l}\text { Down } \\
\text { length }\end{array}$ & CTE & CTEK & $\begin{array}{l}\text { Delta } \\
\text { CTEK }\end{array}$ & $\begin{array}{c}\text { CTE } \\
\text { adjusted }\end{array}$ & $\begin{array}{l}\text { Delta } \\
\text { Up }\end{array}$ & $\begin{array}{c}\text { Up } \\
\text { length }\end{array}$ & CTE & CTEK & $\begin{array}{l}\text { Delta } \\
\text { CTEK }\end{array}$ & $\begin{array}{c}\text { CTE } \\
\text { adjusted }\end{array}$ \\
\hline 0 & 1.47 & 2.05 & 0 & 0.9815 & & & & & 0 & 0.9815 & & & & \\
\hline 100 & 2.44 & 2.75 & 0.7 & 0.9819 & 3.57E-06 & $4.05 E-06$ & $4.84 \mathrm{E}-07$ & 4.07E-06 & 0.97 & 0.9820 & $4.94 \mathrm{E}-06$ & $5.40 E-06$ & 4.59E-07 & $5.44 \mathrm{E}-06$ \\
\hline 200 & 3.30 & 3.62 & 1.57 & 0.9823 & 4.00E-06 & $4.48 E-06$ & 4.81E-07 & $4.50 \mathrm{E}-06$ & 1.83 & 0.9824 & 4.66E-06 & $5.12 E-06$ & 4.59E-07 & 5.16E-06 \\
\hline 300 & 4.03 & 4.30 & 2.25 & 0.9826 & $3.82 \mathrm{E}-06$ & $4.30 E-06$ & 4.79E-07 & $4.32 \mathrm{E}-06$ & 2.56 & 0.9828 & 4.35E-06 & $4.82 E-06$ & $4.73 \mathrm{E}-07$ & 4.85E-06 \\
\hline 400 & 4.70 & 4.90 & 2.85 & 0.9829 & $3.63 \mathrm{E}-06$ & 4.11E-06 & $4.80 \mathrm{E}-07$ & 4.13E-06 & 3.23 & 0.9831 & 4.11E-06 & $4.59 E-06$ & 4.76E-07 & 4.61E-06 \\
\hline 500 & 5.50 & 5.62 & 3.57 & 0.9833 & $3.64 \mathrm{E}-06$ & 4.12E-06 & $4.83 \mathrm{E}-07$ & 4.14E-06 & 4.03 & 0.9835 & 4.11E-06 & $4.58 E-06$ & $4.74 \mathrm{E}-07$ & $4.61 \mathrm{E}-06$ \\
\hline
\end{tabular}

\begin{tabular}{|c|c|c|c|c|c|c|c|c|c|c|c|c|c|c|}
\hline $\begin{array}{l}\text { Capsule } \\
\text { Column }\end{array}$ & $\begin{array}{l}\text { OC4 } \\
\text { West }\end{array}$ & $\begin{array}{l}\text { Specimen } \\
\text { Length }\end{array}$ & $\begin{array}{c}56 \\
0.9776\end{array}$ & $\begin{array}{l}\text { Material } \\
\text { Scale }\end{array}$ & $\begin{array}{l}\text { H-451 } \\
1 "\end{array}$ & $5.00 \mathrm{E}-04$ & & & & & & & & \\
\hline $\begin{array}{c}\text { Temperature } \\
\left({ }^{\circ} \mathrm{C}\right)\end{array}$ & Up & Down & $\begin{array}{l}\text { Delta } \\
\text { Down }\end{array}$ & $\begin{array}{l}\text { Down } \\
\text { length }\end{array}$ & CTE & CTEK & $\begin{array}{l}\text { Delta } \\
\text { CTEK }\end{array}$ & $\begin{array}{c}\text { CTE } \\
\text { adjusted }\end{array}$ & $\begin{array}{l}\text { Delta } \\
\text { Up }\end{array}$ & $\begin{array}{c}\text { Up } \\
\text { length }\end{array}$ & CTE & CTEK & $\begin{array}{l}\text { Delta } \\
\text { CTEK }\end{array}$ & $\begin{array}{c}\text { CTE } \\
\text { adjusted }\end{array}$ \\
\hline 0 & 1.05 & 1.47 & 0 & 0.9776 & & & & & 0 & 0.9776 & & & & \\
\hline 100 & 1.87 & 2.25 & 0.78 & 0.9780 & 3.99E-06 & 4.45E-06 & 4.61E-07 & 4.49E-06 & 0.82 & 0.9780 & 4.19E-06 & $4.65 E-06$ & 4.56E-07 & 4.69E-06 \\
\hline 200 & 2.65 & 3.05 & 1.58 & 0.9784 & $4.04 \mathrm{E}-06$ & $4.50 E-06$ & 4.59E-07 & 4.54E-06 & 1.6 & 0.9784 & 4.09E-06 & $4.55 E-06$ & $4.58 \mathrm{E}-07$ & 4.59E-06 \\
\hline 300 & 3.45 & 4.02 & 2.55 & 0.9789 & $4.35 \mathrm{E}-06$ & 4.80E-06 & $4.53 \mathrm{E}-07$ & $4.85 \mathrm{E}-06$ & 2.4 & 0.9788 & 4.09E-06 & $4.55 E-06$ & $4.58 \mathrm{E}-07$ & 4.59E-06 \\
\hline 400 & 4.40 & 4.90 & 3.43 & 0.9793 & 4.39E-06 & $4.84 E-06$ & $4.54 \mathrm{E}-07$ & 4.89E-06 & 3.35 & 0.9793 & $4.28 \mathrm{E}-06$ & $4.74 E-06$ & 4.57E-07 & $4.78 \mathrm{E}-06$ \\
\hline 500 & 5.42 & 5.87 & 4.4 & 0.9798 & $4.50 \mathrm{E}-06$ & $4.95 E-06$ & $4.49 \mathrm{E}-07$ & $5.00 \mathrm{E}-06$ & 4.37 & 0.9798 & 4.47E-06 & $4.92 E-06$ & $4.50 \mathrm{E}-07$ & 4.97E-06 \\
\hline
\end{tabular}

\begin{tabular}{|c|c|c|c|c|c|c|c|c|c|c|c|c|c|c|}
\hline $\begin{array}{l}\text { Capsule } \\
\text { Column }\end{array}$ & $\begin{array}{r}\text { OC4 } \\
\text { West }\end{array}$ & $\begin{array}{c}\text { Specimen } \\
\text { Length }\end{array}$ & $\begin{array}{c}58 \\
0.9751 \\
\end{array}$ & $\begin{array}{c}\text { Material } \\
\text { Scale }\end{array}$ & $\begin{array}{c}\text { H-451 } \\
1 " \\
\end{array}$ & $5.00 \mathrm{E}-04$ & & & & & & & & \\
\hline $\begin{array}{c}\text { Temperature } \\
\left({ }^{\circ} \mathrm{C}\right)\end{array}$ & Up & Down & $\begin{array}{l}\text { Delta } \\
\text { Down }\end{array}$ & $\begin{array}{l}\text { Down } \\
\text { length }\end{array}$ & CTE & CTEK & $\begin{array}{l}\text { Delta } \\
\text { CTEK }\end{array}$ & $\begin{array}{c}\text { CTE } \\
\text { adjusted }\end{array}$ & $\begin{array}{l}\text { Delta } \\
\text { Up }\end{array}$ & $\begin{array}{c}\text { Up } \\
\text { length }\end{array}$ & CTE & CTEK & $\begin{array}{l}\text { Delta } \\
\text { CTEK }\end{array}$ & $\begin{array}{c}\text { CTE } \\
\text { adjusted }\end{array}$ \\
\hline 0 & 0.82 & 1.08 & 0 & 0.9751 & & & & & 0 & 0.9751 & & & & \\
\hline 100 & 1.60 & 1.80 & 0.72 & 0.9755 & $3.69 \mathrm{E}-06$ & $4.15 E-06$ & $4.58 \mathrm{E}-07$ & 4.19E-06 & 0.78 & 0.9755 & 4.00E-06 & $4.45 E-06$ & 4.50E-07 & $4.50 \mathrm{E}-06$ \\
\hline 200 & 2.45 & 2.62 & 1.54 & 0.9759 & $3.95 \mathrm{E}-06$ & 4.40E-06 & $4.52 \mathrm{E}-07$ & 4.45E-06 & 1.63 & 0.9759 & $4.18 \mathrm{E}-06$ & $4.62 E-06$ & 4.41E-07 & $4.68 \mathrm{E}-06$ \\
\hline 300 & 3.35 & 3.50 & 2.42 & 0.9763 & 4.14E-06 & $4.58 E-06$ & 4.44E-07 & 4.64E-06 & 2.53 & 0.9764 & $4.32 \mathrm{E}-06$ & 4.77E-06 & 4.46E-07 & $4.82 \mathrm{E}-06$ \\
\hline 400 & 4.31 & 4.45 & 3.37 & 0.9768 & $4.32 \mathrm{E}-06$ & 4.76E-06 & 4.40E-07 & $4.82 \mathrm{E}-06$ & 3.49 & 0.9768 & 4.47E-06 & $4.91 E-06$ & 4.36E-07 & 4.97E-06 \\
\hline 500 & 5.30 & 5.42 & 4.34 & 0.9773 & 4.45E-06 & 4.89E-06 & 4.39E-07 & 4.95E-06 & 4.48 & 0.9773 & 4.59E-06 & $5.03 E-06$ & 4.36E-07 & 5.09E-06 \\
\hline
\end{tabular}




\begin{tabular}{|c|c|c|c|c|c|c|c|c|c|c|c|c|c|c|}
\hline $\begin{array}{l}\text { Capsule } \\
\text { Column }\end{array}$ & $\begin{array}{c}\text { OC4 } \\
\text { West }\end{array}$ & $\begin{array}{l}\text { Specimen } \\
\text { Length }\end{array}$ & $\begin{array}{c}60 \\
0.9768\end{array}$ & $\begin{array}{c}\text { Material } \\
\text { Scale }\end{array}$ & $\begin{array}{c}\text { H-451 } \\
1 "\end{array}$ & $5.00 \mathrm{E}-04$ & & & & & & & & \\
\hline $\begin{array}{c}\text { Temperature } \\
\left({ }^{\circ} \mathrm{C}\right) \\
\end{array}$ & Up & Down & $\begin{array}{l}\text { Delta } \\
\text { Down }\end{array}$ & $\begin{array}{l}\text { Down } \\
\text { length }\end{array}$ & CTE & CTEK & $\begin{array}{l}\text { Delta } \\
\text { CTEK }\end{array}$ & $\begin{array}{c}\text { CTE } \\
\text { adjusted }\end{array}$ & $\begin{array}{l}\text { Delta } \\
\text { Up }\end{array}$ & $\begin{array}{c}\text { Up } \\
\text { length }\end{array}$ & CTE & CTEK & $\begin{array}{l}\text { Delta } \\
\text { CTEK }\end{array}$ & $\begin{array}{c}\text { CTE } \\
\text { adjusted }\end{array}$ \\
\hline 0 & 0.92 & 1.21 & 0 & 0.9768 & & & & & 0 & 0.9768 & & & & \\
\hline 100 & 1.68 & 1.95 & 0.74 & 0.9772 & 3.79E-06 & $4.25 E-06$ & $4.62 \mathrm{E}-07$ & 4.29E-06 & 0.76 & 0.9772 & 3.89E-06 & $4.05 E-06$ & $1.60 \mathrm{E}-07$ & 4.39E-06 \\
\hline 200 & 2.51 & 2.78 & 1.57 & 0.9776 & $4.02 \mathrm{E}-06$ & 4.48E-06 & 4.62E-07 & $4.52 \mathrm{E}-06$ & 1.59 & 0.9776 & 4.07E-06 & $4.52 E-06$ & 4.51E-07 & 4.57E-06 \\
\hline 300 & 3.40 & 3.67 & 2.46 & 0.9780 & 4.20E-06 & $4.65 E-06$ & $4.53 \mathrm{E}-07$ & 4.70E-06 & 2.48 & 0.9780 & 4.23E-06 & $4.68 E-06$ & 4.48E-07 & 4.73E-06 \\
\hline 400 & 4.39 & 4.60 & 3.39 & 0.9785 & 4.34E-06 & 4.79E-06 & $4.52 \mathrm{E}-07$ & 4.84E-06 & 3.47 & 0.9785 & $4.44 \mathrm{E}-06$ & $4.88 E-06$ & 4.39E-07 & $4.94 \mathrm{E}-06$ \\
\hline 500 & 5.38 & 5.60 & 4.39 & 0.9790 & 4.49E-06 & $4.94 E-06$ & 4.46E-07 & 4.99E-06 & 4.46 & 0.9790 & 4.57E-06 & $5.01 E-06$ & 4.44E-07 & 5.07E-06 \\
\hline
\end{tabular}

\begin{tabular}{|c|c|c|c|c|c|c|c|c|c|c|c|c|c|c|}
\hline $\begin{array}{l}\text { Capsule } \\
\text { Column }\end{array}$ & $\begin{array}{l}\text { OC4 } \\
\text { North }\end{array}$ & $\begin{array}{c}\text { Specimen } \\
\text { Length }\end{array}$ & $\begin{array}{c}78 \\
0.9958\end{array}$ & $\begin{array}{c}\text { Material } \\
\text { Scale }\end{array}$ & $\begin{array}{c}\text { H-451 } \\
1 "\end{array}$ & $5.00 \mathrm{E}-04$ & & & & & & & & \\
\hline $\begin{array}{c}\text { Temperature } \\
\left({ }^{\circ} \mathrm{C}\right)\end{array}$ & Up & Down & $\begin{array}{l}\text { Delta } \\
\text { Down }\end{array}$ & $\begin{array}{l}\text { Down } \\
\text { length }\end{array}$ & CTE & CTEK & $\begin{array}{l}\text { Delta } \\
\text { CTEK }\end{array}$ & $\begin{array}{c}\text { CTE } \\
\text { adjusted }\end{array}$ & $\begin{array}{l}\text { Delta } \\
\text { Up }\end{array}$ & $\begin{array}{c}\text { Up } \\
\text { length }\end{array}$ & CTE & CTEK & $\begin{array}{l}\text { Delta } \\
\text { CTEK }\end{array}$ & $\begin{array}{c}\text { CTE } \\
\text { adjusted }\end{array}$ \\
\hline 0 & 0.58 & 0.93 & 0 & 0.9958 & & & & & 0 & 0.9958 & & & & \\
\hline 100 & 1.24 & 1.49 & 0.56 & 0.9961 & $2.81 \mathrm{E}-06$ & $2.85 E-06$ & $3.82 \mathrm{E}-08$ & $3.31 \mathrm{E}-06$ & 0.66 & 0.9961 & $3.31 \mathrm{E}-06$ & $4.00 E-06$ & $6.86 \mathrm{E}-07$ & $3.81 \mathrm{E}-06$ \\
\hline 200 & 1.90 & 2.02 & 1.09 & 0.9963 & $2.74 \mathrm{E}-06$ & $3.12 E-06$ & $3.84 \mathrm{E}-07$ & $3.24 \mathrm{E}-06$ & 1.32 & 0.9965 & $3.31 \mathrm{E}-06$ & $3.95 E-06$ & $6.36 \mathrm{E}-07$ & $3.81 \mathrm{E}-06$ \\
\hline 300 & 2.57 & 2.60 & 1.67 & 0.9966 & $2.80 \mathrm{E}-06$ & $3.15 E-06$ & $3.55 \mathrm{E}-07$ & 3.30E-06 & 1.99 & 0.9968 & $3.33 \mathrm{E}-06$ & $3.82 E-06$ & 4.89E-07 & 3.83E-06 \\
\hline 400 & 3.12 & 3.22 & 2.29 & 0.9969 & $2.87 \mathrm{E}-06$ & $3.01 E-06$ & $1.35 \mathrm{E}-07$ & 3.37E-06 & 2.54 & 0.9971 & 3.19E-06 & $3.84 E-06$ & $6.52 \mathrm{E}-07$ & $3.69 \mathrm{E}-06$ \\
\hline 500 & 3.98 & 3.92 & 2.99 & 0.9973 & $3.00 \mathrm{E}-06$ & $3.18 E-06$ & $1.77 \mathrm{E}-07$ & $3.50 \mathrm{E}-06$ & 3.4 & 0.9975 & $3.41 \mathrm{E}-06$ & $3.89 E-06$ & 4.76E-07 & $3.91 \mathrm{E}-06$ \\
\hline
\end{tabular}

\begin{tabular}{|c|c|c|c|c|c|c|c|c|c|c|c|c|c|c|}
\hline $\begin{array}{l}\text { Capsule } \\
\text { Column }\end{array}$ & $\begin{array}{c}\text { OC4 } \\
\text { North }\end{array}$ & $\begin{array}{l}\text { Specimen } \\
\text { Length }\end{array}$ & $\begin{array}{c}79 \\
0.9961\end{array}$ & $\begin{array}{c}\text { Material } \\
\text { Scale }\end{array}$ & $\begin{array}{c}\text { H-451 } \\
1 "\end{array}$ & $5.00 \mathrm{E}-04$ & & & & & & & & \\
\hline $\begin{array}{c}\text { Temperature } \\
\left({ }^{\circ} \mathrm{C}\right)\end{array}$ & Up & Down & $\begin{array}{l}\text { Delta } \\
\text { Down }\end{array}$ & $\begin{array}{l}\text { Down } \\
\text { length }\end{array}$ & CTE & CTEK & $\begin{array}{l}\text { Delta } \\
\text { CTEK }\end{array}$ & $\begin{array}{c}\text { CTE } \\
\text { adjusted }\end{array}$ & $\begin{array}{l}\text { Delta } \\
\text { Up }\end{array}$ & $\begin{array}{l}\text { Up } \\
\text { length }\end{array}$ & CTE & CTEK & $\begin{array}{l}\text { Delta } \\
\text { CTEK }\end{array}$ & $\begin{array}{c}\text { CTE } \\
\text { adjusted }\end{array}$ \\
\hline 0 & 0.81 & 0.84 & 0 & 0.9961 & & & & & 0 & 0.9961 & & & & \\
\hline 100 & 1.40 & 1.38 & 0.54 & 0.9964 & $2.71 \mathrm{E}-06$ & $3.25 E-06$ & 5.39E-07 & $3.21 \mathrm{E}-06$ & 0.59 & 0.9964 & $2.96 \mathrm{E}-06$ & 3.50E-06 & $5.38 \mathrm{E}-07$ & $3.46 \mathrm{E}-06$ \\
\hline 200 & 1.94 & 1.90 & 1.06 & 0.9966 & $2.66 \mathrm{E}-06$ & $3.20 E-06$ & $5.40 \mathrm{E}-07$ & $3.16 \mathrm{E}-06$ & 1.13 & 0.9967 & $2.84 \mathrm{E}-06$ & 3.38E-06 & $5.44 \mathrm{E}-07$ & $3.34 \mathrm{E}-06$ \\
\hline 300 & 2.49 & 2.54 & 1.7 & 0.9970 & $2.84 \mathrm{E}-06$ & $3.38 E-06$ & $5.36 \mathrm{E}-07$ & $3.34 \mathrm{E}-06$ & 1.68 & 0.9969 & $2.81 \mathrm{E}-06$ & 3.35E-06 & $5.39 \mathrm{E}-07$ & $3.31 \mathrm{E}-06$ \\
\hline 400 & 3.02 & 3.24 & 2.4 & 0.9973 & $3.01 \mathrm{E}-06$ & $3.55 E-06$ & $5.38 \mathrm{E}-07$ & $3.51 \mathrm{E}-06$ & 2.21 & 0.9972 & $2.77 \mathrm{E}-06$ & $3.31 E-06$ & 5.37E-07 & $3.27 \mathrm{E}-06$ \\
\hline 500 & 3.63 & 3.82 & 2.98 & 0.9976 & 2.99E-06 & $3.53 E-06$ & $5.38 \mathrm{E}-07$ & 3.49E-06 & 2.82 & 0.9975 & $2.83 \mathrm{E}-06$ & $3.37 E-06$ & 5.39E-07 & 3.33E-06 \\
\hline
\end{tabular}




\begin{tabular}{|c|c|c|c|c|c|c|c|c|c|c|c|c|c|c|}
\hline $\begin{array}{l}\text { Capsule } \\
\text { Column }\end{array}$ & $\begin{array}{l}\text { OC4 } \\
\text { North }\end{array}$ & $\begin{array}{c}\text { Specimen } \\
\text { Length }\end{array}$ & $\begin{array}{c}83 \\
0.9957\end{array}$ & $\begin{array}{c}\text { Material } \\
\text { Scale }\end{array}$ & $\begin{array}{c}\text { H-451 } \\
1 "\end{array}$ & $5.00 \mathrm{E}-04$ & & & & & & & & \\
\hline $\begin{array}{c}\text { Temperature } \\
\left({ }^{\circ} \mathrm{C}\right) \\
\end{array}$ & Up & Down & $\begin{array}{l}\text { Delta } \\
\text { Down }\end{array}$ & $\begin{array}{l}\text { Down } \\
\text { length }\end{array}$ & CTE & CTEK & $\begin{array}{l}\text { Delta } \\
\text { CTEK }\end{array}$ & $\begin{array}{c}\text { CTE } \\
\text { adjusted }\end{array}$ & $\begin{array}{l}\text { Delta } \\
\text { Up }\end{array}$ & $\begin{array}{c}\text { Up } \\
\text { length }\end{array}$ & CTE & CTEK & $\begin{array}{l}\text { Delta } \\
\text { CTEK }\end{array}$ & $\begin{array}{c}\text { CTE } \\
\text { adjusted }\end{array}$ \\
\hline 0 & 1.32 & 1.77 & 0 & 0.9957 & & & & & 0 & 0.9957 & & & & \\
\hline 100 & 2.07 & 2.42 & 0.65 & 0.9960 & $3.26 \mathrm{E}-06$ & $3.80 E-06$ & $5.36 \mathrm{E}-07$ & $3.76 \mathrm{E}-06$ & 0.75 & 0.9961 & $3.77 \mathrm{E}-06$ & $4.30 E-06$ & $5.34 \mathrm{E}-07$ & 4.27E-06 \\
\hline 200 & 2.72 & 2.98 & 1.21 & 0.9963 & $3.04 \mathrm{E}-06$ & $3.58 E-06$ & $5.42 \mathrm{E}-07$ & $3.54 \mathrm{E}-06$ & 1.4 & 0.9964 & $3.52 \mathrm{E}-06$ & $4.05 E-06$ & $5.35 \mathrm{E}-07$ & 4.02E-06 \\
\hline 300 & 3.14 & 3.50 & 1.73 & 0.9966 & $2.90 \mathrm{E}-06$ & $3.43 E-06$ & $5.34 \mathrm{E}-07$ & $3.40 \mathrm{E}-06$ & 1.82 & 0.9966 & $3.05 \mathrm{E}-06$ & $3.58 E-06$ & $5.34 \mathrm{E}-07$ & $3.55 \mathrm{E}-06$ \\
\hline 400 & 3.64 & 4.02 & 2.25 & 0.9968 & $2.82 \mathrm{E}-06$ & $3.36 E-06$ & $5.35 \mathrm{E}-07$ & $3.32 \mathrm{E}-06$ & 2.32 & 0.9969 & $2.91 \mathrm{E}-06$ & $3.45 E-06$ & $5.37 \mathrm{E}-07$ & $3.41 \mathrm{E}-06$ \\
\hline 500 & 4.20 & 4.48 & 2.71 & 0.9971 & $2.72 \mathrm{E}-06$ & $3.26 E-06$ & $5.38 \mathrm{E}-07$ & $3.22 \mathrm{E}-06$ & 2.88 & 0.9971 & $2.89 \mathrm{E}-06$ & $3.43 E-06$ & $5.38 \mathrm{E}-07$ & $3.39 \mathrm{E}-06$ \\
\hline
\end{tabular}

\begin{tabular}{|c|c|c|c|c|c|c|c|c|c|c|c|c|c|c|}
\hline $\begin{array}{l}\text { Capsule } \\
\text { Column }\end{array}$ & $\begin{array}{l}\text { OC4 } \\
\text { North }\end{array}$ & $\begin{array}{l}\text { Specimen } \\
\text { Length }\end{array}$ & $\begin{array}{c}84 \\
0.9947\end{array}$ & $\begin{array}{l}\text { Material } \\
\text { Scale }\end{array}$ & $\begin{array}{l}\text { H-451 } \\
1 "\end{array}$ & $5.00 \mathrm{E}-04$ & & & & & & & & \\
\hline $\begin{array}{c}\text { Temperature } \\
\left({ }^{\circ} \mathrm{C}\right)\end{array}$ & Up & Down & $\begin{array}{l}\text { Delta } \\
\text { Down }\end{array}$ & $\begin{array}{l}\text { Down } \\
\text { length }\end{array}$ & CTE & CTEK & $\begin{array}{l}\text { Delta } \\
\text { CTEK }\end{array}$ & $\begin{array}{c}\text { CTE } \\
\text { adjusted }\end{array}$ & $\begin{array}{l}\text { Delta } \\
\text { Up }\end{array}$ & $\begin{array}{c}\text { Up } \\
\text { length }\end{array}$ & CTE & CTEK & $\begin{array}{l}\text { Delta } \\
\text { CTEK }\end{array}$ & $\begin{array}{c}\text { CTE } \\
\text { adjusted }\end{array}$ \\
\hline 0 & 0.72 & 0.60 & 0 & 0.9947 & & & & & 0 & 0.9947 & & & & \\
\hline 100 & 1.41 & 1.15 & 0.55 & 0.9950 & $2.76 \mathrm{E}-06$ & $3.30 E-06$ & 5.35E-07 & $3.26 \mathrm{E}-06$ & 0.69 & 0.9950 & $3.47 \mathrm{E}-06$ & $4.00 E-06$ & $5.32 \mathrm{E}-07$ & 3.97E-06 \\
\hline 200 & 2.03 & 1.72 & 1.12 & 0.9953 & $2.81 \mathrm{E}-06$ & $3.35 E-06$ & $5.35 \mathrm{E}-07$ & $3.31 \mathrm{E}-06$ & 1.31 & 0.9954 & $3.29 \mathrm{E}-06$ & 3.83E-06 & $5.38 \mathrm{E}-07$ & 3.79E-06 \\
\hline 300 & 2.45 & 2.35 & 1.75 & 0.9956 & 2.93E-06 & 3.47E-06 & $5.38 \mathrm{E}-07$ & $3.43 \mathrm{E}-06$ & 1.73 & 0.9956 & $2.90 \mathrm{E}-06$ & $3.43 E-06$ & 5.31E-07 & $3.40 \mathrm{E}-06$ \\
\hline 400 & 3.02 & 3.02 & 2.42 & 0.9959 & $3.04 \mathrm{E}-06$ & $3.58 E-06$ & 5.39E-07 & $3.54 \mathrm{E}-06$ & 2.3 & 0.9959 & $2.89 \mathrm{E}-06$ & $3.43 E-06$ & $5.40 \mathrm{E}-07$ & 3.39E-06 \\
\hline 500 & 3.65 & 3.70 & 3.1 & 0.9963 & $3.12 \mathrm{E}-06$ & $3.65 E-06$ & $5.33 \mathrm{E}-07$ & $3.62 \mathrm{E}-06$ & 2.93 & 0.9962 & 2.95E-06 & $3.48 E-06$ & $5.34 \mathrm{E}-07$ & $3.45 \mathrm{E}-06$ \\
\hline
\end{tabular}

\begin{tabular}{|c|c|c|c|c|c|c|c|c|c|c|c|c|c|c|}
\hline $\begin{array}{l}\text { Capsule } \\
\text { Column }\end{array}$ & $\begin{array}{c}\text { OC4 } \\
\text { North } \\
\end{array}$ & $\begin{array}{c}\text { Specimen } \\
\text { Length }\end{array}$ & $\begin{array}{c}88 \\
0.9961 \\
\end{array}$ & $\begin{array}{c}\text { Material } \\
\text { Scale }\end{array}$ & $\begin{array}{c}\text { H-451 } \\
1 " \\
\end{array}$ & $5.00 \mathrm{E}-04$ & & & & & & & & \\
\hline $\begin{array}{c}\text { Temperature } \\
\left({ }^{\circ} \mathrm{C}\right)\end{array}$ & Up & Down & $\begin{array}{l}\text { Delta } \\
\text { Down }\end{array}$ & $\begin{array}{l}\text { Down } \\
\text { length }\end{array}$ & CTE & CTEK & $\begin{array}{l}\text { Delta } \\
\text { CTEK }\end{array}$ & $\begin{array}{c}\text { CTE } \\
\text { adjusted }\end{array}$ & $\begin{array}{l}\text { Delta } \\
\text { Up }\end{array}$ & $\begin{array}{c}\text { Up } \\
\text { length }\end{array}$ & CTE & CTEK & $\begin{array}{l}\text { Delta } \\
\text { CTEK }\end{array}$ & $\begin{array}{c}\text { CTE } \\
\text { adjusted }\end{array}$ \\
\hline 0 & 1.05 & 1.99 & 0 & 0.9939 & & & & & 0 & 0.9939 & & & & \\
\hline 100 & 1.78 & 2.49 & 0.5 & 0.9942 & $2.52 \mathrm{E}-06$ & $3.05 E-06$ & 5.35E-07 & $3.02 \mathrm{E}-06$ & 0.73 & 0.9943 & 3.67E-06 & 4.20E-06 & $5.28 \mathrm{E}-07$ & 4.17E-06 \\
\hline 200 & 2.40 & 2.95 & 0.96 & 0.9944 & $2.41 \mathrm{E}-06$ & 2.95E-06 & 5.35E-07 & $2.91 \mathrm{E}-06$ & 1.35 & 0.9946 & $3.40 \mathrm{E}-06$ & $3.92 E-06$ & $5.24 \mathrm{E}-07$ & $3.90 \mathrm{E}-06$ \\
\hline 300 & 2.86 & 3.31 & 1.32 & 0.9946 & $2.21 \mathrm{E}-06$ & $2.75 E-06$ & $5.36 \mathrm{E}-07$ & $2.71 \mathrm{E}-06$ & 1.81 & 0.9948 & $3.04 \mathrm{E}-06$ & 3.57E-06 & $5.35 \mathrm{E}-07$ & $3.54 \mathrm{E}-06$ \\
\hline 400 & 3.48 & 3.72 & 1.73 & 0.9948 & $2.18 \mathrm{E}-06$ & $2.55 E-06$ & $3.74 \mathrm{E}-07$ & $2.68 \mathrm{E}-06$ & 2.43 & 0.9951 & $3.06 \mathrm{E}-06$ & $3.59 E-06$ & $5.34 \mathrm{E}-07$ & $3.56 \mathrm{E}-06$ \\
\hline 500 & 4.18 & 4.28 & 2.29 & 0.9950 & $2.30 \mathrm{E}-06$ & $2.24 E-06$ & $-6.41 \mathrm{E}-08$ & 2.80E-06 & 3.13 & 0.9955 & 3.15E-06 & $3.68 E-06$ & $5.31 \mathrm{E}-07$ & $3.65 \mathrm{E}-06$ \\
\hline
\end{tabular}




\begin{tabular}{|c|c|c|c|c|c|c|c|c|c|c|c|c|c|c|}
\hline $\begin{array}{l}\text { Capsule } \\
\text { Column }\end{array}$ & $\begin{array}{l}\text { OC4 } \\
\text { North }\end{array}$ & $\begin{array}{c}\text { Specimen } \\
\text { Length }\end{array}$ & $\begin{array}{c}89 \\
0.9940\end{array}$ & $\begin{array}{c}\text { Material } \\
\text { Scale }\end{array}$ & $\begin{array}{c}\text { H-451 } \\
1 "\end{array}$ & $5.00 \mathrm{E}-04$ & & & & & & & & \\
\hline $\begin{array}{c}\text { Temperature } \\
\left({ }^{\circ} \mathrm{C}\right) \\
\end{array}$ & Up & Down & $\begin{array}{l}\text { Delta } \\
\text { Down }\end{array}$ & $\begin{array}{l}\text { Down } \\
\text { length }\end{array}$ & CTE & CTEK & $\begin{array}{l}\text { Delta } \\
\text { CTEK }\end{array}$ & $\begin{array}{c}\text { CTE } \\
\text { adjusted }\end{array}$ & $\begin{array}{l}\text { Delta } \\
\text { Up }\end{array}$ & $\begin{array}{c}\text { Up } \\
\text { length }\end{array}$ & CTE & CTEK & $\begin{array}{l}\text { Delta } \\
\text { CTEK }\end{array}$ & $\begin{array}{c}\text { CTE } \\
\text { adjusted }\end{array}$ \\
\hline 0 & 0.86 & 0.55 & 0 & 0.9940 & & & & & 0 & 0.9940 & & & & \\
\hline 100 & 1.40 & 1.04 & 0.49 & 0.9942 & $2.46 \mathrm{E}-06$ & $3.00 E-06$ & $5.35 \mathrm{E}-07$ & $2.96 \mathrm{E}-06$ & 0.54 & 0.9943 & $2.72 \mathrm{E}-06$ & $3.25 E-06$ & $5.34 \mathrm{E}-07$ & $3.22 \mathrm{E}-06$ \\
\hline 200 & 1.97 & 1.60 & 1.05 & 0.9945 & $2.64 \mathrm{E}-06$ & $3.18 E-06$ & 5.39E-07 & $3.14 \mathrm{E}-06$ & 1.11 & 0.9946 & $2.79 \mathrm{E}-06$ & $3.33 E-06$ & $5.38 \mathrm{E}-07$ & 3.29E-06 \\
\hline 300 & 2.39 & 2.22 & 1.67 & 0.9948 & $2.80 \mathrm{E}-06$ & $3.33 E-06$ & $5.30 \mathrm{E}-07$ & $3.30 \mathrm{E}-06$ & 1.53 & 0.9948 & $2.57 \mathrm{E}-06$ & 3.10E-06 & $5.35 \mathrm{E}-07$ & 3.07E-06 \\
\hline 400 & 2.83 & 2.87 & 2.32 & 0.9952 & $2.92 \mathrm{E}-06$ & $3.45 E-06$ & $5.32 \mathrm{E}-07$ & $3.42 \mathrm{E}-06$ & 1.97 & 0.9950 & $2.48 \mathrm{E}-06$ & $3.01 E-06$ & $5.33 \mathrm{E}-07$ & $2.98 \mathrm{E}-06$ \\
\hline 500 & 3.46 & 3.50 & 2.95 & 0.9955 & $2.97 \mathrm{E}-06$ & $3.50 E-06$ & $5.32 \mathrm{E}-07$ & $3.47 \mathrm{E}-06$ & 2.6 & 0.9953 & $2.62 \mathrm{E}-06$ & $3.15 E-06$ & $5.34 \mathrm{E}-07$ & $3.12 \mathrm{E}-06$ \\
\hline
\end{tabular}

\begin{tabular}{|c|c|c|c|c|c|c|c|c|c|c|c|c|c|c|}
\hline $\begin{array}{l}\text { Capsule } \\
\text { Column }\end{array}$ & $\begin{array}{l}\text { OC4 } \\
\text { North }\end{array}$ & $\begin{array}{l}\text { Specimen } \\
\text { Length }\end{array}$ & $\begin{array}{c}90 \\
0.995\end{array}$ & $\begin{array}{l}\text { Material } \\
\text { Scale }\end{array}$ & $\begin{array}{l}\text { H-451 } \\
1 "\end{array}$ & $5.00 \mathrm{E}-04$ & & & & & & & & \\
\hline $\begin{array}{c}\text { Temperature } \\
\left({ }^{\circ} \mathrm{C}\right)\end{array}$ & Up & Down & $\begin{array}{l}\text { Delta } \\
\text { Down }\end{array}$ & $\begin{array}{l}\text { Down } \\
\text { length }\end{array}$ & CTE & CTEK & $\begin{array}{l}\text { Delta } \\
\text { CTEK }\end{array}$ & $\begin{array}{c}\text { CTE } \\
\text { adjusted }\end{array}$ & $\begin{array}{l}\text { Delta } \\
\text { Up }\end{array}$ & $\begin{array}{c}\text { Up } \\
\text { length }\end{array}$ & CTE & CTEK & $\begin{array}{l}\text { Delta } \\
\text { CTEK }\end{array}$ & $\begin{array}{c}\text { CTE } \\
\text { adjusted }\end{array}$ \\
\hline 0 & 1.25 & 1.45 & 0 & 0.9950 & & & & & 0 & 0.9950 & & & & \\
\hline 100 & 1.98 & 2.00 & 0.55 & 0.9953 & $2.76 \mathrm{E}-06$ & $3.30 E-06$ & 5.36E-07 & $3.26 \mathrm{E}-06$ & 0.73 & 0.9954 & $3.67 \mathrm{E}-06$ & $4.20 E-06$ & $5.32 \mathrm{E}-07$ & 4.17E-06 \\
\hline 200 & 2.56 & 2.56 & 1.11 & 0.9956 & $2.79 \mathrm{E}-06$ & $3.32 E-06$ & $5.31 \mathrm{E}-07$ & $3.29 \mathrm{E}-06$ & 1.31 & 0.9957 & $3.29 \mathrm{E}-06$ & 3.83E-06 & 5.39E-07 & $3.79 \mathrm{E}-06$ \\
\hline 300 & 3.10 & 3.22 & 1.77 & 0.9959 & 2.96E-06 & 3.50E-06 & $5.35 \mathrm{E}-07$ & $3.46 \mathrm{E}-06$ & 1.85 & 0.9959 & 3.10E-06 & $3.63 E-06$ & 5.31E-07 & $3.60 \mathrm{E}-06$ \\
\hline 400 & 3.75 & 3.90 & 2.45 & 0.9962 & $3.08 \mathrm{E}-06$ & $3.61 E-06$ & $5.32 \mathrm{E}-07$ & $3.58 \mathrm{E}-06$ & 2.5 & 0.9963 & $3.14 \mathrm{E}-06$ & $3.68 E-06$ & 5.39E-07 & $3.64 \mathrm{E}-06$ \\
\hline 500 & 4.48 & 4.60 & 3.15 & 0.9966 & 3.17E-06 & $3.70 E-06$ & $5.34 \mathrm{E}-07$ & $3.67 \mathrm{E}-06$ & 3.23 & 0.9966 & $3.25 \mathrm{E}-06$ & $3.78 E-06$ & $5.34 \mathrm{E}-07$ & $3.75 \mathrm{E}-06$ \\
\hline
\end{tabular}

\begin{tabular}{|c|c|c|c|c|c|c|c|c|c|c|c|c|c|c|}
\hline $\begin{array}{l}\text { Capsule } \\
\text { Column }\end{array}$ & $\begin{array}{c}\text { OC4 } \\
\text { North } \\
\end{array}$ & $\begin{array}{c}\text { Specimen } \\
\text { Length }\end{array}$ & $\begin{array}{c}93 \\
0.995 \\
\end{array}$ & $\begin{array}{c}\text { Material } \\
\text { Scale }\end{array}$ & $\begin{array}{c}\text { H-451 } \\
1 " \\
\end{array}$ & $5.00 \mathrm{E}-04$ & & & & & & & & \\
\hline $\begin{array}{c}\text { Temperature } \\
\left({ }^{\circ} \mathrm{C}\right)\end{array}$ & Up & Down & $\begin{array}{l}\text { Delta } \\
\text { Down }\end{array}$ & $\begin{array}{l}\text { Down } \\
\text { length }\end{array}$ & CTE & CTEK & $\begin{array}{l}\text { Delta } \\
\text { CTEK }\end{array}$ & $\begin{array}{c}\text { CTE } \\
\text { adjusted }\end{array}$ & $\begin{array}{l}\text { Delta } \\
\text { Up }\end{array}$ & $\begin{array}{c}\text { Up } \\
\text { length }\end{array}$ & CTE & CTEK & $\begin{array}{l}\text { Delta } \\
\text { CTEK }\end{array}$ & $\begin{array}{c}\text { CTE } \\
\text { adjusted }\end{array}$ \\
\hline 0 & 0.90 & 1.39 & 0 & 0.9950 & & & & & 0 & 0.9950 & & & & \\
\hline 100 & 1.65 & 2.05 & 0.66 & 0.9953 & $3.32 \mathrm{E}-06$ & $3.85 E-06$ & $5.33 \mathrm{E}-07$ & $3.82 \mathrm{E}-06$ & 0.75 & 0.9954 & 3.77E-06 & $3.75 E-06$ & $-1.88 \mathrm{E}-08$ & 4.27E-06 \\
\hline 200 & 2.44 & 2.72 & 1.33 & 0.9957 & $3.34 \mathrm{E}-06$ & $3.88 E-06$ & $5.38 \mathrm{E}-07$ & $3.84 \mathrm{E}-06$ & 1.54 & 0.9958 & 3.87E-06 & $3.85 E-06$ & $-1.93 \mathrm{E}-08$ & 4.37E-06 \\
\hline 300 & 3.28 & 3.50 & 2.11 & 0.9961 & $3.53 \mathrm{E}-06$ & 4.07E-06 & $5.36 \mathrm{E}-07$ & 4.03E-06 & 2.38 & 0.9962 & $3.99 \mathrm{E}-06$ & 3.97E-06 & $-1.66 \mathrm{E}-08$ & 4.49E-06 \\
\hline 400 & 4.19 & 4.28 & 2.89 & 0.9964 & $3.63 \mathrm{E}-06$ & 4.16E-06 & $5.29 \mathrm{E}-07$ & 4.13E-06 & 3.29 & 0.9966 & 4.13E-06 & $4.11 E-06$ & $-2.32 \mathrm{E}-08$ & 4.63E-06 \\
\hline 500 & 5.25 & 5.14 & 3.75 & 0.9969 & $3.77 \mathrm{E}-06$ & 4.30E-06 & $5.31 \mathrm{E}-07$ & 4.27E-06 & 4.35 & 0.9972 & 4.37E-06 & $4.35 E-06$ & $-2.19 \mathrm{E}-08$ & 4.87E-06 \\
\hline
\end{tabular}




\begin{tabular}{|c|c|c|c|c|c|c|c|c|c|c|c|c|c|c|}
\hline $\begin{array}{l}\text { Capsule } \\
\text { Column }\end{array}$ & $\begin{array}{l}\text { OC4 } \\
\text { South }\end{array}$ & $\begin{array}{c}\text { Specimen } \\
\text { Length }\end{array}$ & $\begin{array}{c}94 \\
0.9959 \\
\end{array}$ & $\begin{array}{c}\text { Material } \\
\text { Scale }\end{array}$ & $\begin{array}{c}\text { H-451 } \\
1 "\end{array}$ & $5.00 \mathrm{E}-04$ & & & & & & & & \\
\hline $\begin{array}{c}\text { Temperature } \\
\left({ }^{\circ} \mathrm{C}\right) \\
\end{array}$ & Up & Down & $\begin{array}{l}\text { Delta } \\
\text { Down }\end{array}$ & $\begin{array}{l}\text { Down } \\
\text { length }\end{array}$ & CTE & CTEK & $\begin{array}{l}\text { Delta } \\
\text { CTEK }\end{array}$ & $\begin{array}{c}\text { CTE } \\
\text { adjusted }\end{array}$ & $\begin{array}{l}\text { Delta } \\
\text { Up }\end{array}$ & $\begin{array}{c}\text { Up } \\
\text { length }\end{array}$ & CTE & CTEK & $\begin{array}{l}\text { Delta } \\
\text { CTEK }\end{array}$ & $\begin{array}{c}\text { CTE } \\
\text { adjusted }\end{array}$ \\
\hline 0 & 0.75 & 0.73 & 0 & 0.9959 & & & & & 0 & 0.9959 & & & & \\
\hline 100 & 1.46 & 1.40 & 0.67 & 0.9962 & $3.36 \mathrm{E}-06$ & $3.90 E-06$ & $5.36 \mathrm{E}-07$ & $3.86 \mathrm{E}-06$ & 0.71 & 0.9963 & $3.56 \mathrm{E}-06$ & 4.10E-06 & $5.35 \mathrm{E}-07$ & 4.06E-06 \\
\hline 200 & 2.32 & 2.09 & 1.36 & 0.9966 & $3.41 \mathrm{E}-06$ & $3.95 E-06$ & 5.36E-07 & $3.91 \mathrm{E}-06$ & 1.57 & 0.9967 & $3.94 \mathrm{E}-06$ & $4.48 E-06$ & $5.39 \mathrm{E}-07$ & 4.44E-06 \\
\hline 300 & 2.88 & 2.83 & 2.1 & 0.9970 & $3.51 \mathrm{E}-06$ & $1.05 E-06$ & $-2.46 \mathrm{E}-06$ & $4.01 \mathrm{E}-06$ & 2.13 & 0.9970 & $3.56 \mathrm{E}-06$ & 4.10E-06 & $5.35 \mathrm{E}-07$ & 4.06E-06 \\
\hline 400 & 3.51 & 3.62 & 2.89 & 0.9973 & $3.63 \mathrm{E}-06$ & 4.16E-06 & $5.33 \mathrm{E}-07$ & 4.13E-06 & 2.76 & 0.9973 & $3.46 \mathrm{E}-06$ & $4.00 E-06$ & $5.36 \mathrm{E}-07$ & $3.96 \mathrm{E}-06$ \\
\hline 500 & 4.28 & 4.38 & 3.65 & 0.9977 & $3.67 \mathrm{E}-06$ & 4.20E-06 & $5.35 \mathrm{E}-07$ & 4.17E-06 & 3.53 & 0.9977 & $3.54 \mathrm{E}-06$ & $4.08 E-06$ & $5.35 \mathrm{E}-07$ & 4.04E-06 \\
\hline
\end{tabular}

\begin{tabular}{|c|c|c|c|c|c|c|c|c|c|c|c|c|c|c|}
\hline $\begin{array}{l}\text { Capsule } \\
\text { Column }\end{array}$ & $\begin{array}{l}\text { OC4 } \\
\text { North }\end{array}$ & $\begin{array}{l}\text { Specimen } \\
\text { Length }\end{array}$ & $\begin{array}{c}103 \\
0.9961\end{array}$ & $\begin{array}{l}\text { Material } \\
\text { Scale }\end{array}$ & $\begin{array}{l}\text { H-451 } \\
1 "\end{array}$ & $5.00 \mathrm{E}-04$ & & & & & & & & \\
\hline $\begin{array}{c}\text { Temperature } \\
\left({ }^{\circ} \mathrm{C}\right)\end{array}$ & Up & Down & $\begin{array}{l}\text { Delta } \\
\text { Down }\end{array}$ & $\begin{array}{l}\text { Down } \\
\text { length }\end{array}$ & CTE & CTEK & $\begin{array}{l}\text { Delta } \\
\text { CTEK }\end{array}$ & $\begin{array}{c}\text { CTE } \\
\text { adjusted }\end{array}$ & $\begin{array}{l}\text { Delta } \\
\text { Up }\end{array}$ & $\begin{array}{c}\text { Up } \\
\text { length }\end{array}$ & CTE & CTEK & $\begin{array}{l}\text { Delta } \\
\text { CTEK }\end{array}$ & $\begin{array}{c}\text { CTE } \\
\text { adjusted }\end{array}$ \\
\hline 0 & 1.20 & 1.76 & 0 & 0.9961 & & & & & 0 & 0.9961 & & & & \\
\hline 100 & 2.00 & 2.22 & 0.46 & 0.9963 & $2.31 \mathrm{E}-06$ & $2.85 E-06$ & $5.41 \mathrm{E}-07$ & $2.81 \mathrm{E}-06$ & 0.8 & 0.9965 & $4.02 \mathrm{E}-06$ & $4.55 E-06$ & $5.34 \mathrm{E}-07$ & 4.52E-06 \\
\hline 200 & 2.56 & 2.70 & 0.94 & 0.9966 & $2.36 \mathrm{E}-06$ & 2.90E-06 & $5.41 \mathrm{E}-07$ & $2.86 \mathrm{E}-06$ & 1.36 & 0.9968 & $3.41 \mathrm{E}-06$ & $3.95 E-06$ & 5.37E-07 & $3.91 \mathrm{E}-06$ \\
\hline 300 & 3.02 & 3.20 & 1.44 & 0.9968 & $2.41 \mathrm{E}-06$ & 2.95E-06 & $5.41 \mathrm{E}-07$ & $2.91 \mathrm{E}-06$ & 1.82 & 0.9970 & 3.05E-06 & $3.58 E-06$ & $5.35 \mathrm{E}-07$ & $3.55 \mathrm{E}-06$ \\
\hline 400 & 3.50 & 3.71 & 1.95 & 0.9971 & $2.45 \mathrm{E}-06$ & 2.99E-06 & $5.43 \mathrm{E}-07$ & $2.95 \mathrm{E}-06$ & 2.3 & 0.9973 & $2.89 \mathrm{E}-06$ & $3.42 E-06$ & 5.34E-07 & 3.39E-06 \\
\hline 500 & 4.10 & 4.27 & 2.51 & 0.9974 & $2.52 \mathrm{E}-06$ & $3.06 E-06$ & $5.40 \mathrm{E}-07$ & $3.02 \mathrm{E}-06$ & 2.9 & 0.9976 & $2.91 \mathrm{E}-06$ & $3.45 E-06$ & 5.39E-07 & $3.41 \mathrm{E}-06$ \\
\hline
\end{tabular}

\begin{tabular}{|c|c|c|c|c|c|c|c|c|c|c|c|c|c|c|}
\hline $\begin{array}{l}\text { Capsule } \\
\text { Column }\end{array}$ & $\begin{array}{c}\text { OC4 } \\
\text { South }\end{array}$ & $\begin{array}{c}\text { Specimen } \\
\text { Length }\end{array}$ & $\begin{array}{c}104 \\
0.9976 \\
\end{array}$ & $\begin{array}{c}\text { Material } \\
\text { Scale }\end{array}$ & $\begin{array}{c}\text { H-451 } \\
1 " \\
\end{array}$ & $5.00 \mathrm{E}-04$ & & & & & & & & \\
\hline $\begin{array}{c}\text { Temperature } \\
\left({ }^{\circ} \mathrm{C}\right)\end{array}$ & Up & Down & $\begin{array}{l}\text { Delta } \\
\text { Down }\end{array}$ & $\begin{array}{l}\text { Down } \\
\text { length }\end{array}$ & CTE & CTEK & $\begin{array}{l}\text { Delta } \\
\text { CTEK }\end{array}$ & $\begin{array}{c}\text { CTE } \\
\text { adjusted }\end{array}$ & $\begin{array}{l}\text { Delta } \\
\text { Up }\end{array}$ & $\begin{array}{c}\text { Up } \\
\text { length }\end{array}$ & CTE & CTEK & $\begin{array}{l}\text { Delta } \\
\text { CTEK }\end{array}$ & $\begin{array}{c}\text { CTE } \\
\text { adjusted }\end{array}$ \\
\hline 0 & 0.83 & 1.10 & 0 & 0.9976 & & & & & 0 & 0.9976 & & & & \\
\hline 100 & 1.71 & 1.62 & 0.52 & 0.9979 & $2.61 \mathrm{E}-06$ & $3.15 E-06$ & $5.44 \mathrm{E}-07$ & $3.11 \mathrm{E}-06$ & 0.88 & 0.9980 & 4.41E-06 & $4.95 E-06$ & 5.39E-07 & $4.91 \mathrm{E}-06$ \\
\hline 200 & 2.48 & 2.22 & 1.12 & 0.9982 & $2.81 \mathrm{E}-06$ & $3.35 E-06$ & $5.43 \mathrm{E}-07$ & $3.31 \mathrm{E}-06$ & 1.65 & 0.9984 & 4.13E-06 & $4.68 E-06$ & $5.45 \mathrm{E}-07$ & $4.63 \mathrm{E}-06$ \\
\hline 300 & 2.88 & 2.80 & 1.7 & 0.9985 & $2.84 \mathrm{E}-06$ & $3.35 E-06$ & $5.10 \mathrm{E}-07$ & $3.34 \mathrm{E}-06$ & 2.05 & 0.9986 & $3.42 \mathrm{E}-06$ & 3.97E-06 & $5.45 \mathrm{E}-07$ & $3.92 \mathrm{E}-06$ \\
\hline 400 & 3.33 & 3.36 & 2.26 & 0.9987 & $2.83 \mathrm{E}-06$ & $3.38 E-06$ & $5.48 \mathrm{E}-07$ & $3.33 \mathrm{E}-06$ & 2.5 & 0.9989 & $3.13 \mathrm{E}-06$ & $3.68 E-06$ & 5.47E-07 & $3.63 \mathrm{E}-06$ \\
\hline 500 & 3.94 & 3.94 & 2.84 & 0.9990 & $2.85 \mathrm{E}-06$ & $3.39 E-06$ & $5.43 \mathrm{E}-07$ & $3.35 \mathrm{E}-06$ & 3.11 & 0.9992 & $3.12 \mathrm{E}-06$ & $3.66 E-06$ & 5.43E-07 & $3.62 \mathrm{E}-06$ \\
\hline
\end{tabular}




\begin{tabular}{|c|c|c|c|c|c|c|c|c|c|c|c|c|c|c|}
\hline $\begin{array}{l}\text { Capsule } \\
\text { Column }\end{array}$ & $\begin{array}{l}\text { OC4 } \\
\text { North }\end{array}$ & $\begin{array}{c}\text { Specimen } \\
\text { Length }\end{array}$ & $\begin{array}{c}105 \\
0.9768\end{array}$ & $\begin{array}{c}\text { Material } \\
\text { Scale }\end{array}$ & $\begin{array}{c}\text { H-451 } \\
1 "\end{array}$ & $5.00 \mathrm{E}-04$ & & & & & & & & \\
\hline $\begin{array}{c}\text { Temperature } \\
\left({ }^{\circ} \mathrm{C}\right) \\
\end{array}$ & Up & Down & $\begin{array}{l}\text { Delta } \\
\text { Down }\end{array}$ & $\begin{array}{l}\text { Down } \\
\text { length }\end{array}$ & CTE & CTEK & $\begin{array}{l}\text { Delta } \\
\text { CTEK }\end{array}$ & $\begin{array}{c}\text { CTE } \\
\text { adjusted }\end{array}$ & $\begin{array}{l}\text { Delta } \\
\text { Up }\end{array}$ & $\begin{array}{c}\text { Up } \\
\text { length }\end{array}$ & CTE & CTEK & $\begin{array}{l}\text { Delta } \\
\text { CTEK }\end{array}$ & $\begin{array}{c}\text { CTE } \\
\text { adjusted }\end{array}$ \\
\hline 0 & 1.14 & 1.32 & 0 & 0.9952 & & & & & 0 & 0.9952 & & & & \\
\hline 100 & 1.80 & 1.85 & 0.53 & 0.9955 & $2.66 \mathrm{E}-06$ & $4.95 E-06$ & $2.29 \mathrm{E}-06$ & $3.16 \mathrm{E}-06$ & 0.66 & 0.9955 & $3.32 \mathrm{E}-06$ & $3.85 E-06$ & $5.34 \mathrm{E}-07$ & $3.82 \mathrm{E}-06$ \\
\hline 200 & 2.39 & 2.35 & 1.03 & 0.9957 & $2.59 \mathrm{E}-06$ & 4.68E-06 & 2.09E-06 & $3.09 \mathrm{E}-06$ & 1.25 & 0.9958 & $3.14 \mathrm{E}-06$ & $3.68 E-06$ & $5.40 \mathrm{E}-07$ & 3.64E-06 \\
\hline 300 & 2.85 & 2.95 & 1.63 & 0.9960 & $2.73 \mathrm{E}-06$ & 3.97E-06 & $1.24 \mathrm{E}-06$ & $3.23 \mathrm{E}-06$ & 1.71 & 0.9961 & $2.86 \mathrm{E}-06$ & $3.40 E-06$ & $5.36 \mathrm{E}-07$ & $3.36 \mathrm{E}-06$ \\
\hline 400 & 3.27 & 3.51 & 2.19 & 0.9963 & $2.75 \mathrm{E}-06$ & $3.68 E-06$ & $9.29 \mathrm{E}-07$ & $3.25 \mathrm{E}-06$ & 2.13 & 0.9963 & $2.68 \mathrm{E}-06$ & $3.22 E-06$ & $5.45 \mathrm{E}-07$ & $3.18 \mathrm{E}-06$ \\
\hline 500 & 3.95 & 4.10 & 2.78 & 0.9966 & $2.79 \mathrm{E}-06$ & $3.66 E-06$ & 8.67E-07 & $3.29 \mathrm{E}-06$ & 2.81 & 0.9966 & $2.82 \mathrm{E}-06$ & $3.36 E-06$ & $5.36 \mathrm{E}-07$ & $3.32 \mathrm{E}-06$ \\
\hline
\end{tabular}

\begin{tabular}{|c|c|c|c|c|c|c|c|c|c|c|c|c|c|c|}
\hline $\begin{array}{l}\text { Capsule } \\
\text { Column }\end{array}$ & $\begin{array}{l}\text { OC4 } \\
\text { South }\end{array}$ & $\begin{array}{l}\text { Specimen } \\
\text { Length }\end{array}$ & $\begin{array}{c}106 \\
0.9946\end{array}$ & $\begin{array}{l}\text { Material } \\
\text { Scale }\end{array}$ & $\begin{array}{l}\text { H-451 } \\
1 "\end{array}$ & $5.00 \mathrm{E}-04$ & & & & & & & & \\
\hline $\begin{array}{c}\text { Temperature } \\
\left({ }^{\circ} \mathrm{C}\right)\end{array}$ & Up & Down & $\begin{array}{l}\text { Delta } \\
\text { Down }\end{array}$ & $\begin{array}{l}\text { Down } \\
\text { length }\end{array}$ & CTE & CTEK & $\begin{array}{l}\text { Delta } \\
\text { CTEK }\end{array}$ & $\begin{array}{c}\text { CTE } \\
\text { adjusted }\end{array}$ & $\begin{array}{l}\text { Delta } \\
\text { Up }\end{array}$ & $\begin{array}{c}\text { Up } \\
\text { length }\end{array}$ & CTE & CTEK & $\begin{array}{l}\text { Delta } \\
\text { CTEK }\end{array}$ & $\begin{array}{c}\text { CTE } \\
\text { adjusted }\end{array}$ \\
\hline 0 & 0.85 & 1.11 & 0 & 0.9946 & & & & & 0 & 0.9946 & & & & \\
\hline 100 & 1.54 & 1.67 & 0.56 & 0.9949 & $2.82 \mathrm{E}-06$ & $3.35 E-06$ & 5.35E-07 & $3.32 \mathrm{E}-06$ & 0.69 & 0.9949 & $3.47 \mathrm{E}-06$ & $4.00 E-06$ & $5.31 \mathrm{E}-07$ & 3.97E-06 \\
\hline 200 & 2.22 & 2.22 & 1.11 & 0.9952 & $2.79 \mathrm{E}-06$ & $3.32 E-06$ & $5.30 \mathrm{E}-07$ & $3.29 \mathrm{E}-06$ & 1.37 & 0.9953 & $3.44 \mathrm{E}-06$ & $3.98 E-06$ & $5.36 \mathrm{E}-07$ & $3.94 \mathrm{E}-06$ \\
\hline 300 & 2.65 & 2.84 & 1.73 & 0.9955 & 2.90E-06 & $3.43 E-06$ & 5.31E-07 & $3.40 \mathrm{E}-06$ & 1.8 & 0.9955 & $3.02 \mathrm{E}-06$ & $3.55 E-06$ & $5.34 \mathrm{E}-07$ & $3.52 \mathrm{E}-06$ \\
\hline 400 & 3.22 & 3.40 & 2.29 & 0.9957 & $2.88 \mathrm{E}-06$ & $3.41 E-06$ & $5.32 \mathrm{E}-07$ & $3.38 \mathrm{E}-06$ & 2.37 & 0.9958 & $2.98 \mathrm{E}-06$ & $3.51 E-06$ & $5.31 \mathrm{E}-07$ & $3.48 \mathrm{E}-06$ \\
\hline 500 & 3.81 & 3.95 & 2.84 & 0.9960 & $2.86 \mathrm{E}-06$ & $3.39 E-06$ & $5.35 \mathrm{E}-07$ & $3.36 \mathrm{E}-06$ & 2.96 & 0.9961 & $2.98 \mathrm{E}-06$ & $3.51 E-06$ & $5.34 \mathrm{E}-07$ & $3.48 \mathrm{E}-06$ \\
\hline
\end{tabular}

\begin{tabular}{|c|c|c|c|c|c|c|c|c|c|c|c|c|c|c|}
\hline $\begin{array}{l}\text { Capsule } \\
\text { Column }\end{array}$ & $\begin{array}{c}\text { OC4 } \\
\text { South }\end{array}$ & $\begin{array}{c}\text { Specimen } \\
\text { Length }\end{array}$ & $\begin{array}{c}107 \\
0.9961 \\
\end{array}$ & $\begin{array}{c}\text { Material } \\
\text { Scale }\end{array}$ & $\begin{array}{c}\text { H-451 } \\
1 " \\
\end{array}$ & $5.00 \mathrm{E}-04$ & & & & & & & & \\
\hline $\begin{array}{c}\text { Temperature } \\
\left({ }^{\circ} \mathrm{C}\right)\end{array}$ & Up & Down & $\begin{array}{l}\text { Delta } \\
\text { Down }\end{array}$ & $\begin{array}{l}\text { Down } \\
\text { length }\end{array}$ & CTE & CTEK & $\begin{array}{l}\text { Delta } \\
\text { CTEK }\end{array}$ & $\begin{array}{c}\text { CTE } \\
\text { adjusted }\end{array}$ & $\begin{array}{l}\text { Delta } \\
\text { Up }\end{array}$ & $\begin{array}{c}\text { Up } \\
\text { length }\end{array}$ & CTE & CTEK & $\begin{array}{l}\text { Delta } \\
\text { CTEK }\end{array}$ & $\begin{array}{c}\text { CTE } \\
\text { adjusted }\end{array}$ \\
\hline 0 & 0.79 & 1.29 & 0 & 0.9976 & & & & & 0 & 0.9976 & & & & \\
\hline 100 & 1.57 & 1.85 & 0.56 & 0.9979 & $2.81 \mathrm{E}-06$ & $3.35 E-06$ & $5.43 \mathrm{E}-07$ & $3.31 \mathrm{E}-06$ & 0.78 & 0.9980 & 3.91E-06 & $4.45 E-06$ & $5.41 \mathrm{E}-07$ & 4.41E-06 \\
\hline 200 & 2.19 & 2.35 & 1.06 & 0.9981 & $2.66 \mathrm{E}-06$ & $3.20 E-06$ & $5.44 \mathrm{E}-07$ & $3.16 \mathrm{E}-06$ & 1.4 & 0.9983 & $3.51 \mathrm{E}-06$ & $4.05 E-06$ & $5.42 \mathrm{E}-07$ & $4.01 \mathrm{E}-06$ \\
\hline 300 & 2.40 & 2.88 & 1.59 & 0.9984 & $2.66 \mathrm{E}-06$ & $3.20 E-06$ & $5.44 \mathrm{E}-07$ & $3.16 \mathrm{E}-06$ & 1.61 & 0.9984 & $2.69 \mathrm{E}-06$ & $3.23 E-06$ & $5.40 \mathrm{E}-07$ & 3.19E-06 \\
\hline 400 & 2.92 & 3.30 & 2.01 & 0.9986 & $2.52 \mathrm{E}-06$ & $3.06 E-06$ & $5.41 \mathrm{E}-07$ & $3.02 \mathrm{E}-06$ & 2.13 & 0.9987 & $2.67 \mathrm{E}-06$ & $3.21 E-06$ & $5.41 \mathrm{E}-07$ & 3.17E-06 \\
\hline 500 & 3.60 & 3.75 & 2.46 & 0.9988 & 2.47E-06 & $3.01 E-06$ & 5.44E-07 & 2.97E-06 & 2.81 & 0.9990 & $2.82 \mathrm{E}-06$ & $3.36 E-06$ & 5.43E-07 & $3.32 \mathrm{E}-06$ \\
\hline
\end{tabular}




\begin{tabular}{|c|c|c|c|c|c|c|c|c|c|c|c|c|c|c|}
\hline $\begin{array}{l}\text { Capsule } \\
\text { Column }\end{array}$ & $\begin{array}{l}\text { OC4 } \\
\text { South }\end{array}$ & $\begin{array}{l}\text { Specimen } \\
\text { Length }\end{array}$ & $\begin{array}{c}108 \\
0.998\end{array}$ & $\begin{array}{l}\text { Material } \\
\text { Scale }\end{array}$ & $\begin{array}{c}\text { H-451 } \\
1 "\end{array}$ & $5.00 \mathrm{E}-04$ & & & & & & & & \\
\hline $\begin{array}{c}\text { Temperature } \\
\left({ }^{\circ} \mathrm{C}\right) \\
\end{array}$ & Up & Down & $\begin{array}{l}\text { Delta } \\
\text { Down }\end{array}$ & $\begin{array}{l}\text { Down } \\
\text { length }\end{array}$ & CTE & CTEK & $\begin{array}{l}\text { Delta } \\
\text { CTEK }\end{array}$ & $\begin{array}{c}\text { CTE } \\
\text { adjusted }\end{array}$ & $\begin{array}{l}\text { Delta } \\
\text { Up }\end{array}$ & $\begin{array}{c}\text { Up } \\
\text { length }\end{array}$ & CTE & CTEK & $\begin{array}{l}\text { Delta } \\
\text { CTEK }\end{array}$ & $\begin{array}{c}\text { CTE } \\
\text { adjusted }\end{array}$ \\
\hline 0 & 1.07 & 1.65 & 0 & 0.9980 & & & & & 0 & 0.9980 & & & & \\
\hline 100 & 1.85 & 2.25 & 0.6 & 0.9983 & $3.01 \mathrm{E}-06$ & $3.55 E-06$ & 5.44E-07 & $3.51 \mathrm{E}-06$ & 0.78 & 0.9984 & $3.91 \mathrm{E}-06$ & 4.45E-06 & $5.42 \mathrm{E}-07$ & $4.41 \mathrm{E}-06$ \\
\hline 200 & 2.53 & 2.85 & 1.2 & 0.9986 & $3.01 \mathrm{E}-06$ & $3.55 E-06$ & $5.44 \mathrm{E}-07$ & $3.51 \mathrm{E}-06$ & 1.46 & 0.9987 & $3.66 \mathrm{E}-06$ & 4.72E-06 & $1.06 \mathrm{E}-06$ & 4.16E-06 \\
\hline 300 & 3.05 & 3.45 & 1.8 & 0.9989 & $3.01 \mathrm{E}-06$ & $3.55 E-06$ & $5.44 \mathrm{E}-07$ & $3.51 \mathrm{E}-06$ & 1.98 & 0.9990 & $3.31 \mathrm{E}-06$ & $3.85 E-06$ & $5.43 \mathrm{E}-07$ & $3.81 \mathrm{E}-06$ \\
\hline 400 & 3.72 & 4.08 & 2.43 & 0.9992 & $3.04 \mathrm{E}-06$ & 3.59E-06 & $5.46 \mathrm{E}-07$ & 3.54E-06 & 2.65 & 0.9993 & $3.32 \mathrm{E}-06$ & $3.86 E-06$ & $5.41 \mathrm{E}-07$ & $3.82 \mathrm{E}-06$ \\
\hline 500 & 4.50 & 4.70 & 3.05 & 0.9995 & 3.06E-06 & $3.60 E-06$ & $5.44 \mathrm{E}-07$ & 3.56E-06 & 3.43 & 0.9997 & $3.44 \mathrm{E}-06$ & $3.98 E-06$ & $5.43 \mathrm{E}-07$ & $3.94 \mathrm{E}-06$ \\
\hline
\end{tabular}

\begin{tabular}{|c|c|c|c|c|c|c|c|c|c|c|c|c|c|c|}
\hline $\begin{array}{l}\text { Capsule } \\
\text { Column }\end{array}$ & $\begin{array}{l}\text { OC4 } \\
\text { South }\end{array}$ & $\begin{array}{c}\text { Specimen } \\
\text { Length }\end{array}$ & $\begin{array}{c}110 \\
0.9958\end{array}$ & $\begin{array}{c}\text { Material } \\
\text { Scale }\end{array}$ & $\begin{array}{c}\text { H-451 } \\
1 "\end{array}$ & $5.00 \mathrm{E}-04$ & & & & & & & & \\
\hline $\begin{array}{c}\text { Temperature } \\
\left({ }^{\circ} \mathrm{C}\right)\end{array}$ & Up & Down & $\begin{array}{l}\text { Delta } \\
\text { Down }\end{array}$ & $\begin{array}{l}\text { Down } \\
\text { length }\end{array}$ & CTE & CTEK & $\begin{array}{l}\text { Delta } \\
\text { CTEK }\end{array}$ & $\begin{array}{c}\text { CTE } \\
\text { adjusted }\end{array}$ & $\begin{array}{l}\text { Delta } \\
\text { Up }\end{array}$ & $\begin{array}{c}\text { Up } \\
\text { length }\end{array}$ & CTE & CTEK & $\begin{array}{l}\text { Delta } \\
\text { CTEK }\end{array}$ & $\begin{array}{c}\text { CTE } \\
\text { adjusted }\end{array}$ \\
\hline 0 & 0.78 & 1.50 & 0 & 0.9944 & & & & & 0 & 0.9944 & & & & \\
\hline 100 & 1.60 & 2.02 & 0.52 & 0.9947 & $2.61 \mathrm{E}-06$ & $3.15 E-06$ & $5.35 \mathrm{E}-07$ & $3.11 \mathrm{E}-06$ & 0.82 & 0.9948 & 4.12E-06 & $4.65 E-06$ & 5.27E-07 & 4.62E-06 \\
\hline 200 & 2.36 & 2.50 & 1 & 0.9949 & $2.51 \mathrm{E}-06$ & $3.05 E-06$ & $5.36 \mathrm{E}-07$ & $3.01 \mathrm{E}-06$ & 1.58 & 0.9952 & 3.97E-06 & 4.50E-06 & $5.28 \mathrm{E}-07$ & 4.47E-06 \\
\hline 300 & 2.52 & 3.01 & 1.51 & 0.9952 & $2.53 \mathrm{E}-06$ & $3.07 E-06$ & 5.39E-07 & 3.03E-06 & 1.74 & 0.9953 & $2.92 \mathrm{E}-06$ & $3.45 E-06$ & 5.34E-07 & 3.42E-06 \\
\hline 400 & 3.12 & 3.42 & 1.92 & 0.9954 & $2.41 \mathrm{E}-06$ & $2.95 E-06$ & 5.36E-07 & 2.91E-06 & 2.34 & 0.9956 & 2.94E-06 & $3.48 E-06$ & 5.39E-07 & $3.44 \mathrm{E}-06$ \\
\hline 500 & 3.78 & 3.90 & 2.4 & 0.9956 & $2.41 \mathrm{E}-06$ & $2.95 E-06$ & $5.36 \mathrm{E}-07$ & 2.91E-06 & 3 & 0.9959 & $3.02 \mathrm{E}-06$ & $3.55 E-06$ & $5.33 \mathrm{E}-07$ & $3.52 \mathrm{E}-06$ \\
\hline
\end{tabular}

\begin{tabular}{|c|c|c|c|c|c|c|c|c|c|c|c|c|c|c|}
\hline $\begin{array}{l}\text { Capsule } \\
\text { Column }\end{array}$ & $\begin{array}{c}\text { OC4 } \\
\text { North }\end{array}$ & $\begin{array}{l}\text { Specimen } \\
\text { Length }\end{array}$ & $\begin{array}{c}118 \\
0.9944\end{array}$ & $\begin{array}{c}\text { Material } \\
\text { Scale }\end{array}$ & $\begin{array}{c}\text { H-451 } \\
1 "\end{array}$ & $5.00 \mathrm{E}-04$ & & & & & & & & \\
\hline $\begin{array}{c}\text { Temperature } \\
\left({ }^{\circ} \mathrm{C}\right)\end{array}$ & Up & Down & $\begin{array}{l}\text { Delta } \\
\text { Down }\end{array}$ & $\begin{array}{l}\text { Down } \\
\text { length }\end{array}$ & CTE & CTEK & $\begin{array}{l}\text { Delta } \\
\text { CTEK }\end{array}$ & $\begin{array}{c}\text { CTE } \\
\text { adjusted }\end{array}$ & $\begin{array}{l}\text { Delta } \\
\text { Up }\end{array}$ & $\begin{array}{l}\text { Up } \\
\text { length }\end{array}$ & CTE & CTEK & $\begin{array}{l}\text { Delta } \\
\text { CTEK }\end{array}$ & $\begin{array}{c}\text { CTE } \\
\text { adjusted }\end{array}$ \\
\hline 0 & 1.40 & 1.60 & 0 & 0.9944 & & & & & 0 & 0.9944 & & & & \\
\hline 100 & 2.25 & 2.10 & 0.5 & 0.9947 & $2.51 \mathrm{E}-06$ & $3.05 E-06$ & $5.36 \mathrm{E}-07$ & $3.01 \mathrm{E}-06$ & 0.85 & 0.9948 & 4.27E-06 & $4.80 E-06$ & $5.26 \mathrm{E}-07$ & 4.77E-06 \\
\hline 200 & 2.80 & 2.62 & 1.02 & 0.9949 & $2.56 \mathrm{E}-06$ & $3.10 E-06$ & $5.36 \mathrm{E}-07$ & 3.06E-06 & 1.4 & 0.9951 & $3.52 \mathrm{E}-06$ & $4.05 E-06$ & $5.30 \mathrm{E}-07$ & $4.02 \mathrm{E}-06$ \\
\hline 300 & 3.20 & 3.22 & 1.62 & 0.9952 & $2.72 \mathrm{E}-06$ & $3.25 E-06$ & $5.35 \mathrm{E}-07$ & $3.22 \mathrm{E}-06$ & 1.8 & 0.9953 & $3.02 \mathrm{E}-06$ & $3.55 E-06$ & $5.33 \mathrm{E}-07$ & $3.52 \mathrm{E}-06$ \\
\hline 400 & 3.75 & 3.80 & 2.2 & 0.9955 & $2.77 \mathrm{E}-06$ & $3.30 E-06$ & $5.35 \mathrm{E}-07$ & $3.27 \mathrm{E}-06$ & 2.35 & 0.9956 & $2.95 \mathrm{E}-06$ & $3.49 E-06$ & $5.36 \mathrm{E}-07$ & $3.45 \mathrm{E}-06$ \\
\hline 500 & 4.35 & 4.40 & 2.8 & 0.9958 & $2.82 \mathrm{E}-06$ & $3.35 E-06$ & $5.34 \mathrm{E}-07$ & 3.32E-06 & 2.95 & 0.9959 & 2.97E-06 & $3.50 E-06$ & $5.33 \mathrm{E}-07$ & 3.47E-06 \\
\hline
\end{tabular}




\begin{tabular}{|c|c|c|c|c|c|c|c|c|c|c|c|c|c|c|}
\hline $\begin{array}{l}\text { Capsule } \\
\text { Column }\end{array}$ & $\begin{array}{l}\text { OC4 } \\
\text { North }\end{array}$ & $\begin{array}{c}\text { Specimen } \\
\text { Length }\end{array}$ & $\begin{array}{c}120 \\
0.9942\end{array}$ & $\begin{array}{c}\text { Material } \\
\text { Scale }\end{array}$ & $\begin{array}{c}\text { H-451 } \\
1 "\end{array}$ & $5.00 \mathrm{E}-04$ & & & & & & & & \\
\hline $\begin{array}{c}\text { Temperature } \\
\left({ }^{\circ} \mathrm{C}\right) \\
\end{array}$ & Up & Down & $\begin{array}{l}\text { Delta } \\
\text { Down }\end{array}$ & $\begin{array}{l}\text { Down } \\
\text { length }\end{array}$ & CTE & CTEK & $\begin{array}{l}\text { Delta } \\
\text { CTEK }\end{array}$ & $\begin{array}{c}\text { CTE } \\
\text { adjusted }\end{array}$ & $\begin{array}{l}\text { Delta } \\
\text { Up }\end{array}$ & $\begin{array}{c}\text { Up } \\
\text { length }\end{array}$ & CTE & CTEK & $\begin{array}{l}\text { Delta } \\
\text { CTEK }\end{array}$ & $\begin{array}{c}\text { CTE } \\
\text { adjusted }\end{array}$ \\
\hline 0 & 1.11 & 0.91 & 0 & 0.9942 & & & & & 0 & 0.9942 & & & & \\
\hline 100 & 1.73 & 1.53 & 0.62 & 0.9945 & $3.12 \mathrm{E}-06$ & $3.65 E-06$ & $5.32 \mathrm{E}-07$ & $3.62 \mathrm{E}-06$ & 0.62 & 0.9945 & $3.12 \mathrm{E}-06$ & $3.65 E-06$ & $5.32 \mathrm{E}-07$ & $3.62 \mathrm{E}-06$ \\
\hline 200 & 2.40 & 2.13 & 1.22 & 0.9948 & 3.07E-06 & $3.60 E-06$ & $5.32 \mathrm{E}-07$ & $3.57 \mathrm{E}-06$ & 1.29 & 0.9948 & $3.24 \mathrm{E}-06$ & $3.78 E-06$ & $5.36 \mathrm{E}-07$ & 3.74E-06 \\
\hline 300 & 2.85 & 2.78 & 1.87 & 0.9951 & $3.13 \mathrm{E}-06$ & 3.67E-06 & $5.35 \mathrm{E}-07$ & $3.63 \mathrm{E}-06$ & 1.74 & 0.9951 & $2.92 \mathrm{E}-06$ & $3.45 E-06$ & $5.33 \mathrm{E}-07$ & $3.42 \mathrm{E}-06$ \\
\hline 400 & 3.38 & 3.30 & 2.39 & 0.9954 & $3.00 \mathrm{E}-06$ & $3.54 E-06$ & $5.35 \mathrm{E}-07$ & $3.50 \mathrm{E}-06$ & 2.27 & 0.9953 & $2.85 \mathrm{E}-06$ & $3.39 E-06$ & $5.36 \mathrm{E}-07$ & 3.35E-06 \\
\hline 500 & 3.98 & 4.03 & 3.12 & 0.9958 & $3.14 \mathrm{E}-06$ & $3.67 E-06$ & $5.32 \mathrm{E}-07$ & $3.64 \mathrm{E}-06$ & 2.87 & 0.9956 & $2.89 \mathrm{E}-06$ & $3.42 E-06$ & $5.33 \mathrm{E}-07$ & $3.39 \mathrm{E}-06$ \\
\hline
\end{tabular}

\begin{tabular}{|c|c|c|c|c|c|c|c|c|c|c|c|c|c|c|}
\hline $\begin{array}{l}\text { Capsule } \\
\text { Column }\end{array}$ & $\begin{array}{l}\text { OC4 } \\
\text { South }\end{array}$ & $\begin{array}{l}\text { Specimen } \\
\text { Length }\end{array}$ & $\begin{array}{c}121 \\
0.9946\end{array}$ & $\begin{array}{l}\text { Material } \\
\text { Scale }\end{array}$ & $\begin{array}{l}\text { H-451 } \\
1 "\end{array}$ & $5.00 \mathrm{E}-04$ & & & & & & & & \\
\hline $\begin{array}{c}\text { Temperature } \\
\left({ }^{\circ} \mathrm{C}\right)\end{array}$ & Up & Down & $\begin{array}{l}\text { Delta } \\
\text { Down }\end{array}$ & $\begin{array}{l}\text { Down } \\
\text { length }\end{array}$ & CTE & CTEK & $\begin{array}{l}\text { Delta } \\
\text { CTEK }\end{array}$ & $\begin{array}{c}\text { CTE } \\
\text { adjusted }\end{array}$ & $\begin{array}{l}\text { Delta } \\
\text { Up }\end{array}$ & $\begin{array}{c}\text { Up } \\
\text { length }\end{array}$ & CTE & CTEK & $\begin{array}{l}\text { Delta } \\
\text { CTEK }\end{array}$ & $\begin{array}{c}\text { CTE } \\
\text { adjusted }\end{array}$ \\
\hline 0 & 1.15 & 1.90 & 0 & 0.9946 & & & & & 0 & 0.9946 & & & & \\
\hline 100 & 1.97 & 2.48 & 0.58 & 0.9949 & $2.92 \mathrm{E}-06$ & $3.45 E-06$ & 5.34E-07 & $3.42 \mathrm{E}-06$ & 0.82 & 0.9950 & 4.12E-06 & $4.65 E-06$ & $5.28 \mathrm{E}-07$ & 4.62E-06 \\
\hline 200 & 2.72 & 3.10 & 1.2 & 0.9952 & $3.02 \mathrm{E}-06$ & $3.55 E-06$ & $5.34 \mathrm{E}-07$ & $3.52 \mathrm{E}-06$ & 1.57 & 0.9954 & $3.95 \mathrm{E}-06$ & $4.48 E-06$ & $5.34 \mathrm{E}-07$ & 4.45E-06 \\
\hline 300 & 3.37 & 3.76 & 1.86 & 0.9955 & $3.12 \mathrm{E}-06$ & $3.65 E-06$ & 5.33E-07 & $3.62 \mathrm{E}-06$ & 2.22 & 0.9957 & $3.72 \mathrm{E}-06$ & $4.25 E-06$ & $5.30 \mathrm{E}-07$ & $4.22 \mathrm{E}-06$ \\
\hline 400 & 4.28 & 4.50 & 2.6 & 0.9959 & $3.27 \mathrm{E}-06$ & $3.80 E-06$ & $5.32 \mathrm{E}-07$ & 3.77E-06 & 3.13 & 0.9962 & $3.93 \mathrm{E}-06$ & $4.46 E-06$ & $5.26 \mathrm{E}-07$ & 4.43E-06 \\
\hline 500 & 5.20 & 5.32 & 3.42 & 0.9963 & $3.44 \mathrm{E}-06$ & $3.97 E-06$ & $5.31 \mathrm{E}-07$ & $3.94 \mathrm{E}-06$ & 4.05 & 0.9966 & 4.07E-06 & $4.60 E-06$ & $5.28 \mathrm{E}-07$ & 4.57E-06 \\
\hline
\end{tabular}

\begin{tabular}{|c|c|c|c|c|c|c|c|c|c|c|c|c|c|c|}
\hline $\begin{array}{l}\text { Capsule } \\
\text { Column }\end{array}$ & $\begin{array}{c}\text { OC4 } \\
\text { North } \\
\end{array}$ & $\begin{array}{c}\text { Specimen } \\
\text { Length }\end{array}$ & $\begin{array}{c}122 \\
0.9948 \\
\end{array}$ & $\begin{array}{c}\text { Material } \\
\text { Scale }\end{array}$ & $\begin{array}{c}\text { H-451 } \\
1 " \\
\end{array}$ & $5.00 \mathrm{E}-04$ & & & & & & & & \\
\hline $\begin{array}{c}\text { Temperature } \\
\left({ }^{\circ} \mathrm{C}\right)\end{array}$ & Up & Down & $\begin{array}{l}\text { Delta } \\
\text { Down }\end{array}$ & $\begin{array}{l}\text { Down } \\
\text { length }\end{array}$ & CTE & CTEK & $\begin{array}{l}\text { Delta } \\
\text { CTEK }\end{array}$ & $\begin{array}{c}\text { CTE } \\
\text { adjusted }\end{array}$ & $\begin{array}{l}\text { Delta } \\
\text { Up }\end{array}$ & $\begin{array}{c}\text { Up } \\
\text { length }\end{array}$ & CTE & CTEK & $\begin{array}{l}\text { Delta } \\
\text { CTEK }\end{array}$ & $\begin{array}{c}\text { CTE } \\
\text { adjusted }\end{array}$ \\
\hline 0 & 1.50 & 1.88 & 0 & 0.9948 & & & & & 0 & 0.9948 & & & & \\
\hline 100 & 2.24 & 2.45 & 0.57 & 0.9951 & $2.86 \mathrm{E}-06$ & $3.40 E-06$ & 5.35E-07 & $3.36 \mathrm{E}-06$ & 0.74 & 0.9952 & $3.72 \mathrm{E}-06$ & $4.25 E-06$ & 5.31E-07 & $4.22 \mathrm{E}-06$ \\
\hline 200 & 2.90 & 3.02 & 1.14 & 0.9954 & $2.86 \mathrm{E}-06$ & 3.40E-06 & 5.35E-07 & $3.36 \mathrm{E}-06$ & 1.4 & 0.9955 & $3.52 \mathrm{E}-06$ & $4.05 E-06$ & $5.32 \mathrm{E}-07$ & $4.02 \mathrm{E}-06$ \\
\hline 300 & 3.32 & 3.60 & 1.72 & 0.9957 & $2.88 \mathrm{E}-06$ & $3.42 E-06$ & $5.38 \mathrm{E}-07$ & $3.38 \mathrm{E}-06$ & 1.82 & 0.9957 & $3.05 \mathrm{E}-06$ & $3.58 E-06$ & $5.31 \mathrm{E}-07$ & $3.55 \mathrm{E}-06$ \\
\hline 400 & 3.86 & 4.12 & 2.24 & 0.9959 & $2.81 \mathrm{E}-06$ & $3.35 E-06$ & $5.35 \mathrm{E}-07$ & $3.31 \mathrm{E}-06$ & 2.36 & 0.9960 & 2.97E-06 & $3.50 E-06$ & $5.35 \mathrm{E}-07$ & 3.47E-06 \\
\hline 500 & 4.52 & 4.72 & 2.84 & 0.9962 & $2.85 \mathrm{E}-06$ & $3.39 E-06$ & $5.35 \mathrm{E}-07$ & 3.35E-06 & 3.02 & 0.9963 & 3.04E-06 & $3.57 E-06$ & $5.34 \mathrm{E}-07$ & $3.54 \mathrm{E}-06$ \\
\hline
\end{tabular}




\begin{tabular}{|c|c|c|c|c|c|c|c|c|c|c|c|c|c|c|}
\hline $\begin{array}{l}\text { Capsule } \\
\text { Column }\end{array}$ & $\begin{array}{l}\text { OC4 } \\
\text { South }\end{array}$ & $\begin{array}{c}\text { Specimen } \\
\text { Length }\end{array}$ & $\begin{array}{c}123 \\
0.9953\end{array}$ & $\begin{array}{c}\text { Material } \\
\text { Scale }\end{array}$ & $\begin{array}{c}\text { H-451 } \\
1 "\end{array}$ & $5.00 \mathrm{E}-04$ & & & & & & & & \\
\hline $\begin{array}{c}\text { Temperature } \\
\left({ }^{\circ} \mathrm{C}\right) \\
\end{array}$ & Up & Down & $\begin{array}{l}\text { Delta } \\
\text { Down }\end{array}$ & $\begin{array}{l}\text { Down } \\
\text { length }\end{array}$ & CTE & CTEK & $\begin{array}{l}\text { Delta } \\
\text { CTEK }\end{array}$ & $\begin{array}{c}\text { CTE } \\
\text { adjusted }\end{array}$ & $\begin{array}{l}\text { Delta } \\
\text { Up }\end{array}$ & $\begin{array}{c}\text { Up } \\
\text { length }\end{array}$ & CTE & CTEK & $\begin{array}{l}\text { Delta } \\
\text { CTEK }\end{array}$ & $\begin{array}{c}\text { CTE } \\
\text { adjusted }\end{array}$ \\
\hline 0 & 1.59 & 1.79 & 0 & 0.9953 & & & & & 0 & 0.9953 & & & & \\
\hline 100 & 2.32 & 2.40 & 0.61 & 0.9956 & $3.06 \mathrm{E}-06$ & $3.60 E-06$ & $5.36 \mathrm{E}-07$ & $3.56 \mathrm{E}-06$ & 0.73 & 0.9957 & $3.67 \mathrm{E}-06$ & $4.20 E-06$ & $5.33 \mathrm{E}-07$ & 4.17E-06 \\
\hline 200 & 2.05 & 2.98 & 1.19 & 0.9959 & 2.99E-06 & $3.52 E-06$ & $5.31 \mathrm{E}-07$ & $3.49 \mathrm{E}-06$ & 0.46 & 0.9955 & $1.16 \mathrm{E}-06$ & $4.20 E-06$ & $3.04 \mathrm{E}-06$ & $1.66 \mathrm{E}-06$ \\
\hline 300 & 3.48 & 3.55 & 1.76 & 0.9962 & $2.95 \mathrm{E}-06$ & $3.48 E-06$ & $5.33 \mathrm{E}-07$ & $3.45 \mathrm{E}-06$ & 1.89 & 0.9962 & $3.16 \mathrm{E}-06$ & 3.70E-06 & $5.35 \mathrm{E}-07$ & $3.66 \mathrm{E}-06$ \\
\hline 400 & 4.02 & 4.05 & 2.26 & 0.9964 & $2.84 \mathrm{E}-06$ & $3.38 E-06$ & $5.42 \mathrm{E}-07$ & $3.34 \mathrm{E}-06$ & 2.43 & 0.9965 & $3.05 \mathrm{E}-06$ & $3.59 E-06$ & $5.38 \mathrm{E}-07$ & $3.55 \mathrm{E}-06$ \\
\hline 500 & 4.72 & 4.67 & 2.88 & 0.9967 & $2.89 \mathrm{E}-06$ & $3.43 E-06$ & $5.36 \mathrm{E}-07$ & $3.39 \mathrm{E}-06$ & 3.13 & 0.9969 & $3.14 \mathrm{E}-06$ & $3.68 E-06$ & $5.35 \mathrm{E}-07$ & $3.64 \mathrm{E}-06$ \\
\hline
\end{tabular}

\begin{tabular}{|c|c|c|c|c|c|c|c|c|c|c|c|c|c|c|}
\hline $\begin{array}{l}\text { Capsule } \\
\text { Column }\end{array}$ & $\begin{array}{l}\text { OC4 } \\
\text { South }\end{array}$ & $\begin{array}{l}\text { Specimen } \\
\text { Length }\end{array}$ & $\begin{array}{c}4 \\
0.9883\end{array}$ & $\begin{array}{l}\text { Material } \\
\text { Scale }\end{array}$ & $\begin{array}{c}H-327 \\
1 "\end{array}$ & $5.00 \mathrm{E}-04$ & & & & & & & & \\
\hline $\begin{array}{c}\text { Temperature } \\
\left({ }^{\circ} \mathrm{C}\right)\end{array}$ & Up & Down & $\begin{array}{l}\text { Delta } \\
\text { Down }\end{array}$ & $\begin{array}{l}\text { Down } \\
\text { length }\end{array}$ & CTE & CTEK & $\begin{array}{l}\text { Delta } \\
\text { CTEK }\end{array}$ & $\begin{array}{c}\text { CTE } \\
\text { adjusted }\end{array}$ & $\begin{array}{l}\text { Delta } \\
\text { Up }\end{array}$ & $\begin{array}{c}\text { Up } \\
\text { length }\end{array}$ & CTE & CTEK & $\begin{array}{l}\text { Delta } \\
\text { CTEK }\end{array}$ & $\begin{array}{c}\text { CTE } \\
\text { adjusted }\end{array}$ \\
\hline 0 & 1.78 & 1.92 & 0 & 0.9883 & & & & & 0 & 0.9883 & & & & \\
\hline 100 & 2.15 & 2.05 & 0.13 & 0.9884 & $6.58 \mathrm{E}-07$ & $1.20 E-06$ & $5.42 \mathrm{E}-07$ & $1.16 \mathrm{E}-06$ & 0.37 & 0.9885 & $1.87 \mathrm{E}-06$ & $2.40 E-06$ & $5.28 \mathrm{E}-07$ & 2.37E-06 \\
\hline 200 & 2.49 & 2.22 & 0.3 & 0.9885 & $7.59 \mathrm{E}-07$ & $1.30 E-06$ & $5.41 \mathrm{E}-07$ & $1.26 \mathrm{E}-06$ & 0.71 & 0.9887 & $1.80 \mathrm{E}-06$ & $2.32 E-06$ & $5.24 \mathrm{E}-07$ & $2.30 \mathrm{E}-06$ \\
\hline 300 & 2.72 & 2.44 & 0.52 & 0.9886 & 8.77E-07 & $1.42 E-06$ & $5.43 \mathrm{E}-07$ & $1.38 \mathrm{E}-06$ & 0.94 & 0.9888 & 1.59E-06 & $2.12 E-06$ & $5.35 \mathrm{E}-07$ & $2.09 \mathrm{E}-06$ \\
\hline 400 & 2.87 & 2.72 & 0.8 & 0.9887 & $1.01 \mathrm{E}-06$ & $1.55 E-06$ & $5.38 \mathrm{E}-07$ & $1.51 \mathrm{E}-06$ & 1.09 & 0.9888 & $1.38 \mathrm{E}-06$ & $1.91 E-06$ & 5.31E-07 & $1.88 \mathrm{E}-06$ \\
\hline 500 & 3.18 & 3.12 & 1.2 & 0.9889 & $1.21 \mathrm{E}-06$ & $1.75 E-06$ & $5.36 \mathrm{E}-07$ & $1.71 \mathrm{E}-06$ & 1.4 & 0.9890 & $1.42 \mathrm{E}-06$ & $1.95 E-06$ & $5.33 \mathrm{E}-07$ & $1.92 \mathrm{E}-06$ \\
\hline
\end{tabular}

\begin{tabular}{|c|c|c|c|c|c|c|c|c|c|c|c|c|c|c|}
\hline $\begin{array}{l}\text { Capsule } \\
\text { Column }\end{array}$ & $\begin{array}{c}\text { OC4 } \\
\text { North } \\
\end{array}$ & $\begin{array}{c}\text { Specimen } \\
\text { Length }\end{array}$ & $\begin{array}{c}65 \\
0.9968 \\
\end{array}$ & $\begin{array}{c}\text { Material } \\
\text { Scale }\end{array}$ & $\begin{array}{c}\text { H-451 } \\
1 " \\
\end{array}$ & $5.00 \mathrm{E}-04$ & & & & & & & & \\
\hline $\begin{array}{c}\text { Temperature } \\
\left({ }^{\circ} \mathrm{C}\right)\end{array}$ & Up & Down & $\begin{array}{l}\text { Delta } \\
\text { Down }\end{array}$ & $\begin{array}{l}\text { Down } \\
\text { length }\end{array}$ & CTE & CTEK & $\begin{array}{l}\text { Delta } \\
\text { CTEK }\end{array}$ & $\begin{array}{c}\text { CTE } \\
\text { adjusted }\end{array}$ & $\begin{array}{l}\text { Delta } \\
\text { Up }\end{array}$ & $\begin{array}{c}\text { Up } \\
\text { length }\end{array}$ & CTE & CTEK & $\begin{array}{l}\text { Delta } \\
\text { CTEK }\end{array}$ & $\begin{array}{c}\text { CTE } \\
\text { adjusted }\end{array}$ \\
\hline 0 & 1.32 & 0.99 & 0 & 0.9968 & & & & & 0 & 0.9968 & & & & \\
\hline 100 & 1.33 & 1.12 & 0.13 & 0.9969 & $6.52 \mathrm{E}-07$ & $1.20 E-06$ & $5.48 \mathrm{E}-07$ & 1.15E-06 & 0.01 & 0.9968 & $5.02 \mathrm{E}-08$ & $6.00 E-07$ & $5.50 \mathrm{E}-07$ & $5.50 \mathrm{E}-07$ \\
\hline 200 & 1.50 & 1.23 & 0.24 & 0.9969 & $6.02 \mathrm{E}-07$ & $1.15 E-06$ & $5.48 \mathrm{E}-07$ & $1.10 \mathrm{E}-06$ & 0.18 & 0.9969 & $4.51 \mathrm{E}-07$ & $1.00 E-06$ & $5.49 \mathrm{E}-07$ & $9.51 \mathrm{E}-07$ \\
\hline 300 & 1.26 & 1.20 & 0.21 & 0.9969 & $3.51 \mathrm{E}-07$ & $9.00 E-07$ & $5.49 \mathrm{E}-07$ & $8.51 \mathrm{E}-07$ & -0.06 & 0.9968 & $-1.00 \mathrm{E}-07$ & 4.50E-07 & $5.50 \mathrm{E}-07$ & 4.00E-07 \\
\hline 400 & 1.50 & 1.18 & 0.19 & 0.9969 & $2.38 \mathrm{E}-07$ & 7.80E-07 & $5.42 \mathrm{E}-07$ & $7.38 \mathrm{E}-07$ & 0.18 & 0.9969 & $2.26 \mathrm{E}-07$ & $7.75 E-07$ & 5.49E-07 & $7.26 \mathrm{E}-07$ \\
\hline 500 & 1.50 & 1.40 & 0.41 & 0.9970 & 4.11E-07 & 9.60E-07 & 5.49E-07 & $9.11 \mathrm{E}-07$ & 0.18 & 0.9969 & $1.81 \mathrm{E}-07$ & 7.30E-07 & 5.49E-07 & $6.81 \mathrm{E}-07$ \\
\hline
\end{tabular}




\begin{tabular}{|c|c|c|c|c|c|c|c|c|c|c|c|c|c|c|}
\hline $\begin{array}{l}\text { Capsule } \\
\text { Column }\end{array}$ & $\begin{array}{l}\text { OC4 } \\
\text { South }\end{array}$ & $\begin{array}{c}\text { Specimen } \\
\text { Length }\end{array}$ & $\begin{array}{c}67 \\
0.9989 \\
\end{array}$ & $\begin{array}{c}\text { Material } \\
\text { Scale }\end{array}$ & $\begin{array}{c}\text { H-451 } \\
1 "\end{array}$ & $5.00 \mathrm{E}-04$ & & & & & & & & \\
\hline $\begin{array}{c}\text { Temperature } \\
\left({ }^{\circ} \mathrm{C}\right) \\
\end{array}$ & Up & Down & $\begin{array}{l}\text { Delta } \\
\text { Down }\end{array}$ & $\begin{array}{l}\text { Down } \\
\text { length }\end{array}$ & CTE & CTEK & $\begin{array}{l}\text { Delta } \\
\text { CTEK }\end{array}$ & $\begin{array}{c}\text { CTE } \\
\text { adjusted }\end{array}$ & $\begin{array}{l}\text { Delta } \\
\text { Up }\end{array}$ & $\begin{array}{c}\text { Up } \\
\text { length }\end{array}$ & CTE & CTEK & $\begin{array}{l}\text { Delta } \\
\text { CTEK }\end{array}$ & $\begin{array}{c}\text { CTE } \\
\text { adjusted }\end{array}$ \\
\hline 0 & 1.20 & 0.80 & 0 & 0.9989 & & & & & 0 & 0.9989 & & & & \\
\hline 100 & 1.42 & 0.95 & 0.15 & 0.9990 & 7.51E-07 & $1.30 E-06$ & $5.49 \mathrm{E}-07$ & $1.25 \mathrm{E}-06$ & 0.22 & 0.9990 & $1.10 \mathrm{E}-06$ & $1.65 E-06$ & $5.49 \mathrm{E}-07$ & $1.60 \mathrm{E}-06$ \\
\hline 200 & 1.62 & 1.09 & 0.29 & 0.9990 & $7.26 \mathrm{E}-07$ & $1.28 E-06$ & $5.54 \mathrm{E}-07$ & $1.23 \mathrm{E}-06$ & 0.42 & 0.9991 & $1.05 \mathrm{E}-06$ & $1.60 E-06$ & $5.49 \mathrm{E}-07$ & $1.55 \mathrm{E}-06$ \\
\hline 300 & 1.59 & 1.25 & 0.45 & 0.9991 & 7.51E-07 & $1.30 E-06$ & $5.49 \mathrm{E}-07$ & $1.25 \mathrm{E}-06$ & 0.39 & 0.9991 & $6.51 \mathrm{E}-07$ & $1.20 E-06$ & $5.49 \mathrm{E}-07$ & $1.15 \mathrm{E}-06$ \\
\hline 400 & 1.56 & 1.39 & 0.59 & 0.9992 & $7.38 \mathrm{E}-07$ & $1.29 E-06$ & $5.52 \mathrm{E}-07$ & $1.24 \mathrm{E}-06$ & 0.36 & 0.9991 & $4.50 \mathrm{E}-07$ & $1.00 E-06$ & $5.50 \mathrm{E}-07$ & $9.50 \mathrm{E}-07$ \\
\hline 500 & 1.60 & 1.60 & 0.8 & 0.9993 & $8.01 \mathrm{E}-07$ & $1.35 E-06$ & $5.49 \mathrm{E}-07$ & 1.30E-06 & 0.4 & 0.9991 & 4.00E-07 & 9.50E-07 & $5.50 \mathrm{E}-07$ & $9.00 \mathrm{E}-07$ \\
\hline
\end{tabular}

\begin{tabular}{|c|c|c|c|c|c|c|c|c|c|c|c|c|c|c|}
\hline $\begin{array}{l}\text { Capsule } \\
\text { Column }\end{array}$ & $\begin{array}{l}\text { OC4 } \\
\text { North }\end{array}$ & $\begin{array}{l}\text { Specimen } \\
\text { Length }\end{array}$ & $\begin{array}{c}72 \\
0.997\end{array}$ & $\begin{array}{l}\text { Material } \\
\text { Scale }\end{array}$ & $\begin{array}{l}\text { H-451 } \\
1 "\end{array}$ & $5.00 \mathrm{E}-04$ & & & & & & & & \\
\hline $\begin{array}{c}\text { Temperature } \\
\left({ }^{\circ} \mathrm{C}\right)\end{array}$ & Up & Down & $\begin{array}{l}\text { Delta } \\
\text { Down }\end{array}$ & $\begin{array}{l}\text { Down } \\
\text { length }\end{array}$ & CTE & CTEK & $\begin{array}{l}\text { Delta } \\
\text { CTEK }\end{array}$ & $\begin{array}{c}\text { CTE } \\
\text { adjusted }\end{array}$ & $\begin{array}{l}\text { Delta } \\
\text { Up }\end{array}$ & $\begin{array}{c}\text { Up } \\
\text { length }\end{array}$ & CTE & CTEK & $\begin{array}{l}\text { Delta } \\
\text { CTEK }\end{array}$ & $\begin{array}{c}\text { CTE } \\
\text { adjusted }\end{array}$ \\
\hline 0 & 1.45 & 1.22 & 0 & 0.9970 & & & & & 0 & 0.9970 & & & & \\
\hline 100 & 1.92 & 1.88 & 0.66 & 0.9973 & $3.31 \mathrm{E}-06$ & $3.85 E-06$ & $5.40 \mathrm{E}-07$ & $3.81 \mathrm{E}-06$ & 0.47 & 0.9972 & $2.36 \mathrm{E}-06$ & 2.90E-06 & $5.43 \mathrm{E}-07$ & $2.86 \mathrm{E}-06$ \\
\hline 200 & 2.35 & 2.41 & 1.19 & 0.9976 & $2.98 \mathrm{E}-06$ & $3.52 E-06$ & $5.36 \mathrm{E}-07$ & $3.48 \mathrm{E}-06$ & 0.9 & 0.9975 & $2.26 \mathrm{E}-06$ & $2.80 E-06$ & $5.43 \mathrm{E}-07$ & $2.76 \mathrm{E}-06$ \\
\hline 300 & 2.72 & 2.80 & 1.58 & 0.9978 & $2.64 \mathrm{E}-06$ & 3.18E-06 & 5.39E-07 & 3.14E-06 & 1.27 & 0.9976 & $2.12 \mathrm{E}-06$ & 2.67E-06 & $5.47 \mathrm{E}-07$ & $2.62 \mathrm{E}-06$ \\
\hline 400 & 3.18 & 3.30 & 2.08 & 0.9980 & $2.61 \mathrm{E}-06$ & $3.15 E-06$ & $5.42 \mathrm{E}-07$ & $3.11 \mathrm{E}-06$ & 1.73 & 0.9979 & 2.17E-06 & $2.71 E-06$ & 5.41E-07 & 2.67E-06 \\
\hline 500 & 3.77 & 3.85 & 2.63 & 0.9983 & $2.64 \mathrm{E}-06$ & $3.18 E-06$ & $5.42 \mathrm{E}-07$ & $3.14 \mathrm{E}-06$ & 2.32 & 0.9982 & $2.33 \mathrm{E}-06$ & 2.87E-06 & $5.43 \mathrm{E}-07$ & $2.83 \mathrm{E}-06$ \\
\hline
\end{tabular}

\begin{tabular}{|c|c|c|c|c|c|c|c|c|c|c|c|c|c|c|}
\hline $\begin{array}{l}\text { Capsule } \\
\text { Column }\end{array}$ & $\begin{array}{c}\text { OC4 } \\
\text { South }\end{array}$ & $\begin{array}{c}\text { Specimen } \\
\text { Length }\end{array}$ & $\begin{array}{c}73 \\
0.9964 \\
\end{array}$ & $\begin{array}{c}\text { Material } \\
\text { Scale }\end{array}$ & $\begin{array}{c}\text { H-451 } \\
1 " \\
\end{array}$ & $5.00 \mathrm{E}-04$ & & & & & & & & \\
\hline $\begin{array}{c}\text { Temperature } \\
\left({ }^{\circ} \mathrm{C}\right)\end{array}$ & Up & Down & $\begin{array}{l}\text { Delta } \\
\text { Down }\end{array}$ & $\begin{array}{l}\text { Down } \\
\text { length }\end{array}$ & CTE & CTEK & $\begin{array}{l}\text { Delta } \\
\text { CTEK }\end{array}$ & $\begin{array}{c}\text { CTE } \\
\text { adjusted }\end{array}$ & $\begin{array}{l}\text { Delta } \\
\text { Up }\end{array}$ & $\begin{array}{c}\text { Up } \\
\text { length }\end{array}$ & CTE & CTEK & $\begin{array}{l}\text { Delta } \\
\text { CTEK }\end{array}$ & $\begin{array}{c}\text { CTE } \\
\text { adjusted }\end{array}$ \\
\hline 0 & 1.12 & 1.80 & 0 & 0.9964 & & & & & 0 & 0.9964 & & & & \\
\hline 100 & 1.21 & 2.19 & 0.39 & 0.9966 & $1.96 \mathrm{E}-06$ & $2.50 E-06$ & $5.43 \mathrm{E}-07$ & $2.46 \mathrm{E}-06$ & 0.09 & 0.9964 & $4.52 \mathrm{E}-07$ & $1.00 E-06$ & $5.48 \mathrm{E}-07$ & $9.52 \mathrm{E}-07$ \\
\hline 200 & 2.32 & 2.52 & 0.72 & 0.9968 & $1.81 \mathrm{E}-06$ & $2.35 E-06$ & $5.43 \mathrm{E}-07$ & $2.31 \mathrm{E}-06$ & 1.2 & 0.9970 & $3.01 \mathrm{E}-06$ & $3.55 E-06$ & $5.39 \mathrm{E}-07$ & $3.51 \mathrm{E}-06$ \\
\hline 300 & 2.65 & 2.98 & 1.18 & 0.9970 & $1.97 \mathrm{E}-06$ & $2.52 E-06$ & $5.46 \mathrm{E}-07$ & $2.47 \mathrm{E}-06$ & 1.53 & 0.9972 & $2.56 \mathrm{E}-06$ & $3.10 E-06$ & $5.41 \mathrm{E}-07$ & 3.06E-06 \\
\hline 400 & 3.20 & 3.40 & 1.6 & 0.9972 & $2.01 \mathrm{E}-06$ & $2.55 E-06$ & $5.43 \mathrm{E}-07$ & $2.51 \mathrm{E}-06$ & 2.08 & 0.9974 & $2.61 \mathrm{E}-06$ & $3.15 E-06$ & $5.41 \mathrm{E}-07$ & $3.11 \mathrm{E}-06$ \\
\hline 500 & 3.78 & 3.84 & 2.04 & 0.9974 & $2.05 \mathrm{E}-06$ & 2.59E-06 & 5.43E-07 & $2.55 \mathrm{E}-06$ & 2.66 & 0.9977 & 2.67E-06 & $3.21 E-06$ & 5.40E-07 & 3.17E-06 \\
\hline
\end{tabular}




\begin{tabular}{|c|c|c|c|c|c|c|c|c|c|c|c|c|c|c|}
\hline $\begin{array}{l}\text { Capsule } \\
\text { Column }\end{array}$ & $\begin{array}{l}\text { OC4 } \\
\text { West }\end{array}$ & $\begin{array}{c}\text { Specimen } \\
\text { Length }\end{array}$ & $\begin{array}{c}2 G \\
0.992\end{array}$ & $\begin{array}{c}\text { Material } \\
\text { Scale }\end{array}$ & $\begin{array}{c}\text { Poco } \\
1 "\end{array}$ & $5.00 \mathrm{E}-04$ & & & & & & & & \\
\hline $\begin{array}{c}\text { Temperature } \\
\left({ }^{\circ} \mathrm{C}\right) \\
\end{array}$ & Up & Down & $\begin{array}{l}\text { Delta } \\
\text { Down }\end{array}$ & $\begin{array}{l}\text { Down } \\
\text { length }\end{array}$ & CTE & CTEK & $\begin{array}{l}\text { Delta } \\
\text { CTEK }\end{array}$ & $\begin{array}{c}\text { CTE } \\
\text { adjusted }\end{array}$ & $\begin{array}{l}\text { Delta } \\
\text { Up }\end{array}$ & $\begin{array}{c}\text { Up } \\
\text { length }\end{array}$ & CTE & CTEK & $\begin{array}{l}\text { Delta } \\
\text { CTEK }\end{array}$ & $\begin{array}{c}\text { CTE } \\
\text { adjusted }\end{array}$ \\
\hline 0 & 0.68 & 0.82 & 0 & 0.9920 & & & & & 0 & 0.9920 & & & & \\
\hline 100 & 1.58 & 1.77 & 0.95 & 0.9925 & 4.79E-06 & $5.30 E-06$ & $5.12 \mathrm{E}-07$ & $5.29 \mathrm{E}-06$ & 0.9 & 0.9925 & $4.54 \mathrm{E}-06$ & $5.05 E-06$ & $5.14 \mathrm{E}-07$ & $5.04 \mathrm{E}-06$ \\
\hline 200 & 2.53 & 2.75 & 1.93 & 0.9930 & 4.86E-06 & $5.38 E-06$ & $5.16 \mathrm{E}-07$ & $5.36 \mathrm{E}-06$ & 1.85 & 0.9929 & 4.66E-06 & $5.18 E-06$ & $5.18 \mathrm{E}-07$ & 5.16E-06 \\
\hline 300 & 3.49 & 3.71 & 2.89 & 0.9934 & $4.86 \mathrm{E}-06$ & 5.37E-06 & $5.14 \mathrm{E}-07$ & $5.36 \mathrm{E}-06$ & 2.81 & 0.9934 & $4.72 \mathrm{E}-06$ & $5.23 E-06$ & $5.09 \mathrm{E}-07$ & $5.22 \mathrm{E}-06$ \\
\hline 400 & 4.52 & 4.75 & 3.93 & 0.9940 & 4.95E-06 & $5.46 E-06$ & $5.08 \mathrm{E}-07$ & $5.45 \mathrm{E}-06$ & 3.84 & 0.9939 & $4.84 \mathrm{E}-06$ & $5.35 E-06$ & $5.11 \mathrm{E}-07$ & $5.34 \mathrm{E}-06$ \\
\hline 500 & 5.60 & 5.80 & 4.98 & 0.9945 & $5.02 \mathrm{E}-06$ & $5.53 E-06$ & $5.10 \mathrm{E}-07$ & $5.52 \mathrm{E}-06$ & 4.92 & 0.9945 & 4.96E-06 & 5.47E-06 & $5.10 \mathrm{E}-07$ & $5.46 \mathrm{E}-06$ \\
\hline
\end{tabular}

\begin{tabular}{|c|c|c|c|c|c|c|c|c|c|c|c|c|c|c|}
\hline $\begin{array}{l}\text { Capsule } \\
\text { Column }\end{array}$ & $\begin{array}{l}\text { OC4 } \\
\text { South }\end{array}$ & $\begin{array}{l}\text { Specimen } \\
\text { Length }\end{array}$ & $\begin{array}{c}\mathrm{P} 12 \\
0.9815\end{array}$ & $\begin{array}{l}\text { Material } \\
\text { Scale }\end{array}$ & $\begin{array}{l}\text { Poco } \\
1 "\end{array}$ & $5.00 \mathrm{E}-04$ & & & & & & & & \\
\hline $\begin{array}{c}\text { Temperature } \\
\left({ }^{\circ} \mathrm{C}\right)\end{array}$ & Up & Down & $\begin{array}{l}\text { Delta } \\
\text { Down }\end{array}$ & $\begin{array}{l}\text { Down } \\
\text { length }\end{array}$ & CTE & CTEK & $\begin{array}{l}\text { Delta } \\
\text { CTEK }\end{array}$ & $\begin{array}{c}\text { CTE } \\
\text { adjusted }\end{array}$ & $\begin{array}{l}\text { Delta } \\
\text { Up }\end{array}$ & $\begin{array}{c}\text { Up } \\
\text { length }\end{array}$ & CTE & CTEK & $\begin{array}{l}\text { Delta } \\
\text { CTEK }\end{array}$ & $\begin{array}{c}\text { CTE } \\
\text { adjusted }\end{array}$ \\
\hline 0 & 0.76 & 0.89 & 0 & 0.9815 & & & & & 0 & 0.9815 & & & & \\
\hline 100 & 1.60 & 1.83 & 0.94 & 0.9820 & 4.79E-06 & $5.25 E-06$ & 4.61E-07 & $5.29 \mathrm{E}-06$ & 0.84 & 0.9819 & $4.28 \mathrm{E}-06$ & $4.75 E-06$ & 4.71E-07 & 4.78E-06 \\
\hline 200 & 2.70 & 2.85 & 1.96 & 0.9825 & 4.99E-06 & $5.45 E-06$ & $4.58 \mathrm{E}-07$ & $5.49 \mathrm{E}-06$ & 1.94 & 0.9825 & 4.94E-06 & $5.40 E-06$ & 4.59E-07 & $5.44 \mathrm{E}-06$ \\
\hline 300 & 3.80 & 3.88 & 2.99 & 0.9830 & $5.08 \mathrm{E}-06$ & 5.53E-06 & $4.53 \mathrm{E}-07$ & $5.58 \mathrm{E}-06$ & 3.04 & 0.9830 & $5.16 \mathrm{E}-06$ & $5.62 E-06$ & $4.58 \mathrm{E}-07$ & 5.66E-06 \\
\hline 400 & 4.88 & 4.92 & 4.03 & 0.9835 & $5.13 \mathrm{E}-06$ & 5.59E-06 & $4.58 \mathrm{E}-07$ & $5.63 \mathrm{E}-06$ & 4.12 & 0.9836 & $5.25 \mathrm{E}-06$ & $5.70 E-06$ & 4.53E-07 & 5.75E-06 \\
\hline 500 & 5.98 & 6.05 & 5.16 & 0.9841 & $5.26 \mathrm{E}-06$ & $5.71 E-06$ & 4.53E-07 & $5.76 \mathrm{E}-06$ & 5.22 & 0.9841 & $5.32 \mathrm{E}-06$ & $5.77 E-06$ & $4.52 \mathrm{E}-07$ & $5.82 \mathrm{E}-06$ \\
\hline
\end{tabular}

\begin{tabular}{|c|c|c|c|c|c|c|c|c|c|c|c|c|c|c|}
\hline $\begin{array}{l}\text { Capsule } \\
\text { Column }\end{array}$ & $\begin{array}{c}\text { OC4 } \\
\text { North } \\
\end{array}$ & $\begin{array}{c}\text { Specimen } \\
\text { Length }\end{array}$ & $\begin{array}{c}\text { P13 } \\
0.9822 \\
\end{array}$ & $\begin{array}{c}\text { Material } \\
\text { Scale }\end{array}$ & $\begin{array}{c}\text { Poco } \\
1 " \\
\end{array}$ & $5.00 \mathrm{E}-04$ & & & & & & & & \\
\hline $\begin{array}{c}\text { Temperature } \\
\left({ }^{\circ} \mathrm{C}\right)\end{array}$ & Up & Down & $\begin{array}{l}\text { Delta } \\
\text { Down }\end{array}$ & $\begin{array}{l}\text { Down } \\
\text { length }\end{array}$ & CTE & CTEK & $\begin{array}{l}\text { Delta } \\
\text { CTEK }\end{array}$ & $\begin{array}{c}\text { CTE } \\
\text { adjusted }\end{array}$ & $\begin{array}{l}\text { Delta } \\
\text { Up }\end{array}$ & $\begin{array}{c}\text { Up } \\
\text { length }\end{array}$ & CTE & CTEK & $\begin{array}{l}\text { Delta } \\
\text { CTEK }\end{array}$ & $\begin{array}{c}\text { CTE } \\
\text { adjusted }\end{array}$ \\
\hline 0 & 0.85 & 0.98 & 0 & 0.9822 & & & & & 0 & 0.9822 & & & & \\
\hline 100 & 1.60 & 1.85 & 0.87 & 0.9826 & $4.43 \mathrm{E}-06$ & 4.90E-06 & 4.71E-07 & 4.93E-06 & 0.75 & 0.9826 & $3.82 \mathrm{E}-06$ & 4.30E-06 & $4.82 \mathrm{E}-07$ & $4.32 \mathrm{E}-06$ \\
\hline 200 & 2.60 & 2.78 & 1.8 & 0.9831 & $4.58 \mathrm{E}-06$ & $5.05 E-06$ & $4.68 \mathrm{E}-07$ & $5.08 \mathrm{E}-06$ & 1.75 & 0.9831 & 4.45E-06 & 4.93E-06 & 4.76E-07 & $4.95 \mathrm{E}-06$ \\
\hline 300 & 3.60 & 3.72 & 2.74 & 0.9836 & 4.65E-06 & $5.12 E-06$ & 4.71E-07 & $5.15 \mathrm{E}-06$ & 2.75 & 0.9836 & 4.67E-06 & 5.13E-06 & 4.64E-07 & 5.17E-06 \\
\hline 400 & 4.60 & 4.69 & 3.71 & 0.9841 & $4.72 \mathrm{E}-06$ & 5.18E-06 & $4.58 \mathrm{E}-07$ & $5.22 \mathrm{E}-06$ & 3.75 & 0.9841 & 4.77E-06 & $5.24 E-06$ & $4.68 \mathrm{E}-07$ & 5.27E-06 \\
\hline 500 & 5.57 & 5.65 & 4.67 & 0.9845 & $4.75 \mathrm{E}-06$ & $5.22 E-06$ & 4.65E-07 & $5.25 \mathrm{E}-06$ & 4.72 & 0.9846 & 4.81E-06 & $5.27 E-06$ & 4.64E-07 & $5.31 \mathrm{E}-06$ \\
\hline
\end{tabular}


ORNL/TM-2009/176

\begin{tabular}{|c|c|c|c|c|c|c|c|c|c|c|c|c|c|c|}
\hline $\begin{array}{l}\text { Capsule } \\
\text { Column }\end{array}$ & $\begin{array}{l}\text { OC4 } \\
\text { South }\end{array}$ & $\begin{array}{l}\text { Specimen } \\
\text { Length }\end{array}$ & $\begin{array}{c}\text { P22 } \\
0.9799\end{array}$ & $\begin{array}{c}\text { Material } \\
\text { Scale }\end{array}$ & $\begin{array}{c}\text { Poco } \\
1 "\end{array}$ & $5.00 \mathrm{E}-04$ & & & & & & & & \\
\hline $\begin{array}{c}\text { Temperature } \\
\left({ }^{\circ} \mathrm{C}\right) \\
\end{array}$ & Up & Down & $\begin{array}{l}\text { Delta } \\
\text { Down }\end{array}$ & $\begin{array}{l}\text { Down } \\
\text { length }\end{array}$ & CTE & CTEK & $\begin{array}{l}\text { Delta } \\
\text { CTEK }\end{array}$ & $\begin{array}{c}\text { CTE } \\
\text { adjusted } \\
\end{array}$ & $\begin{array}{c}\text { Delta } \\
\text { Up }\end{array}$ & $\begin{array}{c}\text { Up } \\
\text { length }\end{array}$ & CTE & CTEK & $\begin{array}{l}\text { Delta } \\
\text { CTEK }\end{array}$ & $\begin{array}{c}\text { CTE } \\
\text { adjusted }\end{array}$ \\
\hline 0 & 0.95 & 1.22 & 0 & 0.9799 & & & & & 0 & 0.9799 & & & & \\
\hline 100 & 1.87 & 2.06 & 0.84 & 0.9803 & 4.29E-06 & $4.75 E-06$ & 4.64E-07 & 4.79E-06 & 0.92 & 0.9804 & 4.69E-06 & $5.15 E-06$ & 4.56E-07 & 5.19E-06 \\
\hline 200 & 2.98 & 2.98 & 1.76 & 0.9808 & 4.49E-06 & $4.95 E-06$ & 4.60E-07 & 4.99E-06 & 2.03 & 0.9809 & $5.18 \mathrm{E}-06$ & $5.63 E-06$ & $4.51 \mathrm{E}-07$ & $5.68 \mathrm{E}-06$ \\
\hline 300 & 3.96 & 3.95 & 2.73 & 0.9813 & $4.64 \mathrm{E}-06$ & 5.10E-06 & 4.57E-07 & 5.14E-06 & 3.01 & 0.9814 & 5.12E-06 & 5.57E-06 & 4.50E-07 & 5.62E-06 \\
\hline 400 & 4.98 & 4.98 & 3.76 & 0.9818 & 4.80E-06 & 5.25E-06 & 4.54E-07 & 5.30E-06 & 4.03 & 0.9819 & 5.14E-06 & 5.59E-06 & 4.49E-07 & 5.64E-06 \\
\hline 500 & 5.98 & 6.02 & 4.8 & 0.9823 & 4.90E-06 & $5.35 E-06$ & 4.52E-07 & 5.40E-06 & 5.03 & 0.9824 & 5.13E-06 & $5.58 E-06$ & 4.47E-07 & $5.63 \mathrm{E}-06$ \\
\hline
\end{tabular}




\section{Appendix C Creep Corrections}

\section{KFA Correction to Creep Data}

The following correction to creep data was presented to ORNL during a German/US creep programme exchange (ref X).

\section{$1 \quad$ Available Data}

$1.1 \quad$ Measured sample length at R.T. without load

1.1.1 Reference Sample length

1.1.2 Reference dimensional strain

1.1.3 Stressed Sample length

1.1.4 Stressed dimensional strain

1.2 Creep Strain

$2 \quad$ Wanted Data

2.1

2.1 .1

Length values at Temperature, $\mathrm{T}$ under load, $\sigma$ Input data

CTE (what cte value assumed - mean 20-T?)

(n.b. factorial change in CTE assumed)

CTE change due to creep (comp or tensile), $\varepsilon_{\mathrm{c}}(\%)$

$$
\begin{aligned}
& I^{R}\left(\gamma, \theta_{r t}, 0\right) \\
& \varepsilon^{R}\left(\gamma, \theta_{r t}, 0\right)=\frac{I^{R}\left(\gamma, \theta_{r t}, 0\right)}{I^{R}\left(0, \theta_{r t}, 0\right)}-1 \\
& I^{S}\left(\gamma, \theta_{r t}, 0\right) \\
& \varepsilon^{S}\left(\gamma, \theta_{r t}, 0\right)=\frac{I^{S}\left(\gamma, \theta_{r t}, 0\right)}{I^{S}\left(0, \theta_{r t}, 0\right)}-1 \\
& \varepsilon_{c}\left(\gamma, \theta_{r t}, 0\right)=\frac{I^{S}\left(\gamma, \theta_{r t}, 0\right)}{I^{S}\left(0, \theta_{r t}, 0\right)}-\frac{I^{R}\left(\gamma, \theta_{r t}, 0\right)}{I^{R}\left(0, \theta_{r t}, 0\right)}
\end{aligned}
$$

$\alpha\left(0, \theta_{T}, 0\right)$

$\alpha\left(\gamma, \theta_{T}, 0\right)$

$a(\gamma)=\frac{\alpha\left(\gamma, \theta_{T}, 0\right)}{\alpha\left(0, \theta_{T}, 0\right)}$

$\alpha_{c}\left(\gamma, \theta_{T}, \sigma\right)=\alpha\left(\gamma, \theta_{T}, 0\right)\left(1 \pm 0.15 \varepsilon_{c}\left(\gamma, \theta_{r t}, 0\right)\right)$ 
$E\left(0, \theta_{T}, 0\right)$

$$
E\left(\gamma, \theta_{T}, 0\right)
$$

\section{Strain data corrected for creep conditions}

2.2.1 Modified Reference Length at Temperature

$e(\gamma)=\frac{E\left(\gamma, \theta_{T}, 0\right)}{E\left(0, \theta_{T}, 0\right)}$

Modified Reference dimensional strain

Modified Stressed Length at Temperature

Modified Stressed dimensional strain

Modified Creep strain
$I^{R}\left(0, \theta_{T}, 0\right)=[1+\alpha(0) \Delta T]\left(I^{R}\left(0, \theta_{r t}, 0\right)\right)$

$I^{R}\left(\gamma, \theta_{T}, 0\right)=[1+a(\gamma) \alpha(0) \Delta T]\left(I^{R}\left(\gamma, \theta_{r t}, 0\right)\right)$

$\left.\varepsilon^{R}\left(\gamma, \theta_{T}, 0\right)+1=\left[\frac{1+a(\gamma) \alpha(0) \Delta T}{1+\alpha(0) \Delta T}\right] \frac{I^{R}\left(\gamma, \theta_{r t}, 0\right)}{I^{R}\left(0, \theta_{r t}, 0\right)}\right)$

$I^{S}\left(0, \theta_{T}, \sigma\right)=\left\lfloor 1+\frac{\sigma}{E\left(0, \theta_{T}, 0\right)}\right\rfloor[1+\alpha(0) \Delta T]\left(I^{S}\left(0, \theta_{r t}, 0\right)\right)$

$I^{S}\left(\gamma, \theta_{T}, \sigma\right)=\left[1+\frac{\sigma}{e(\gamma) E\left(0, \theta_{T}, 0\right)}\right]\left[1+a(\gamma) \alpha_{c}(\gamma) \Delta T\right]\left(I^{S}\left(\gamma, \theta_{r t}, 0\right)\right)$

$\left.\varepsilon^{S}\left(\gamma, \theta_{T}, \sigma\right)+1=\left\lfloor\frac{\left(1+\frac{\sigma}{e(\gamma) E\left(0, \theta_{T}, 0\right)}\right)\left(1+a(\gamma) \alpha_{c}(\gamma) \Delta T\right)}{\left(1+\frac{\sigma}{E\left(0, \theta_{T}, 0\right)}\right)(1+\alpha(0) \Delta T)}\right\rfloor \frac{I^{S}\left(\gamma, \theta_{r t}, 0\right)}{I^{S}\left(0, \theta_{r t}, 0\right)}\right)$

$\varepsilon_{c}\left(\gamma, \theta_{T}, \sigma\right)=\varepsilon^{S}\left(\gamma, \theta_{T}, \sigma\right)-\varepsilon^{R}\left(\gamma, \theta_{T}, 0\right)$ 


\section{UK correction to creep data}

Within the UK methodology (Ref X), the irradiation induced creep strain is defined as the additional length change arising from an applied stress as measured at temperature in the stressed condition and the measured dimensions are corrected for difference in:

- Elastic recovery upon load removal;

- Change of length upon cooling from the irradiation temperature.

The method applied is as follows

If:

$l_{0}$ is the measured length of the specimen unirradiated at room temperature

$l$ is the measured length of the specimen irradiated at room temperature

Then, assuming a mean irradiation temperature of $\mathrm{T}$

$$
\begin{aligned}
& l_{0}^{\prime}=l_{0}\left(1+\alpha_{0}(T)+\sigma / E_{0}\right) \text { is the length of specimen at the start of irradiation } \\
& I^{\prime}=l\left(1+\alpha_{i}(T)+\sigma / E_{i}\right) \text { is the length of specimen at the end of irradiation }
\end{aligned}
$$

The in-reactor strain is then given by:

$$
\left(\frac{L^{\prime}-L_{0}^{\prime}}{L_{0}^{\prime}}\right)=\left(\frac{L-L_{0}}{L_{0}}\right)+\left(\alpha_{i}-\alpha_{0}\right)(T)-\left(\frac{\sigma}{E_{0}}\right)\left[1-\frac{E_{0}}{E_{i}}\right]
$$

For the unstressed control specimen: 


$$
\left(\frac{l^{\prime}-l_{0}^{\prime}}{l_{0}^{\prime}}\right)=\left(\frac{l-l_{0}}{l_{0}}\right)+\left(\alpha_{c}-\alpha_{0}\right)(T)
$$

Therefore, creep strain should be defined as:

$$
\varepsilon_{c}=\left(\frac{L^{\prime}-L_{0}^{\prime}}{L_{0}^{\prime}}\right)-\left(\frac{l^{\prime}-l_{0}^{\prime}}{l_{0}^{\prime}}\right)=\left(\frac{L-L_{0}}{L_{0}}\right)-\left(\frac{l-l_{0}}{l_{0}}\right)+\left(\alpha_{i}-\alpha_{c}\right)(T)-\left(\frac{\sigma}{E_{0}}\right)\left[1-\frac{E_{0}}{E_{i}}\right]
$$

\section{Discussion}

KFA have used the length change induced by thermal strain to be multiplicative to the length change induced by elastic strain i.e. the strain is sequential. The UK methodology assumes that both the load and thermal strain apply to the length measured at room temperature. For the OC-Series, the UK methodology applies a larger correction than the KFA methodology.

Whilst the corrections are small, of the order of $0.1 \%$ on creep strain, there is a significant difference between the two methodologies, which warrants further investigation. 


\section{Distribution}

\section{$\underline{\text { ORNL }}$}

Tim Burchell, ORNL

Gene Ice, ORNL

Mark Vance, ORNL

INL

Robert Bratton, INL

Dave Petti, INL

Will Windes, INL

MARAD Co. Ltd.

Mark A. Davies 